\title{
Finite Element Analyses for Seismic Shear Wall International Standard Problem
}

Manuscript Completed: February 1998

Date Published: April 1998

Prepared by

Y. J. Park, C. H. Hofmayer

Brookhaven National Laboratory

Upton, NY 11973-5000

N. C. Chokshi, NRC Project Manager

DISTRIBUTION OF THIS DOCUMENT IS UNLIMTTED

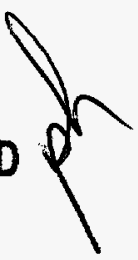

Prepared for

Division of Engineering Technology

Office of Nuclear Regulatory Research

U.S. Nuclear Regulatory Commission

Washington, DC 20555-0001

NRC Job Code W6249 


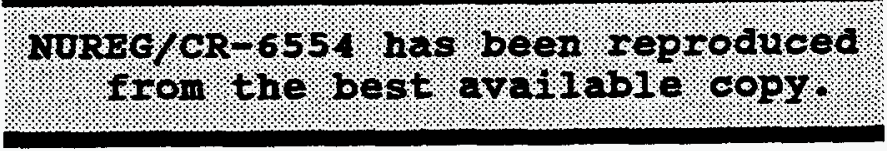




\section{DISCLAIMER}

This report was prepared as an account of work sponsored by an agency of the United States Government. Neither the United States Government nor any agency thereof, nor any of their employees, makes any warranty, express or implied, or assumes any legal liability or responsibility for the accuracy, completeness, or usefulness of any information, apparatus, product, or process disclosed, or represents that its use would not infringe privately owned rights. Reference herein to any specific commercial product, process, or service by trade name, trademark, manufacturer, or otherwise does not necessarily constitute or imply its endorsement, recommendation, or favoring by the United States Government or any agency thereof. The views and opinions of authors expressed herein do not necessarily state or reflect those of the United States Government or any agency thereof. 


\section{DISCLAIMER}

Portions of this document may be illegible electronic image products. Images are produced from the best available original document. 


\begin{abstract}
Two identical reinforced concrete $(R C)$ shear walls, which consist of web, flanges and massive top and bottom slabs, were tested up to ultimate failure under earthquake motions at the Nuclear Power Engineering Corporation's (NUPEC) Tadotsu Engineering Laboratory, Japan. NUPEC provided the dynamic test results to the OECD (Organization for Economic Cooperation and Development), Nuclear Energy Agency (NEA) for use as an International Standard Problem (ISP). The shear walls were intended to be part of a typical reactor building. One of the major objectives of the Seismic Shear Wall ISP (SSWISP) was to evaluate various seismic analysis methods for concrete structures used for design and seismic margin assessment. It also offered a unique opportunity to assess the state-of-the-art in nonlinear dynamic analysis of reinforced concrete shear wall structures under severe earthquake loadings. As a participant of the SSWISP workshops, Brookhaven National Laboratory (BNL) performed finite element analyses under the sponsorship of the U.S. Nuclear Regulatory Commission (USNRC). Three types of analysis were performed, i.e., monotonic static (push-over), cyclic static and dynamic analyses. Additional monotonic static analyses were performed by two consultants, $F$. Vecchio of the University of Toronto (UT) and F. Filippou of the University of California at Berkeley (UCB).
\end{abstract}

The analysis results by BNL and the consultants were presented during the second workshop in Yokohama, Japan in 1996. A total of 55 analyses were presented during the workshop by 30 participants from 11 different countries. The major findings on the presented analysis methods, as well as engineering insights regarding the applicability and reliability of the FEM codes are described in detail in this report. 



\section{TABLE OF CONTENTS}

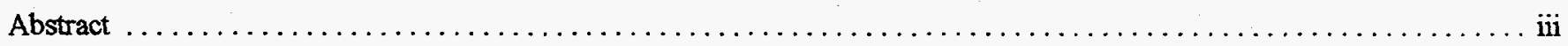

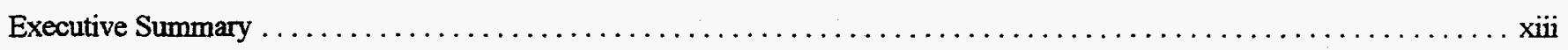

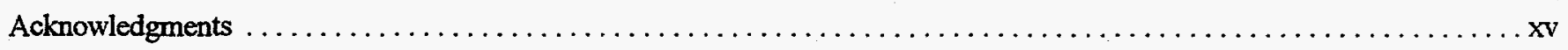

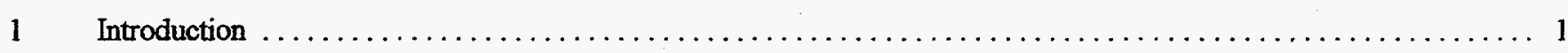

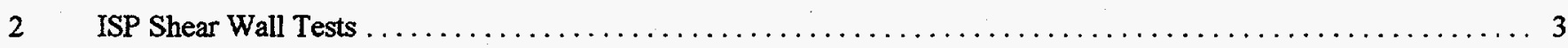

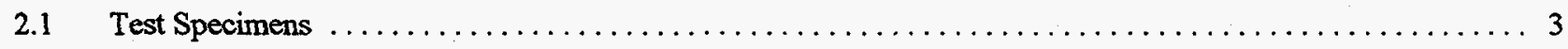

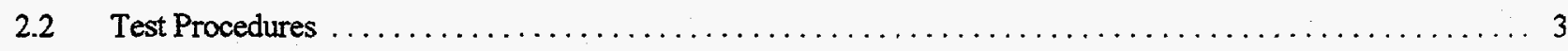

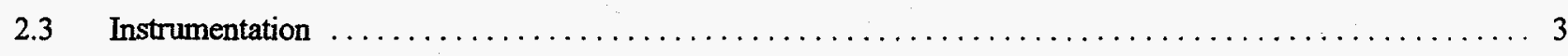

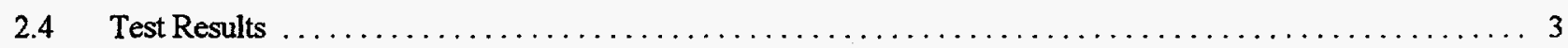

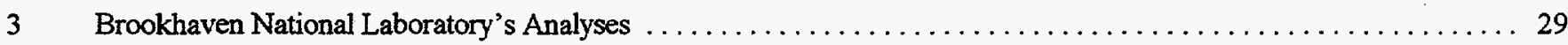

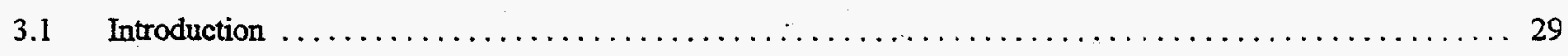

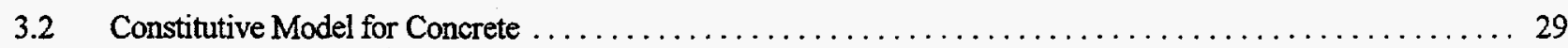

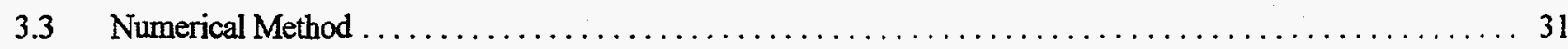

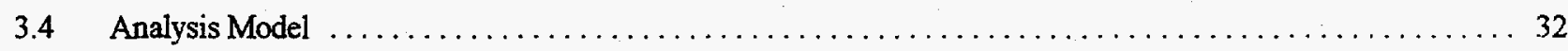

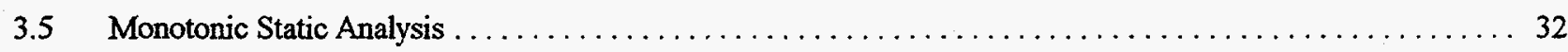

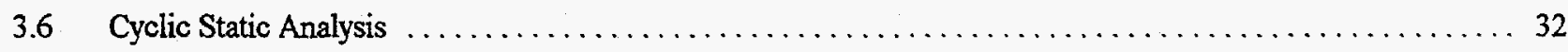

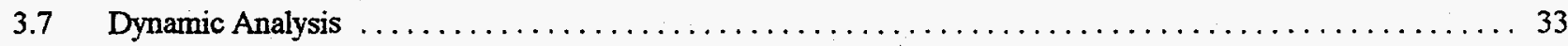

3.7.1 Linear Dynamic Analysis for RUN-1 $\ldots \ldots \ldots \ldots \ldots \ldots \ldots \ldots \ldots \ldots \ldots \ldots \ldots \ldots \ldots \ldots \ldots \ldots \ldots \ldots \ldots$

3.7.2 Nonlinear Dynamic Analysis for RUN-4 $\ldots \ldots \ldots \ldots \ldots \ldots \ldots \ldots \ldots \ldots \ldots \ldots \ldots \ldots \ldots \ldots \ldots$

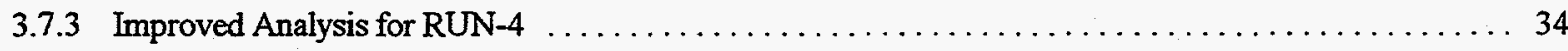

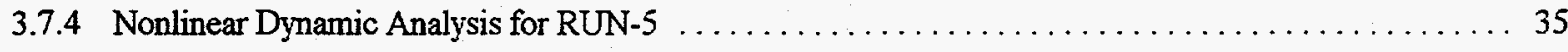




\section{CONTENTS}

(continued)

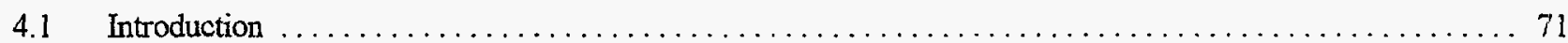

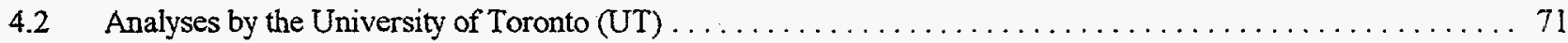

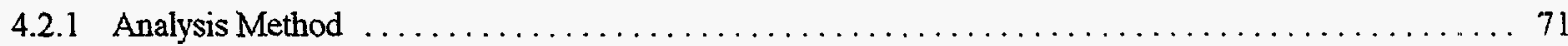

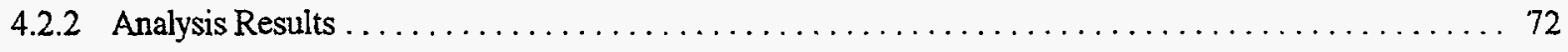

4.3 Analysis by the University of California at Berkeley $(\mathrm{UCB}) \ldots \ldots \ldots \ldots \ldots \ldots \ldots \ldots \ldots \ldots \ldots \ldots$

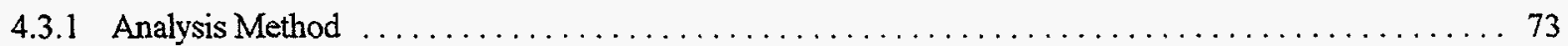

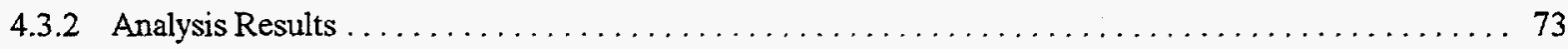

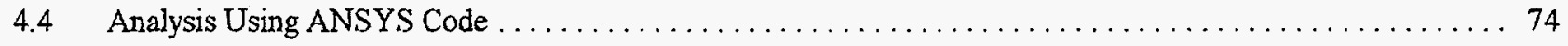

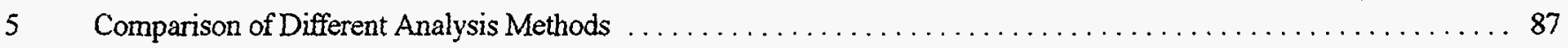

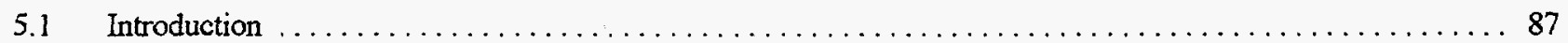

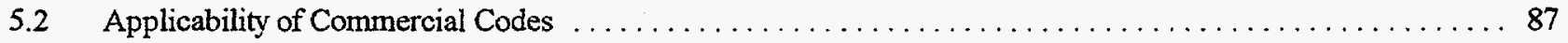

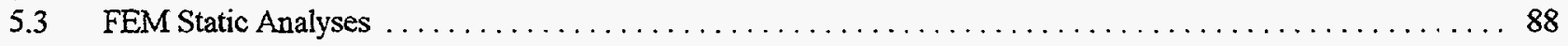

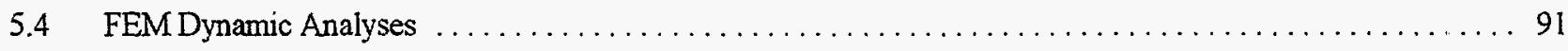

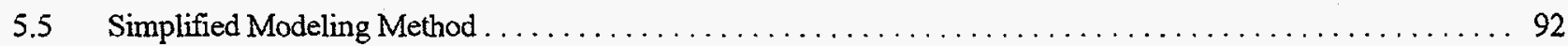

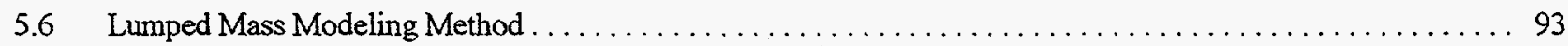

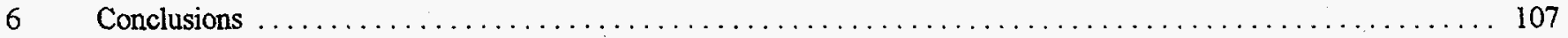

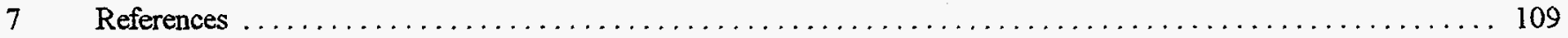




\section{CONTENTS}

(continued)

\section{Appendices}

A Static Finite Element Analysis of Seismic Shear Wall ISP (by F. Vecchio) $\ldots \ldots \ldots \ldots \ldots \ldots \ldots \ldots \ldots \ldots \ldots$

B Monotonic Load to Collapse Analysis of NUPEC Shear Wall ISP Specimen (by F. Filippou and T. Balan) ........ B-1

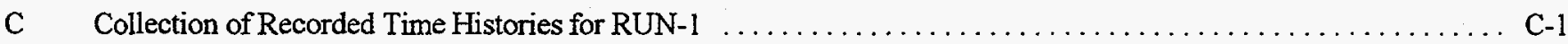

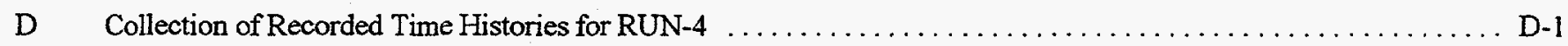

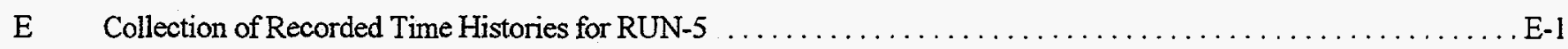

\section{Figures}

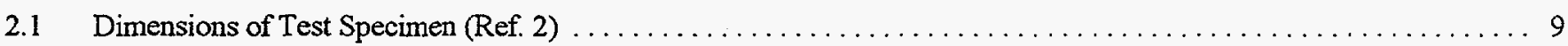

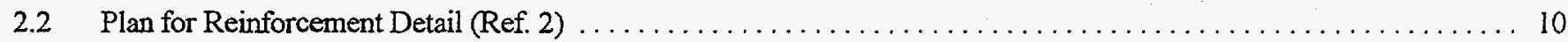

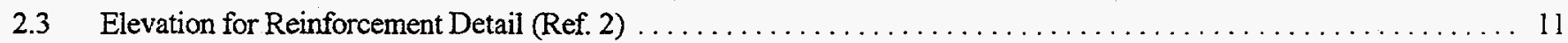

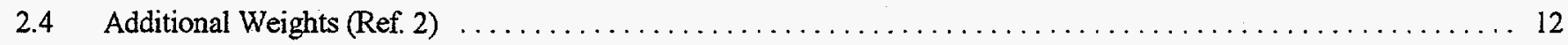

2.5 Stress-Strain Relationships of Concrete of Web and Flange Walls (Ref. 2$) \ldots \ldots \ldots \ldots \ldots \ldots \ldots \ldots \ldots$

2.6 Accelerogram of Horizontal Input Table Motion, AXB, Recorded During RUN-5 $\ldots \ldots \ldots \ldots \ldots \ldots \ldots \ldots$

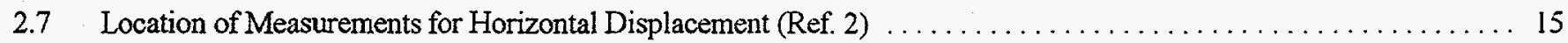

2.8 Location of Measurement for Relative Vertical Displacement (Ref. 2$) \ldots \ldots \ldots \ldots \ldots \ldots \ldots \ldots \ldots \ldots \ldots \ldots$

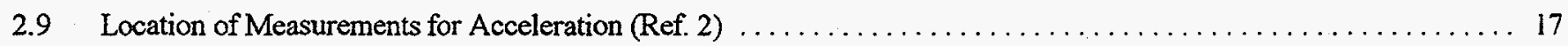

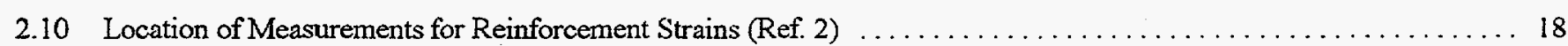

2.11 Maximum Inertial Force-Maximum Displacement Relationsihps (Ref. 2) $\ldots \ldots \ldots \ldots \ldots \ldots \ldots \ldots \ldots \ldots$

2.12 Inertial Force-Top Slab Horizontal Displacement Relationships (Ref. 2$) \ldots \ldots \ldots \ldots \ldots \ldots \ldots \ldots \ldots \ldots \ldots$

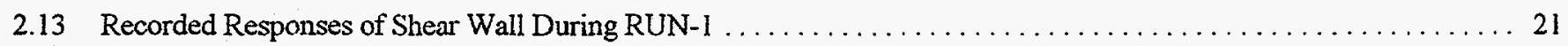

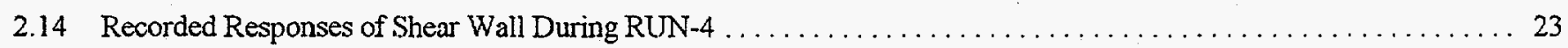

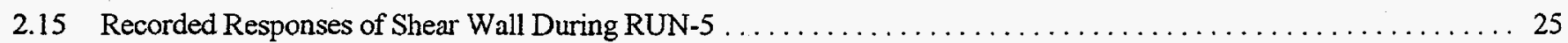

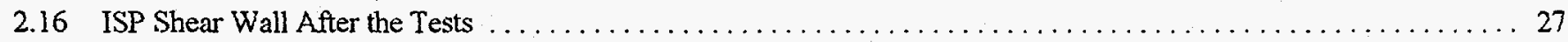




\section{CONTENTS}

(continued)

Page

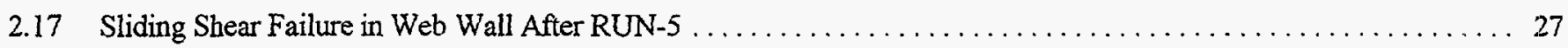

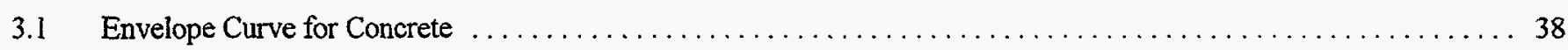

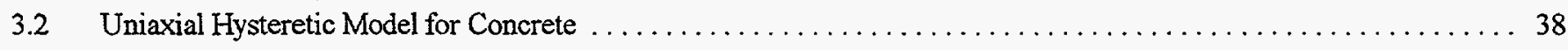

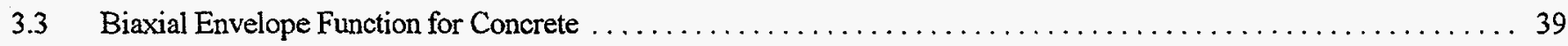

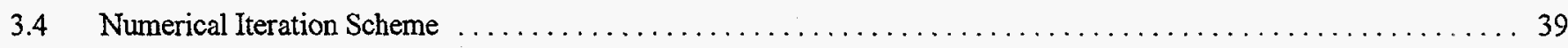

3.5 Additional Consideration for Uniaxial Hysteretic Model for Concrete $\ldots \ldots \ldots \ldots \ldots \ldots \ldots \ldots \ldots \ldots \ldots$

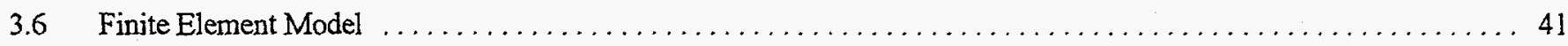

3.7 Load-Deformation Relationships from Monotonic Static Analyses $\ldots \ldots \ldots \ldots \ldots \ldots \ldots \ldots \ldots \ldots \ldots \ldots \ldots . \ldots 2$

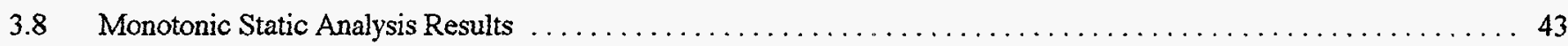

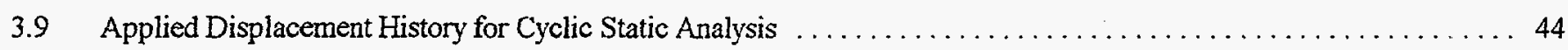

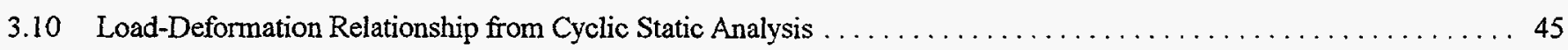

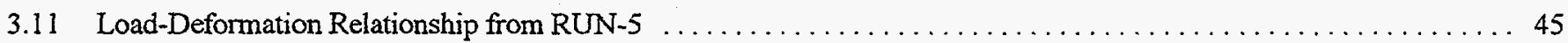

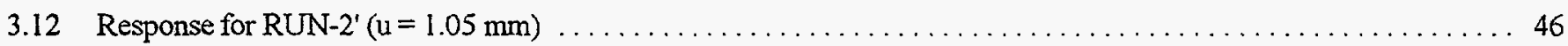

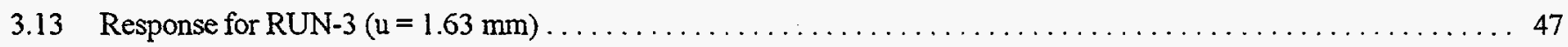

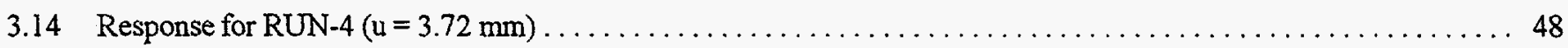

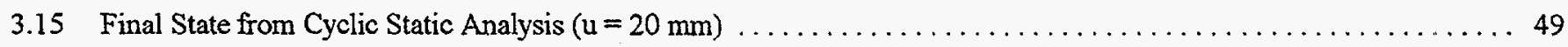

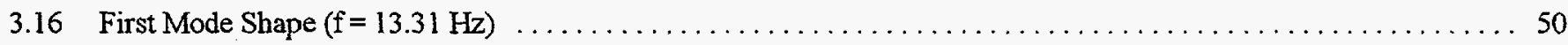

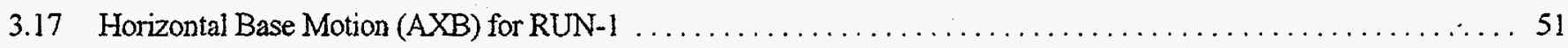

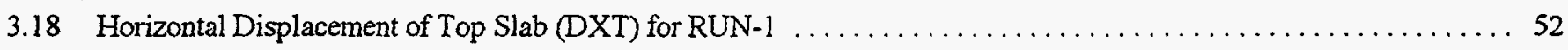

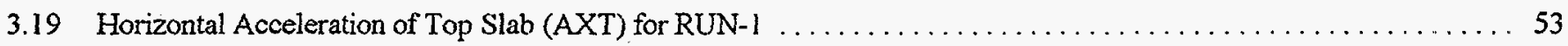

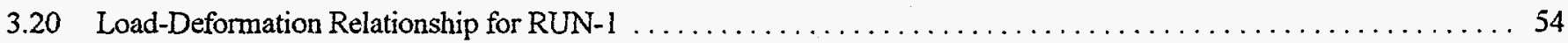

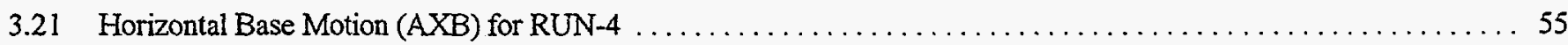

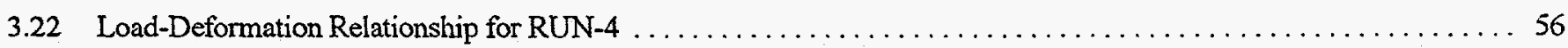

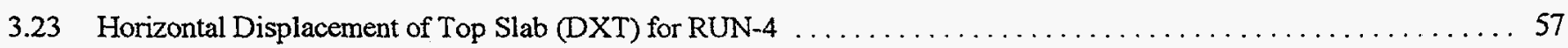




\section{CONTENTS}

(continued)

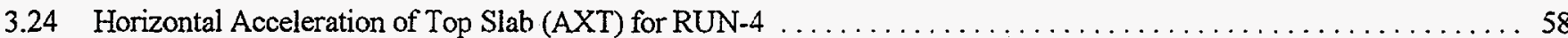

3.25 Vertical Displacement of Right Side Top Slab (DVRF) for RUN-4, Analysis Result $\ldots \ldots \ldots \ldots \ldots \ldots \ldots \ldots$. 59

3.26 Relative Vertical Displacement of Flange Wall (DV16) for RUN-4, Test Result . . . . . . . . . . . . . . . . . . 59

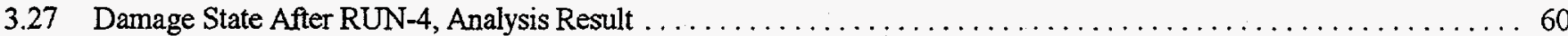

3.28 Load-Deformation Relationship from Improved Analysis for RUN-4 $\ldots \ldots \ldots \ldots \ldots \ldots \ldots \ldots \ldots \ldots \ldots \ldots \ldots$

3.29 Horizontal Displacement of Top Slab (DXT) from Improved Analysis for RUN $-4 \ldots \ldots \ldots \ldots \ldots \ldots \ldots \ldots \ldots$

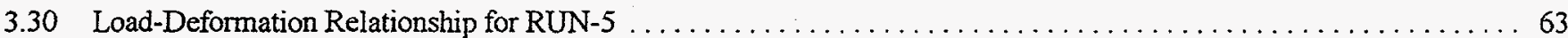

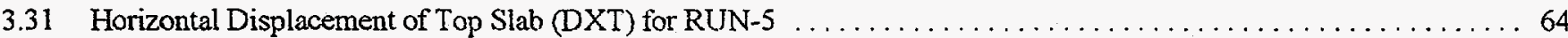

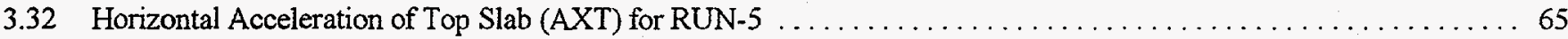

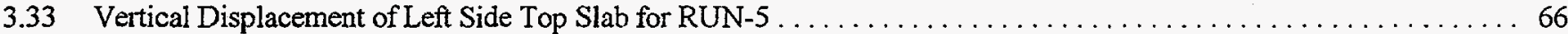

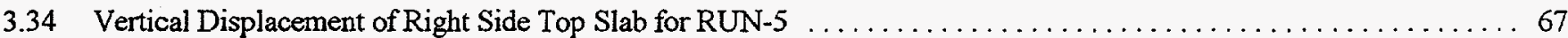

3.35 Vertical Acceleration of Left Side Top Slab (AZTL) for RUN-5 $\ldots \ldots \ldots \ldots \ldots \ldots \ldots \ldots \ldots \ldots \ldots \ldots$

3.36 Vertical Acceleration of Right Side Top Slab (AZTR) for RUN $-5 \ldots \ldots \ldots \ldots \ldots \ldots \ldots \ldots \ldots \ldots \ldots \ldots \ldots$

$4.1 \quad$ Uniaxial Stress-Strain Envelope Curve for Cracked Concrete (by UT) $\ldots \ldots \ldots \ldots \ldots \ldots \ldots \ldots \ldots \ldots \ldots \ldots$

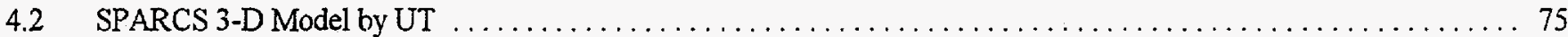

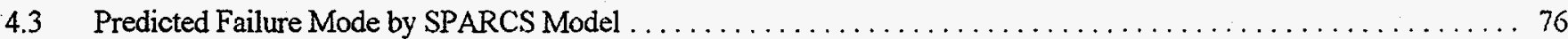

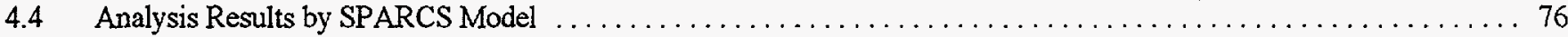

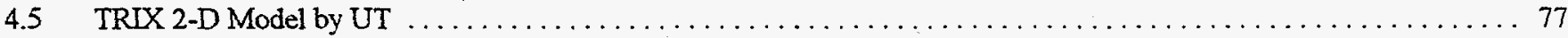

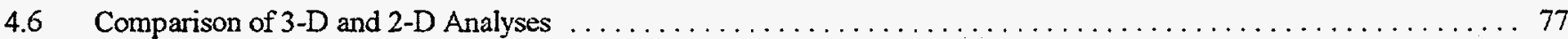

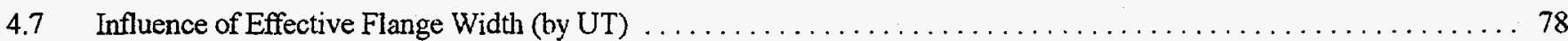

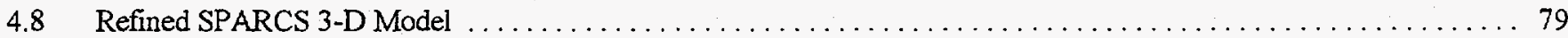

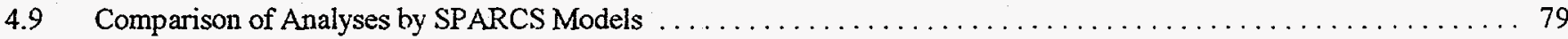

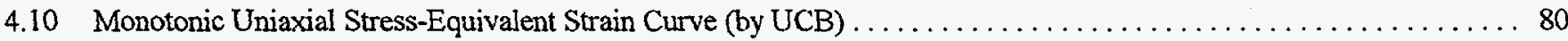

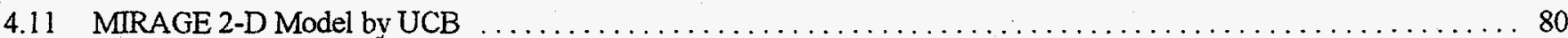




\section{CONTENTS}

(continued)

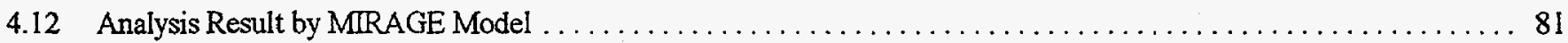

4.13 Predicted Crack Patterns in the Web and Flange Walls by MTRAGE Model $\ldots \ldots \ldots \ldots \ldots \ldots \ldots \ldots \ldots \ldots$

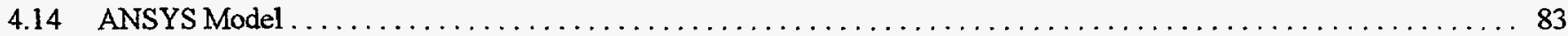

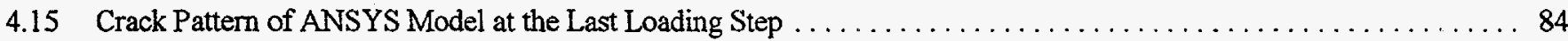

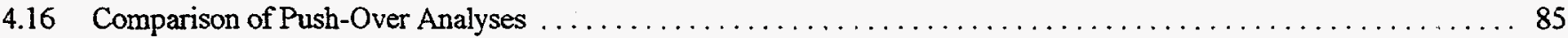

$5.1 \quad$ Push-Over Analysis Results Using Commercially Available FEM Codes $\ldots \ldots \ldots \ldots \ldots \ldots \ldots \ldots \ldots \ldots \ldots$

5.2 Comparison of Load-Displacement Relationship Obtained from Monotonic Loading Analyses (Ref. 2) . . . . . . . . . 99

5.3 Distribution of Displacement at Loads of Every $10 \mathrm{kN}$ Obtained from Analyses (Ref. 2) $\ldots \ldots \ldots \ldots \ldots$

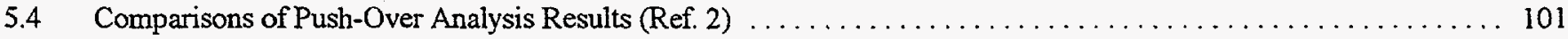

5.5 Uniaxial Hysteretic Models for Concrete for FEM Dynamic Analyses $($ Ref. 2$) \ldots \ldots \ldots \ldots \ldots \ldots \ldots \ldots \ldots$

5.6 Typical Example of Simplified Hysteretic Model for Concrete $\ldots \ldots \ldots \ldots \ldots \ldots \ldots \ldots \ldots \ldots \ldots \ldots \ldots \ldots \ldots \ldots$

$5.7 \quad$ Hysteretic Models Used for Lumped Mass Models (Ref. 2$) \quad \ldots \ldots \ldots \ldots \ldots \ldots \ldots \ldots \ldots \ldots \ldots \ldots \ldots \ldots \ldots$

\section{Tables}

$2.1 \quad$ Tensile Strength Test Results of D6 Deformed Bar (Ref. 2) $\ldots \ldots \ldots \ldots \ldots \ldots \ldots \ldots \ldots \ldots \ldots \ldots \ldots \ldots$

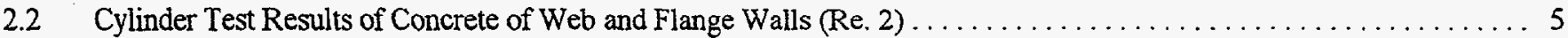

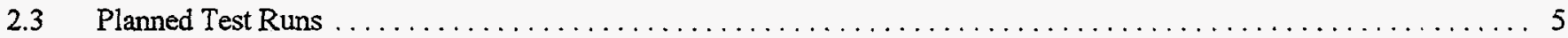

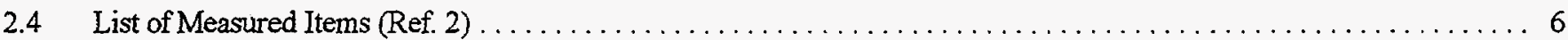

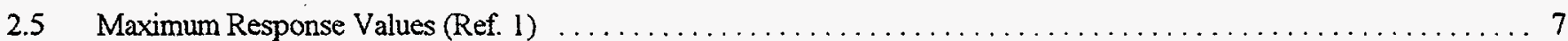

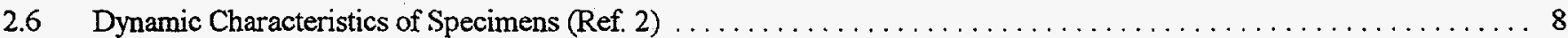

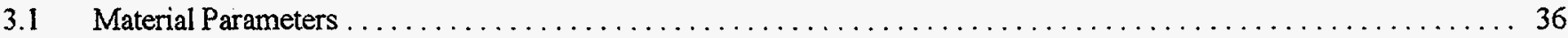

3.2 Summary of Calculated Results (Monotonic Static Analysis) $\ldots \ldots \ldots \ldots \ldots \ldots \ldots \ldots \ldots \ldots \ldots \ldots \ldots$

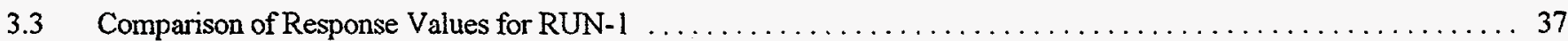

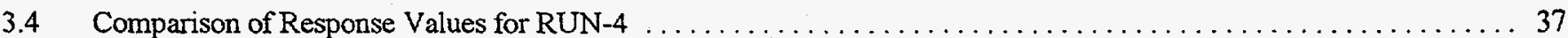




\section{CONTENTS}

(continued)

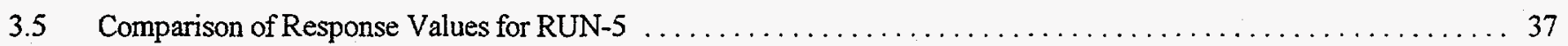

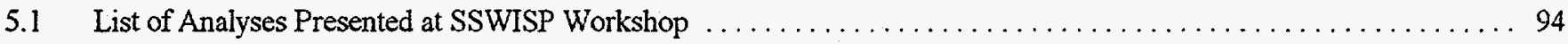

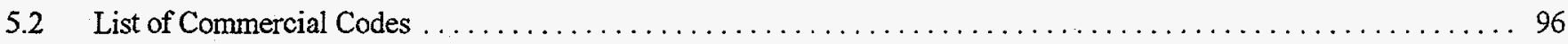

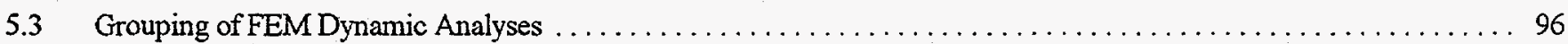

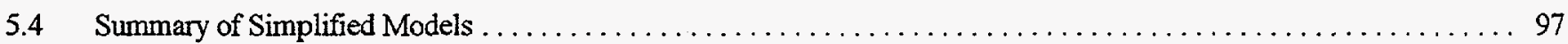

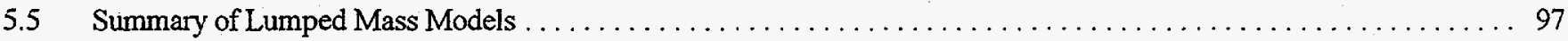





\section{EXECUTIVE SUMMARY}

Two identical reinforced concrete (RC) shear walls, which consists of web, flanges and massive top and bottom slabs, were tested up to ultimate failure under earthquake motions at the Nuclear Power Engineering Corporation's (NUPEC) Tadotsu Engineering Laboratory, Japan. NUPEC provided the dynamic test results to the OECD (Organization for Economic Cooperation and Development), Nuclear Energy Agency (NEA) for use as an International Standard Problem (ISP). The shear walls were intended to be part of a typical reactor building. One of the major objectives of the Seismic Shear Wall ISP (SSWISP) was to evaluate various seismic analysis methods for concrete structures used for design and seismic margin assessment. It also offered a unique opportunity to assess the state-of-the-art in nonlinear dynamic analysis of reinforced concrete shear wall structures under severe earthquake loadings.

The detailed information on the ISP tests, including the test specimens, test procedures and dynamic test results, was provided during the first SSWISP workshop in Paris in 1994. The shear wall specimens were designed to fail in shear. The tests were conducted at six amplitude levels, starting from the lower elastic run to the ultimate failure. During the highest amplitude test run, the shear wall failed catastrophically due to a sudden sliding shear failure. Before each test run, a small-amplitude vibration test was performed to evaluate the vibration frequency and the equivalent viscous damping values.

As a participant in the SSWISP workshops, Brookhaven National Laboratory (BNL) performed finite element analyses under the sponsorship of the U.S. Nuclear Regulatory Commission (USNRC). Three types of analyses were performed, i.e., monotonic static (push-over), cyclic static, and dynamic analyses. No significant differences were observed in the predicted envelope curves of the load-displacement relationship and the final failure modes between the three types of analyses. In addition, the nonlinear analyses accurately predicted the dynamic response of the shear walls, as well as the timing of the observed sliding shear failure.

Additional monotonic static analyses were performed by two consultants, F. Vecchio of the University of Toronto (UT) and F. Filippou of the University of California at Berkeley (UCB). The analyses by UT used 3-D brick elements, whereas 2-D solid elements were used in the analysis by UCB. By using 3-D elements, UT's model accurately predicted the location of the sliding shear failure since the additional confinement at the boundaries was accounted for.

The analysis results by BNL and the consultants were presented during the second SSWISP workshop in Yokohama, Japan in 1996. A total of 55 analyses were presented during the workshop by 30 participants from 11 different countries. The submitted analyses were classified as FEM static, FEM dynamic, simplified modeling method and lumped mass modeling method. The highlight of the workshop was the FEM dynamic analyses. The SSWISP workshop probably was the first occasion that the application of nonlinear FEM dynamic analysis to shear wall structures was discussed as the main theme in an international conference. For many participants, the SSWISP was their first opportunity to perform this type of nonlinear dynamic analysis, and a wide variety of technical issues were discussed for improving the prediction accuracy. Several participants presented remarkable analysis results using originally developed in-house codes, which were at various stages of development. The major findings on the presented analysis methods, as well as engineering insights regarding the applicability and reliability of the FEM codes are described in detail in this report.

Presently, the structural design methods of reinforced concrete structures used in the nuclear industry have been developed and evaluated based on a set of laboratory test data. Efforts have been on-going for the last two decades to develop powerful analysis tools which could improve accuracy and eliminate or significantly reduce the need for additional costly laboratory testing as new issues arise. The SSWISP has provided valuable test data to evaluate such analysis tools, as well as to develop new analysis approaches.

The specific objectives of this study were to perform nonlinear dynamic analyses of R.C. shear wall structures under severe earthquake loading, to identify limitations of the currently available analysis methods, and to collect information on the 


\section{Executive Summary}

analysis methods worldwide. These objectives have been achieved through a series of analyses using both in-house and commercial codes, and comparisons with test results as well as with analysis results performed by other SSWISP workshop participants. The SSWISP workshop provided a unique opportunity to review the reliability and applicability of various analysis methods to predict the dynamic behavior of shear wall structures under severe earthquake loads. 


\section{ACKNOWLEDGMENTS}

The Seismic Shear Wall International Standard Problem (SSWISP) was conducted by Nuclear Power Engineering Corporation (NUPEC) with the sponsorship of the Ministry of International Trade and Industry (MITI), and it was organized by the OECD NEA Committee on the Safety of Nuclear Installations (CSNI).

This research program was performed with the sponsorship of the Office of Nuclear Regulatory Research of the U.S. NRC. Dr. N. Chokshi was the NRC Project Manager.

The authors wish to acknowledge the NUPEC staff for providing the information on the test results of ISP shear walls, and organizing two SSWISP workshops. Efforts made by Dr. A. Miller of OECD and the SSWISP Committee chaired by Prof. H. Aoyama to bring this ISP to a highly successful conclusion are deeply appreciated.

The authors wish to thank two consultants, Prof. F. Vecchio of the University of Toronto and Prof. F. Filippou of the University of California at Berkeley, for performing independent analysis using their own in-house codes.

The authors also wish to thank Dr. K. Akino of NUPEC and Dr. P. Bezler of BNL for their review comments and Mr. J. Braverman for analytical support in applying the ANSYS code.

The authors would like to express special thanks to B. Roland and A. Melocoton for their secretarial help throughout this program and dedication to the preparation of this report. 


\section{INTRODUCTION}

The finite element method (FEM) is now widely used as a practical structural design tool to analyze complex structures in both the nuclear and non-nuclear industries. In the seismic design of shear wall structures, e.g., nuclear reactor buildings, a linear FEM analysis is frequently used to quantify the stresses under the design loading condition. The final design decisions, however, are still based on empirical design rules established over decades from accumulated laboratory test data.

Over the last two decades, the application of nonlinear FEM to reinforced concrete structures has been considered an alternate analysis/design tool for the seismic structural design. In recent years, significant improvements have been made in Europe, Japan and the United States in both the numerical techniques and the development of constitutive model for concrete. Although many research results are available in open publication, engineers generally do not have easy access to the computer codes as they are mostly proprietary in-house codes. Commercial finite element codes, such as ANSYS and ABAQUS, also have some options for the nonlinear analysis of R.C. structures, however, most are limited to the consideration of monotonic push over type loading only. Under seismic loadings, structural components are subjected to repeated cyclic loading reversals. Most available commercial codes cannot deal with this type of loading for reinforced concrete structures. Under such loading, repeated shear stress reversals occur and the concrete experiences a combination of cracking and compression softening/crushing with the principal stress directions constantly rotating. Improvements in the constitutive model of concrete material are needed to model this phenomena and produce a more reliable result for various concrete structures. The Seismic Shear Wall International Standard Problem (SSWISP) offered a unique opportunity to perform state-of-the-art nonlinear dynamic analyses of shear wall structures under earthquake loadings, as well as, to collect information on the currently available analysis methods worldwide.

Nuclear Power Engineering Corporation (NUPEC) offered the dynamic test results of shear wall structures to the OECD/NEA/CSNI (Organization for Economic Cooperation and Development/Nuclear Energy Agency/Committee on the Safety of Nuclear Installation) for use as an International Standard Problem (ISP). Two identical shear walls, which consisted of a web ( 3 meter wide, $75 \mathrm{~mm}$ thick, and 2.02 meter high), flanges ( 2.98 meter wide, $100 \mathrm{~mm}$ thick and 2.02 meter high), and massive top and bottom slabs, were tested to ultimate failure under earthquake motions at NUPEC's Tadotsu Engineering Laboratory. The shear walls simulated a part of a typical reactor building.

The test results and detailed information on the test conditions were made available during the first workshop in Paris in 1994 (Ref. 1), and the participants were asked to perform structural analyses to reproduce the test results. As a participant in this effort, Brookhaven National Laboratory (BNL) performed finite element analyses under the sponsorship of the U.S. Nuclear Regulatory Commission (USNRC). Two consultants, F. Vecchio of the University of Toronto (UT) and F. Filippou of the University of California at Berkeley (UCB), also performed finite element analyses. The analysis results by BNL/NRC and the consultants were presented during the second workshop in Yokohama, Japan in 1996 (Ref. 3). The analysis results of all the participants of the SSWISP Workshop are summarized in the comparison report published by OECD/NEA (Ref. 17).

The objectives of this study are to perform nonlinear dynamic analyses of R.C. shear wall structures under severe earthquake loading based on the best knowledge currently available in the areas of material constitutive modeling and numerical procedure, to identify limitations of the analysis methods, and to collect information on the currently available analysis methods worldwide.

This report presents BNL's and consultants' analysis results. The findings and information obtained during the workshops and detailed descriptions of promising analysis methods presented by other participants are summarized. 


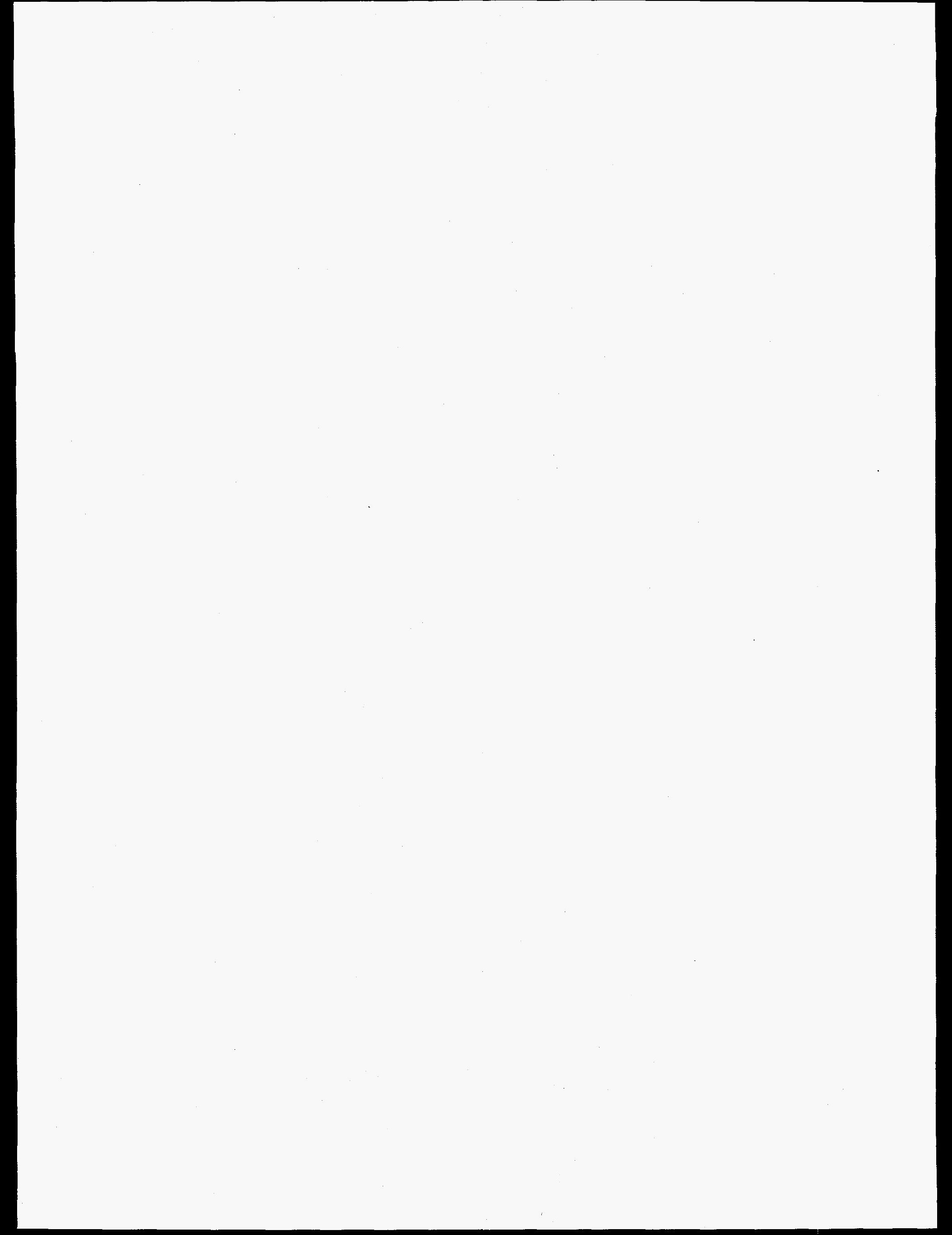




\section{ISP SHEAR WALL TESTS}

\subsection{Test Specimens}

A complete description of the ISP shear wall tests can be found in References 1 and 2 (NUPEC). Two identical shear wall specimens (called U-1 and U-2) were constructed and dynamically tested using the same input motions to confirm the reliability and reproducibility of the test. Figures. 2.1 through 2.4 provide detailed descriptions of the specimen. The shear walls are intended to be part of a typical reactor building, and consist of a web, two flanges, massive top and bottom slabs and additional weights. The wall has a shear span ratio of 0.8 , and together with a high bending strength provided by the two massive flanges, the design is intended to be predominantly a shear failure type.

The reinforcement ratio is $1.2 \%$ for both web and flanges in both the horizontal and vertical directions except with additional vertical reinforcement at the intersection between the web and flange walls (see Figure 2.2). For the reinforcement, D6 deformed rebars (nominal diameter $6.35 \mathrm{~mm}$ ) were used.

For additional weight, lead blocks with a total of 92.9 tonf were fixed to the top slab. The total weight of the top slab was 122.0 tonf, and the average vertical stress in the walls is $0.15 \mathrm{~kg} / \mathrm{mm}^{2}$.

Material test results were also made available as summarized in Tables 2.1 and 2.2, and in Figure 2.5.

\subsection{Test Procedures}

NUPEC's large shake table at the Tadotsu Engineering Laboratory was used for the dynamic tests. The table has a 1.000 tonf maximum load capacity, a 3000 tonf horizontal maximum excitation capacity $(3,300$ ton $\mathrm{f}$ vertically) and can accommodate a $15 \mathrm{~m}$ by $15 \mathrm{~m}$ sized specimen. It is a two-directional (horizontal and vertical) vibration table, although only horizontal motions were applied during the ISP tests.

Figure 2.6 shows the acceleration time history and the response spectra of the input horizontal motion (AXB) recorded during the maximum amplitude test run (RUN-5). In the dynamic tests, the amplitude of the input motion was the only parameter varied. A total of five (5) test runs were planned including RUN-1 for the elastic responses and RUN-5 for the ultimate response, as shown in Table 2.3 .

\subsection{Instrumentation}

Figures 2.7 through 2.10 and Table 2.6 show the location of the test instrumentation which consisted of displacement transducers, accelerometers and strain gages. To represent the overall response of the shear walls, the average of DXT1 and DXT2 is used for the horizontal relative displacement (see Figure 2.7), and the average of AXT1 and AXT2 is used for the horizontal acceleration (see Figure 2.9). The horizontal inertia force is estimated by multiplying the value of AXT (average of AXT1 and AXT2) by the total weight of the top slab, 122 tonf. The sampling time of the data acquisition was 0.001 second.

\subsection{Test Results}

The peak responses of the two shear walls, $U-1$ and U-2, are summarized in Figure 2.11 in terms of the maximum inertia force and displacement relationship. The U-2 specimen produced slightly lower responses compared with the U-1 specimen. 
ISP Shear Wall Tests

An additional RUN-2' was performed to obtain the response right after the shear cracking run for the U-1 specimen, as indicated in Figure 2.11.

Table 2.5 summarizes the recorded peak response values. During RUN-5 for the U-1 specimen, the top horizontal displacement (UXT) far exceeded the measurable range of the displacement transducer of $20 \mathrm{~mm}$ due to a sudden sliding shear failure. For all the analyses described in the following chapters, the response results of the U-1 specimen are used exclusively.

Figure 2.12 shows the top-slab horizontal force-deformation relationships for all the test runs of the U-1 specimen. It can be observed that RUN-1 and RUN-2 are in the elastic range; RUN-2' and RUN-3 are in moderately nonlinear ranges; significant nonlinear responses start to appear in RUN-4; and the ultimate response is shown in RUN-5. Figures 2.13 through 2.15 show the force-deformation relationship of the top slab and the recorded responses of the top and bottom slabs for RUN-1, RUN-4 and RUN-5. More complete collections of the recorded time histories can be found in Appendices C, D and E of this report.

Initial shear cracks were found at the mid-lower portion of the web wall after RUN-2'. Horizontal bending cracks in the flange walls were found after RUN-4. During RUN-5, a sliding shear failure occurred at the bottom of the web wall as shown in Figures 2.16 and 2.17.

Before each test run, a small-amplitude vibration test was performed to evaluate the vibration frequency and the equivalent viscous damping values. The results are listed in Table 2.6. When the specimens were in the elastic range, the calculated damping value was slightly higher than $1 \%$ of critical. 
Table 2.1 Tensile strength test results of D6 deformed bar (Ref. 2)

\begin{tabular}{|c|c|c|c|c|c|}
\hline $\begin{array}{c}\text { JIS } \\
\text { Designation }\end{array}$ & Sample No. & $\begin{array}{c}\text { Yield } \\
\text { Strength } \\
\left(\mathrm{kgf} / \mathbf{m m}^{2}\right)\end{array}$ & $\begin{array}{c}\text { Tensile } \\
\text { Strength } \\
\left(\mathrm{kgf} / \mathbf{m m}^{2}\right)\end{array}$ & $\begin{array}{c}\text { Modulus of } \\
\text { Elasticity } \\
\left.\text { (x } 10^{3} \mathrm{kgf} / \mathrm{mm}^{2}\right)\end{array}$ & $\begin{array}{c}\text { Elongation } \\
(\%)\end{array}$ \\
\hline \multirow{4}{*}{$D 6^{* 11}$} & 1 & 38.7 & 49.8 & 18.9 & $* *$ \\
\hline & 2 & 39.3 & 49.1 & 18.6 & 29.5 \\
\hline & 3 & 39.3 & 49.5 & 18.9 & 28.7 \\
\hline & mean & 39.1 & 49.5 & 18.8 & 29.1 \\
\hline $\begin{array}{l}\text { Nominal } \\
\text { Nominal } \\
\text { Nominal }\end{array}$ & $\begin{array}{l}\text { ection area: } \\
\text { ter length: } \\
\text { er: }\end{array}$ & & & \multicolumn{2}{|c|}{ (** Break outside gauge length) } \\
\hline
\end{tabular}

Table 2.2 Cylinder test results of concrete of web and flange walls (Ref. 2)

\begin{tabular}{|c|c|c|c|c|c|}
\hline \multicolumn{2}{|c|}{ Specimen } & $\begin{array}{c}\text { Compressive } \\
\text { Strength } \\
\left(\text { kgf/mm }^{2}\right)\end{array}$ & $\begin{array}{c}\text { Modulus of } \\
\text { Elasticity } \\
\left(\mathbf{k g f}^{* 2} / \mathbf{m m}^{2}\right)\end{array}$ & $\begin{array}{c}\text { Poisson's } \\
\text { ratio }^{-3)}\end{array}$ & $\begin{array}{c}\text { Splitting } \\
\text { Tens. Strength } \\
\left(\mathbf{k g f}^{2} / \mathbf{m m}^{2}\right)\end{array}$ \\
\hline \multirow{3}{*}{ U-1 } & No. $1^{* 1)}$ & 2.88 & 23.2 & 0.167 & 0.244 \\
\cline { 2 - 6 } & No. 2 & 3.04 & 22.8 & 0.157 & 0.221 \\
\cline { 2 - 6 } & No. 3 & 2.83 & 24.1 & 0.139 & 0.219 \\
\cline { 2 - 6 } & mean & 2.92 & 23.4 & 0.155 & 0.228 \\
\hline
\end{tabular}

*1) Material test specimen geometry: Cylinder with $100 \mathrm{~mm}$ dia. and $200 \mathrm{~mm}$ height

*2) Secant modulus of stiffness at $1 / 3$ value of compressive strength

*3) Ratio at $1 / 3$ value of compressive strength

Table 2.3 Planned test runs

\begin{tabular}{|c|c|c|}
\hline Name of Test Run & Target Amplitude Level & Comments \\
\hline RUN-1 & About $0.05 \mathrm{~g}$ & Elastic Response \\
RUN-2 & $0.05 \mathrm{~g}-0.1 \mathrm{~g}$ & Shear Crack Initiation \\
RUN-3 & $0.25 \mathrm{~g}-0.3 \mathrm{~g}$ & 3 times of RUN-2 \\
RUN-4 & $0.4 \mathrm{~g}-0.5 \mathrm{~g}$ & $\gamma=2 / 1000 \mathrm{rad}$ \\
RUN-5 & $0.6 \mathrm{~g}-0.7 \mathrm{~g}$ & Uitimate Strength \\
\hline
\end{tabular}


ISP Shear Wall Tests

Table 2.4 List of measured items (Ref. 2)

\begin{tabular}{|c|c|c|}
\hline Measured Item & Direction & Number of Measurements \\
\hline - Horizontal displacement $" 1\rangle$ & $\begin{array}{l}X \\
Y\end{array}$ & $\begin{array}{l}2 \\
2\end{array}$ \\
\hline - Vertical displacement ${ }^{* 2}$ & 2 & 16 \\
\hline - Acceleration & $\begin{array}{r}\text { Top slab X } \\
Y \\
Z\end{array}$ & $\begin{array}{l}2 \\
2 \\
4\end{array}$ \\
\hline & $\begin{array}{r}\text { Base slab X } \\
Y \\
Z\end{array}$ & $\begin{array}{l}2 \\
2 \\
4\end{array}$ \\
\hline - Rebar strain & $\begin{array}{l}\text { Web:horizontal } \\
\text { Web:vertical } \\
\text { Flange:vertical }\end{array}$ & $\begin{array}{c}11 \\
9 \\
24\end{array}$ \\
\hline \multicolumn{2}{|c|}{ Total } & 80 \\
\hline
\end{tabular}

*1)

Relative horizontal displacement between the top slab and the base slab

*2) Relative vertical displacements of segmental parts of flange wails 
Table 2.5 Maximum response values (Ref. 1)

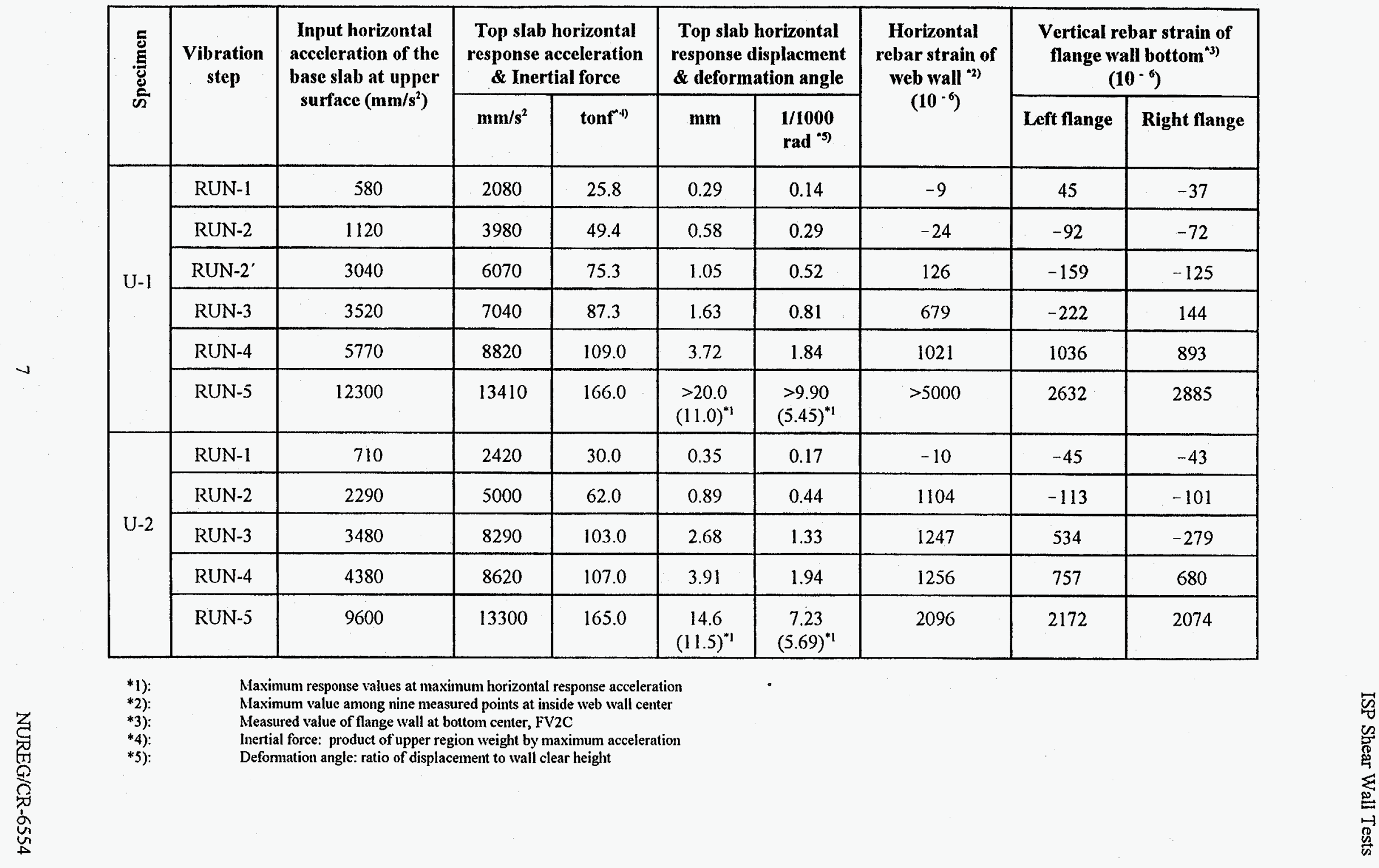


Table 2.6 Dynamic characteristics of specimens (Ref. 2)

\section{(U-1 Specimen)}

\begin{tabular}{|l|c|c|}
\hline & $f_{0}(\mathrm{~Hz})$ & $\mathrm{h}_{\mathrm{eq}}(\%)$ \\
\hline Before RUN-1 & 13.2 & 1.1 \\
\hline Before RUN-3 & 11.3 & 2.5 \\
\hline Before RUN-4 & 9.0 & 3.0 \\
\hline Before RUN-5 & 7.7 & 4.0 \\
\hline
\end{tabular}

(U-2 Specimen)

\begin{tabular}{|l|c|c|}
\hline & $\mathrm{f}_{0}(\mathrm{~Hz})$ & $\mathrm{h}_{\mathrm{eq}}(\%)$ \\
\hline Before RUN-1 & 13.1 & 1.2 \\
\hline Before RUN-3 & 11.3 & 2.6 \\
\hline Before RUN-4 & 8.3 & 4.2 \\
\hline Before RUN-5 & 7.1 & 4.2 \\
\hline
\end{tabular}

$\mathrm{f}_{0}$ : Natural frequency

$\mathrm{h}_{\mathrm{eq}}$ : Equivalent damping ratio 
ISP Shear Wall Tests
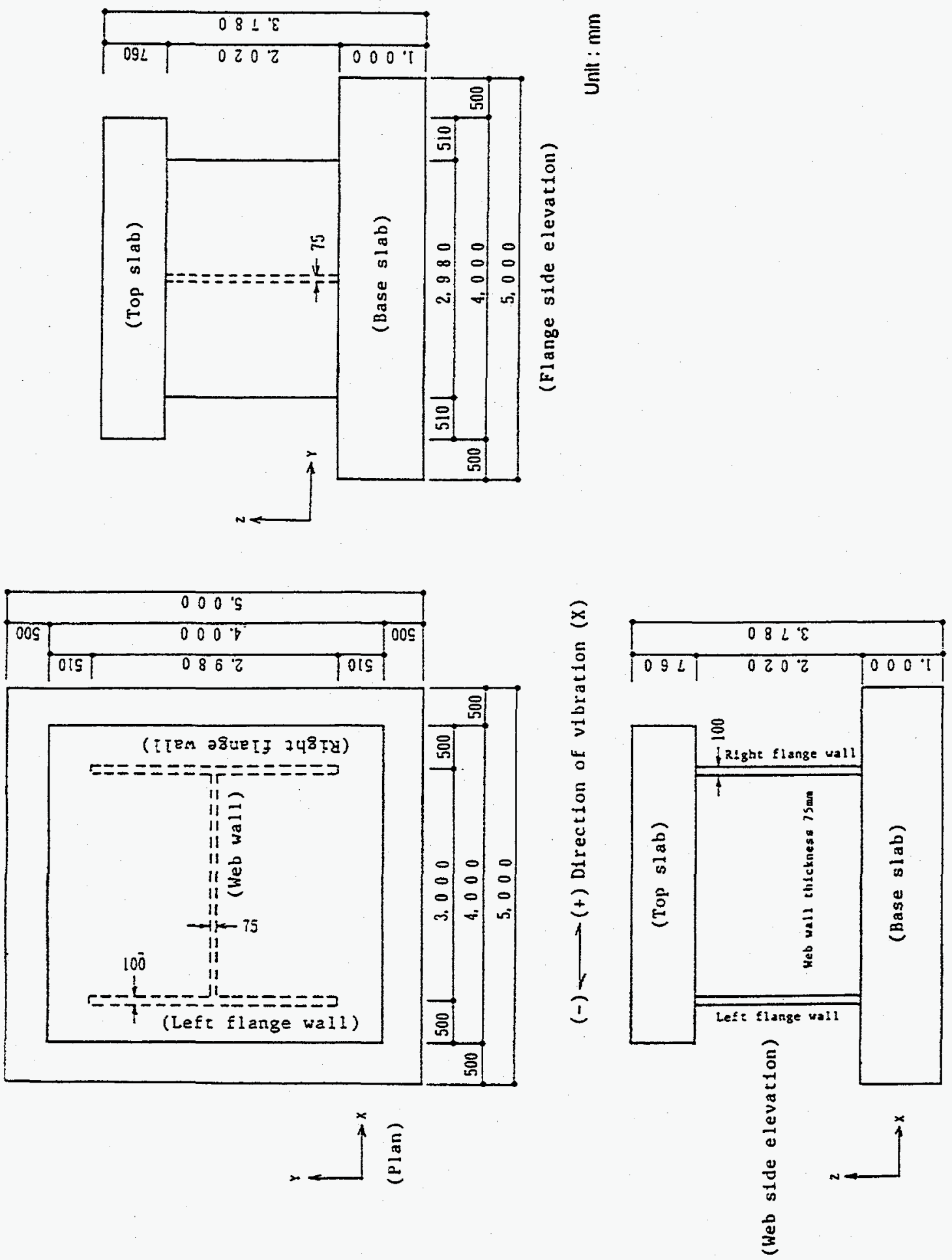



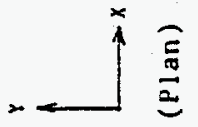


ISP Shear Wall Tests

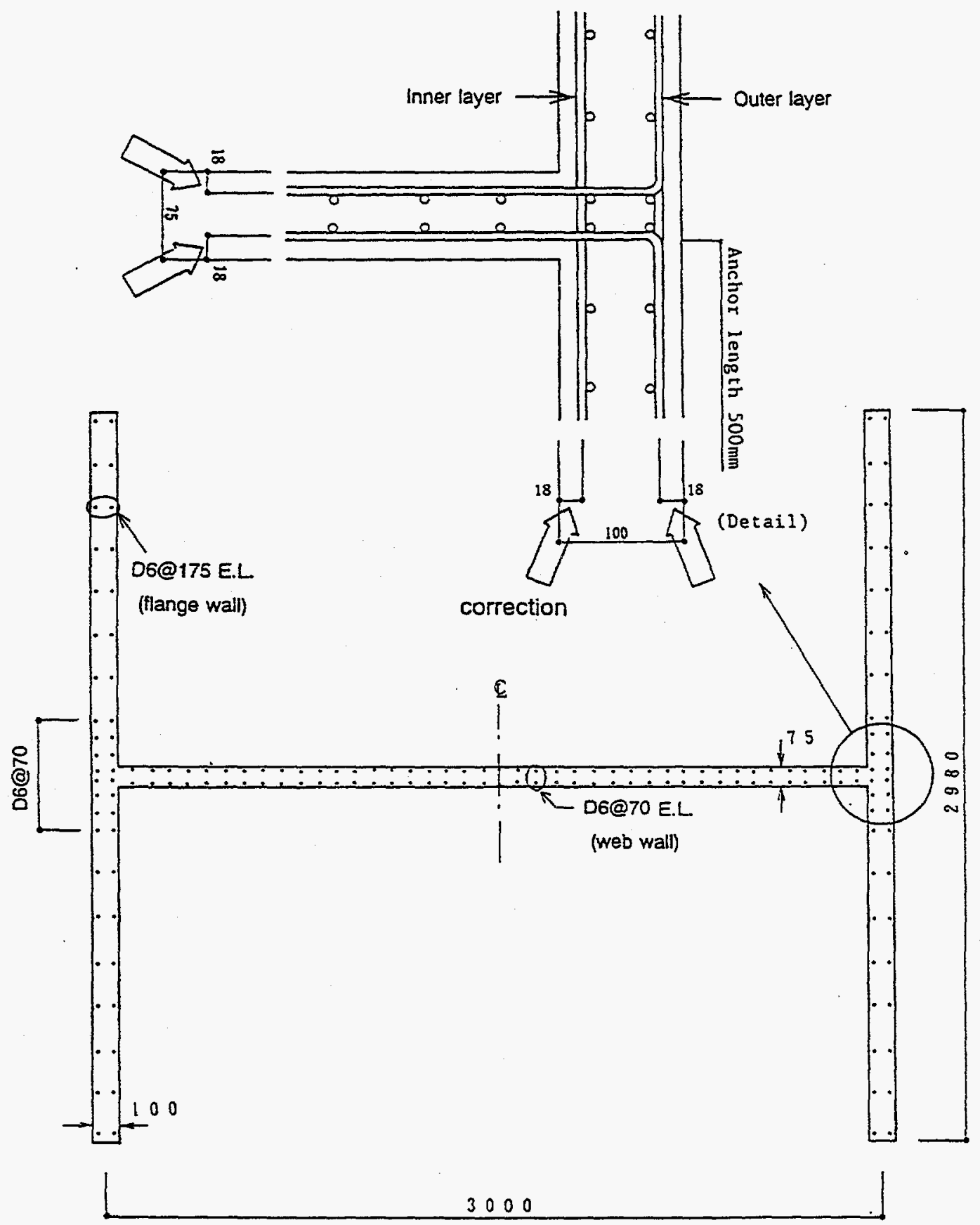

EL : each layer

Unit : $\mathrm{mm}$

Fig. 2.2 Plan for Reinforcement Detail (Ref. 2) 



Fig. 2.3 Elevation for Reinforcement Detail (Ref. 2) 

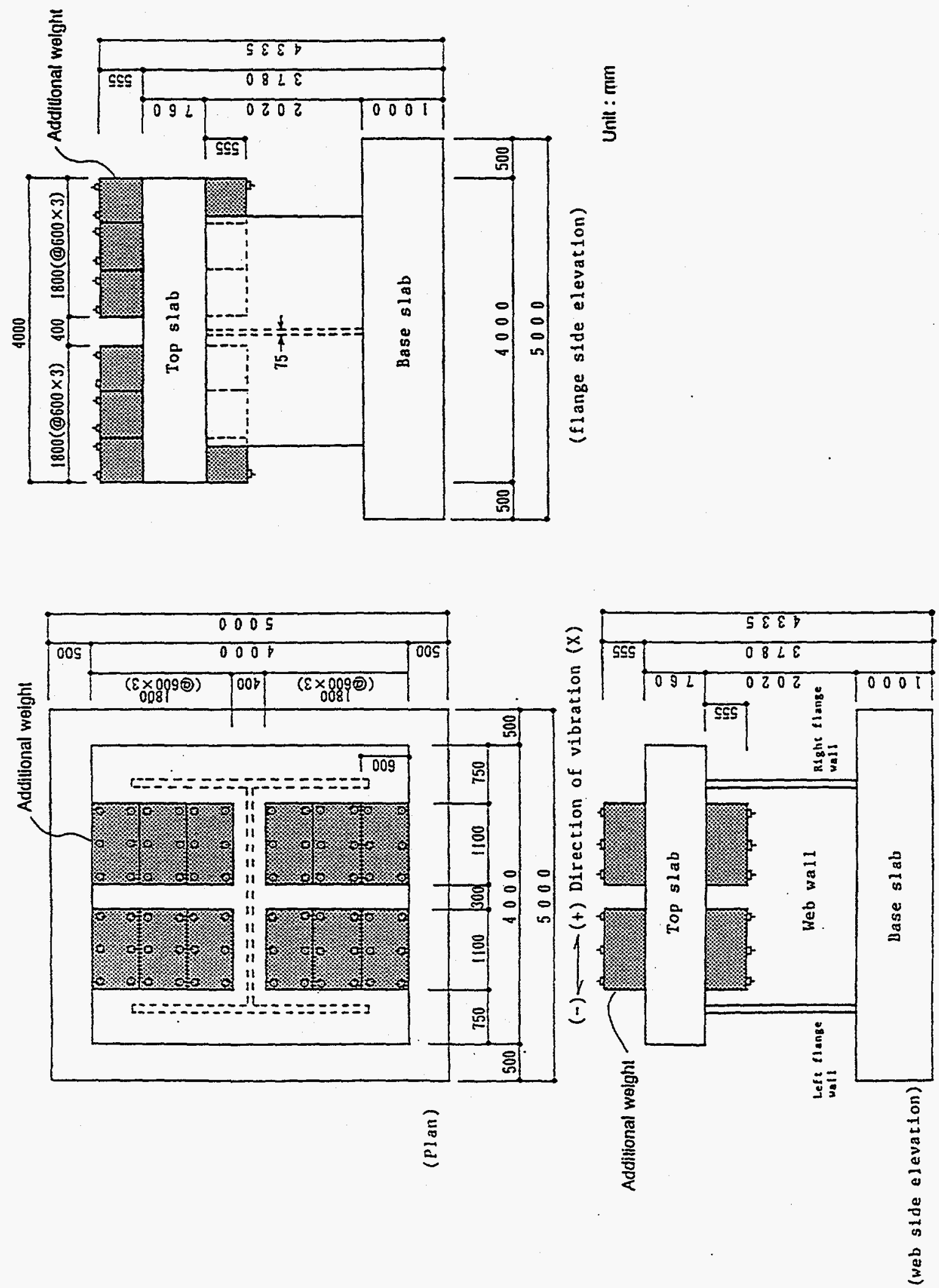
ISP Shear Wall Tests

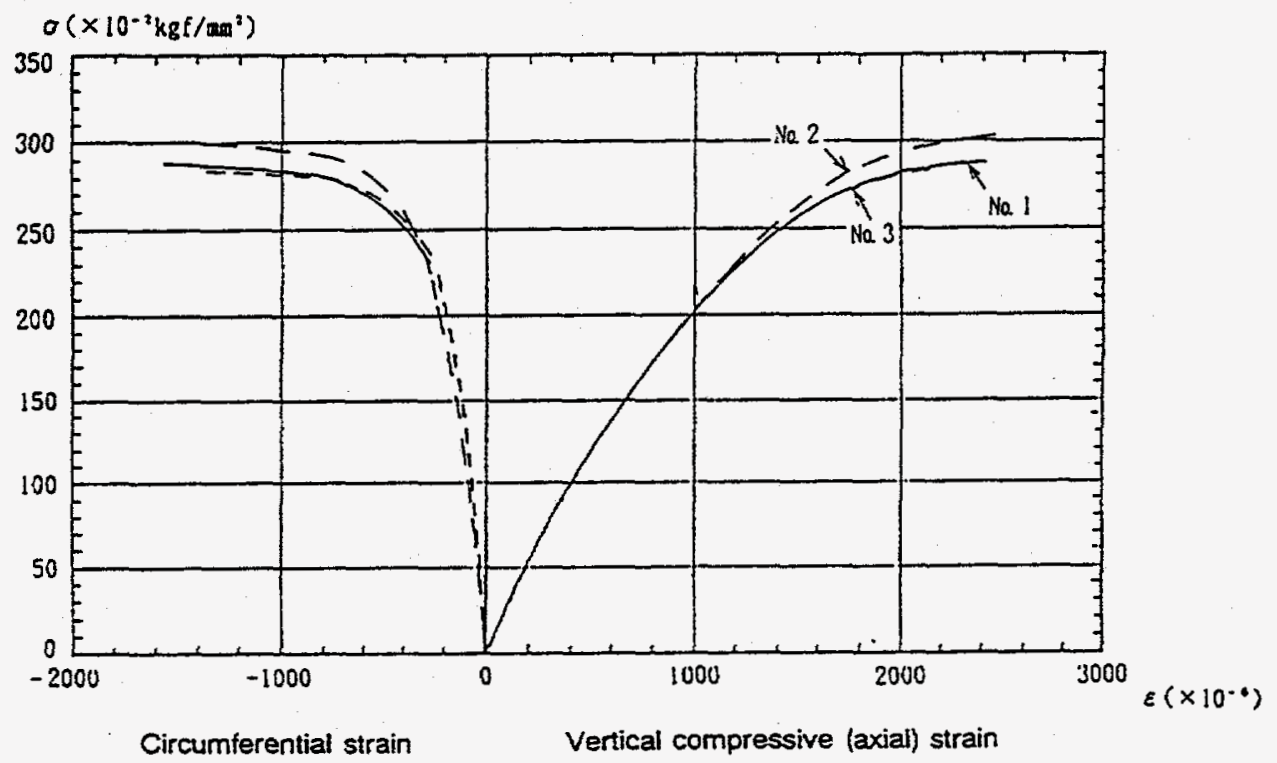

Fig. 2.5 Stress - Strain Relationships of Concrete of Web and Flange Walls (Ref. 2) 
ISP Shear Wall Tests
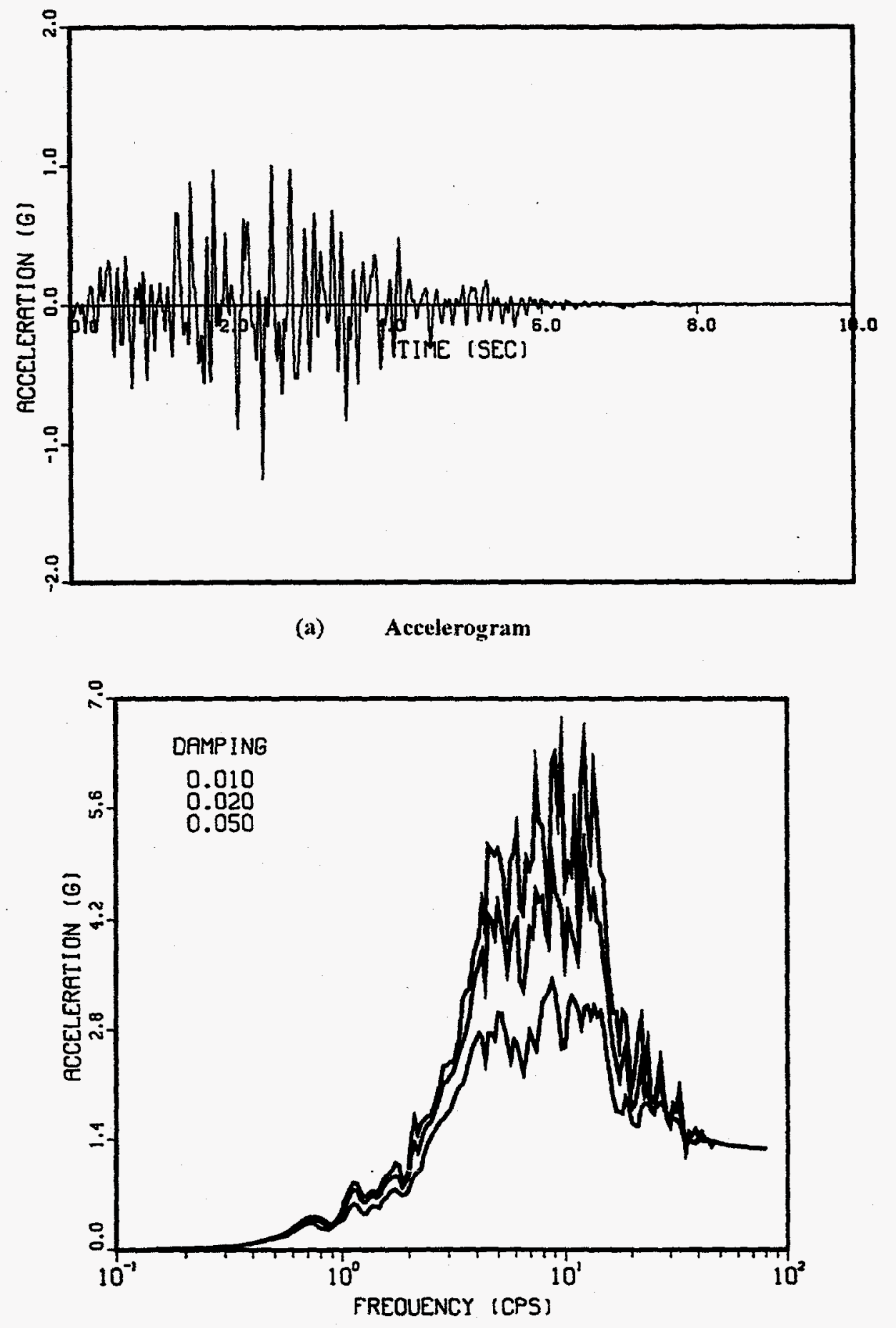

(b) Response Spectra

Fig. 2.6 Accelerogram of Horizontal Input Table Motion, AXB, Recorded During RUN-5 
$(-) \longrightarrow(+)$ Direction of vibration

$(x)$
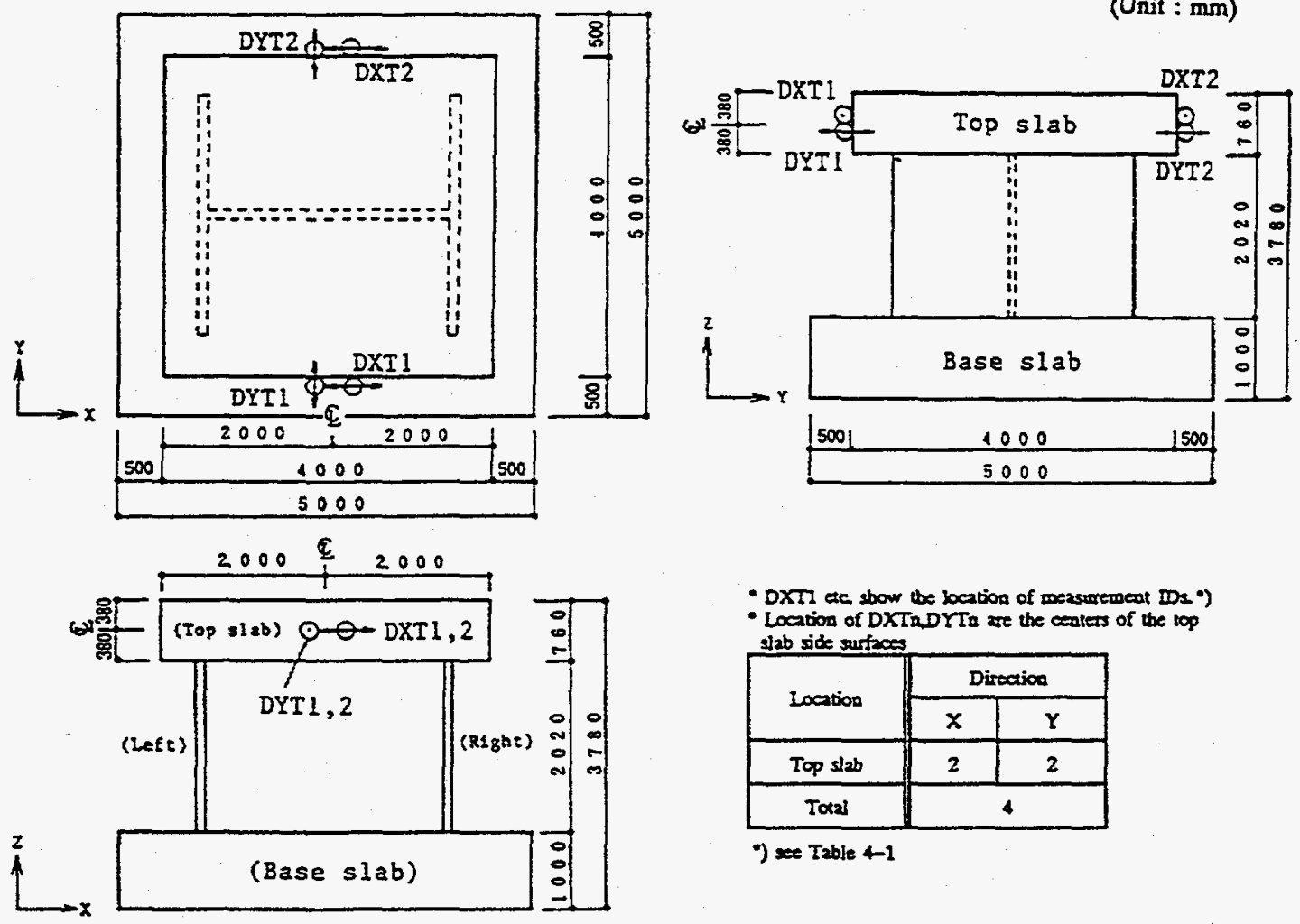

- DXTI ace show the beation of measuremeat $\mathrm{DI}$ ")

- Location of DXTaDYTa are the conters of the top

\begin{tabular}{|c|c|c|}
\hline \multirow{2}{*}{ jab side sarfoces } & \multicolumn{2}{|c|}{ Direction } \\
\hline & $X$ & $Y$ \\
\hline Top slab & 2 & 2 \\
\hline Tokal & & 4 \\
\hline
\end{tabular}

) see Table $4-1$

Fig. 2.7 Location of Measurements for Horizontal Displacement (Ref. 2) 
ISP Shear Wall Tests

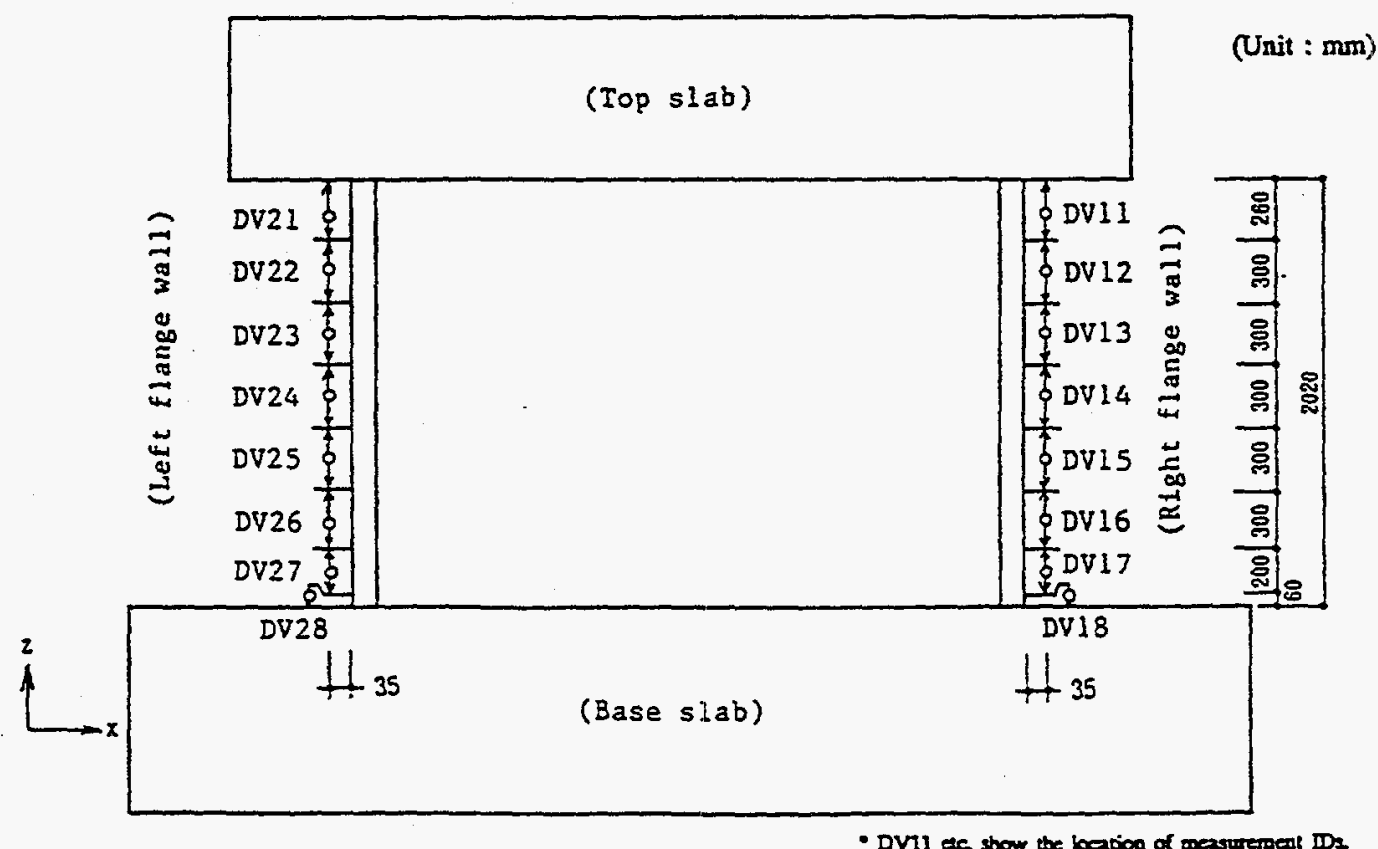

Fig. 2.8 Location of Measurements for Relative Vertical Displacement (Ref. 2) 


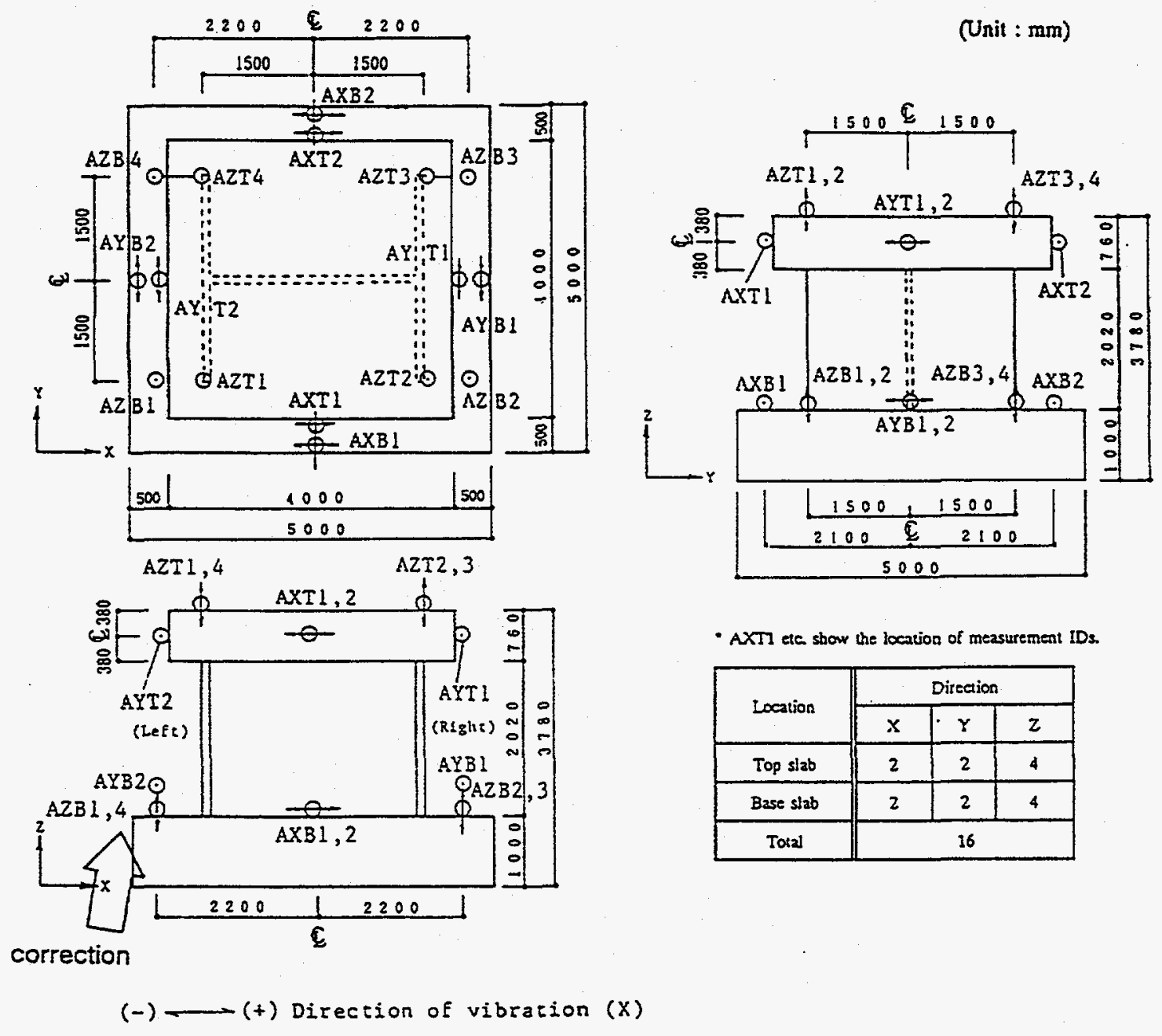

Fig. 2.9 Location of Measurements for Acceleration (Ref. 2) 
ISP Shear Wall Tests

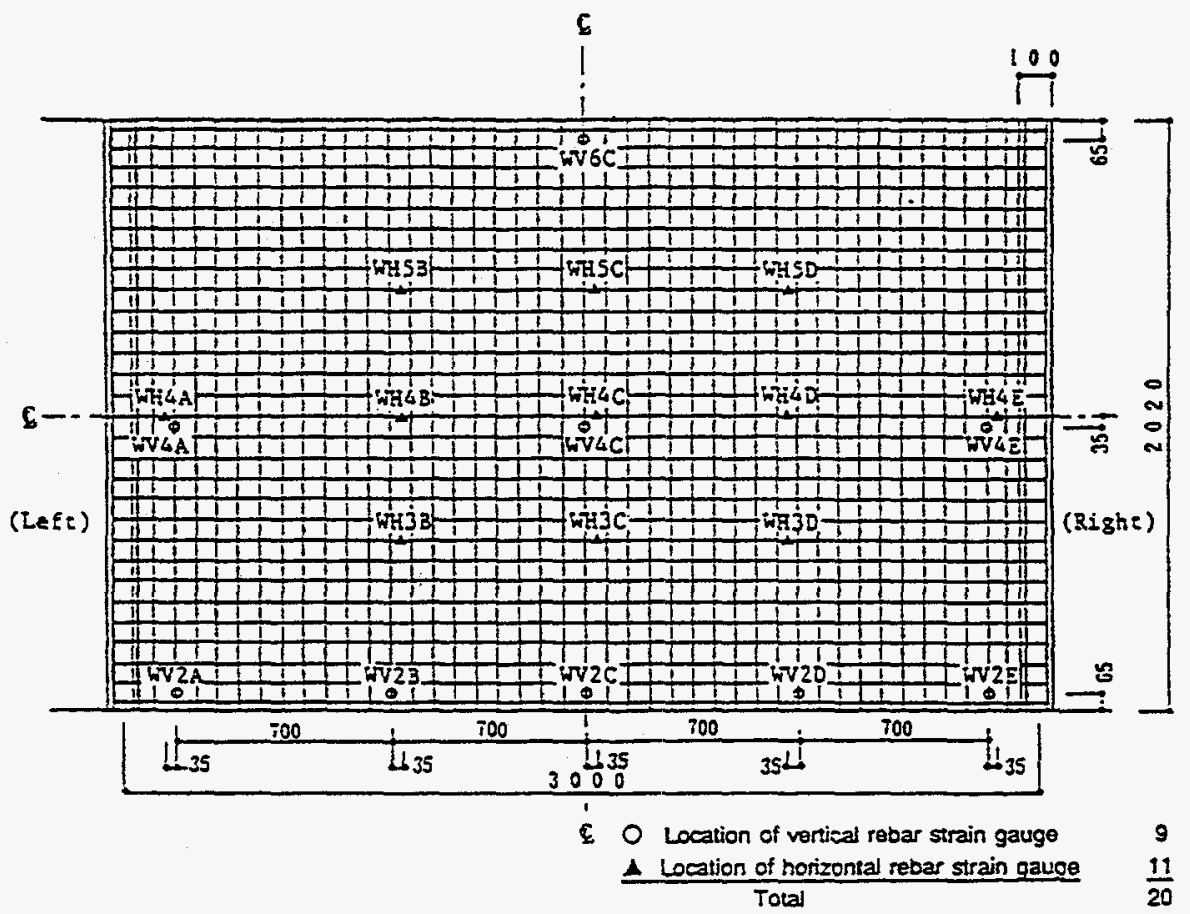

(a) Location of measurements for rebar strain of web wall

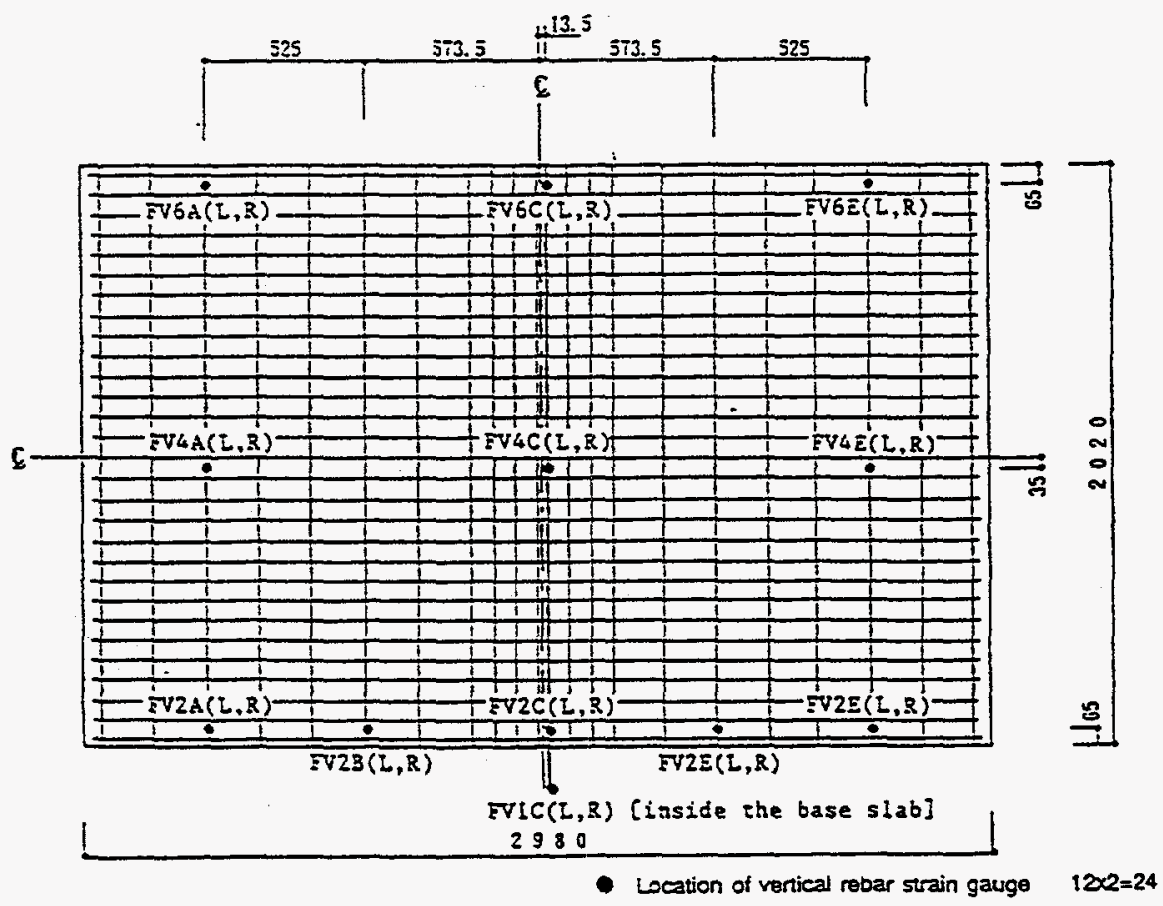

(b) Location of measurements for rebar strain of hange wall

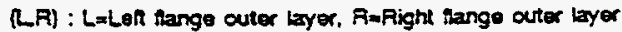

Fig. 2.10 Location of Measurements for Reinforcement Strains (Ref. 2) 


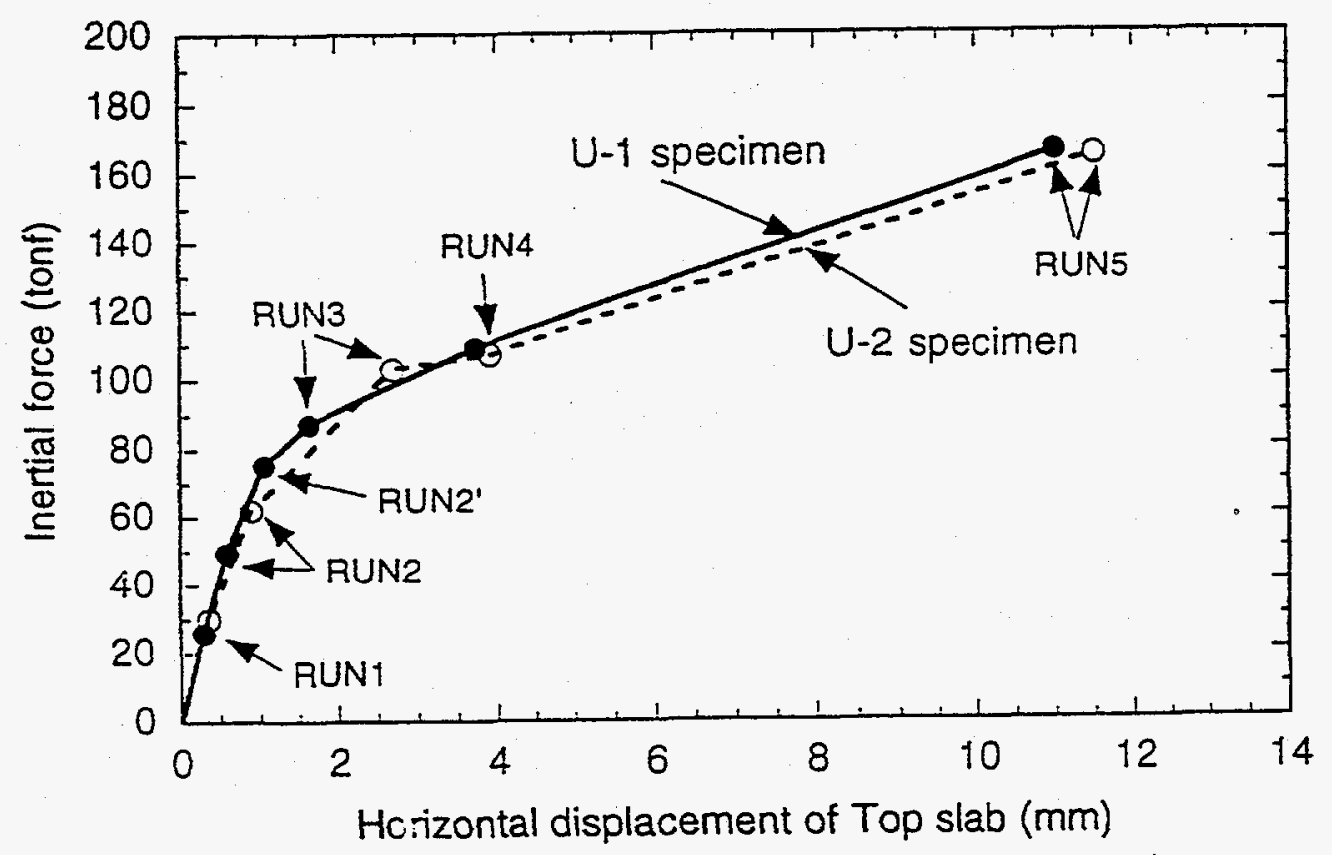

Fig. 2.11 Maximum Inertial Force - Maximum Displacement Relationships (Ref. 2) 
ISP Shear Wall Tests
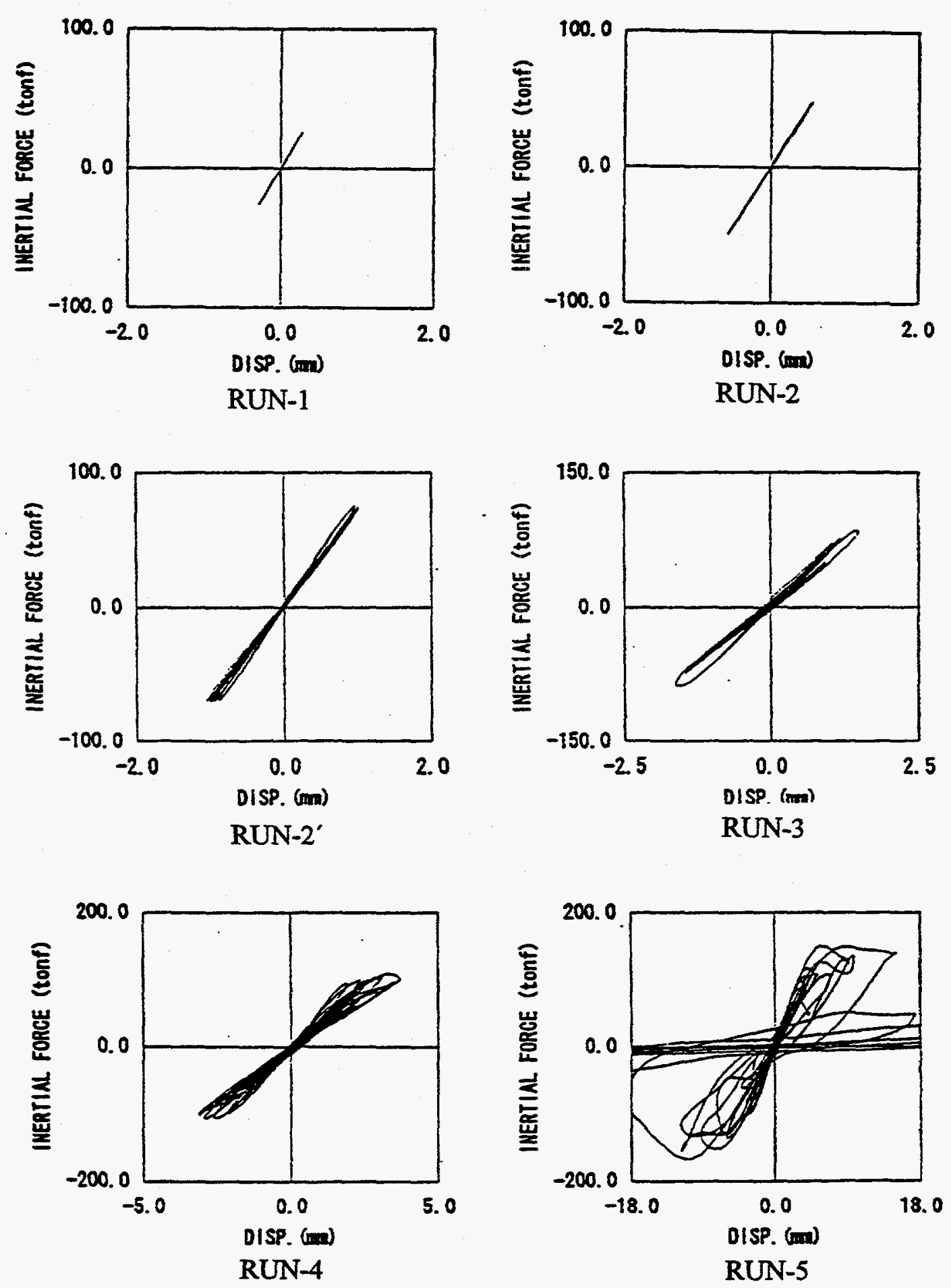

Fig. 2.12 Inertial Force - Top Slab Horizontal Displacement Relationships (Ref. 2) 


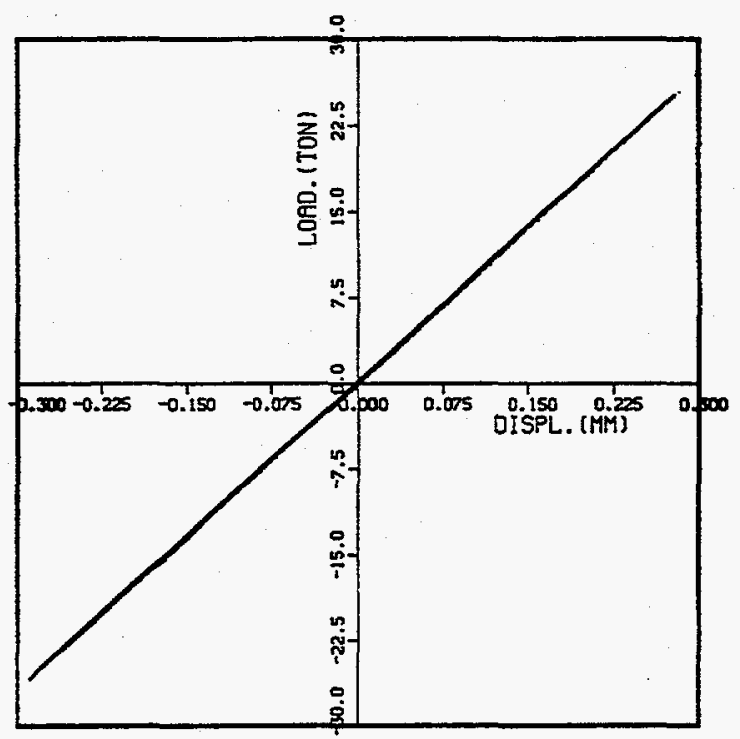

(a) Foree-Displacement Relationship of Top Slab

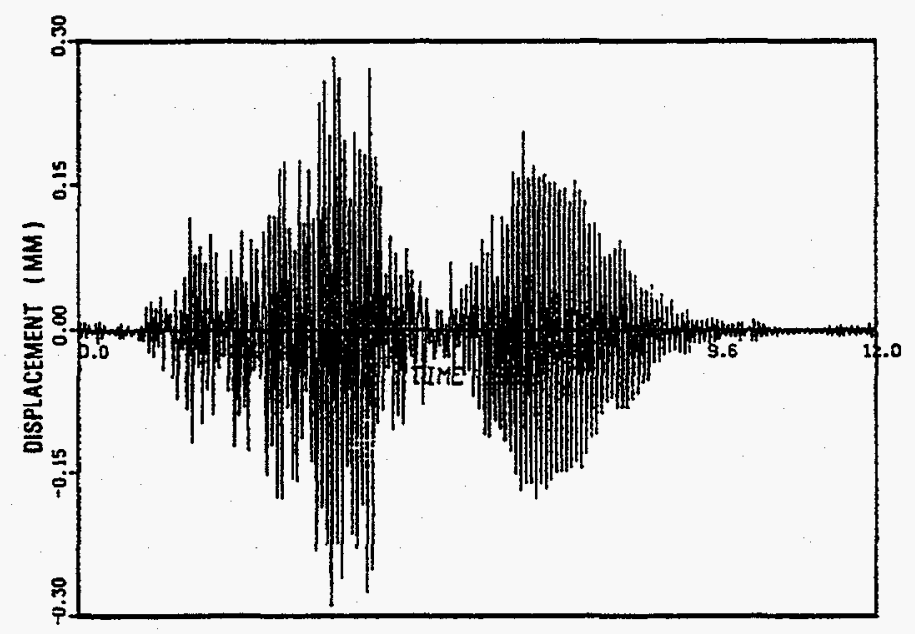

(b) Horizontal Displacement of Top Slabs

Fig. 2.13 Recorded Responses of Shear Wall During RUN-1 
ISP Shear Wall Tests

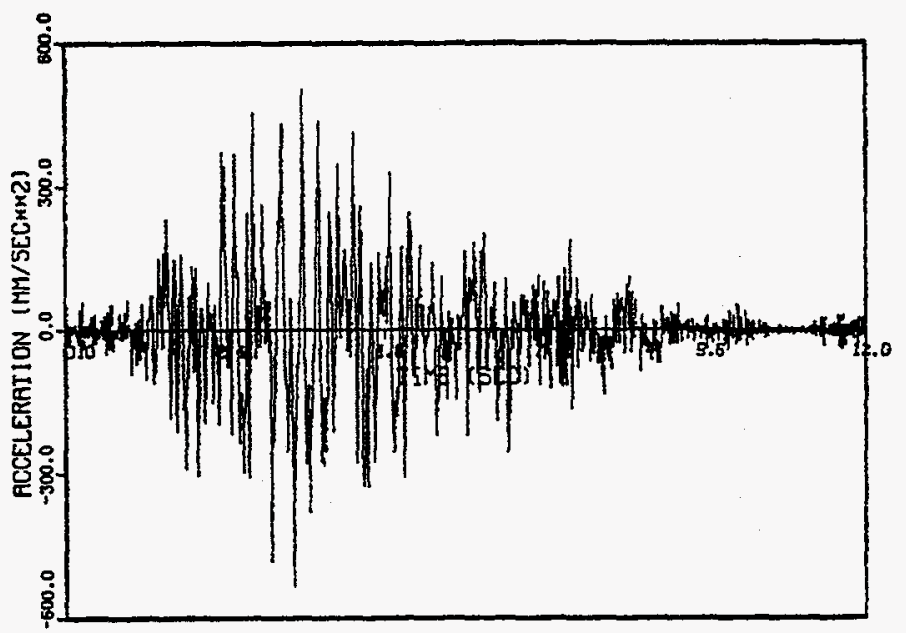

(c) Horizontal Acceleration of Bottom Slab

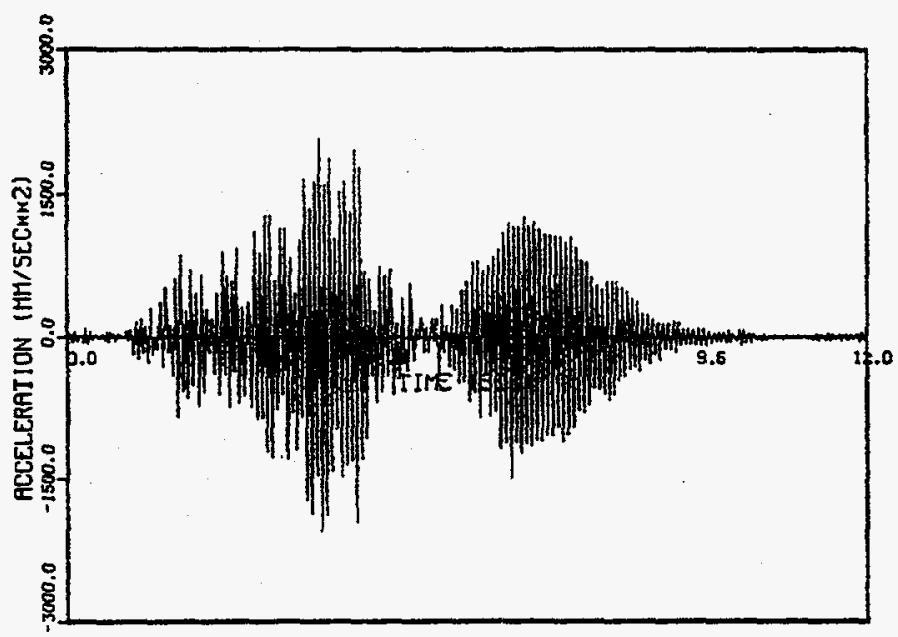

(d) Horizontal Acceleration of Top Slab

Fig. 2.13 Recorded Responses of Shear Wall During RUN-1 (continued) 


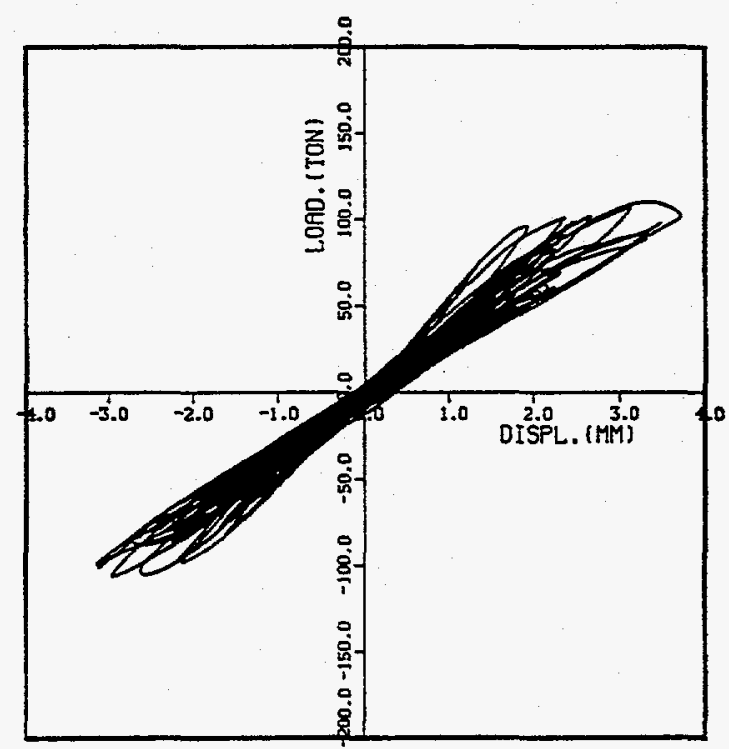

(a) Force-Displacement Relationship of Top Slab

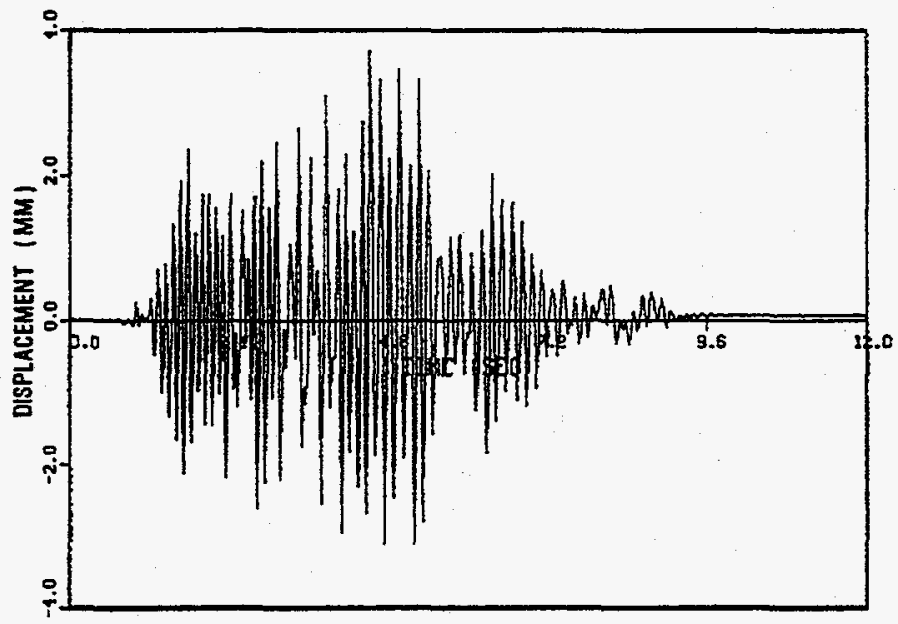

(b) Horizontal Displacement of Top Slab

Fig. 2.14 Recorded Responses of Shear Wall During RUN-4 
ISP Shear Wall Tests

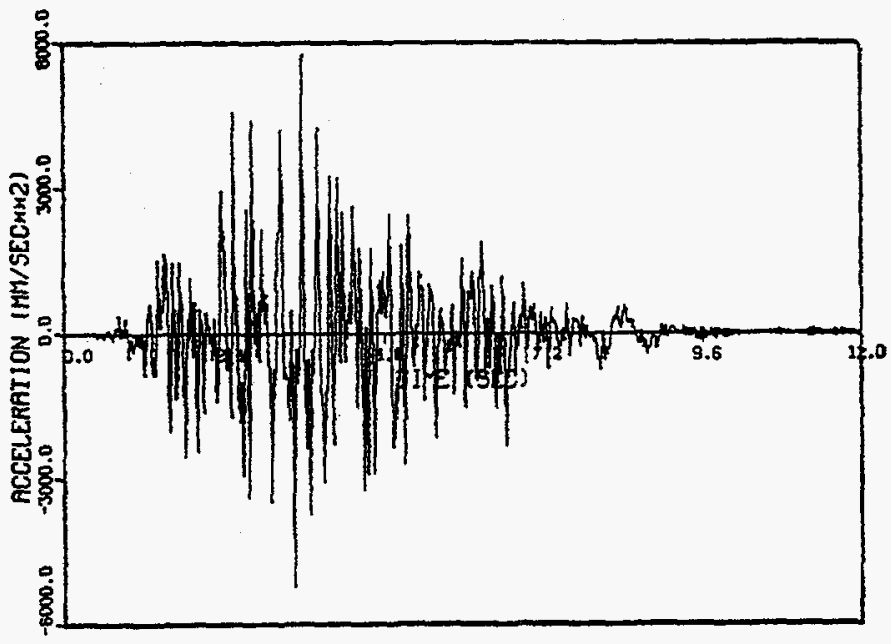

(c) Horizontal Acceleration of Bottom Slab

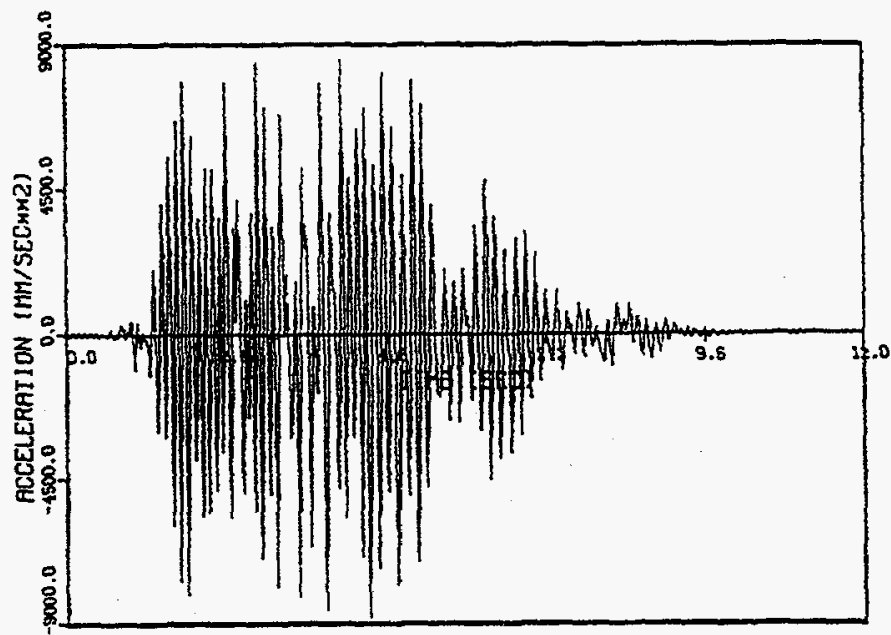

(d) Horizontal Acceleration of Top Slab

Fig. 2.14 Recorded Responses of Shear Wall During RUN-4 (continued) 


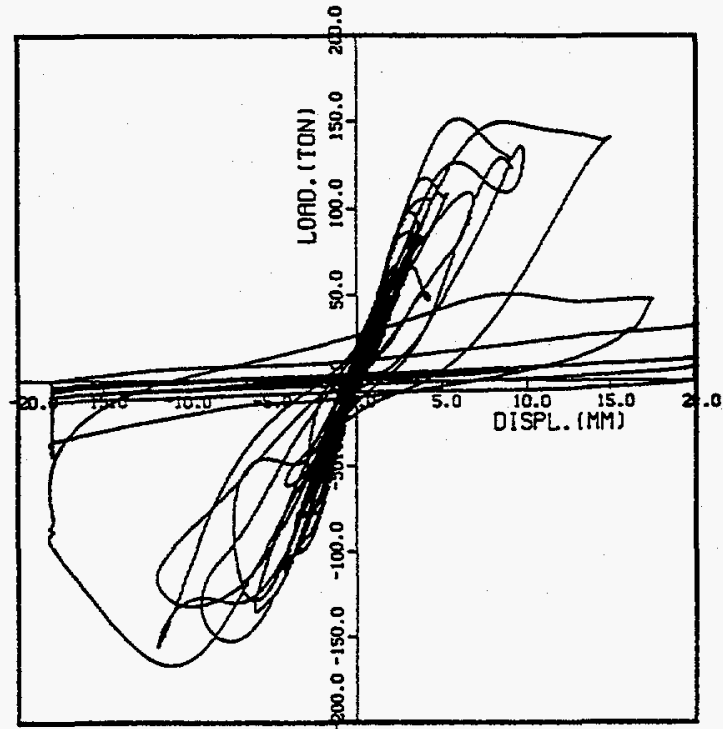

(a) Force-Displacement Relationship of Top Slab

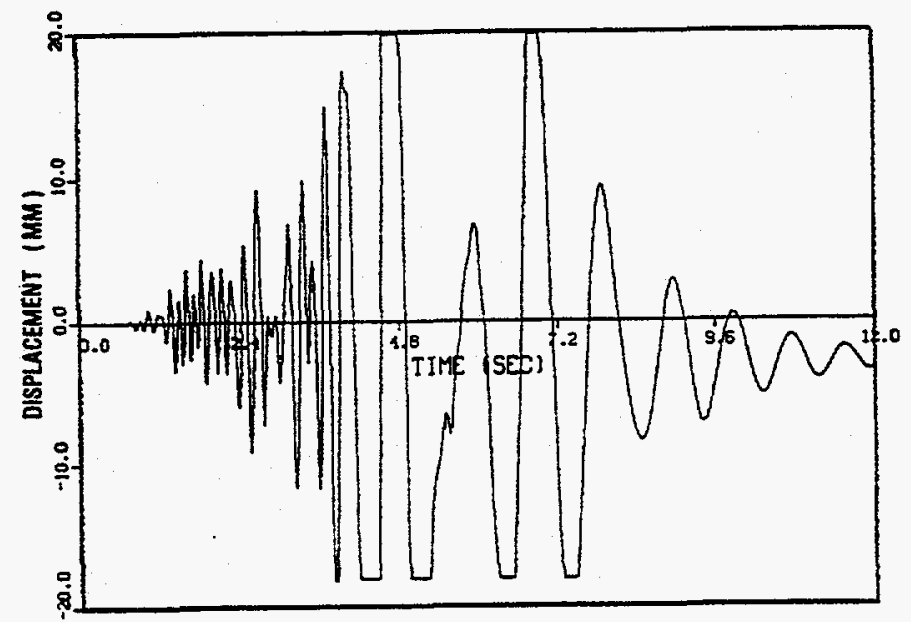

(b) Horizontal Displacement of Top Slab

Fig. 2.15 Recorded Responses of Shear Wall During Run-5 


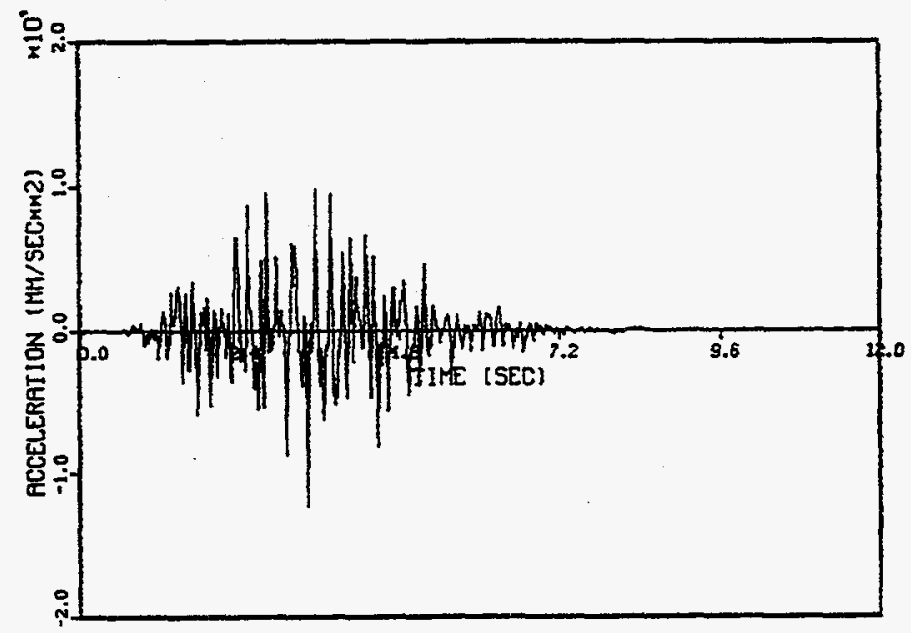

(c) Horizontal Acceleration of Bottom Slab

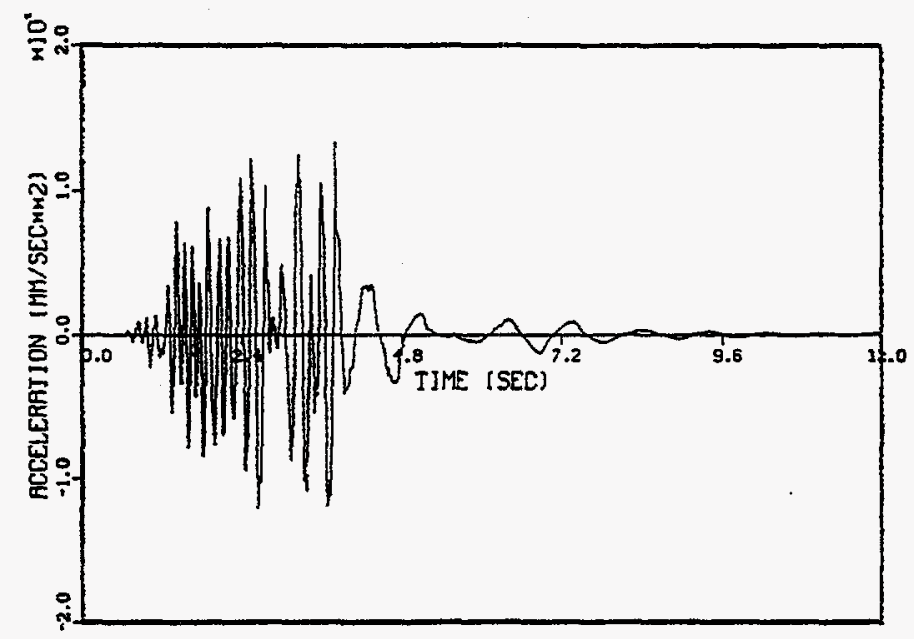

(d) Horizontal Acceleration of Top Slab

Fig. 2.15 Recorded Responses of Shear Wall During Run-5 (continued) 


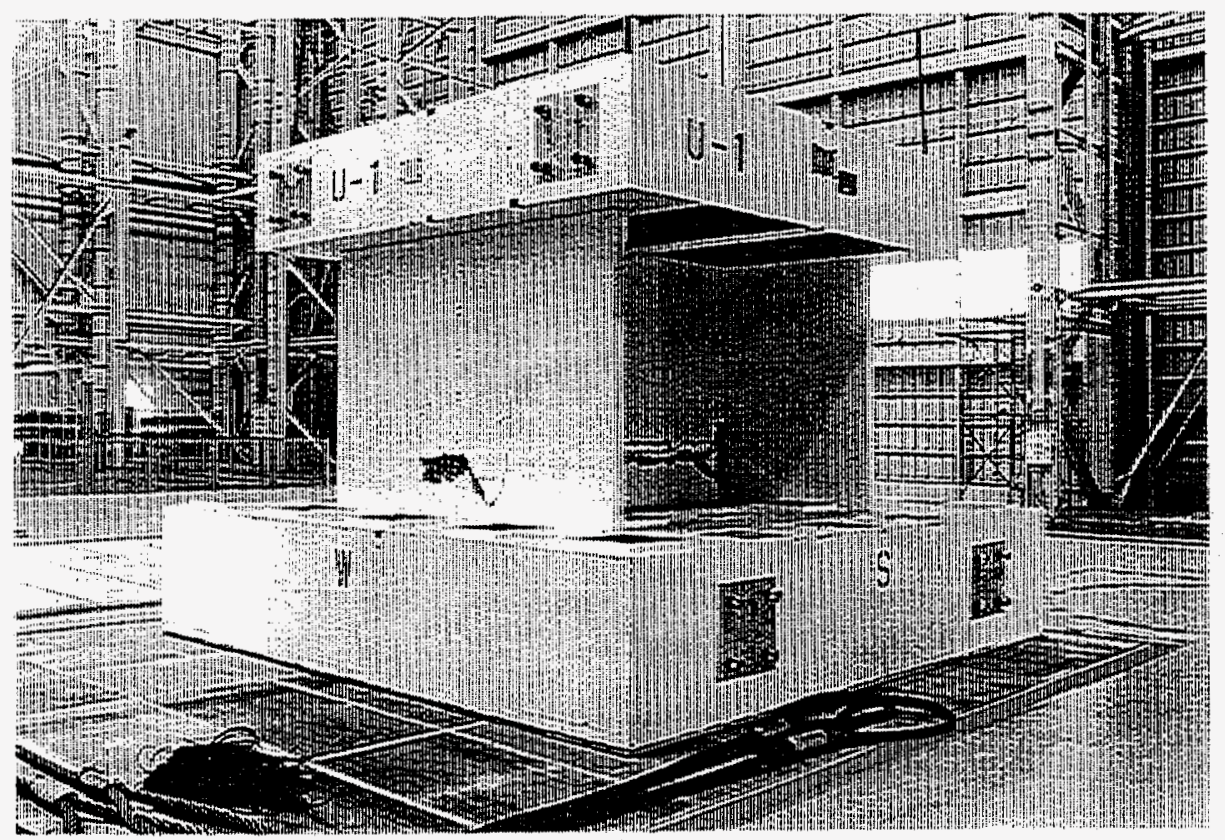

Fig. 2.16 ISP Shear Wall after the Tests

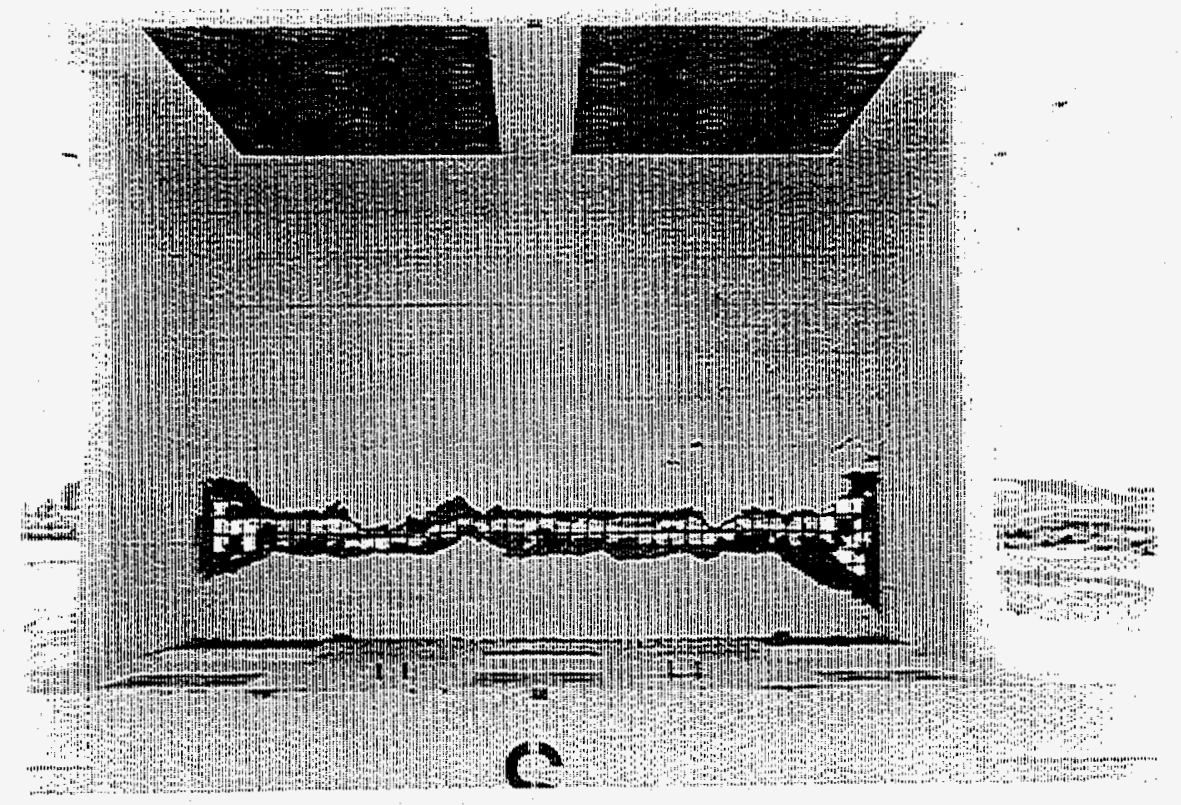

Fig. 2.17 Sliding Shear Failure in Wel Wall after RUN-5 


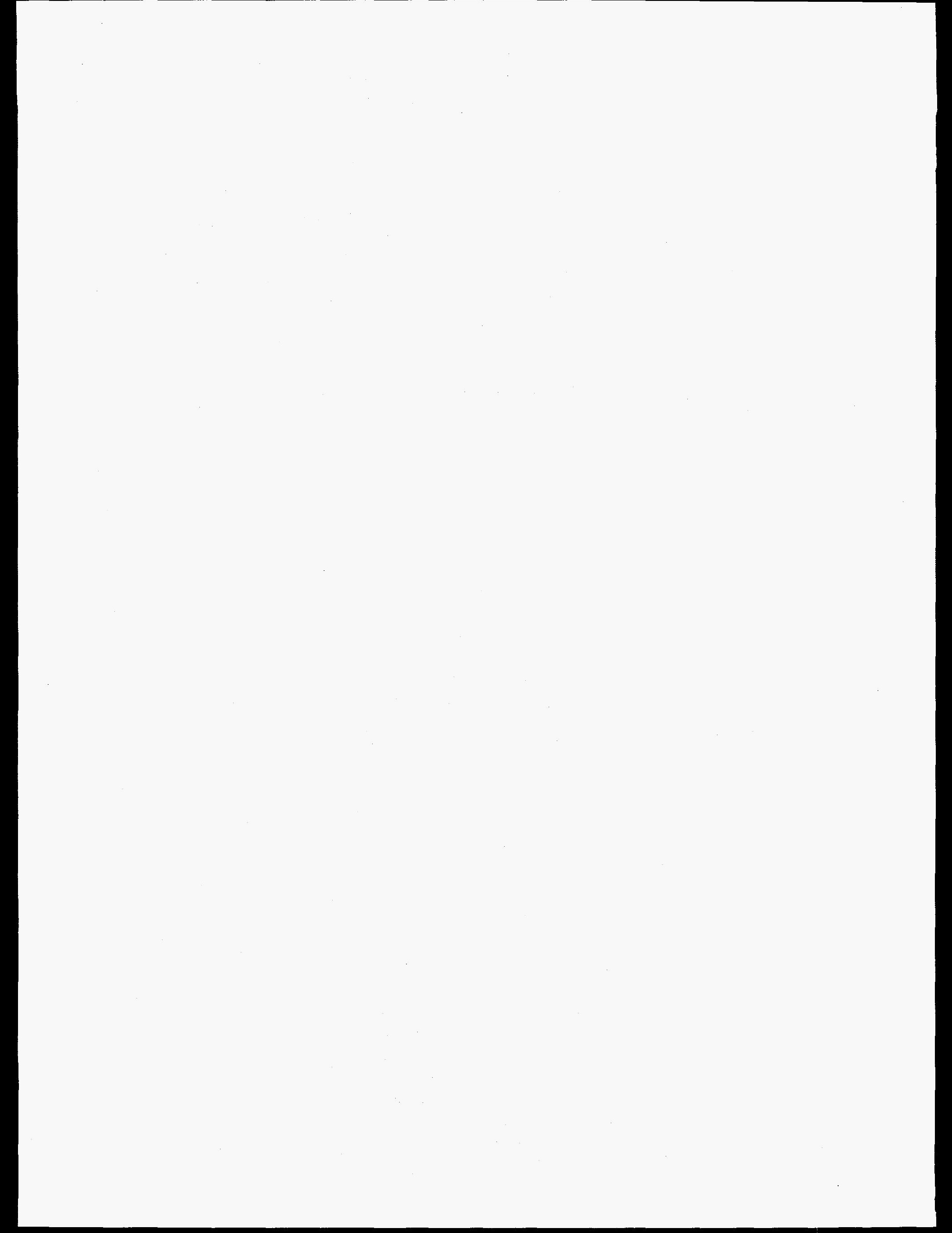




\section{BROOKHAVEN NATIONAL LABORATORY'S ANALYSES}

\subsection{Introduction}

This chapter presents the results of the nonlinear FEM analyses for ISP Shear Walls performed by BNL. The finite element analyses were performed with the ISSAC computer code which was developed by Young J. Park of BNL (Ref. 5). Three types of analyses were performed, i.e., monotonic static (push-over), cyclic static and dynamic analyses.

A serious technical difficulty was encountered in performing the nonlinear dynamic analyses when the analysis model was subjected to highly plastic loading reversals. As a result, the initial analysis results for the higher amplitude tests did not match well with the test results. Additional efforts were made to improve numerical stability by changing analysis conditions/assumptions, including the damping assumption. Both the initial and improved analysis results are presented in this chapter.

\subsection{Constitutive Model for Concrete}

The constitutive model for concrete used in the ISSAC code is based on the concepts of orthotropic plasticity theory and the rotating smeared crack model (e.g., Refs. 6 and 7). In this analysis scheme, rebar can also be smeared (e.g., Ref. 6). The biaxial stress-strain relationship in the principal stress direction is expressed as (smeared rebar not included),

$$
\left\{\begin{array}{l}
d \sigma_{1} \\
d \sigma_{2} \\
d \tau
\end{array}\right\}=\frac{1}{1-v^{2}}\left[\begin{array}{ccc}
E_{1} & v E_{1} \\
v E_{2} & E_{2} & 0 \\
0 & 0 & G
\end{array}\right]\left\{\begin{array}{l}
d \epsilon_{1} \\
d \epsilon_{2} \\
d \gamma
\end{array}\right\}
$$

in which,

$$
\begin{array}{lll}
\mathrm{do}_{1}, \mathrm{do}_{2} & = & \text { incremental principal stresses } \\
\mathrm{d} \epsilon_{1}, \mathrm{~d} \epsilon_{2} & = & \text { incremental strains in the principal stress directions } \\
\mathrm{d} \tau, \mathrm{d} \gamma & = & \text { incremental shear stress and strain } \\
\mathrm{E}_{1}, \mathrm{E}_{2} & = & \text { uniaxial modulus in the principal stress directions } \\
\mathrm{G} & = & \text { shear modulus (set to zero after cracking) } \\
v & = & \text { Poisson's ratio }
\end{array}
$$

Currently, detailed information on the hysteretic behavior of concrete is available only from test results under uniaxial loading conditions. Therefore, a relationship should be established between the uniaxial hysteretic model of concrete and the foregoing principal stresses, $\sigma_{1}$ and $\sigma_{2}$, and associated strains, $\epsilon_{1}$ and $\epsilon_{2}$. The following "independent uniaxial" (IU) concrete stresses, $\sigma_{1}{ }^{*}$ and $\sigma_{2}{ }^{*}$, and strains, $\epsilon_{1}{ }^{*}$ abd $\epsilon_{2}{ }^{*}$, are introduced for this purpose.

$$
\begin{aligned}
& \sigma_{1}^{*}=\sigma_{1} / X_{1} \\
& \sigma_{2}^{*}=\sigma_{2} / X_{2}
\end{aligned}
$$




$$
\begin{aligned}
& d \epsilon_{1}^{*}=\frac{1}{1-v^{2}}\left(d \epsilon_{1}+v d \epsilon_{2}\right) / X_{1} \\
& d \epsilon_{2}^{*}=\frac{1}{1-v^{2}}\left(d \epsilon_{2}+v d \epsilon_{1}\right) / X_{2}
\end{aligned}
$$

In which, $X_{1}$ and $X_{2}$ are the biaxial interaction factors that are determined from the biaxial envelope function. In this scheme, the uniaxial hysteretic behavior is expressed in terms of the $\Pi \mathrm{U}$ stress-strains, $\sigma_{1}{ }^{*}, \sigma_{2}{ }^{*}, \mathrm{~d} \epsilon_{1}{ }^{*}$ and $\mathrm{d} \epsilon_{2}{ }^{*}$; then the biaxial stressstrains are obtained from the above Equations 3.2 through 3.5.

Figures 3.1 and 3.2 illustrate the uniaxial hysteretic model for concrete. The negative slope in compression is assumed to be a straight line up to the specified failure strain, $-\beta \cdot \epsilon_{0}$. The tangential stiffiness of the negative slope, $E_{n}$, is expressed as,

$$
E_{n}=\frac{(1-\lambda) f_{C}^{\prime}}{(1-\beta) \epsilon_{o}}
$$

All the above parameters are defined in Figure 3.1. To account for the so-called tension stiffening effect, the tensile strength of cracked concrete, is expressed by a factor " $\alpha$ " as illustrated in Figure 3.1 .

$$
\alpha=C_{t} \frac{\rho_{s}}{D_{s}}
$$

In which,

$$
\begin{aligned}
& C_{t}=\text { a constant with unit of length }(\sim 75 \mathrm{~mm} \text { based on studies by the University of Toronto, Ref. } 8) . \\
& \rho_{s}=\text { steel ratio } \\
& D_{s}=\text { diameter of rebars }(\mathrm{mm}) .
\end{aligned}
$$

When more than one rebar group is considered (e.g., horizontal and vertical rebars), the values of " $\alpha$ " are added to obtain the overall value, i.e.,

$$
\alpha=\Sigma \alpha_{i}
$$

The biaxial envelope function is shown in Figure 3.3, which is a simplified version of the enveloped curve proposed by Kupfer et al (Ref. 9). In the tension-compression regions, the reduction in the compressive strength is controlled by the socalled compression softening of cracked concrete (Ref. 10), as 


$$
C=\frac{1}{1+0.27\left(\epsilon_{p} / \epsilon_{0}-0.37\right)}
$$

In which, $\epsilon_{\mathrm{p}}$ is the tensile strain in the direction orthogonal to the crack plane, and $\epsilon_{\mathrm{o}}$ is defined in Figure 3.1.

\subsection{Numerical Method}

The equations of motion for a FEM model are expressed as,

$$
[M]\{\ddot{u}\}+[C]\{\ddot{u}\}+[K]\{\Delta u\}+\{R\}=-[M]\{\ddot{z}\}
$$

In which, $[M],[C]$, and $[\mathrm{K}]$ are the mass damping and stiffness matrices; ù, ù and $\Delta \mathrm{u}$ are acceleration, velocity and incremental displacement; $\ddot{z}$ is the input excitation; and $R$ is the restoring force from the previous analysis step. Rayleigh damping is used for the damping assumption,

$$
[C]=\alpha[M]+\beta[K]
$$

Newmark's $\beta$-method is used to solve the above nonlinear dynamic equations.

In the application of nonlinear FEM to concrete structures, either for static or dynamic problems, a key to a successful analysis is the method to suppress the unbalanced forces during the analysis. The unbalanced forces arise due to a mismatch of internal stresses between adjacent elements with different stiffnesses. When the element stiffness changes abruptly, e.g., due to cracking, crushing or unloading/reloading, the unbalanced forces tend to increase sharply. Some form of iterative scheme is necessary to minimize the unbalanced forces during analysis. In the ISSAC code, the following three (3) stages of iterations are employed in succession to achieve this purpose as illustrated in Figure 3.4.

Stage 1 Newton-Raphson method using tangential stiffnesses with sub-step option (similar to the ones used in commercial codes such as ANSYS and ABAQUS).

Stage 2 Modified Newton-Raphson method using the "local secant stiffnesses."

Stage 3 Initial stiffness method using the elastic stiffnesses.

During the main loading steps, i.e., stage 1 and 2 (see Figure 3.4), it is necessary to make all the changes in the element state as smooth as possible including;

- principal stress angle,

- biaxial interaction factors,

- material stiffnesses,

- Poisson's ratio. 
Particularly, a smooth transition of loading paths should be implemented in the uniaxial hysteretic model of concrete as illustrated in Figure 3.5.

During the iteration steps, i.e., stage 3 (see Fig. 3.4), the above changes in the element state should be "frozen" as much as practically possible in order to achieve a faster and successful numerical conversion. In addition, a simplified version of the hysteretic model maybe adapted during the iteration steps to avoid repeated loading/unloading changes as illustrated in Figure 3.5.

\subsection{Analysis Model}

Figure 3.6 shows the analysis model used for both the static and dynamic analyses. Simple 3-node constant-strain 2-D solid elements were used for both the web and flanges. It is a so-called "unfolded model", a modeling scheme frequently used to model a 3-D panel structure using 2-D solid elements. The top slabs of the flanges are constrained in the horizontal direction (direction orthogonal to the web wall), and the vertical displacements are tied to those of the web at the flange/web joints. Therefore, the flange actions, except for the out-of-plane bending of the flanges, including the in-plane shear in flanges due to shear lag, are accounted for. In this modeling scheme, the deformations in the direction orthogonal to the vibration direction are excluded. Since the model is symmetrical, only half of each flange wall is modeled with twice the thickness.

The material parameters are listed in Table 3.1 (see Figure 3.1 for the definition of the parameters). All the rebar were smeared both in the web and flange walls.

\subsection{Monotonic Static Analysis}

Monotonic static analyses were performed to check the adequacy of the analysis model and to estimate the negative slope of the concrete stress-strain relationship. Figure 3.7 shows the load-deformation relationships of three monotonic static analyses in which only the value of $\beta$, which defines the negative slope of concrete (see Figure 3.1), is varied. It appears that when the value of $\beta$ is assumed to be 10 to 20 (i.e. failure strain value is $2.5 \%$ to $5 \%$ ), the load-deformation relationship obtained matches well with that for RUN-5.

Figure 3.8 shows the detailed analysis results for $\beta=20$. It is obvious that the analysis predicts the wall to fail in sliding shear at the bottom of the web. The observed failure plane in the test, however, is located approximately $40 \mathrm{~cm}$ from the bottom. One possible explanation for this difference may be the use of the plain-stress assumption for the concrete elements. The 3-D confinement at the bottom of the web wall may have shifted the failure plane away from the bottom.

Table 3.2 lists the horizontal displacements and forces of the top slab for various damage stages.

\subsection{Cyclic Static Analysis}

A cyclic static analysis was performed under an idealized loading reversal condition. The analysis was performed by controlling the horizontal displacement of the top slab. The objectives of this analyses are as follows:

- Verify the constitutive model for concrete and the numerical method for analyses that involve large loading reversals up to the failure point;

- Characterize the hysteric behavior of the ISP shear wall under a simplified static loading condition.

- Observe the differences in responses between the monotonic and cyclic analyses. 
- Obtain the response of the shear wall after loading reversals which are representative of RUN-2', 3, 4 and 5 .

Figure 3.9 illustrates the applied displacement history. Loading reversals for peak displacements of $1.05 \mathrm{~mm}, 1.63 \mathrm{~mm}$ and $3.72 \mathrm{~mm}$ were first applied, representing RUN-2', RUN-3 and RUN-4, respectively. Then two larger loading reversals were applied to observe the hysteretic behavior beyond the maximum strength point. A total of 1330 analysis steps were necessary to complete the analysis (the average displacement increment is $0.1 \mathrm{~mm}$ per step).

Figures 3.10 and 3.11 compare the load-deformation relationships. Relatively small hysteretic damping (i.e., hysteresis loop area), a significant pinching behavior, as well as sharp strength drop after reaching the maximum point, are observed both in the analysis and the test results. These observed hysteretic characteristics are considered to be consistent with the brittle sliding failure mode of the ISP wall. The maximum strength is $3 \%$ lower than that of the foregoing monotonic analysis. No significant differences are observed between the monotonic and cyclic analysis results.

Figures 3.12 through 3.15 depict the responses of the shear wall for each representative loading reversal. The same sliding shear failure at the bottom of the wall as in the static analysis can be found in Figure 3.15.

\subsection{Dynamic Analysis}

\subsubsection{Linear Dynamic Analysis for RUN-1}

Based on the static analysis results, no element is expected to exceed the tensile strength level during RUN-1, and the shear wall responses are considered to be entirely in the elastic range. Therefore, a conventional modal time history analysis is performed for RUN-1.

Figure 3.16 shows the fundamental mode shape. This mode is considered to be dominant over other higher modes. The analysis conditions/assumptions are as follows:

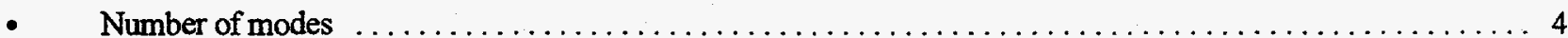

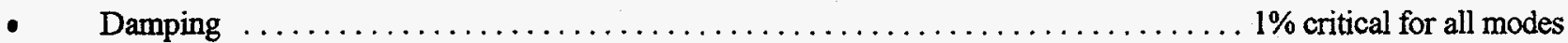

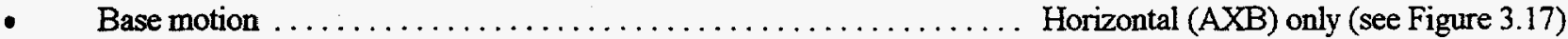

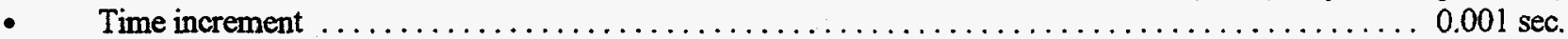

- Numerical method $\ldots \ldots \ldots \ldots \ldots \ldots \ldots \ldots \ldots \ldots \ldots \ldots \ldots \ldots \ldots \ldots \ldots \ldots \ldots \ldots \ldots \ldots$ Newmark's $\beta$-method

Table 3.3 summarizes a comparison of response values. Figures 3.18 through 3.20 show some time history plots and the load-deformation relationship. As vertical base motions are not considered, the vertical accelerations (e.g., AZTL, and AZTR) are underestimated. Other response values, including the fundamental frequency of vibration, agree well with the measured values.

\subsubsection{Nonlinear Dynamic Analysis for RUN-4}

A nonlinear dynamic analysis was performed for RUN-4 using the direct time integration method. As indicated in the foregoing static analyses, the stiffness matrix, $[\mathrm{K}]$ of Equation 3.11 for Rayleigh damping, may take negative values due to the cracking and crushing of concrete. Therefore, the second term of Equation 3.11 could become a negative contribution to the linear damping value. To avoid this anomaly, the value of $\beta$ in Equation 3.11 is assumed to be zero. The corresponding value of $\alpha$ is estimated based on the frequency value of $11.3 \mathrm{~Hz}$ and the equivalent damping value of $2.5 \%$ critical:

$$
\alpha=3.55
$$


The dynamic analysis was preceded by two cycles of static loading with a peak displacement of $1.63 \mathrm{~mm}$ to simulate the effects of the previous test runs. Therefore, residual responses are included in the following dynamic analysis. Other analysis assumptions/conditions are as follows:

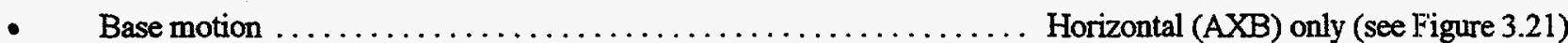

- Time increment ............................... $0.001 \mathrm{sec}$. (max.) to $0.00025 \mathrm{sec}$. (min.)

- Numerical method $\ldots \ldots \ldots \ldots \ldots \ldots \ldots \ldots \ldots \ldots$ Modified Wilson-style Newmark's $\beta$-method

In the course of the direct time integration analysis, a difficulty was encountered as the abrupt changes in stiffness of the concrete (mainly due to crack opening and closing) affected the acceleration responses. Relatively high-frequency components were introduced artificially to the acceleration responses, adversely affecting the numerical stability. The displacement responses, however, were much less affected by the abrupt stiffness changes. Due to this problem, the analysis was terminated at $6.5 \mathrm{sec}$. although the program could have completed the full $12 \mathrm{sec}$. length of analysis. The analysis, however, captured most of the nonlinear response as it is truncated near the end of the main table motions (see Figure 3.21).

Table 3.4 summarizes comparison of response values, and Figures 3.22 through 3.26 compare the load-deformation and time history plots. Figure a 3.27 shows the damage state after the analysis.

The displacement responses agree well with the measured records both in terms of the peak value, the timing of the peak value, and the time history shape. The acceleration responses, however, are overestimated due to the artificial high-frequency components as discussed above. The damage state shown in Figure 3.27 indicates more damage to the wall than in the static analysis result shown in Figure 3.14. Under many cycles of loadings, more elements suffered concrete crushing and rebar yielding.

Figures 3.25 and 3.26 compare the vertical displacement responses. It should be noted that the analysis result shown in Figure 3.25 is a vertical displacement at the top slab, whereas the recorded data shown in Figure 3.26 is a relative vertical displacement measured for a $30 \mathrm{~cm}$ segment of the flange wall (see pp. 3-19 of NU-SSWISP-D008). Both time histories indicate "ratcheting-type" upward movements due to the accumulation of permanent plastic strains under repeated loading reversals.

\subsubsection{Improved Analysis for RUN-4}

Efforts were made to improve the numerical stability in the nonlinear dynamic analysis solutions. It was found that the viscous damping assumption had a significant impact on numerical stability. The foregoing definition of Rayleigh damping was modified as,

$$
[C]=\alpha[M]+\beta\left[K_{e}\right]
$$

where $\left[K_{e}\right]$ is the elastic stiffiness matrix. The values of the parameters, $\alpha, \beta$; were assumed to be,

$$
\alpha=0.97, \quad \beta=0.0001 \text {. }
$$

According to this assumption, the damping value in the elastic range $(13.2 \mathrm{~Hz})$ is $1 \%$ of critical.

This new damping assumption significantly improved the numerical stability, and the artificial high frequency components in the calculated acceleration responses were eliminated. The calculated results are shown in Figures 3.28 and 3.29. Although 

"a perfect match" between the analysis and test results was not achieved, it nevertheless improved the applicability and
accuracy of the nonlinear FEM to dynamic problems.

\subsubsection{Nonlinear Dynamic Analysis for RUN-5}

The same damping assumption was used for the analysis of RUN-5, the highest amplitude test run. The comparisons with test results are given in Table 3.5 and Figures 3.30 through 3.36.

During the course of the analysis, a numerical difficulty was encountered as the analysis model was subjected to large plastic loading reversals. It was observed that as many elements started to crush and enter the negative-slope region, the iterations in stage 3 (see Figure 3.4) tended to aggravate the solutions, rather than improve them. In the analysis, the iterations in stage 3 were turned off at $t=2.5 \mathrm{sec}$. The analysis was terminated at $t=3.616 \mathrm{sec}$., which coincides with the occurrence of the observed shear failure (see Figures 3.30, 3.33 and 3.34). The final failure mode was almost identical with the one from the static analysis (see Figure 3.15). Although some minor differences between the analysis prediction and the test results were observed, the analysis nevertheless predicted the timing of sliding shear failure fairly accurately. 
Table 3.1 Material parameters

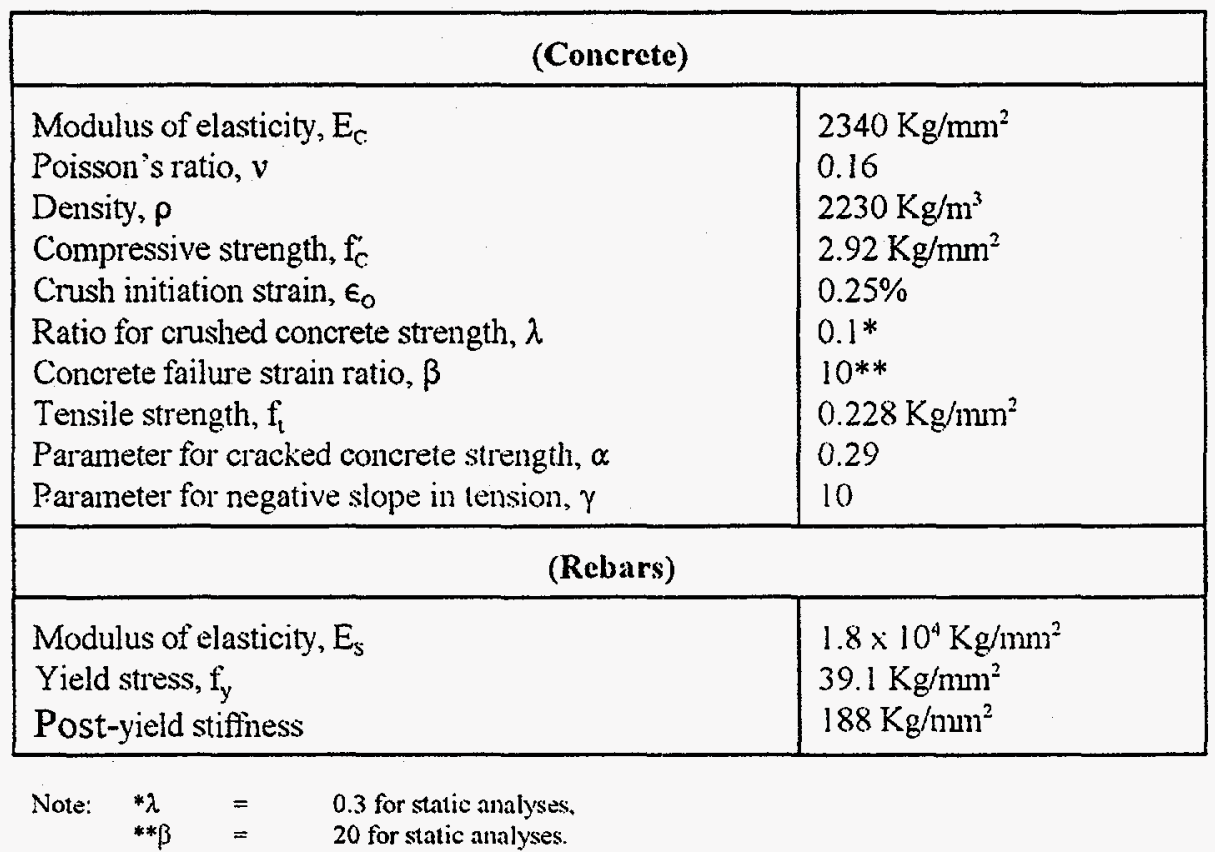

Table 3.2 Summary of calculated results (monotonic static analysis)

\begin{tabular}{|c|c|c|c|}
\hline \multirow{2}{*}{\multicolumn{2}{|c|}{ Phenomena }} & \multicolumn{2}{|c|}{ Top slab } \\
\hline & & $\begin{array}{l}\text { Horizontal displacement } \\
\text { ( } \mathbf{m m})\end{array}$ & $\begin{array}{l}\text { Horizontal force } \\
\text { (N) }\end{array}$ \\
\hline (a) & $\begin{array}{l}\text { Flange wall } \\
\text { Initiation point of bending crack }\end{array}$ & 0.4 & $4.02 \times 10^{5}$ \\
\hline (b) & $\begin{array}{l}\text { Web wall } \\
\text { Initiation point of shear crack }\end{array}$ & 0.5 & $4.90 \times 10^{5}$ \\
\hline (c) $\mathrm{F}$ & $\begin{array}{l}\text { age wall vertical rebar } \\
\text { Initiation point of yield }\end{array}$ & 3.9 & $1.28 \times 10^{\circ}$ \\
\hline (d) & $\begin{array}{l}\text { Web wall vertical rebar } \\
\text { Initiation point of yield }\end{array}$ & 3.9 & $1.28 \times 10^{6}$ \\
\hline (e) & $\begin{array}{l}\text { Web wall horizontal rebar } \\
\text { Initiation point of yield }\end{array}$ & 10.6 & $1.54 \times 10^{6}$ \\
\hline (f) & Crush point & 3.8 & $1.28 \times 10^{6}$ \\
\hline & Maximum load & 8.6 & $1.58 \times 10^{6}$ \\
\hline
\end{tabular}


Table 3.3 Comparison of response values for RUN-1

\begin{tabular}{|l|c|c|c|}
\hline \multicolumn{1}{|c|}{ Items } & Unit & Analysis & Test \\
\hline Fundamental frequency & $\mathrm{Hz}$ & 13.31 & 13.2 \\
Horizontal disp. (DXT) & $\mathrm{mm}$ & 0.291 & 0.29 \\
Horizontal accel. (AXT) & $\mathrm{mm} / \mathrm{s}^{2}$ & 2030 & 2080 \\
Horizontal inertial force & $\mathrm{ton}$ & 25.3 & 25.8 \\
Left vertical accel. (AZTL) & $\mathrm{mm} / \mathrm{s}^{2}$ & 230 & 340 \\
Right vertical accel. (AZTR) & $\mathrm{mm} / \mathrm{s}^{2}$ & 231 & 350 \\
Horizontal rebar strain of web & $10^{-6}$ & 4.5 & 9 \\
Vertical rebar strain of flange & & & \\
- Left flange & $10^{-6}$ & 50.4 & 45 \\
- Right flange & $10^{-6}$ & 50.9 & 37 \\
\hline
\end{tabular}

Table 3.4 Comparison of response values for RUN-4

\begin{tabular}{|l|c|c|c|}
\hline \multicolumn{1}{|c|}{ Items } & Unit & Analysis & Test \\
\hline Horizontal disp. (DXT) & $\mathrm{mm}$ & 3.80 & 3.72 \\
Horizontal accel. (AXT) & $\mathrm{mm} / \mathrm{s}^{2}$ & 11200 & 8820 \\
Horizontal inertial force & tom & $94.4^{-1}$ & $109.0^{* 2}$ \\
Left vertical disp. (DVLF) & $\mathrm{mm}$ & 2.64 & -- \\
Right vertical disp. (DVRF) & $\mathrm{mm}$ & 1.76 & - \\
Left vertical accel. (AZTL) & $\mathrm{mm} / \mathrm{s}^{2}$ & $10330^{-3}$ & 9820 \\
Right vertical accel. (AZTR) & $\mathrm{mm} / \mathrm{s}^{2}$ & $10900^{-3}$ & 8338 \\
\hline
\end{tabular}

\footnotetext{
Note: *1 Program directly evaluates the peak restoring force value

*2 Estimated from acceleration response

*3 Peak values up to 5 sec.
}

Table 3.5 Comparison of response values for RUN-5

\begin{tabular}{|l|c|c|c|}
\hline \multicolumn{1}{|c|}{ Items } & Unit & Analysis & Test \\
\hline Horizontal disp. (DXT) & $\mathrm{mm}$ & 14.3 & $>20$ \\
Horizontal accel. (AXT) & $\mathrm{mm} / \mathrm{s}^{2}$ & 13089 & 13410 \\
Horizontal inertial force & ton & 155 & 166 \\
Left vertical disp. (DVLF) & $\mathrm{mm}$ & 4.35 & -- \\
Right vertical disp. (DVRF) & $\mathrm{mm}$ & 6.70 & -- \\
Left vertical accel. (AZTL) & $\mathrm{mm} / \mathrm{s}^{2}$ & 17690 & 17740 \\
Right vertical accel. (AZTR) & $\mathrm{mm} / \mathrm{s}^{2}$ & 13500 & 20000 \\
\hline
\end{tabular}


BNL's Analyses

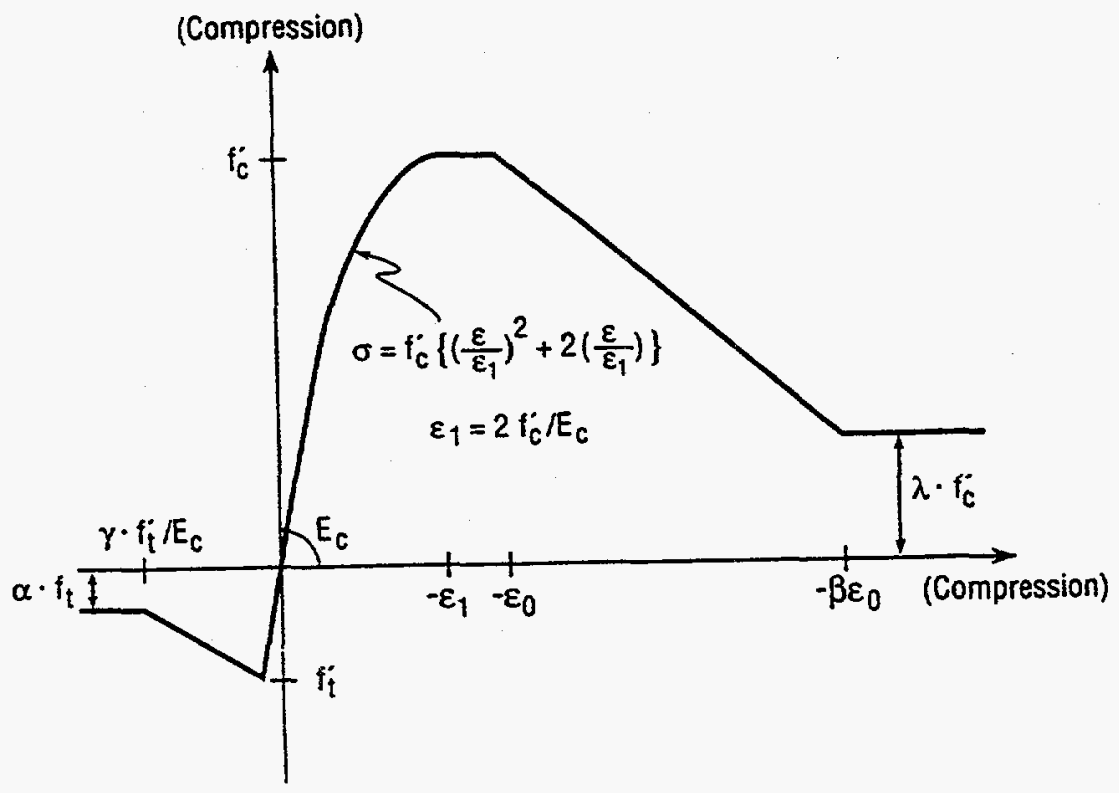

Fig. 3.1 Envelope Curve for Concrete

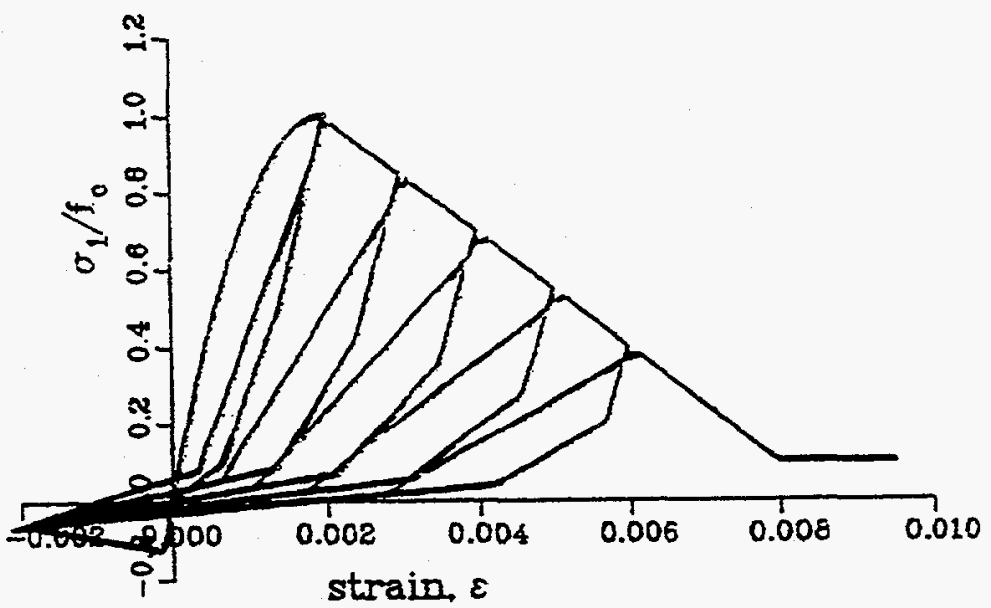

Fig. 3.2 Uniaxial Hysteretic Model for Concrete 


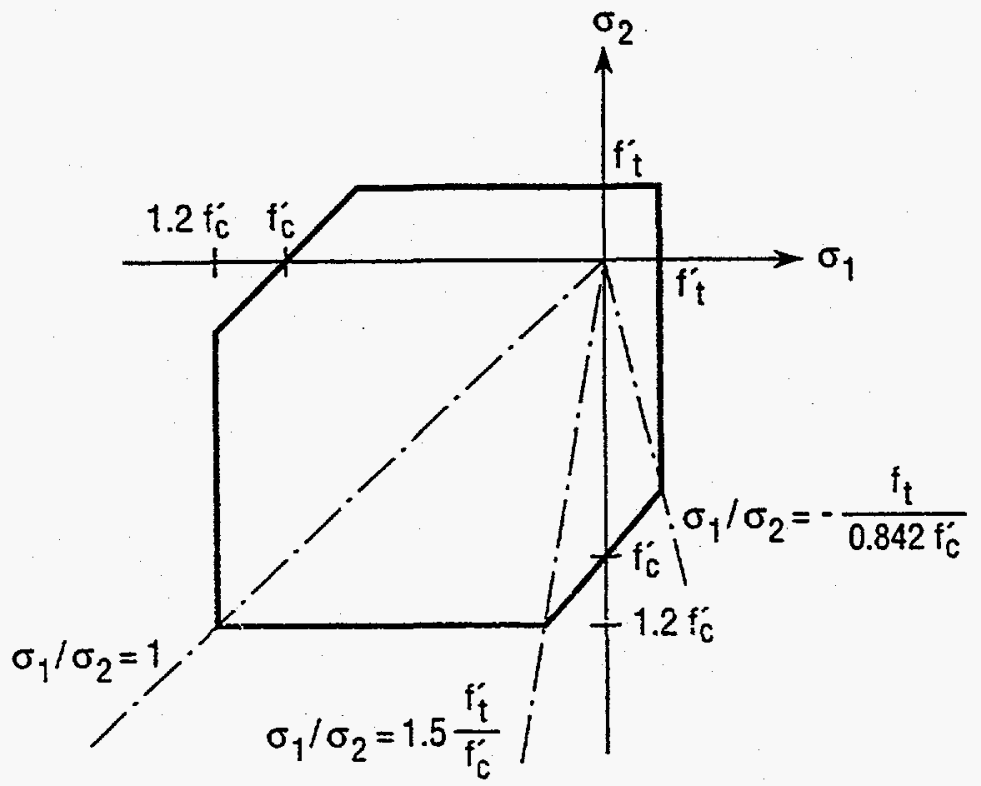

Fig. 3.3 Biaxial Envelope Function for Concrete

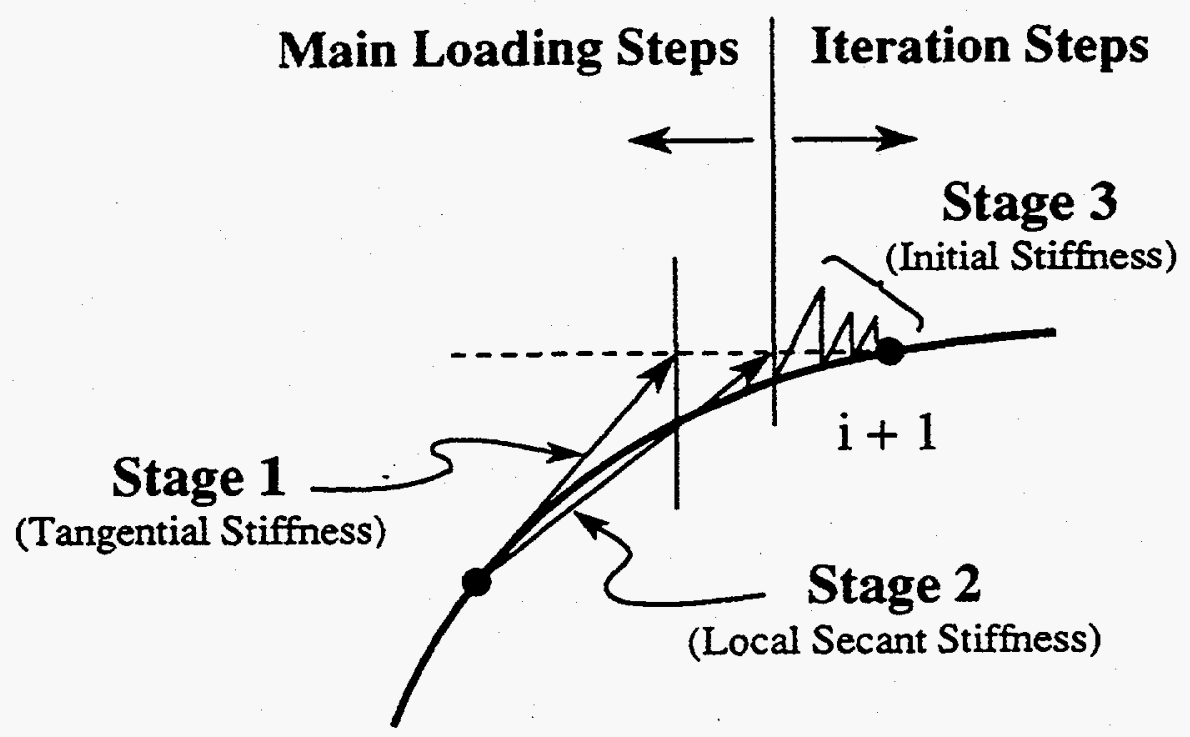

Fig. 3.4 Numerical Iteration Scheme 
BNL's Analyses

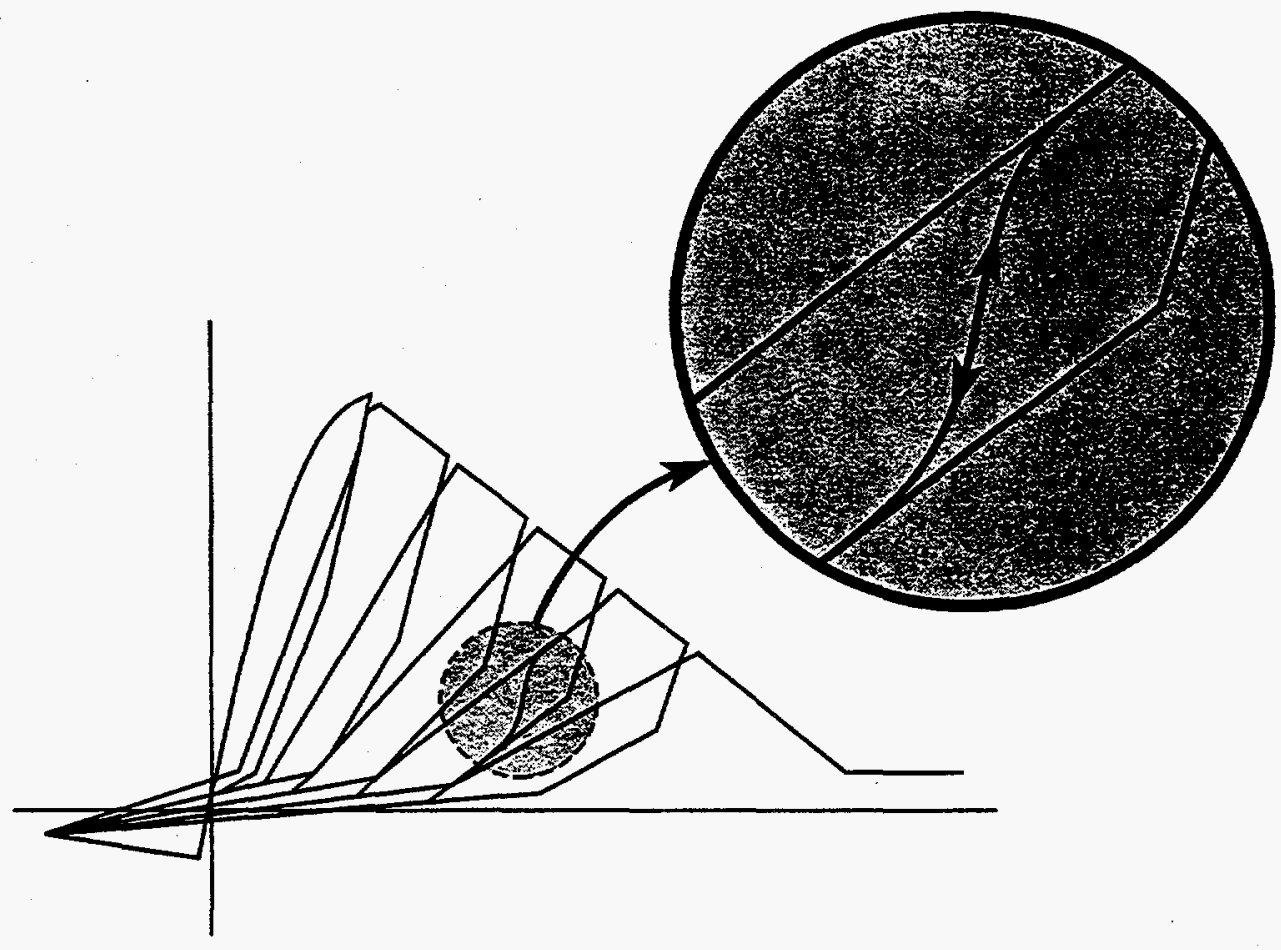

(For Main Loading Steps)

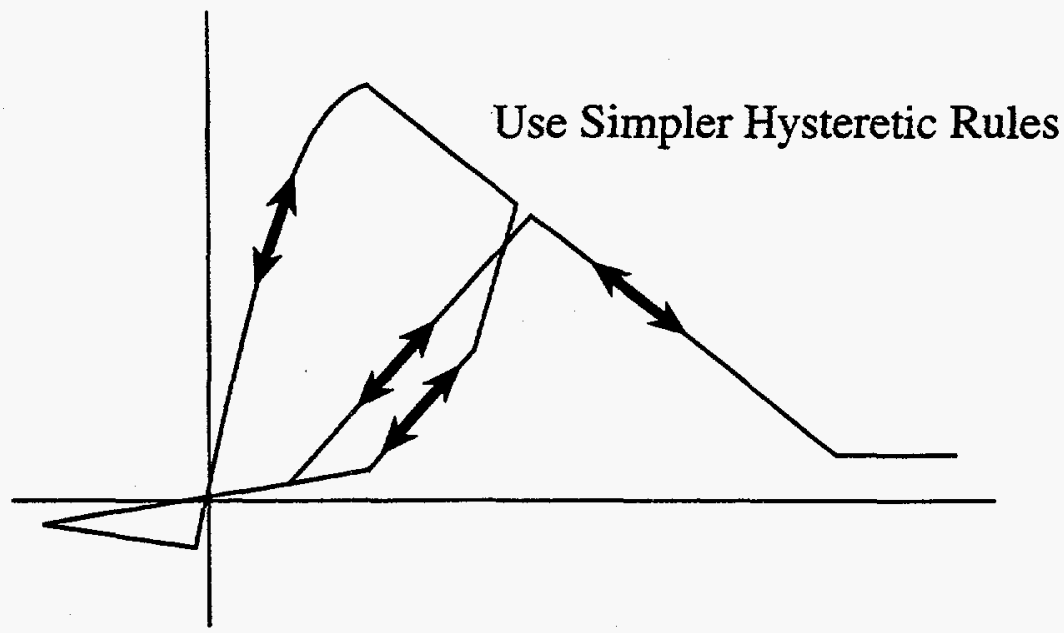

(For Iteration Steps)

Fig. 3.5 Additional Consideration for Uniaxial Hysteretic Model of Concrete 
BNL's Analyses

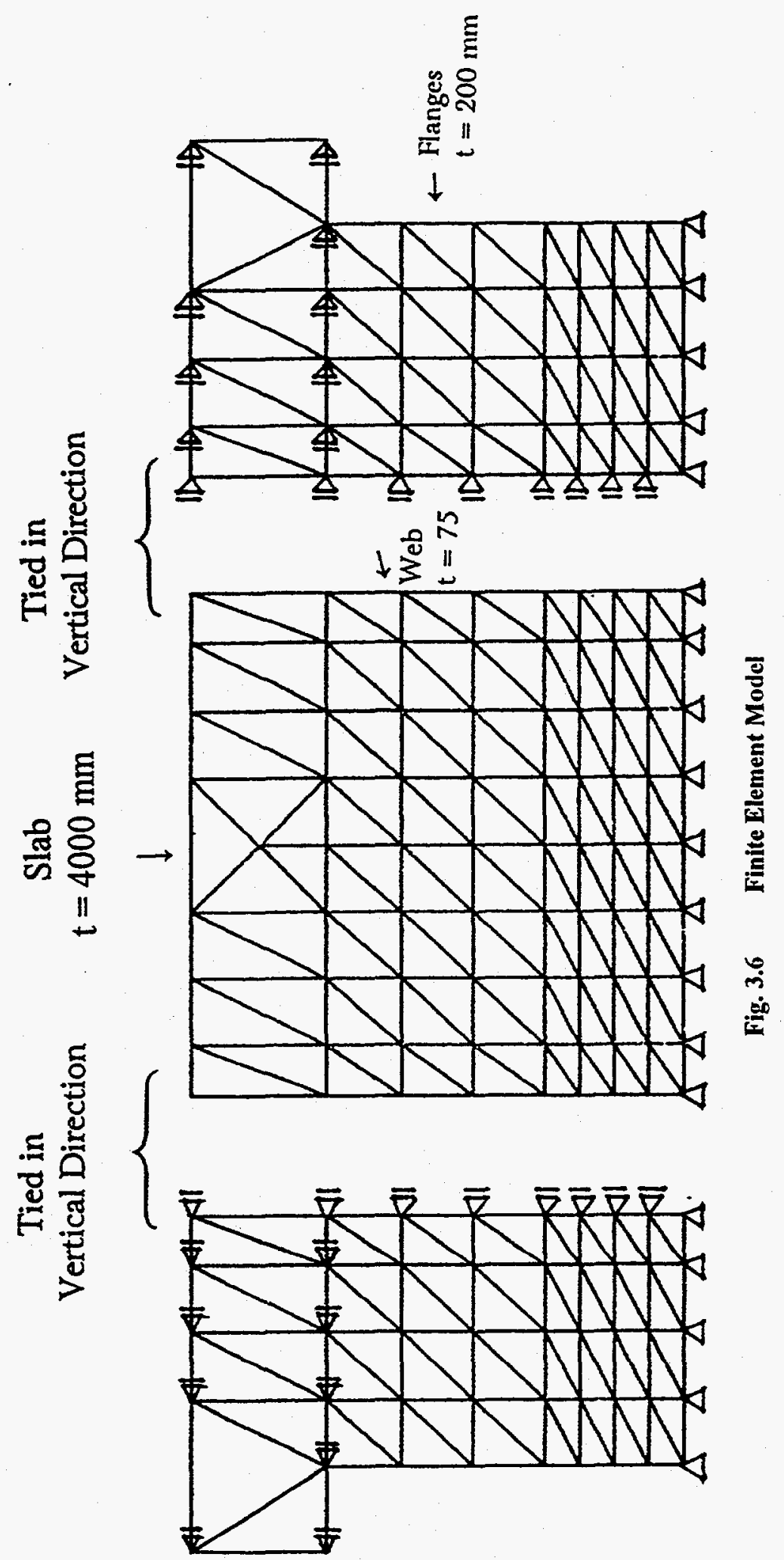


BNL's Analyses

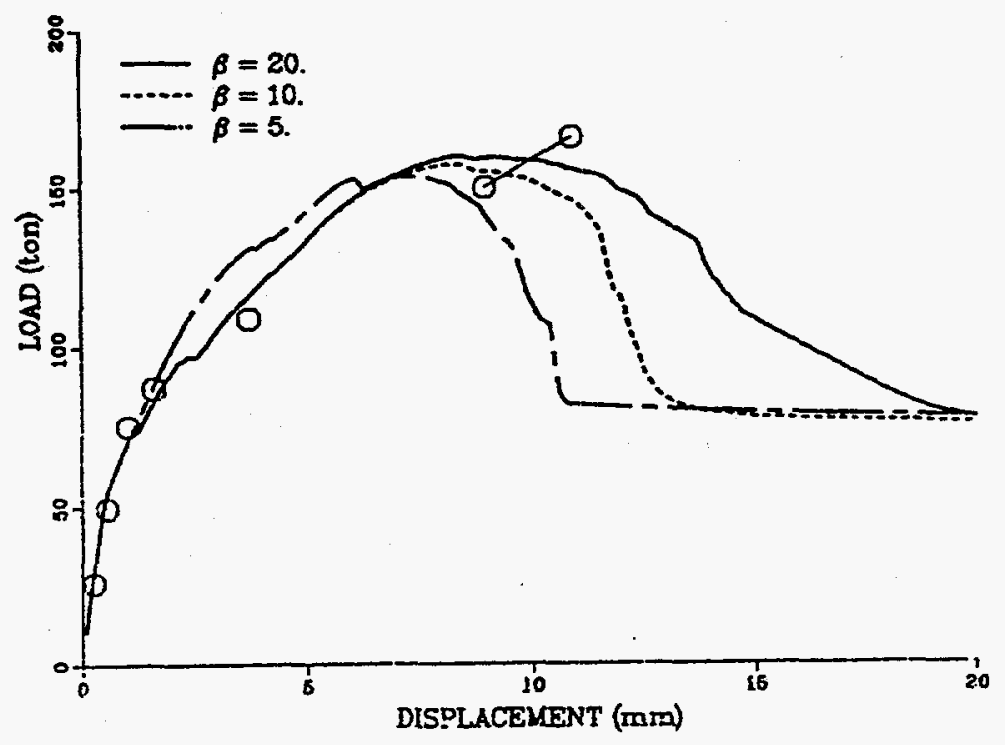

Fig. 3.7 Load-deformation Relationships from Monotonic Static Analyses (Circles Indicate Location of Peak Responses of Six Test Runs) 
BNL's Analyses
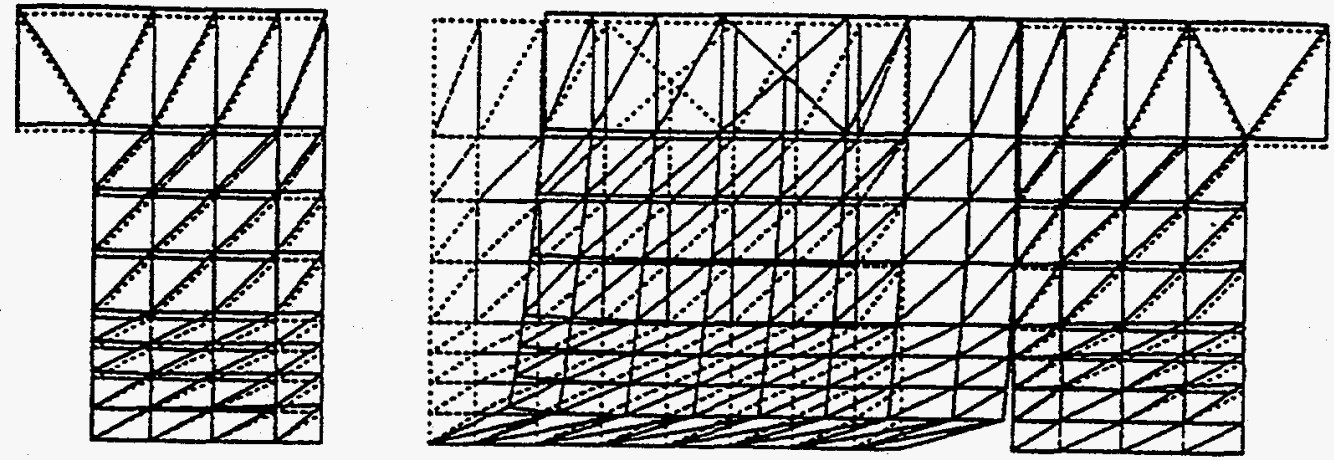

(a) Final Deformation Shape (u $=20 \mathrm{~mm}$ )
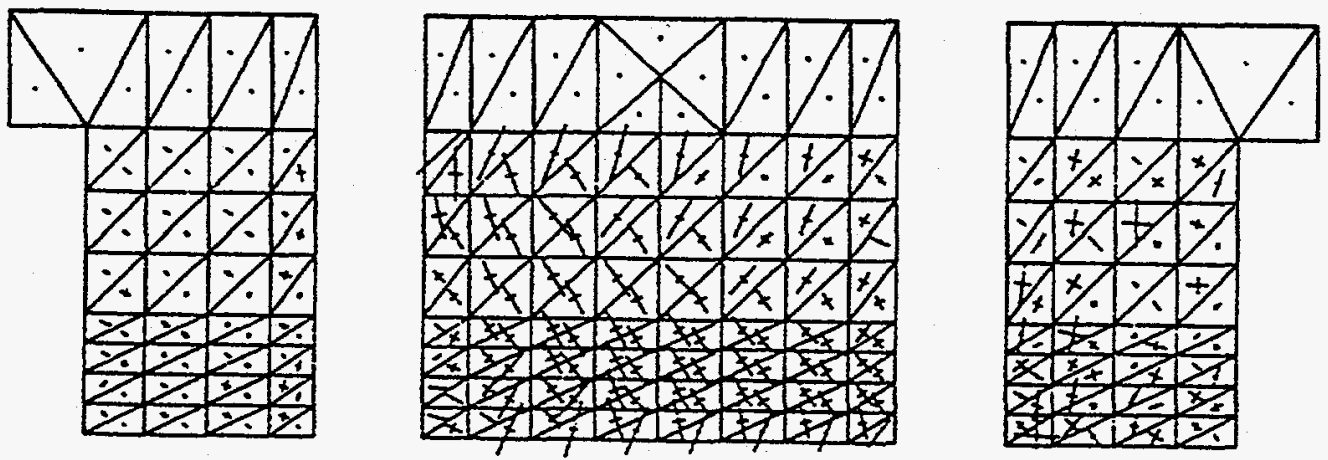

(b) Principal Stresses $(u=10 \mathrm{~mm})$
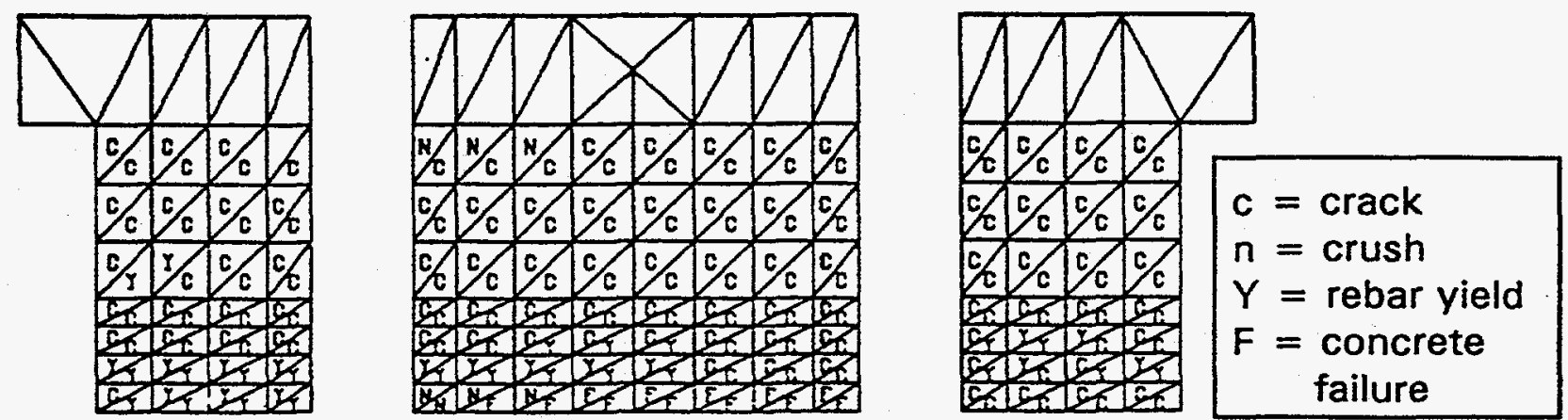

(c) Damage State $(u=20 \mathrm{~mm})$

Figs. 3.8 Monotonic Static Analysis Results 
BNL's Analyses

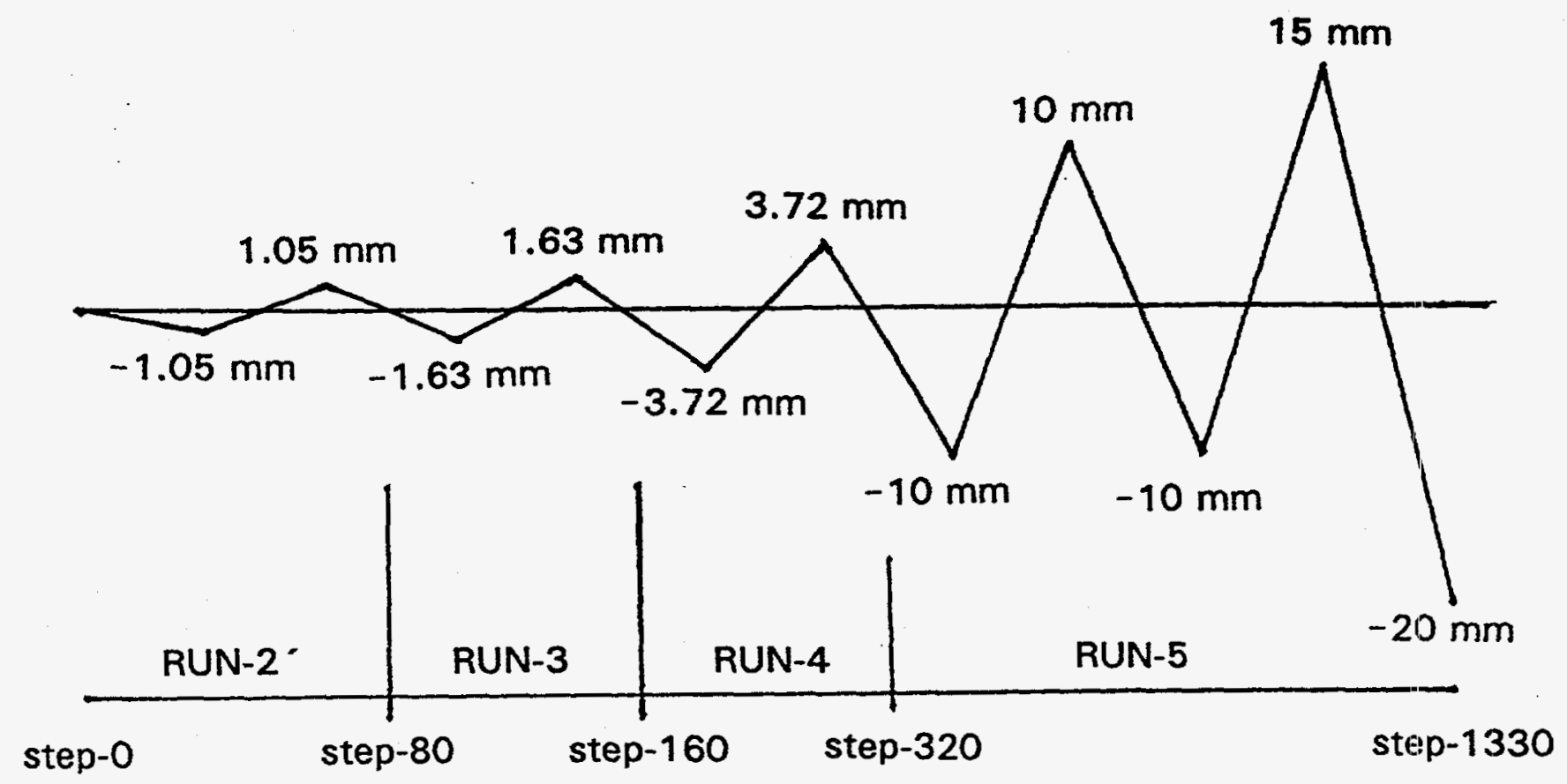

Fig. 3.9 Applied Displacement History for Cyclic Static Analysis 


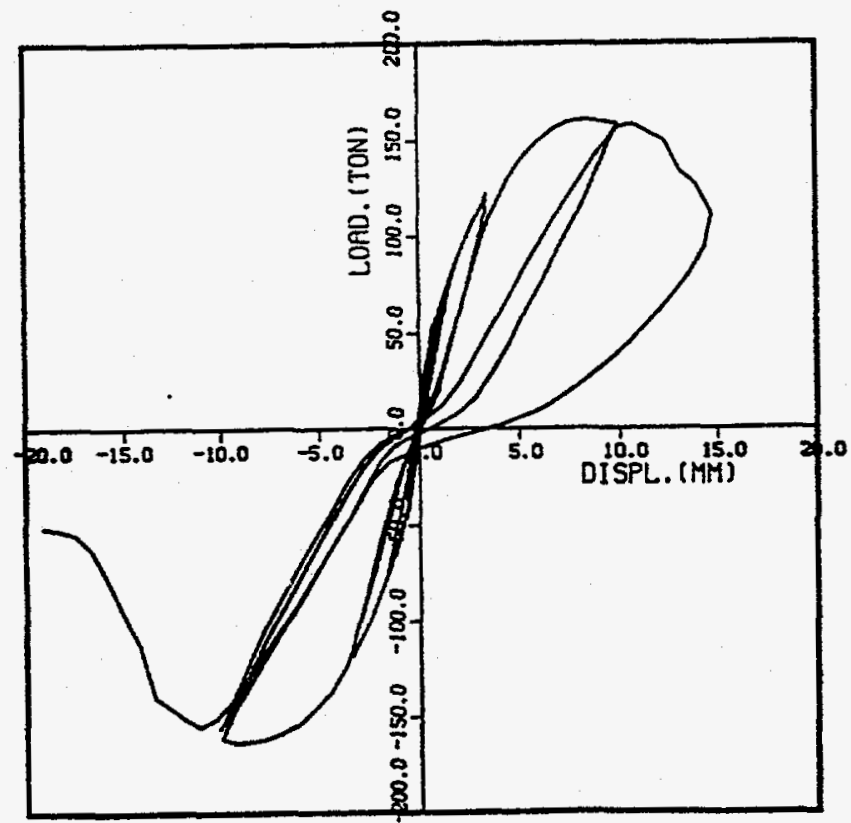

Fig. 3.10 Load-deformation Relationship from Cyclic Static Analysis

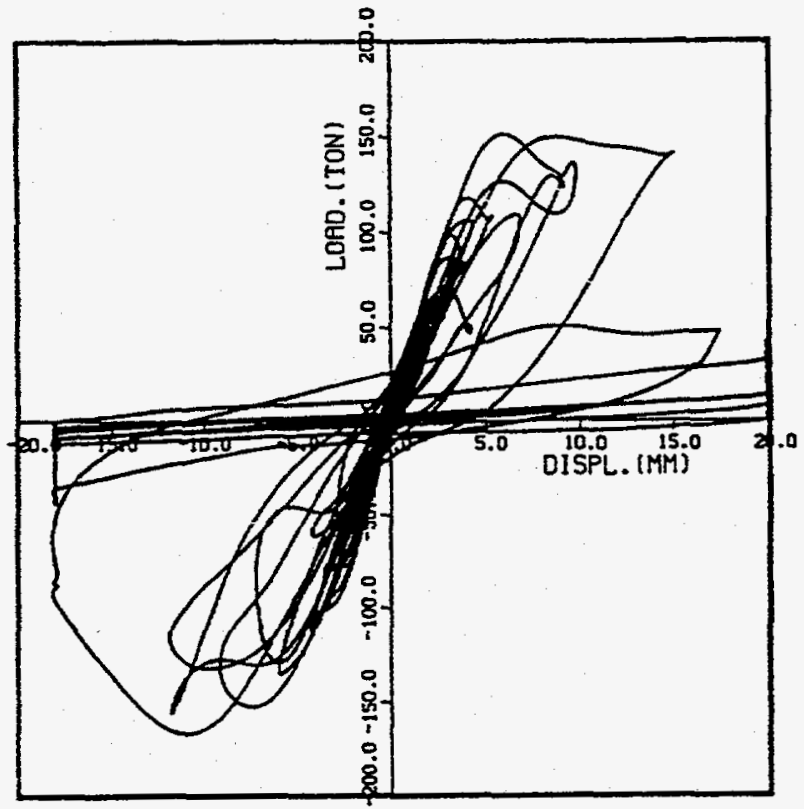

Fig. 3.11 Load-deformation Relationship from RUN-5 

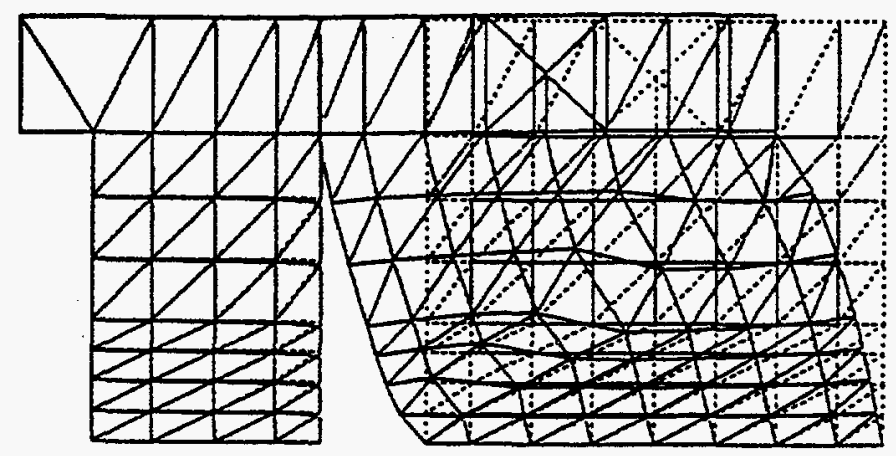

(a) Deformation Shape
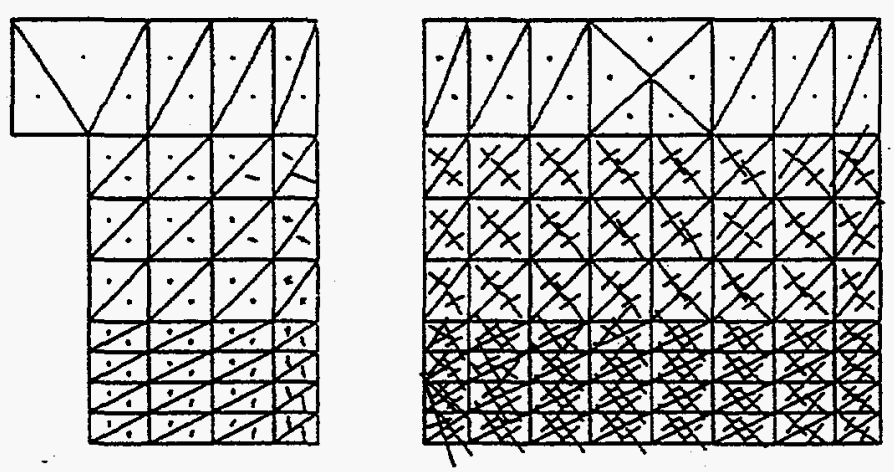

(b) Principal Stresses

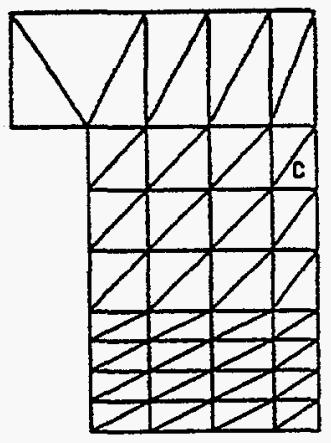

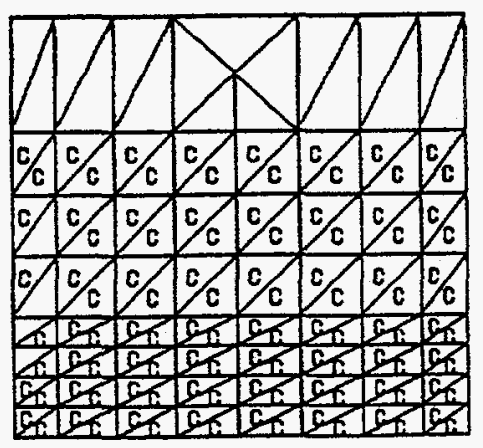

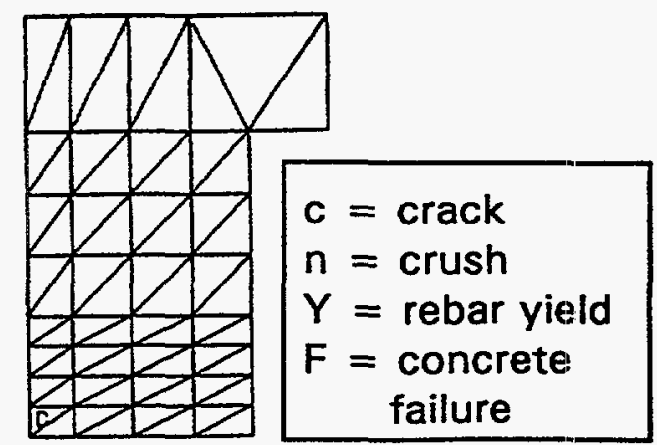

\section{(c) Damage State}

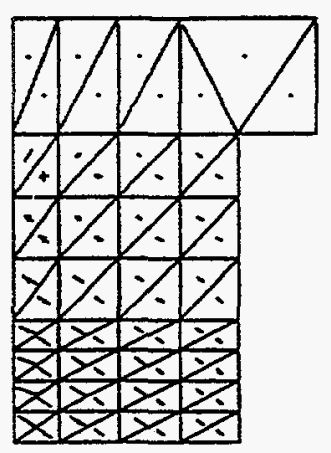

Fig. 3.12 Response for RUN-2' $(u=1.05 \mathrm{~mm}$ ) 

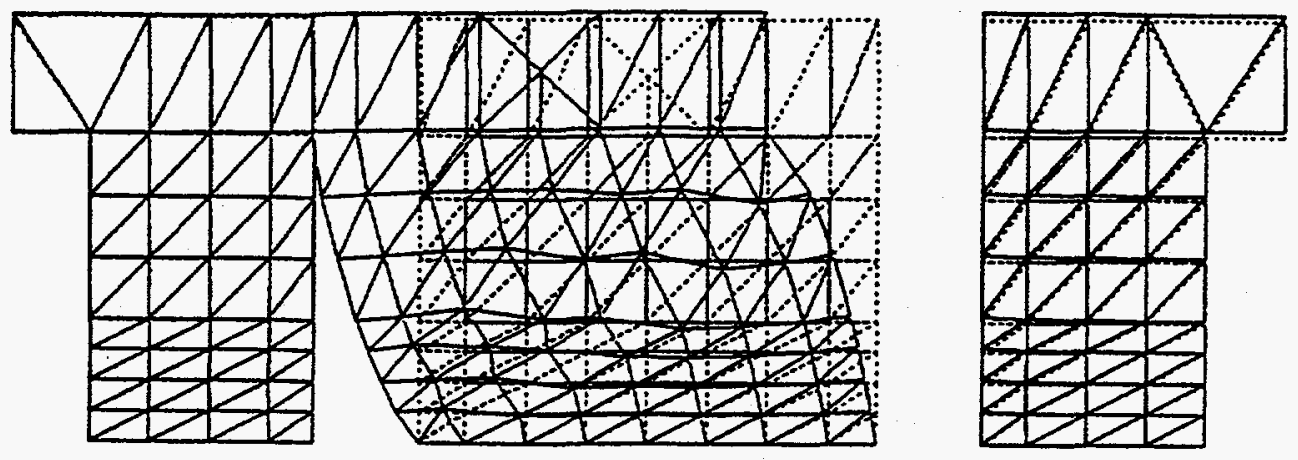

\section{(a) Deformation Shape}
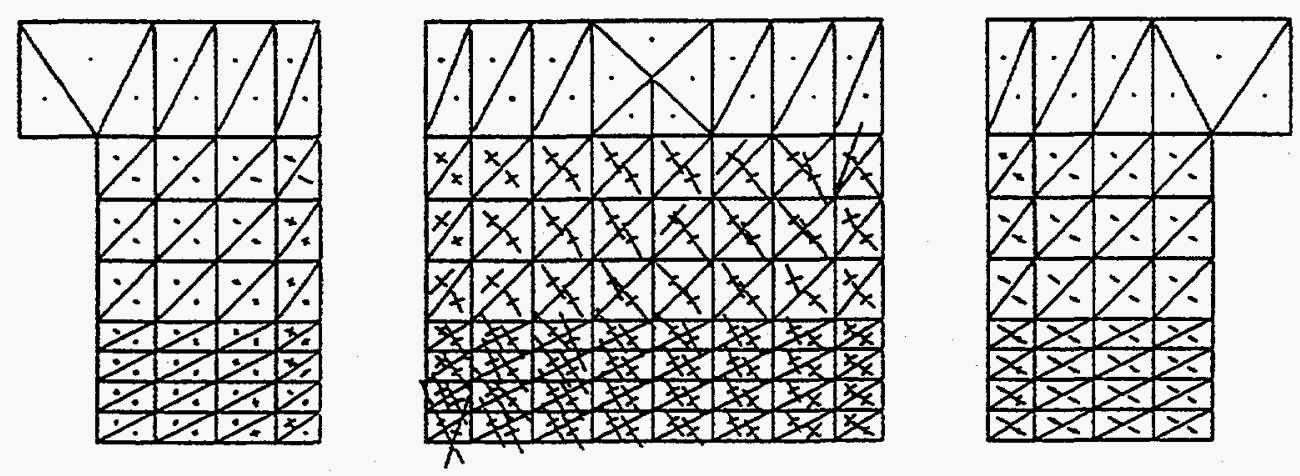

(b) Principal Stresses
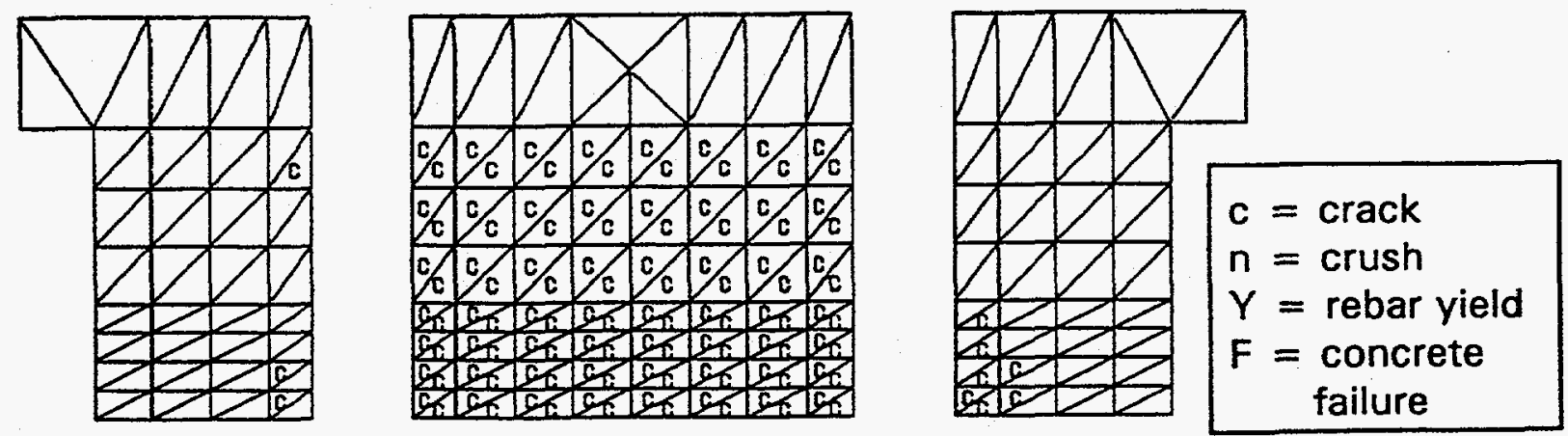

\section{(c) Damage State}

Fig. 3.13 Response for RUN-3 (u=1.63 mm) 

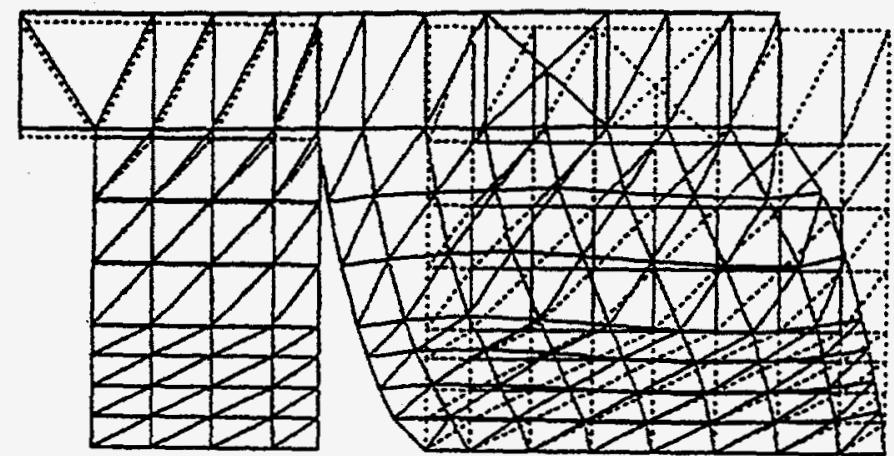

(a)
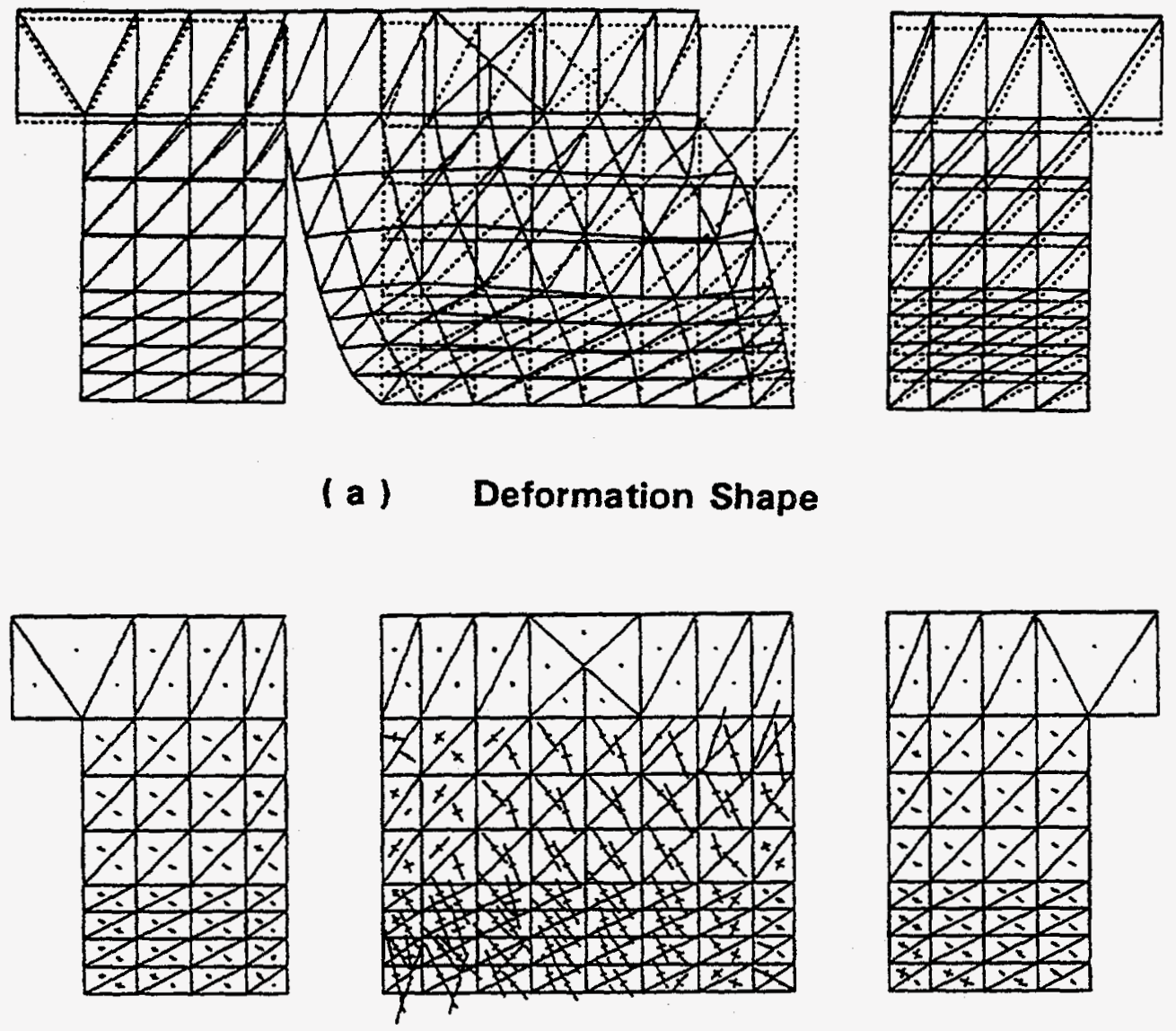

(b) Principal Stresses
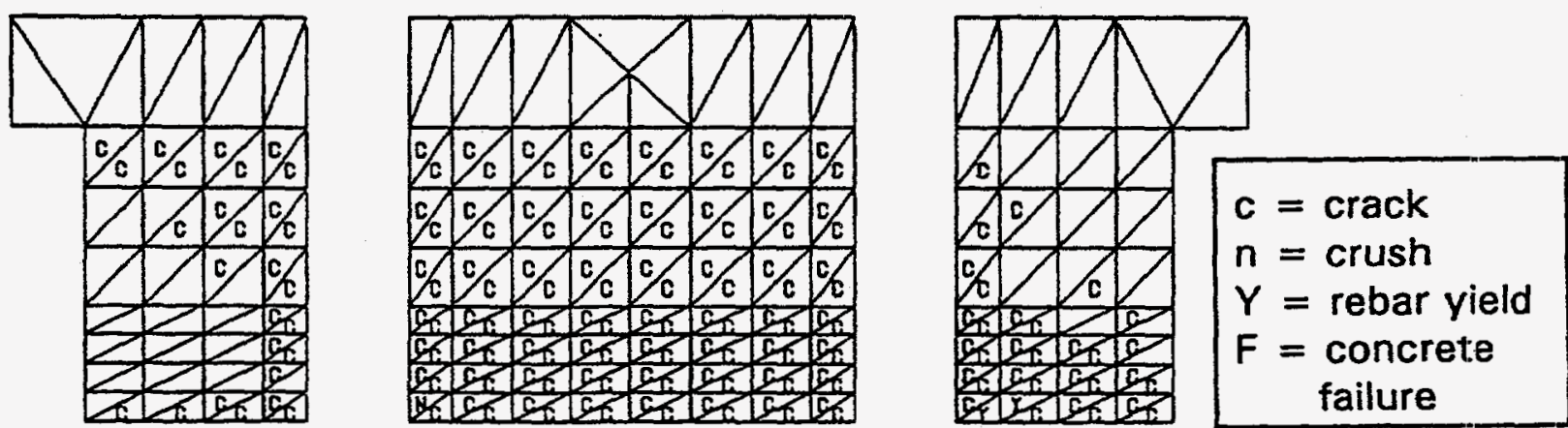

\section{(c) Damage State}

Fig. 3.14 Response for RUN-4 $(u=3.72 \mathrm{~mm})$ 

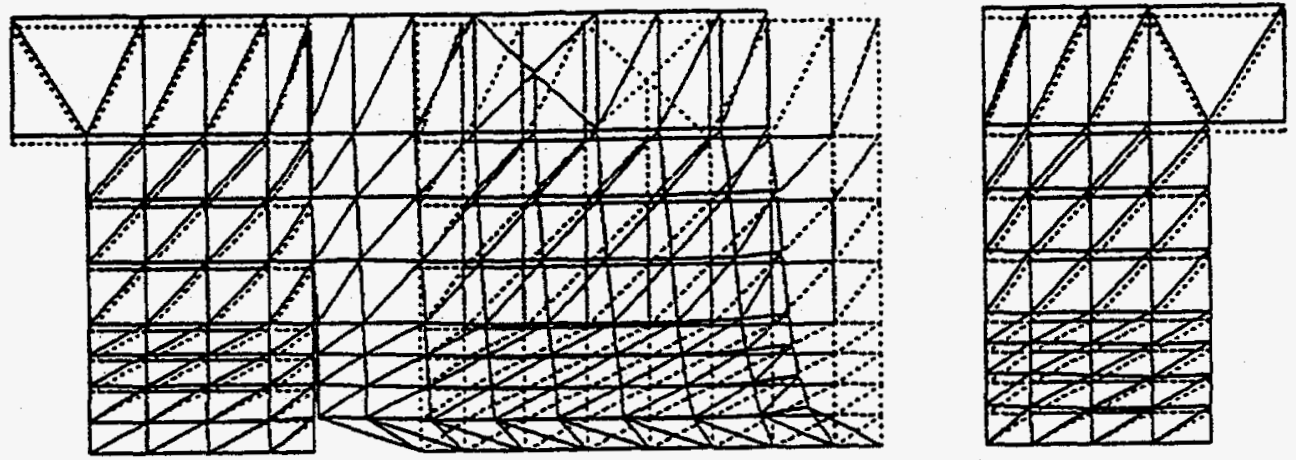

(a) Deformation Shape
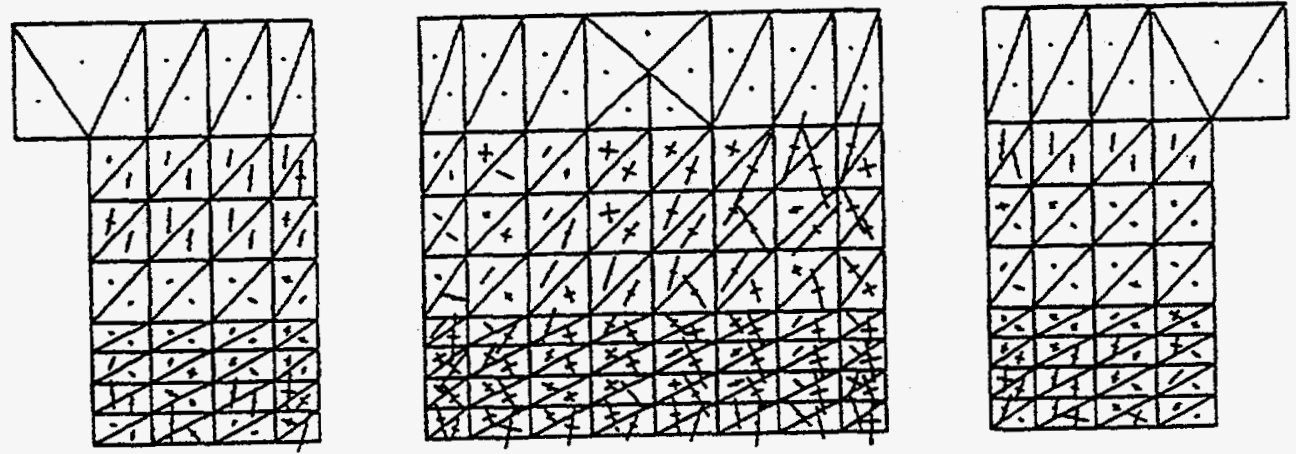

(b) Principal Stresses
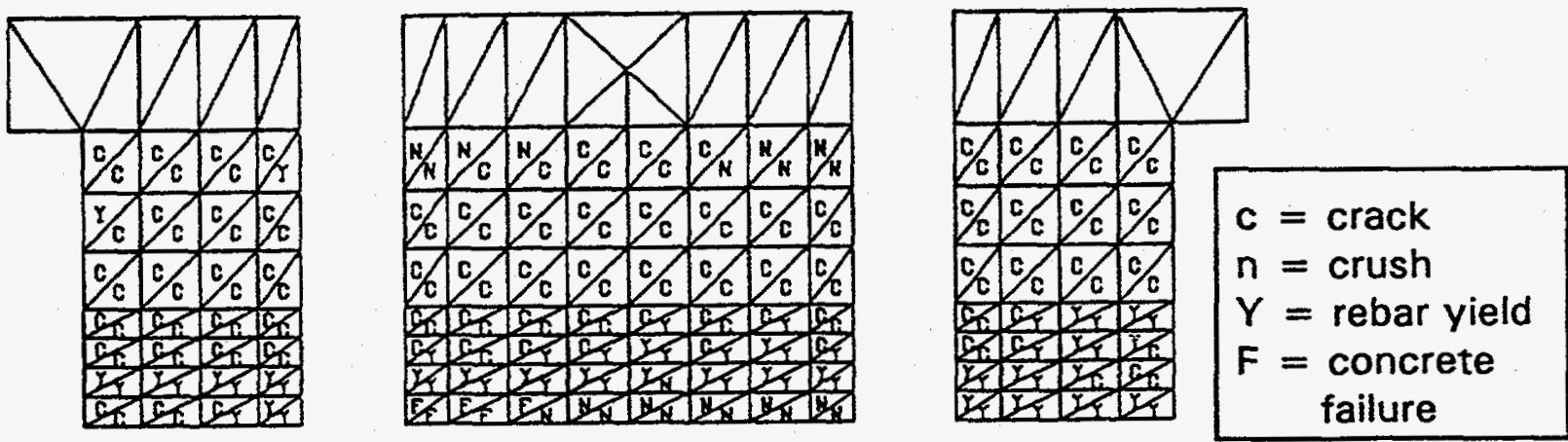

\section{(c) Damage State}

Fig. 3.15 Final State from Cyclic Static Analysis $(u=20 \mathrm{~mm})$ 
BNL's Analyses
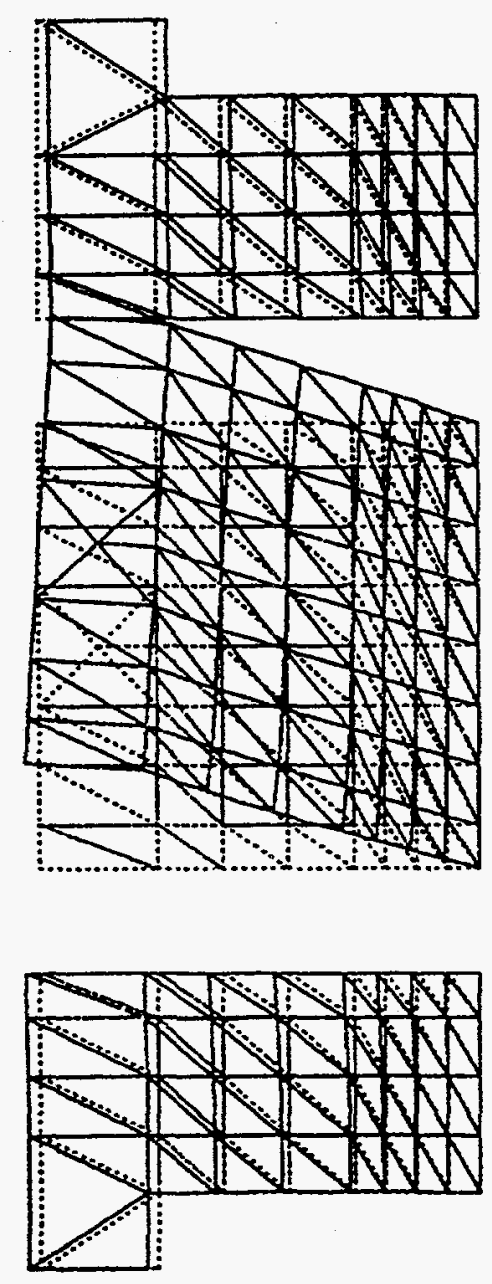

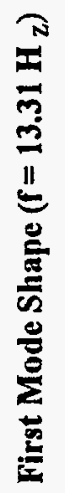

लำ

点

容 


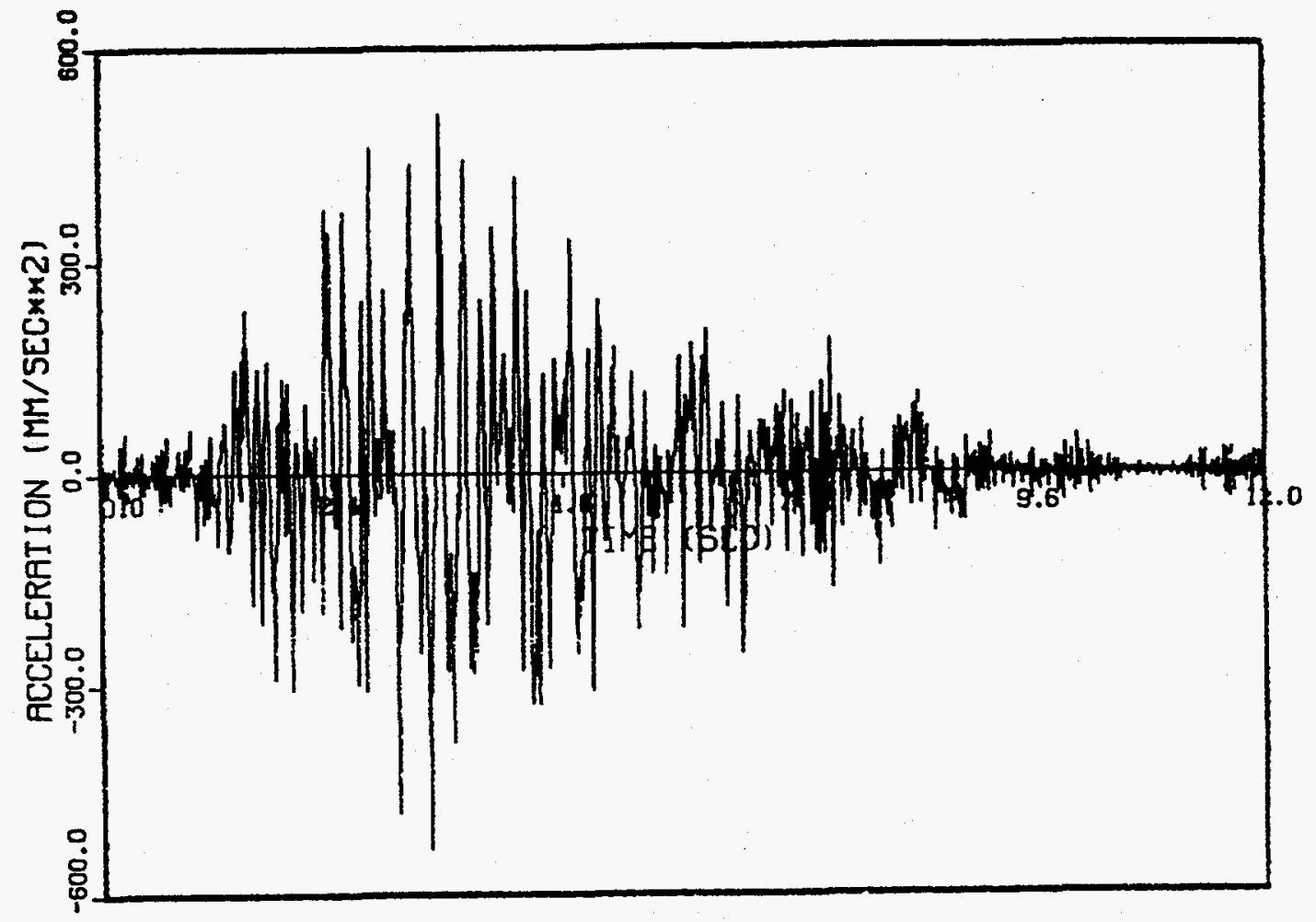

Fig. 3.17 Horizontal Base Motion (AXB) for RUN-1 


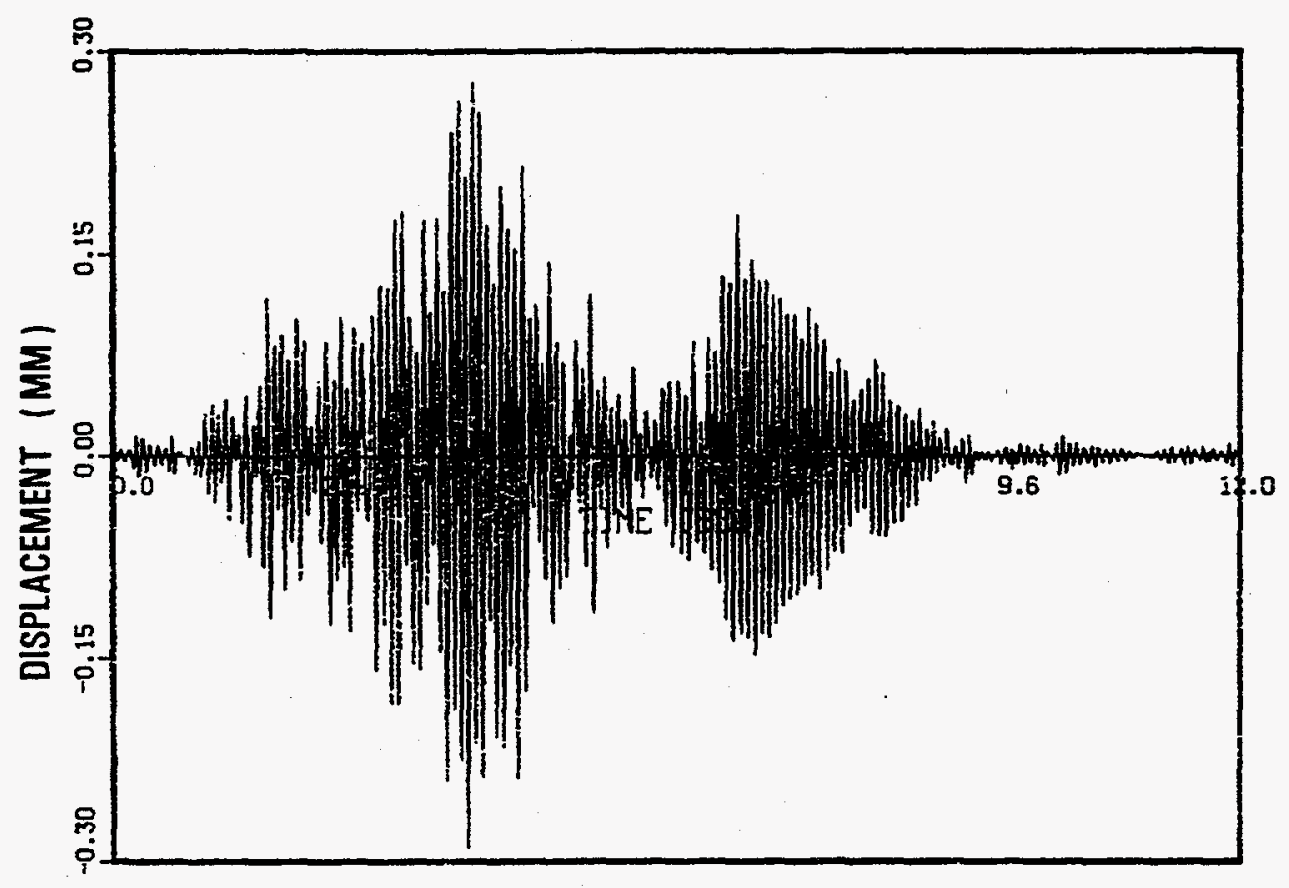

(a) Analysis

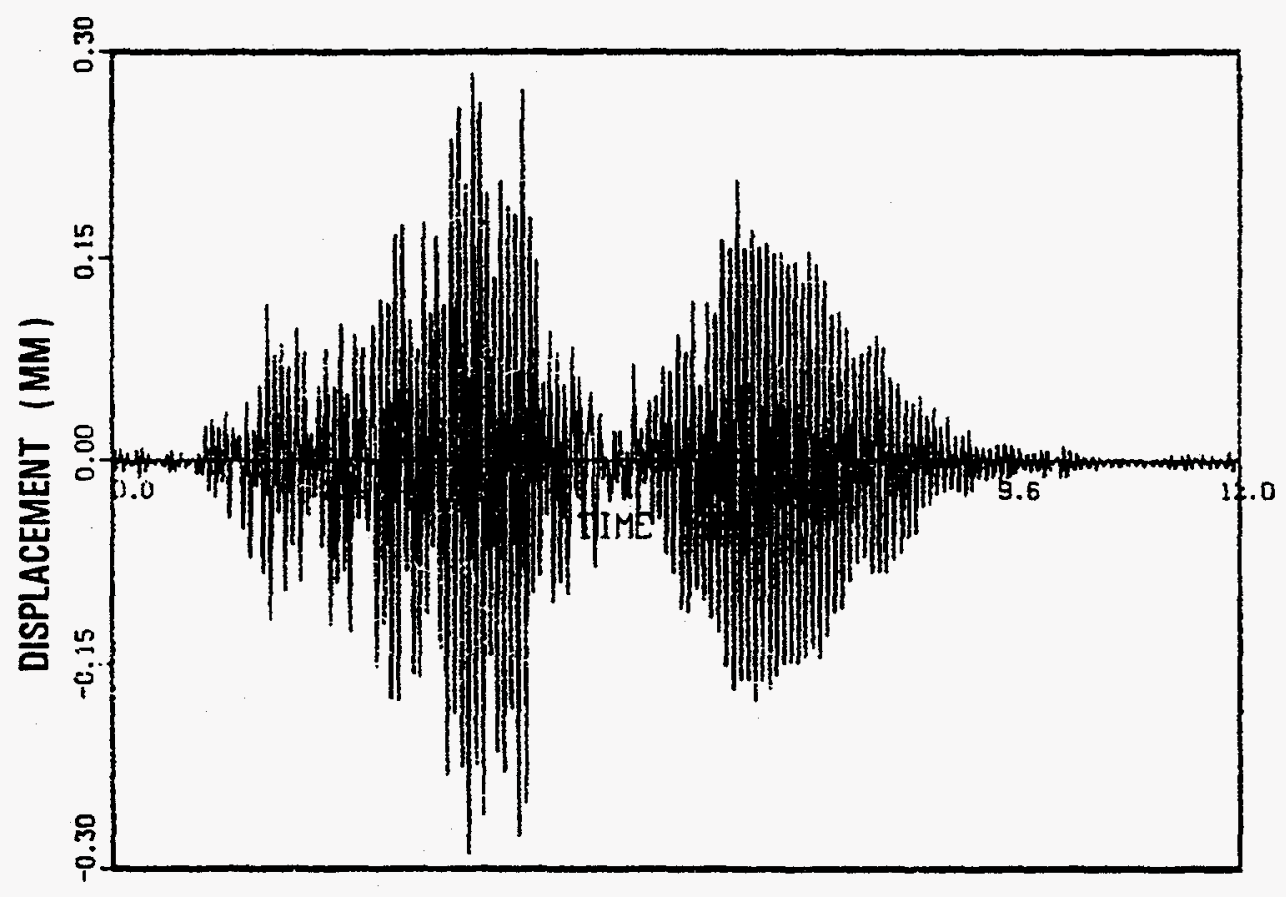

(b) Test

Fig. 3.18 Horizontal Displacement of Top Slab (DXT) for RUN-1 


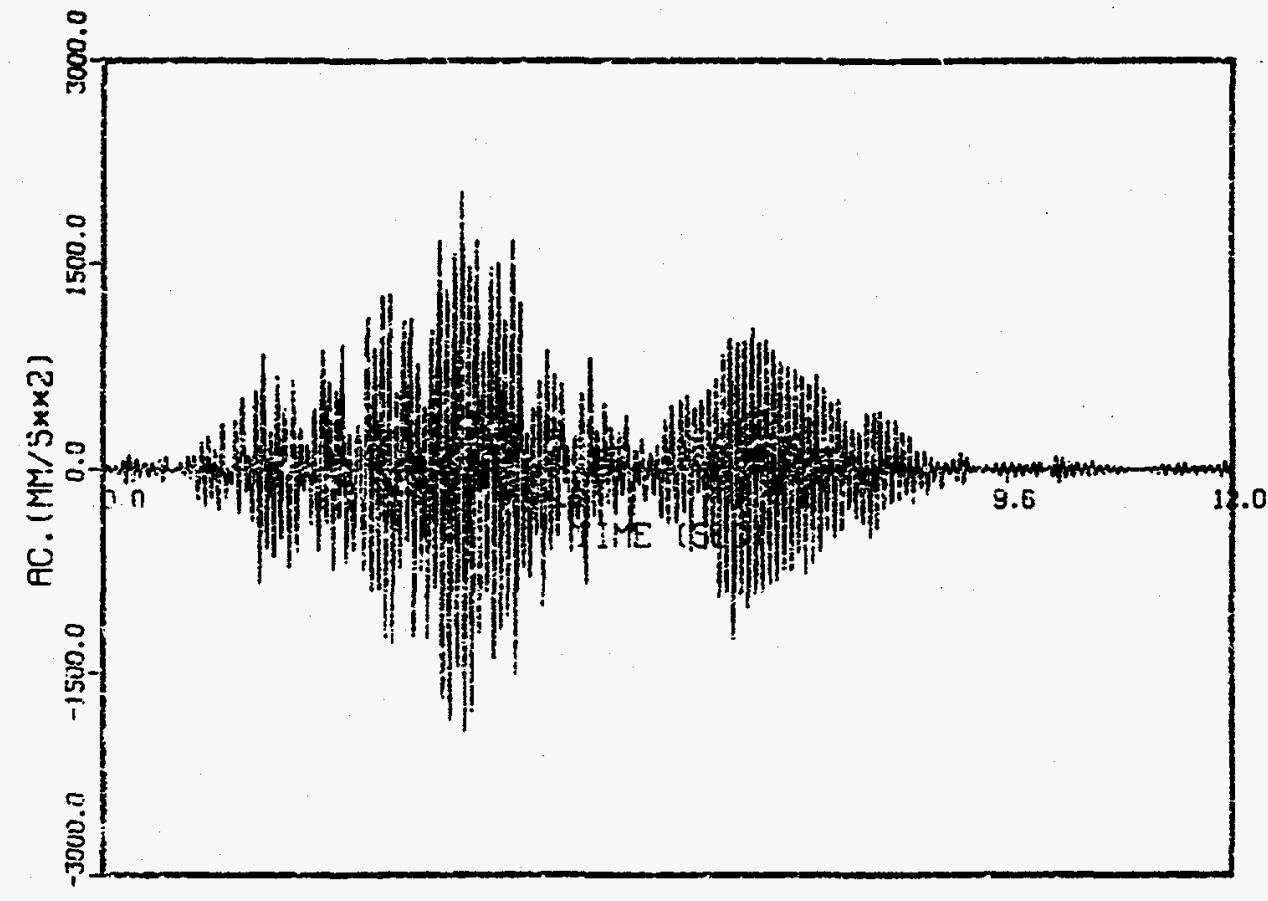

(a) Analysis

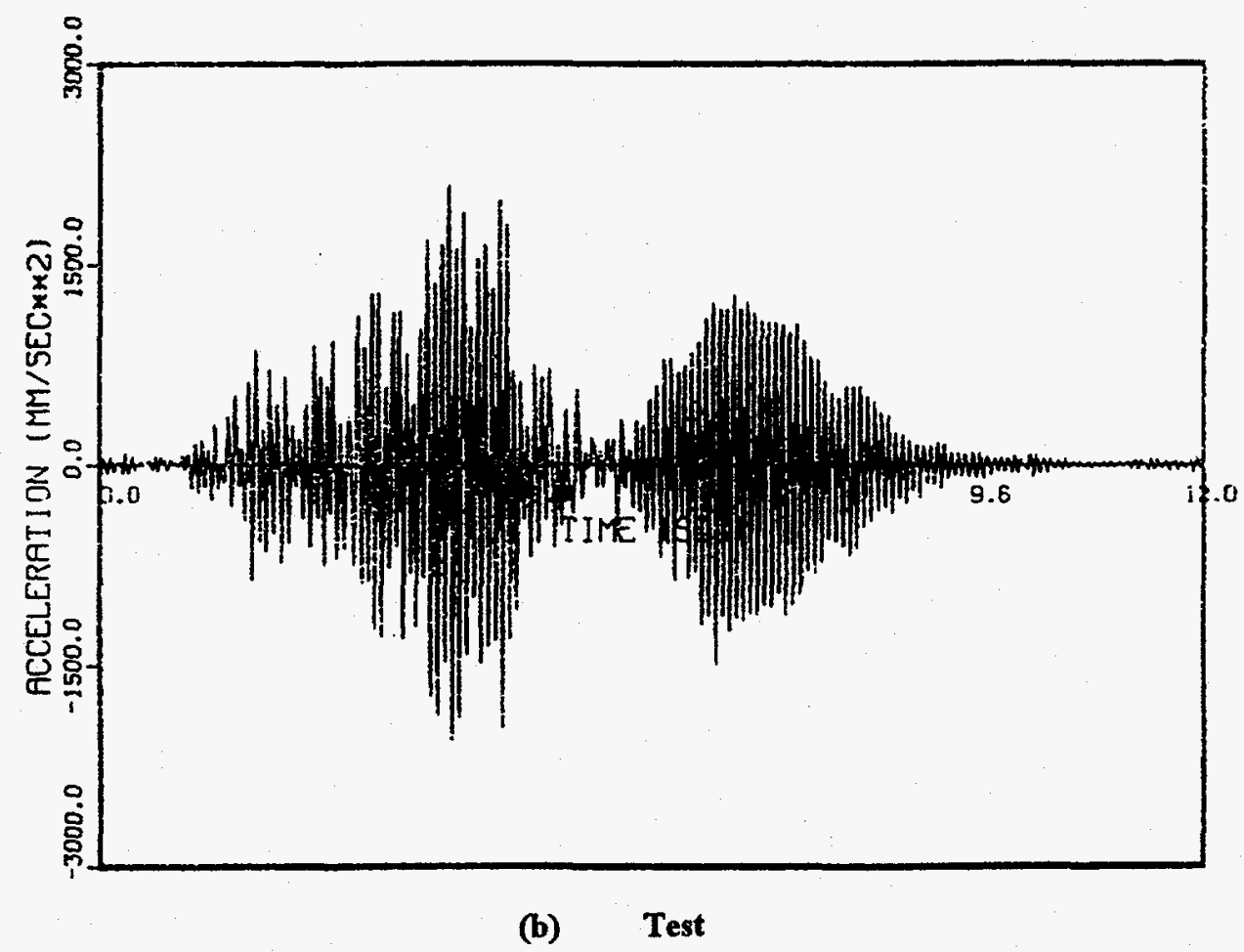

Fig. 3.19 Horizontal Acceleration of Top Slab (AXT) for RUN-1 
BNL's Analyses

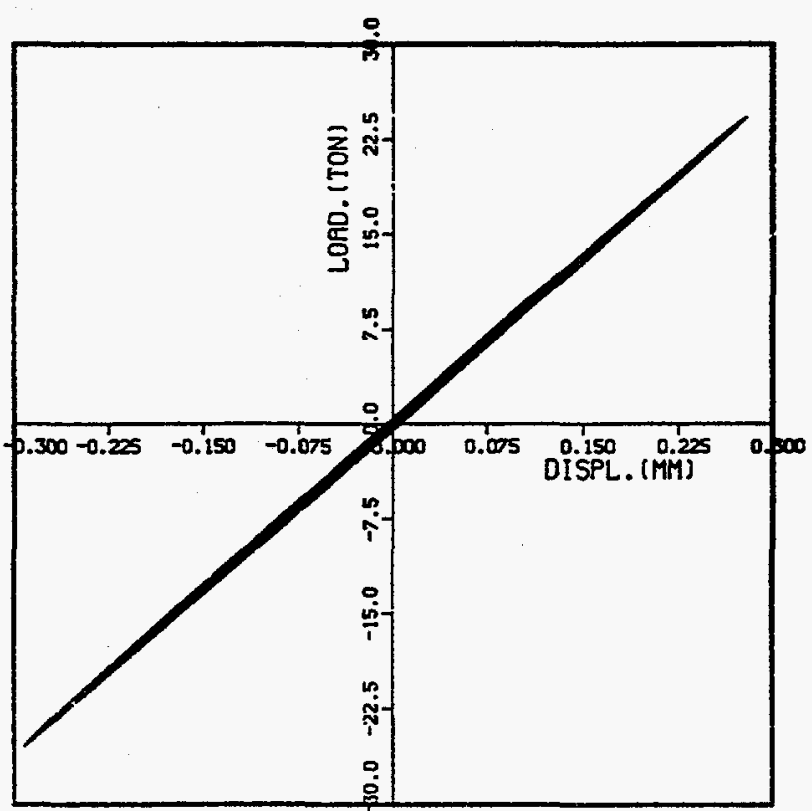

(a) Analysis

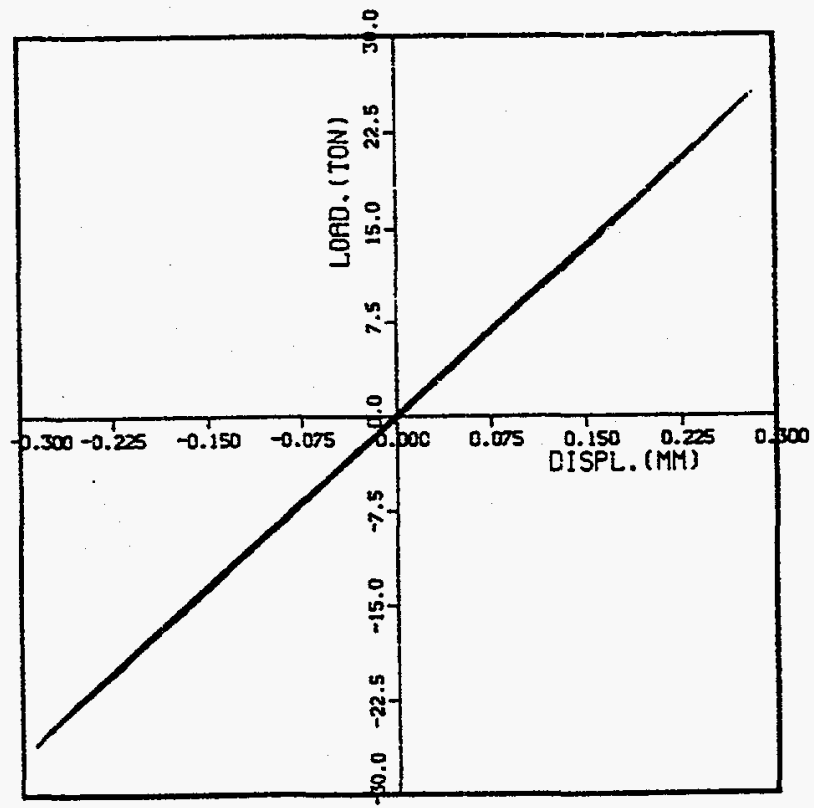

(b) Test

Fig. 3.20 Load-Deformation Relationship for RUN-1 


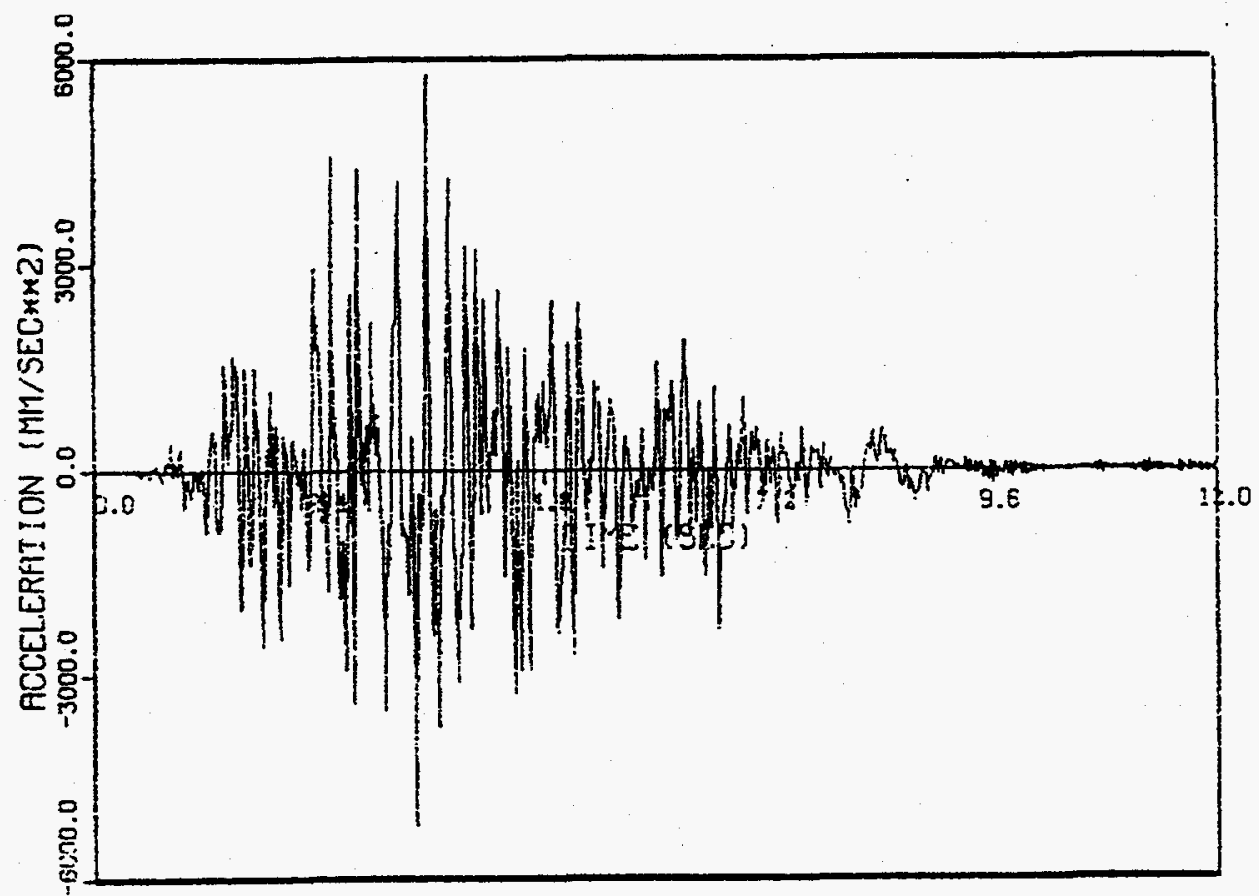

Fig. 3.21 Horizontal Base Motion (AXB) for RUN-4 


\section{BNL`s Analyses}

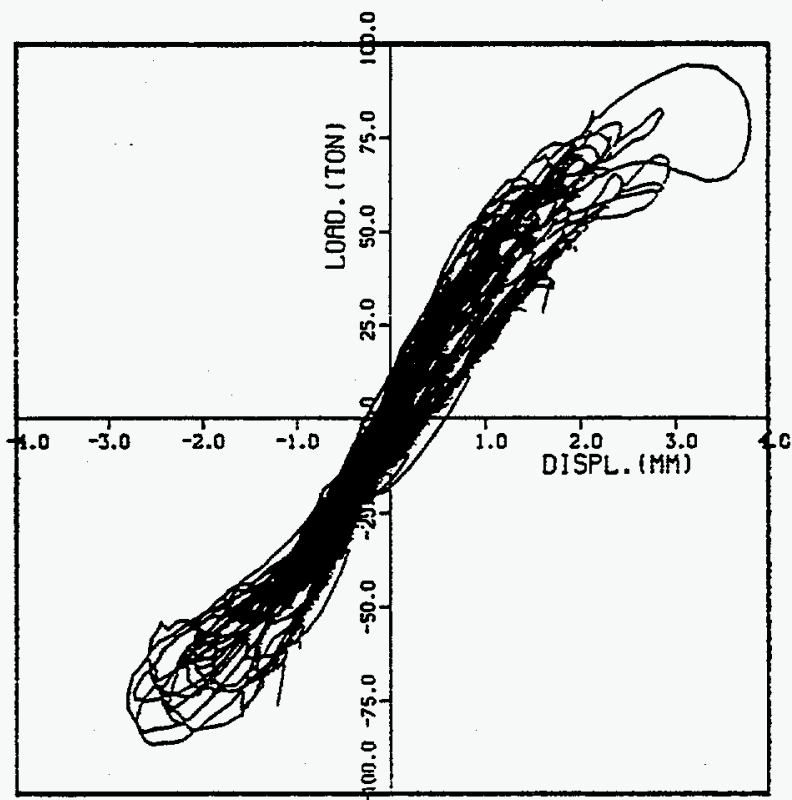

(a) Analysis

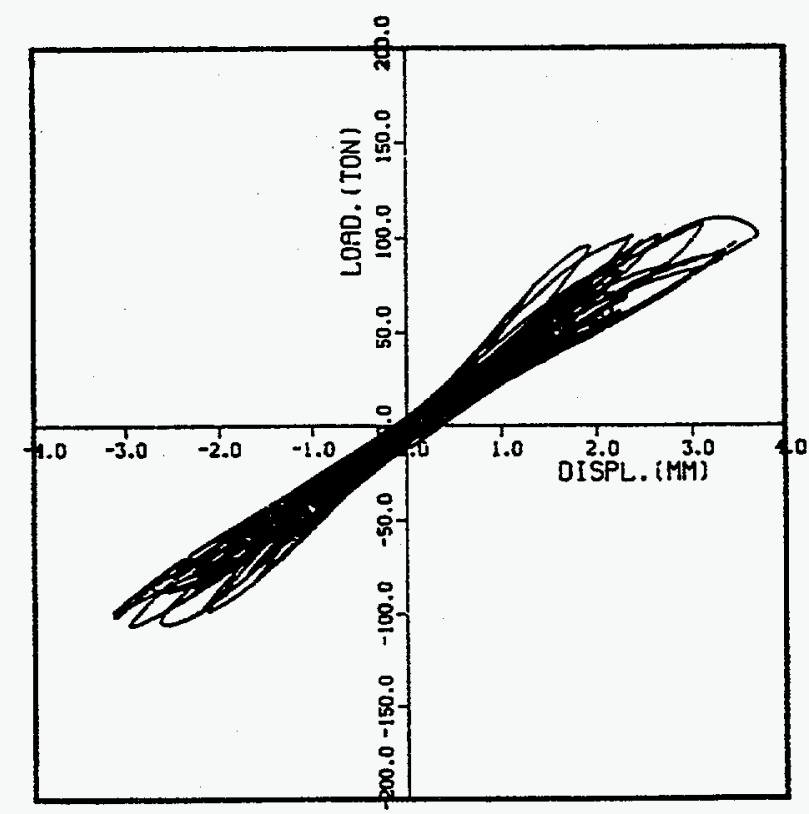

(b) Test

Fig. 3.22 Load-Deformation Relationship for RUN-4 
BNL's Analyses

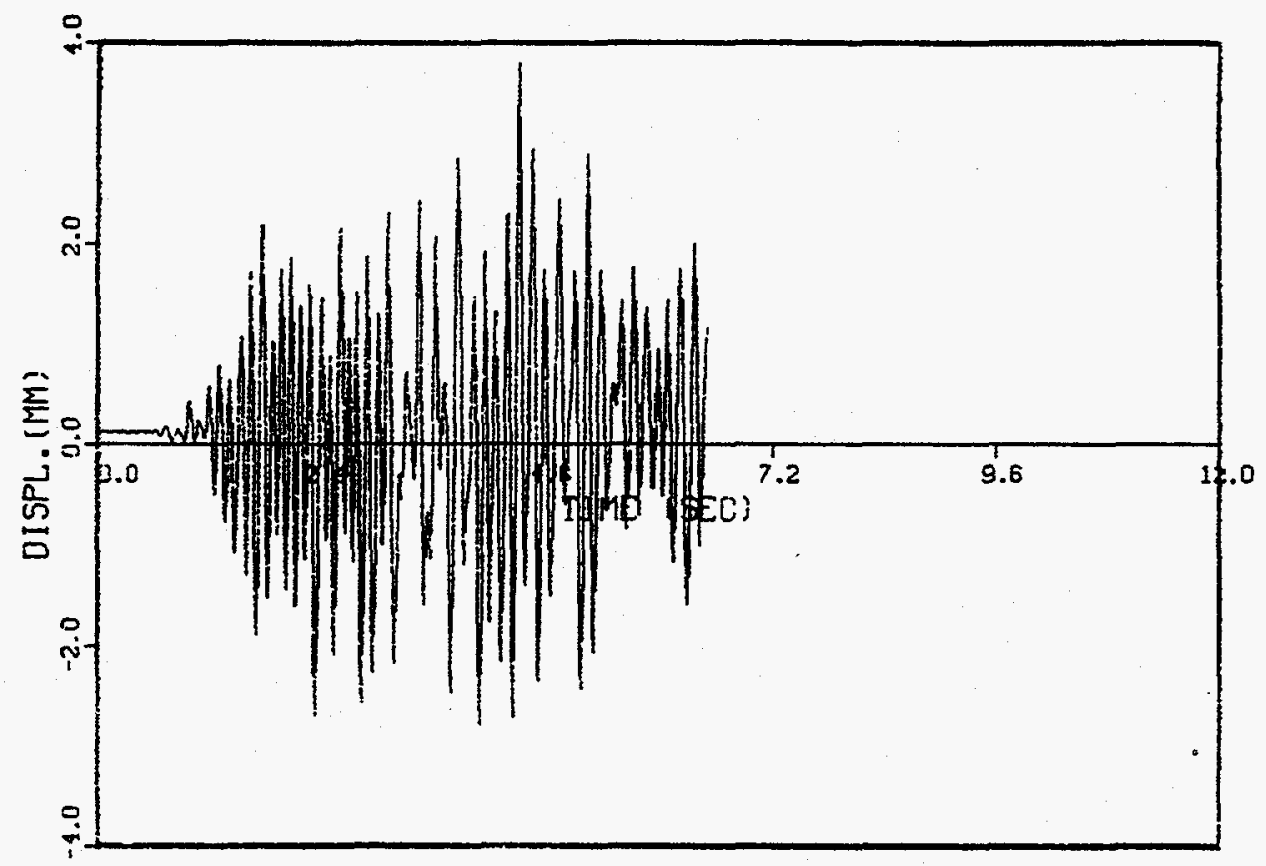

(a) Analysis

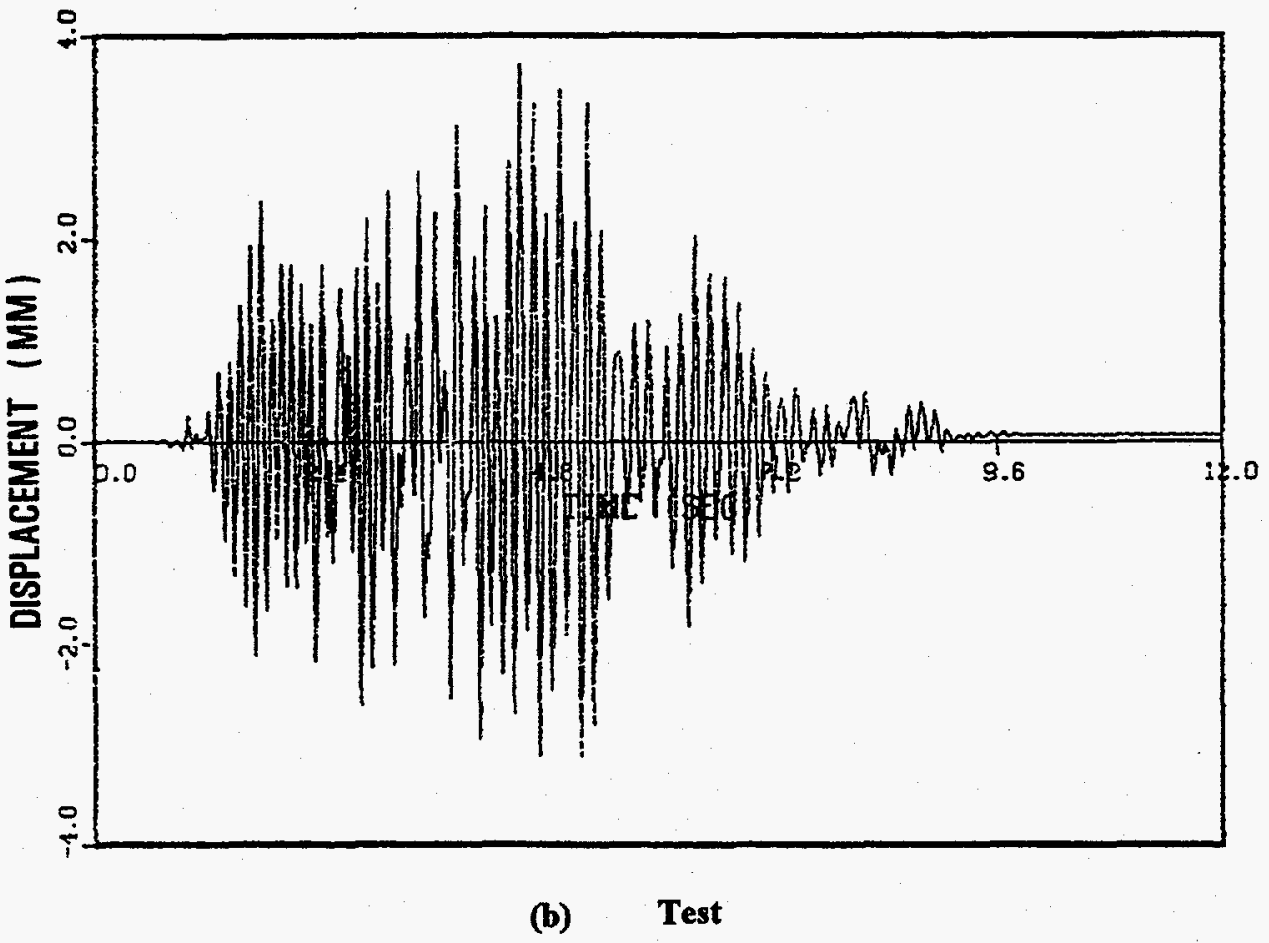

Fig. 3.23 Horizontal Displacement of Top Slab (DXT) for RUN-4 
BNL's Analyses

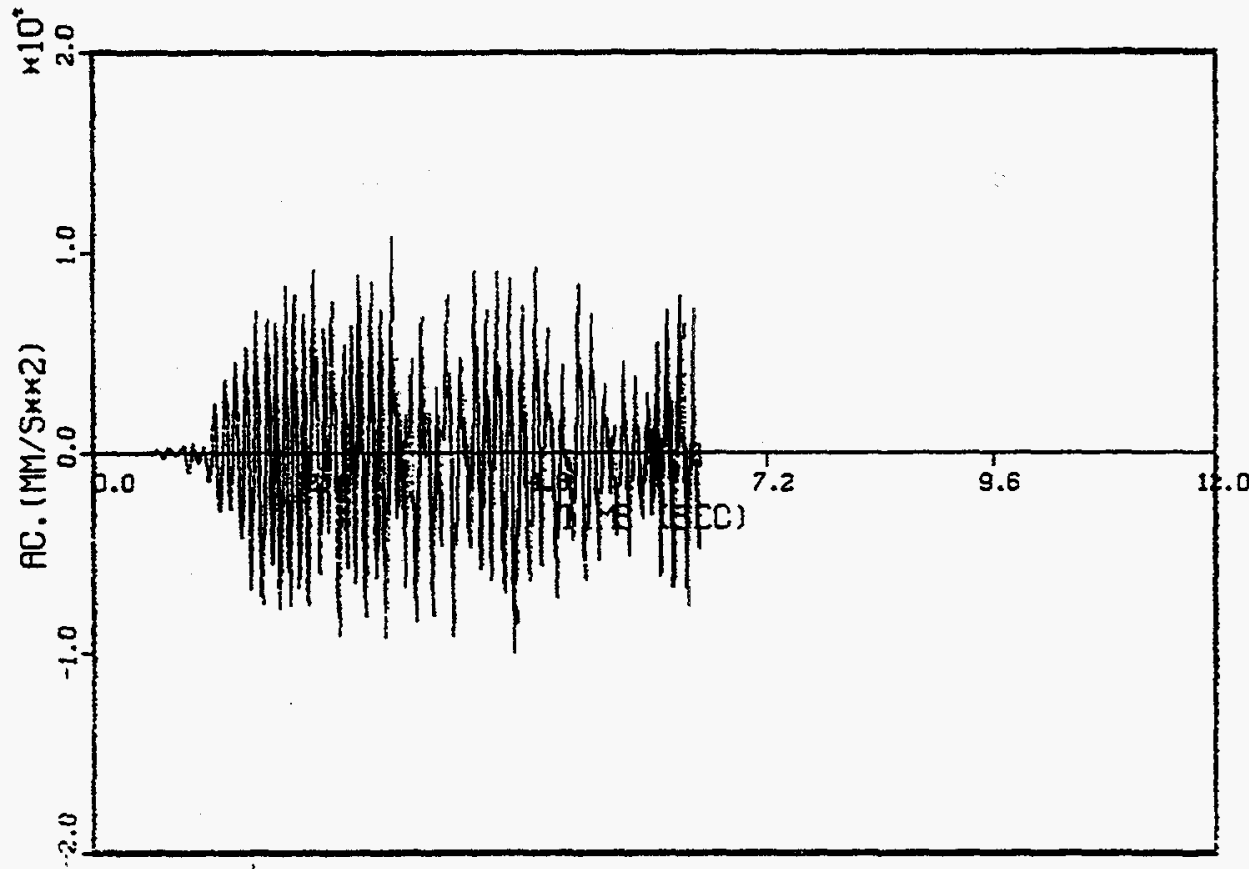

(a) Analysis

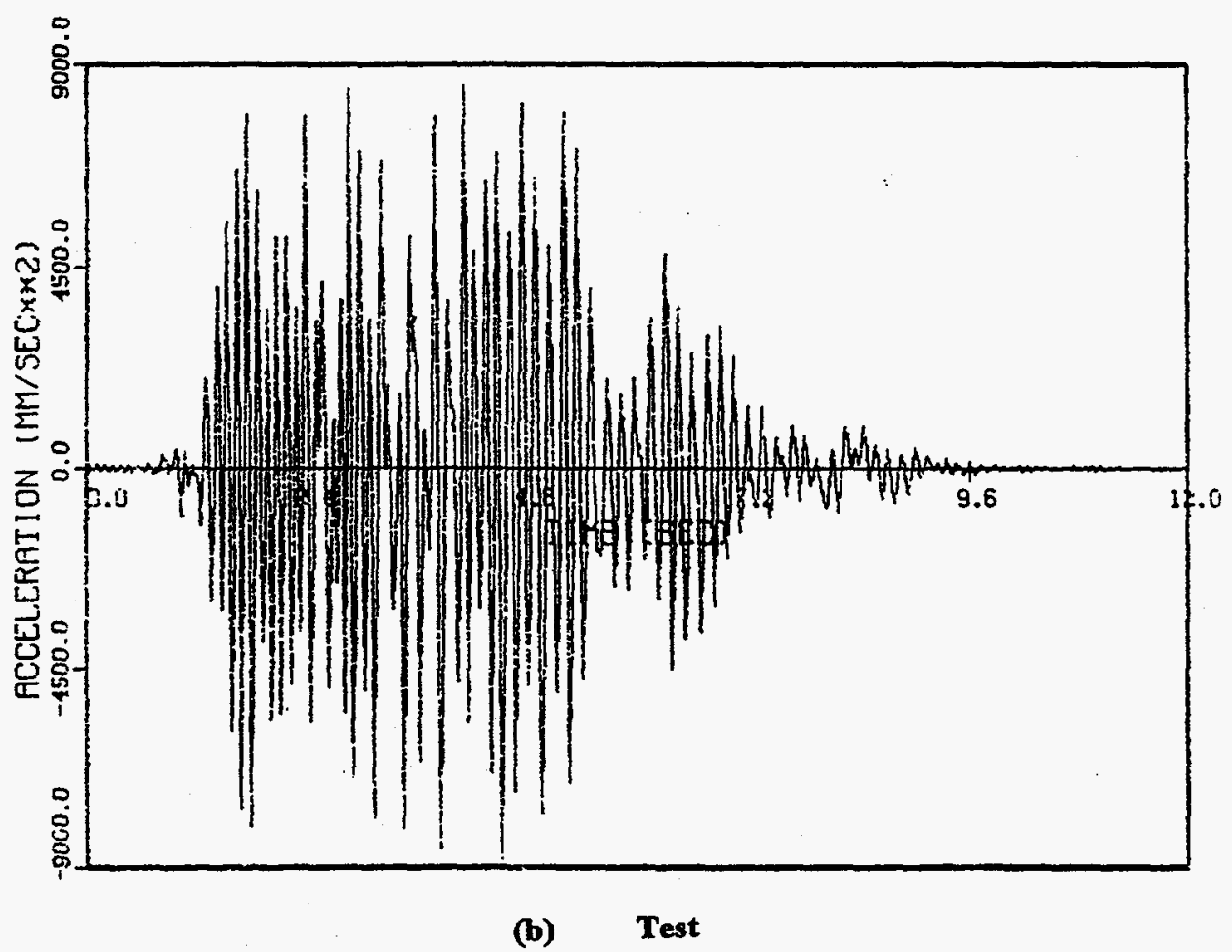

Fig. 3.24 Horizontal Acceleration of Top Slab (AXT) for RUN-4 


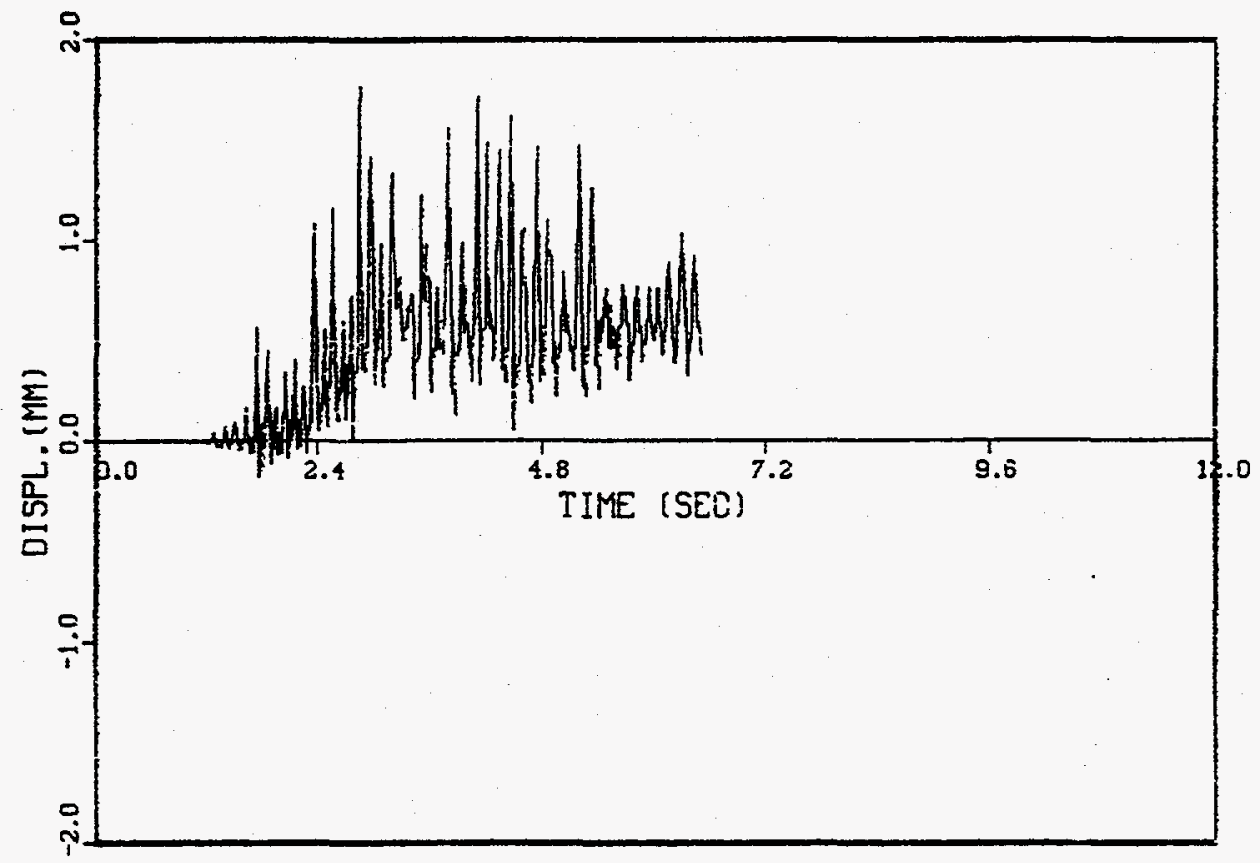

Fig. 3.25 Vertical Displacement of Right Side Top Slab (DVRF) for RUN-4, Analysis Result

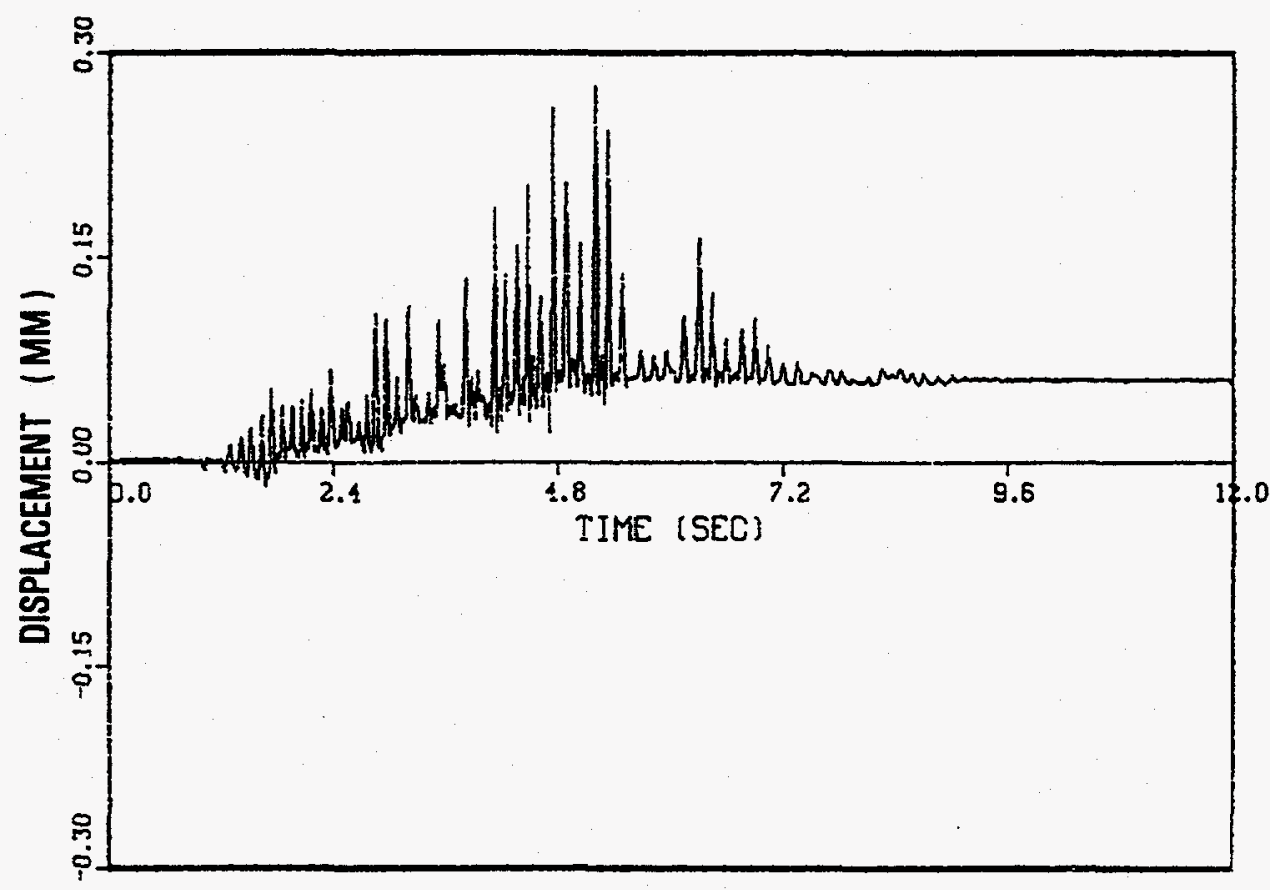

Fig. 3.26 Relative Vertical Displacement of Flange Wall (DV 16) for RUN-4, Test Result 
BNL's Analyses
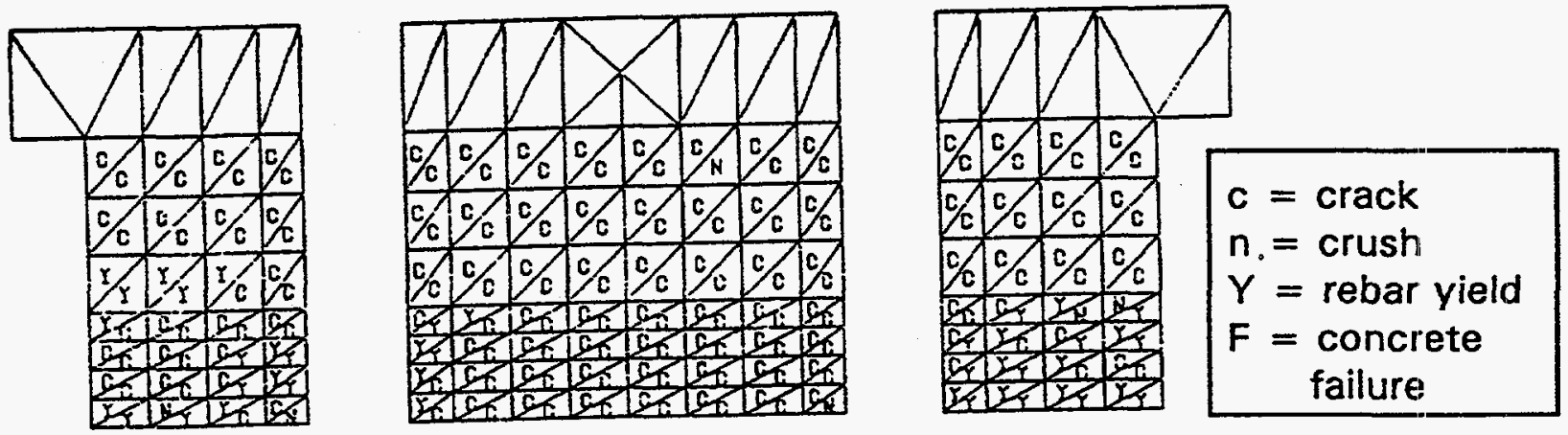

Fig. 3.27 Damage State After RUN-4, Analysis Result 


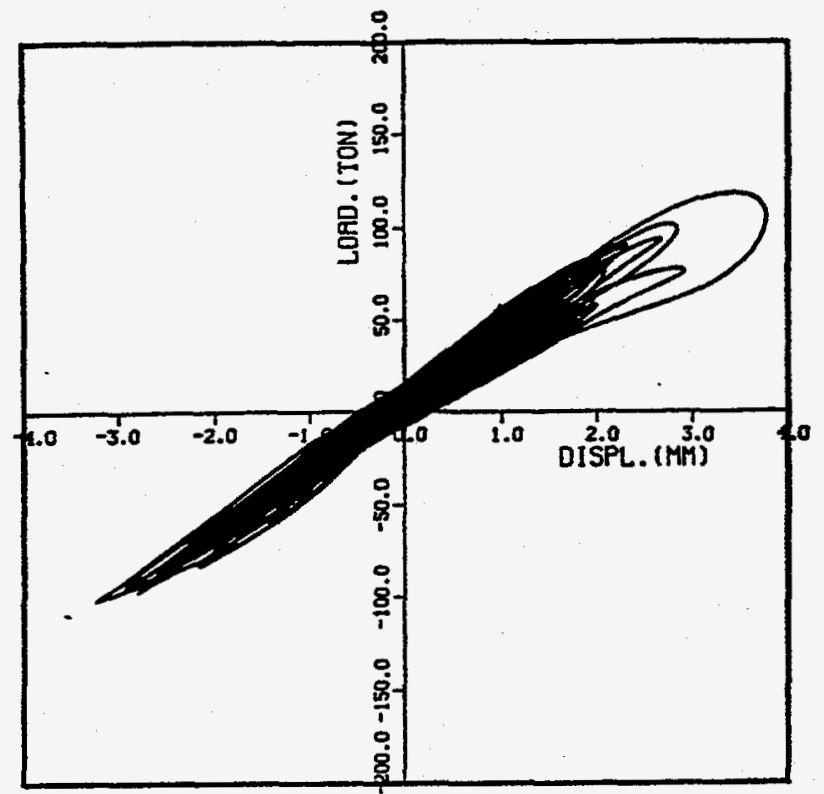

(a) Analysis

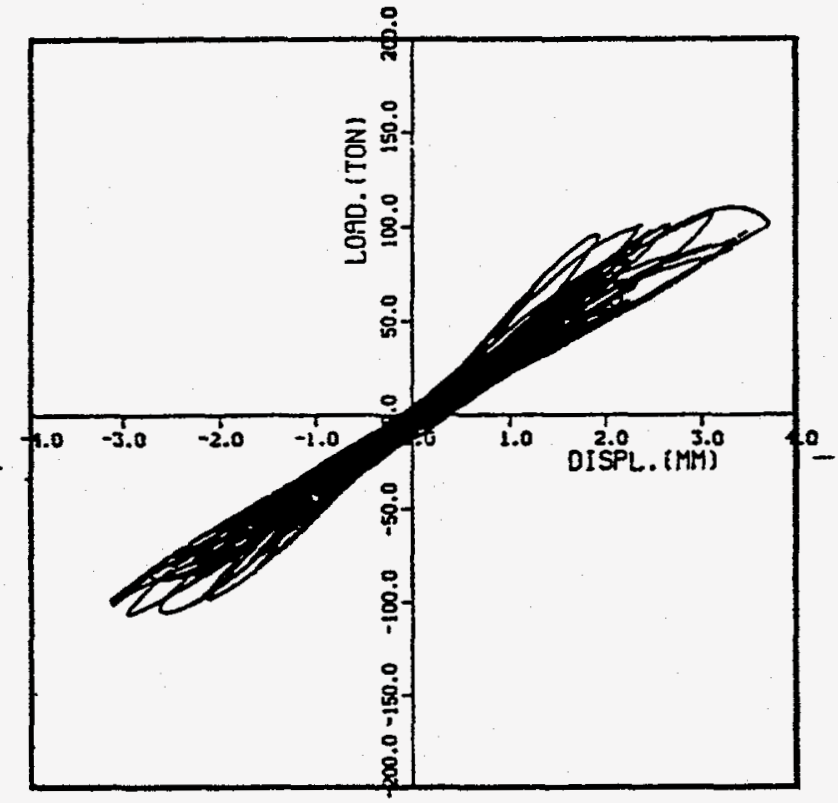

(b) Test

Fig. 3.28 Load-Deformation Relationship from Improved Analysis for RUN-4 
BNL's Analyses

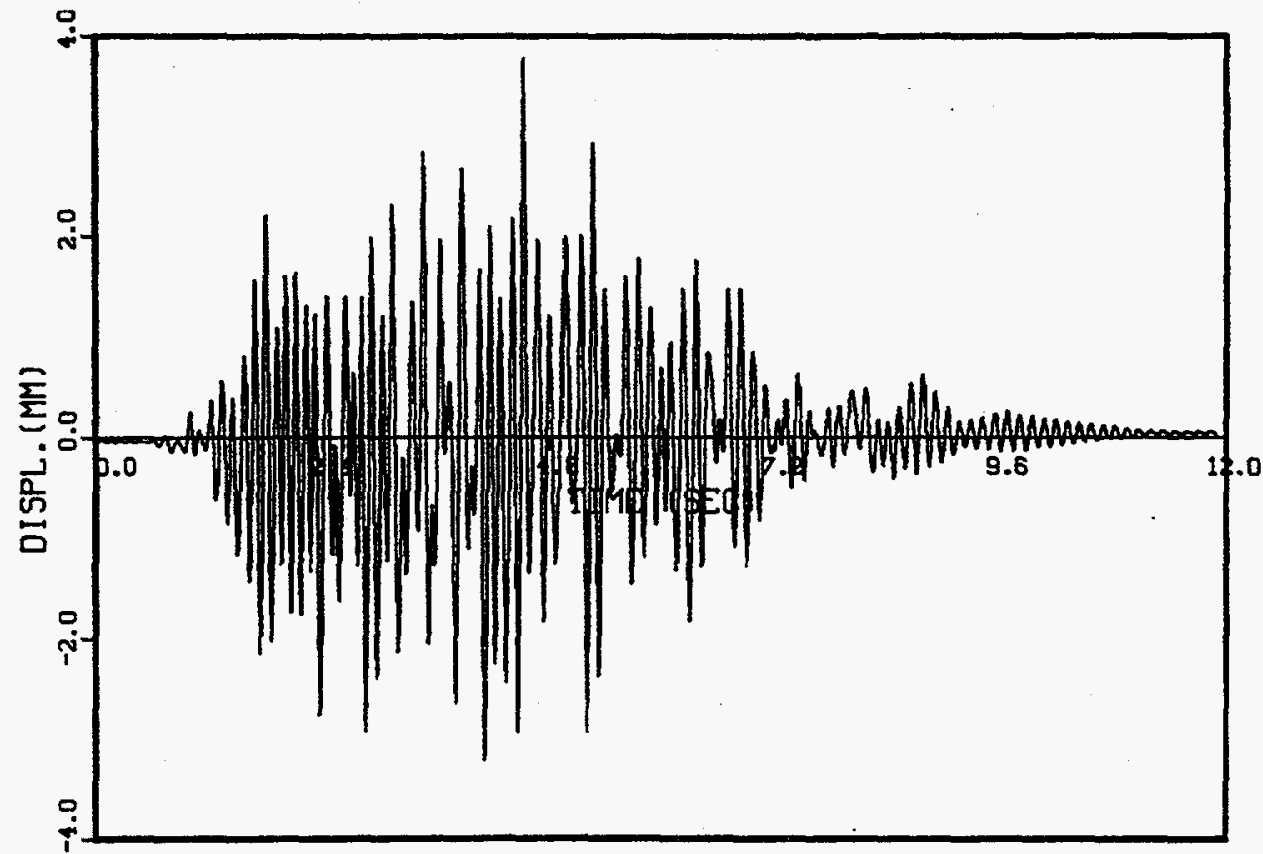

(a) Analysis

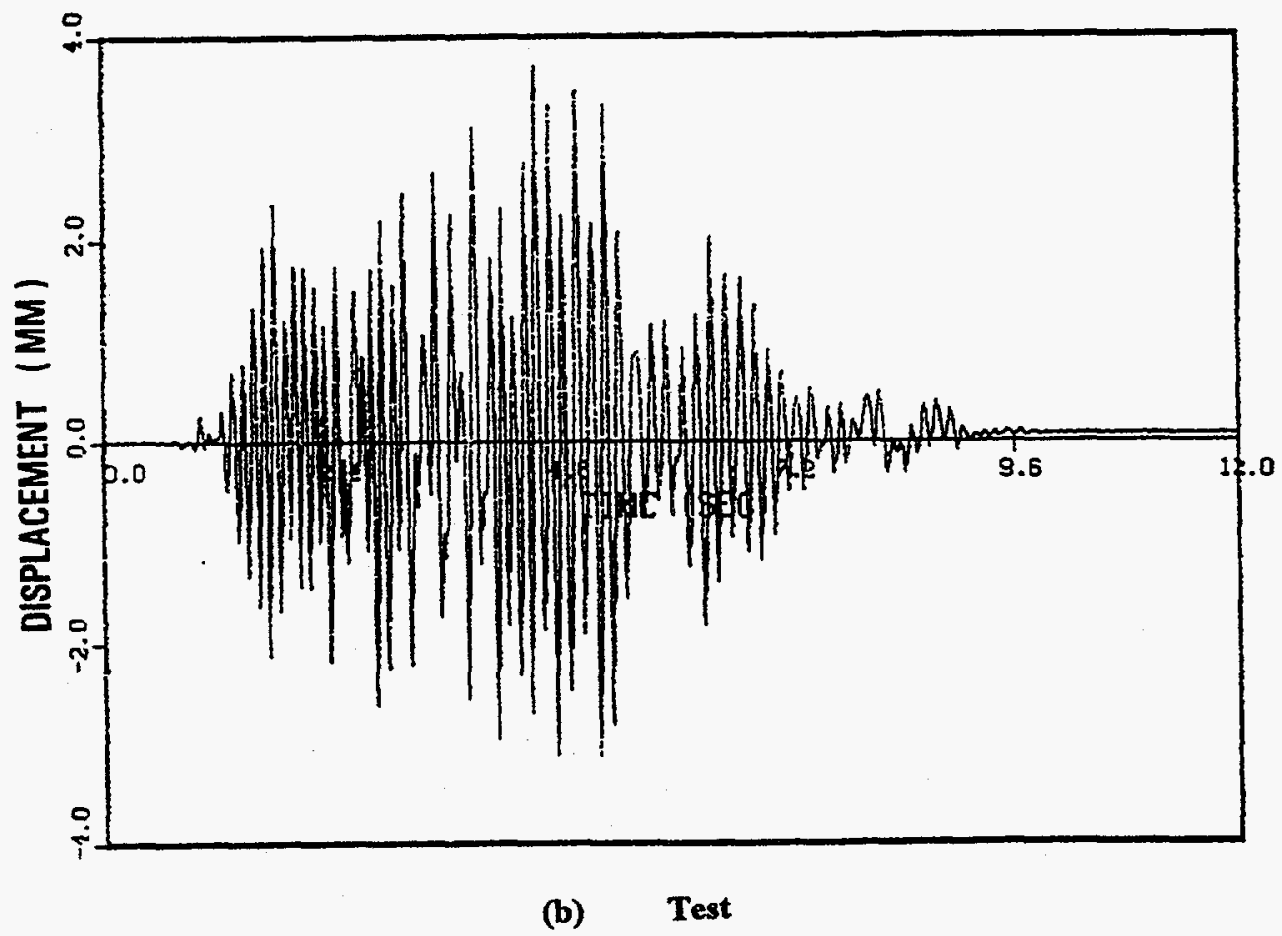

Fig. 3.29 Horizontal Displacement of Top Slab (DXT) from Improved Analysis for RUN-4 
BNL's Analyses

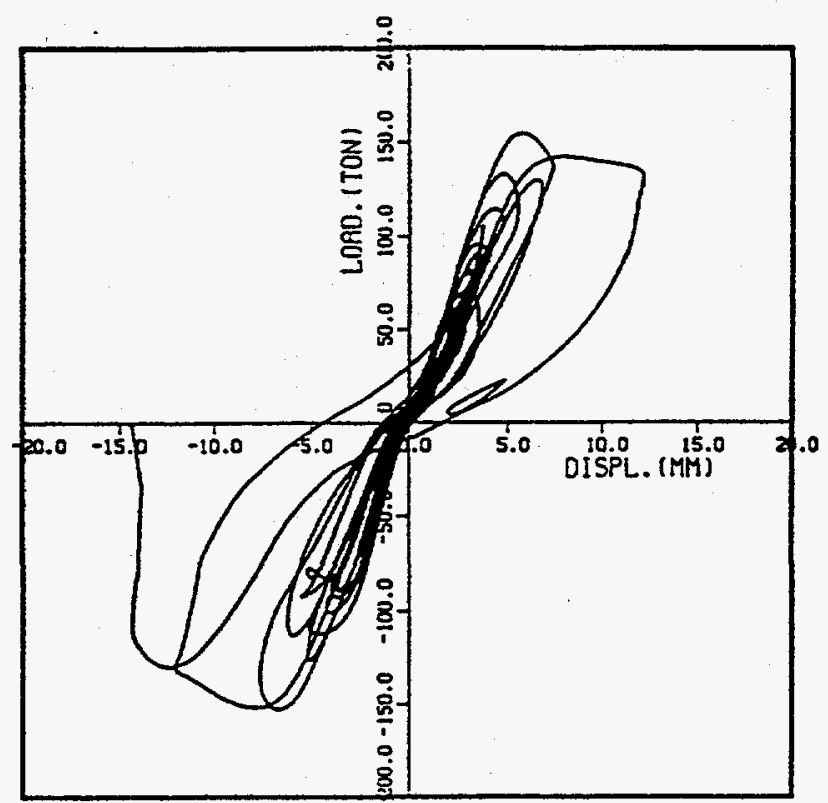

(a) Analysis

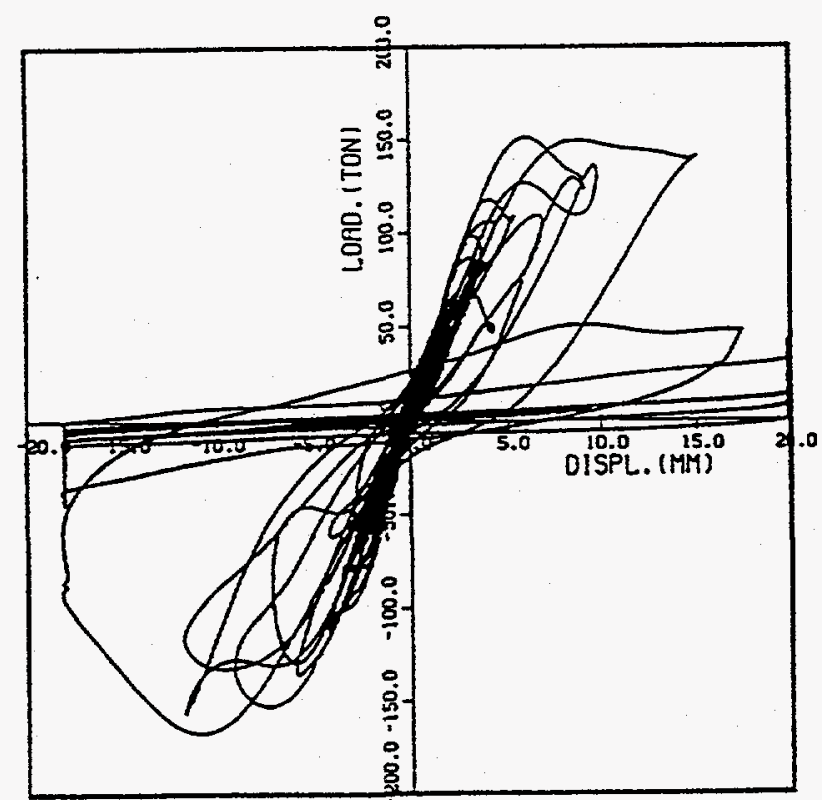

(b) Test

Fig. 3.30 Loal-Deformation Relationship for RUN-5 
BNL's Analyses

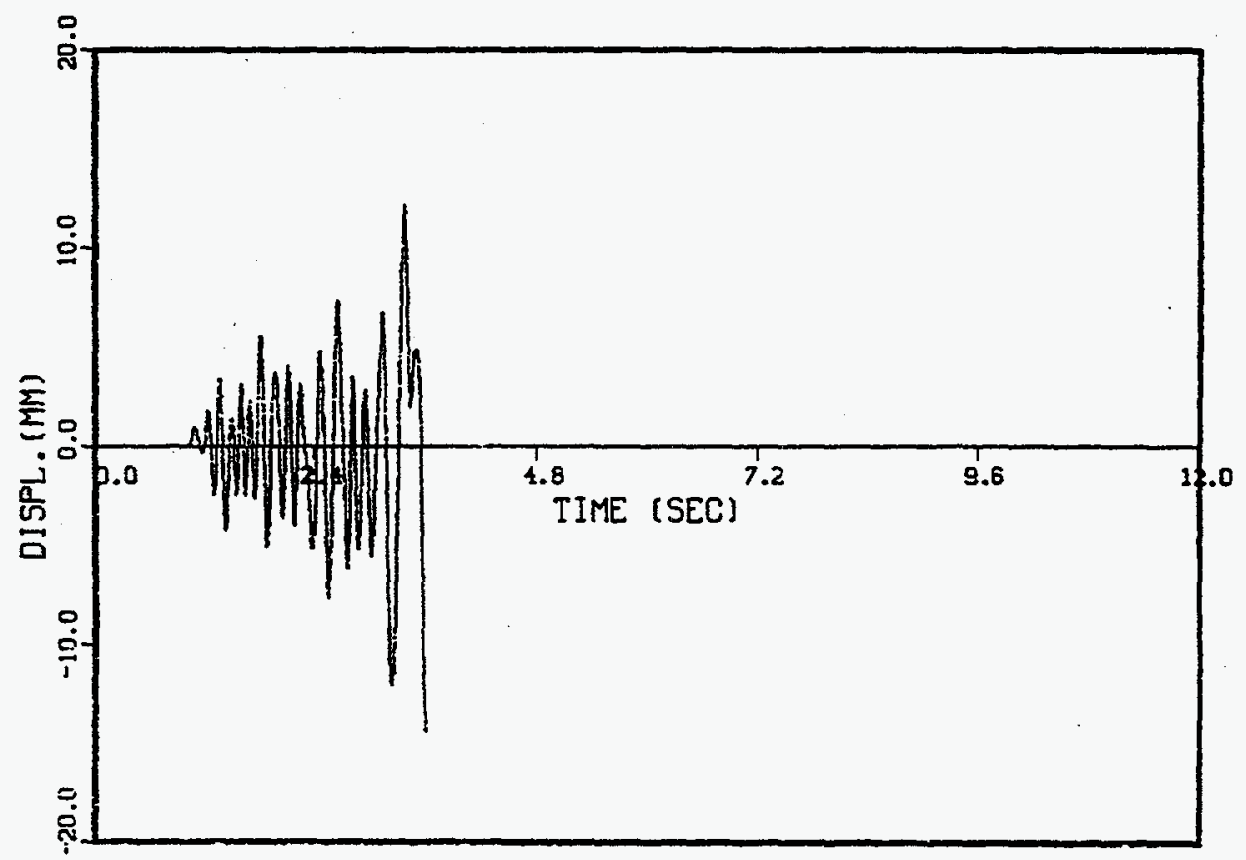

(a) Analysis

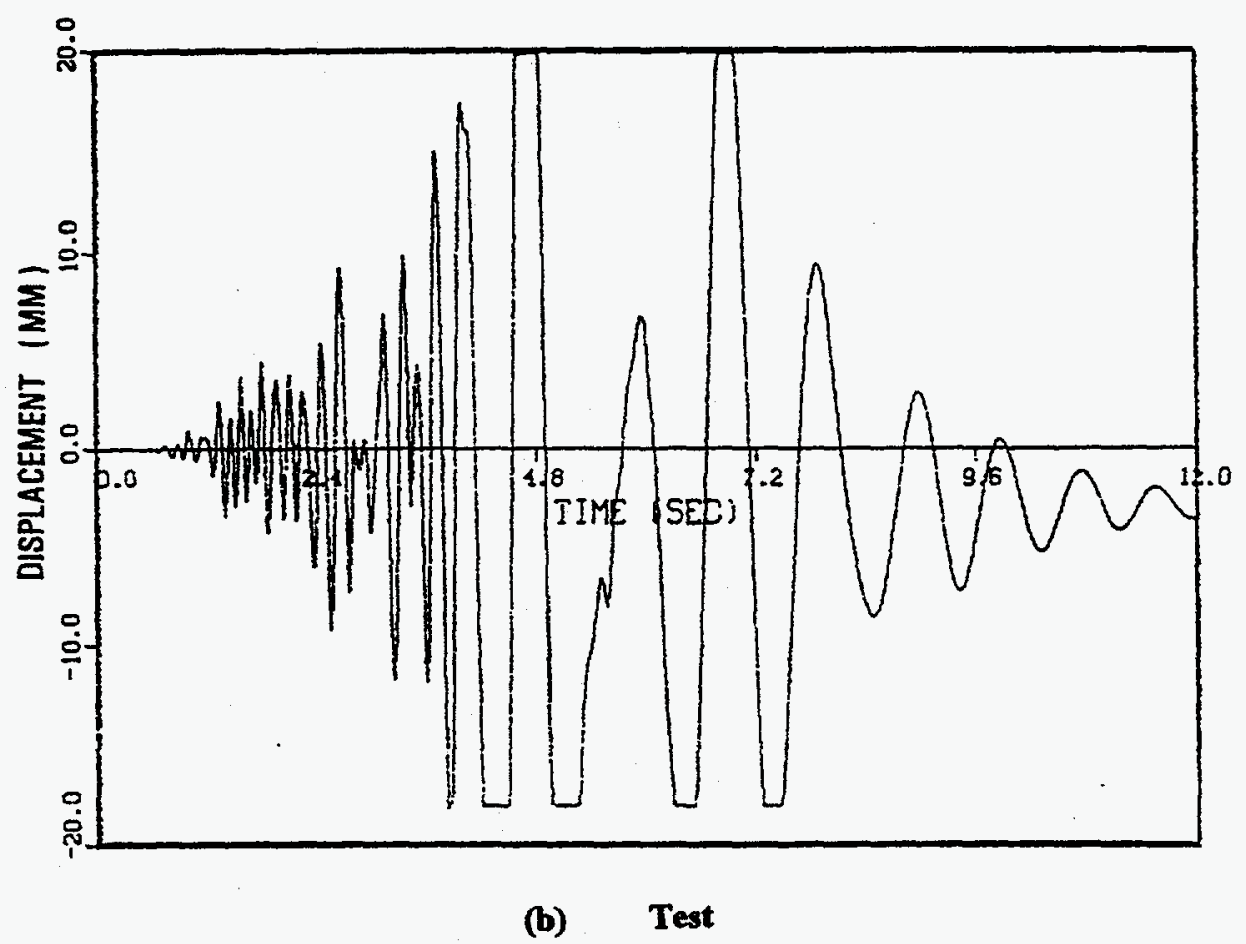

Fig. 3.31 Horizontal Displacement of Top Slab (DXT) for RUN-5 
BNL's Analyses

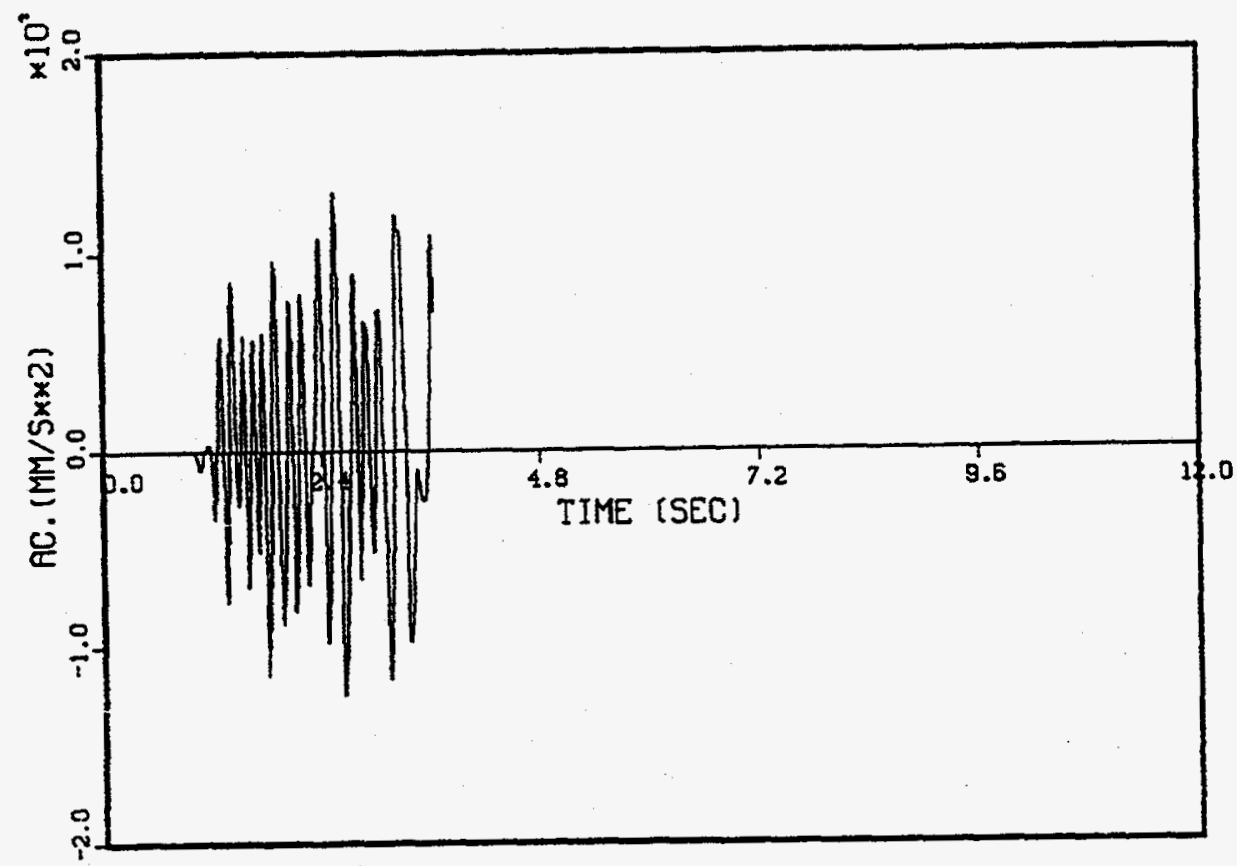

(a) Analysis

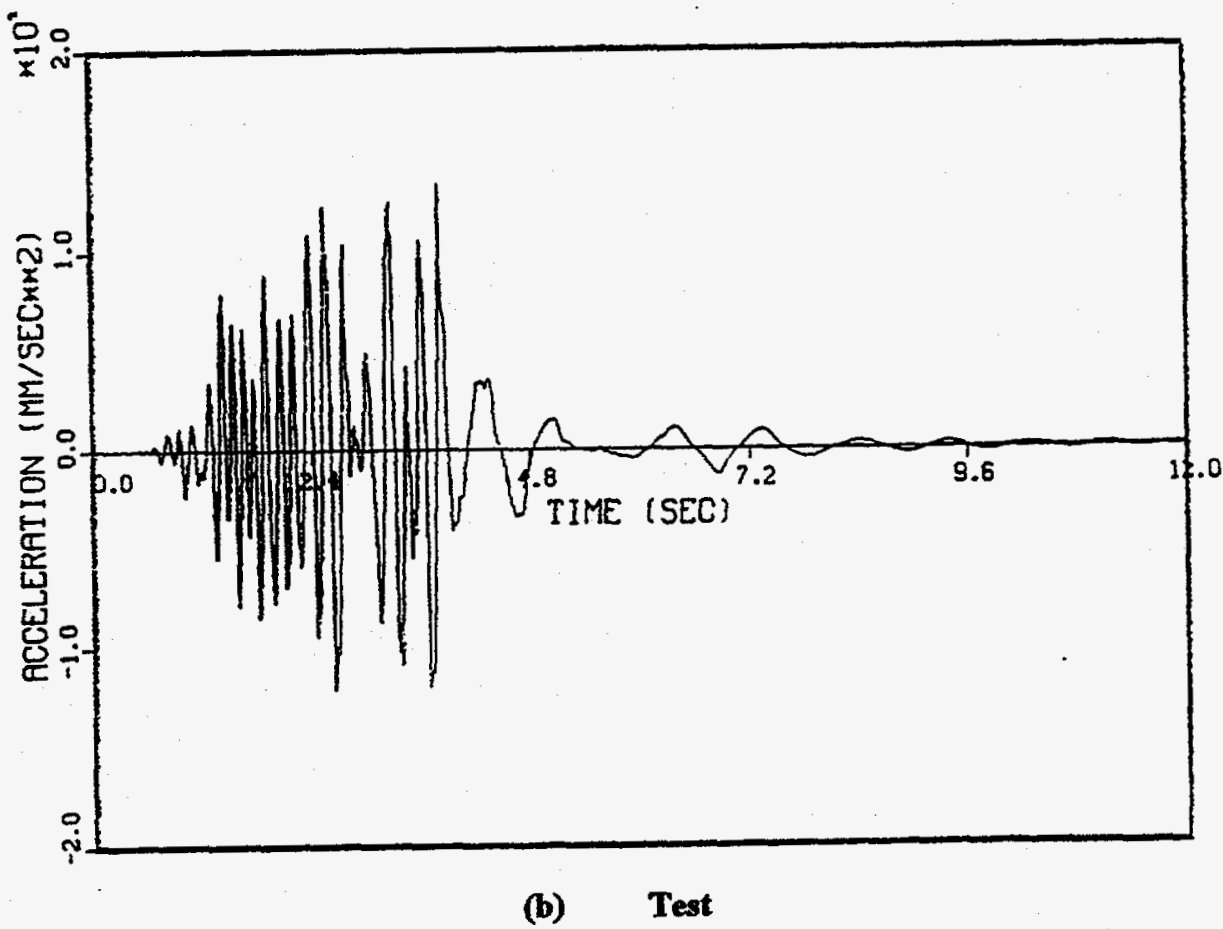

Fig. 3.32 Horizontal Acceleration of Top Slab (AXT) for RUN-5 


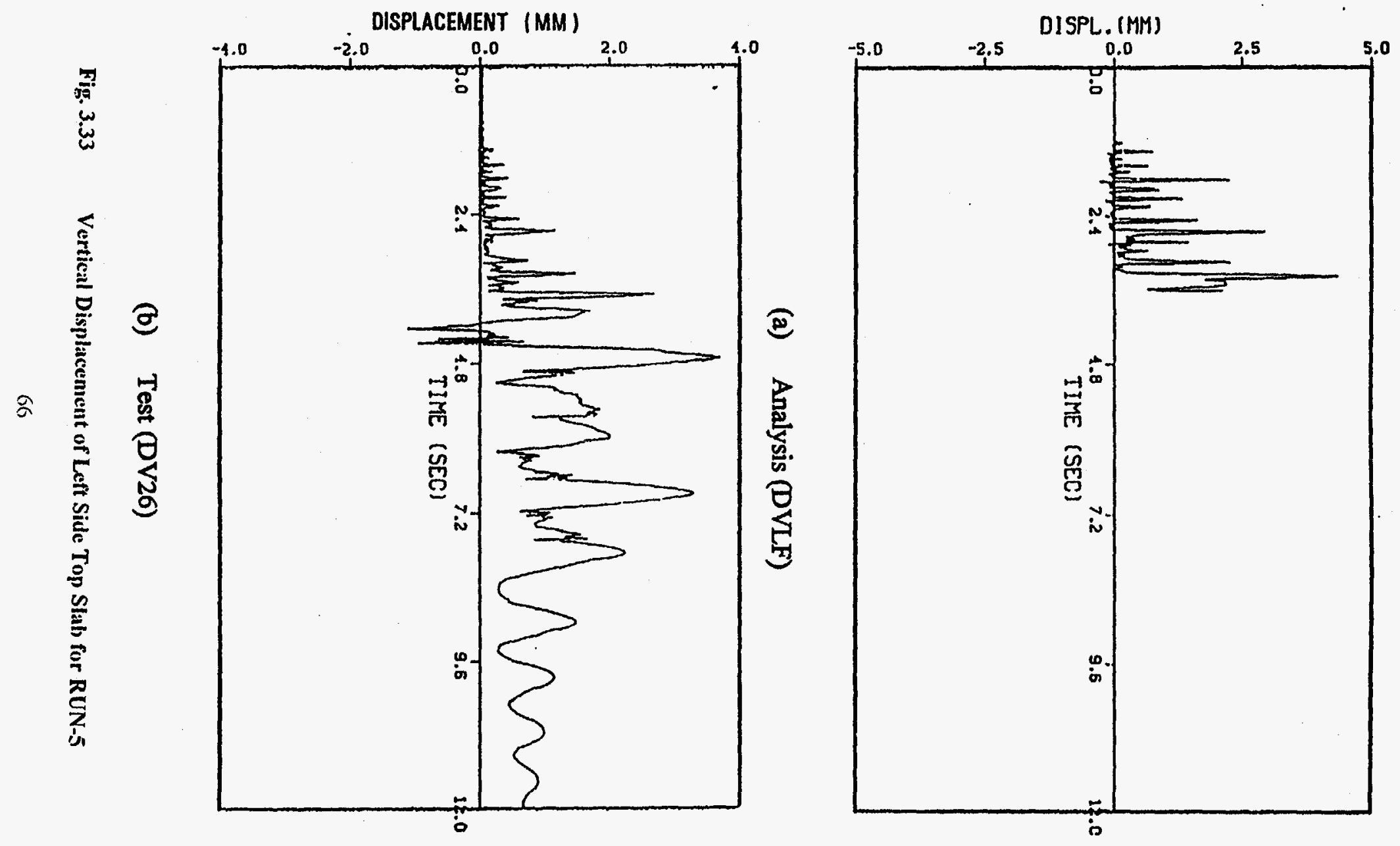




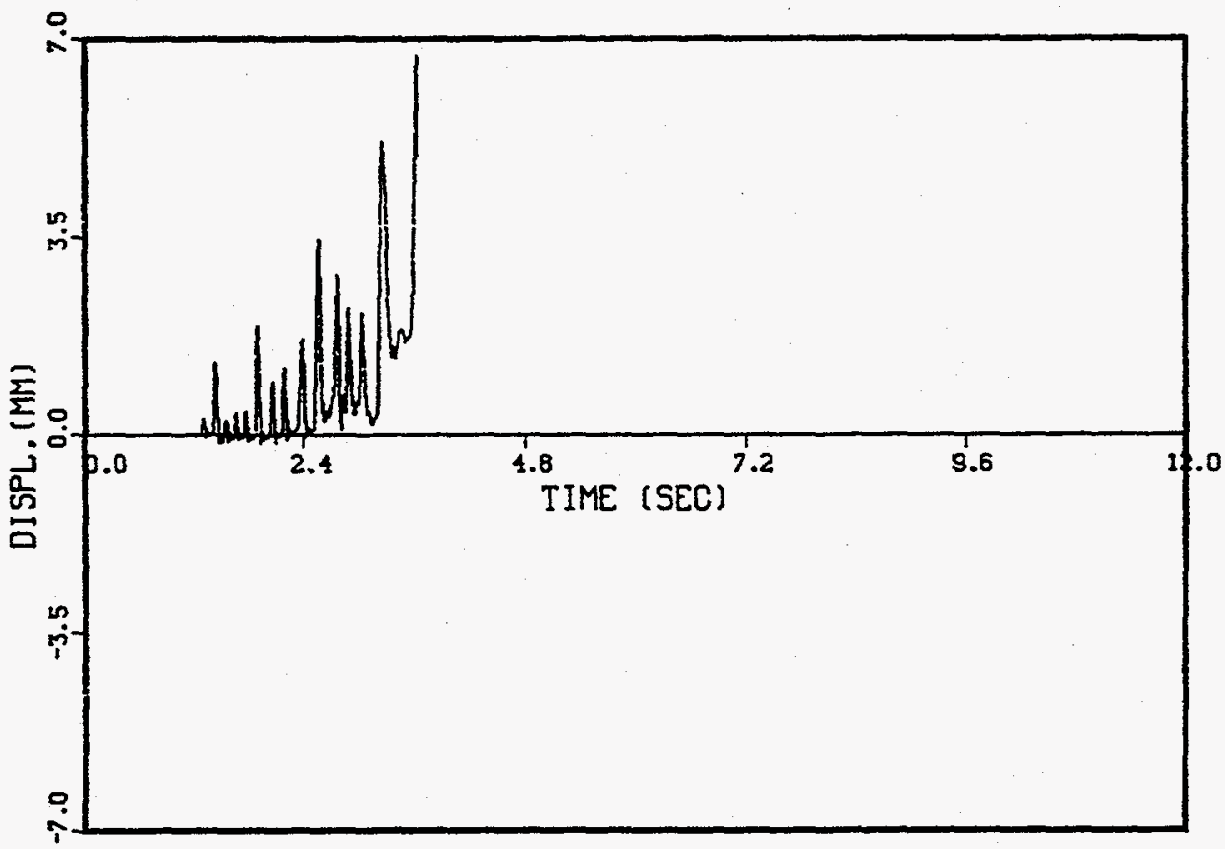

(a) Analysis (DVRF)

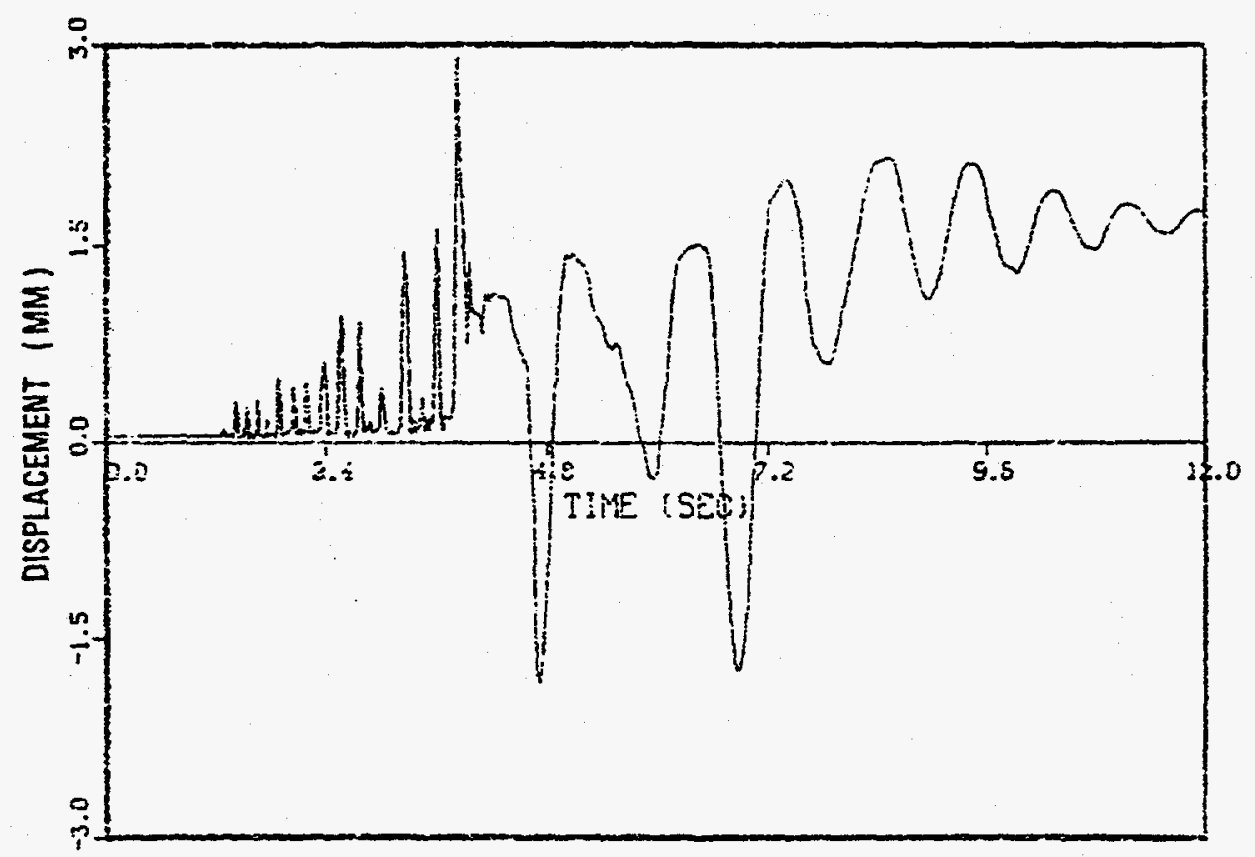

(b) Test (DV1G)

Fig. 3.34 Vertical Displacement of Right Side Top Slab for RUN-5 
BNL's Analyses

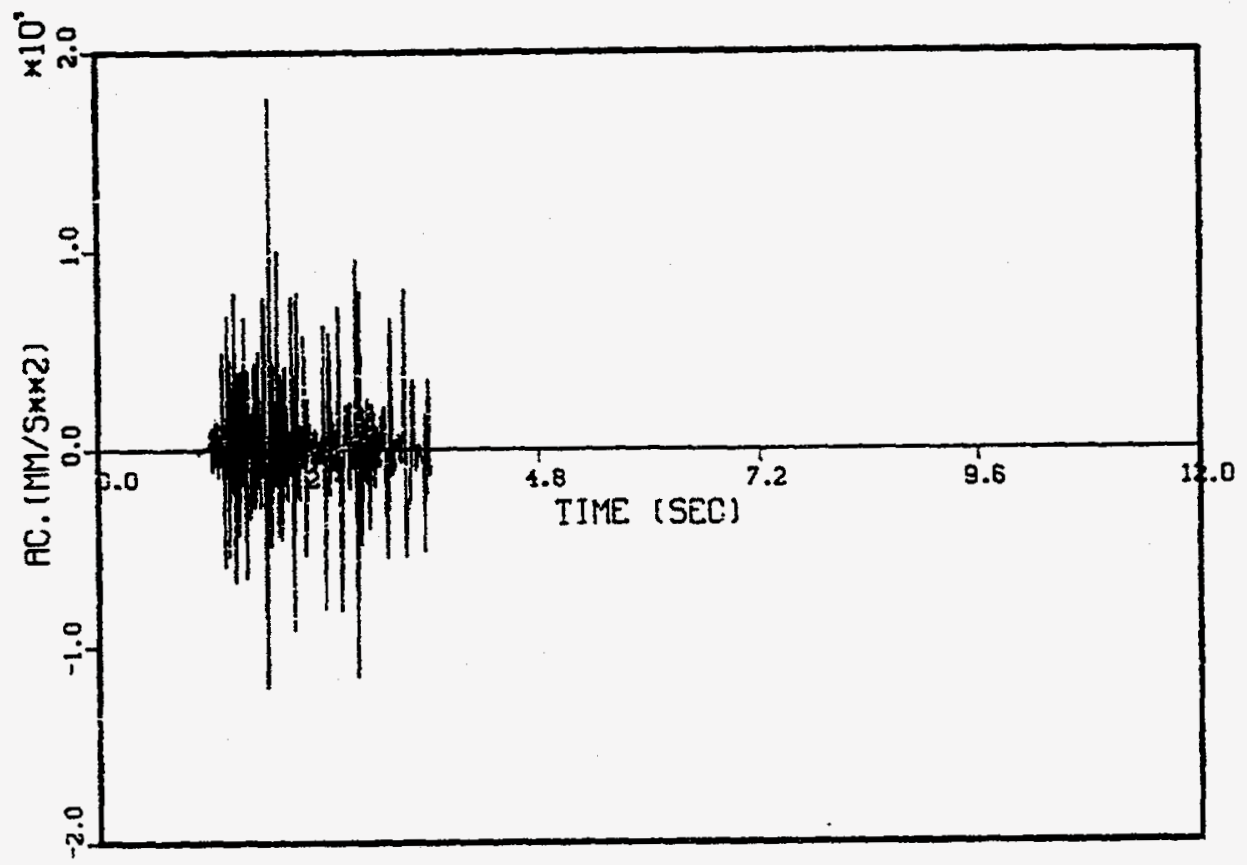

(a) Analysis

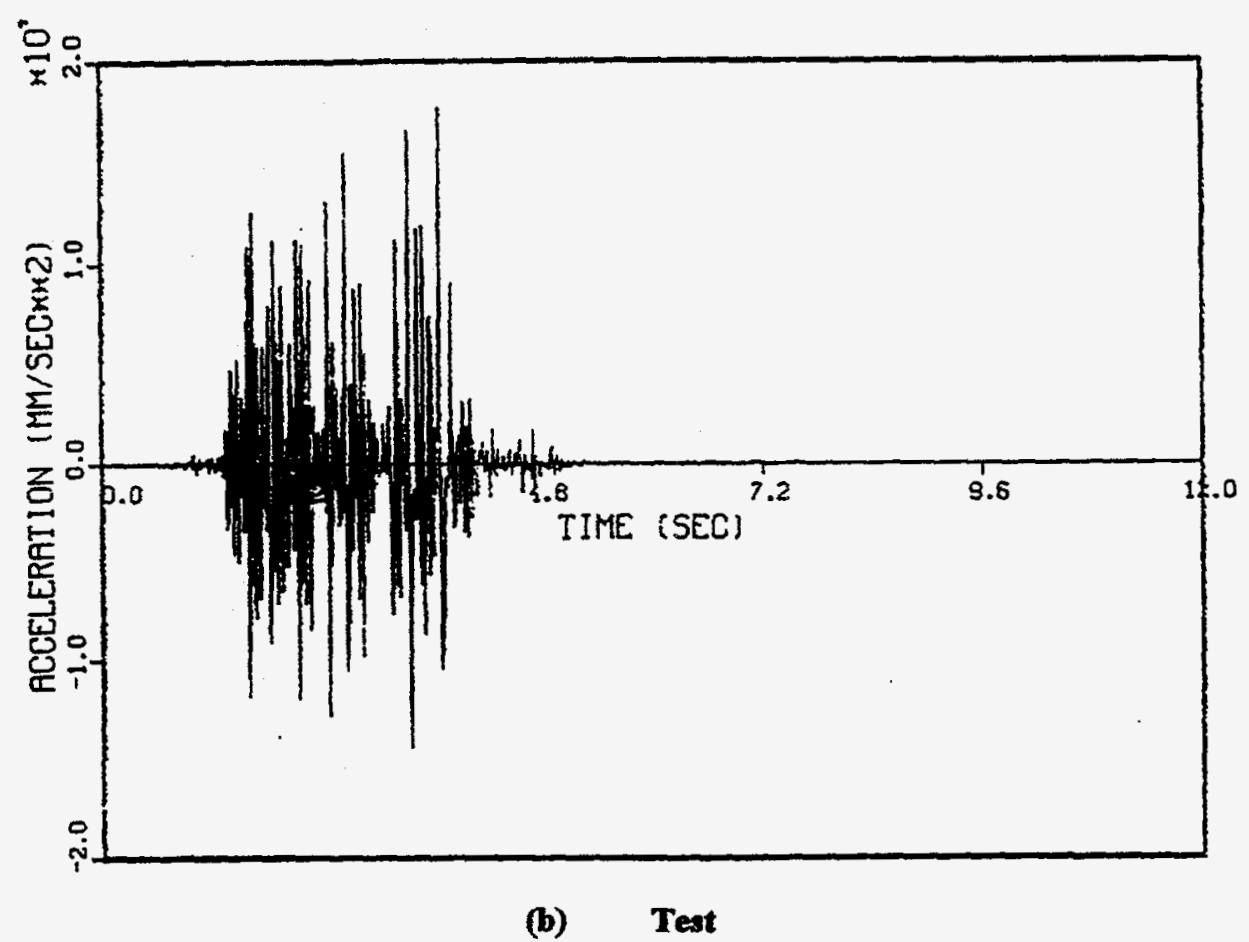

Fig. 3.35 Vertical Acceleration of Left Side Top Slab (AZTL) for RUN-5 


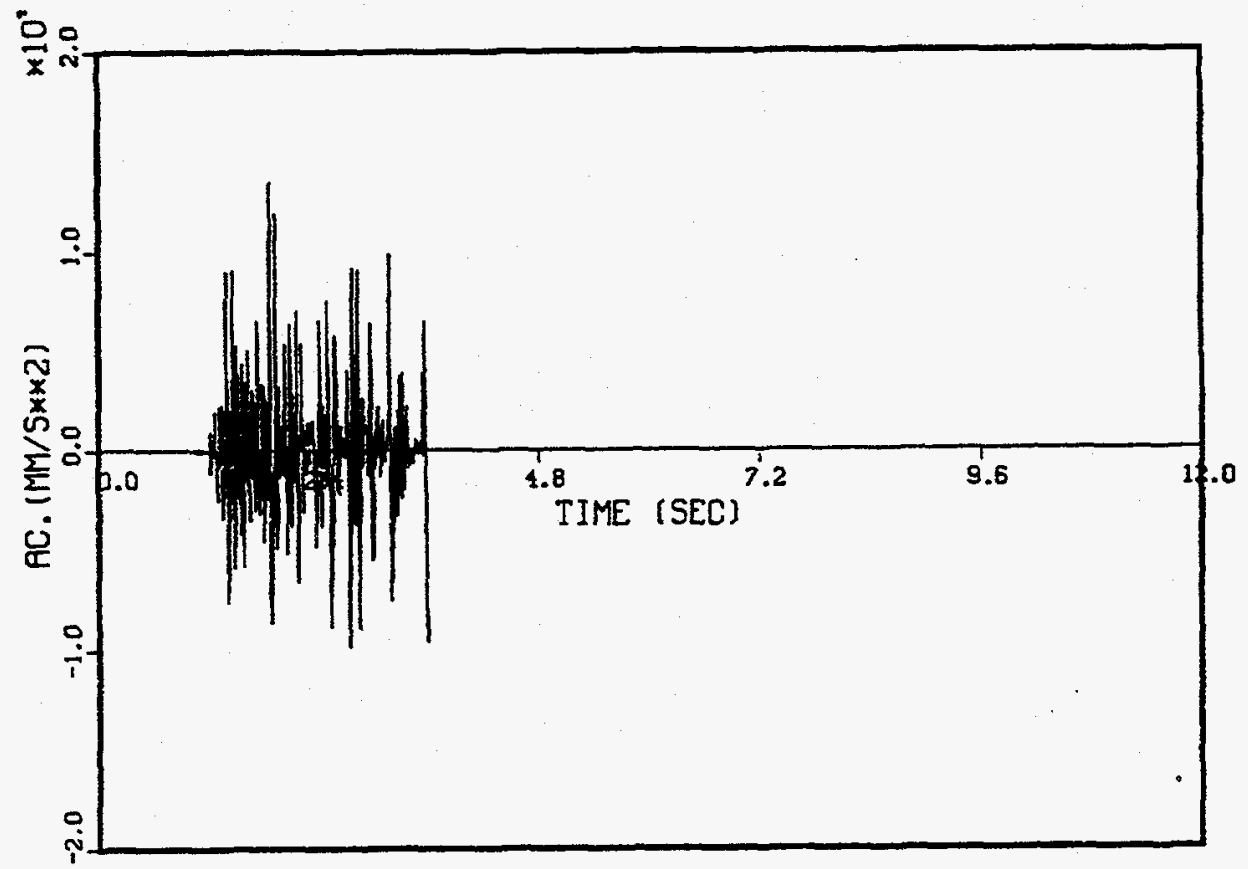

(a) Analysis

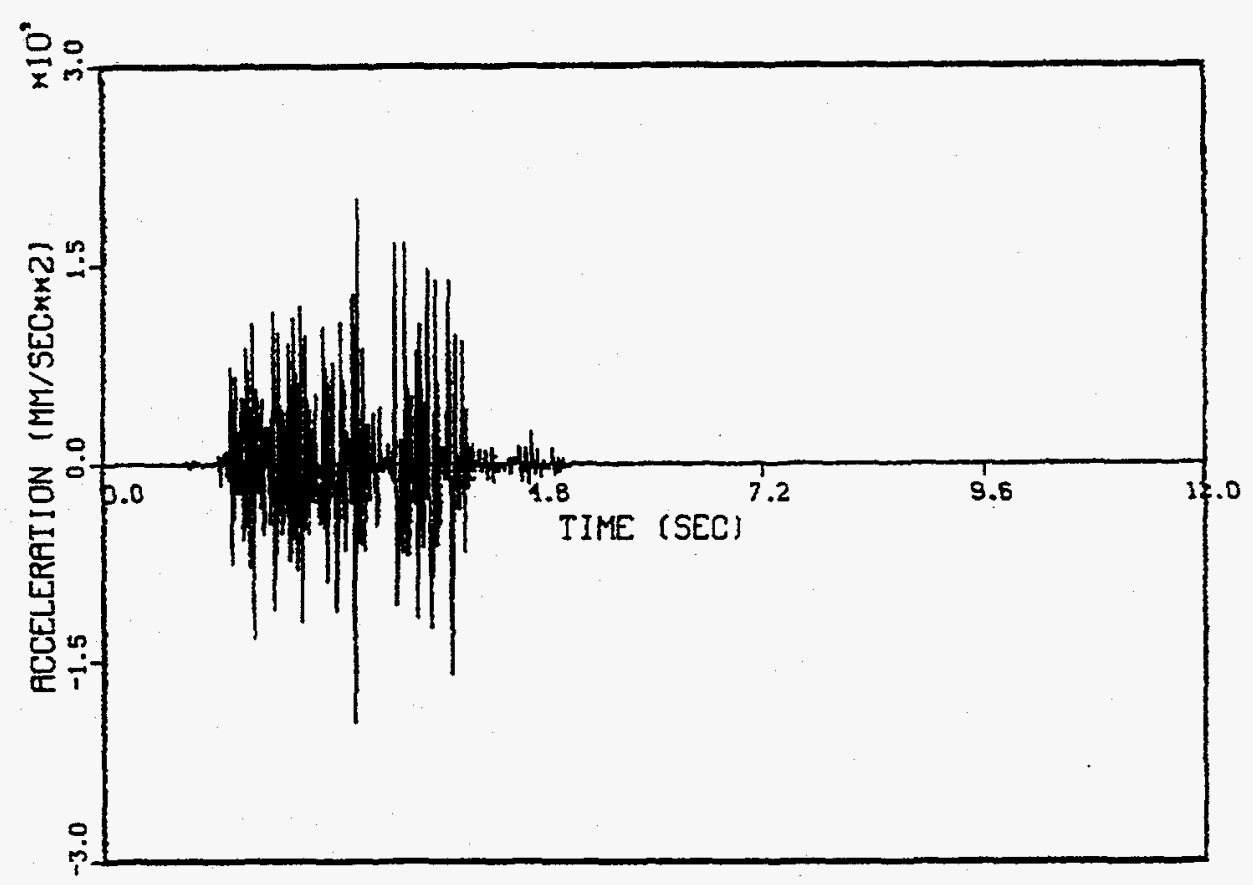

\section{(b) Test}

Fig. 3.36 Vertical Acceleration of Right Side Top Slab (AZTR) for RUN-5 


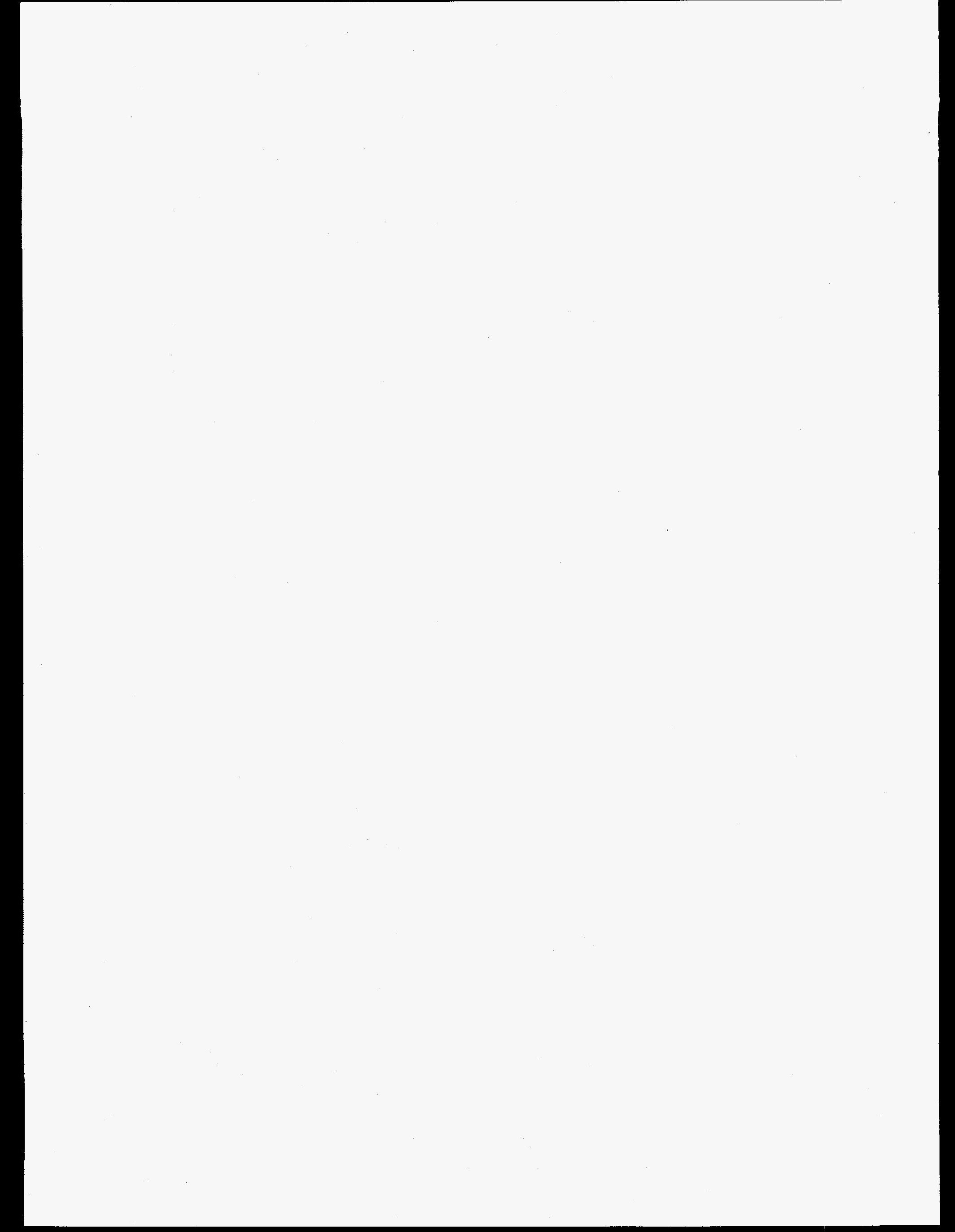




\section{ADDITIONAL PUSH-OVER ANALYSES}

\subsection{Introduction}

As mentioned in Chapter 1 of this report, most of the currently available FEM codes are limited to monotonic push-over type loading conditions for the nonlinear analysis of concrete structures. Push-over type analyses, if properly performed, still provide engineers with useful information in the seismic design of concrete structures, including evaluation of the maximum strength, stiffness, and the dominant failure mode. The reliability of the analysis results using currently available FEM codes, however, is questionable particularly regarding their ability to predict nonlinear behavior near the failure point of complex 3$D$ shear wall structures. Moreover, the response of concrete structures under cyclic loading reversals may be greatly different than that for monotonic static loads. The ISP project offered a unique opportunity to examine the reliability and applicability of currently available FEM codes when applied to dynamically tested shear wall structures.

Additional push-over analysis results, by two consultants, F. Vecchio of the University of Toronto (UT) and F. Filippou of the University of California at Berkeley (UCB), as well as by BNL using the ANSYS code are presented in this chapter. Detailed descriptions of the analyses by UT and UCB are given in Appendices A and B of this report, respectively.

\subsection{Analyses by the University of Toronto (UT)}

\subsubsection{Analysis Method}

The analyses by UT were performed using 3-D models and 2-D models under monotonic static loading conditions. Two (2) in-house computer codes were used, i.e., the SPARCS code for 3-D analyses and the TRIX code for 2-D analyses.

The constitutive model for concrete is based on the Modified Compression Field Theory (Ref. 7), which is frequently referenced by many researchers. The model is based on the orthotopic plasticity theory and rotating smeared cracking. Only monotonically increasing stress conditions are considered both in the material modeling and the numerical procedures. The Hognestad parabola, as illustrated in Fig. 4.1, is used as the envelope curve for the uniaxial stress-strain of cracked concrete.

The compression strength reduction factor, $\beta$, for cracked concrete is based on their own studies, expressed as a function of the ratio of the tensile principal strain, $\epsilon_{1}$, to the compressive principal strain, $\epsilon_{3}$, in triaxial stress-strain condition, as

$$
\beta=\frac{1}{1+K_{S} K_{f}}
$$

where

$$
\begin{aligned}
& K_{S}=0.35\left(\frac{-\epsilon_{1}}{\epsilon_{3}}-0.280\right)^{080} \succeq 1.0 \\
& K_{f}=0.1825 \sqrt{f_{c}^{\prime}} \succeq 1.0
\end{aligned}
$$


and $f_{c}^{\prime}$ is expressed in MPa. The peak stress and strain of the base (envelope) curve, $f_{p}$ and $\epsilon_{p}$, are modified using the factor, $\beta$, to account for this strength degradation effect.

$$
\begin{aligned}
& f_{P}=\beta \cdot f_{C}^{\prime} \\
& \epsilon_{P}=\beta \cdot \epsilon_{O}
\end{aligned}
$$

The crack model used in the Modified Compression Field Theory (MCFT), on which the analyses are based, assumes smeared rotating cracks. Cracking is assumed to occur normal to a principal stress direction, when that stress exceeds the uniaxial cracking strength of the concrete. While the constitutive relationships are formulated in terms of average stresses and average strains, local stresses at crack locations are also considered. The presence of average tensile stresses in the concrete implies local stress increases in the reinforcement at crack locations, and the presence of shear stresses on the concrete crack surfaces. Thus, the crack criteria includes a check that the local stresses in the reinforcement can be tolerated, and that the shear stresses on the crack surfaces are below a limiting value (Ref. 7). Otherwise, a shear slip can occur along the crack surfaces. The model is hypoelastic nonlinear; no attempt is made to reflect load/strain history in determining crack opening and closing. Cracks are assumed to 'heal' if the strains fall below the cracking strain.

The common approach of using a shear retention factor in modeling the shear stiffness of cracked concrete is not employed. Rather, in the manner of a true orthotopic elastic material, the shear stiffnesses are defined by the moduli of elasticity in the three principal directions, and by the associated Poisson's ratios. The Poisson's ratios are variable, modeled according to the data of Kupfer (Ref. 9), and are not necessarily set to zero after cracking.

Due to the influence of bond, post-cracking average tensile stresses can develop in the concrete between cracks. This socalled tension stiffening effect is modeled by adding a descending branch to the constitutive law for concrete in tension.

The so-called smeared rebar modeling is used, therefore, the effects of dowel action and bond-slip of reinforcement are not considered.

SPARCS employs a total load, secant stiffness approach in the formulation of its nonlinear analysis algorithm. For the SPARCS model shown in Fig. 4.2, 8-node (24 DOF) brick elements which have one stress-strain point are used. The model consists of 1090 elements, 1932 nodes and a total of 5796 DOF's to model half of the ISP wall.

\subsubsection{Analysis Results}

The predicted failure mode by the SPARCS code is shown in Fig. 4.3. A sliding shear failure is observed at the 2nd layer of the web wall, which is consistent with the location of the actual failure in the ISP wall (see Figs. 2.16 and 2.17). The ISSAC code 2-D analyses predicted the same sliding shear failure at the bottom of the web wall (see Fig. 3.8). This difference can be explained by the additional confinement in the thickness direction, which strengthens the bottom web wall in the 3-D analysis by the SPARCS code.

Figure 4.4 compares the load-deformation response of the top slab from two (2) SPARCS analyses with the test results. In one analysis, tension softening was considered, and the other, tension softening was deliberately excluded. It seems, tension softening has a vary minor effect on the predicted response of the shear wall. The analyses start to deviate from the test results right after the occurrence of shear cracking, and overestimate the shear strength.

The 2-D analysis model for the TRIX code is shown in Figure 4.5. Plane-stress 4-node elements were used to model both the web and flange walls. Figure 4.6 compares the analysis results of the 2-D (TRIX) and 3-D (SPARCS) runs, in which the 
effective width of flanges was assumed to be $100 \%$ for the 2-D model. A parametric study was performed to examine the influence of effective flange width in the 2-D model as shown in Figure 4.7. It is pointed out that the actual effective width lies between $67 \%$ and $100 \%$ (see Appendix A for a more detailed description).

Additional SPARCS analyses were performed using a finer element discretization as illustrated in Figure 4.8 . The number of layers in the web wall was increased from 10 to 16 , and the number of elements through the flange thickness from 1 to 2 . The finer model shows a slight reduction of shear wall strength, about $5 \%$, when compared with the original SPARCS model results as shown in Figure 4.9.

\subsection{Analysis by the University of California at Berkeley (UCB)}

\subsubsection{Analysis Method}

A push-over analysis was performed by UCB using 2-D solid elements and an in-house code MIRAGE.

The constitutive model of concrete is based on a hypoelastic orthotopic model with a stress-equivalent uniaxial strain relation that is generalized to account for triaxial stress conditions. In the analysis of the ISP shear wall, the constitutive model was simplified to biaxial plane stress conditions by setting the out-of-plane normal stress equal to zero.

Figure 4.10 shows the envelope curve for the uniaxial stress-strain of concrete. In the formulation, the triaxial stress-strain relationship is determined from the uniaxial stress-strain components in three (3) orthogonal directions. The concept of the equivalent uniaxial strain, $\epsilon_{i j}$ is used to convert from the uniaxial to triaxial stress-strain field, which was originally proposed by Darwin and Pecknold (Ref. 6), as

$$
\epsilon_{u i}=\int \frac{d \sigma_{u i}}{E_{i}}(i=1,2,3)
$$

The rotating smeared cracking model is employed. However, the compression strength reduction of cracked concrete is not accounted for. Similar to the foregoing analyses by UT, smeared rebar modeling was used, and the effects of dowel action and bond-slip of reinforcement were not considered. The traditional load increment method based on Newton-Raphson iteration is used in the MIRAGE code.

Figure 4.11 shows the 2-D MIRAGE model. Similar to the foregoing ISSAC model, the unfolded modeling technique is employed to model the ISP shear wall using 2-D solid elements. The model consists of 319 quadrilateral plane stress elements and 360 nodes.

\subsubsection{Analysis Results}

Figure 4.12 compares the analytical with the experimental load-displacement responses. The analysis was terminated at the end of the last branch due to a singularity of the stiffness matrix. The load-displacement response up to ultimate consists of 16 load steps. During the 16th load step, crushing of several concrete elements and yielding of rebars at critical locations led to the singularity of the stiffness matrix with consequent lack of convergence in the Newton-Raphson iteration. Figure 4.13 show the crack pattern and the area of crushed concrete at two (2) loading steps. 


\subsection{Analysis Using ANSYS Code}

ANSYS is one of the most popular FEM codes used in the nuclear industry. An additional push-over analysis was performed to examine the applicability of this commercial code to the ISP shear wall tests. To analyze reinforced concrete structures, only one type of 3-D solid element, "SOLID65", is available in the ANSYS code. It is a 8-node brick element with 8 Gauss points. Rebar can be smeared in three different directions. A very simple material model for concrete is employed as briefly summarized below:

Cracking...........elastic up to tension strength, cracking, then the tangential stiffiness is set to be zero.

Crushing............multi-linear compression softening, then the element "vanishes" after reaching the crushing stress.

Shear Transfer Across Crack............ constant $\beta_{t}$, called shear transfer coefficient, is used to reduce the elastic shear modulus after cracking; $\beta_{t}=0$ indicates elimination of shear modulus after cracking, and $\beta_{t}=1.0$ indicates no reduction in shear modulus.

Figure 4.14 shows the ANSYS model, which is identical with the foregoing SPARCS model, Figure 4.2. For the analysis a shear transfer coefficient, $\beta_{t}=0.5$ was assumed. The push-over analysis was perfomed using the ordinary load increment method based on the Newton-Raphson iteration scheme with substep option.

Figure 4.15 shows the deformed shape and crack locations in the web wall at the last step of the analysis. The analysis survived up to 17 steps (lateral load of about 100 tonf), and then the solution did not converge due to a singularity of the stiffness matrix. Figure 4.16 compares the ANSYS result with the foregoing push-over analyses including the one developed with the ISSAC code (denoted as BNL). It is obvious that right after the initiation of cracking, the ANSYS result starts to deviate from the other analysis results. In order to improve the solution, various attempts were made by changing analysis parameters such as the load increment, convergence criteria, the shear transfer coefficient, as well as the crushing criteria. No significant improvement was observed during the parametric studies. It is concluded that the applicability of this commercial code to complex 3-D shear wall structures is seriously limited due to the simple material modeling and the numerical procedure which does not accept a negative stiffness to simulate cracked or crushed concrete. 


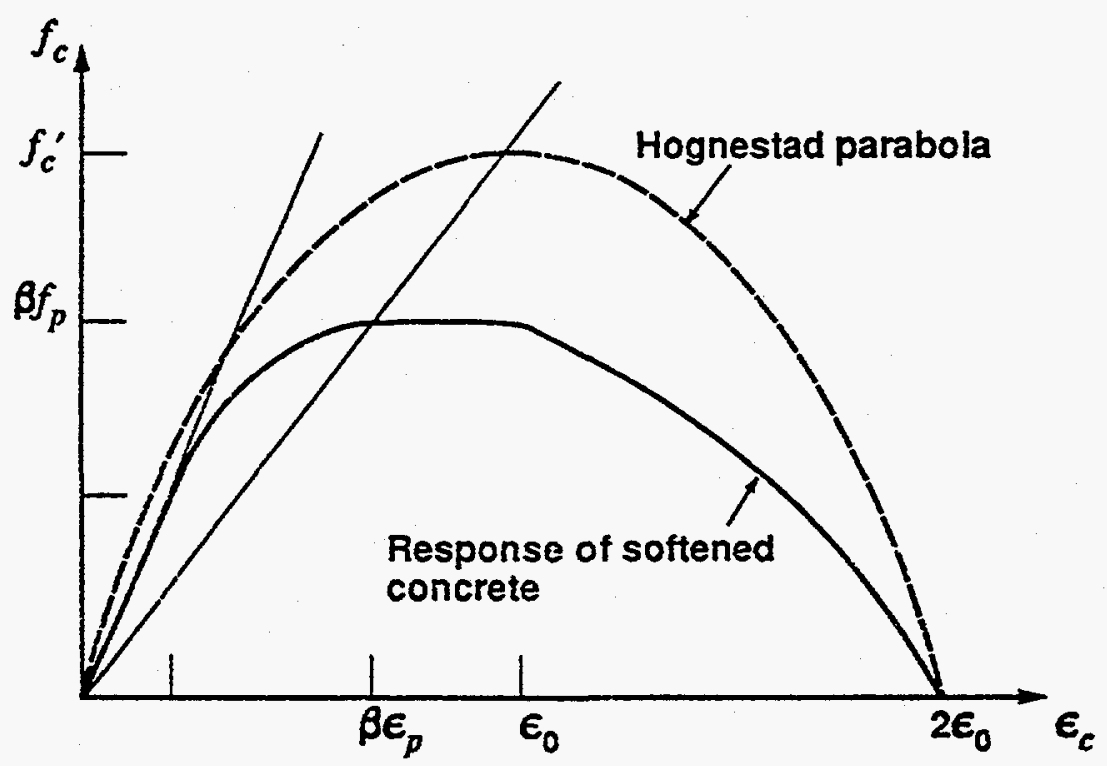

Fig. 4.1 Uniaxial Stress-strain Envelope Curve For Cracked Concrete (by UT)
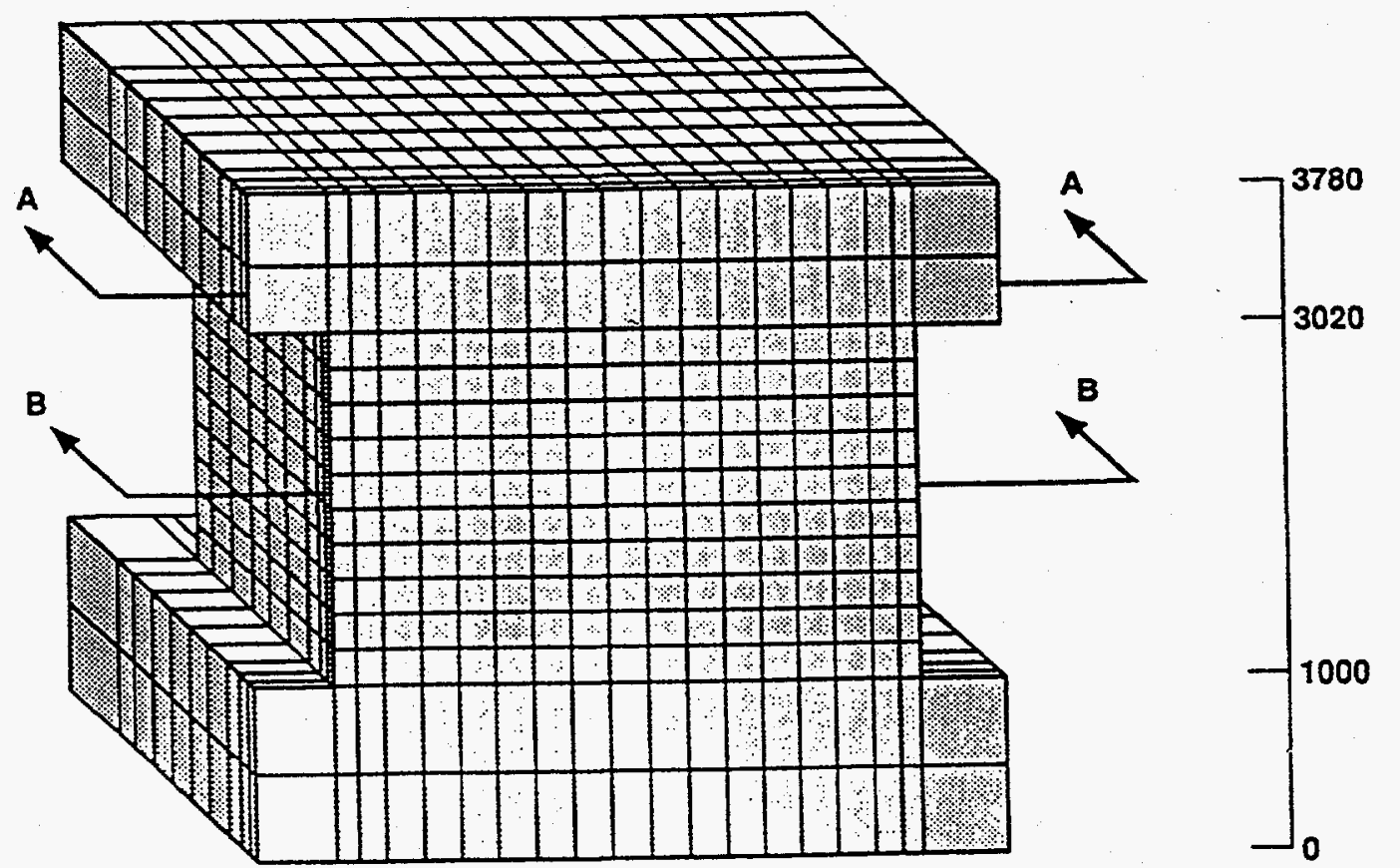

Fig. 4.2 SPARCS 3-D Model by UT 
Push-Over Analyses

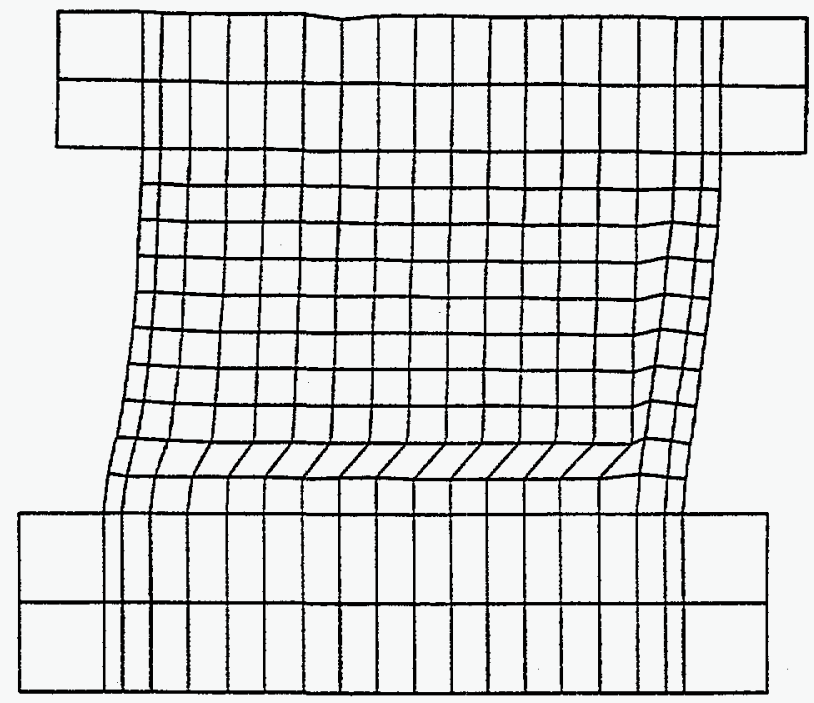

Fig. 4.3 Predicted Failure Mode by SPARCS Model

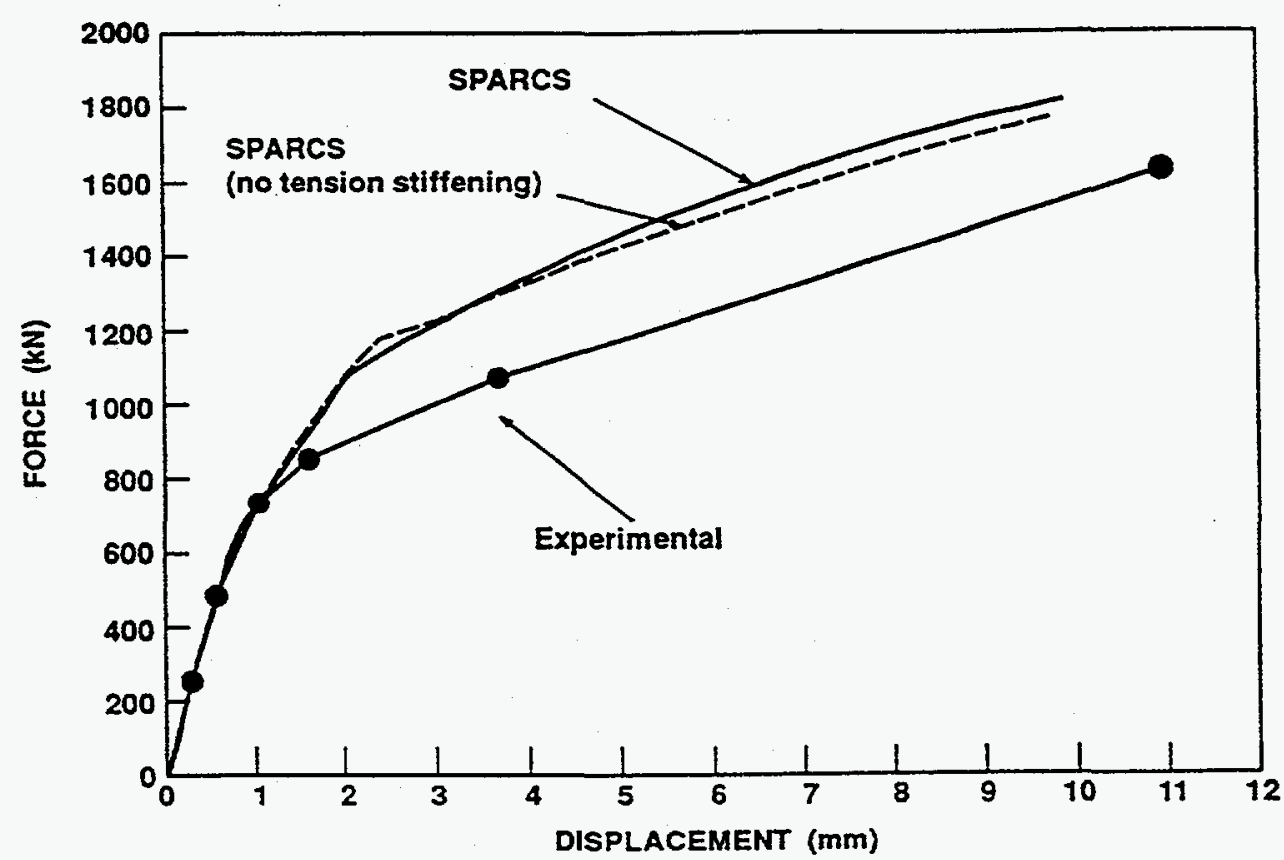

Fig. 4.4 Analysis Results by SPARCS Model 
Push-Over Analyses

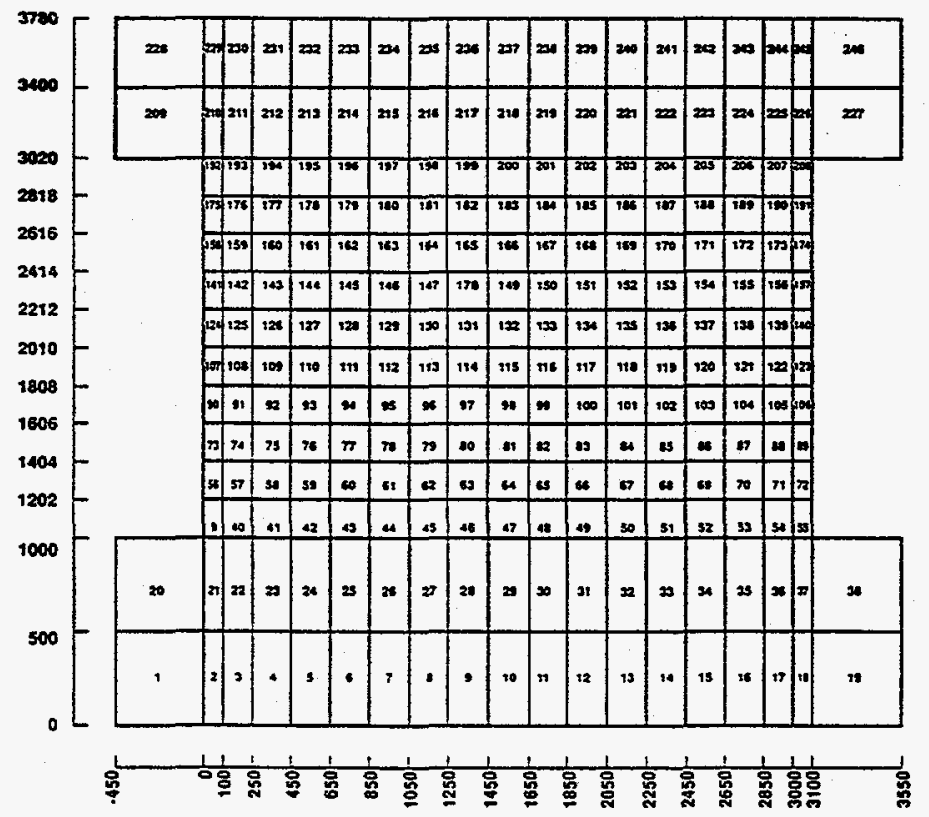

Fig. 4.5 TRIX 2-D Model by UT

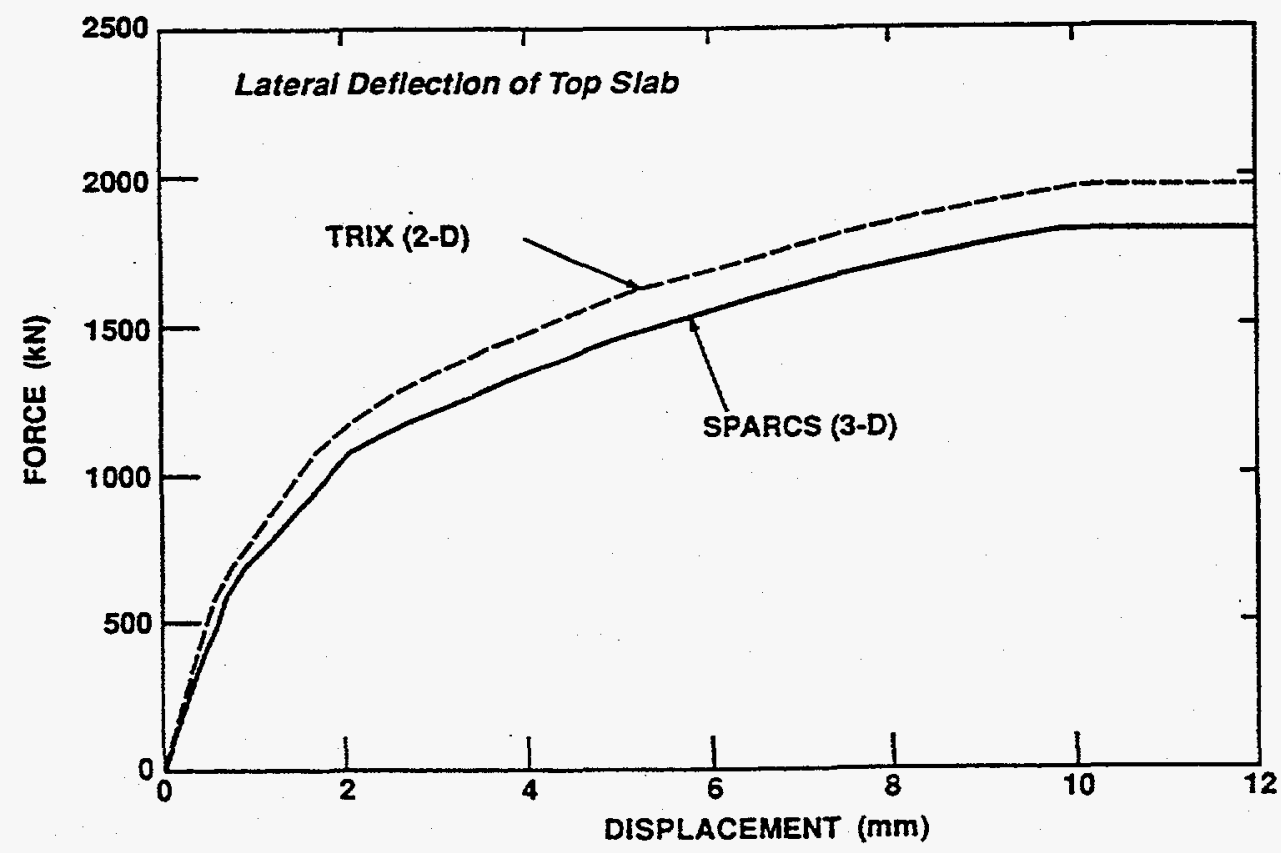

Fig. 4.6 Comparison of 3-D and 2-D Analyses 
Push-Over Analyses

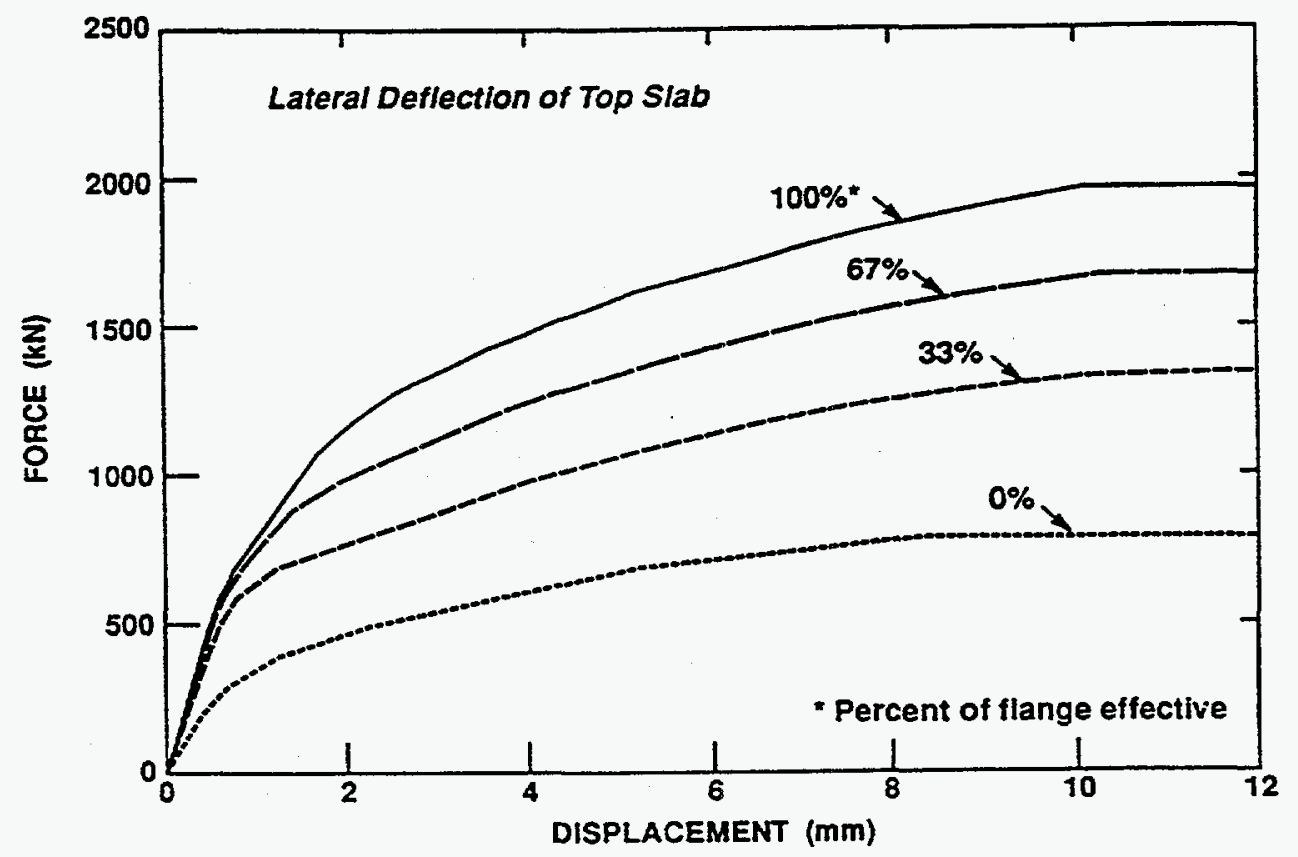

Fig. 4.7 Influence of Effective Flange Width (by UT) 
Push-Over Analyses

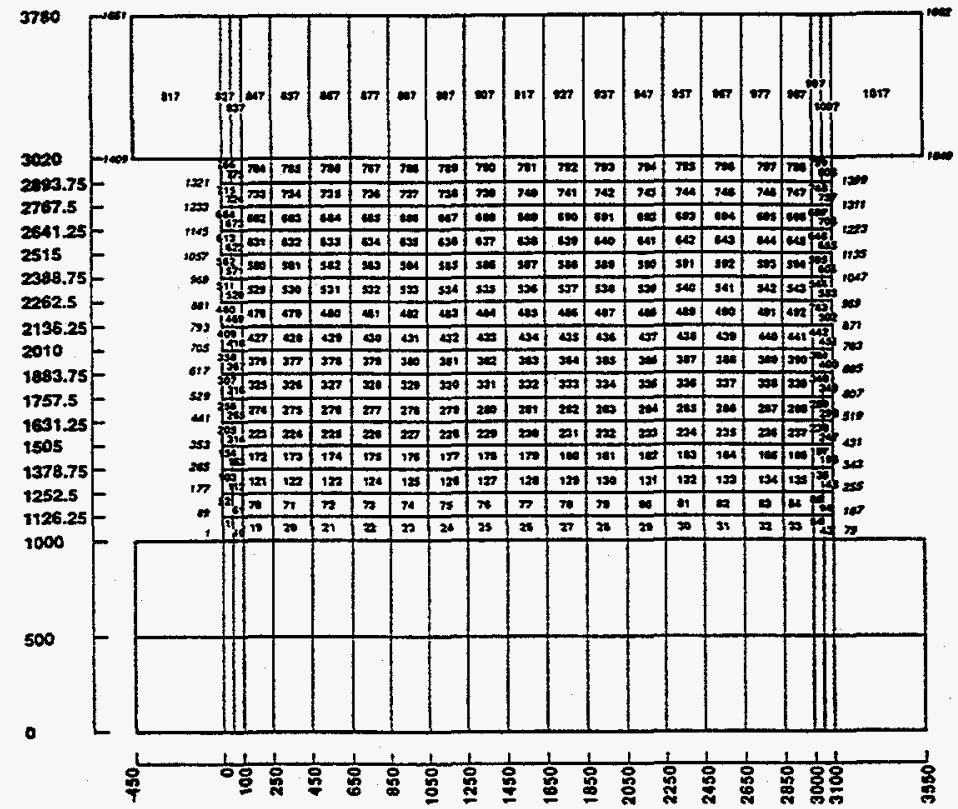

Fig. 4.8 Refined SPARCS 3-D Model

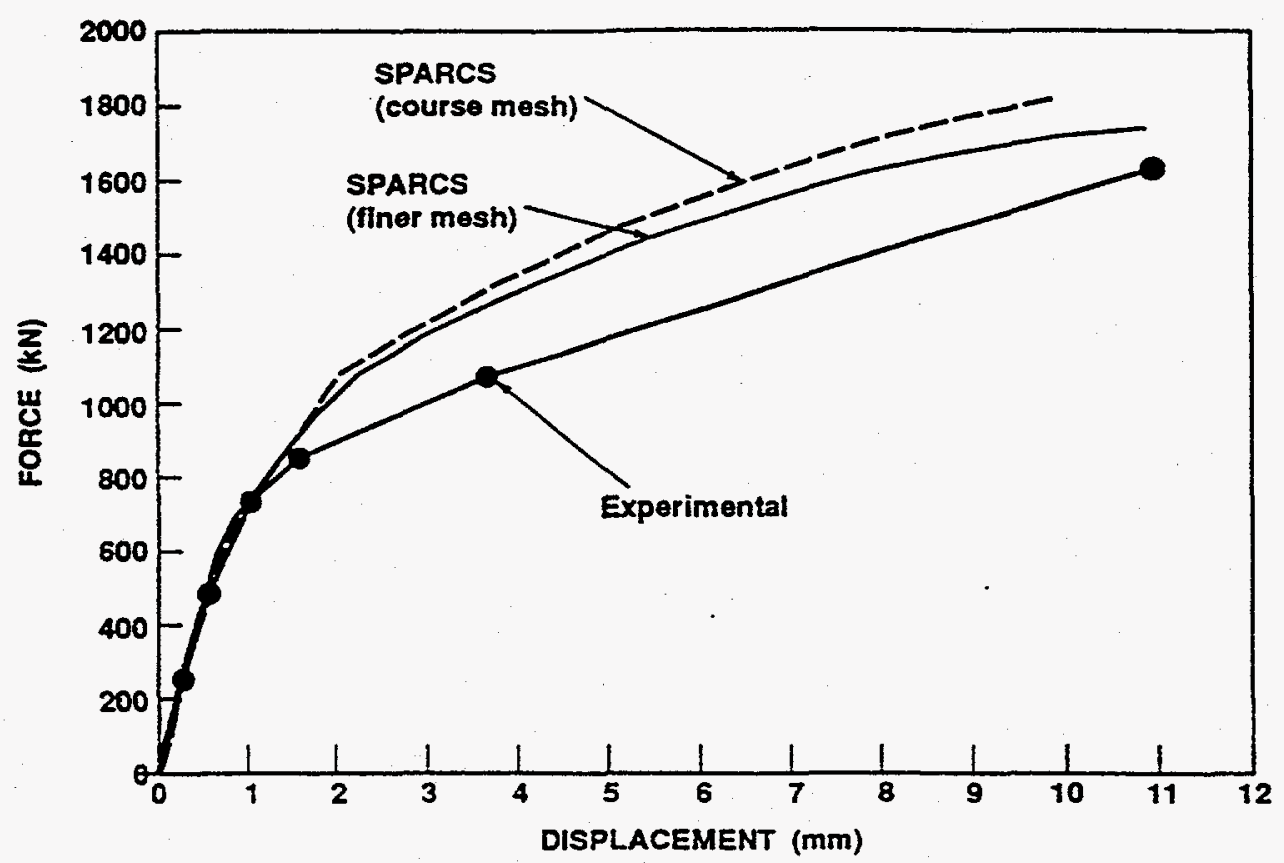

Fig. 4.9 Comparison of Analyses by SPARCS Models 
Push-Over Analyses

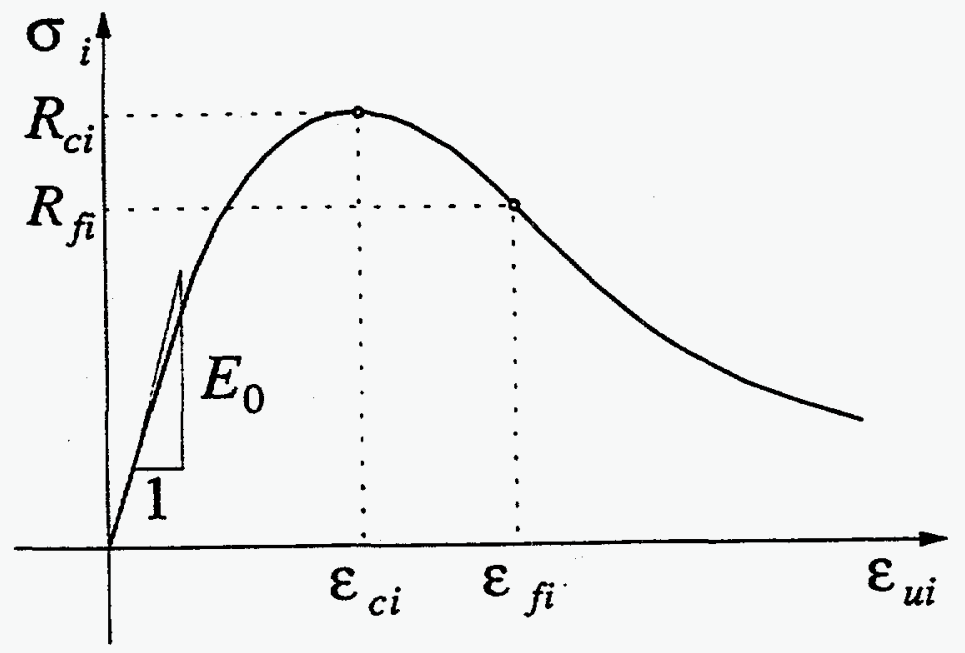

Fig. 4.10 Monotonic Uniaxial Stress-equivalent Strain Curve (by UCB)

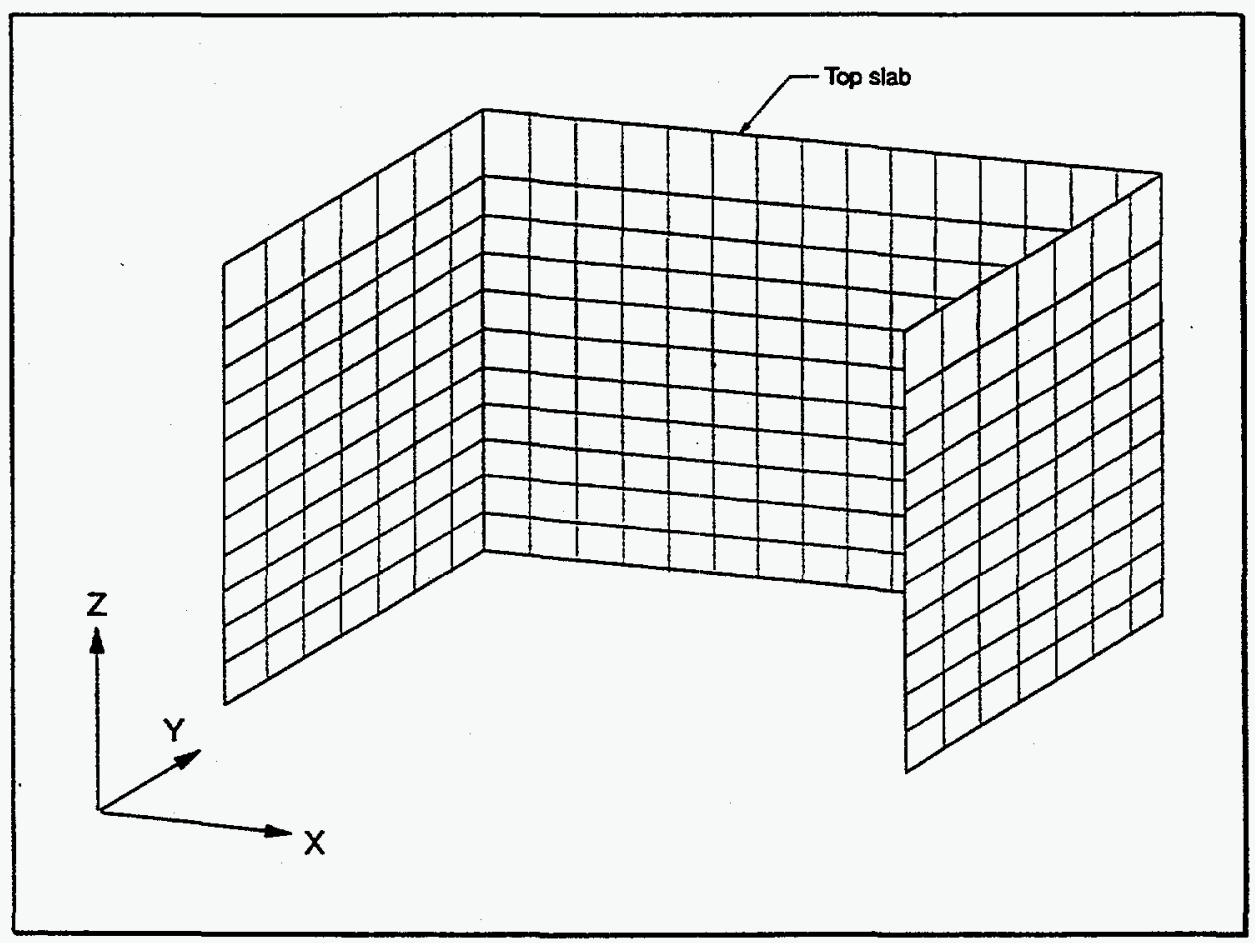

Fig. 4.11 MIRAGE 2-D Model by UCB 


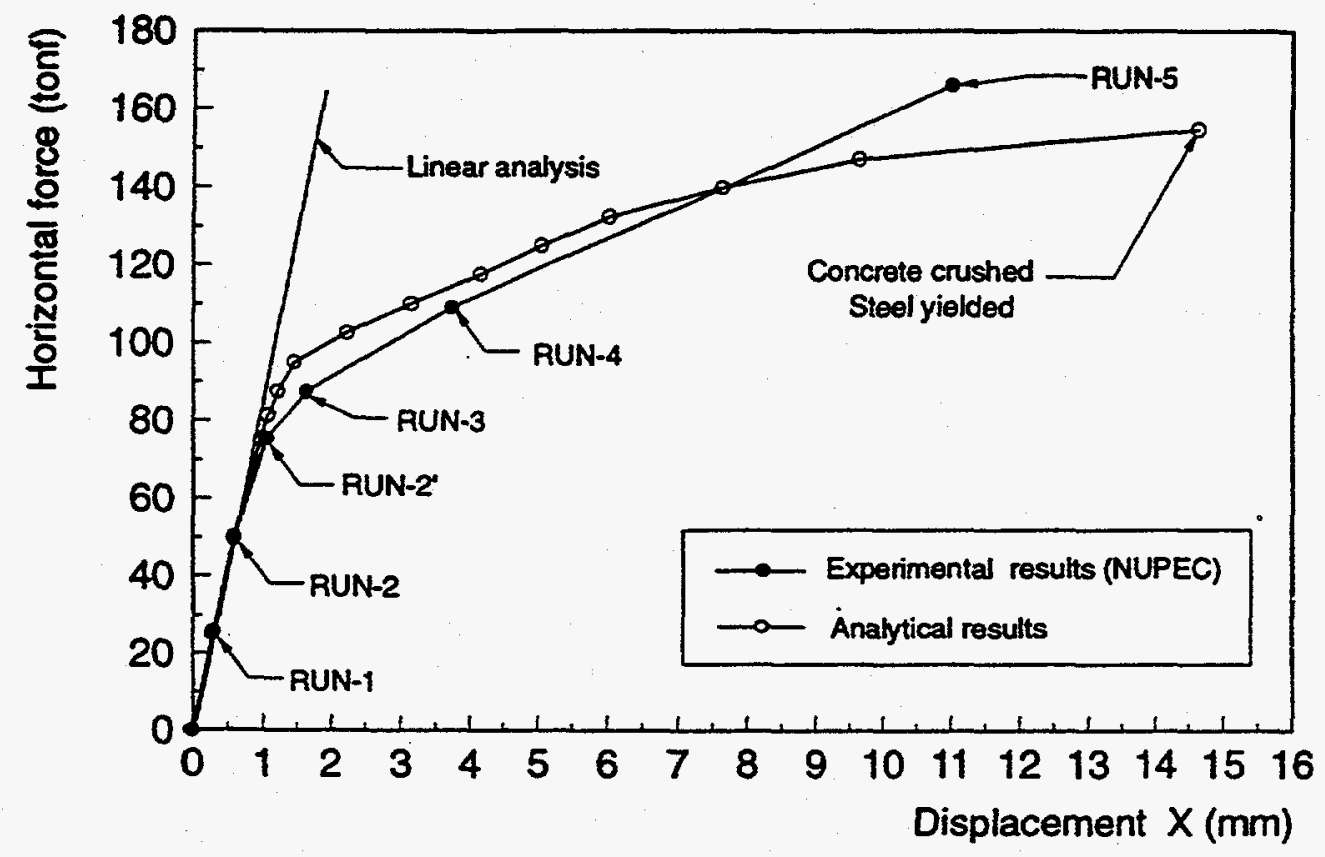

Fig. 4.12 Analysis Result by MIRAGE Model 
Push-Over Analyses
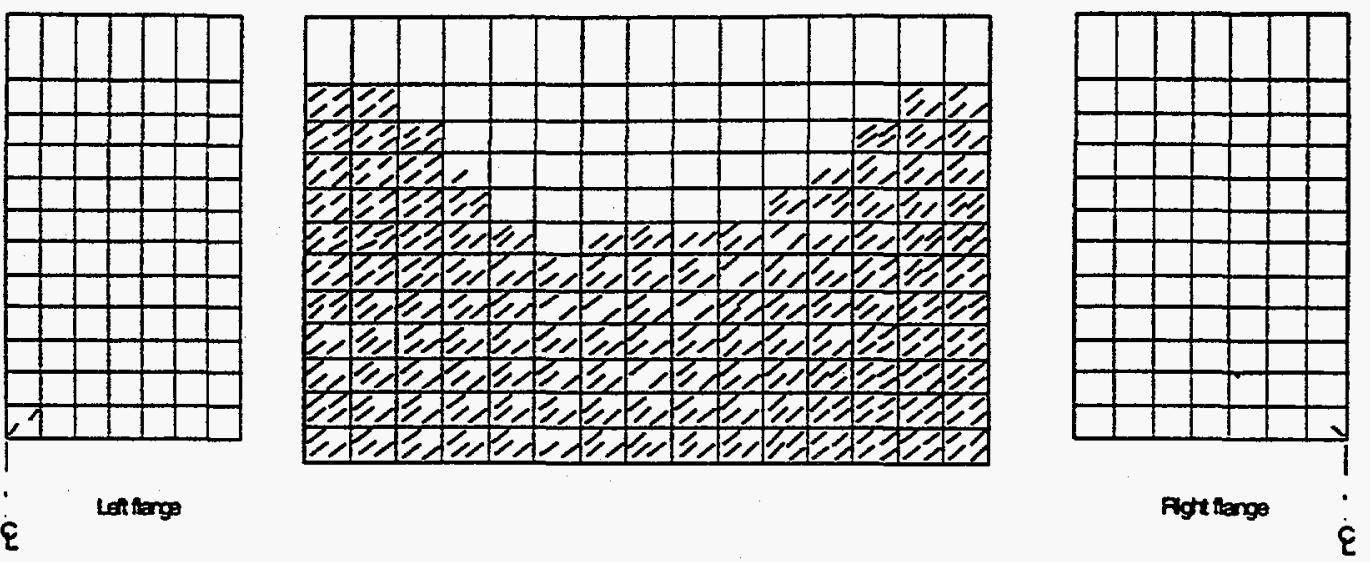

(a) Horizontal force $P_{2}=110.0$ tonf
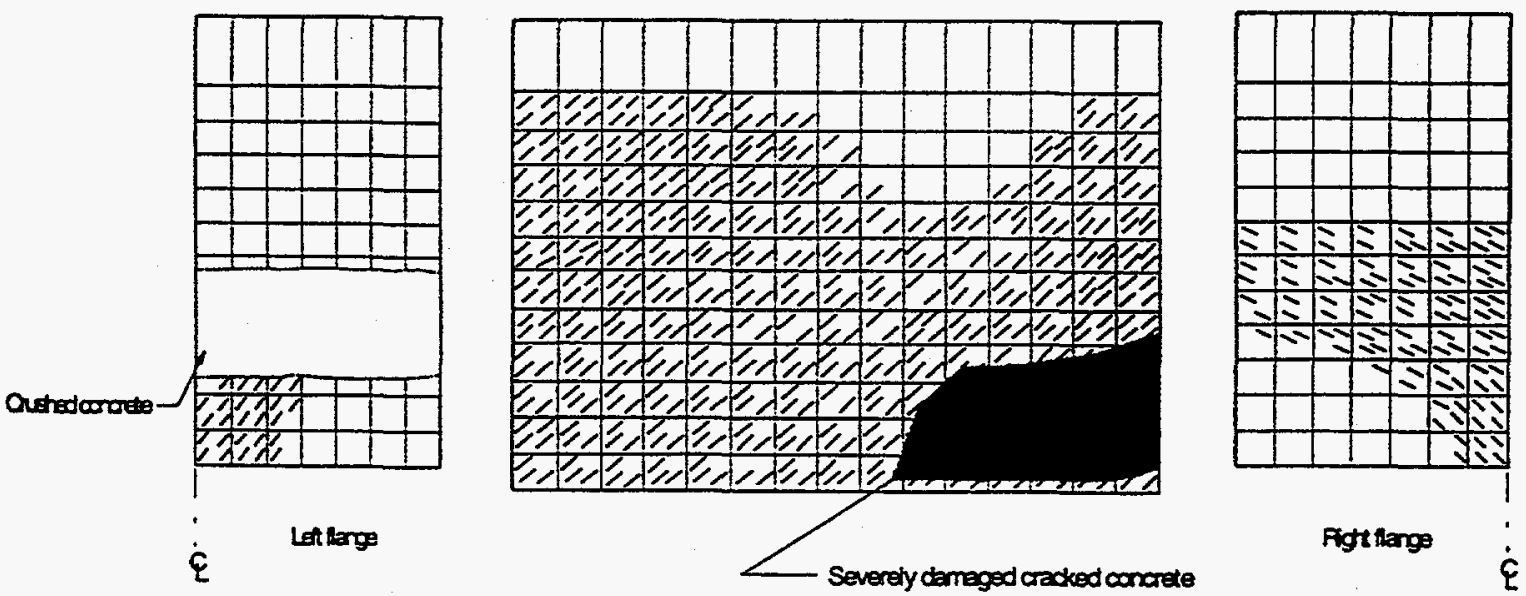

(b) Horizontal force $P_{2}=158.0$ tonf

Fig. 4.13 Predicted Crack Patterns in the Web and Flange Walls by MIRAGE Model 
Push-Over Analyses

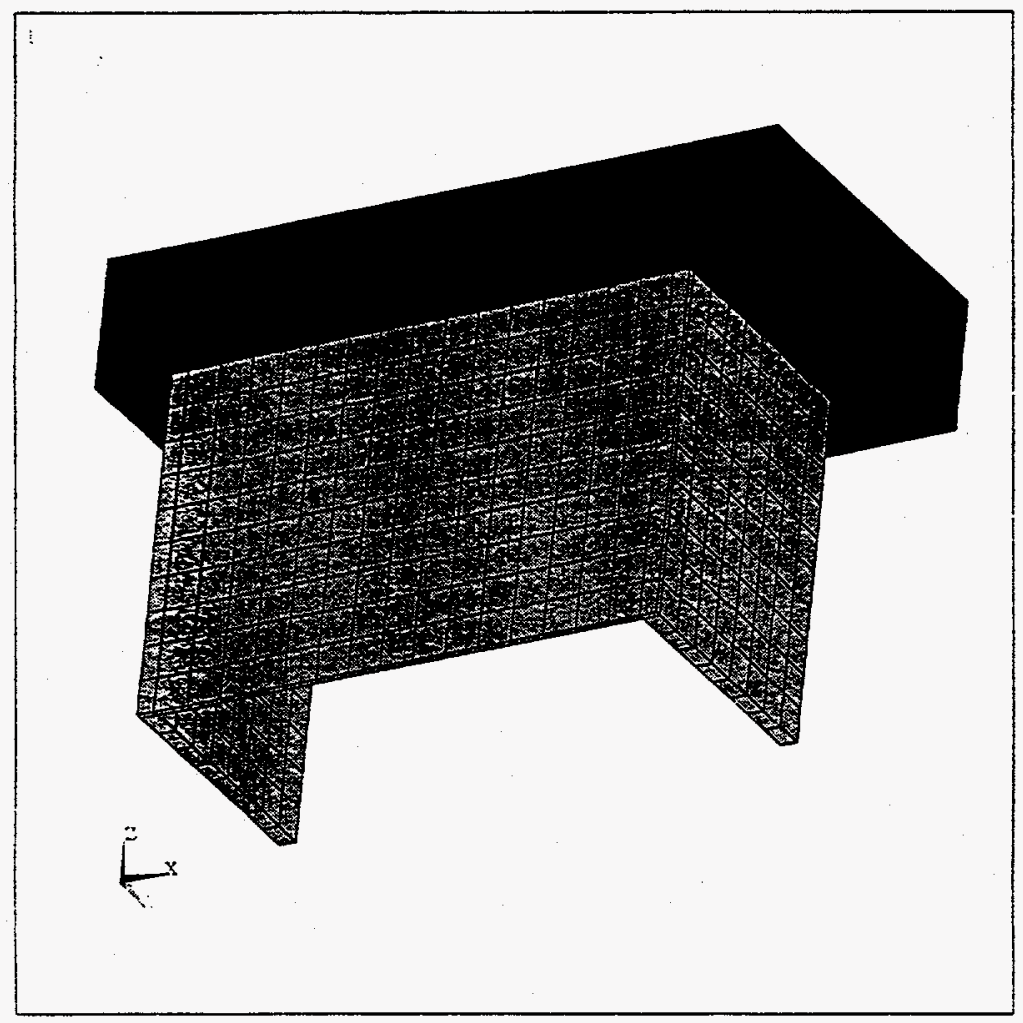

Fig. 4.14 ANSYS Model 
Push-Over Analyses

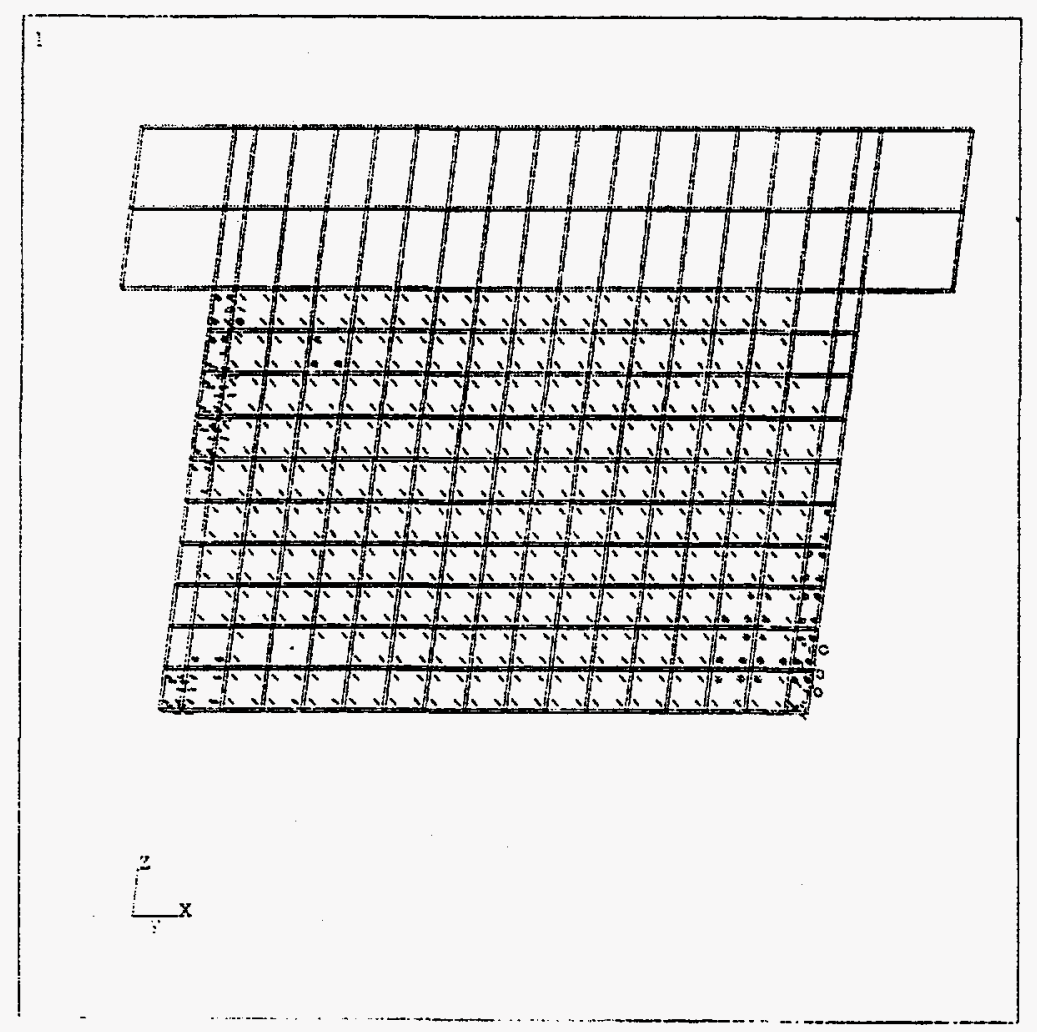

Fig. 4.15 Crack Pattern of ANSYS Model at the Last Loading Step 


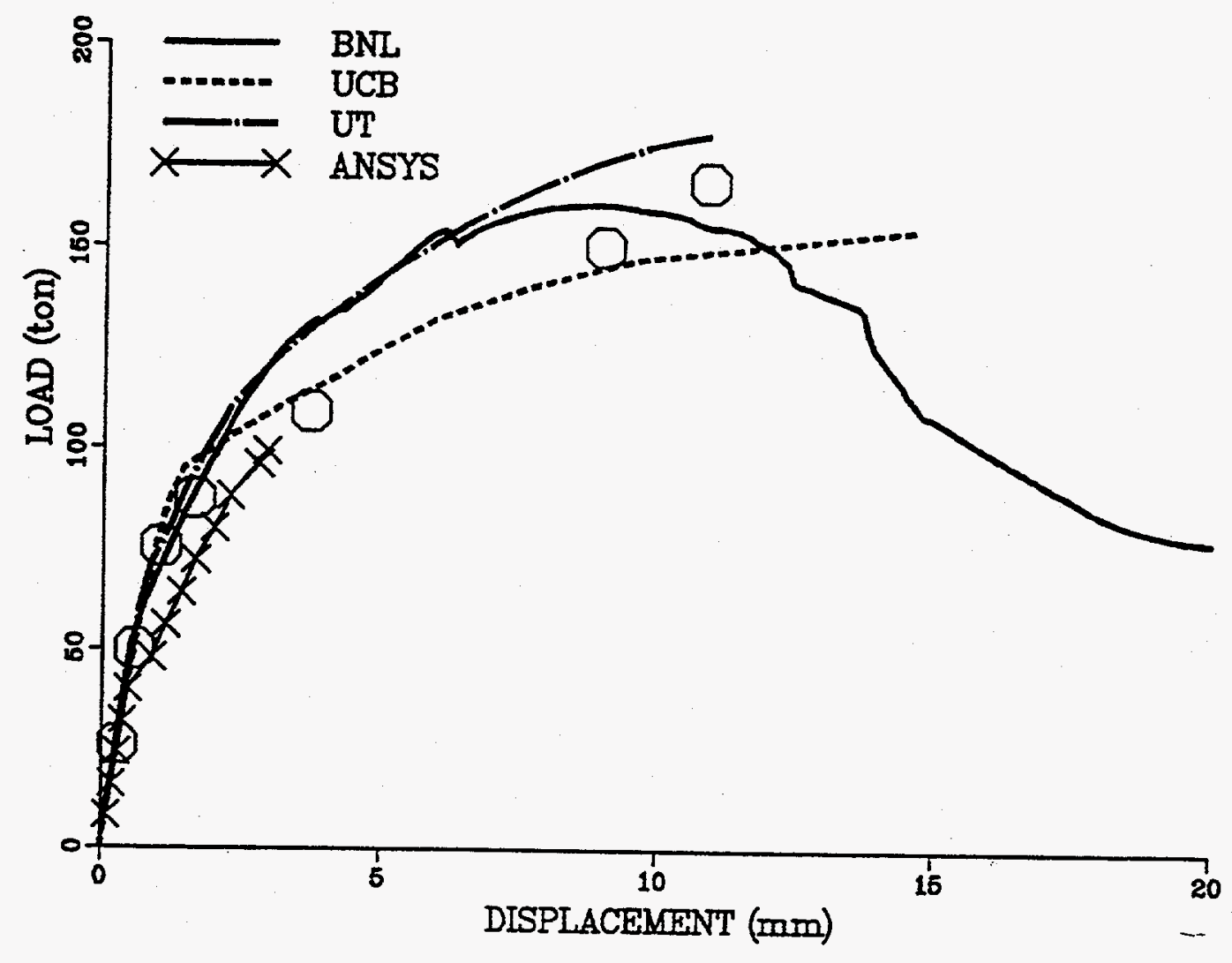

Fig. 4.16 Comparison of Push-over Analyses 


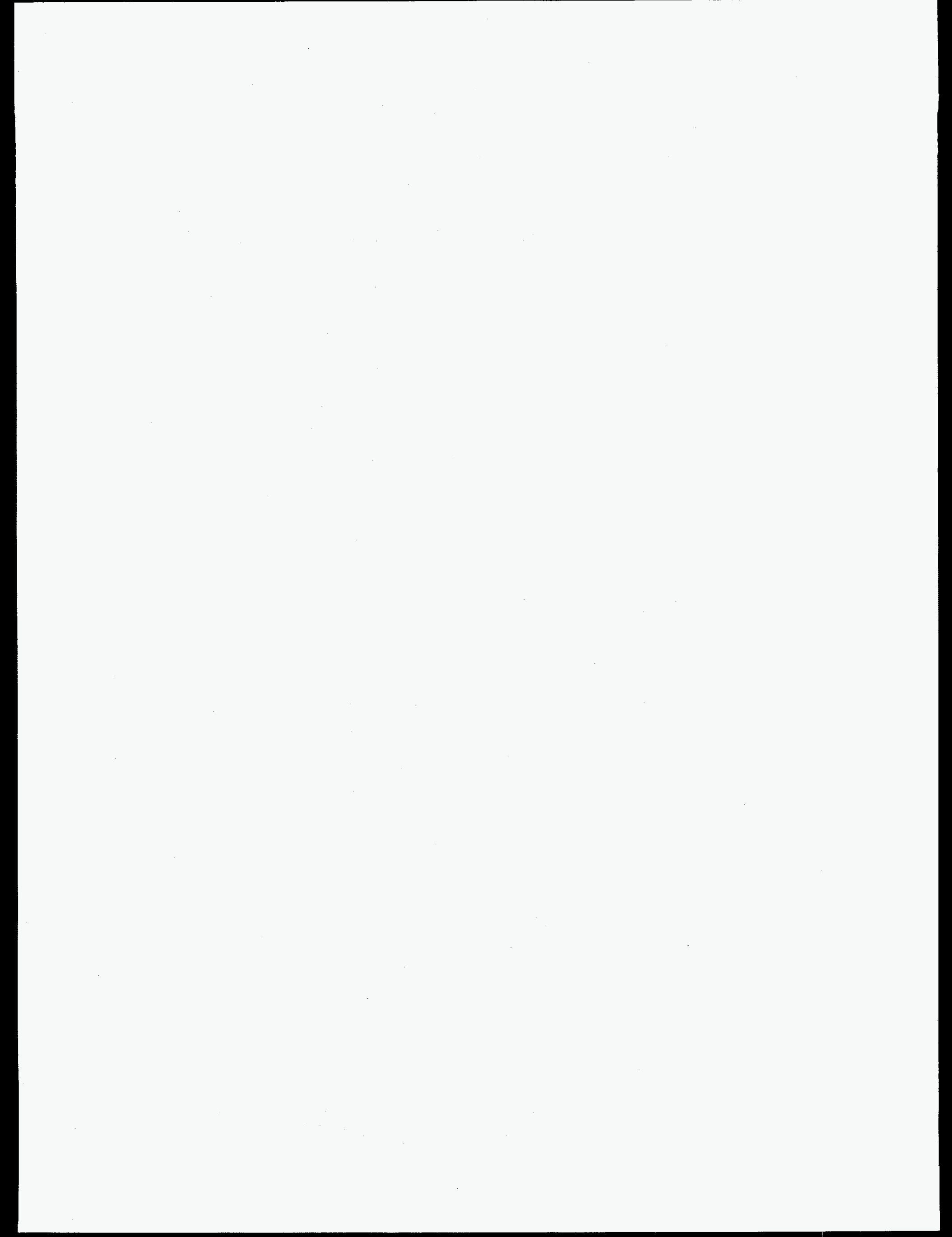




\section{COMPARISON OF DIFFERENT ANALYSIS METHODS}

\subsection{Introduction}

During the week of April 22, 1996, the 2nd SSWISP Workshop was held in Yokohama, Japan. During the workshop, a total of 55 analyses were presented by 30 participants from 11 different countries as listed in Table 5.1. These are classified according to the analysis type as:

\section{(Type of analysis)}

FEM Monotonic static (MS)

FEM Cyclic static (CS)

FEM Dynamic (FD)

Simplified modeling (SM)

Lumped mass modeling (LM)
(Total number of analyses)

20

6

13

8

8

55

NUPEC prepared comparison reports (Ref. 2), as well as a series of updated comparison reports which were also presented during the workshop. The analysis results of all the participants of the SSWISP Workshop are summarized in the comparison report published by OECD/NEA (Reg. 17).

The workshop provided a unique opportunity to assess the state-of-the-art in the nonlinear dynamic analysis of reinforced concrete shear wall structures under severe earthquake loadings. Major findings obtained during the workshop are described below.

\subsection{Applicability of Commercial Codes}

Several participants of the workshop attempted to use commercially available FEM codes to evaluate the ISP shear walls. Table 5.2 lists the names of the codes, the number of participants, and the types of analyses performed:

How well these commercial codes performed in comparison to in-house or proprietary codes is an interesting question since the commercial codes are often the only analysis tools available to most practical engineers.

In general, the correlation of dynamic analysis results achieved with these code may be considered poor even though a few participants tried to improve the solutions by implementing original material models with user subroutines (i.e., participants P5, P19 and P22). One exception may be the participant P19, who implemented a simple constitutive model for concrete in the commercially available DIANA code. According to their formulation, significant simplifications in material modeling were made using the Von Mises yield criterion and straight lines for the unloading/reloading branches of the uniaxial hysteretic model of concrete. Good correlations with recorded test results were achieved in the dynamic analyses up to RUN4. The correlation in the RUN-5 analysis, however, is not as good probably because of the simplifications made in material modeling.

As indicated in Table 5.2, most of the applications of commercial codes were for push-over type analyses. Fig. 5.1 compares the eight (8) analysis results (Ref. 2). Various modeling schemes were used by different analysts, i.e., use of shell elements 
Comparison

(in all the ABAQUS cases), 3-D brick elements (in ANACAP and ANSYS cases), and 2-D solid elements (in ADINA and FEM-I cases). As the solutions approach the maximum strength point, large scatter is observed among the analysis results even for those using the same code (e.g., ABAQUS cases). Except for the analyses performed with ADINA, which has a displacement control option, most of the push-over analyses were performed by an ordinary load-increment method. As a result, the analyses usually terminated due to the development of a singularity in the stiffness matrix. In these analysis, it is difficult to identify the "failure point", as the location of the last loading step is usually controlled by the numerical stability problems and analysis assumptions/conditions, rather than the actual failure.

\subsection{FEM Static Analyses}

A total of 20 push-over (monotonic static) analyses and 6 cyclic static analyses were submitted to the ISP workshop; among them, 8 push-over analyses were performed using commercially available codes. Figs. 5.2 through 5.4 show some of the comparison studies performed by NUPEC (Ref. 2). Much greater scatter is observed in the prediction of the ultimate displacement than in the prediction of the maximum strength. Various issues were discussed during the workshop at Yokohama. Some key issues, which are considered to have a major impact on the static analysis results, are discussed below.

\section{Analysis Control}

As mentioned, the load-increment method is seriously deficient in predicting the shear wall behavior near the failure point. All the better quality push-over analysis results (e.g., P11, P17, P20 and P34a) are obtained using displacement-control analyses, which predict not only the maximum strength point but also post-peak response with a negative slope. The secant stiffness method, used by participant $\mathrm{P} 34 \mathrm{~b}$, is considered to be a practical approach to trace the monotonic static behavior of reinforced concrete structures, as the negative slopes in the stress-strain relationships of concrete (due to cracking and crushing) can be naturally accounted for. However, the approach cannot be applied with cyclic static or dynamic loading conditions.

\section{Compressive Strength Reduction}

Since the early 1980 's, it has been recognized that the compressive strength in the direction parallel to the cracks of cracked concrete tends to be lower than the uniaxial compressive strength, $f_{c}$. How to quantify this compressive strength reduction is still a matter of controversy, and various assumptions/models were presented during the workshop. The approaches may be classified as,

(i) Simply ignored........ This is the case for most of the commercial codes which were developed in the 1960's and 1970's, and therefore do not reflect the most recent findings in concrete mechanics.

(ii) Use of a constant reduction factor........Factors ranging from 0.6 to 0.7 were assumed by varous participants. This approach may be considered to be a practical way of accounting for the strength reduction in light of the large scatter observed in the laboratory test data.

(iii) Use of empirical equations........Various empirical equations were presented which expressed the reduction factor as a function of the principal tensile strain, $\epsilon_{\mathrm{t}}$ Among them, the equations developed by Vecchio and Collins (see Eq. 4.1 of this report) and by H. Noguchi (Ref. 12) were the most popular choices among the participants. Noguchi's formulation expresses the reduction factor, $\lambda$, as follows:

$$
\lambda=\frac{1}{0.27+0.96\left(\epsilon_{t} / \epsilon_{o}\right)^{.167}}<1.0
$$


in which $\epsilon_{\mathrm{t}}$ is the average tensile strain; and $\epsilon_{\mathrm{o}}$ is the strain at the compressive strength.

The impact of the different assumptions on the predicted overall behavior of the shear wall, however, are not clearly evident as many factors in the material models are inter-related, and it is difficult to isolate one particular factor from the others

(Ref. 2).

\section{Shear Transfer Across Cracks}

A wide variety of formulations were presented at the workshop to account for the shear retention of cracked concrete due to aggregate interlocking. Some notable formulations are summarized below:

(i) Yamada-Aoyagi model........This model (Ref. 13) is one of the most popular choices among the presented analyses, probably owing to the simplicity of the formulation. The shear modulus, $G$, is expressed as a function of the elastic shear modulus, $G_{o}$, and the maximum tensile strain, $\epsilon_{\max }$ as

$$
G\left(\mathrm{~kg} / \mathrm{cm}^{2}\right)=\frac{1}{1 / G_{o}+\epsilon_{\max } / 36}
$$

(ii) Orthotropic model........Based on orthotropic plasticity theory, the shear modulus is expressed as a function of the uniaxial moduli, $E_{i}$ 's, and Poisson's ratio, $v$, as

$$
G=f\left(E_{i}, v\right)
$$

Examples of this approach can be found in Appendices A and B of this report. An advantage of this approach is that an independent hysteretic model for the shear stress-shear strain is not needed as it can be represented by those of the uniaxial normal stress-strain relationships.

(iii) Maekawa's model........According to the experimental study by B.Li and K. Maekawa (Ref. 14), the shear resistance across cracks due to aggregate interlocking can be expressed as an independent hysteretic model for the shear stress, $\tau$, and the shear strain, $\gamma$. The envelope curve is defined as,

$$
\tau / m=\frac{\left(\gamma / \epsilon_{t}\right)^{2}}{1+\left(\gamma / \epsilon_{t}\right)^{p}}
$$

in which, $\epsilon_{\mathrm{t}}$ is the normal tensile strain; and $\mathrm{m}$ is the direct shear strength,

$$
m(M P a)=3.83 f_{C}^{1 / 3}
$$

This shear retention assumption is considered to have a greater influence on the predicted results than the foregoing strength reduction factor. However, it was not possible to assess this influence independently from the presented analysis results. 


\section{Tension Softening}

In most of the analyses, tension softening of concrete was considered by assigning a negative stiffness after cracking. It was the opinion by most of the participants at the workshop that this factor had a very minor influence on the overall shear wall behavior. A similar conclusion was reached by F. Vecchio from a parametric study (see Fig. 16 in Appendix A of this report).

\section{Finite Element Model}

The following modeling schemes were used in the 20 finite element models for the push-over analyses:

$\begin{array}{cc}\text { (Type of elements) } & \text { (No. of models) } \\ \text { 2-D solid } & 11 \\ \text { Shell } & 6 \\ \text { 3-D solid } & 3\end{array}$

For the analyses using 2-D solid elements, the determination of the effective flange width was a significant issue and it was frequently discussed during the workshop. Due to the large flanges of the ISP shear walls, it was observed that the effective flange width would have to be increased as the plasticity in the shear walls progressed. In fact, in some analyses (e.g., Pl6) the effective flange width was changed for different test runs; with a progressively larger width for a higher amplitude test run. To alleviate this problem, the use of the unfolded model was proposed by several analysts (i.e., P22, P34a and P34c), to account for the complex shear-lag behavior of flanges in the nonlinear analysis.

The use of shell elements was probably the most reasonable modeling approach for the ISP shear walls. The 2-D (planestress) constitutive model can still be applied when the elements are formulated by layered membranes. It was pointed out however, that a reliable method to account for the out-of-plane shear failure is not currently available for layered shell elements. In most of the analyses presented, a simple linear stiffness was assumed for the out-of-plane shear deformation.

A few participants presented a complete 3-D model formed with brick solid elements, which is considered to be the most realistic modeling method as the out-of-plane shear failure can be fully accounted for. An apparent drawback of this modeling approach is the fact that the constitutive model for concrete for truely 3-D stress-strain conditions is still in the developmental stage. The 3-D constitutive model developed by the University of Toronto (see Appendix A of this report) is probably one of the most advanced material modeling methods, although it is limited only to push-over type analyses.

\section{Cyclic Loading}

Among the six (6) cyclic static analyses presented during the workshop, only three (3) were considered to be truely credible, i.e., particpants P11, P17 and P34a. Significant technical problems exist in expanding an FEM code to treat cyclic loading conditions for reinforced concrete. These may include:

- A "complete" hysteretic model for concrete, including a sophisticated logic for controlling repeated crack operings and closings, is required for at least biaxial stress-strain reversals.

- The analysis should account for negative-slope (i.e., negative stiffiness) due to the cracking and crushing of concrete.

- The solutions tend to be highly unstable when most of the elements enter the negative slope regions, and the loading direction is reversed. The ordinary Newton-Raphson iteration scheme is not sufficient for this type of problem. 
- To keep the unbalanced forces under control, some form of additional numerical iterations are necessary. During repeated numerical iterations, elements tend to be subjected to artificial unloading/reloading reversals, which could aggravate the solutions.

- The so-called numerical rachetting in an analysis may cause significant numerical problems under repeated cyclic loading reversals.

Some of these problems were discussed during the workshop. It should be noted that all successul [three (3)] cyclic static analyses were performed with original in-house codes. Currently, no commercially available FEM codes can deal with the above problems, without significant modifications being made to the material model and analysis procedures through user subroutines.

\subsection{FEM Dynamic Analyses}

A total of thirteen (13) FEM dynamic analyses were presented during the workshop, and were considered to be the highlight of the meeting. The SSWISP workshop was probably the first occasion that the application of nonlinear FEM dynamic analyses to shear wall structures was discussed as the main theme in an international conference. For many participants, the SSWISP was the first opportunity to perform these types of nonlinear dynamic analyses, and a wide variety of technical issues for improving the prediction accuracies were discussed.

Most of the issues described in the foregoing section also apply to the dynamic analyses. In addition, the following issues and unique problems associated with the application of the FEM to the dynamic analysis of concrete structures were raised:

- Determination of linear (viscous) damping;

- Consideration of hysteretic damping;

- Whether or not to account for the strain rate effect;

- Numerical stability problems;

- Differences in responses (e.g., envelope response) between static and dynamic analyses;

- Whether or not the ultimate failure mode is different between static and dynamic analysis results.

Although no definitive answers were obtained to any of the above issues, the workshop nevertheless provided a unique opportunity to review the state-of-the-art in these areas.

Participants were asked to submit the dynamic analysis results for RUN-4 and RUN-5, and the submission of results for the lower-amplitude test runs was encouraged. Most of the participants experienced technical difficulties in performing the RUN-5 analysis, during which the shear wall failed catastrophically. A detailed comparison of the accuracies of prediction is described in Ref. 2. To gain a further insight, the 13 analysis results are classified into three groups according to the originality of the computer codes and the degree of sophistication of the hysteretic models for concrete, as listed in Table 5.3.

Most of the analyses in Group A showed good correlation for both RUN-4 and RUN-5. It seems that the use of an original computer code was an important factor in a successful analysis. Apparently because of the complexity of the analysis and the technical difficulties, a through knowledge and total control of the computer program are necessary for successful analysis. Another cirtical factor is the modeling of the hysteretic property of the concrete in the constitutive model. Fig. 5.5 shows the uniaxial hysteretic models of concrete used in the Group A analyses. All these models are very detailed and realistic, and they have the following features in common:

- Strength deterioration due to cycling in compression zone;

- Hysteresis damping in unloading/reloading path;

- Negative slope both in tension and compression zones;

- Multi-linear or curved lines for unloading path in compression zone. 
The dynamic analysis results for RUN-4 from Group B are considered to be as good as those from Group A, except for those of participant P24 who reported that the computer code used was still in an early stage of development. The analysis correlations for RUN-5 from Group B however are considered to be somehow inferior to Group A. All the analyses in Group $B$ are based on simpler hysteretic models of concrete as illustrated in Fig. 5.6. Further simplifications are also implemented in some analyses, such as the elimination of Poisson's effects (e.g., P14) and the use of a simple Von-Mises yield surface (e.g., P19).

It seems that the use of a simplified hysteretic model does not have a significant impact on the moderately plastic responses for RUN-4, but is a critical factor in predicting responses with very large plastic deformations, i.e., for RUN-5.

All the analyses in Group C, were developed using commercial codes, and were considered not successful even for the moderately nonlinear response case, RUN-4.

\subsection{Simplified Modeling Method}

In the nonlinear dynamic analysis of building structures, shear wall components are frequently modeled using truss elements or a combination of shear and bending springs. A total of 8 analyses based on such simplified modeling schemes were presented during the workshop, as summarized in Table 5.4 .

For the models using either beam elements (P6 and P33) or a combination of springs (P25 and P28), the characterization of the nonlinear shear spring was important since shear deformation dominated over flexural deformation in the ISP shear wall tests.

For the analyses using truss models (P12, P15, P23 and P31), the shear deformation behavior is controlled by the diagonal struts, which account for the shear strength/deformation of the concrete portion of the web wall. In all the cases, a simple uniaxial spring element is used for the diagonal struts. The cross-sectional area of the element is usually determined from the equivalent overall shear stiffness of the wall, which can be obtained either from an empirical equation or a linear static FEM analysis. Since the diagonal struts represent the plain concrete of the web wall, a conventional uniaxial hysteretic model for concrete can be used to define the nonlinear characteristics of the struts. The compression strength, $\mathrm{P}_{\mathrm{c}}$, can be defined as,

$$
P_{c}=v \cdot f_{c}^{\prime} \cdot A_{e}
$$

where, $v$ is a strength reduction factor, $\mathrm{f}_{c}^{\prime}$ is the concrete strength, and $\mathrm{A}_{e}$ is the cross-sectional area of a strut. According to earlier studies with similar simplified models of shear walls (Ref. 15), constant values around 0.5 to 0.6 were suggested for the foregoing reduction factor, $v$. The following values were used by the ISP participants using truss models:

(Participants)

P12

P15

P23

P31

\section{(Reduction factor)}

$$
\begin{aligned}
& v=0.55 \\
& v=0.65 \\
& v=0.75 \\
& v=1.0
\end{aligned}
$$


Since the strength reduction factor was not considered by participant P31, the shear strength of the wall was grossly overestimated (by about 20\%). A good correlation in the dynamic analysis results are observed for P12 and P15, who used a relatively low reduction factor.

\subsection{Lumped Mass Modeling Method}

A total of eight (8) participants presented dynamic analysis results developed using a single-degree-of-freedom (SDOF) system, as listed in Table 5.5. The analyses may be characterized by the following items:

- Way to determine the envelope curve,

- Shape of envelope curve,

- Whether or not the shear and bending deformations are separately modeled using two springs,

- Choice of hysteretic model,

- Viscous damping assumption.

The envelope curves were modeled as either a multi-linear or continuous curve. The following methods were used to determine the envelope curve:

- Use of ISP test results (P4, P37),

- $\quad$ Empirical equations (P4, P32)

- $\quad$ Static analysis using simplified model (P11, P27),

- $\quad$ FEM static analysis (P2, P16, P20).

The order of the above list reflects the order of analysis reliability (i.e., use of test results is the most reliable, and the use of FEM analysis is the least reliable), as suggested by several experts during the workshop. Participant P4 used an empirical equation to calculate the strength, and the ISP test results to estimate the deformation of turning points. Whether or not a negative slope was included after the maximum point seems to have an impact on the predicted responses for RUN-5.

As indicated in Table 5.5, several participants (P11, P20 and P37) used two nonlinear springs to separate the shear and bending deformations, which obviously improved the prediction accuracy. Relatively ductile hysteresis models were used for the bending spring, and less ductile models for the shear spring. Fig. 5.7 shows the hysteresis models used by the participants (Ref. 2). It seems that the models used by participants P2 and P27 have too large a hysteresis area (i.e., excessive energy absorption), and the model used by participant $\mathrm{P} 32$ has an excessive pinching characteristic in comparison with the observed hysteretic responses of the ISP shear walls.

Among all the presented response results, the ones by participants $\mathrm{P} 11$ and $\mathrm{P} 20$, who used a combination of bending and shear springs as well as a negative slope after the maximum strength point, showed a good correlation with the recorded responses for both RUN-4 and RUN-5.

Regarding the viscous damping assumption, no definitive conclusions were reached during the workshop. However, according to participant P16, the prediction accuracy improved significantly when the damping value was reduced from $4 \%$ to $2 \%$ for RUN-5. 
Table 5.1 List of Analyses Presented at SSWISP Workshop

\begin{tabular}{|c|c|c|c|}
\hline Participant I.D. & Type of Analysis ${ }^{(-1)}$ & Computer Code ${ }^{(2)}$ & Analysis Model \\
\hline P1 & $\mathrm{CS}, \mathrm{FD}$ & SOLVIA & 2-D solid elements with fine mesh \\
\hline $\mathrm{P} 2$ & $\mathrm{MS}, \mathrm{LM}(\mathrm{F})$ & original & 2-D solid element \\
\hline $\mathrm{P} 4$ & $\mathrm{LM}(\mathrm{E})$ & original & slip model \\
\hline P5 & FD & CASTEM (M) & simplified hysteretic model \\
\hline P6 & SM & original & used beam elements \\
\hline P9 & $\mathrm{MS}, \mathrm{FD}$ & ABAQUS & 3-D shell elements \\
\hline P11 & $\begin{array}{l}\mathrm{MS}, \mathrm{CS}, \mathrm{FD} \\
\mathrm{LM}(\mathrm{S})\end{array}$ & $\begin{array}{l}\text { original } \\
\text { original }\end{array}$ & $\begin{array}{l}\text { 3-D shell element } \\
\text { bending and shear springs }\end{array}$ \\
\hline P12 & SM & original & strain rate effect considered \\
\hline $\mathrm{P} 13$ & FD & original & 2-D solid elements \\
\hline P14 & $\mathrm{FD}$ & original & simpler material model, 2-D solid elements \\
\hline P15 & SM & original & truss elements \\
\hline P16 & MS, LM(F) & ADNA & displacement control with small increment \\
\hline P17 & $\mathrm{MS}, \mathrm{CS}, \mathrm{FD}$ & original & 3-D shell elements \\
\hline P18 & MS, FD & original & 2-D solid elements \\
\hline P19 & $\mathrm{FD}$ & DIANA (M) & 3-D shell elements, simpler material model \\
\hline P20 & $\mathrm{MS}, \mathrm{LM}(\mathrm{F})$ & original & shell elements \\
\hline $\mathrm{P} 21$ & MS & original & 2-D solid elements \\
\hline $\mathrm{P} 22$ & $\begin{array}{c}\text { MS } \\
\text { MS, FD }\end{array}$ & $\begin{array}{c}\text { original } \\
\text { ABAQUS }(M)\end{array}$ & $\begin{array}{l}\text { unfolded model } \\
3-D \text { shell elements }\end{array}$ \\
\hline P23 & SM & original & truss madel \\
\hline $\mathrm{P} 24$ & $\mathrm{MS}, \mathrm{CS}, \mathrm{FD}$ & original & 2-D solid elements \\
\hline $\mathrm{P} 25$ & SM & original & macro-model \\
\hline $\mathrm{P} 27$ & $\mathrm{LM}(\mathrm{S})$ & original & flanges were ignored \\
\hline P28 & $\begin{array}{l}\text { FD } \\
\text { SM }\end{array}$ & $\begin{array}{l}\text { ABAQUS } \\
\text { original }\end{array}$ & $\begin{array}{l}\text { 3-D shell elements } \\
\text { Kabeyazawa model }\end{array}$ \\
\hline P30 & MS & ABAQUS & shell elements \\
\hline
\end{tabular}


Table 5.1 List of Analyses Presented at SSWISP Workshop

\begin{tabular}{|c|c|c|c|}
\hline Participant I.D. & Type of Analysis ("1) & Computer Code ${ }^{(-2)}$ & Analysis Model \\
\hline P31 & SM & original & truss model \\
\hline P32 & $\mathrm{LM}(\mathrm{E})$ & original & pinching model \\
\hline P33 & $\begin{array}{l}\text { MS } \\
\text { MS } \\
\text { SM }\end{array}$ & $\begin{array}{c}\text { FEM-I } \\
\text { ADINA } \\
\text { DARC-2D }\end{array}$ & $\begin{array}{l}\text { 2-D solid elements } \\
\text { 2-D solid elements } \\
\text { beam elements }\end{array}$ \\
\hline P34a & $\begin{array}{l}\text { MS, CS, FD } \\
\quad \text { MS }\end{array}$ & $\begin{array}{l}\text { original } \\
\text { ANSYS }\end{array}$ & $\begin{array}{l}\text { unfolded model } \\
\text { 3-D brick elements }\end{array}$ \\
\hline P34b & $\begin{array}{l}\text { MS } \\
\text { MS }\end{array}$ & $\begin{array}{l}\text { original } \\
\text { original }\end{array}$ & $\begin{array}{l}\text { 3-D brick elements } \\
\text { parametric study by } 2-D \text { model }\end{array}$ \\
\hline P34c & MS & original & unfolded model \\
\hline P35 & MS & ANACAP & 3-D brick elements \\
\hline $\mathrm{P} 37$ & $\begin{array}{c}\mathrm{CS} \\
\mathrm{LM}(\mathrm{T})\end{array}$ & $\begin{array}{l}\text { original } \\
\text { original }\end{array}$ & $\begin{array}{l}\text { shell elements } \\
\text { JEAG model }\end{array}$ \\
\hline
\end{tabular}

Note (*1): $\quad$ MS......monotonic static analysis by FEM

CS......cyclic static analysis by FEM

FD.......dynamic analysis by FEM

SM......simplified modeling approach

LMC ).lumped-mass analysis, the ( ) indicates the method to determine envelope curve

(F)......from FEM anlaysis

(E)......from empirical equations

(S).......from simplified analysis

(T)......from ISP test results

Note (*2): only the names of publically available codes are given

(M)......indicates the modification of material model using a user subroutine 
Table 5.2 List of Commercial Codes

\begin{tabular}{|c|c|c|c|}
\hline Computer Code & No. of Participants & Types of Analysis & Finite Elements \\
\hline ABAQUS & 4 & monotonic static, dynamic & 3-D shell \\
\hline ADINA & 2 & monotonic static & 2-D solid \\
\hline ANACAP & 1 & monotonic static & 3-D brick solid \\
\hline ANSYS & 1 & monotonic static & 3-D brick solid \\
\hline CASTEM & 1 & dynamic & 2-D solid \\
\hline DIANA & 1 & dynamic & 2-D solid \\
\hline FEM-I & 1 & monotonic static & 2-D solid \\
\hline IDARC & 1 & monotonic static, dynamic & beam element \\
\hline SOLVIA & 1 & cyclic static, dynamic & 2-D solid \\
\hline
\end{tabular}

Table 5.3 Grouping of FEM Dynamic Analyses

\begin{tabular}{|c|c|c|c|}
\hline Group & Computer Code & $\begin{array}{c}\text { Hysteretic Model for } \\
\text { Concrete }\end{array}$ & Participants \\
\hline A & Original & Detailed and realistic & P11, P13, P17, P18, P34a \\
\hline B & Original or commercial with significant \\
changes & Simplified & P5, P14, P19, P24 \\
\hline C & Commercial & Simplified & P1, P9, P22, P28 \\
\hline
\end{tabular}


Table 5.4 Summary of Simplified Models

\begin{tabular}{|c|c|c|}
\hline Participants & Structural Model & Constitutive Model \\
\hline P6 & Fiber model using beam elements & Material - level \\
\hline P12 & Truss model & Material - level \\
\hline P15 & Truss model & Material - level \\
\hline P23 & Truss model & Material - level \\
\hline P25 & Axial and shear springs & Component - level \\
\hline P28 & Axial, shear and bending springs & Component - level \\
\hline P31 & Truss model & Material - level \\
\hline P33 & Beam element & Component - level \\
\hline
\end{tabular}

Table 5.5 Summary of Lumped Mass Models

\begin{tabular}{|c|c|c|c|c|c|}
\hline \multirow{2}{*}{ Participants } & \multicolumn{2}{|c|}{ Skelton Curve } & \multirow{2}{*}{$\begin{array}{l}\text { Comb. of Shear } \\
\text { \& Bending } \\
\text { Springs }\end{array}$} & \multicolumn{2}{|c|}{ Damping (\%) } \\
\hline & Method & Negative Slope & & RUN-4 & RUN-5 \\
\hline $\mathrm{P} 2$ & FEM Analysis & no & no & 3 & 4 \\
\hline P4 & Empirical Eq. & no & no & 1 & 1 \\
\hline P11 & $\begin{array}{l}\text { Simplified } \\
\text { method }\end{array}$ & yes & yes & 2 & 2 \\
\hline $\mathrm{P} 16$ & FEM Analysis & yes & no & 3.5 & 2 \\
\hline $\mathrm{P} 20$ & FEM Analysis & yes & yes & 0.8 & 0.8 \\
\hline $\mathrm{P} 27$ & $\begin{array}{l}\text { Simplified } \\
\text { method }\end{array}$ & yes & no & 3 & 4 \\
\hline P32 & Empirical Eq. & yes & no & 1 & 1 \\
\hline P37 & ISP test & no & yes & 1 & 1 \\
\hline
\end{tabular}




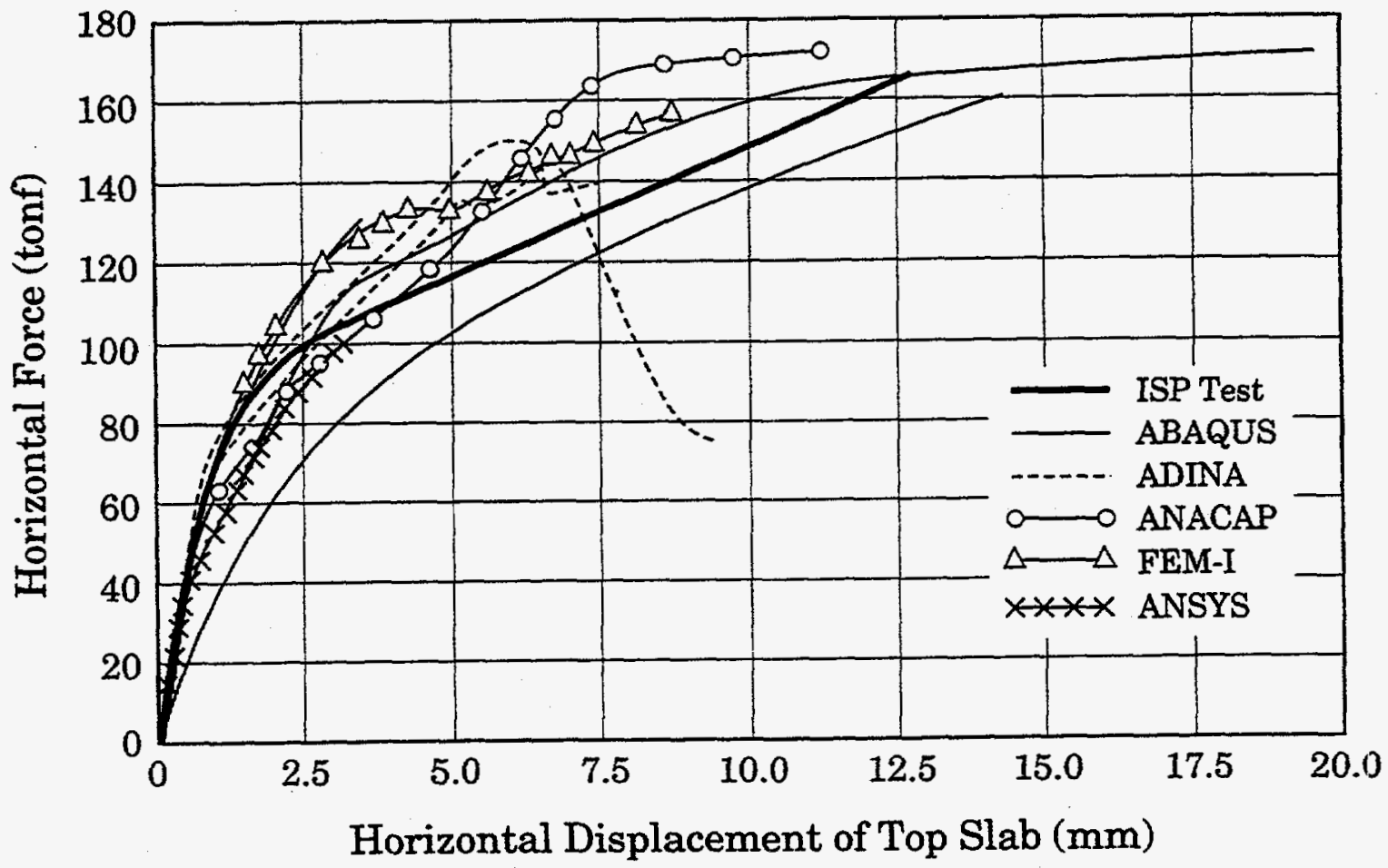

Fig. 5.1 Push-over Analysis Results Using Commercially Available FEM Codes 


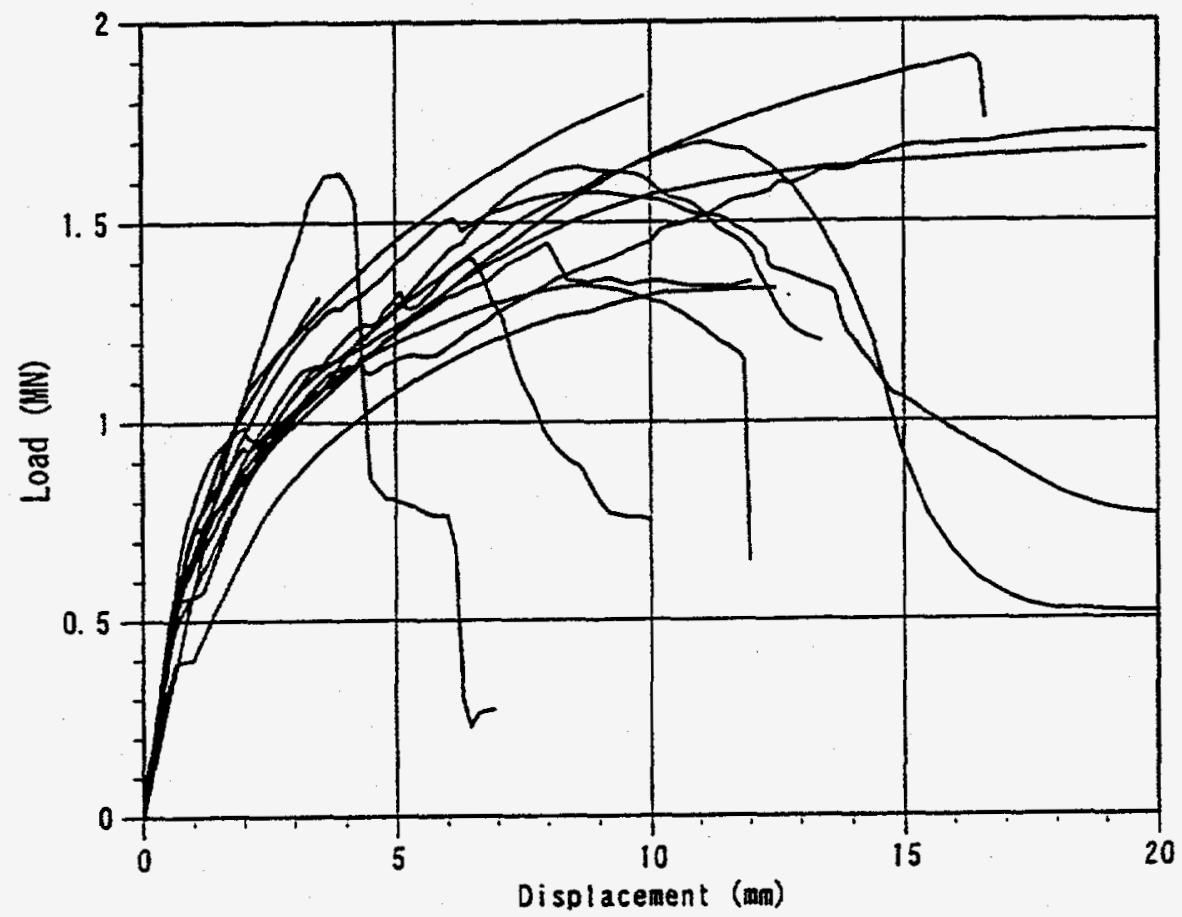

Fig. 5.2 Comparison of Load-displacement Relationship Obtained from Monotonic Loading Analyses (Ref. 2) 

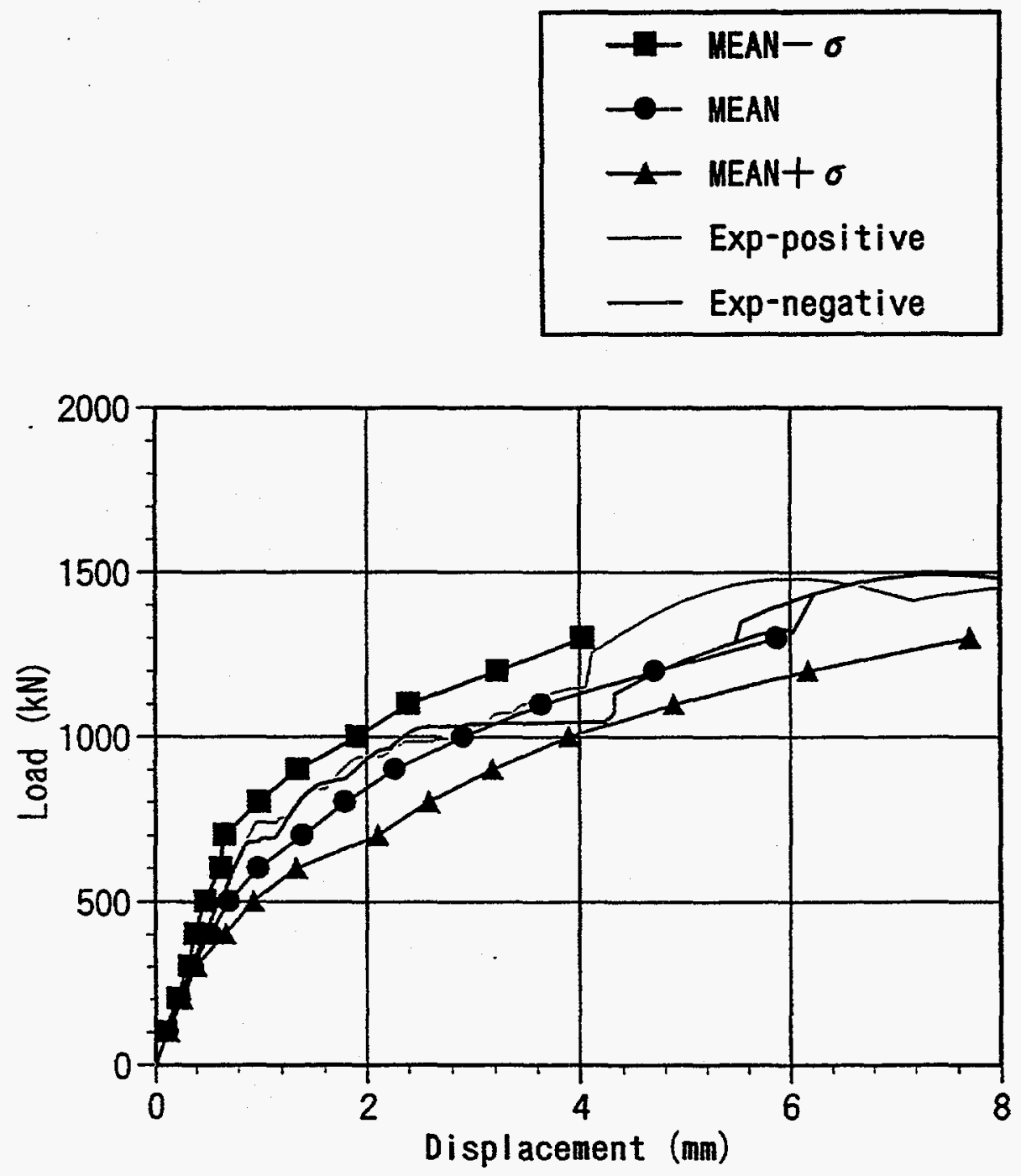

Fig. 5.3 Distribution of Displacement at Loads of Every 10kN Obtained from Analyses (Ref. 2) 




\section{Stress}

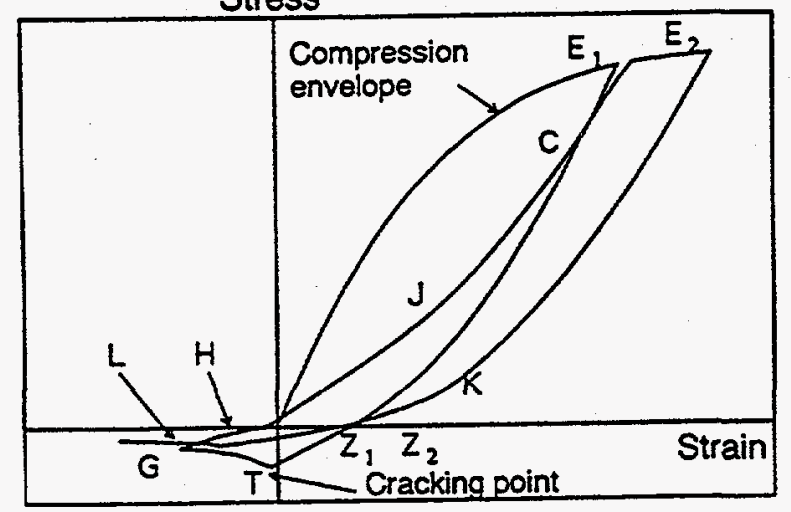

(a) P11

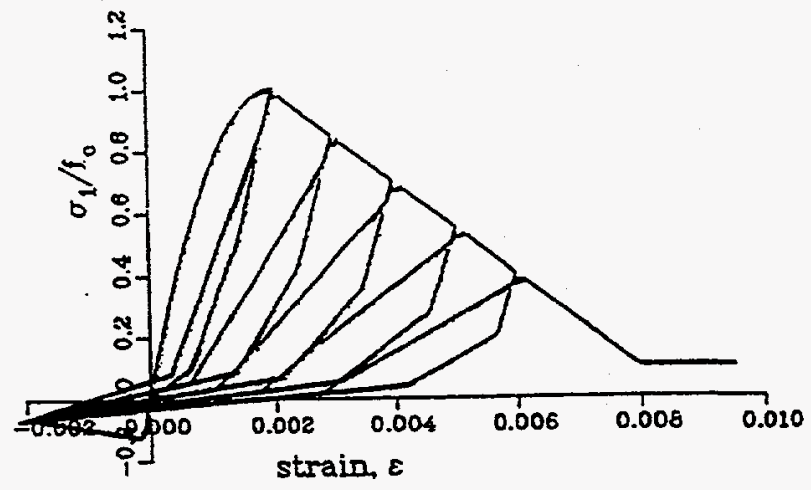

(c) P34a

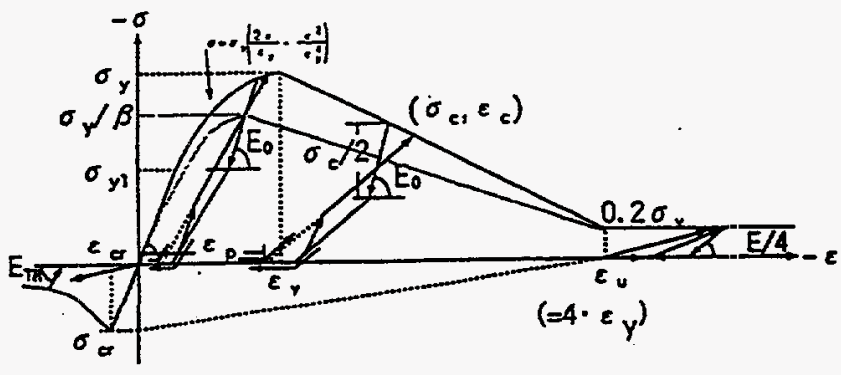

(b) $\mathbf{P 1 3}$

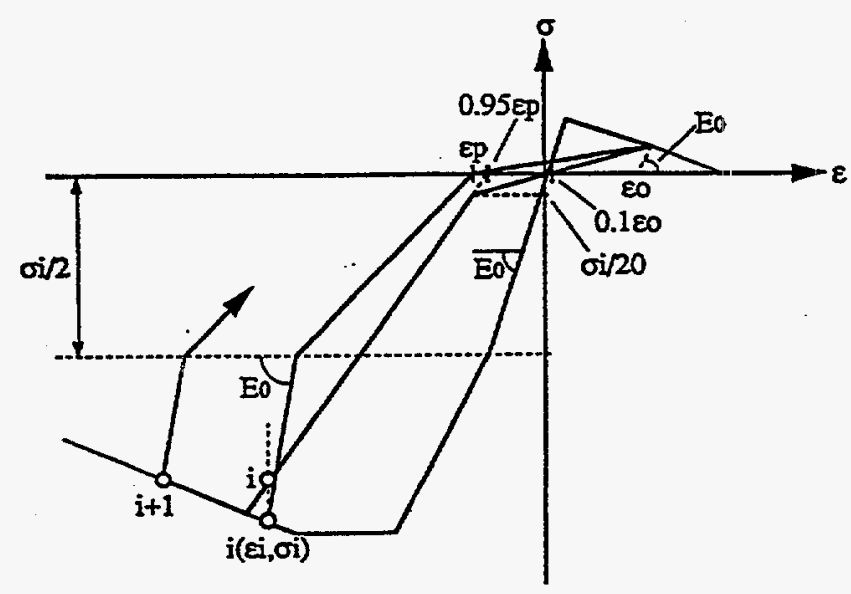

(d) $\mathbf{P 1 7}$

Fig. 5.5 Uniaxial Hysteretic Models for Concrete for FEM Dynamic Analyses (Ref. 2) 


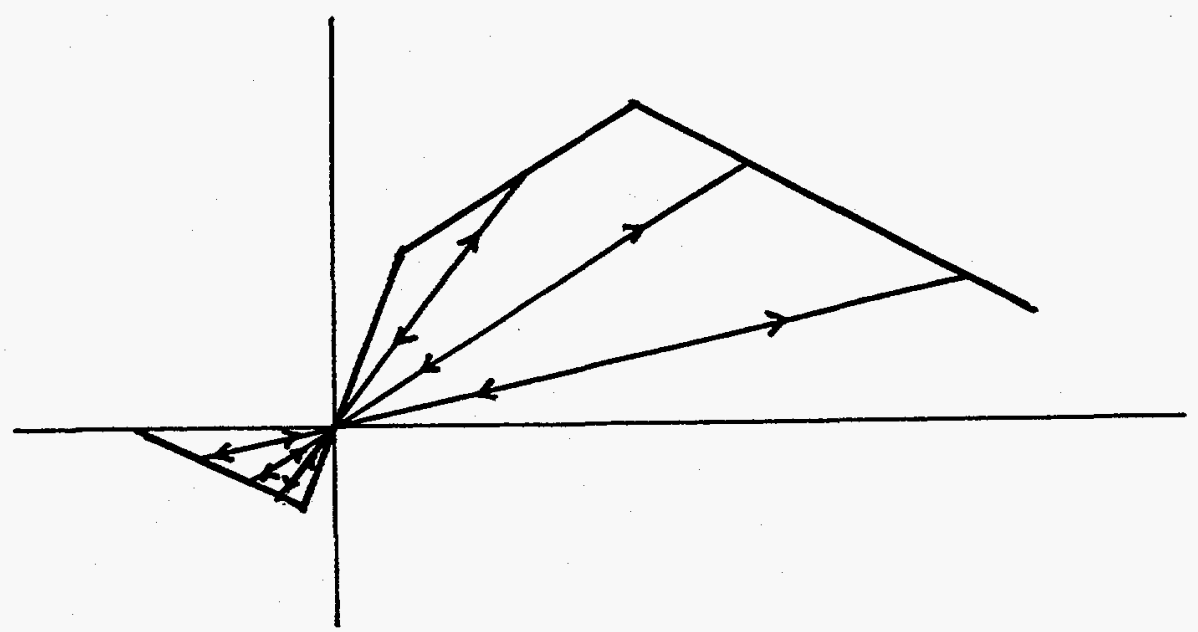

Fig. 5.6 Typical Example of Simplified Hysteretic Model for Concrete 


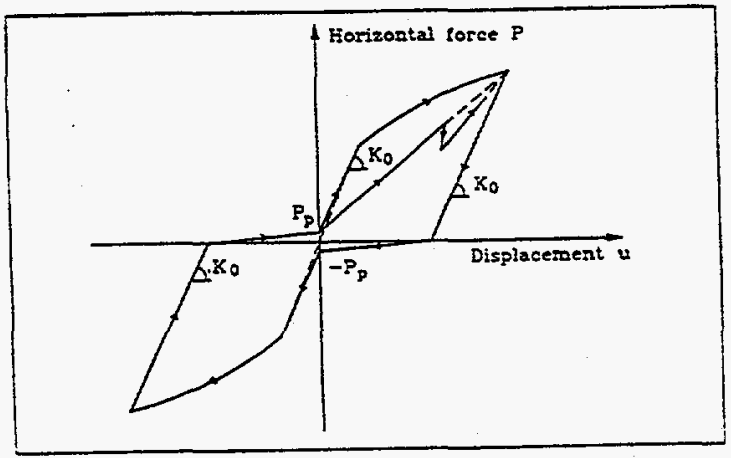

P2

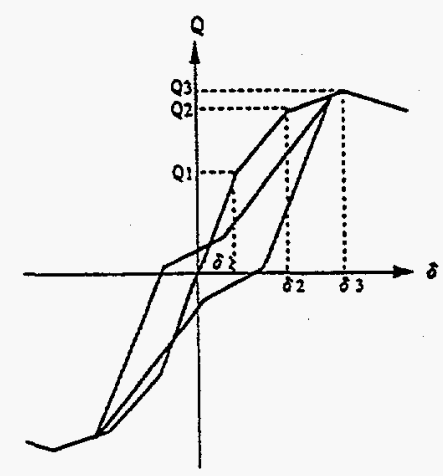

P11

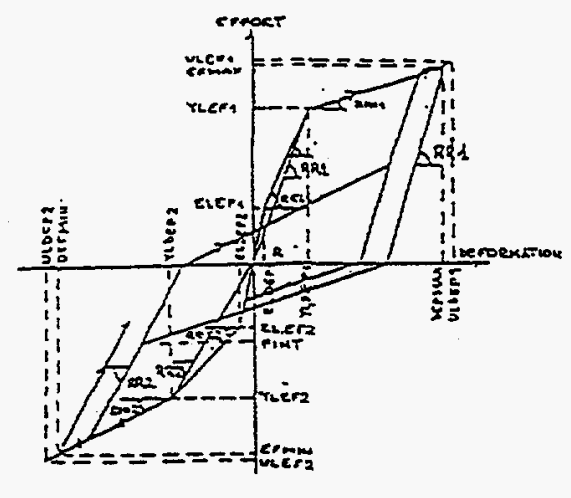

P4

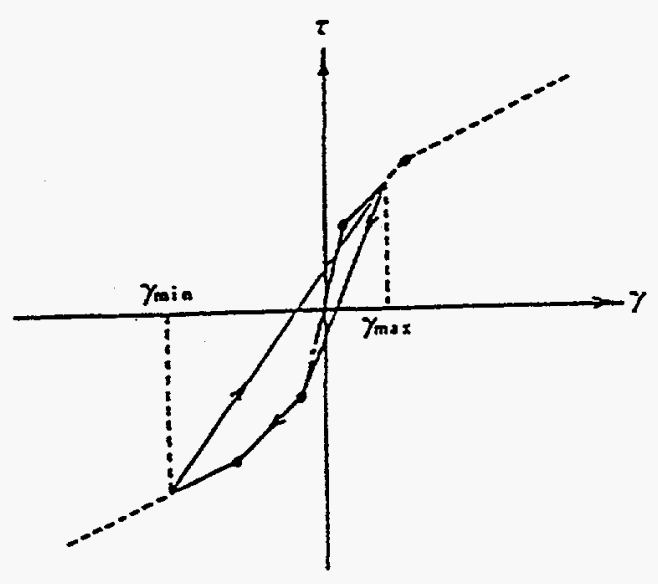

P16

Fig. 5.7 Hysteretic Models Used for Lumped Mass Models (When Both Shear and Bending Models Are Used in Analyses, Only Shear Model Is Shown) (Ref. 2) 


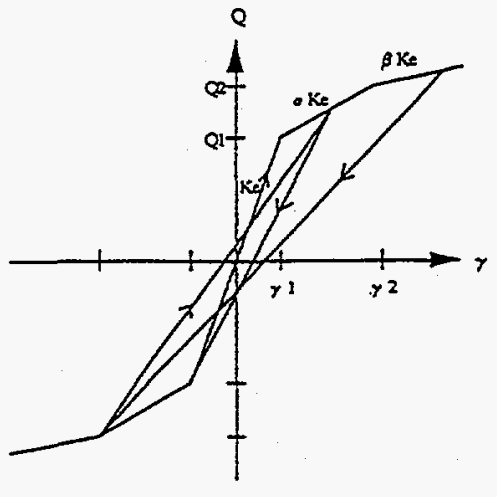

P20

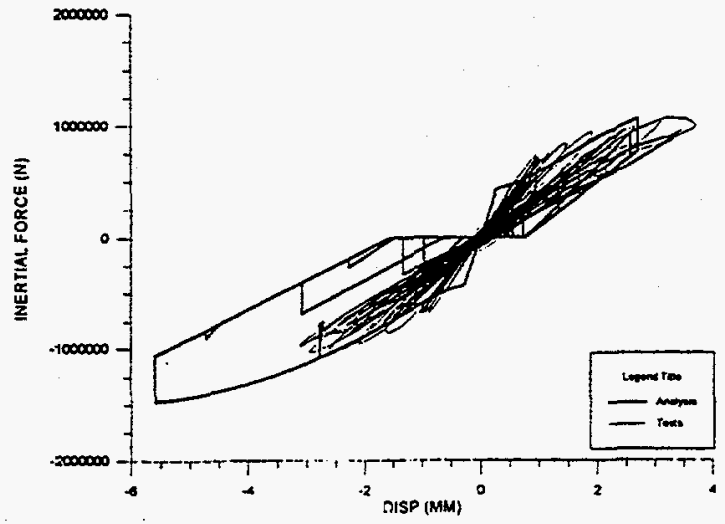

P27

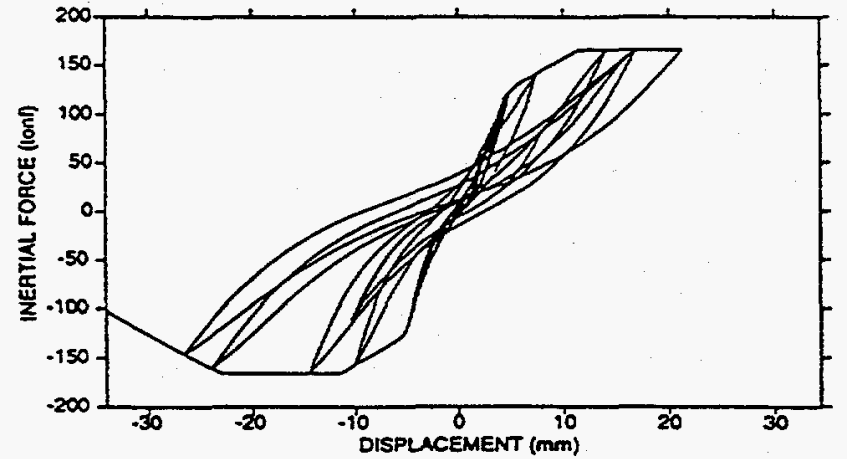

P32

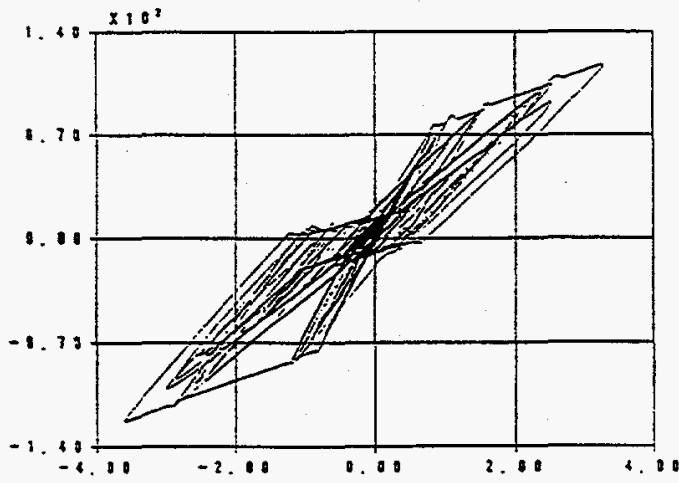

P37

Fig. 5.7 Hysteretic Models Used for Lumped Mass Models (When Both Shear and

Bending Models Are Used in Analyses, Only Shear Model Is Shown) (Ref. 2) (continued) 


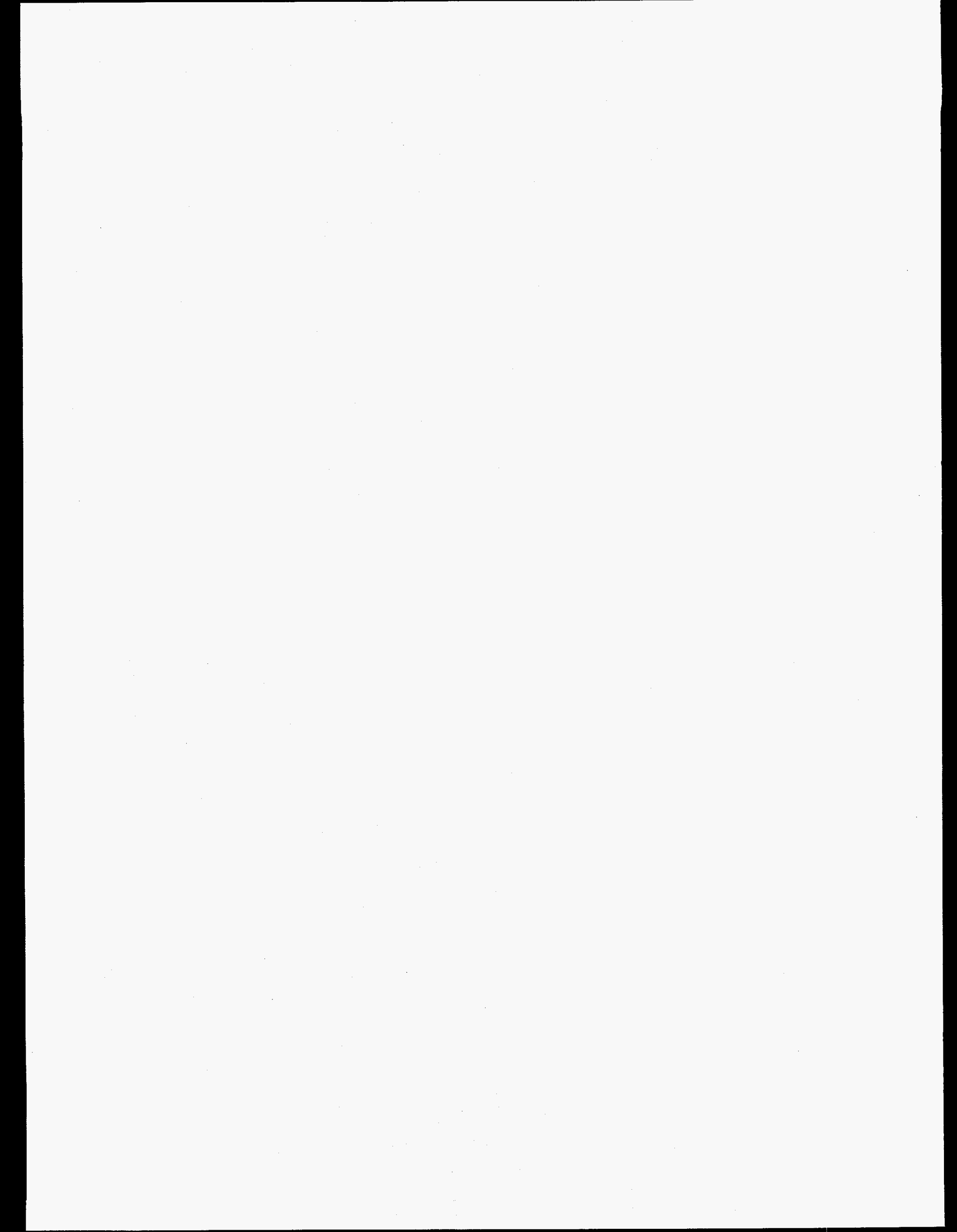




\section{CONCLUSIONS}

In the nuclear industry, reinforced concrete (RC) structures are typically designed using linear elastic structural analysis methods and empirical design formulations. Nonlinear analyses of any kind are rarely used in the seismic design of RC structures. One exception may be in the seismic fragility analysis of structures and components, where critical RC structures, such as turbine buildings, may be evaluated using nonlinear time history methods (e.g., Ref. 16). For such an occasion, engineers tend to use a very simplified approach, modeling highly complex RC structures with a few well-defined nonlinear elements. This may be due to the fact that more sophisticated analysis methods, such as those described in the foregoing chapters, are generally perceived to be less reliable, and also most engineers do not have easy access to such analysis tools.

Presently, the structural design methods of reinforced concrete structures used in the nuclear industry have been developed and evaluated based on a set of laboratory test data. Efforts have been on-going for the last two decades to develop powerful analysis tools which could eliminate or significantly reduce the need for additional costly laboratory testing as new issues arise. The SSWISP has provided valuable test data to evaluate such analysis tools, as well as to develop new analysis approaches.

The specific objectives of this study were to perform nonlinear dynamic analyses of $\mathrm{RC}$ shear wall structures under severe earthquake loading, to identify limitations of the currently available analysis methods, and to collect information on the analysis methods worldwide. These objectives have been achieved through a series of analyses using both in-house and commercial codes, and comparisons with test resuits as well as with analysis results performed by other SSWISP workshop participants. The SSWISP workshop provided a unique opportunity to review the reliability and applicability of various analysis methods to predict the dynamic behavior of shear wall structures under severe earthquake loads. Based on the correlation and comparison studies presented in the previous chapters, the following conclusions may be drawn:

\section{Commercial FEM Codes}

It seems the applicability and reliability of commercially available FEM codes to RC shear wall structures are limited. This may be due to the fact that most of these codes were developed in the 1970's, and therefore, do not reflect the latest understanding of concrete mechanics, such as compressive strength reduction and shear transfer in cracked concrete. Several participants attempted to improve the prediction accuracies by introducing original material models through user subroutines. For some of these cases, the errors in prediction for push-over type static analyses were significantly reduced However, for nonlinear dynamic analysis, the commercial codes were considered to be only partially successful at best.

\section{FEM Dynamic Analysis}

In a sense this is the "simplest" analysis approach since many of the engineering judgments and analysis assumptions, necessary to allow modeling both structural systems and seismic loads in simplified approaches, can be eliminated. Several participants presented remarkable analysis results using originally developed in-house codes, which were considered to be in various stages of development. At the moment of this writing, the applicability and reliability of these codes to full-size RC structures are still seriously limited. However, the quality and computational power of these codes seems to be improving rapidly in the last few years. It was suggested by several participants at the workshop that, in the near future, the FEM may be utilized to visualize the complex damage process of RC structures in severe earthquake events; a far cry from the current engineering analysis practice. 


\section{FEM Static Analysis}

Three participants, P11, P17 and P34, presented results based on three alternate analysis methods, i.e., monotonic static, cyclic static and dynamic analyses. With these, there are no significant differences in the predicted envelope curves, hysteresis loops (between dynamic and cyclic static), and the predicted failure modes. It should be noted that all the goodquality monotonic static analyses were performed using the displacement-control procedure, which clearly predicts the location of the maximum strength point and traces post-peak behavior. Some of the analysis methods presented during the workshop may be considered to be reliable analysis tools, although additional correlation studies using available test data may be still needed to confirm their applicability in unique design conditions. The results of cyclic static analyses are particularly useful in seismic design as the energy absorbing characteristics (i.e., equivalent damping values) can be evaluated.

\section{Simplified Modeling Approach}

Two types of simplified modeling approaches were presented during the workshop, i.e., truss element model and beam-type element model. For application to typical shear wall structures in nuclear power plants, e.g., reactor buildings, the truss model is considered to have a greater potential. It was observed that the assignment of the properties of each truss component, particularly those of diagonal struts, is judgmental, and may require additional studies.

\section{Lumped Mass Modeling Approach}

In a typical simplified nonlinear dynamic analysis of a building, the weights are lumped at each floor level and the norlinear behavior is represented by the nonlinear springs for the story shear and story drift relationship. Based on several analysis results developed using such modeling schemes, it was observed that the bending and shear deformations should be separated and modeled with separate springs with different hysteretic characteristics. Also, the specification of the envelope curves and the selection of hysteretic models were considered to be key factors for successfully reproducing the nonlinear dynamic response. 


\section{REFERENCES}

1. NUPEC, "Specification Report of Seismic Shear Wall ISP on NUPEC's Seismic Ultimate Dynamic Response Test," NU-SSWISP-D008, NUPEC, Aug. 1994.

2. NUPEC, “Comparison Report, Seismic Shear Wall ISP, NUPEC's Seismic Ultimate Dynamic Response Test," NUSSWISP-D014, NUPEC, March 1996.

3. Y.J. Park, C.H. Hofmayer and N.C. Chokshi, "Finite Element Analyses for Seismic Shear Wall International Standard Problem," Brookhaven National Laboratory, Sept. 1995, Nov. 1995 (Rev. 1).

4. Y.J. Park, "Additional Dynamic Analysis for RUN-5," Informal Report, Brookhaven National Laboratory, February 1996.

5. Y.J. Park, "ISSAC User's Manual," Informal Report, Brookhaven National Laboratory, February 1995.

6. D. Darwin and D.A.W. Pecknold, "Inelastic Model for Cyclic Biaxial Loading of Reinforced Concrete," University of Illinois at Urbana-Champaign, SRS No. 409, Urbana, Illinois, 1974.

7. F.J. Vecchio and M.P. Collins, "The Modified Compression Field Theory for Reinforced Concrete Elements Subjected to Shear," ACI Journal, Vol. 83, No. 2, pp 219-231, 1986.

8. N.J. Stevens, S.M. Uzumeri and M.P. Collins, "Analytical Modeling of Reinforced Concrete Subjected to Monotonic and Reversed Loadings," Publication No. 87-1, Department of Civil Engineering, University of Toronto, Jan. 1987.

9. H. Kupfer, HK. Hilsdorf and H. Rusch, "Behavior of Concrete Under Biaxial Stresses," ACI Journal, V. 66, No. 8, pp. 656-666, Aug. 1969.

10. F.J. Vecchio and M.P. Collins, "Compression Response of Cracked Reinforced Concrete," Journal of Structural Engineering, ASCE, Vol. 119, No. 12, pp. 3590-3610, December 1993.

11. ANSYS User's Manual for Revision 5.1, Swanson Analysis Systems, Inc.

12. S. Hamada, M. Ohkubo and H. Noguchi, "Basic Experiments on the Degradation of Cracked Concrete under Biaxial Compression and Tension," Annual Conference of AIJ, pp. 919-920, Oct. 1989.

13. Y. Aoyagi and K. Yamada, "Strength and Deformation Characteristics of Reinforced Concrete Shell Elements Subjected to In-plane Forces," Transactions of JSCE, Japan Society of Civil Engineers, Vol. 331, pp. 167-180, March 1983.

14. B. Li, K. Maekawa, and H. Okamura, "Contact Density Model for Stress Transfer Across Cracks in Concrete," Journal of the Faculty of Engineering, The University of Tokyo, Vol. XXX, No. 1, pp. 9-52, 1989.

15. Young J. Park, "Survey on the Experimental Studies and Design Practice of Reinforced Concrete Shear Wall Structures in Japan," Technical Report, A-3301-2/95, Brookhaven National Laboratory, Feb. 1995.

16. NTS Engineering, "Seismic Fragilities of Civil Structures and Equipment Components at the Diablo Canyon Power Plant," Report No. 164302, Prepared for PG\&E Company, September 1988. 


\title{
APPENDIX $A$
}

\section{STATIC FINITE ELEMENT ANALYSIS \\ OF SEISMIC SHEAR WALL ISP}

\author{
By \\ F.J. Vecchio \\ Department of Civil Engineering \\ University of Toronto \\ Performed for \\ Brookhaven National Laboratory \\ Under the Sponsorship of the \\ U.S. Nuclear Regulatory Commission
}

\author{
September 1995 \\ and \\ November 1995 Addendum
}




\title{
STATIC FINITE ELEMENT ANALYSIS \\ OF SEISMIC SHEAR WALL ISP
}

\author{
F.J. Vecchio \\ Department of Civil Engineering \\ University of Toronto
}

September 25, 1995

\section{INTRODUCTION}

In a study of the seismic design of shear walls in nuclear reactor buildings, the Nuclear Power Engineering Corporation of Japan (NUPEC) recently conducted an extensive experimental investigation. Two large-scale flanged shear walls (designated as ISP series) were subjected to dynamic loading conditions using a high performance shaking table. The results of the tests were made available to participants of the Seismic Shear Wall International Standard Problem (SSWISP) Workshop, so that the data might be used for verification of seismic response analysis codes.

At the request of the Brookhaven National Laboratory, a three-dimensional nonlinear static finite element analysis of the test specimen was undertaken. The shear wall was analyzed using the conceptual models, constitutive relations, and analysis software developed in-house at the University of Toronto. Previous application of these analysis tools to monotonically loaded shear walls were found to yield reasonably accurate results (Vecchio, 1992).

This report summarizes the analytical formulations, finite element models, analysis results, interpretation of results, and relevant discussion pertaining to the ISP shear wall analyses. A parametric study of the factors influencing the behaviour of the test structure, using two-dimensional finite element analyses, is also presented.

\section{CONSTITUTTVE MODELS}

\subsection{CONCRETE:}

Concrete is modelled as an orthotropic nonlinear elastic material according to the Modified Compression Field Theory (Vecchio and Collins, 1986). A complete description of the model is provided in Appendix I. A condensed description follows. 
The constitutive equations for multiaxial stress states are based on modifications to a uniaxial stress-strain base curve. For the analysis discussed herein, the compressive stress-strain diagram used for the base curve is the Hognestad parabola, shown in Figure 1. It provides a simple and reasonably accurate representation for normal strength concretes $(<30 \mathrm{MPa})$. The strength enhancement arising from confining stresses is modelled by modifying the peak: stress, and the strain at peak stress, of the parabolic base curve. The yield envelope proposed by Hseih et al (1979) is used to determine the modifying peak stress factor. The modifying peak strain factor is determined from a fit to the data of Kupfer et al (1969) for low peak stresses, and from a relationship according to Richart et al (1928) for higher peak stress ratios. The negative gradient of the descending branch of the stress strain curve is based on the Modified Kent-Park model (Scott et al 1982).

In tension-compression stress states, a compression reduction factor (or compression softening factor) is applied to the strength of the concrete after cracking. The compression reduction factor used is that proposed by Vecchio and Collins (1992). Note that according to the Vecchio/Collins model, both the peak stress and peak strain of the base curve are modified. The reduction factor is a variable primarily dependent on the magnitudes of the strains transverse to the compression direction (ie, by the degree of cracking).

The crack model used in the Modified Compression Field Theory (MCFT), on which the analyses are based, assumes smeared rotating cracks. Cracking is assumed to occur normal to a principal stress direction, when that stress exceeds the uniaxial cracking strength of the concrete. While the constitutive relationships are formulated in terms of average stresses and average strains, local stresses at crack locations are also considered. The presence of average tensile stresses in the concrete implies local stress increases in the reinforcement at crack locations, and the presence of shear stresses on the concrete crack surfaces. Thus, the crack criteria includes a check that the local stresses in the reinforcement can be tolerated, and that the shear stresses on the crack surfaces are below a limiting value (Vecchio and Collins, 1986). Otherwise, a shear slip can occur along the crack surfaces. The model is hypoelastic norlinear; no attempt is made to reflect load/strain history in determining crack opening and closing. Cracks are assumed to 'heal' if the strains fall below the cracking strain.

The common approach of using a shear retention factor in modelling the shear stiffness of cracked concrete is not employed. Rather, in the manner of a true orthotropic elastic material, the shear stiffnesses are defined by the moduli of elasticity in the three principal directions, and by the associated Poisson's ratios. The Poisson's ratios are variable, modelled according to the data of Kupfer (1969), and are not necessarily set to zero after cracking.

Due to the influence of bond, post-cracking average tensile stresses can develop in the concrete between cracks. This so-called tension stiffening effect is modelled by adding a descending branch to the constitutive law for concrete in tension. The model proposed by Izumo et al (1992) is used for the three-dimensional analysis presented herein. 


\subsection{REINFORCEMENT:}

Average stresses in the reinforcement are related to average strains using the standard elastic-plastic stress-strain relationship. A tri-linear curve is used to model strain hardening, although strain hardening was not relevant to the analyses herein. The MCFT currently does not model shear stiffness due to dowel action.

\subsection{BOND AND SLIP:}

The MCFT, currently formulated for monotonically increasing quasi-static load conditions, assumes perfect bond to exist between the concrete and the reinforcement. Hence, no explicit bond-slip model is used, and no slip of embedded rebar is assumed to occur in a standard analysis. However, tests indicate that the degradation in the bond between concrete and reinforcement, prevalent in dynamic and reversed cyclic loading conditions, has the effect of diminishing the development of post-cracking tensile stresses in the concrete. Thus, the effects of bond-slip can be approximated to some extent by discounting the tension stiffening effect; that is, by assuming no post-cracking tensile stresses in the concrete.

\section{FINITE ELEMENT ANALYSIS PROCEDURE}

\subsection{THREE-DIMENSIONAL ANALYSES:}

Analyses were undertaken using program SPARCS, a three-dimensional nonlinear finite element program developed at the University of Toronto (Vecchio and Selby, 1991). SPARCS incorporates the constitutive relationships and conceptual models of the Modified Compression Field Theory (Vecchio and Collins, 1986).

SPARCS employs a total load, secant stiffness approach in the formulation of its nonlinear analysis algorithm. An analysis usually begins by assuming linear elastic isotropic material properties. Element stiffness matrices are calculated and then the global stiffness is assembled. The load vector, which includes prestrain and expansion effects if any, is then formed. Nodal displacements are determined, from which one strain tensor is calculated for each element. Principal strains and corresponding directions are then found. Evaluation of the concrete and steel stresses using the constitutive models permits determination of secant moduli and, in turn, new material stiffness matrices. Average secant moduli are calculated; if they have converged to the specified limit, then the load stage is complete. Otherwise, the newly calculated material stiffness values are used to perform another linear elastic analysis. Normally, satisfactory convergence is achieved within 10 to 25 iterations. The control procedure in developing a load-deformation response history for a structure is by load control, with increments of load applied in each load stage.

SPARCS employs an 8-noded ( 24 degree of freedom) brick element which assumes linear displacement fields. As well, a 6-noded wedge element and a truss-bar element are available. 
Reinforcement is typically modelled as smeared within the elements, although it can be discretely represented using the truss-bar elements.

\subsection{TWO-DIMENSIONAL ANALYSES:}

The equivalent two-dimensional nonlinear finite element program is TRIX (Vecchio, 1989). It incorporates essentially the same material models and analytical formulations as SPARCS.

TRIX was used for the 2-D parametric studies described later.

\section{MODELLING OF TEST SPECIMEN}

Taking advantage of symmetry, one-half of the structure was modelled using the element mesh shown in Figure 2. A total of 1090 8-noded brick elements were used, requiring 1932 nodes and 5796 degrees of freedom. However, it should be noted that the large number of elements was a consequence of using an automatic mesh generating facility available, resulting in a disproportionate and unnecessary number of elements in the top slab and bottom slab regions. The top slab consumed 380 elements; the base slab required a similar amount; each flange consumed 90 elements, and the web was modelled with 150 elements. A significantly more efficient analysis, with no deterioration in accuracy, could be achieved through a coarser discretization of the top and bottom slabs. The element numbering scheme for the web portion of the structure is given in Figure 3.

The structure was assumed fully restrained at all nodes along the bottom surface of the base slab. The total vertical load, having a constant value of $600 \mathrm{kN}$, was uniformly distributed among the interior nodes at the mid-depth of the top slab. Similarly, the lateral loads were applied as uniformly distributed along the mid-depth of the top slab.

The concrete material properties used were as provided in the NUPEC Report of September 1994. The concrete in the web/flange regions was modelled as having a compressive strength of $28.6 \mathrm{MPa}$, and a tensile strength of $1.77 \mathrm{MPa}$. The strain at peak stress, taken from the cylinder curves provided, was 0.0025 . The initial modulus of elasticity and the Poisson's ratio were $22,900 \mathrm{MPa}$ and 0.155 , respectively.

The reinforcement ratios in the web were $1.219 \%$ in both the horizontal and vertical directions. The reinforcement in the flange regions varied with position. At the intersection of the web and flange (a $75 \times 100 \mathrm{~mm}$ area), the vertical reinforcement ratio was $1.707 \%$ (ie 4 rebars). For a distance of $250 \mathrm{~mm}$ out from the flange/web corner, the vertical reinforcement ratio was $0.760 \%$. Beyond that point, to the outer tips, the vertical reinforcement ratio used was $0.366 \%$. The horizontal reinforcement in the flanges was set at $1.371 \%$ to a distance $250 \mathrm{~mm}$ out from the web/flange corner, and at $0.914 \%$ in the areas beyond. The top and base slabs were nominally reinforced in all three directions. 
The reinforcement was modelled as elastic-plastic, with a yield stress of $384 \mathrm{MPa}$ and a modulus of elasticity of $184,400 \mathrm{MPa}$.

Lateral load was applied in increments of $98.1 \mathrm{kN}$ (10 tonf) per load stage, up to $981 \mathrm{kN}$. Beyond that point, the load step size was reduced to $49.0 \mathrm{kN}$ (5 tonf). A tight convergence criteria was selected, requiring up to 30 iterations at each load stage. Using a MIPS Challenge M R4400/150 workstation, each load stage required up to $175 \mathrm{~min}$ CPU time, and approximately 8 hours real time to execute. Determination of maximum load was made from the flattening deformation response curves (e.g. displacements, strains in the reinforcement, shear strains in the concrete). At ultimate load, strains and displacements increased to extreme values (eg horizontal displacement of the top slab in excess of $200 \mathrm{~mm}$ ), and was accompanied by an inability to converge to stable values. Execution of the program required a disk storage memory size of approximately $120 \mathrm{MB}$.

\section{ANALYSIS RESULTS}

\subsection{RUN 1 - TENSION STIFFENING CONSIDERED:}

The initial analysis of the ISP shear wall was done considering the full effects of tension stiffening, as would be appropriate under monotonically increasing load conditions. Also, it should be emphasized that all the modelling decisions were made and all the analysis parameters were set before this initial (and only) analysis was executed. No 'fine-tuning' of the analysis was done subsequently in an attempt to obtain a better fit to the experimental results.

Under a monotonic lateral load applied at the mid-depth of the top slab, the shear wall was predicted to experience a brittle flexural/shear failure near the base. The predicted maximum strength of the wall was $1815 \mathrm{kN}$ (185 tonf). The mode of failure, represented in Figure 4, involved a shear/crushing failure of the concrete web leading to a shear failure plane forming approximately 300 to $500 \mathrm{~mm}$ above the base. Crushing of the concrete was also prevalent in the web regions adjoining the right flange. The web failure was partially initiated by the prior yielding of the left flange. Yielding of the flange resulted in a deterioration of the clamping force, and thus a deterioration in the confinement effects, from which the web had benefitted.

The relatively brittle response of the wall is reflected in the load-deformation response curves. Shown in Figure 5 is the horizontal displacement of the top slab (at its mid-depth). Figures 6 and 7 provide the vertical relative displacements of the left and right flanges, respectively. Figure 8 documents the predicted locations of first cracking, first yielding and first crushing.

The first cracking sustained by the structure was predicted to be shear cracking in the web, in the lower left region centered around element 457 (see Figure 3), at a load of $490 \mathrm{kN}$. By a load of $590 \mathrm{kN}$, the shear cracking had propagated essentially across the full width and full height of the web. At a load of $785 \mathrm{kN}$, the first bending crack developed in the left flange, at 
the web-flange junction, just above the base (element 381). As loading increased, the flexural crack zone propagated outward and upward. At $1175 \mathrm{kN}$ load, the horizontal flexural cracks had extended across the full width of the flange; the crack zone extended approximately one-third of the way up the flange. With increased loading, the crack zone continued to expand up the flange. Figures 9 (a) and 9 (b) provide the crack patterns at $67 \%$ capacity $(1220 \mathrm{kN})$ and $100 \%$ capacity $(1815 \mathrm{kN})$, respectively. It should be noted that the right flange, and the top and bottom slabs, remained essentially uncracked.

The flange wall vertical rebar was the first reinforcement to yield, occurring at a load of $1420 \mathrm{kN}$. Interestingly, however, the location of yielding was towards the outer tip of the flange, just above the base (elements 387-389). As load increased, the yielding zone gradually moved in toward the web. Yielding across the full width of the flange did not occur until a load of $1570 \mathrm{kN}$. The zone of yielding also propagated up the flange such that by ultimate load, yielding was widespread in the left flange. Figure 10 shows the straining of the vertical reinforcement in the flange, at the web junction and just above the base (ie element 381). As ultimate load was approached, rapid and uncontrolled yielding of the reinforcement was observed to occur. This ultimately contributed to the failure of the structure.

Yielding of the vertical reinforcement eventually migrated into the web zone. First yielding of the web wall vertical rebar occurred at a load of $1665 \mathrm{kN}$, in the bottom left corner area (elements 390,391, 423). As load increased, the yield zone extended inward along the base. Failure of the wall ensued shortly after.

The web wall horizontal rebar was not highly stressed and did not yield. The greatest demand on the horizontal reinforcement was in the area adjacent to the right flange, about $700 \mathrm{~mm}$ above the base (elements 500 to 503). Here, the horizontal reinforcement acted together with the right flange to contain the outward thrust developed in the concrete compression strut that formed. Just prior to failure, the average stress in the horizontal reinforcement was about $67 \%$ of the yield stress. At crack locations, the maximum local stress in the horizontal reinforcement was approximately $75 \%$ of the yield stress.

The concrete in the web zone was highly stressed. The internal load-carrying mechanism that prevailed can be visualized as a simple strut-and-tie system, with the left flange representing a tension tie, the top and bottom slabs representing stiff chords, and a diagonal compression strut forming in the web from the top left corner to the bottom right corner. While the most highly stressed zone was that in the vicinity of the compression toe (elements 436,469 ), the web was nearly uniformly stressed throughout, as it would be in the case of pure shear. It should also be noted that the zones immediately adjacent to the top and bottom slabs experienced less distress because of the out-of-plane confinement provided by the massive slabs. First crushing occurred in a zone about $400 \mathrm{~mm}$ above the base and $300 \mathrm{~mm}$ in from the right flange (elements 436 , $437,469)$, at a load of $1620 \mathrm{kN}$. The zone of crushing quickly expanded inward and upward. At $1720 \mathrm{kN}$, the concrete was extensively damaged in a large area spanning the lower right quadrant of the web. The concrete at about 300 to $500 \mathrm{~mm}$ up from the base (elements 434 to 437,464 to 469 ) was in post-peak response, indicating the formation of a potential failure plane. 
The shear strain response of the concrete web, at element 437, is shown in Figure 11. Large shear strains were apparent as the ultimate load was approached, indicating that a concrete shear failure was imminent.

The concrete in the compression flange was not highly stressed. At failure, the stress at the base of the flange was about $50 \%$ of the crushing strength, dropping rapidly to less than $10 \%$ of the crushing strength at distances $500 \mathrm{~mm}$ and greater above the base.

The analysis procedure assumed perfect bond between concrete and reinforcement. Hence, no predictions could be made of the vertical slip of rebar at the base of the flanges.

\subsection{RUN 2 - TENSION STIFFENING IGNORED:}

The analysis was repeated with all parameters unchanged except that no tension stiffening effects were considered in cracked concrete. For concrete structures subjected to longterm or repeated loads, it has been suggested that post-cracking tensile stresses in concrete be reduced or ignored (Collins and Mitchell, 1991).

The consequences of ignoring tension stiffening effects were minor. The ultimate load capacity of the wall was only slightly reduced, by $3 \%$ to $1765 \mathrm{kN}$ (180 tonf), and the failure mode was essentially unchanged. At the intermediate and later load stages, the wall did exhibited more flexibility, thus better representing the deflection responses observed in the test specimens. The horizontal deflections of the top slab, obtained from the two analyses, are compared in Figure 5. The vertical relative defiections of the flanges are given in Figures 6 and .7; the strains in the vertical reinforcement at the base of the left flange are shown in Figure 10; and the shear strains in the web near the bottom right corner (element 437 ) are compared in Figure 11. The absence of tension stiffening resulted in a loss of stiffness of approximately 10 percent beginning at about $1275 \mathrm{kN}$ load (approximately $70 \%$ of ultimate).

The sequence and locations of cracking, yielding, and crushing, and the loads at which they occurred, were also essentially unchanged. The only significant deviations from the behaviour chronicled previously were that: i) yielding of the vertical reinforcement in the left flange, at the base near the tip, occurred at an earlier load; $1275 \mathrm{kN}$ versus $1420 \mathrm{kN}$ with tension stiffening considered; ii) cracking of the left flange, in the tip regions, was more concentrated towards the base; and iii) the horizontal reinforcement in the web showed slightly higher stress. The absence of tension stiffening impaired the formation of a well-distributed crack pattern in the flange regions away from the web, concentrating the deformations near the base. In the web, the absence of post-cracking tensile stresses in the concrete placed a slightly higher demand on the reinforcement.

\subsection{DISCUSSION:}

It must be emphasized that the analyses discussed simulate behaviour under the supposition of static monotonically increasing load. That the specimens were tested under 
dynamic cyclically-reversing loads raises some important factors that must be considered in trying to understand and correlate the predicted and observed behaviours.

Firstly, it is observed that the experimentally measured load-deformation responses are significantly less stiff than those predicted. The loss of tension stiffening effects under reversed cyclic load conditions has already been suggested as one possible reason for the discrepancy, accounting for a portion of the difference as evidenced by the results of the two analyses. However, a probably more significant factor is the degradation in the bond and anchorage of the reinforcement, particularly at the base. This behaviour is seen with cyclic loading conditions only, and would not have manifested itself had the wall been subjected to monotonic loads. The analyses conducted made no allowances for bond and anchorage slip at the base.

The analyses showed that the vertical reinforcement yielded across the full width of the left flange at approximately $1570 \mathrm{kN}$, and that yielding of the vertical reinforcement in the lower left region of the web commenced shortly after at about $1620 \mathrm{kN}$. If the loads were then reversed, the vertical reinforcement in the right flange and right lower regions of the web could be expected to yield at similar load levels. However, the reinforcement on the left side, having undergone plastic yielding previously, would have retained some permanent offset strain. Through each cycle of loading, the offset strains resulting from yielding would tend to grow; this phenomenon is well-known and commonly referred to as the 'ratcheting effect'. The consequence is two-fold: i) a concentrated zone of damage is created near the base, in the yield regions, leading to a potential shear failure plane being established; ii) the increased strains result in a diminished confinement of the web, and an increased influence from compression softening, lowering the concrete web's ability to resist load. Thus, in situations where conditions lend themselves to this ratcheting effect, the reversed loading can result in a lowering of the ultimate load capacity and a change in the failure mode. It is interesting to note that the analyses predicted yielding of the vertical reinforcement in the flange and web at about the same load at which failure was observed in the test specimens. This would be consistent with observations from other experimental investigations; most notably with beam-column joints where this ratcheting behaviour leading to a shear-failure plane at the joint interface is well known. Thus, that the static analysis predicts a somewhat higher failure load (by approximately $12 \%$ ), and a failure mode more in the nature of a shear-crushing of the web, is not unexpected. Further, one might speculate that a similar wall specimen tested under static loads would exhibit, to some degree, these variances relative to the dynamically tested specimens.

\section{PARAMETRIC STUDY}

\subsection{OVERVIEW OF STUDY:}

A parametric study was undertaken in an effort to more fully understand the behaviour of the test specimens, the factors influencing behaviour, and the factors influencing the aralysis results. Due to the heavy demands on resources imposed by the three-dimensional models previously discussed, a two-dimensional model was used for the study. The finite element mesh 
used is illustrated in Figure 12; note that the exact same number and arrangement of elements was used in modelling the web portion of the wall as was used with the three-dimensional model. The flange portions, of course, were not modelled out-of-plane and thus were represented by a single element of appropriate thickness. The material models and analysis parameters were as specified with the three-dimensional model. Analyses were performed using program TRIX, as previously described. It is interesting to note that the TRIX analyses required approximately $1 / 50$ th the CPU time as a comparable three-dimensional analysis.

\subsection{INFLUENCE OF THREE-DIMENSIONAL EFFECTS:}

A two-dimensional analysis of the wall indicated a behaviour somewhat similar to that obtained from the three-dimensional analysis. The predicted lateral deflections of the top slab, from the two analyses, are compared in Figure 13. The predicted failure mode from the 2-D analysis is shown in Figure 14. The following observations can be made:

i) the 2-D analysis predicts an ultimate load capacity of $1960 \mathrm{kN}$, approximately $8 \%$ higher than that predicted by the 3-D analysis;

ii) the 2-D analysis predicts a substantially stiffer response; and

iii) the failure mode in the 2-D analysis involves a sliding shear failure of the concrete web at an elevation of about $300 \mathrm{~mm}$ above the base.

Concentrating the full width of the flanges into a single element in the two-dimensional model has several significant implications. The thick, and thus very stiff, flange elements are assumed fully connected to the web elements. Thus, the degree of lateral and vertical confinement they provide to the web is over-estimated. Further, the shear lag effect that occurs in the out-of-plane direction in the three-dimensional model, and in reality, is not considered. Finally, the ability of the flange elements to carry a lateral shear is over-stated when full fixity to the web is assumed. These factors contribute to over-estimating both the strength and stiffness of the wall, relative to the three-dimensional analysis.

Not considering out-of-plane effects negates the triaxial confinement that occurs in the web regions adjoining the base slab. The ensuing strength enhancement in these regions is thus not fully taken into account. However, the effect quickly dissipates as one moves away from the base. As a result, the failure plane is unchanged relative to the results of the three-dimensional analyses.

\subsection{INFLUENCE OF EFFECTIVE FLANGE WIDTH:}

In developing a two-dimensional model of the test structure, the question arises as to how much of the flange width is effective in contributing to the lateral load resistance of the wall. Four series of analysis were run, with the effective flange width (hence element thickness) modelled variously as $100 \%(2980 \mathrm{~mm}), 67 \%(2012 \mathrm{~mm}), 33 \%(1043 \mathrm{~mm})$ and $0 \%(75 \mathrm{~mm})$ 
effective. The corresponding lateral deflection responses at the top slab level are compared in Figure 15.

It can be seen that the assumption of a reduced effective flange width has a pronounced effect on the predicted response. Strength and stiffness are greatly diminished with each successive reduction in width. When the flange is considered totally ineffective, for example, wall strength is reduced by $55 \%$. Also significant is the change in predicted failure mode. With a decreasing flange width, the failure mode becomes more flexural in nature. Increasing tensile strains and pronounced yielding are observed in the left flange at earlier loads, and eventually capacity is governed by a concrete crushing failure in the right flange.

In comparing the results of these analyses to those of the three-dimensional analysis, and to the test results, the indication is the flanges are nearly fully effective in contributing to the response of the wall in some manner. In a 2-D analysis of this wall, an effective flange width of somewhere between $67 \%$ and $100 \%$ seems most appropriate. As described above, however, considering the flanges $100 \%$ effective results in an over-estimation of strength and stiffness.

\subsection{INFLUENCE OF TENSION STIFFENING MODEL:}

The assumption of post-cracking tensile stresses in the concrete has a measurable impact on the computed stiffness of the structure and, to a lesser extent, on the computed strength. This was observed in the results of the two series of three-dimensional analyses previously described. To get a firmer understanding of the significance of this mechanism, and to test the sensitivity of the analysis results to the type of tension stiffening model used, four analysis series were undertaken. The analyses differed in the tension stiffening models used, employing the following: i) Izumo et al model, as was used in the 3-D analyses; experience has shown this model to provide reasonable results in flexure-dominated conditions; ii) Vecchio-Collins model, which seems to provide the most accurate simulations in membrane stress conditions but tends to overestimate stiffness under flexural conditions; iii) Collins-Mitchell model, which serves as a good general purpose model and provides reduction factors for longterm and cyclic loading conditions; and iv) no tension stiffening effects after cracking.

The resulting lateral load-deflection responses are compared in Figure 16. As can be seen, the responses predicted using the three alternative tension stiffening models exhibit only minor differences. The Vecchio-Collins model is stiffest, and the Izumo et al model is least stiff, as would be expected. However, the differences in deflections never exceed $10 \%$ at any load level. Conversely, ignoring tension stiffening effects results in deflections increased by as much as $30 \%$ at intermediate and higher load levels. The predicted strength of the wall is also affected somewhat, since the degree of deflection sustained impacts on the degree of containment afforded the web. However, the influences are minor, except when tension stiffening is discounted altogether. In the latter case, the predicted strength is reduced by about $5 \%$. There is no influence on the predicted failure mode. 


\subsection{INFLUENCE OF COMPRESSION SOFTENING MODEL:}

As is well documented in the literature, the effective compressive strength of concrete is reduced in the presence of transverse tensile strains. For elements subjected to membrane shear, as is the web wall of the structure in question, compression softening can significantly affect the strength, stiffness and failure mode of the structure. To investigate the magnitude of the effect in this case, two analyses were done: one using the Vecchio-Collins 1992 model for compression softening; and one in which no compression softening effects were considered.

The predicted load-deformation responses are compared in Figure 17. When compression softening is considered, only a slight degradation in stiffness is observed at later load stages. Further, the ultimate load capacity is reduced by a mere $3 \%$. Thus, for this structure, the compression softening effect is not significant. The influence is minimal because neither the vertical nor horizontal reinforcement in the highly stressed regions of the web yielded. The transverse tensile strains remained relatively small, and hence the reductions in compressive strength and stiffness were small. The compression softening effect becomes much more pronounced in situations where the tensile strains become large, as when there is yielding of either the horizontal or vertical reinforcement in the membrane elements.

\subsection{INFLUENCE OF CONCRETE CONFINEMENT:}

The analysis results discussed previously indicated that concrete strength enhancement due to confinement affected the strength and failure mode of the wall to some degree. Recalling the resuits of the both the 2-D and 3-D models, confinement effects introduced by the massive base stiffened the adjoining web regions and pushed the failure plane up the wall away from the base. And the vertical and lateral confinement provided by the stiff flange regions allowed the concrete in the web to achieve a higher strength before failing.

To further test the role of confinement effects in the response of the wall, an analysis was done in which the concrete confinement effects were removed. That is, no strength or stiffness enhancement was allowed due to biaxial or triaxial compression effects. The resulting loaddeformation response of the wall is compared in Figure 18 to the standard case (which allows for confinement effects).

It is seen that neglecting confinement effects results in a modest decrease in the load capacity of the wall, with a reduction of about $5 \%$. The failure mode is essentially unchanged. Also, there is virtually no effect on the stiffness of the load-deformation response prior to failure.

\subsection{INFLUENCE OF AMOUNT OF VERTICAL REINFORCEMENT IN FLANGES:}

The three-dimensional analyses suggested that the failure of the wall was governed by the shear-crushing failure of the concrete in the web, occurring after yielding of the vertical reinforcement in the left flange. Once the flange lost its ability to clamp down on the web, the 
reduced confinement of the web precipitated failure. Thus, the question remains: Was the failure brought on by yielding of the reinforcement in the flanges? Would averting yield in the flanges, by increasing the amount of reinforcement provided, significantly increase the strength and stiffness of the wall? To address these questions, the standard 2-D analysis was coupled with two additional analyses: one with $25 \%$ more reinforcement provided in the flanges; and one with $25 \%$ less reinforcement. The results are shown in Figure 19.

It is seen that the strength and stiffness are not affected in a degree proportional to the amount of reinforcement provided. The increase of $25 \%$ in reinforcement area results in an increase in load capacity of about $5 \%$ relative to the standard case. Similarly, a decrease in reinforcement of $25 \%$ results in a decrease in capacity of about $3 \%$. In all cases, the reinforcement in the flanges continues to yield and the failure mode remains unchanged. Deflections are influenced to only minor degrees as well. Thus, while yielding of the reinforcement in the flanges contributes ultimately to a shear failure of the web, it is not a major influencing factor.

\subsection{INFLUENCE OF CONCRETE STRENGTH:}

From earlier analyses it has been concluded that failure of the wall was dictated by a shear failure of the concrete web, and that compression softening effects and loss of confinement due to yielding of the tension flange played only minor roles. This then suggests that the concrete failure in the web was a result of the direct compressive strength being exceeded. If so, changes in the strength of the concrete should have a noticeable effect on the strength of the wall. To test this hypothesis, another set of analyses was undertaken. In addition to the standard case, two other analyses were performed: one with the compressive strength of the concrete in the wall increased by $15 \%$; and another with the strength decreased by $15 \%$. The resulting lateral load-deformation responses are given in Figure 20.

It is seen that the wall strength is influenced by the concrete strength to a much larger degree than it was by the flange reinforcement amount. A $15 \%$ increase in concrete strength brings an $8 \%$ increase in the wall's load capacity; a $15 \%$ decrease in concrete strength results in a $8 \%$ decrease in wall capacity. Deflections are similarly affected. Given that the wall capacity is influenced by other structural parameters as well, including the amounts of vertical and horizontal reinforcement in the web and the area of the compression flange, the correlation between concrete strength and wall load capacity is strong. The indication is that the concrete strength is being fully utilized.

\subsection{INFLUENCE OF MESH SIZE:}

With respect to the discretization of the web wall, the finite element mesh used for the two-dimensional analyses was purposely made similar to that used for the three-dimensional analyses. Considering the stiff nature of the top and bottom slabs, and the low height-to-width ratio of the wall, one can expect boundary effects to be significant. Given that the finite elements used were low-powered, one must consider whether the mesh used was sufficiently fine. To this 
end, two additional 2-D analyses were undertaken with progressively finer meshes. Recall that the original 2-D mesh contained 246 elements. The additional analyses employed meshes of 540 elements and 984 elements, respectively; roughly, 2 times and 4 times the density of the original mesh.

Compared in Figure 21 are the predicted load-deflection responses of the top slab. It is immediately evident that there are significant differences in the computed responses. The finer meshes result in: i) lower cracking stresses; ii) reduced stiffness after cracking; and iii) a lower ultimate load capacity. Post-cracking deflections are increased by as much as $50 \%$ in the later load stages, and the ultimate load is reduced by $17 \%$. Note that the 'doubled mesh' and 'quadrupled mesh' give essentially similar results, suggesting that a further refinement of the mesh is not necessary.

The finer meshes are better able to capture the lateral expansions of the web wall that occur away from the constrained top and bottom ends. Shown in Figure 22 is the predicted bulging of the wall at mid-height; that is, the relative horizontal displacement between the two flanges in the plane of the web. The finer meshes predict considerably higher expansions (i.e. tensile strains). The result is an increased compression softening effect in the web concrete, higher stresses in the web reinforcement, and higher stresses in the restraining flanges. These effects result in a decreased stiffness, and an earlier failure of the concrete. The predicted modes of failure are not significantly different, however, as can be seen in Figures 23 and 24 .

\section{CONCLUSIONS}

From the three-dimensional nonlinear static finite element analyses undertaken of the ISP shear wall, the following conclusions may be drawn:

i) The wall strength is predicted to be $1815 \mathrm{kN}$ under monotonically increasing lateral load; this is approximately $12 \%$ greater than the experimentally observed value with the wall tested under dynamic cyclically reversing loads.

ii) The predicted load-deformation responses agree well with the observed behaviour before cracking; however, the cracking load and post-cracking stiffness are overestimated. The degradation of tension stiffening effects partially accounts for the decreased stiffnesses observed in the test specimens. Deterioration in the bond and anchorage of the reinforcement, which was not considered in the analyses, is likely a contributing factor as well.

iii) The predicted failure mode is one involving a shear-crushing of the concrete web, resulting in a shear plane approximately 300 to $500 \mathrm{~mm}$ above the base, occurring after yielding of the reinforcement in the tension flange. The predicted mode of failure is in reasonable agreement with the observed failure. 
iv) Three-dimensional effects are significant. The flanges are near fully effective in contributing load resistance to the structure. The massive top and bottom slabs provide out-of-plane confinement to the web wall, enhancing the strength of the adjoining concrete web elements.

v) The sequence and location of yielding in the flange and web regions were such that likely 'ratcheting effects' influenced the failure mode and load capacity of the wall. This phenomenon is commonly observed in beam-column joints subjected to cyclic loads. Its effect is to produce a well-defined shear failure plane across the zone of yielding, at loads slightly higher than those required to yield the reinforcement, sequentially, on both faces of the element.

From the parametric study undertaken, using two-dimensional nonlinear static finite element analyses, the following conclusions are supported:

i) Two-dimensional analyses fail to capture some important three-dimensional effects, such as the out-of-plane confinement provided by the base slab and shear lag effects in the flange walls. They also lead to an over-estimation of the lateral and horizontal confinement of the web provided by the flanges, and an over-estimation of the contribution of the flange elements to the lateral shear stress distribution. The result is a slightly stronger and stiffer response than obtained using a three-dimensional analysis. However, the loss in accuracy is not severe, and the failure mode remains predicted well.

ii) In modelling the thickness of the flange elements, an effective thickness of between $67 \%$ and $100 \%$ of the width of the flanges appears to be appropriate. The flanges are nearly fully effective.

iii) The choice of a tension stiffening model, among several available, does not significantly affect behaviour. However, ignoring tension stiffening effects results in substantial increases in post-cracking deflections, and a slight lowering of the ultimate load capacity.

iv) Compression softening effects (ie the degradation in compressive strength of cracked concrete due to the influence of transverse tensile strains) is not a significant influencing factor for this test specimen.

v) Confinement effects have a minor influence, resulting in a slightly increased load capacity and a positioning of the failure plane up away from the base.

vi) The amount of vertical reinforcement provided in the flanges is not a significant influencing factor, although yielding of this reinforcement immediately precedes the web shear failure. 
vii) The compressive strength of the concrete in the web is fully utilized at ultimate load. Increasing or decreasing the concrete strength significantly affects the predicted failure load of the structure. Thus, the strength of the wall is most governed by the strength and thickness of the web. The vertical and horizontal reinforcement in the web are of sufficient amounts to not yield. The flanges are of sufficient width, and sufficiently reinforced, to not precipitate a flexural failure.

viii) The element mesh used to model the web wall, in both the standard two- and threedimensional analyses, was somewhat too coarse. Analytically, there was insufficient freedom for the mid-height regions of the web to overcome the restraint imposed by the stiff top and bottom slabs. A finer mesh predicts a considerably less stiff response, and a significantly lower load capacity.

\section{RECOMMENDATIONS}

Given the results of the sensitivity study pertaining to mesh size, the question arises whether a similar order effect would occur in the three-dimensional analyses. If reductions of the same proportion were realized in the 3-D analyses, the resulting ultimate load capacity and load-deflection responses would be very close to those measured experimentally. Thus, it is recommended that the three-dimensional analysis be repeated using an improved mesh for the web and flange regions. The total degrees of freedom could be maintained at a reasonable number by adopting a coarser discretization for the top and bottom slabs.

Some aspects of the parametric study, conducted using two-dimensional finite element analyses, might also be repeated using a finer mesh. It is possible that factors such as compression softening may assume more significance, given the reductions in strength and stiffness previously seen with the finer meshes.

\section{REFERENCES}

Collins, M.P., and Mitchell,D. (1991). Prestressed Concrete Structures, Prentice-Hall, New Jersey.

Hsieh,S.S., Ting,E.C., and Chen,W.F. (1979). "An Elastic-Fracture Model For Concrete". Proc. 3rd Eng. Mech. Div. Spec. Conf., ASCE, Austin, Texas, pp.437-440.

Izumo,J., Shin,H., Maekawa,K., and Okamura,H. (1992). "An Analytical Model for RC Panels Subjected to In-Plane Loads", Concrete Shear in Earthquake, Elsevier Applied Science, London, pp. 206-215.

Kupfer,H., Hilsdorf, K.H., and Rusch,H. (1969). "Behaviour of Concrete Under Biaxial Stress". J. Amer. Concrete Inst., Vol. 66, No.8, pp.656-666. 
Richart,F.E., Brandzaeg,A., and Brown,R.L. (1928). "A Study of the Failure of Concrete Under Combined Compressive Stresses". Bulletin No. 185, Univ. of Illinois Engineering Experimental Station, Urbana, Illinois.

Scott,B.D., Park,R., and Priestley,M.J.N. (1982). "Stress-Strain Behaviour of Concrete Confined by Overlapping Hoops at Low and High Strain Rates". J. Amer. Concretie Inst., Vol.79, No.1, pp.13-27.

Vecchio,F.J., and Collins,M.P. (1986). "The Modified Compression Field Theory for Reinforced Concrete Elements Subjected to Shear". J. Amer. Concrete Inst., Vol.83, No.2, pp.219-231.

Vecchio,F.J. (1989). "Nonlinear Finite Element Analysis of Reinforced Concrete Membranes". Amer. Conc. Inst. Structural J., Vol.86, No.1, pp.26-35.

Vecchio,F.J., and Selby,R.G. (1991). "Towards Compression Field Analysis of Reinforced Concrete Solids". J. Struct. Engrg., ASCE, Vol.117, No.6, pp.1740-1758.

Vecchio, F.J. (1992). "Finite Element Modelling Of Concrete Expansion and Confinement". J. Struct. Engrg., ASCE, Vol. 118, No. 9, pp. 2390-2406. 
Table 1

\section{ANALYSIS SUMMARY}

1. General information
(1) Contact person
: F.J. VECCHIO
(2) Organization
: Dept. of Civil Engineering, University of Toronto
(3) Address
(4) Tel. No.
: 35 St. George Street, Toronto, Canada
(5) Fax. No.
: (416) $978-5910$
: (416) 978-7046
(6) E-mail address
: fjv@civ.utoronto.ca

2. Computer information
(1) Type of computer
: MIPS Challenge M Workstation R4400/150
(2) Memory size used
: $120 \mathrm{MB}$

3. Code information
(1) Category of analytical method
: Static F.E.M.
(2) Code name and version
: SPARCS (original)
(3) User specific changes to code
: $\mathrm{n} / \mathrm{a}$
(4) Total number of degrees of freedom : 5796
(5) Non-linear analysis algorithm : total load, secant stiffness

4-1. Dynamic analysis condition
(1) RUN No. (1-5)
: $\mathbf{n} / \mathbf{a}$
(2) Time step size
: $\mathbf{n} / \mathbf{a}$
(3) CPU time for 12 seconds analysis period
: $\mathrm{n} / \mathrm{a}$
(4) Consideration of vertical input
: $\mathbf{n} / \mathbf{a}$

4-2. Static analysis condition

(1) Control procedure of load increment: load control

(2) CPU time: 175 minutes per load stage

(3) Load step size (Max. and Min. value): $98 \mathrm{kN} / 49 \mathrm{kN}$

(4) Determination procedure of maximum load: response curves 


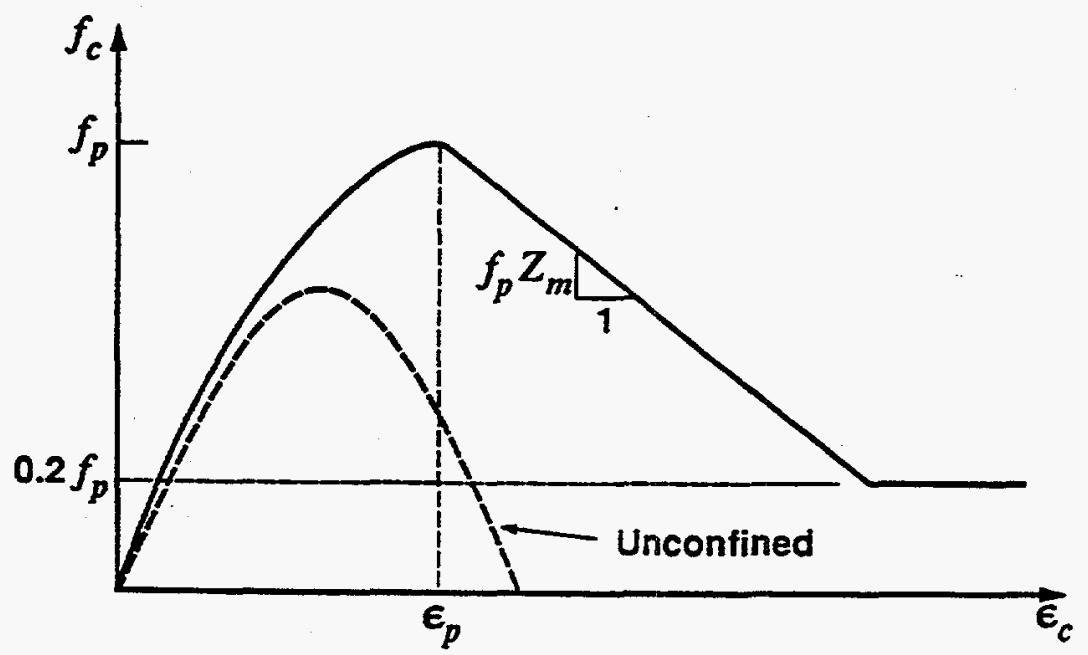

(a) Confined Concrete

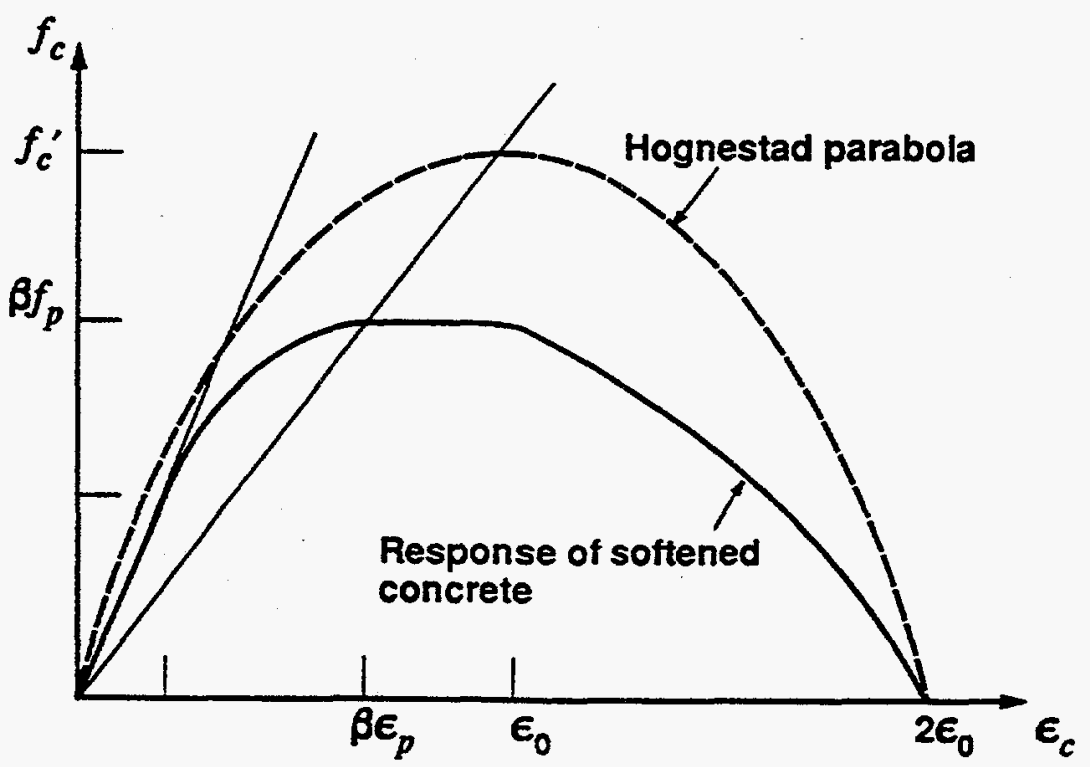

(b) Cracked Concrete

Figure 1

Concrete Compressive Stress-Strain Relationships. (see Appendix I for equations of curves) 

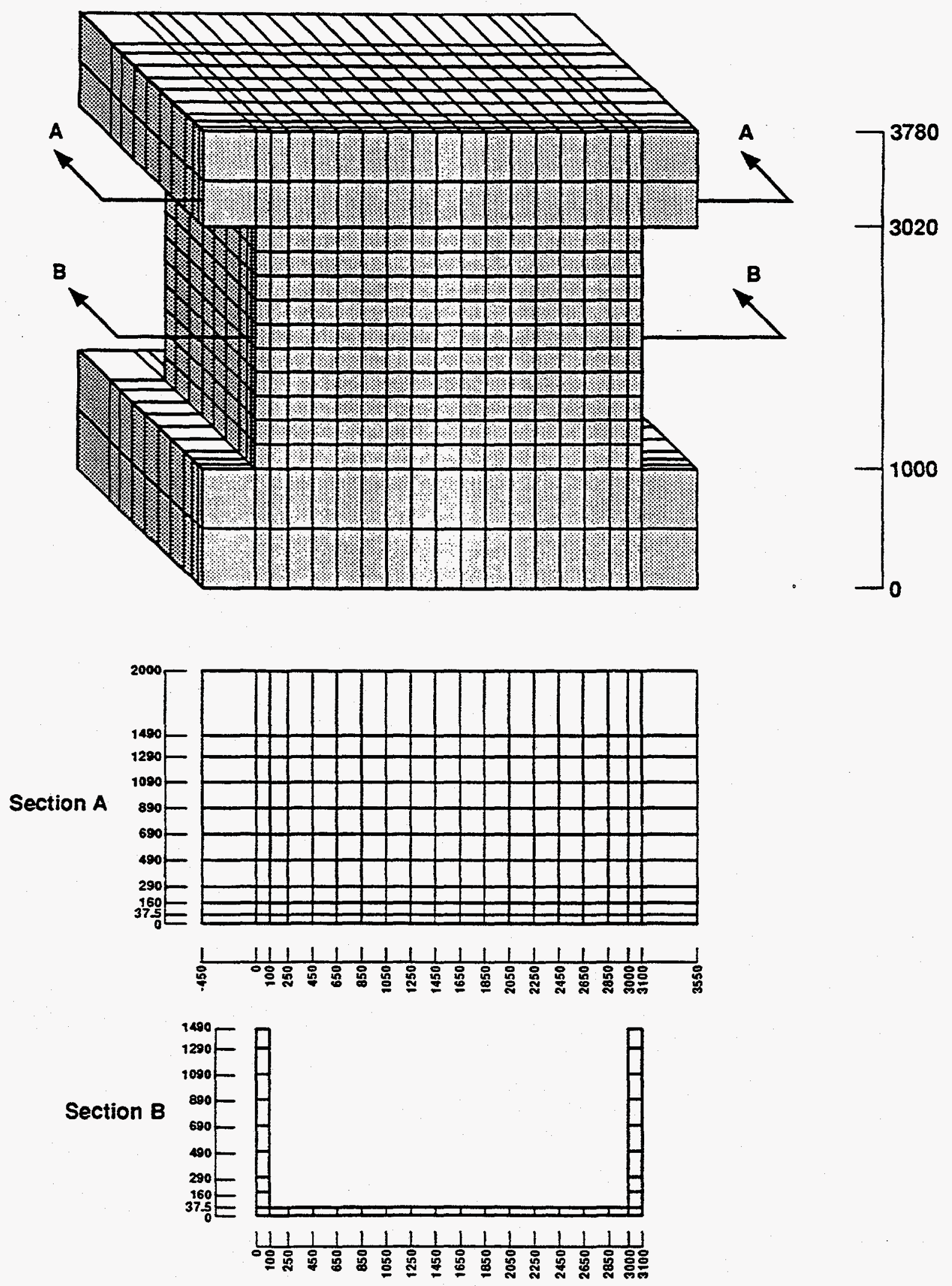

Figure 2

SPARCS Model of ISP Shear Wall 


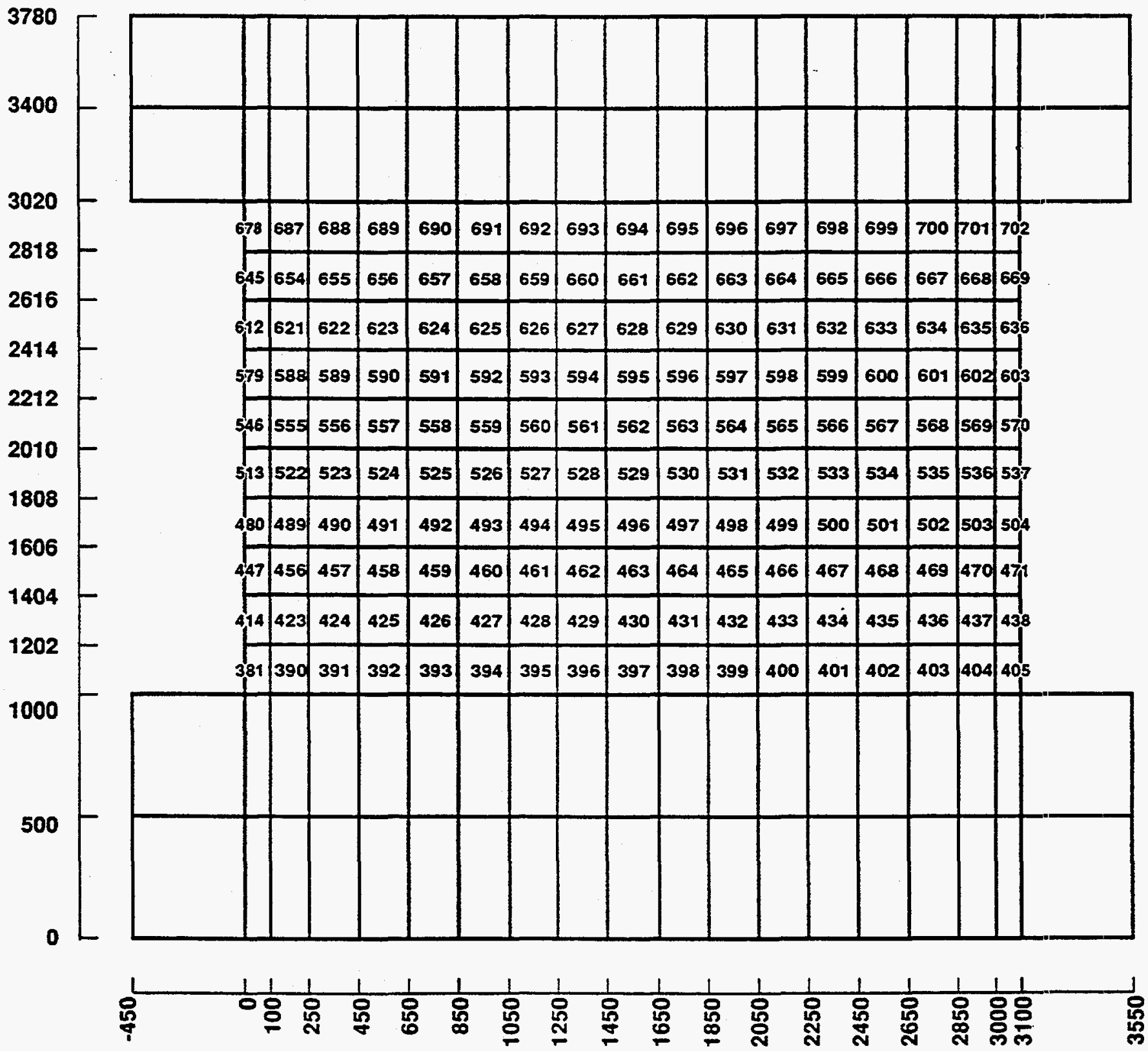

Figure 3

Element Scheme for SPARCS Model 


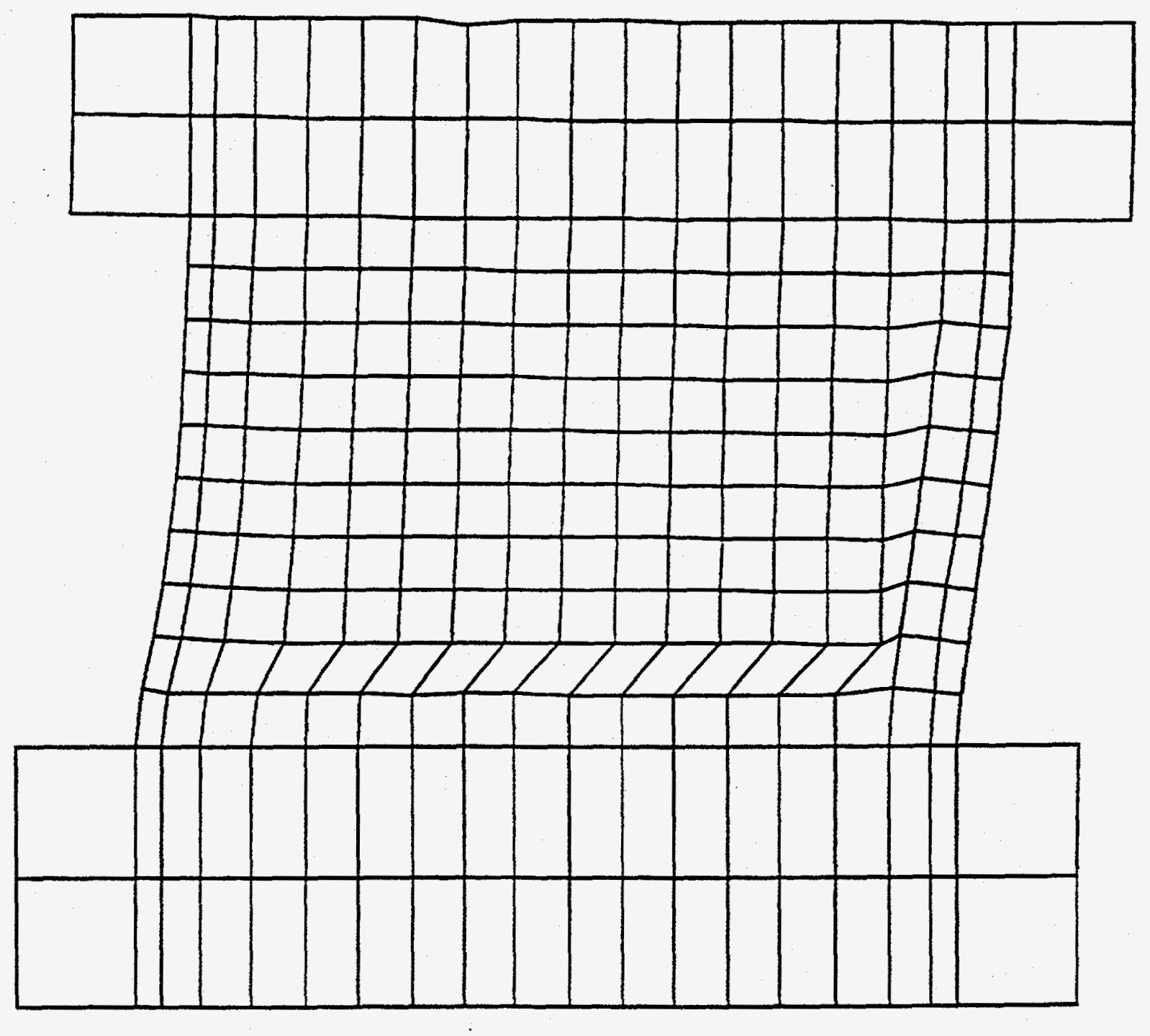

Figure 4

Predicted Failure Mode From 3-D Analysis 


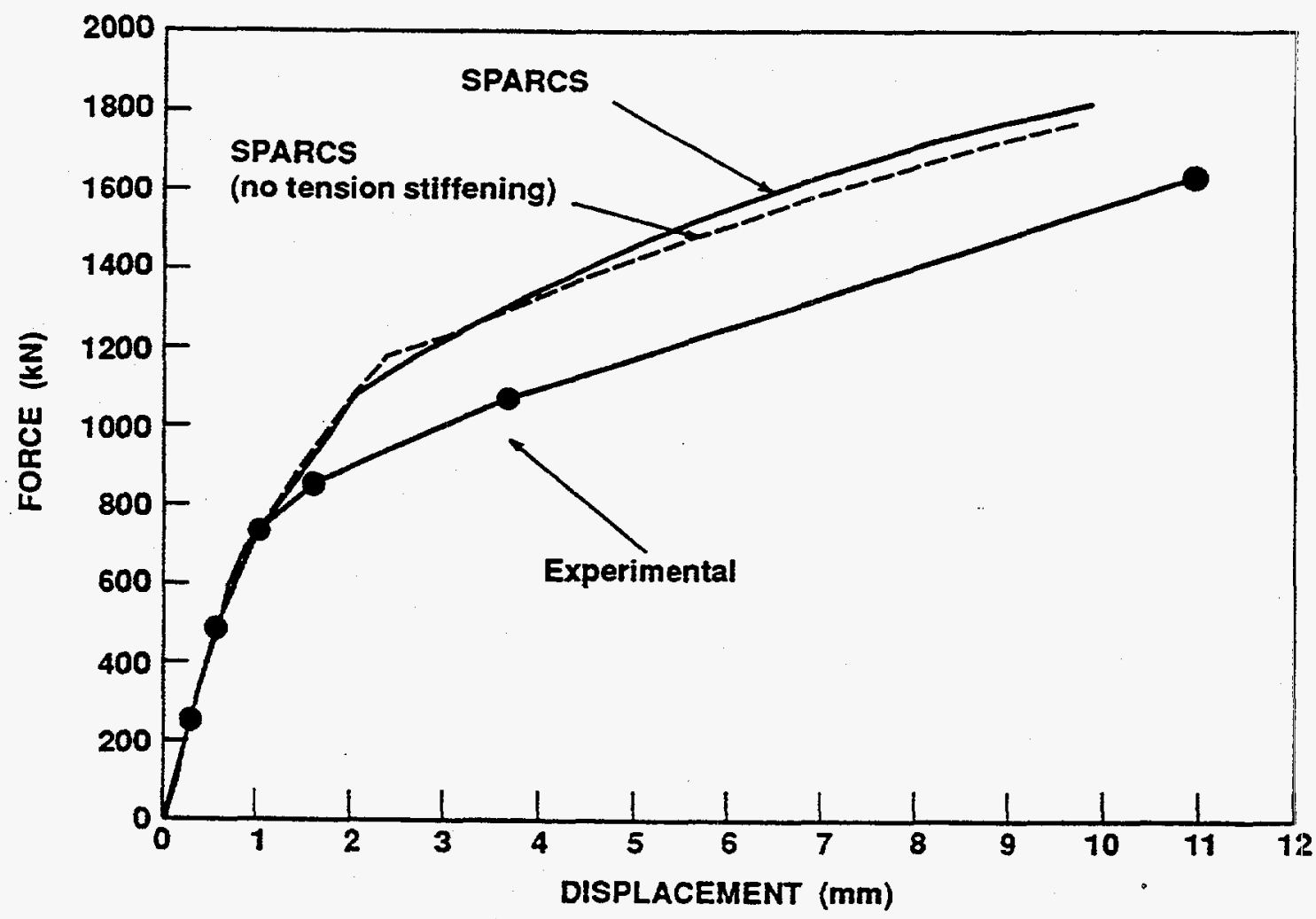

Figure 5

Horizontal Displacement of Top Slab

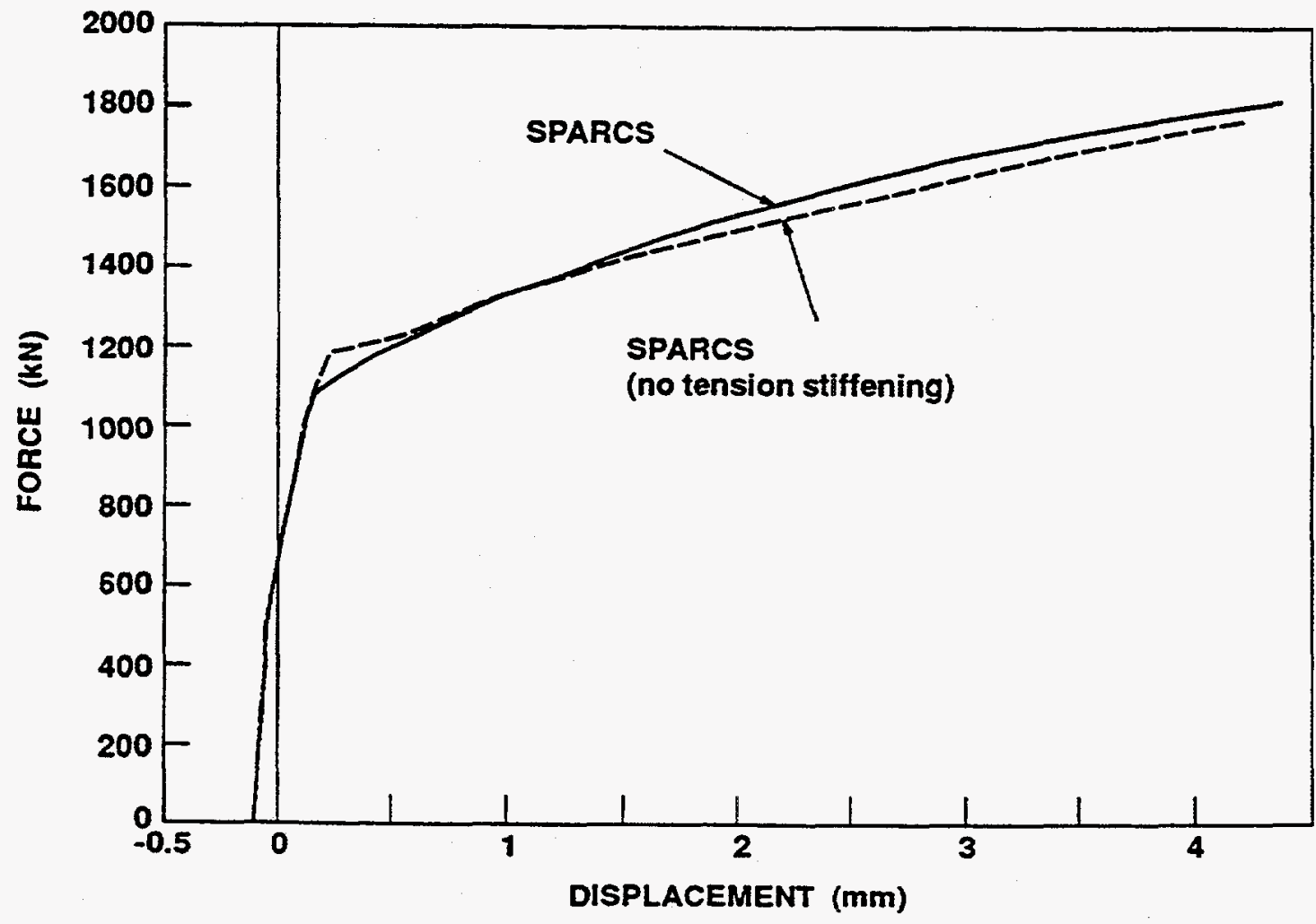

Figure 6

Vertical Relative Displacement - Left Flange 


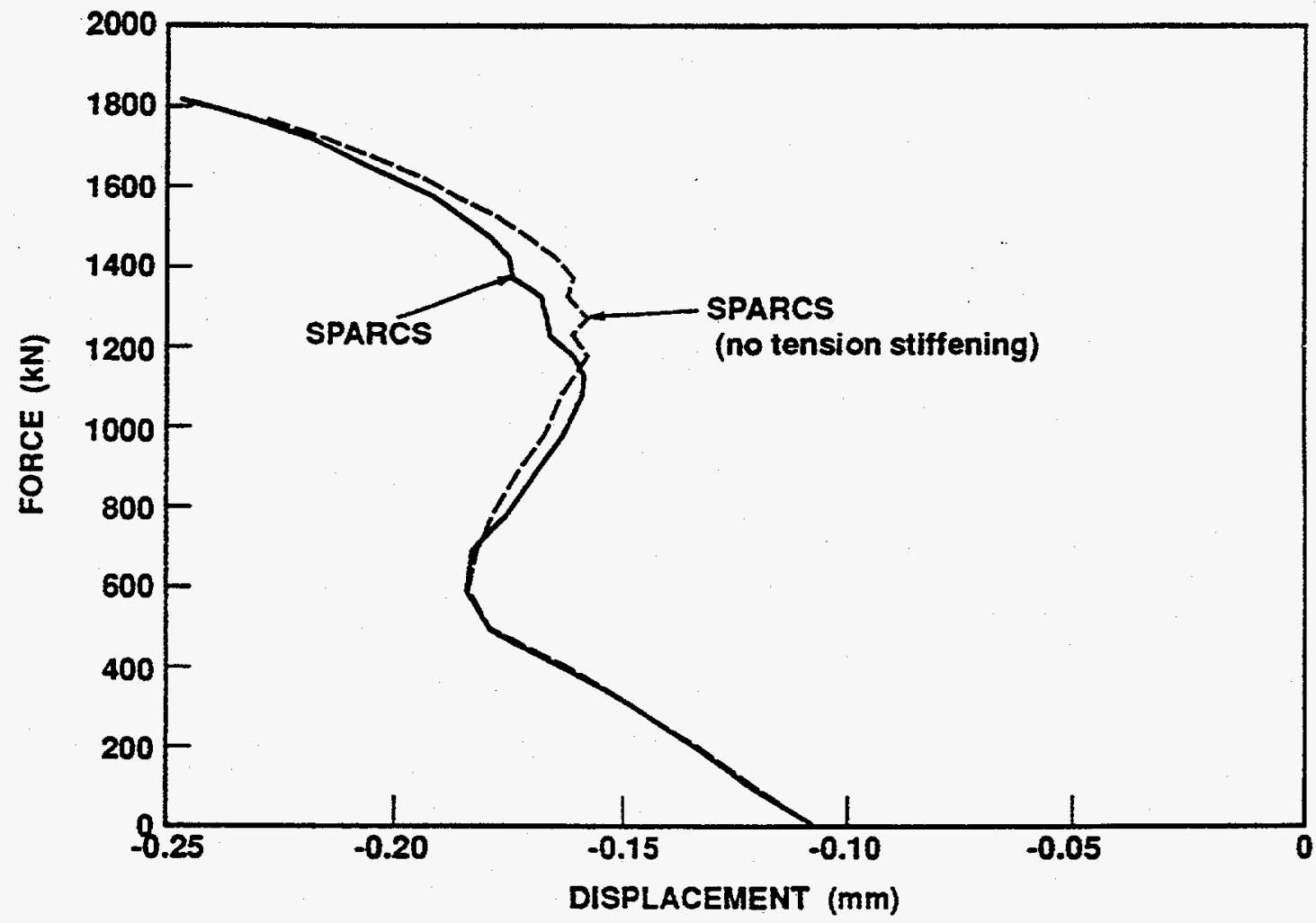

Figure 7

Vertical Relative Displacement - Right Flange 


\begin{tabular}{|c|c|c|}
\hline \multirow[t]{2}{*}{ Phenomena } & \multicolumn{2}{|c|}{ Top Slab } \\
\hline & $\begin{array}{l}\text { Horizontal Displacement } \\
\qquad(\mathrm{mm})\end{array}$ & $\begin{array}{l}\text { Horizontal Force } \\
(\mathrm{N})\end{array}$ \\
\hline $\begin{array}{l}\text { (a) Flange wall } \\
\text { Initiation point of bending crack }\end{array}$ & 1.200 & $785 \times 10^{3}$ \\
\hline $\begin{array}{l}\text { (b) Web wall } \\
\text { Initiation point of shear crack }\end{array}$ & 0.614 & $490 \times 10^{3}$ \\
\hline $\begin{array}{l}\text { (c) Flange wall vertical rebar } \\
\text { Initiation point of yield crack }\end{array}$ & 4.684 & $1420 \times 10^{3}$ \\
\hline $\begin{array}{l}\text { (d) Web wall vertical rebar } \\
\text { Initiation point of yield crack }\end{array}$ & 7.453 & $1665 \times 10^{3}$ \\
\hline $\begin{array}{l}\text { (e) Web wall horizontal rebar } \\
\text { Initiation point of yield crack }\end{array}$ & $n / a$ & $n / a$ \\
\hline (f) Crush point & 6.814 & $1620 \times 10^{3}$ \\
\hline Maximum load & 9.862 & $1815 \times 10^{3}$ \\
\hline
\end{tabular}

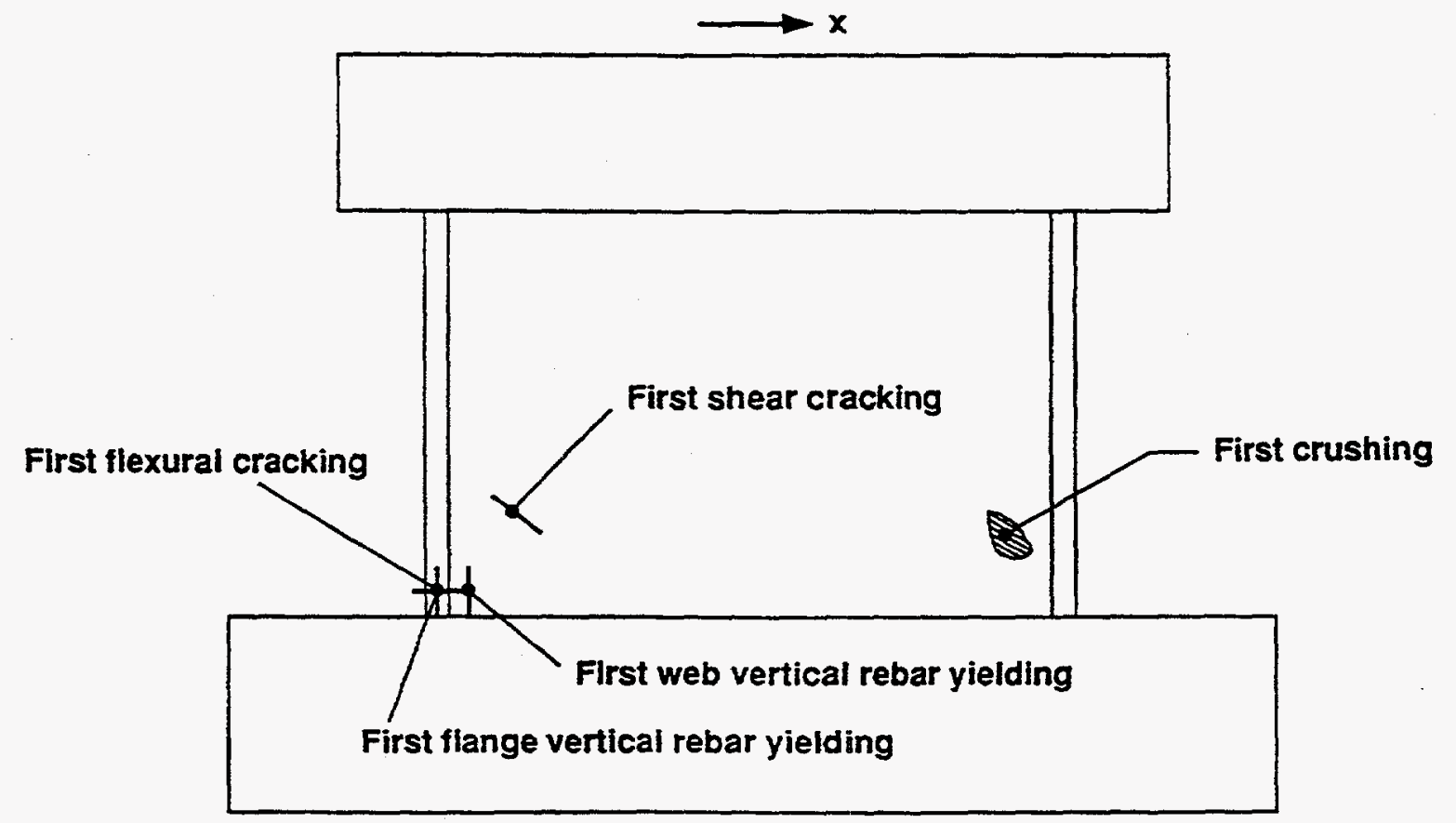

Figure 8 

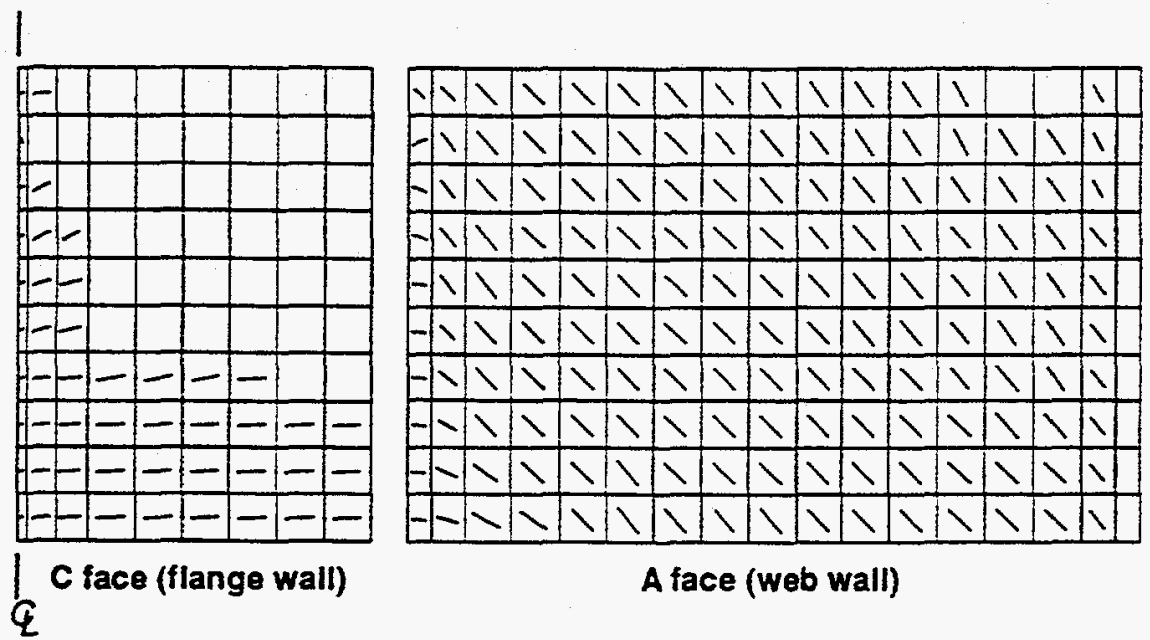

A face (web wall)

(a) At 2/3 of Maximum Load (1220 kN)
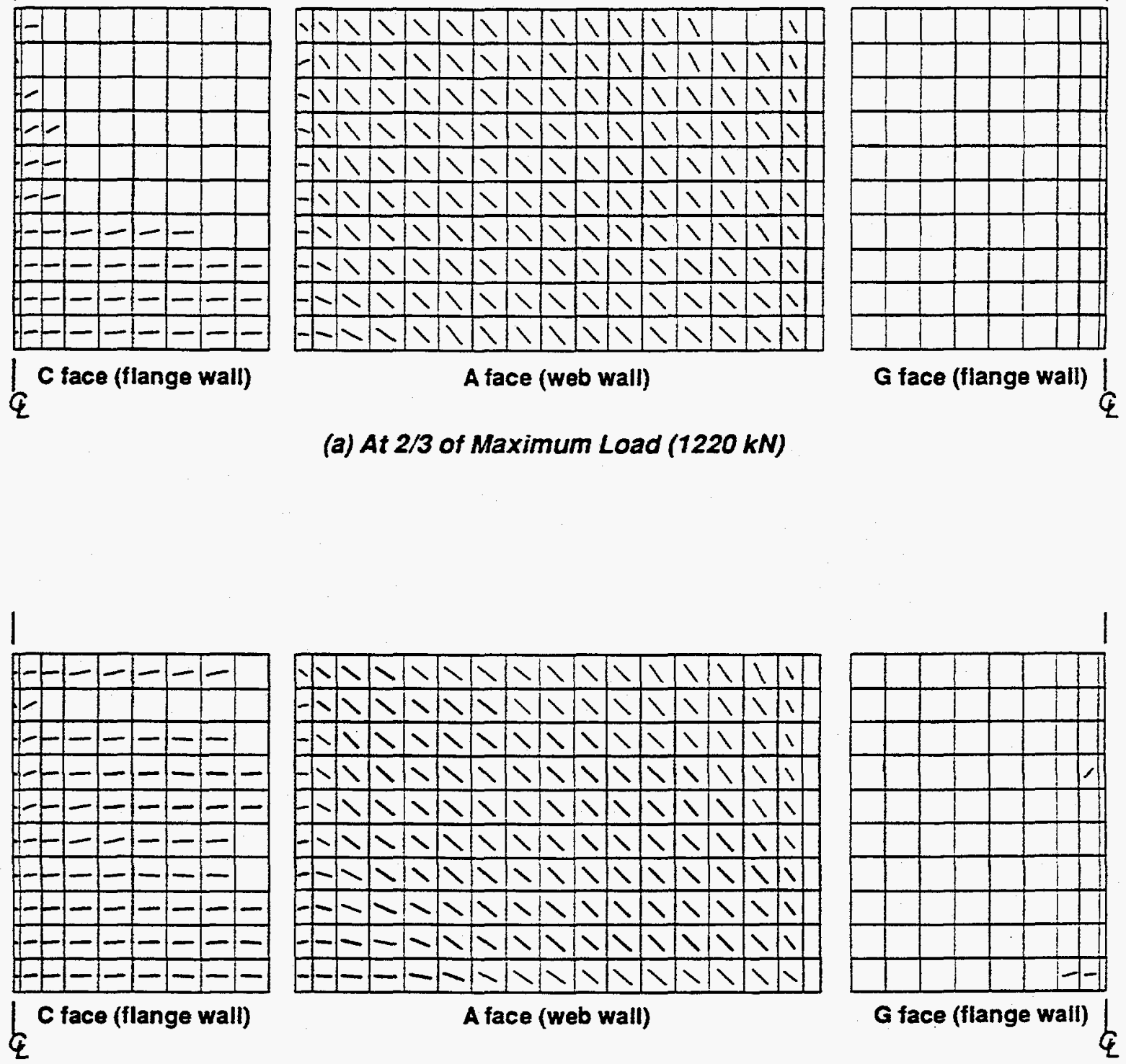

(b) At Maximum Load (1815 kN)

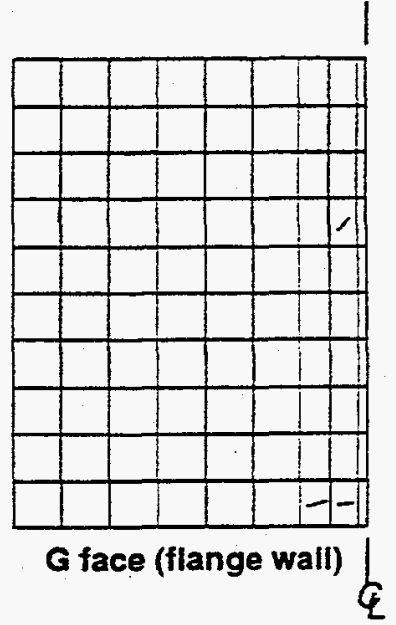

Figure 9

Predicted Crack Patterns 


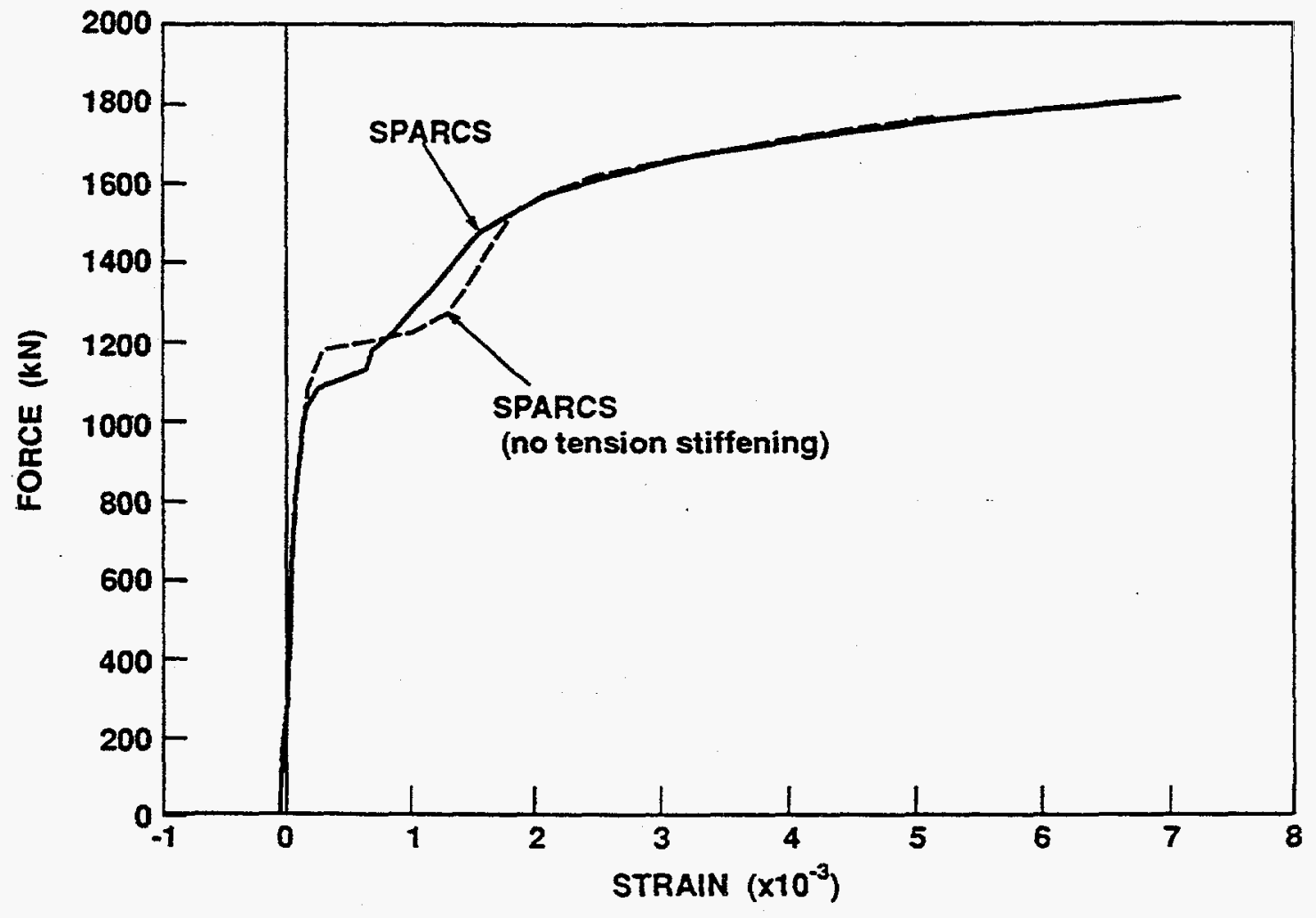

Figure 10

Vertical Rebar Strain - Base of Left Flange

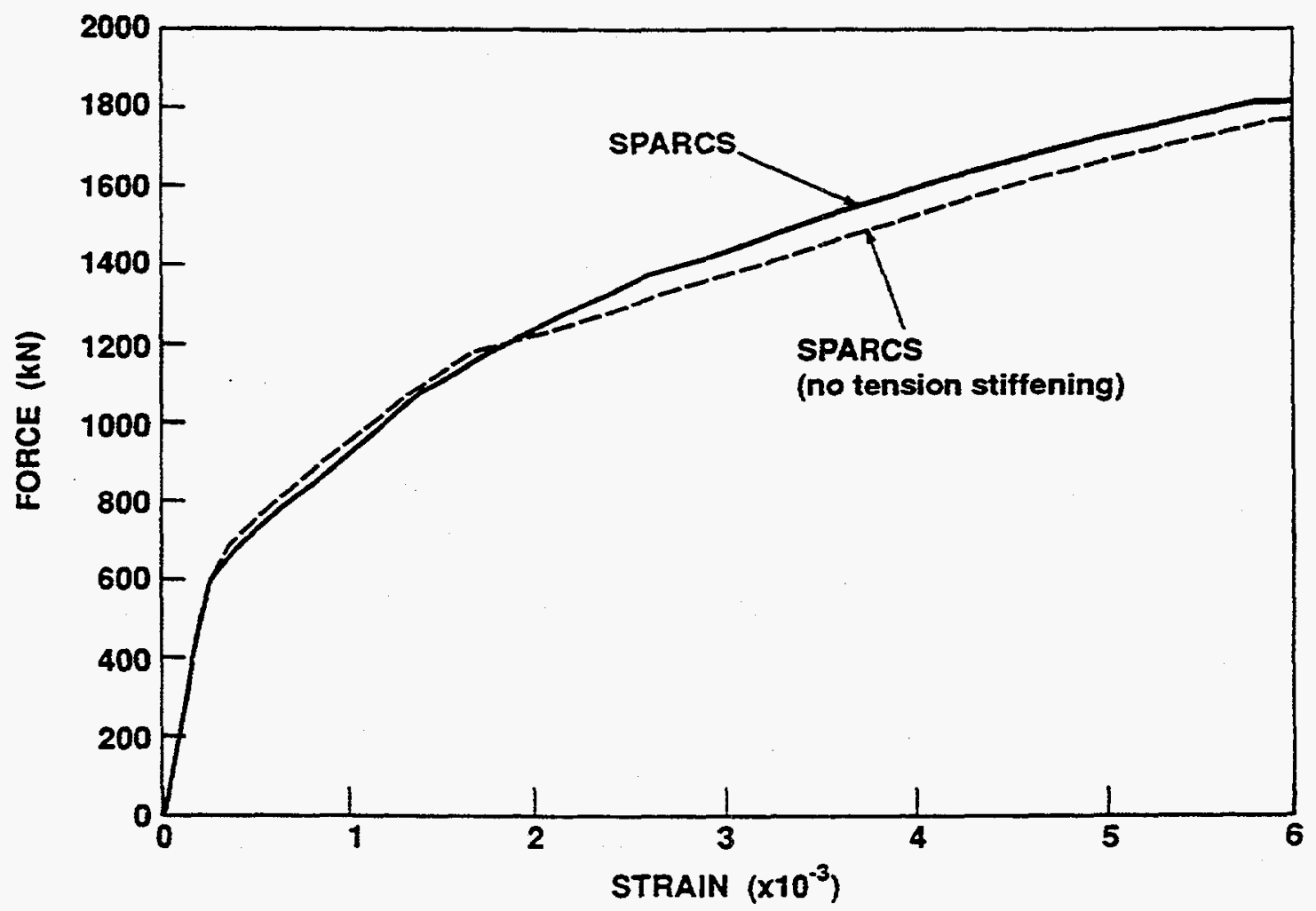

Figure 11

Shear Strain in Web 


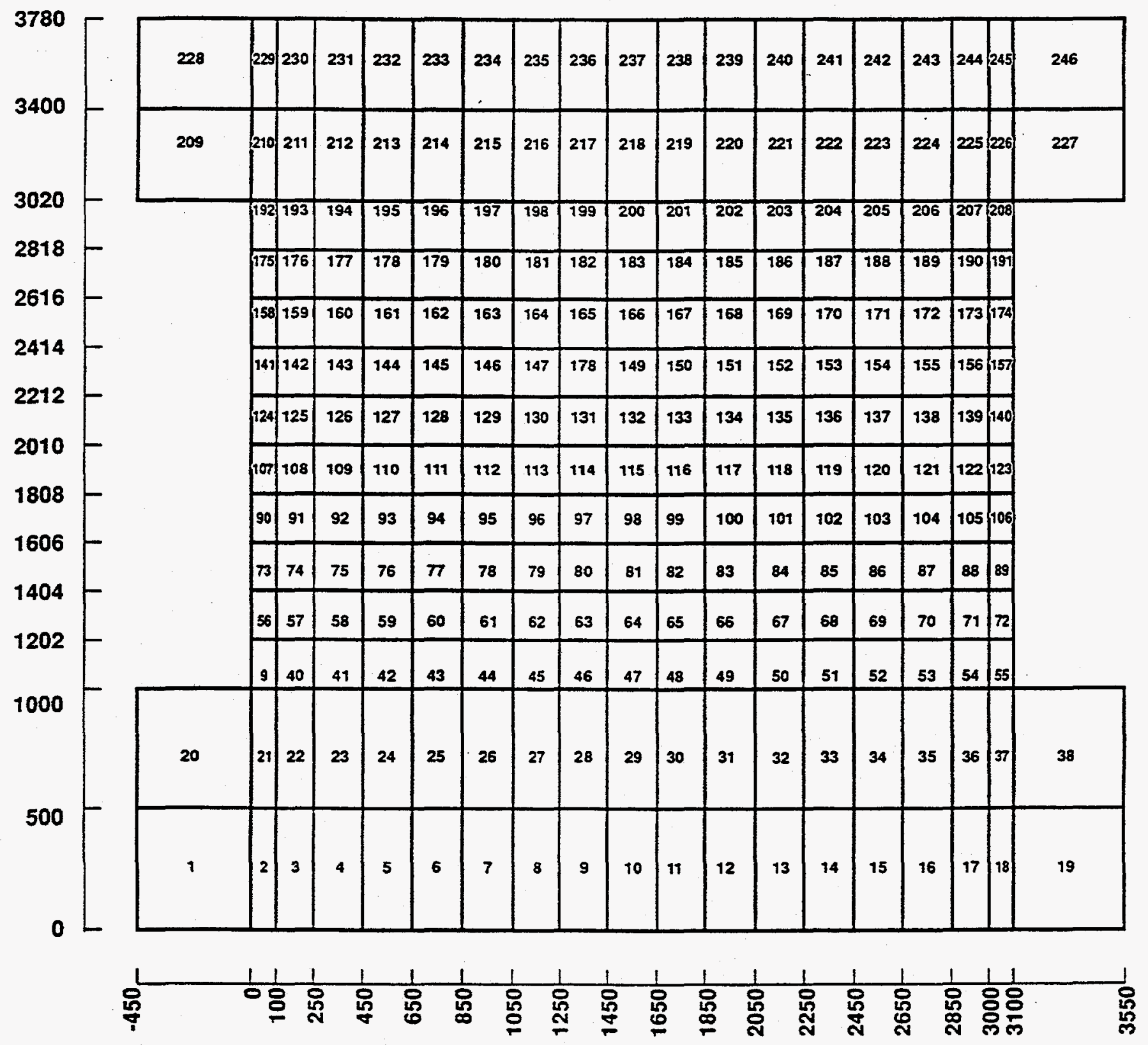

Figure 12

Element Scheme for TRIX Model of ISP Wall 


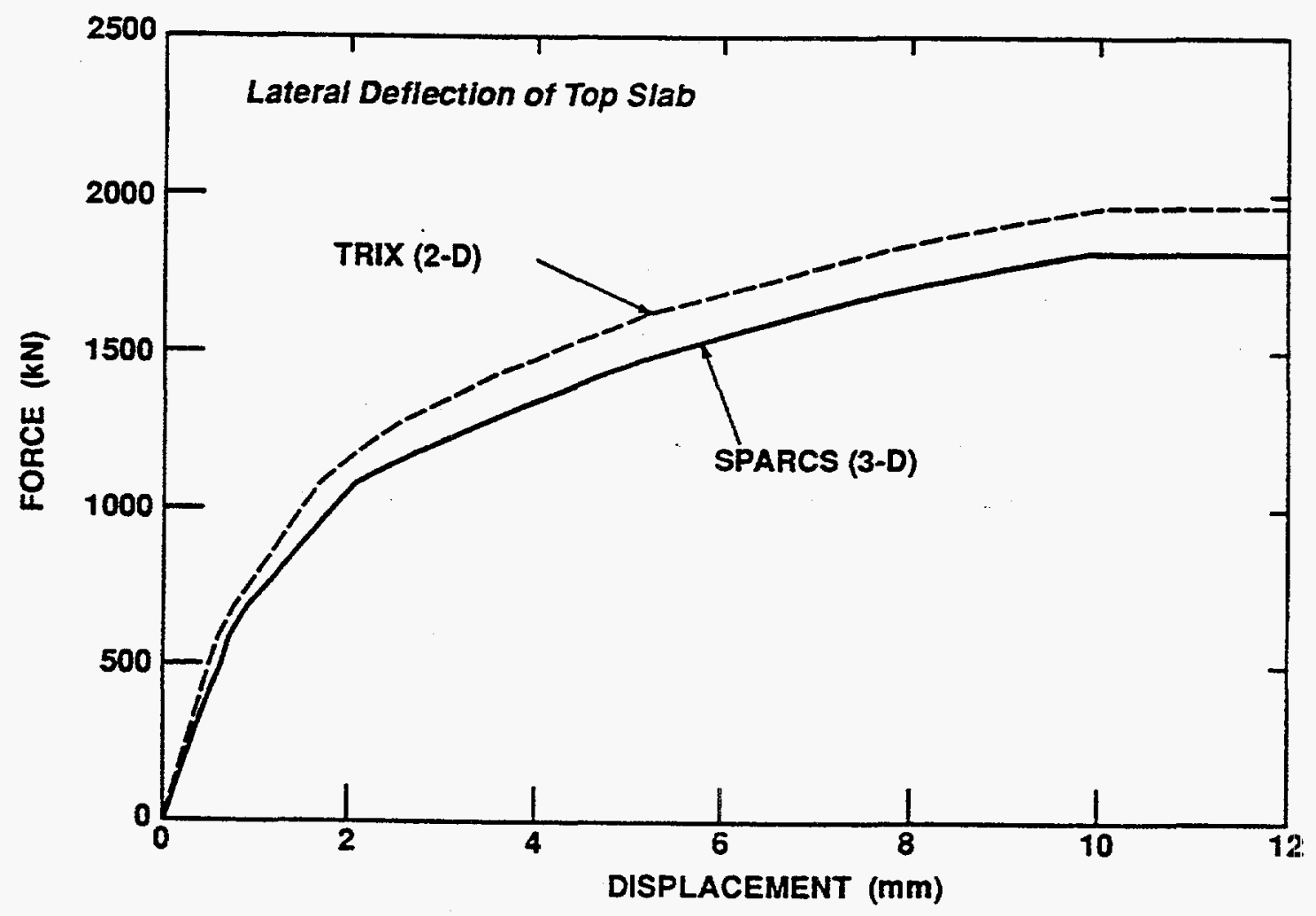

Figure 13

Influence of Three-Dimensional Effects 


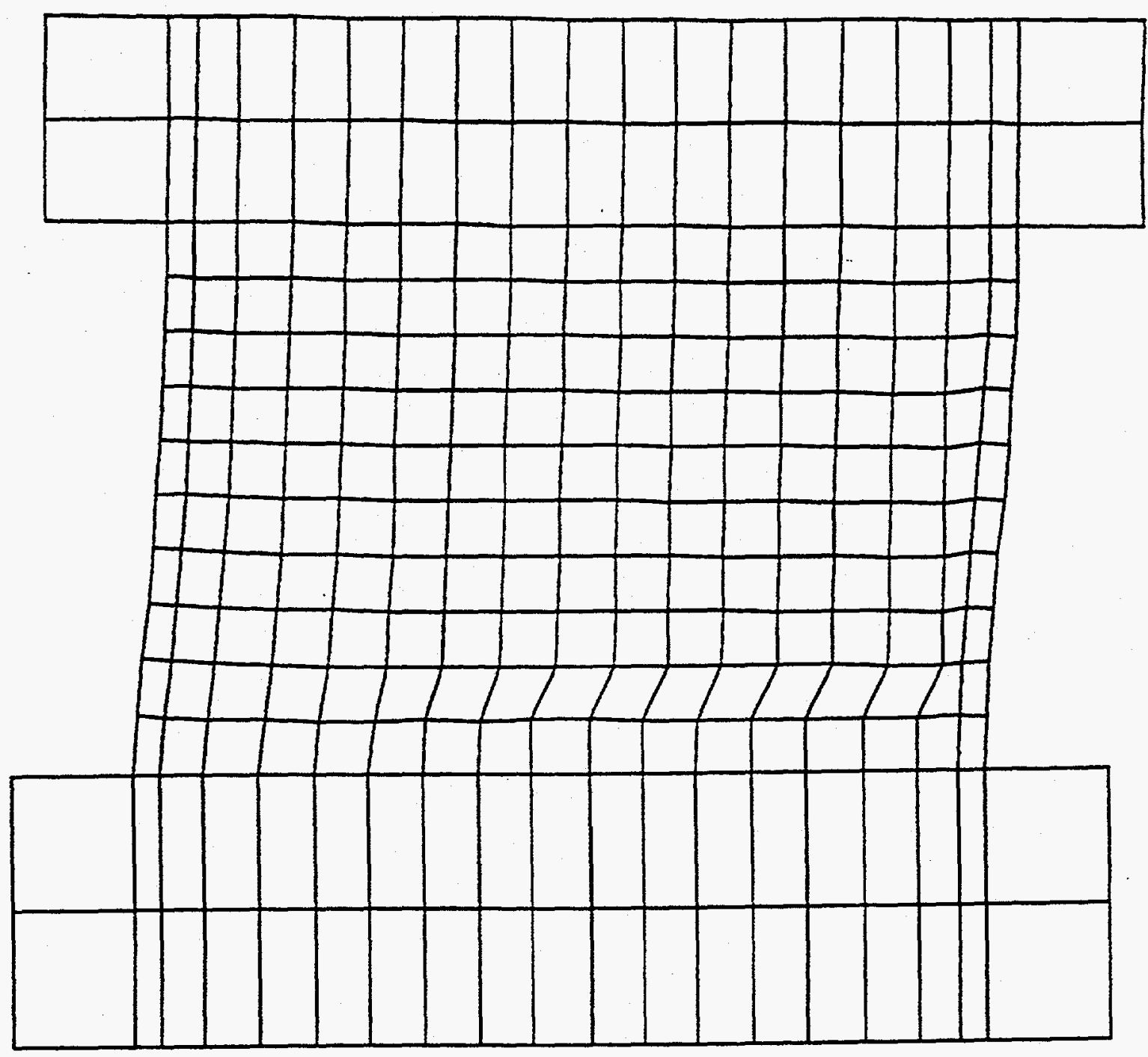

Figure 14

Predicted Failure Mode From 2-D Analysis 


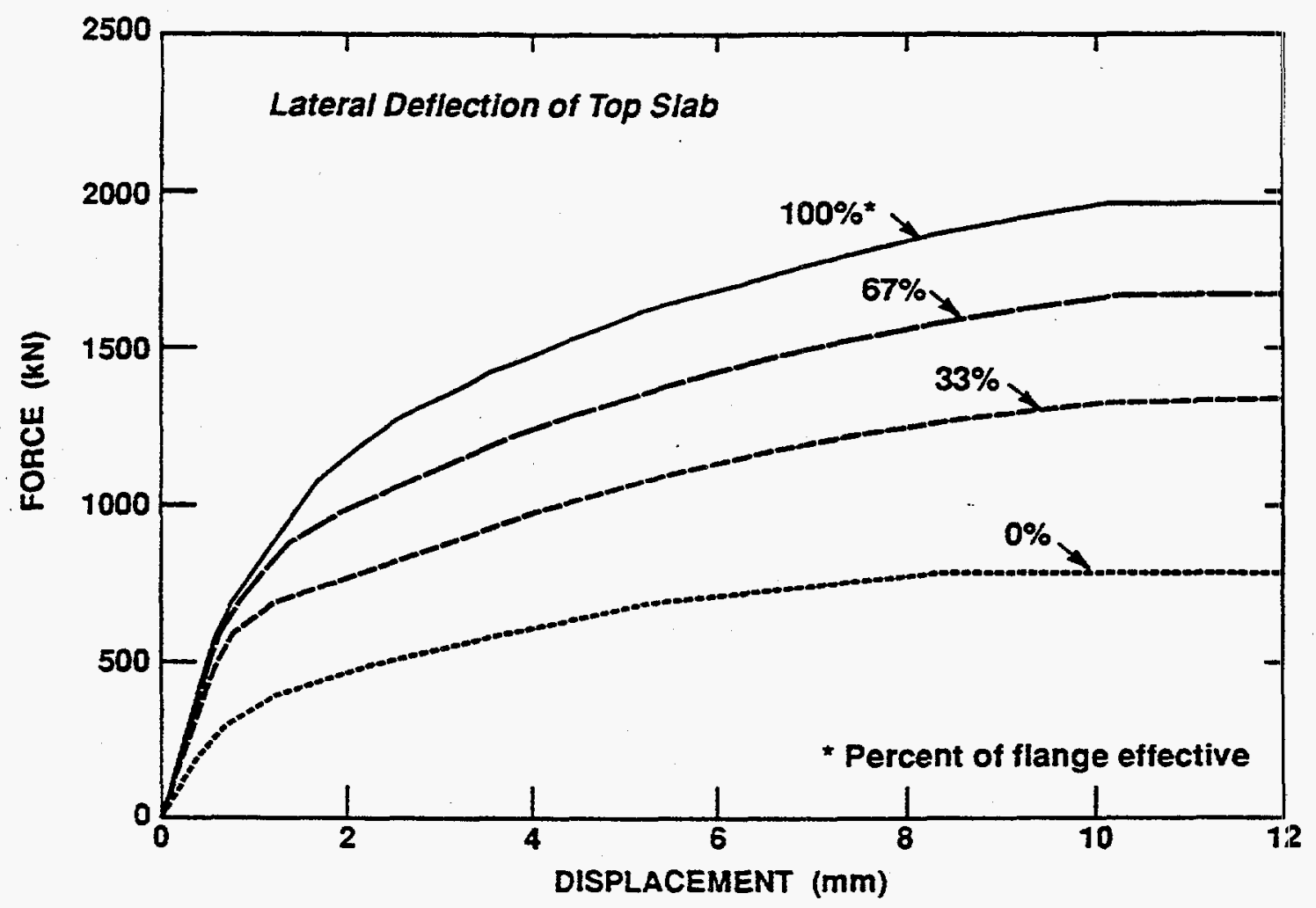

Figure 15

Influence of Effective Flange Width

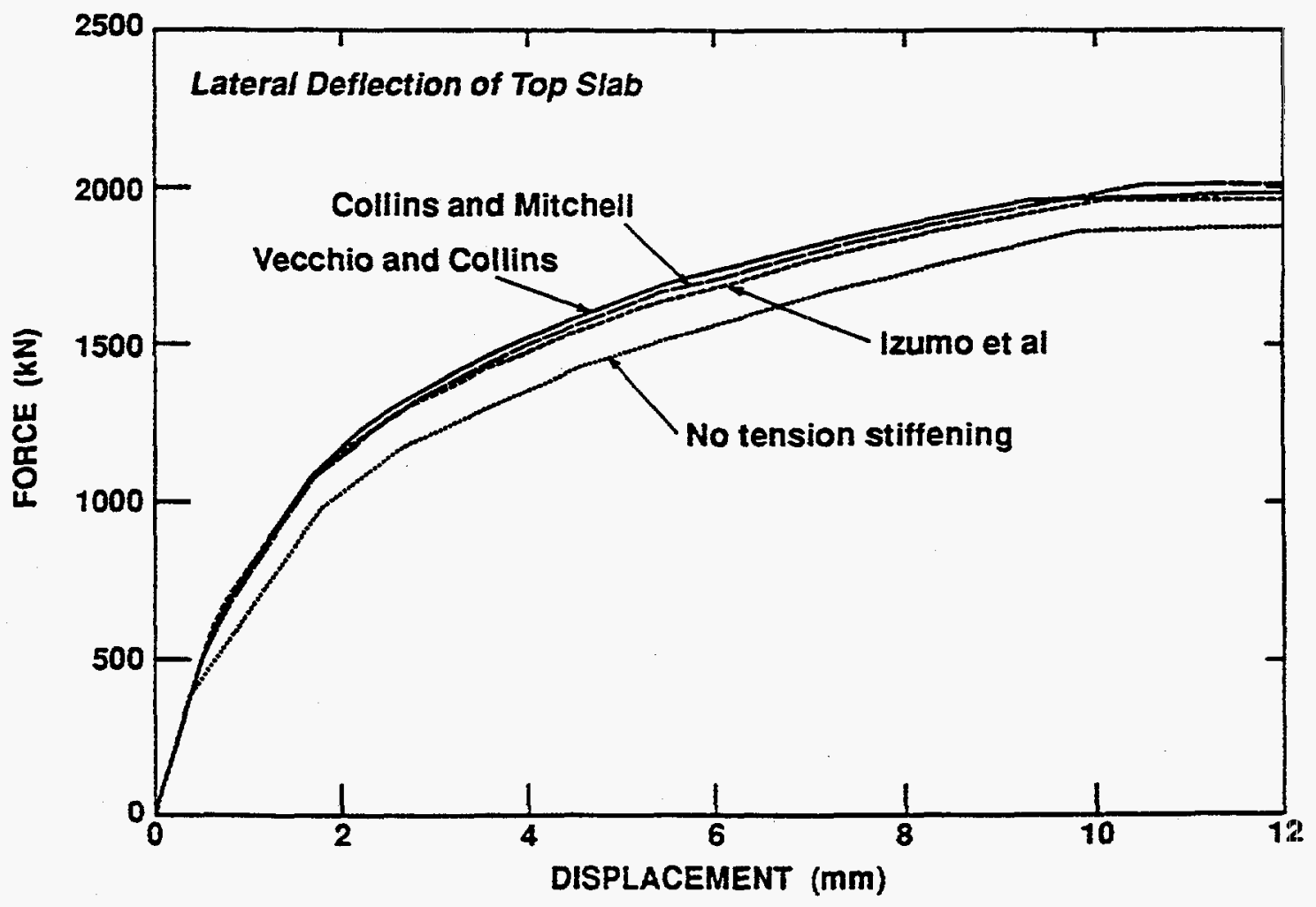

Figure 16

Influence of Tension Stiffening 


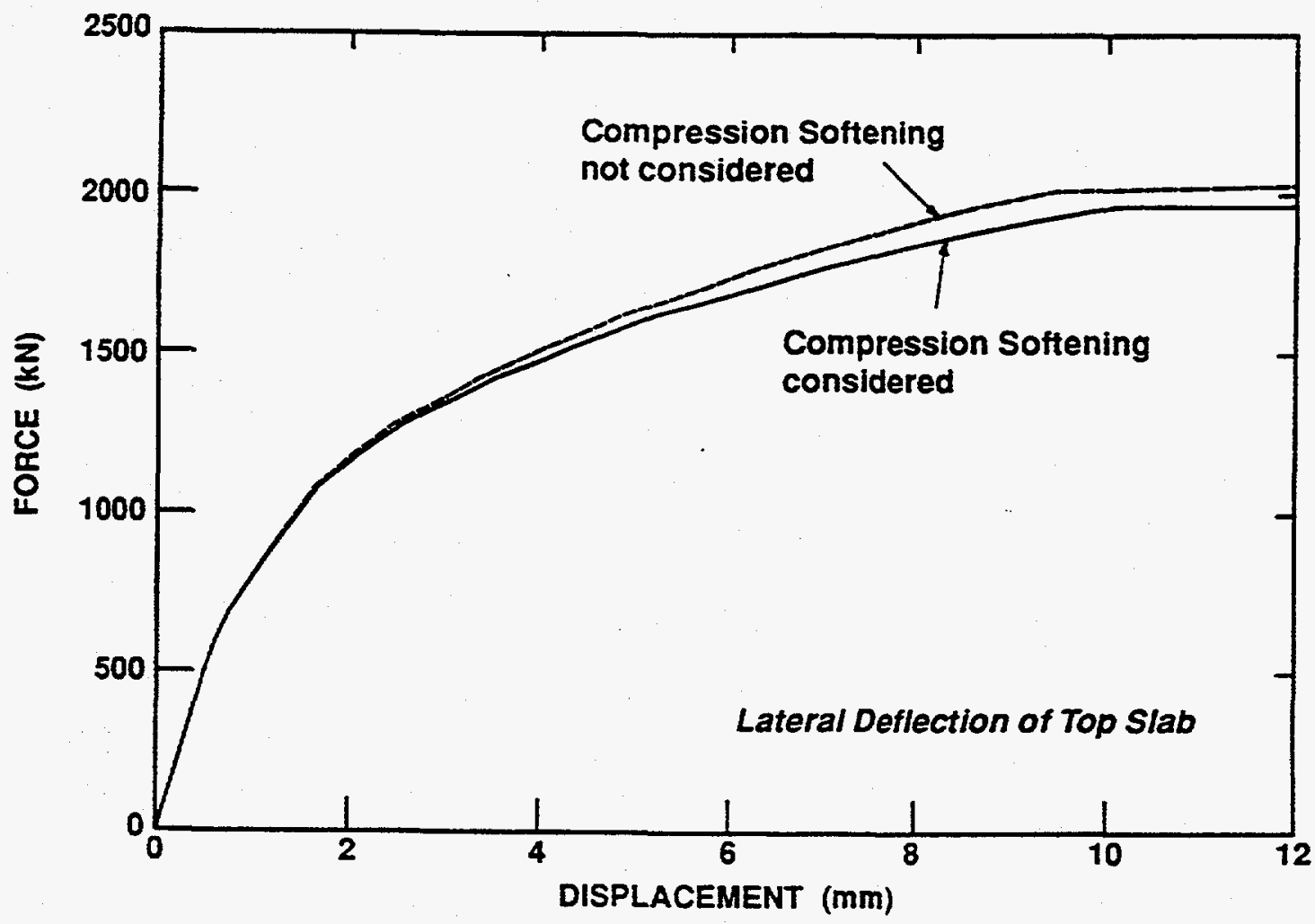

Figure 17

Influence of Compressive Softening

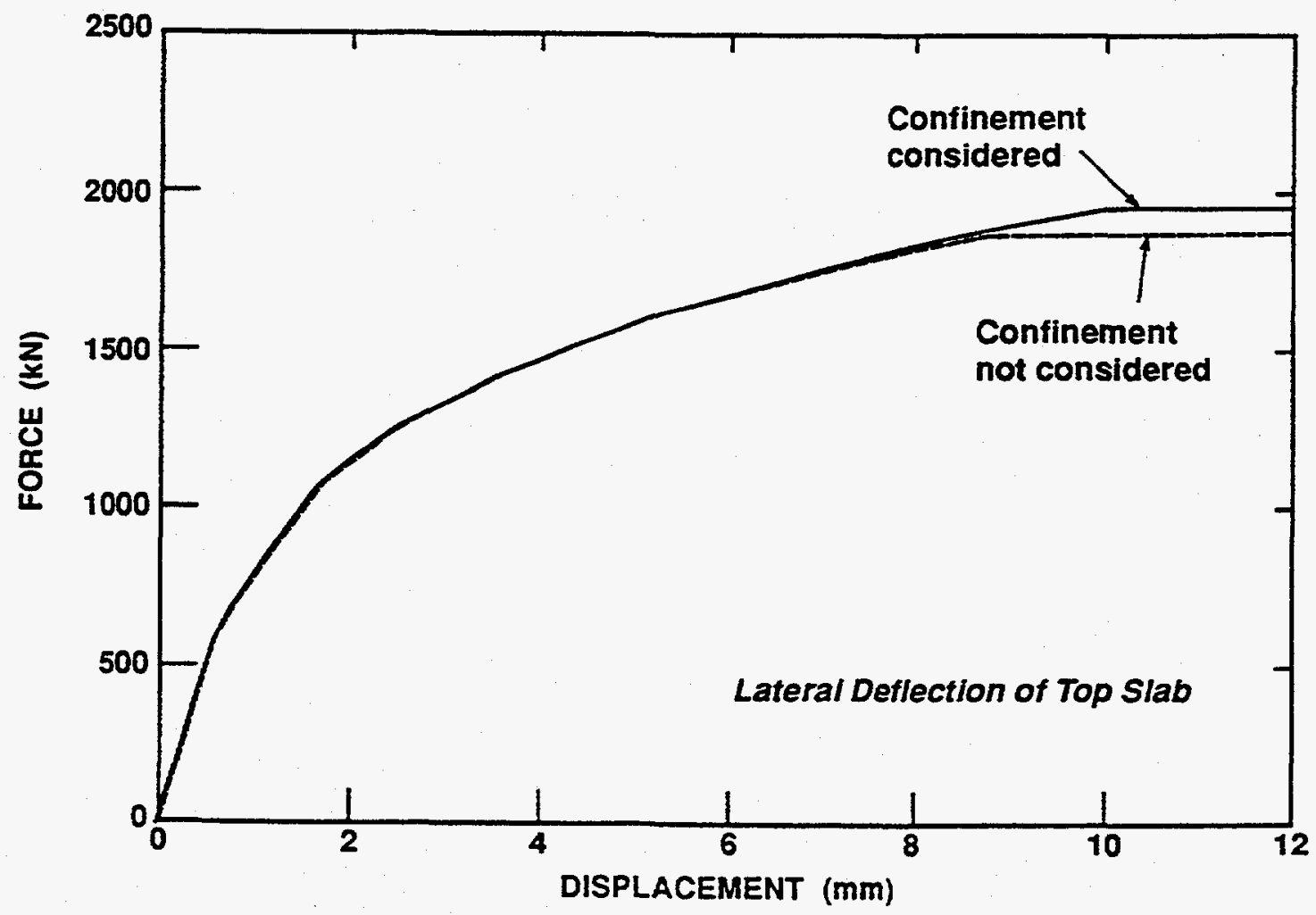

Figure 18

Influence of Confinement 


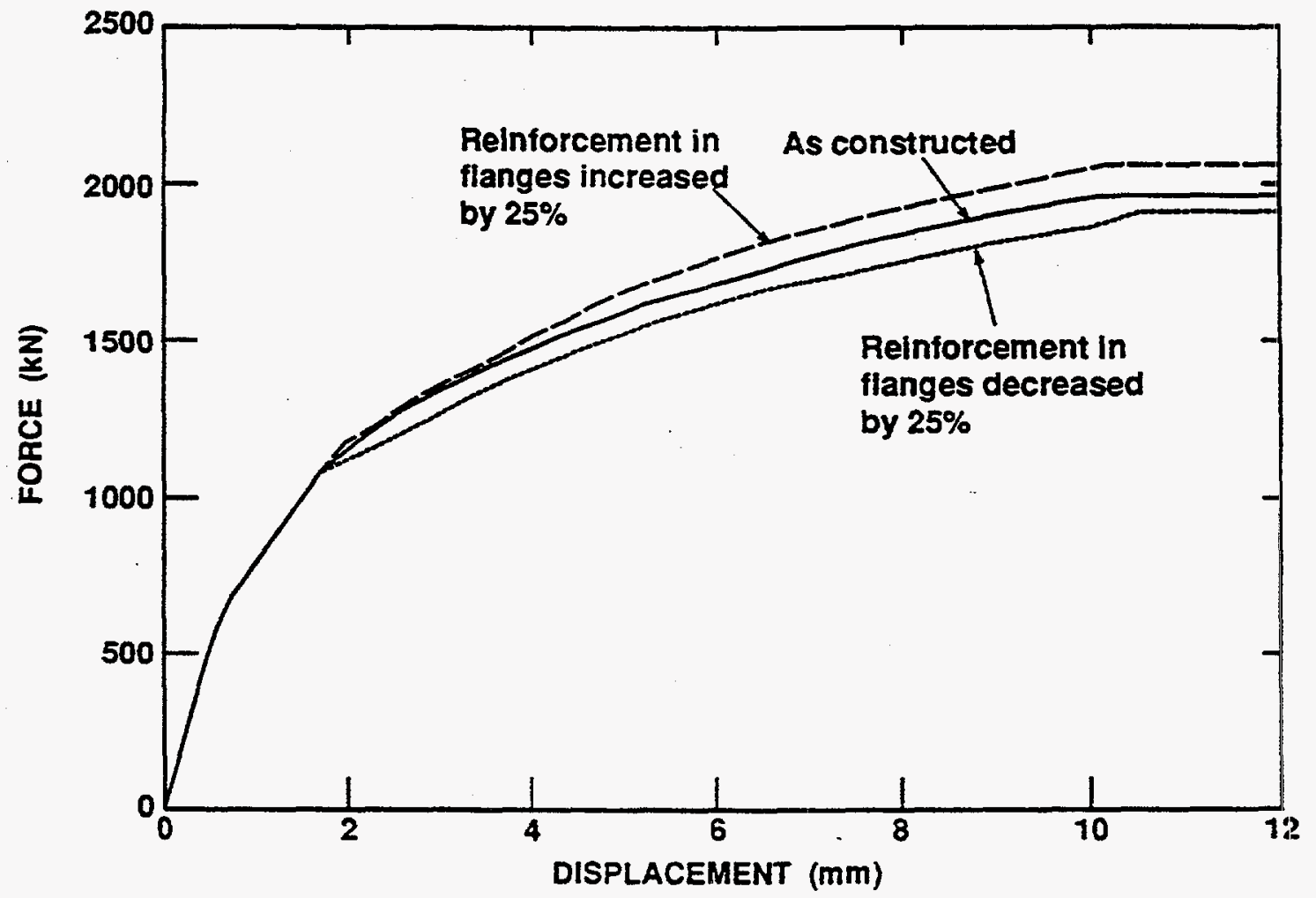

Figure 19

Influence of Vertical Reinforcement in Flange

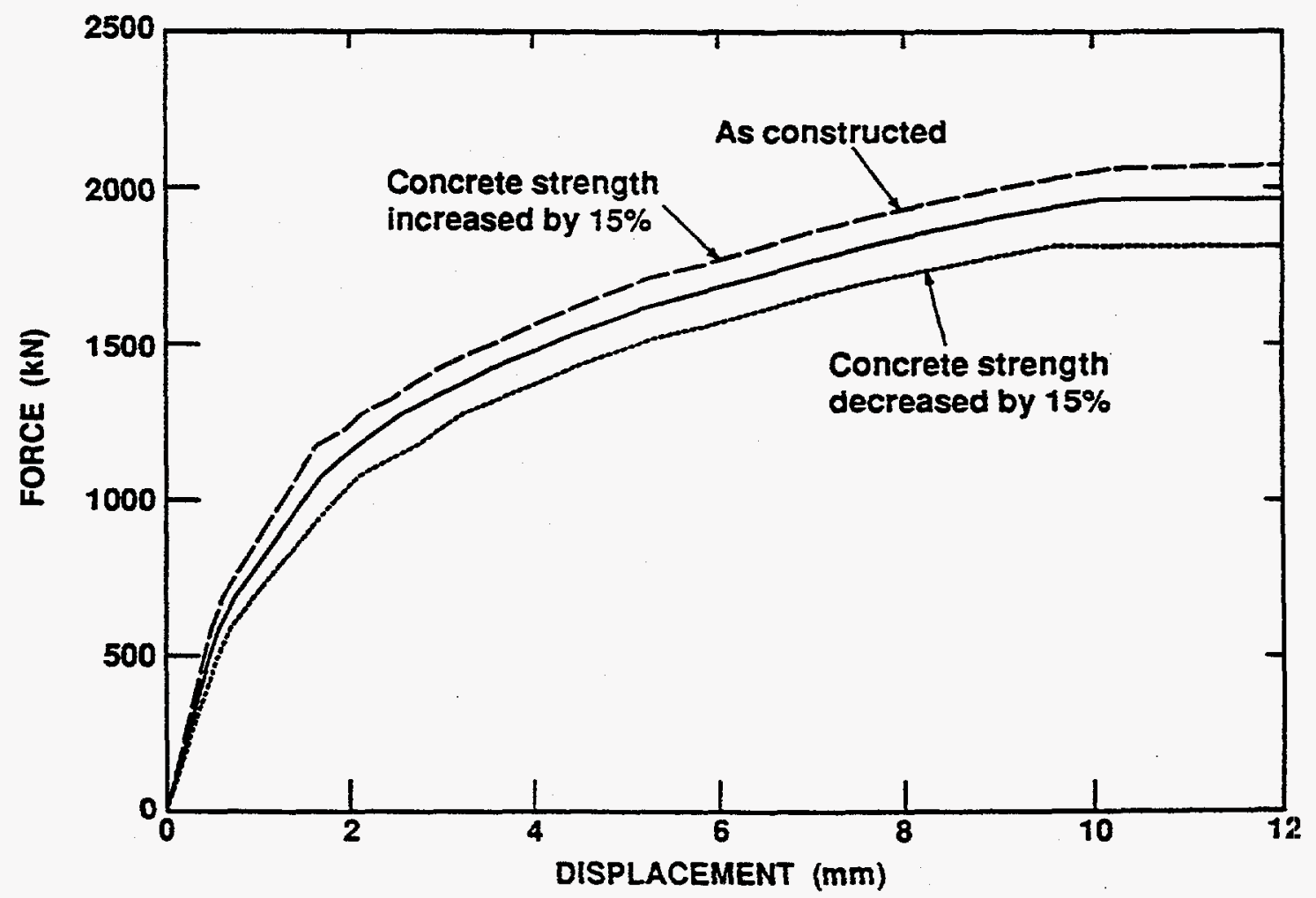

Figure 20

Influence of Concrete Strength 


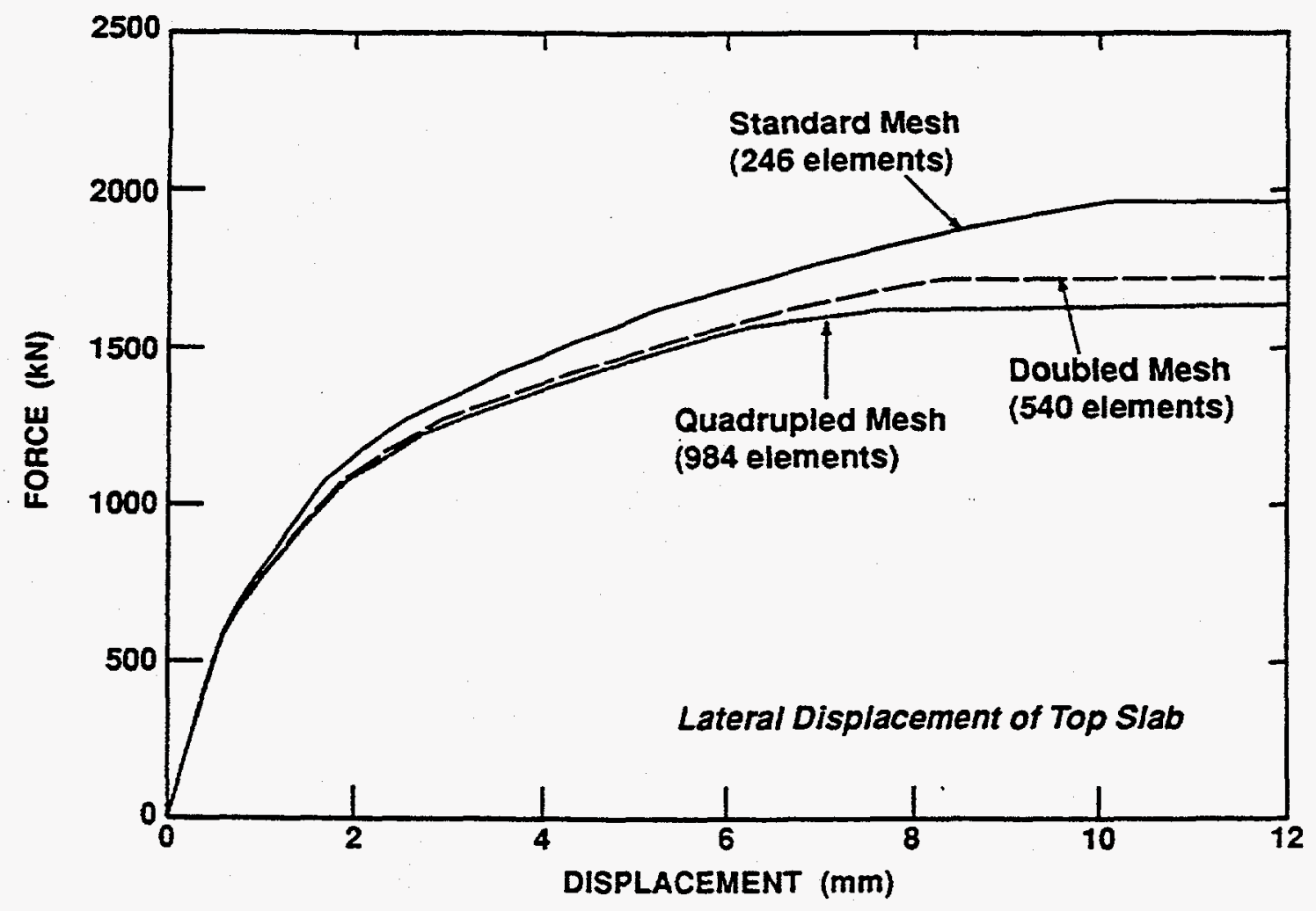

Flgure 21

Influence of Mesh Size

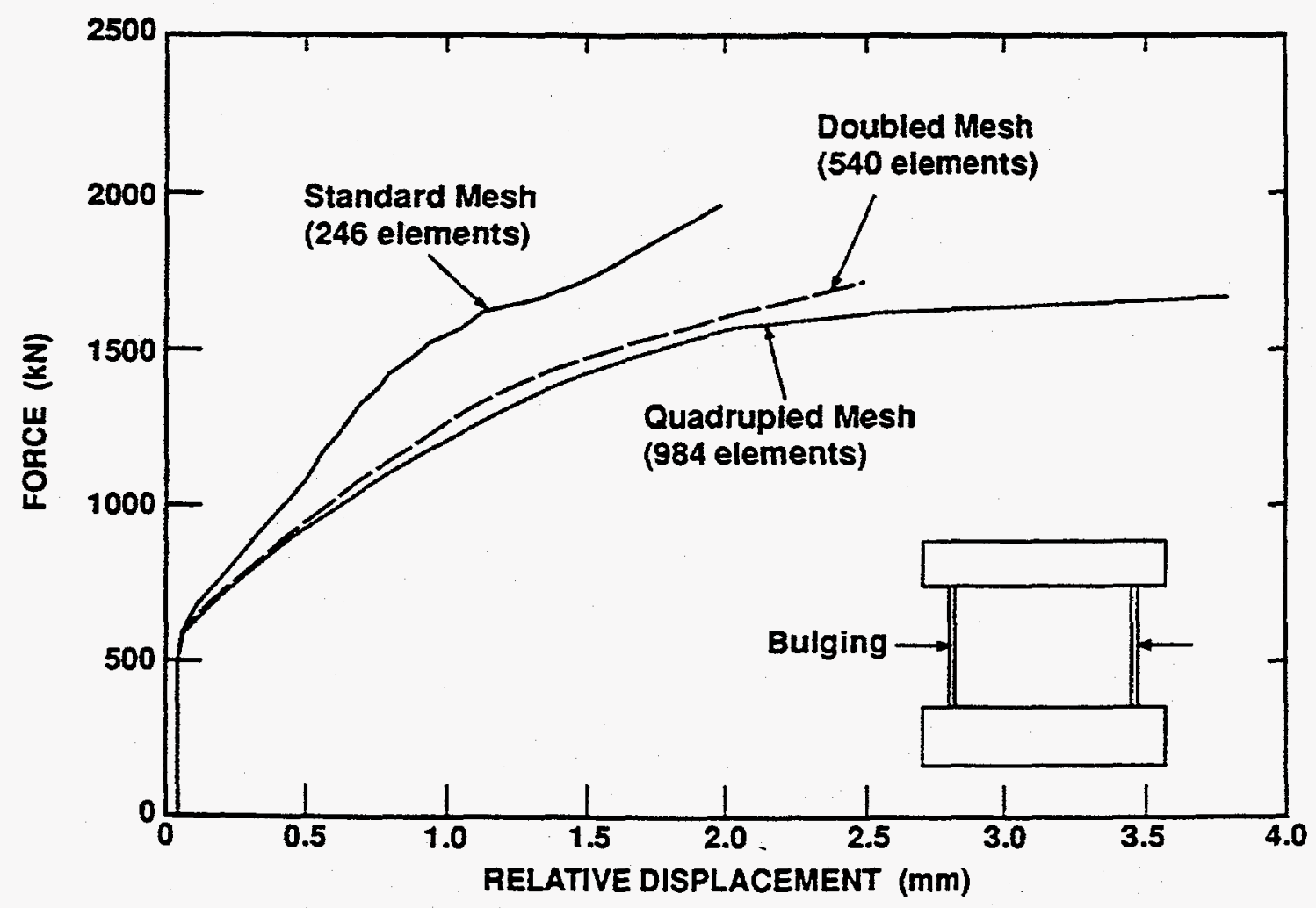

Figure 22

Predicted Bulging of Web at Mid-Height 


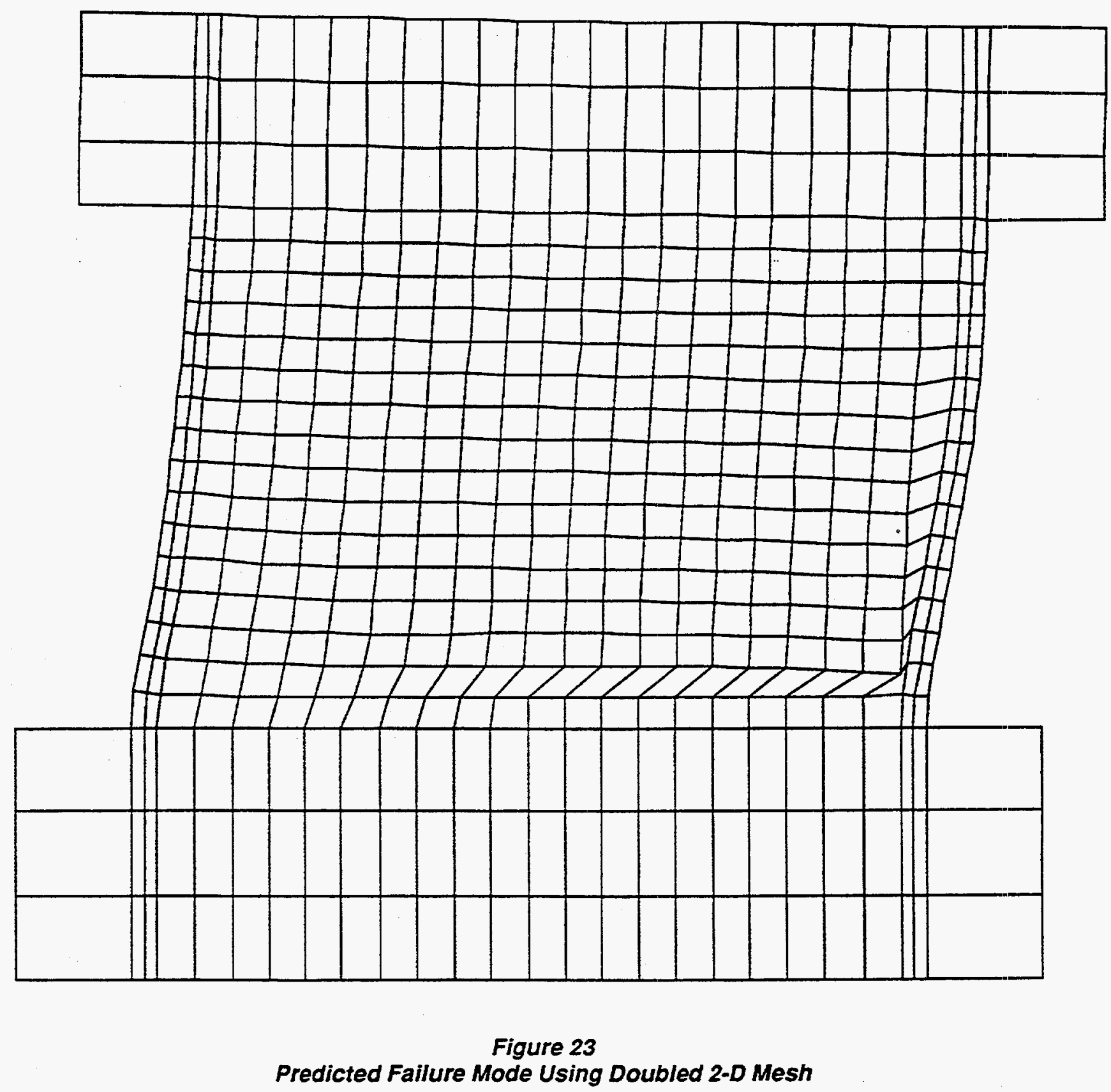




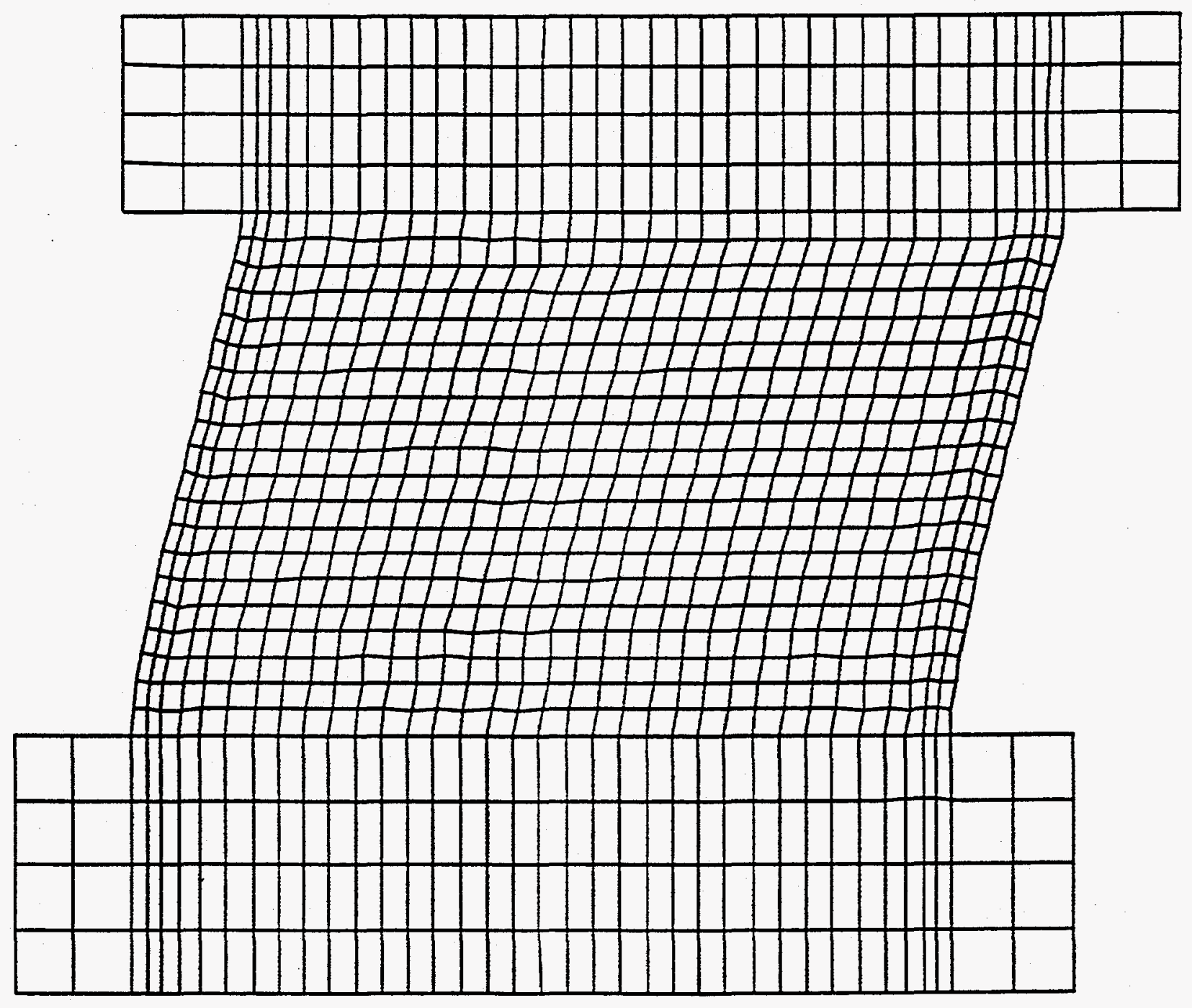

Figure 24

Predicted Failure Mode Using Qudrupled 2-D Mesh 
APPENDIX I

Constitutive Modelling (Manuscript Submitted For Publication) 


\title{
A Constitutive Model for Analysis of Reinforced Concrete Solids
}

\author{
R.G. Selby* \\ F.J. Vecchio ${ }^{\dagger}$
}

October 16. 1994

\begin{abstract}
This paper describes formulations intended to expand the applicability of the modified compression field theory for general three-dimensional analysis of reinforced concrete solids. Proposed stress-strain relationships are based on an orthotropic nonlinear elastic model that acknowledges the asymmetric response of plain concrete under multiaxial stress conditions. Lateral expansion of concrete subjected to compression is included for all stress states, even those involving tension. The formulation accounts for complex behaviour such as concrete strength enhancement due to confinement, concrete strength degradation due to transverse cracking, tension stiffening and crack slip. Provisions to more accurately reflect the response of high strength concrete are also presented. A sample analysis of a reinforced concrete shear wall is presented to illustrate several features of the stress-strain model.
\end{abstract}

- Research Engineer, Morrison Hershfield Ltd., Toronto

'Professor, University of Toronto, Toronto 


\section{Introduction}

In an effort to determine more realistic constitutive relationships for cracked reinforced concrete, Vecchio and Collins (1982) tested a series of reinforced concrete panels. From these tests, the modified compression field theory (MCFT), which included stress-strain relationships for cracked reinforced concrete under plane stress conditions, was derived. In this theory, directions of principal stress and directions of principal strain were assumed to coincide. Cracked concrete was treated as an orthotropic material with its own stress-strain relationships in terms of average stress and average strain. Of particular significance was that these relationships reflected the reduced compressive strength of concrete in the presence of large transverse tensile strains, and accounted for the significant tensile stresses that exist in the concrete between cracks. Local stress conditions at cracks were also examined. The modified compression field theory was shown to be capable of accurately predicting the response of reinforced concrete elements subjected to in-plane shear and normal stresses.

Adeghe (1986) and Stevens (1987) were the first to use the constitutive models of the modified compression field theory in finite element work. Each of these programs used a plane stress element and was cast in a tangent stifness algorithm. Some numerical difficulties were encountered in modelling the softening branches of the concrete stress-strain curves in tension and compression. Also, the tangent stiffiness matrix, computed according to the modified compression field theory, is unsymmetric. In an attempt to overcome these problems, both Adeghe and Stevens were forced to compromise the formulations of the modified compression field theory.

To avoid the problems encountered by these tangent stiffness approaches, Vecchio (1989) developed a secant stiffness algorithm for analysis of reinforced concrete subject to conditions of plane stress. By using a secant stiffness based approach, the asymmetry of the stiffness matrix was avoided while still completely representing the modified compression field theory. The program proved to be numerically robust, while predicting the strength: deformation 
pattern and failure mode with good accuracy for a variety of membrane structures (Vecchio 1992, 1990, 1989).

The constitutive relationships of the modified compression field theory were first used in a three-dimensional finite element context by Vecchio and Selby (1991). The plane stress constitutive relationships were tentatively extrapolated to three dimensions. In accordance with the two dimensional formulation of Vecchio, the program (SPARCS) used a secant stiffness algorithm with a smeared, rotating crack model. As a check on the proposed algorithm, a series of reinforced concrete beams subjected to varying conditions of flexure and torsion were modelled. The program was able to provide realistic predictions of ultimate load, failure mode, load-deformation response and locai strain conditions.

This paper discusses some of the recent research work (Selby 1993) that has been directed at improving these tentative three dimensional constitutive reiationships. In particular, models to include the effects of lateral expansion and confinement are formulated to be compatible with the existing cracked concrete theory. The confined concrete theory is implemented in an orthotropic nonlinear elastic model, consistent with the crack model. Simple confined concrete stress-strain relationships are developed from basic experimentally observed behaviour of concrete specimens subjected to multiaxial compressive stresses. These constitutive relationships represent a first attempt at developing triaxial theory to be used with the modified compression field theory.

A sample full scale finite element analysis of a shear wall is also presented. The analysis examines the effects of accounting for confinement and lateral expansion in a compression field based analysis. 


\section{Finite Element Formulation}

The finite element program SPARCS was developed (Vecchio and Selby 1991) to allow for compression field analysis of reinforced concrete solids. A relatively simple approach is taken with respect to the finite elements and the solution techniques that are employed. The program is based on an iterative linear elastic formulation in which secant moduli are defined and progressively refined according to current local stress/strain states. The program uses relatively low powered brick, wedge and truss elements based on linear displacement functions.

The stiffness matrix. [ $k]$, for a particular element is found from the well known expression

$$
[k]=\int[B]^{T}[D][B] d V
$$

where $[D]$ is the composite material stiffness matrix and $[B]$ is the strain displacement matrix which is derived from the assumed element displacement functions. Note that this integral was evaluated for each of the three different elements and the results built into the program, thus avoiding costly numerical integration procedures. Small displacements and infinitesimal rotations were assumed in the derivation of $[B]$.

The element stiffness matrices are assembled to form the giobal stiffiness matrix [K]. Nodal forces, $\{F\}$, are related to the nodal displacements, $\{r\}$, through $[K]$ :

$$
[K]\{r\}=\{F\}
$$

This system of equations is solved for the nodal displacements using a blocked bandwidth technique.

Element strains, $\{\epsilon\}$, and element stresses, $\{f\}$, are evaluated once the nodal displacernents are known.

$$
\begin{aligned}
& \{\epsilon\}=[B]\{r\} \\
& \{f\}=[D]\{\epsilon\}
\end{aligned}
$$


The composite material stiffness matrix $[D]$ must account for the contributions from concrete and any smeared steel, and for the directional dependence of each material. The matrix is evaluated as

$$
[D]=\left[D_{c}\right]+\sum_{i=1}^{n}\left[D_{s}\right]_{i}
$$

where the concrete stiffness matrix defined in the principal directions, $\left[D_{c}\right]^{\prime}$, is transformed to the global axes by

$$
\left[D_{c}\right]=\left[\mathcal{T}_{c}\right]^{T}\left[D_{c}\right]^{\prime}\left[T_{c}\right]
$$

and the stiffness matrix for each smeared reinforcement component in the local axes, $\left[D_{s}\right]_{i}^{\prime}$, is transiormed to the global axes according to

$$
\left[D_{s}\right]_{i}=\left\{T_{s}\right]_{i}^{T}\left[D_{s}\right]_{i}^{\prime}\left[T_{s}\right]_{i}
$$

The transformation matrix $[\mathrm{T}]$ is given by

$$
[T]=\left[\begin{array}{cccccc}
k_{1}{ }^{2} & l_{1}{ }^{2} & m_{1}{ }^{2} & k_{1} l_{1} & l_{1} m_{1} & m_{1} k_{1} \\
k_{2}{ }^{2} & l_{2}{ }^{2} & m_{2}{ }^{2} & k_{2} l_{2} & l_{2} m_{2} & m_{2} k_{2} \\
k_{3}{ }^{2} & l_{3}{ }^{2} & m_{3}{ }^{2} & k_{3} l_{3} & l_{3} m_{3} & m_{3} k_{3} \\
2 k_{1} k_{2} & 2 l_{1} l_{2} & 2 m_{1} m_{2} & k_{1} l_{2}+k_{2} l_{1} & l_{1} m_{2}+l_{2} m_{1} & m_{1} k_{2}+m_{2} k_{1} \\
2 k_{2} k_{3} & 2 l_{2} l_{3} & 2 m_{2} m_{3} & k_{2} l_{3}+k_{3} l_{2} & l_{2} m_{3}+l_{3} m_{2} & m_{2} k_{3}+m_{3} k_{2} \\
2 k_{3} k_{1} & 2 l_{3} l_{1} & 2 m_{3} m_{1} & k_{3} l_{1}+k_{1} l_{3} & l_{3} m_{1}+l_{1} m_{3} & m_{3} k_{1}+m_{1} k_{3}
\end{array}\right]
$$

where $k, l, m$ are the direction cosines of either the principal concrete strains (Equation 6) or the smeared reinforcement component (Equation 7 ). The composite material stiffness matrix will be fully populated and symmetric.

The constitutive relationships of the modified compression field theory are best implemented into secant stiffness based algorithms. In the proposed constitutive models, orthotropic theory is used for concrete in all stress states. Cracked concrete treated by the smeared crack approach is inherently modelled as an orthotropic material. In confined concrete, the adoption of an orthotropic model allows for the consideration of anisotropic behaviour near ultimate. 
The three-dimensional orthotropic materiai stiffness matrix can be written in the principal directions as

$$
\left[\mathrm{D}_{c}\right]^{\prime}=\frac{1}{\phi}\left[\begin{array}{cccccc}
E_{c 1}\left(1-\nu_{32} \nu_{23}\right) & E_{\mathrm{cl}}\left(\nu_{12}+\nu_{13} \nu_{32}\right) & E_{\mathrm{cl}}\left(\nu_{13}+\nu_{12} \nu_{23}\right) & 0 & 0 & 0 \\
E_{\Omega}\left(\nu_{21}+\nu_{31} \nu_{23}\right) & E_{c 2}\left(1-\nu_{31} \nu_{13}\right) & E_{c 2}\left(\nu_{23}+\nu_{11} \nu_{13}\right) & 0 & 0 & 0 \\
E_{c 3}\left(\nu_{31}+\nu_{21} \nu_{32}\right) & E_{c 3}\left(\nu_{32}+\nu_{12} \nu_{31}\right) & E_{e 3}\left(\nu_{23}+\nu_{11} \nu_{13}\right) & 0 & 0 & 0 \\
0 & 0 & 0 & \phi G_{c 12} & 0 & 0 \\
0 & 0 & 0 & 0 & \phi G_{c 23} & 0 \\
0 & 0 & 0 & 0 & 0 & \phi G_{c 31}
\end{array}\right]
$$

where $E_{c i}$ is the modulus of elasticity in the i-direction, the Poisson's ratio $\nu_{i j}$ is the component of strain in the $\mathrm{i}$-direction due to a stress in the $\mathrm{j}$-direction and

$$
\dot{0}=1-\nu_{32} \nu_{23}-\nu_{21} \nu_{12}-\nu_{31} \nu_{13}-\nu_{21} \nu_{32} \nu_{13}-\nu_{31} \nu_{12} \nu_{23}
$$

The three shear moduli are given by

$$
\begin{aligned}
& G_{\mathrm{c} 12}=\frac{E_{\mathrm{c} 1} E_{\mathrm{c} 2}}{E_{\mathrm{c} 1}\left(1+\nu_{12}\right)+E_{\mathrm{c} 2}\left(1+\nu_{21}\right)} \\
& G_{\mathrm{c} 23}=\frac{E_{\mathrm{c} 2} E_{\mathrm{c} 3}}{E_{\mathrm{c} 2}\left(1+\nu_{23}\right)+E_{\mathrm{c} 3}\left(1+\nu_{32}\right)} \\
& G_{\mathrm{c} 13}=\frac{E_{\mathrm{c} 1} E_{\mathrm{c} 3}}{E_{\mathrm{c} 1}\left(1+\nu_{13}\right)+E_{\mathrm{c} 3}\left(1+\nu_{31}\right)}
\end{aligned}
$$

To maintain symmetry in the stiffness matrix. the following three conditions must be satisfied:

$$
\begin{aligned}
& E_{c 1} \nu_{12}=E_{c 2} \nu_{21} \\
& E_{c 2} \nu_{23}=E_{c 3} \nu_{32} \\
& E_{c 1} \nu_{13}=E_{c 3} \nu_{31}
\end{aligned}
$$

For cracked concrete, most orthotropic material descriptions (i.e., smeared crack models) have assumed that the Poisson's effects are negligible (e.g., Hu and Schnobrich 1989, Vecchio 1989). All off-diagonal terms in Equation 9 become zero since the six Poisson's ratios are neglected; i.e., $\nu_{i j}=0$. This assumption is relatively good for many situations, but for cases in which 
the tensile strains in cracked concrete are relatively small, the lateral expansion of concrete arising from Poisson's effects can represent a significant portion of the total strains (Vecchio 1992). Too much strength degradation, due to transverse cracking, would be predicted in this situation. Also, in triaxial compressive stress conditions the lateral expansion effects cannot be neglected.

The assumption of symmetry in the secant orthotropic model appears to contradict the observed behaviour of concrete. The constitutive response of concrete in compression is characterized by a progressively softening stress-strain curve (Figure 1). Also, concrete exhibits a progressively higher proportion of lateral expansion as compression is increased. Considering the 2/3-plane of a specimen of triaxially compressed concrete for the case when $f_{c 3}<f_{c 2}$, it will generally be the case that $E_{c 3}<E_{c 2}$ and $\nu_{23}>\nu_{32}$. Thus, in general, Equation 16 cannot be satisfied and an elastic orthotropic formulation for concrete in accordance with the symmetry conditions cannot accurately represent behaviour in which expansion effects are significant. In the proposed model, the symmetry conditions are not enforced, but an approach is developed in which the asymmetry is handled through the material prestrain concept. Thus, the symmetry of the material stiffness matrices, and therefore the global stiffness matrix, is retained.

The three dimensional finite element SPARCS includes provisions to model prestrains arising from strain offset effects such as prestressing of reinforcement, shrinkage or expansion of concrete, and thermal expansion of either concrete or reinforcement. For concrete, a prestrain matrix, $\left\{\epsilon_{c}^{\circ}\right\}$, is defined relative to the giobal $x, y, z$ system

$$
\left\{\epsilon_{c}^{\circ}\right\}=\left\{\epsilon_{c=}^{o} \epsilon_{c y}^{o} \epsilon_{c z}^{\circ} \gamma_{c z}^{\circ} \gamma_{c y}^{0} \gamma_{c z}^{o}\right\}^{T}
$$

to account for all nonstress-related straining. A similar matrix is developed for the reinforcement prestrains. An equivalent force approach is then used to incorporate the prestrain effects. From the known prestrains, free nodal displacements $\left\{r_{c}\right\}$ and $\left\{r_{s}\right\}$ are calculated for 
the concrete and steel, respectively

$$
\begin{aligned}
& \left\{r_{c}\right\}=\int\left\{\epsilon_{c}^{\circ}\right\} d V \\
& \left\{r_{s}\right\}=\int\left\{\epsilon_{s}^{o}\right\} d V
\end{aligned}
$$

The equivalent nodal loads due to the prestrains, $\left\{F^{*}\right\}$ are calculated from

$$
\left\{F^{*}\right\}=\left[k_{c}\right]\left\{r_{e}\right\}+\sum_{i=1}^{n}\left[k_{s}\right]_{i}\left\{r_{s}\right\}_{i}
$$

where $\left[k_{c}\right]$ and $\left[k_{s}\right]_{i}$ are the element stiffness matrices evaluated separately for the concrete and each reinforcement component. These equivalent nodal forces due to the prestrains are then added to the externally applied nodal forces to determine the total nodal forces. The total nodal load vector is updated on each iteration since the equivalent nodal forces depend on the component stiffnesses.

This algorithm was modified to include the expansion effects due to Poisson's effects. The expansion strains in the principal directions are written as

$$
\left\{\epsilon_{c p}^{o}\right\}=\left\{\epsilon_{1}^{o} \epsilon_{2}^{o} \epsilon_{3}^{o}\right\}^{T}
$$

where

$$
\begin{aligned}
& \epsilon_{1}^{0}=-\nu_{12} \frac{f_{c 2}}{E_{c 2}}-\nu_{13} \frac{f_{\mathrm{c}}}{E_{\mathrm{c}}} \\
& \epsilon_{2}^{0}=-\nu_{21} \frac{f_{\mathrm{c} 1}}{E_{\mathrm{cl}}}-\nu_{23} \frac{f_{\mathrm{c}}}{E_{\mathrm{c} 3}} \\
& \epsilon_{3}^{0}=-\nu_{31} \frac{f_{\mathrm{c} 1}}{E_{\mathrm{c} 1}}-\nu_{32} \frac{f_{\mathrm{c} 2}}{E_{\mathrm{c}}}
\end{aligned}
$$

The expansion strains are then transformed to the global $x, y, z$ axes according to

$$
\left\{\epsilon_{c}^{0}\right\}=[T]\left\{\epsilon_{c p}^{0}\right\}
$$

where $[T]$ is the transformation that defines the orientation of the principal axes. Equivalent nodal loads are then calculated as outlined above. This formulation includes all straining arising from expansion in the right hand side of Equation 2. All of the expansion strairs are 
modelled through the prestrain concept so the material stiffness matrix then includes diagonal terms only

$$
\left[D_{\mathrm{c}}^{\prime}\right]=\left[\begin{array}{cccccc}
E_{\mathrm{c} 1} & 0 & 0 & 0 & 0 & 0 \\
0 & E_{\mathrm{c} 2} & 0 & 0 & 0 & 0 \\
0 & 0 & E_{\mathrm{c} 3} & 0 & 0 & 0 \\
0 & 0 & 0 & G_{\mathrm{c} 12} & 0 & 0 \\
0 & 0 & 0 & 0 & G_{\mathrm{c} 23} & 0 \\
0 & 0 & 0 & 0 & 0 & G_{\mathrm{c} 13}
\end{array}\right]
$$

where $E_{\mathrm{c} 1}, E_{\mathrm{c} 2}$ and $E_{\mathrm{c} 3}$ are the secant moduli

$$
\begin{aligned}
& E_{\mathrm{c} 1}=\frac{f_{\mathrm{c1}}}{\epsilon_{1}} \\
& E_{\mathrm{c} 2}=\frac{f_{\mathrm{c} 2}}{\epsilon_{2}} \\
& E_{\mathrm{c} 3}=\frac{f_{\mathrm{c} 3}}{\epsilon_{3}}
\end{aligned}
$$

and the shear moduli are given by

$$
\begin{aligned}
& G_{\mathrm{c} 12}=\frac{E_{\mathrm{c1}} E_{\mathrm{c} 2}}{E_{\mathrm{c} 1}+E_{\mathrm{c} 2}} \\
& G_{\mathrm{c} 23}=\frac{E_{\mathrm{c} 2} E_{\mathrm{c} 3}}{E_{\mathrm{c} 2}+E_{\mathrm{c} 3}} \\
& G_{\mathrm{cl3}}=\frac{E_{\mathrm{c} 1} E_{\mathrm{c} 3}}{E_{\mathrm{c} 1}+E_{\mathrm{c} 3}}
\end{aligned}
$$

The principal strains used in Equations 27-29 are strains due to stress, not total strains. That is, any nonstress-related strains are first subtracted from the total strains before calculating the principal values. The prestrain approach is valid for both uncracked and cracked concrete. An iterative procedure is required for finite element analysis based on this approach.

The concept can be further explained with the example shown in Figure 2. Consider the linear elastic biaxial element subjected to the uniaxial stress $f_{c 2}$. In the traditional approach, Equation 9 would be used to determine the strains corresponding to this stress state. With the prestrain concept, no laterai expansion is included in the material stiffness matrix (see 
Equation 26). The deformation response comprises an axial strain under the applied stress (with no corresponding lateral strain) and a lateral strain produced from the fictitious lateral forces (with no corresponding axial strain). The magnitude of the lateral forces is chosen to produce the lateral strain determined from Equation 22.

The algorithm used to solve for the stresses and strains in a reinforced concrete solid under externally applied loads involves the following steps. An analysis begins by assuming linear elastic isotropic material properties. Element stiffness matrices are calculated and then the global stiffness is assembled. The load vector, which includes prestrain and expansion effects; is formed. Nodal displacements are determined, from which one strain tensor is caiculated for each element. Principal strains and corresponding directions are found. Evaluation of the concrete and steel stresses using the constitutive models permits determination of secant moduli and in turn new material stiffness matrices. Average secant moduli are calculated and if they have converged to the specified limit, then the load stage is complete. Otherwise: the newly calculated material stiffness values are used to perform another linear elastic analysis. Normally satisfactory convergence is achieved within 10 to 25 iterations. A flowchart of this procedure is shown in Figure 3.

\section{Material Modeling}

To describe the response of reinforced concrete, models are required for strength degradation due to cracking, strength enhancement due to confinement, pre- and post-peak stress-strain response in tension and compression, and concrete lateral expansion. These factors must be considered when determining the six material constants $\left(E_{1}, E_{2}, E_{3}, \nu_{12}, \nu_{21}, \nu_{13}\right)$ required for the orthotropic formulation described above.

The constitutive equations for multiaxial stress states are based on modifications to the concrete uniaxial stress-strain curve. The following curve, based on modifications to the work of Thorenfeldt, Tomasewicz and Jensen (1987) and Popovics (1973), was suggested for 
high strength concrete by Collins and Porasz (1989)

$$
f_{a 3}=f_{p} \cdot \frac{\epsilon_{3}}{\epsilon_{p}} \cdot \frac{n}{n-1+\left(\epsilon_{3} / \epsilon_{p}\right)^{n k}}
$$

where $n$ is given by

$$
n=0.80+\frac{f_{p}}{17}
$$

and $\mathrm{k}$ equals $\mathrm{I}$ for ascending branch and

$$
k=0.67+\frac{f_{p}}{62}
$$

for the descending branch (where $f_{c}^{\prime}$ is in $\mathrm{MPa}$ ). Note that $f_{p}$ and $\epsilon_{p}$ are the peak stress and the strain at peak stress, respectively.

For this curve, the strain at peak stress under uniaxial compression is estimated from

$$
\epsilon_{0}=\frac{n}{n-1} \cdot \frac{f_{c}^{\prime}}{E_{c}}
$$

The effect of the cylinder strength on the shape of this curve is shown in Figure 4a. The uniaxial stress-strain response of high strength concrete is more linear in the ascending branch and the descending branch drops off more sharply as the concrete strength increases.

Lateral confining stresses increase the strength, stiffness and strain at peak stress of concrete cylinders. The strength enhancement is modelled by modifying the peak stress of the base curve. The failure surface of Hsieh et al. (1979)

$$
2.0108 \frac{J_{2}}{f_{c}^{\prime 2}}+0.9714 \frac{\sqrt{J_{2}}}{f_{c}^{\prime}} \div 9.1412 \frac{f_{\mathrm{cl}}}{f_{c}^{\prime}}+0.2312 \frac{I_{1}}{f_{c}^{\prime}}-1=0
$$

is used to find the stress required in the major compressive direction to cause failure, $f_{c b}$, in the presence of the stresses $f_{\mathrm{cl}}$ and $f_{\mathrm{c} 2}$. The stress $f_{\mathrm{c} f}$ is used as the peak stress of the base curve and a peak stress factor, $K_{\sigma}$ is defined as

$$
K_{\sigma}=\frac{f_{\mathrm{c} f f}}{f_{c}^{\prime}}
$$

While experimental evidence suggests the same factor can be applied to find the peak stress and the corresponding peak strain in cracked concrete, different factors must be applied to 
$f_{c}^{\prime}$ and $\epsilon_{o}$ for confined concrete. The peak strain increases much more rapidly than the peak stress as confining pressure is increased. To relate the peak stress factor and the strain at peak stress factor, $K_{\epsilon}$ a two part expression is implemented (Figure $4 \mathrm{~b}$ ). For low peak stress ratios $\left(K_{\sigma}<3\right)$, a fit to the data of Kupfer et al. (1969) is used

$$
K_{\epsilon}=0.2036 K_{\sigma}{ }^{4}-2.819 K_{\sigma}{ }^{3}+13.313 K_{\sigma}{ }^{2}-24.42 K_{\sigma}+13.718 \sqrt{K_{\sigma}}+1
$$

For higher peak stress ratios the expression of Richart et al. (1928) is adopted

$$
K_{\epsilon}=5 K_{\sigma}-4
$$

The coordinates of the apex of the base curve become

$$
\begin{gathered}
f_{p}=K_{\sigma} \cdot f_{c}^{\prime} \\
\epsilon_{p}=-\epsilon_{0}\left[K_{\sigma}\left(1-\frac{f_{\mathrm{c}}}{f_{\mathrm{c} 3 f}}\right)+K_{\epsilon}\left(\frac{f_{\mathrm{c}}}{f_{\mathrm{c} f}}\right)\right]
\end{gathered}
$$

The ratio $f_{c 3} / f_{c 3 f}$ is a measure of the degree of noninearity. When this value is low, the strain at peak stress is close to $K_{\sigma} \epsilon_{o}$. Vearer the ultimate strength, the strain at peak stress becomes closer to $K_{\epsilon} \epsilon_{0}$. The modified stress-strain curve is then used to determine all three concrete principal stresses from the corresponding principal strains.

Increased ductility is evident when concrete is confined. To simulate the descending branch (Figure 4c) of the stress-strain curve of confined concrete a modification was made to the modified Kent-Park model (Scott et al. 1982). The descending branch is

$$
f_{\mathrm{c} 3}=-f_{p}\left[1+Z_{m}\left(\epsilon_{\mathrm{c} 3}-\epsilon_{p}\right)\right] \leq-0.2 f_{p}
$$

where

$$
Z_{m}=\frac{0.5}{\frac{3+0.29 f_{c}^{\prime}}{145 f_{c}^{\prime}-1000} \cdot\left(\frac{\epsilon_{0}}{-0.002}\right)+\left(\frac{-I_{1}+f_{c i}}{170}\right)^{0.9}+\epsilon_{p}}
$$

Note that $I_{1}$ is the first stress invariant, $f_{c i}$ is the current stress in the principal direction under consideration, and both $\epsilon_{o}$ and $\epsilon_{p}$ are negative quantities. This equation assumes that $f_{c}^{\prime}$ is expressed in megapascals. 
Lateral expansion increases rapidly near the peak stress (Figure 4d). This behaviour is modelled by a fit to the uniaxial Poisson's ratio data of Kupfer et al. (1969)

$$
\nu_{i j}= \begin{cases}\nu_{0} & , 0>\epsilon_{j}>\frac{K_{\sigma} \epsilon_{0}}{2} \\ \nu_{0}\left[1+1.5\left(\frac{2 \epsilon_{i}}{K_{\sigma} \epsilon_{o}}-1\right)^{2}\right] \leq 0.5 & , \frac{K_{\sigma} \epsilon_{0}}{2}>\epsilon_{j}\end{cases}
$$

where $\nu_{0}$ is the initial Poisson's ratio. This relationship implies that only three Poisson's ratios are independent since

$$
\begin{aligned}
& \nu_{21}=\nu_{31} \\
& \nu_{12}=\nu_{32} \\
& \nu_{13}=\nu_{23}
\end{aligned}
$$

For uncracked concrete in tension, the initial Poisson's ratio is used. Upon cracking in the tensile principal direction, expansion normal to this direction is set to zero only (i.e., $\nu_{21}=$ $\nu_{31}=0$, but all other Poisson's ratios are nonzero). If the intermediate principal direction also cracks, $\nu_{12}$ and $\nu_{32}$ are equated to zero, too. All Poisson's ratios are zero if three orthogonal tensile failures occur.

In cracked concrete, large tensile strains perpendicular to the principal compressive direction reduce the concrete compressive strength. Thus. the compressive stress $f_{c 3}$ is made a function of the tensile principal strain, $\epsilon_{1}$, in addition to the compressive principal strain, $\epsilon_{3}$. The strength reduction factor $\beta$ (Figure 4 ) for cracked concrete is given by

$$
\beta=\frac{1}{1+K_{s} K_{f}}
$$

where

$$
\begin{gathered}
K_{s}=0.35\left(\frac{-\epsilon_{1}}{\epsilon_{3}}-0.280\right)^{0.80} \\
K_{f}=0.1825 \sqrt{f_{c}^{\prime}}
\end{gathered}
$$

and $f_{c}^{\prime}$ is expressed in $\mathrm{MPa}$. The $K_{s}$ factor accounts for the effect of the transverse straining and $K_{f}$ represents the influence of the concrete cylinder strength. High strength concrete 
is thought to exhibit a more pronounced compression softening effect due to the smoother fracture planes.

The peak stress and strain of the base curve are modified to account for this strength clegradation effect

$$
\begin{aligned}
& f_{p}=3 \cdot f_{c}^{\prime} \\
& \epsilon_{p}=3 \cdot \epsilon_{0}
\end{aligned}
$$

A linear tensile stress-strain relationship is used until the concrete tensile principal stress $f_{\mathrm{cl}}$, reaches the concrete tensile strength $f_{t}^{\prime}$, which is estimated from

$$
f_{t}^{\prime}=0.33 \sqrt{f_{c}^{\prime}} \quad(M P a)
$$

Due to the influence of bond, tensile stresses can develop in the concrete between cracks. Adding a descending branch in the post-cracked region accounts for this tension stifiening effect. The response of reinforced concrete in tension (Figure 4f) is given by

$$
f_{\mathrm{cl}}= \begin{cases}E_{\mathrm{c}} \cdot \epsilon_{1} & , 0<\epsilon_{1}<\epsilon_{c r} \\ \frac{f_{t}^{\prime}}{1+\sqrt{200 \cdot \epsilon_{1}}} & , \epsilon_{1}>\epsilon_{\mathrm{cr}}\end{cases}
$$

To determine the stress in the intermediate principal direction, Equations 48 through 52 are used with the appropriate base curve if $\epsilon_{2}$ is compressive, and Equation 54 is used if it is tensile.

The presence of average tensile stresses in the concrete implies local stress increases in the reinforcement at crack locations. Thus, local conditions must be checked to ensure that the concrete tensile stress calculated from Equation 54 can be transmitted across the crack. The average tensile stress in the concrete must not be greater than the sum of the reserve of strength of each reinforcement direction taken in the perpendicular direction to a crack. If this 
condition is violated, then $f_{c 1}$ must be reduced accordingly. This is expressed algebraically as

$$
f_{\mathrm{cl}} \leq \sum_{i=1}^{n} \cos ^{2} \cdot \theta_{c i} \cdot \rho_{i} \cdot\left(f_{y i}-f_{s i}\right)
$$

where $f_{y i}$ is the yield stress of the reinforcement, $f_{s i}$ is the average tensile stress in the reinforcement and $\theta_{c i}$ is the angle between the reinforcement and the normal to the crack surface. This check is made for the principal tensile direction and also the intermediate principal direction, if it is tensile.

Tensile stresses may also be limited if the required shear stresses cannot be supported on the crack surfaces. The local increases in the reinforcement stresses may require shear stresses on the crack surfaces for equilibrium. As the crack width increases, the ability of the concrete to transmit shear by aggregate interlock decreases. This may inhibit the local stress increases in the reinforcement and in turn limit the average concrete tensile stress. Crack slip is said to occur if the required shear stresses cannot be supported at higher crack widths. The crack width, $w$ is estimated from

$$
w=\epsilon_{1} \cdot s
$$

where $s$ is the crack spacing which must be estimated before an analysis is performed. This can be determined from the crack control characteristics of the reinforcement, or estimated from the reinforcement spacing. Vecchio and Collins (1986) suggested that in the absence of compressive stresses on the crack, the limiting shear stress (in MPa) on the crack can be estimated from

$$
v_{c i}^{\max } \leq \frac{0.18 \sqrt{f_{c}^{\prime}}}{0.3+\frac{24 w}{a+16}}
$$

where $a$ is the aggregate size. This equation requires units of $\mathrm{MPa}$ and mm. The calculated shear stress on the crack surface $v_{c i}$ in the tensile principal direction is compared against $v_{c i}^{\max }$. If the limiting shear stress is exceeded, then the average concrete tensile stress $f_{\mathrm{cI}}$ is reduced to $f_{\mathrm{cl}}^{*}$ according to

$$
f_{c 1}^{*}=\frac{v_{c i}^{m a x}}{v_{c i}} \cdot f_{c l}
$$


Reinforcement stresses are related to strains using an elastic-plastic formulation that includes strain hardening behaviour. The constitutive relationship, shown in Figure 5 , is expressed as

$$
f_{s i}= \begin{cases}E_{s} \epsilon_{s i} & , 0>\epsilon_{s i} \leq \epsilon_{y} \\ f_{y} & , \epsilon_{y}<\epsilon_{s i} \leq \epsilon_{s h} \\ f_{y}+E_{s h}\left(\epsilon_{s i}-\epsilon_{s h}\right) \leq f_{u} & , \quad \epsilon_{s i}>\epsilon_{s h}\end{cases}
$$

where $E_{s}$ is the modulus of elasticity, $E_{s h}$ is the modulus of strain hardening, $\epsilon_{s h}$ is the strain at the onset of strain hardening, $f_{y}$ is the yield strength and $f_{2 s}$ is the ultimate strength of the reinforcement. Perfect bond is assumed between the concrete and steel.

\section{Sample Analysis}

Lefas et al. (1990) tested several walls under conditions of constant axial load and monotonically increasing lateral load. Shear wall SW16, chosen for this analysis. had a height to width ratio of 1.0 (see Figure 6). The wall had a rectangular cross section and heavily reinforced, thickened regions at the top and bottom of the wall to facilitate load transfer. The wail was reinforced vertically with two layers of $8 \mathrm{~mm}$ diameter bars spaced at $60 \mathrm{~mm}$ and horizontally with two layers of $6.25 \mathrm{~mm}$ diameter bars spaced at $80 \mathrm{~mm}$. Closed ties were included near the edges of the wall, creating $140 \mathrm{~mm}$ wide concealed columns.

The wall showed a ductile response and was able to resist a higher than expected load. This was attributed to the confinement of the concrete in the concealed column at the base of the wall. Triaxial compressive stress conditions were reported for this region. This wall provides a good test for the proposed confinement model.

The concrete properties input to the program were a cylinder strength of $44.0 \mathrm{MPa}$, an initial secant modulus of $33150 \mathrm{MPa}$ and an initial Poisson's ratio of 0.15 . The reinforcement had yield strengths of $470 \mathrm{MPa}, 520 \mathrm{MPa}$ and $420 \mathrm{MPa}$ for the $8 \mathrm{~mm}, 6.25 \mathrm{~mm}$ and $4 \mathrm{~mm}$ bars, respectively. The modulus of elasticity of the reinforcement was $210000 \mathrm{MPa}$ and strain 
hardening was assumed to begin at a strain of $2.5 \times 10^{-3}$ with a strain hardening modulus of $10000 \mathrm{MPa}$.

A mesh of 310 brick elements was used to model the spreader beams and wall (Figure $7 a$ ). One element was used across the width of a concealed column. Loads were applied to the top spreader beam as equivalent nodal loads. The nodes along the lower edge of the base were fixed. A smeared steel model was used to account for the presence of the reinforcement. Two material zones were defined for the wall and one region each for the spreader beams (Figure 7b).

The uitimate horizontal load of $353 \mathrm{kN}$ obtained from the SPARCS analysis compared well with the experimental value of $355 \mathrm{kN}$. The analytical response involved flexural-shear cracking, followed by yielding of the vertical reinforcement on the tension side, and ultimately crushing of the concrete near the base on the compression side. This was in agreement with the experimentally observed response. The load-deformation response was also well represented. The deflection at the centreline of the top of the wall is plotted against the applied horizontal load in Figure 8a. The shape of the load-deformation curve matches the measured response, but the deflections are somewhat underestimated near ultimate.

Of particular interest is the calculated stress condition near the base on the compression side of the wall. The distribution of principal compressive stresses across the base of the wall at the ultimate load is shown in Figure $8 \mathrm{~b}$. The theoretical principal compressive stress on the compression side of the wall was equal to $1.52 f_{c}^{\prime}$. At this location, triaxial compressive stress conditions resulted because of the passive confinement provided by the hoop steel. The theoretical principal compressive stresses in the concrete were $f_{\mathrm{c} 1}=-1.39 \mathrm{MPa}, f_{\mathrm{c} 2}=-5.10$ $\mathrm{MPa}$ and $f_{\mathrm{c}}=-67.0 \mathrm{MPa}$. The reinforcement in the out-of-plane direction was approaching yield. The principal compressive strain of $-5.80 \times 10^{-3}$ was well into the post-peak regime.

An additional analysis was run without consideration of expansion and strength increase effects. The maximum horizontal applied load was reduced to $285 \mathrm{kN}$ (see Figure $8 \mathrm{a}$ ). There 
was a substantial reduction in the strength and in the ductility near ultimate, but little influence at low to intermediate load levels. This analysis failed by crushing of the concrete at the base on the compression side at a stress equal to the cylinder strength. Therefore, the confinement model proved crucial in providing a realistic response for this wall.

The SPARCS program has also been able to successfully capture the response of a variety of reinforced concrete elements, including shear paneis, beam elëments and shell elements (Selby 1993). The program has also been used to conduct local nonlinear analyses of concrete offshore platforms to investigate reasons for structural failure (Collins et al. 1994), and to confirm the design of critical components (Selby et al. 1994). Work is progressing with regards to application to confined columns.

\section{Conclusions}

A constitutive model was presented for predicting the response of reinforced concrete solids subject to short term, monotonic loading. The model was built into a finite element program thereby permitting three-dimensional noniinear analyses of complex reinforced concrete structures.

A confined concrete model was developed to be compatible with the secant stiffness based modified compression field theory, cracked concrete formulation. The simple constitutive equations proposed for confined concrete were capable of predicting concrete strength enhancement, dilatancy and post-peak compressive response under triaxial compressive stresses.

The orthotropic nonlinear elastic secant algorithm was a successful framework for the confined concrete model. The theory accounted for anisotropic behaviour, but did not require enforcement of the unrealistic symmetry conditions. The prestrain approach handled the asymrnetry of the material stiffness matrices in a numerically stable manner, with only a modest increase in storage and solution time requirements. 
The formulation presented herein is simple and fexible. Any set of realistic constitutive relationships can easily be incorporated into the program. Further work needs to be directed at improving the preliminary confined concrete stress-strain model. Also, there is currently a lack of adequate experimental data from which to calibrate three-dimensional constitutive relationships for cracked reinforced concrete. The influence of the intermediate principal strain on the compression softening and tension stiffening effects could be quantified once suitable data is obtained.

The analysis of a shear wall demonstrated the significance of the confinement model in providing a good estimate of strength. The concealed column at the edge of the wall was subjected to triaxial stress conditions. Ignoring confinement effects resulted in a significant decrease in wall capacity and ductility.

\section{References}

[1] Adeghe, L.N. (1986). "A finite element model for studying reinforced concrete detailing problems." thesis presented to the University of Toronto, at Toronto, Canada, in partial fulfillment of the requirements for the degree of Doctor of Philosophy.

[2] Collins, M.P., and Porasz, A. (1989). "Shear design for high-strength concrete." CEB Bulletin d'Information No. 193, pp77-83.

[3] Collins, M.P., Vecchio, F.J., and Selby, R.G. (1994). "Investigating the failure of an offshore structure." submitted to Concrete International, May 1994.

[4] Hu, H.T., and Schnobrich, W.C. (1990). "Nonlinear analysis of cracked reinforced concrete." American Concrete Institute Structural Journal, v87, n2, pp199-207.

[5] Hsieh, S.S., Ting, E.C., and Chen W.F. (1979). "An elastic-fracture model for concrete." Proc. 3d Eng. Mech. Div. Spec. Conf., ASCE, Austin, Texas, pp437-440. 
[6] Kupfer. H., Hilsdorf, K.H., and Rüsch. H. (1969). "Behaviour of concrete under biaxial stress." Journal of the American Concrete Institute, v66, n8, pp656-666.

[7] Lefas, I.D., Kotsovos, M.D., and Ambraseys, N.N. (1990). "Behaviour of reinforced concrete structural walls: Strength, deformation characteristics and failure mecharism." ACI Structural Journal, v87, n1, pp23-31.

[8] Popovics, S. (1973). "A numerical approach to the complete stress-strain curve of concrete." Cement and Concrete Research, v3, n5, pp553-599.

[9] Richart, F.E., Brandzaeg, A., and Brown, R.L. (1928). "A study of the failure of concrete under combined compressive stresses." Bulletin No. 185. University of Illinois Engineering Experimental Station. Lrbana. Illinois.

[10] Scott, B.D., Park, R, and Priestley, M.J.N. (1982). "Stress-strain behaviour of concrete confined by overlapping hoops at low and high strain rates." Journal of the American Concrete Institute, v79, $\mathrm{nl}, \mathrm{pp} 13-27$.

[11] Selby, R.G. (1993). "Three dimensional constitutive relations for reinforced concrete." thesis presented to the University of Toronto, at Toronto, Canada, in partial fulfillment of the requirements for the degree of Doctor of Philosophy.

[12] Selby, R.G., Vecchio, F.J., and Collins, M.P. (1994). "Analysis of reinforced concrete sections subjected to shear and high axial compression." submitted to Journal of Structural Engineering, ASCE, July 1994.

[13] Stevens, N.J. (1987). "Analytical modelling of reinforced concrete subjected to monotonic and reversed loadings." thesis presented to the University of Toronto, at Toronto, Canada, in partial fulfillment of the requirements for the degree of Doctor of Philosiophy.

[14] Thorenfeldt, E., Tomaszewicz, A., and Jensen, J.J. (1987). "Mechanical properties of high strength concrete and application in design." Proceedings of the Symposium 'Utitization of High Strength Concrete', Tapir, Trondheim, Norway. 
[15] Vecchio, F.J., and Collins, M.P. (1982). "The response of reinforced concrete to inplane shear and normal stresses." Publication No. 82-03, Dept. of Civil Engineering, University of Toronto, Toronto, Canada.

[16] Vecchio, F.J., and Collins, M.P. (1986). "The modified compression field theory for reinforced concrete elements subjected to shear." Journal of the American Concrete Institute, $\mathrm{v} 83, \mathrm{n} 2, \mathrm{pp} 219-231$.

[17] Vecchio, F.J. (1989). "Nonlinear finite element analysis of reinforced concrete membranes." ACI Structural Journal, v86, n1, pp26-35.

[18] Vecchio, F.J., and Selby, R.G. (1991). "Toward compression field analysis of reinforced concrete solids." Journal of Structural Engineering, ASCE, v117, n6, pp1740-1758.

[19] Vecchio, F.J. (1992). "Finite element modelling of concrete expansion and confinement." Journal of Structural Engineering, ASCE, v118, n9, pp2390-2406. 
Notation

$a$

[B]

$[D]$

$[D]_{c}$

$[D]_{c}^{\prime}$

$[D]_{s i}$

$E_{\mathrm{c}}$

$E_{\mathrm{cl}}$

$E_{\mathrm{c2}}$

$E_{\mathrm{c} 3}$

$E_{\text {s }}$

$E_{\text {sh }}$

$f_{c}^{\prime}$

$f_{c 1}$

$f_{2}$

$f_{C 3}$

$f_{c 3 f}$

$f_{p}$

$f_{s i}$

$f_{u i}$

$f_{y i}$

$f_{s i}$

$\{F\}$

$\left\{F^{*}\right\}$

$\{f\}$

$G_{c 12}$

$G_{c 23}$
= aggregate size

$=$ element strain displacement matrix

$=$ composite material stiffness matrix in global axes

$=$ concrete material stiffness matrix in global axes

$=$ concrete material stifiness matrix in principal directions

$=$ material stiffness in global axes of reinforcement component $\mathrm{i}$

= modulus of elasticity of concrete (initial tangent modulus)

$=$ concrete secant modulus in tensile principal strain direction

$=$ concrete secant modulus in intermediate principal strain direction

$=$ concrete secant modulus in compressive principal strain direction

= Young's modulus of reinforcement

$=$ modulus of strain hardening of reinforcement

$=$ compressive strength of concrete cylinder

$=$ tensile principal stress in concrete

$=$ intermediate principal stress in concrete

= compressive principal stress in concrete

$=$ required $f_{\mathrm{c} 3}$ to cause failure in presence of $f_{\mathrm{cl}}$ and $f_{\mathrm{c} 2}$

$=$ peak stress

$=$ average stress in i-direction reinforcement

$=$ ultimate stress of $\mathrm{i}$-direction reinforcement

$=$ yield stress of i-direction reinforcement

$=$ stress in i-direction reinforcement

= structure nodal force matrix

= equivalent nodal loads due to prestrains

$=$ element stress matrix (tensile stresses positive)

$=$ secant shear modulus of concrete relative to 1,2 axes

$=$ secant shear modulus of concrete relative to 2.3 axes 


$$
\begin{aligned}
& G_{\mathrm{el3}} \quad=\text { secant shear modulus of concrete relative to } 1.3 \text { axes } \\
& I_{1} \quad=\text { first invariant of stress tensor } \\
& J_{2} \quad=\text { second invariant of deviatoric stress tensor } \\
& K_{e} \quad=\text { strain at peak stress factor } \\
& K_{\sigma} \quad=\text { peak stress factor } \\
& k \\
& {[K]} \\
& \text { [k] } \\
& {\left[k_{\mathrm{c}}\right]} \\
& {\left[k_{s}\right]_{i}} \\
& l \\
& m \\
& \{r\} \\
& \left\{r_{c}\right\} \\
& \left\{r_{s}\right\} \\
& \boldsymbol{s} \\
& =\text { direction cosine with respect to } x \text {-axis } \\
& \text { = structure stiffness matrix } \\
& =\text { element stiffness matrix } \\
& \text { = element stiffness matrix evaluated for concrete component } \\
& =\text { element stiffness evaluated for } \mathrm{i} \text {-direction reinforcement } \\
& =\text { direction cosine with respect to } y \text {-axis } \\
& =\text { direction cosine with respect to } z \text {-axis } \\
& \text { = structure nodal displacement matrix } \\
& =\text { free nodal displacements due to concrete prestrains } \\
& \text { = free nodal displacements due to steel prestrains } \\
& \text { = crack spacing } \\
& =\text { transformation matrix } \\
& \text { = maximum allowable shear stress on crack surface } \\
& =\text { shear stress on crack surface } \\
& \text { = crack width } \\
& \text { = slope factor for post-peak compressive curve } \\
& =\text { strength reduction factor } \\
& =\text { strain in concrete at cracking } \\
& =\text { concrete expansion strain in 1-direction } \\
& =\text { concrete expansion strain in 2-direction } \\
& =\text { concrete expansion strain in 3-direction } \\
& =\text { strain in concrete cylinder at peak stress } f_{c}^{\prime} \text { (a negative quantity) } \\
& =\text { strain at peak stress (a negative quantity) }
\end{aligned}
$$




$\begin{array}{ll}\epsilon_{s}^{\circ} & =\text { smeared reinforcement prestrains relative to global axes } \\ \epsilon_{s h} & =\text { strain at onset of strain hardening of reinforcement } \\ \epsilon_{s i} & =\text { strain in i-direction reinforcement } \\ \epsilon_{s}^{\circ} & =\text { smeared reinforcement prestrains relative to global axes } \\ \epsilon_{y} & =\text { yield strain of reinforcement } \\ \epsilon_{1} & =\text { tensile principai strain } \\ \epsilon_{1}^{c r} & =\text { tensile principal strain at a crack } \\ \epsilon_{2} & =\text { intermediate principal strain } \\ \epsilon_{3} & =\text { compressive principal strain } \\ \left\{\epsilon_{3}\right. & =\text { element strain matrix (tensile strains positive) } \\ \left\{\epsilon_{\mathrm{c}}^{\circ}\right\} & =\text { concrete prestrains reiative to global axes } \\ \left\{\epsilon_{c}^{\circ}\right\} & =\text { expansion strains in principai directions } \\ \left\{\epsilon_{s}^{\circ}\right\} & =\text { smeared reinforcement prestrains relative to global axes } \\ \rho_{i} & =\text { smeared steel reinforcement ratio in i-direction } \\ \nu_{i j} & =\text { component of strain in i-direction due to a stress in the j-direction } \\ \nu_{0} & =\text { initial Poisson's ratio } \\ \theta_{c i} & =\text { angle between i-direction steel and normal to crack surface }\end{array}$


Figure Captions

Figure 1 - Symmetry conditions in the orthotropic model

Figure 2 - Expansion prestrains concept

Figure 3 - SPARCS solution aigorithm

Figure 4 - Concrete constitutive model

(a) - Effect of $f_{c}^{\prime}$ on the shape of the base curve

(b) - Peak stress and corresponding peak strain in confined concrete

(c) - Compressive stress-strain model for confined concrete

(d) - Increase in Poisson's ratio near ultimate

(e) - Strength reduction in cracked concrete

(f) - Tension stiffening

Figure 5 - Reinforcement stress-strain relationship

Figure 6 - Details of Shear Wall SW16

Figure 7 - Finite element model of SW16

(a) - SPARCS finite element mesh

(b) - Reinforced concrete element type zones

Figure 8 - Finite element analysis results for SW16

(a) - Load-deformation response of SW16

(b) - Predicted stress distributions along base of wall at ultimate

Draft of October 16, 1994 


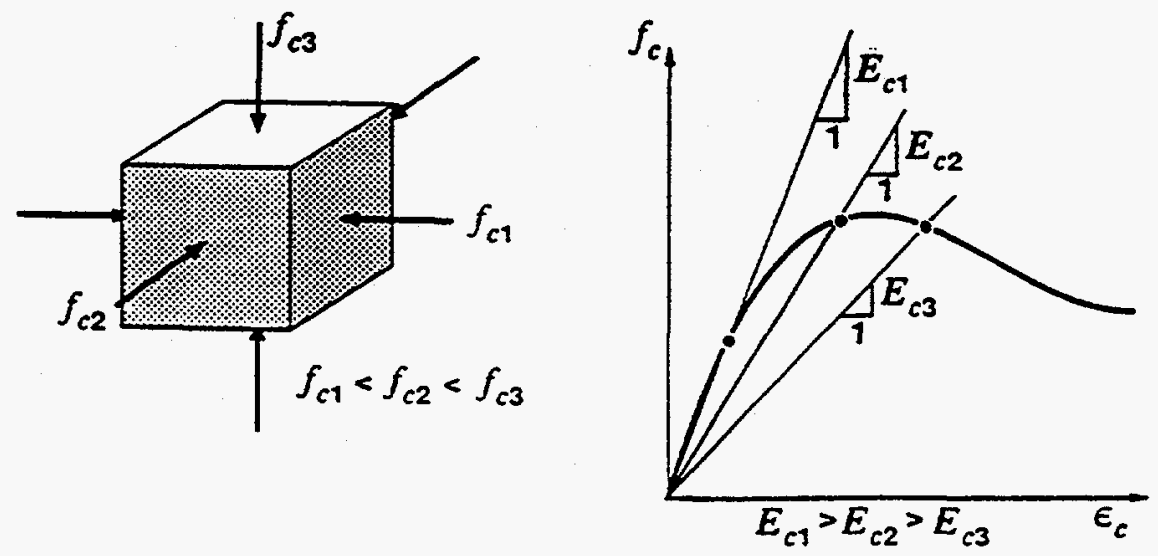

$$
\begin{aligned}
& \epsilon_{1}=\frac{f_{c 1}}{E_{c 1}}-v_{12} \frac{f_{c 2}}{E_{c 2}}-v_{13} \frac{f_{c 3}}{E_{c 3}} \\
& \epsilon_{2}=\frac{f_{c 2}}{E_{c 2}}-v_{21} \frac{f_{c 1}}{E_{c 1}}-v_{23} \frac{f_{c 3}}{E_{c 3}} \\
& \epsilon_{3}=\frac{f_{c 3}}{E_{c 3}}-v_{31} \frac{f_{c 1}}{E_{c 1}}-v_{32} \frac{f_{c 2}}{f_{c 3}}
\end{aligned}
$$

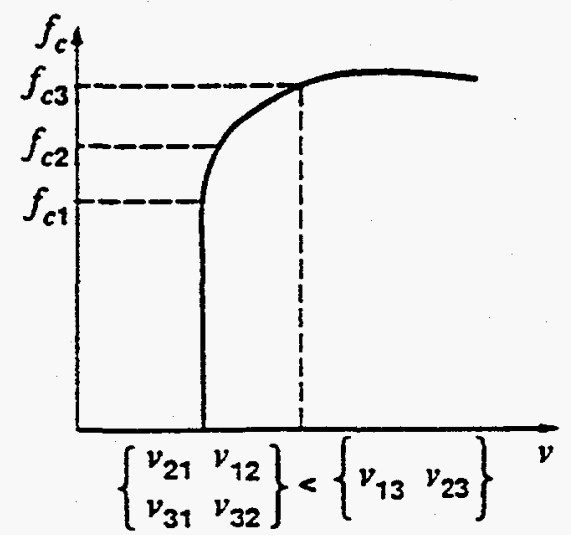




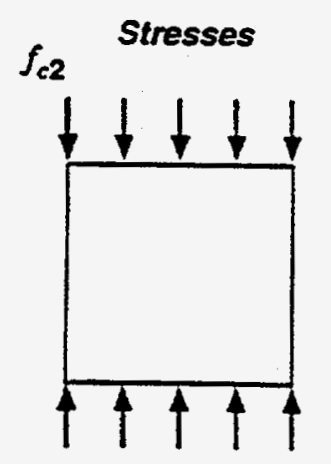

Nodal Forces

Strains
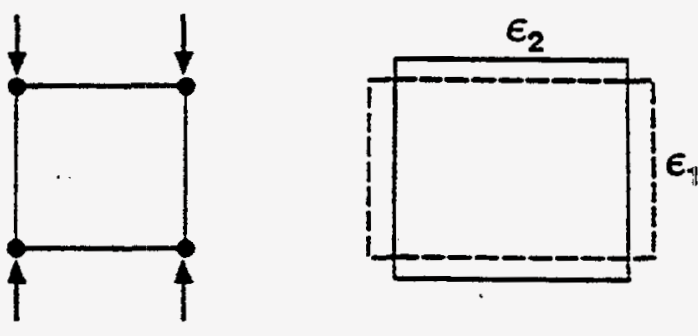

(a) Traditional Approach, $v_{12} \neq 0$

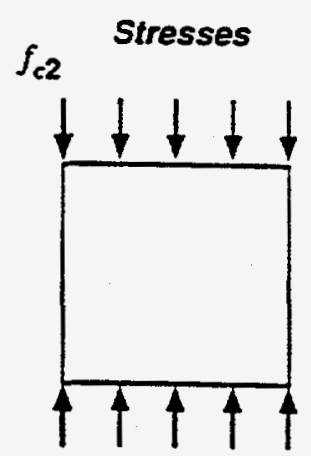

Nodal Forces

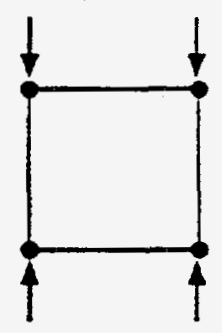

$f_{c 2}=0$

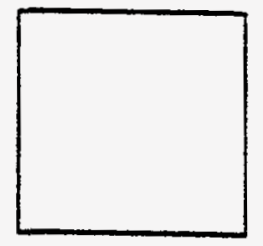

$+$
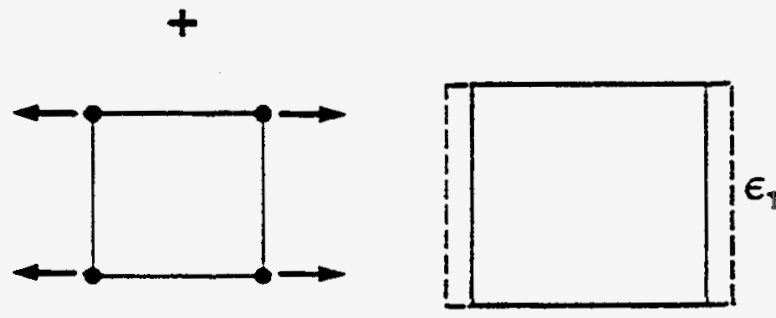

(b) Prestrain Approach, $v_{12}=v_{21}=0$
Strains

$\epsilon_{2}$

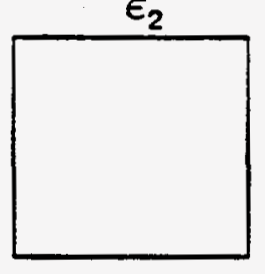

$\epsilon_{1}$

Figure 2 


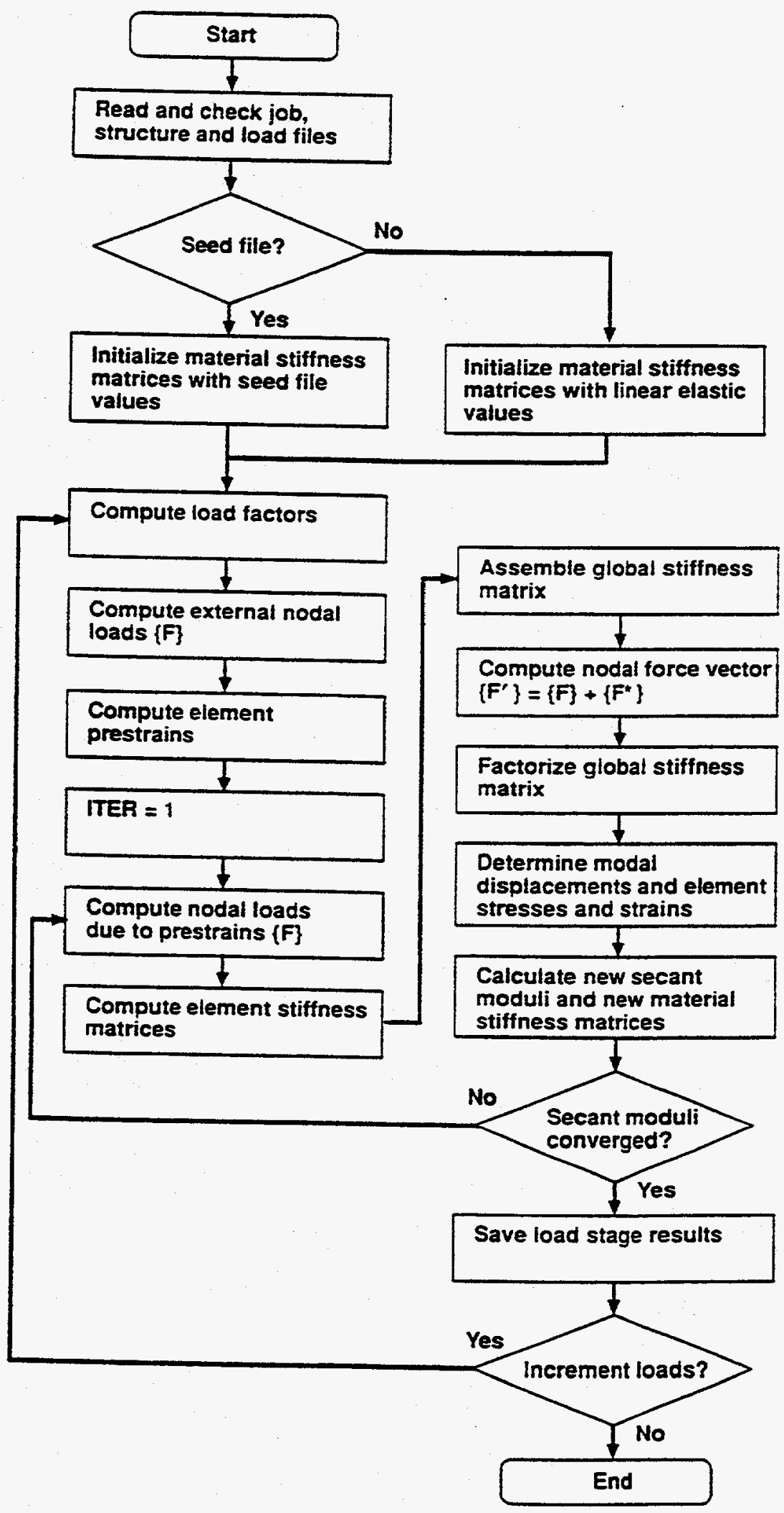

Figure 3 


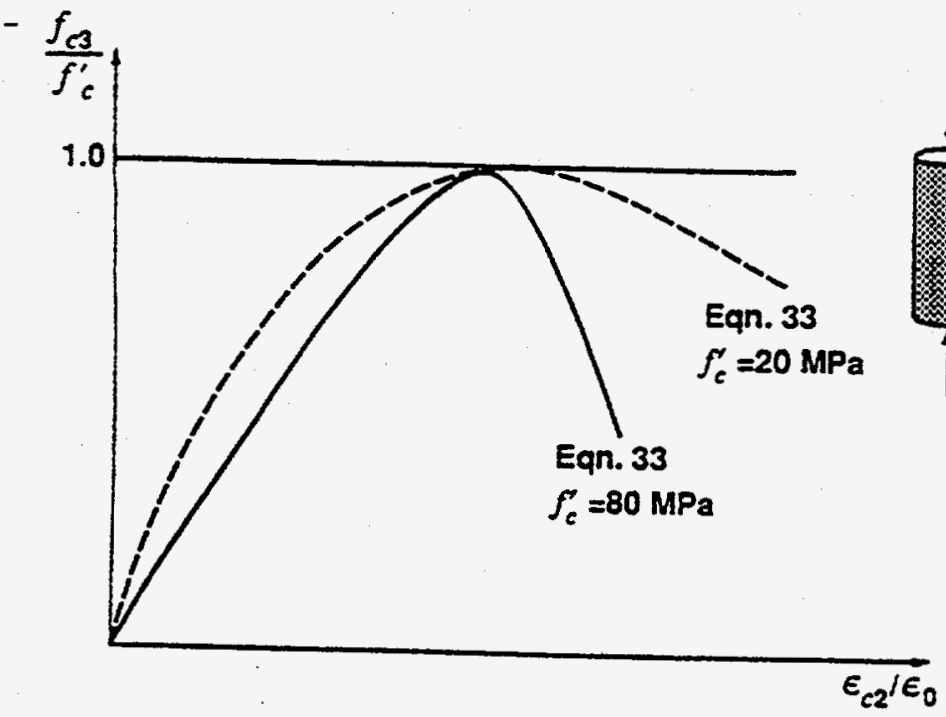

(a)

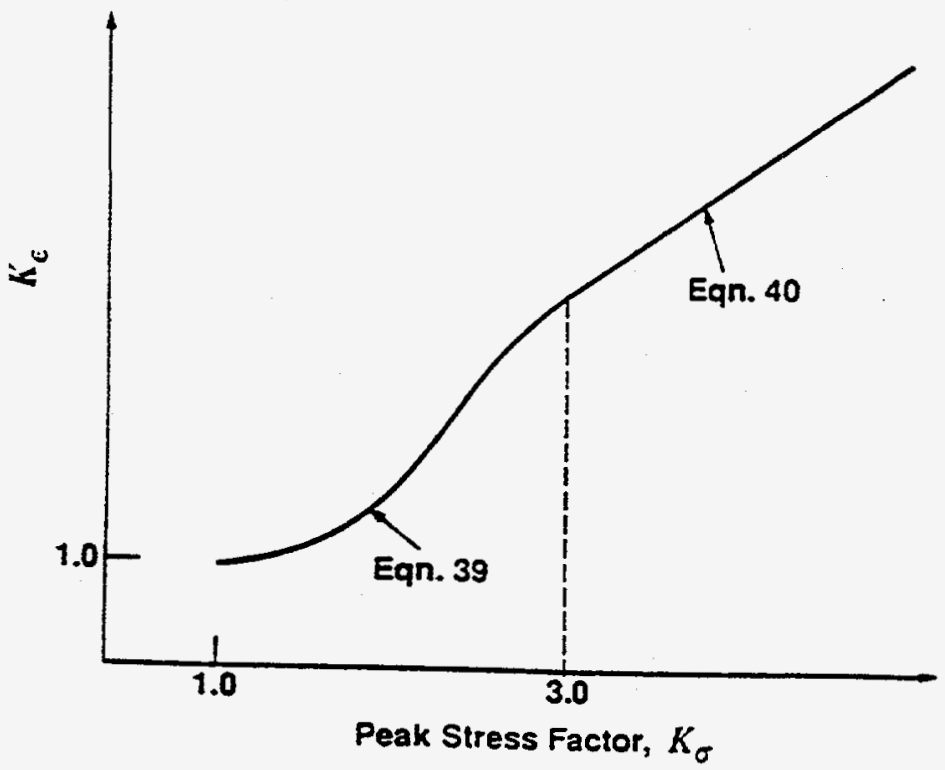

(b)

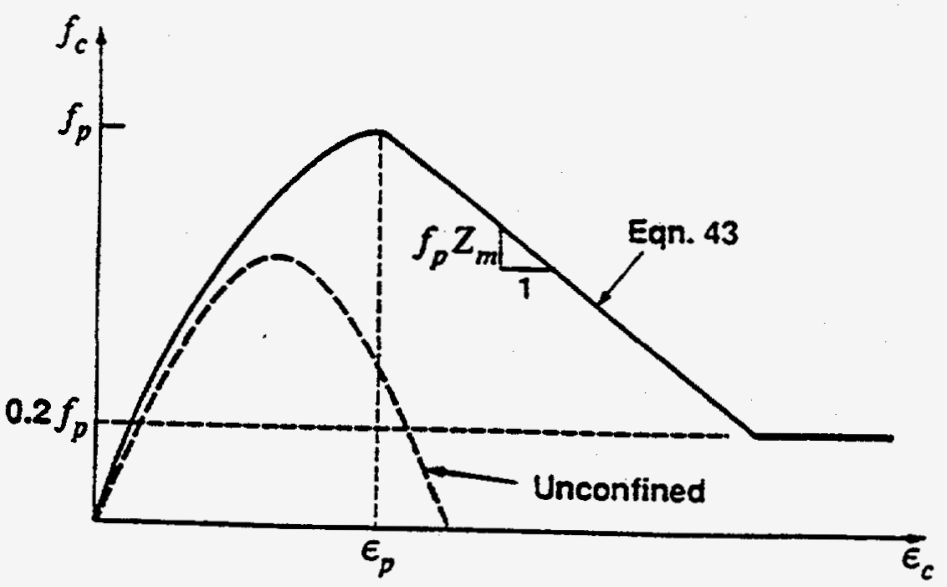

(c)

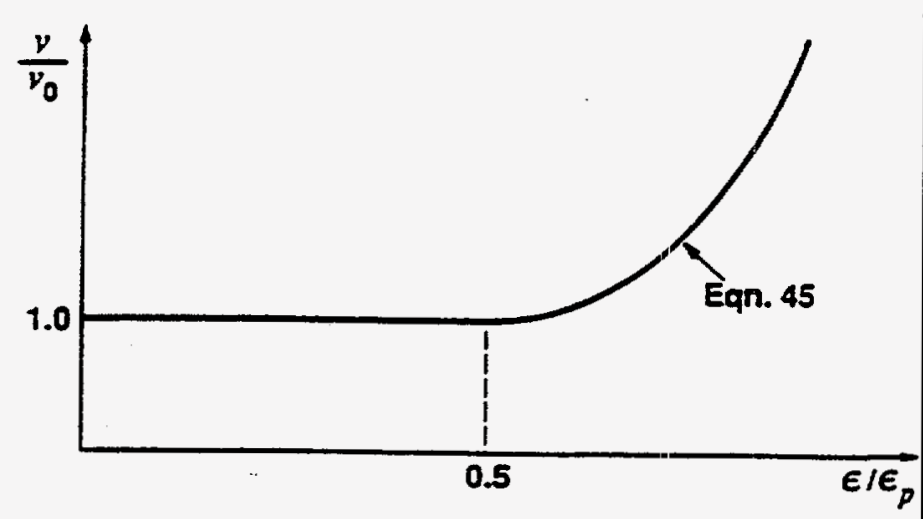

(d)

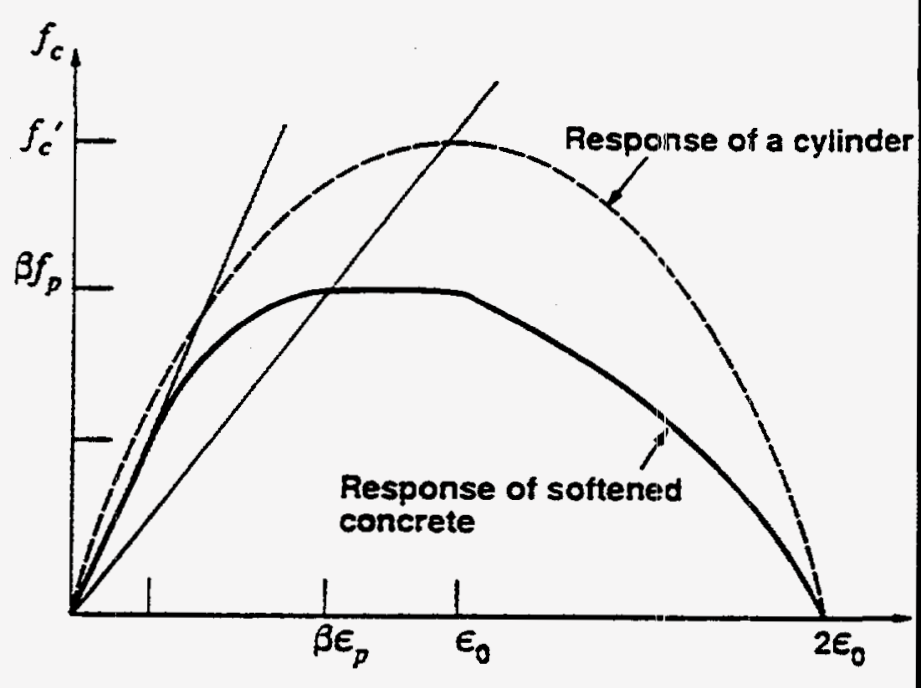

(e)

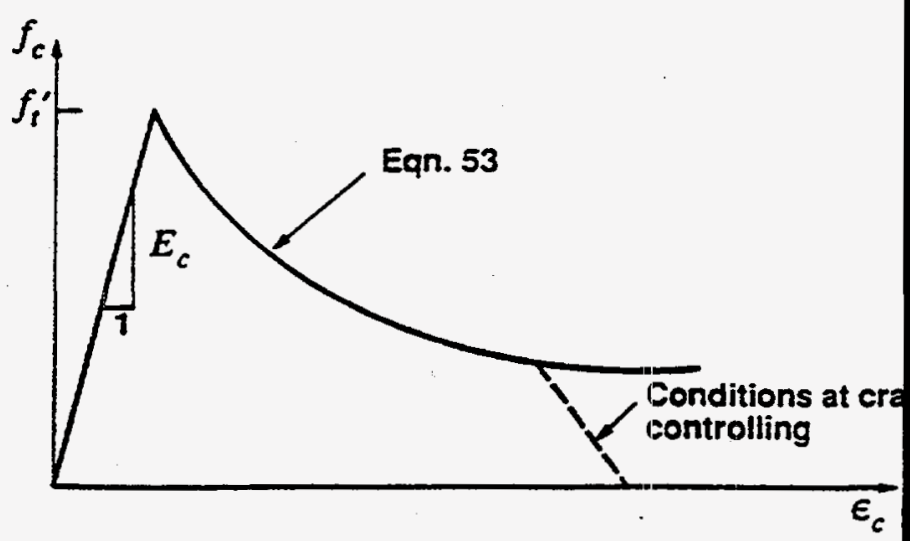

(f) 


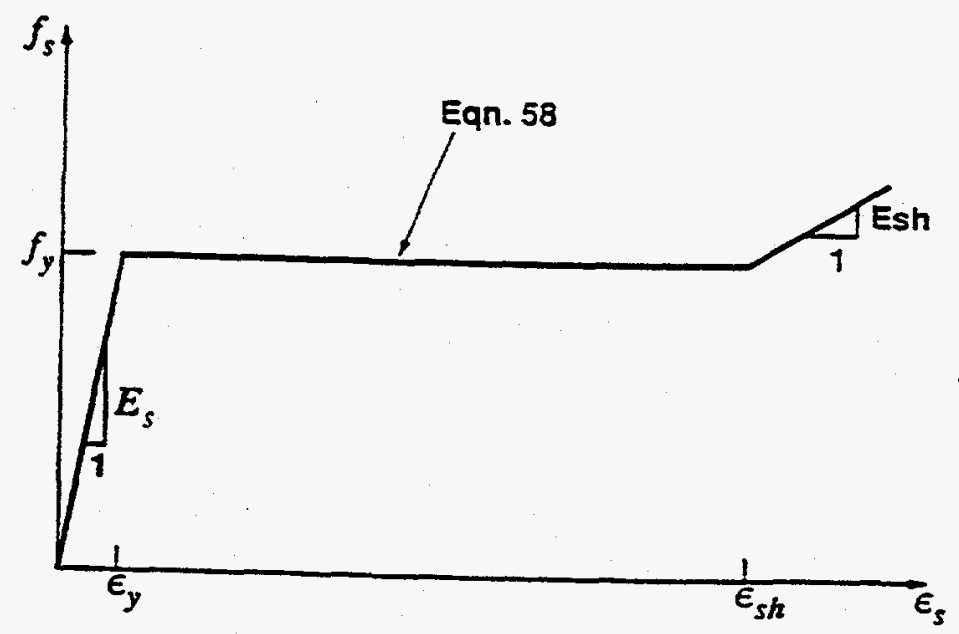

Figure 5 


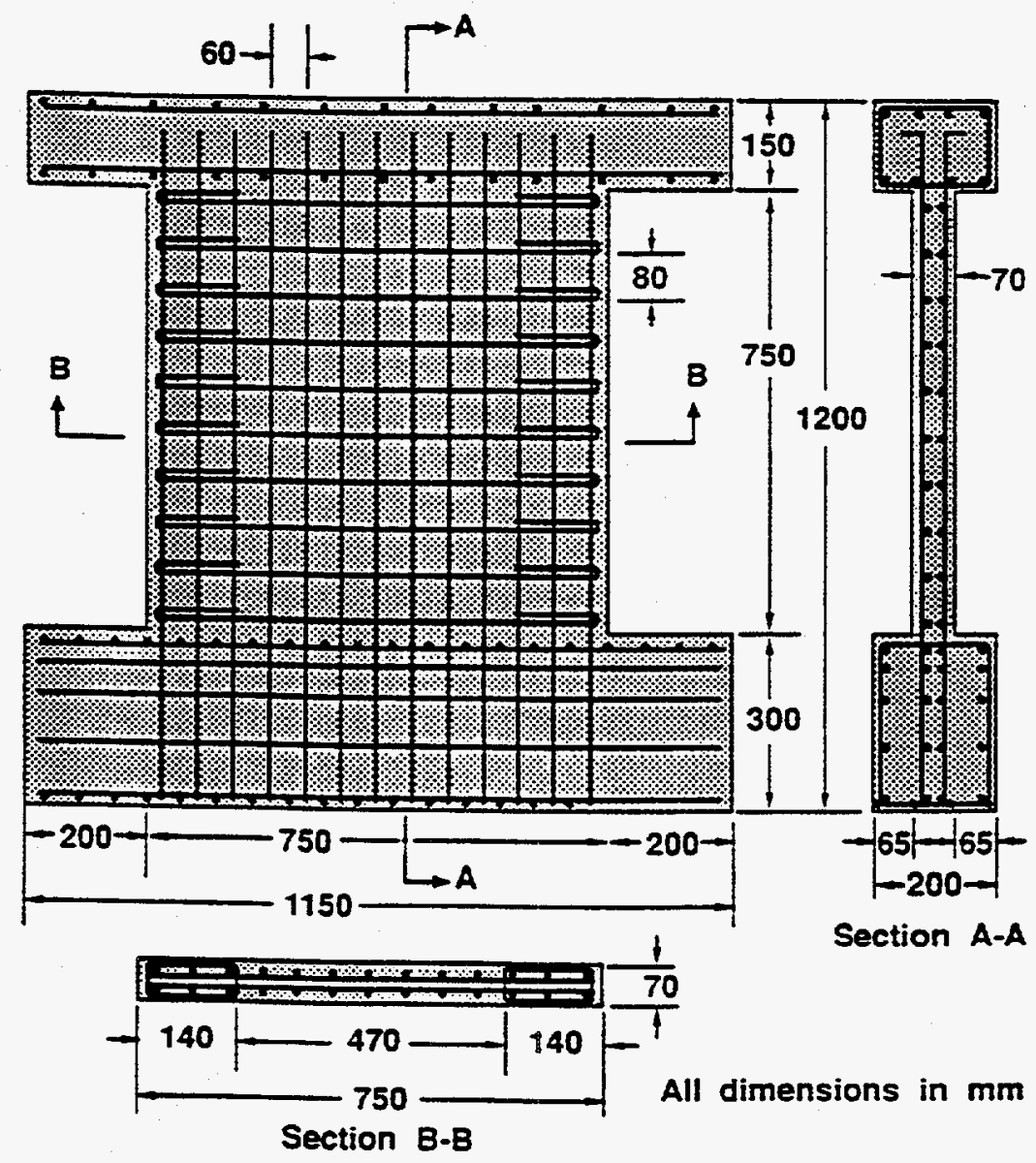

Figure 6 


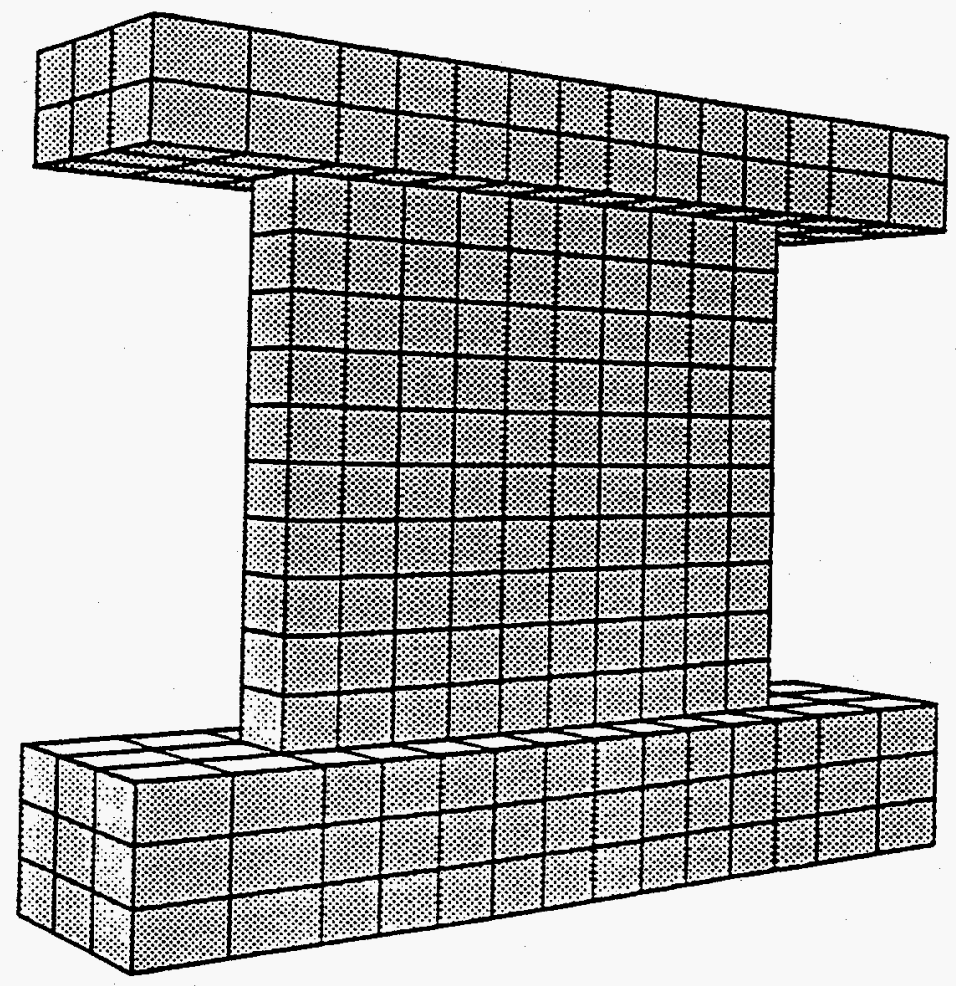

(a)

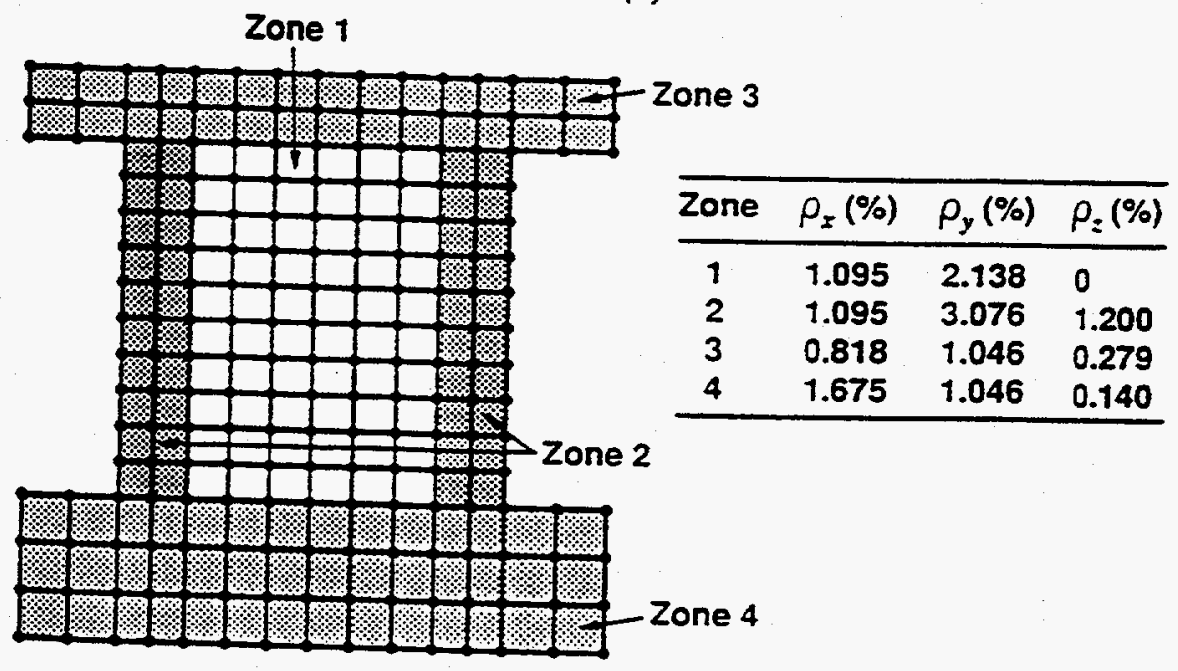

(b)

Figure 7 


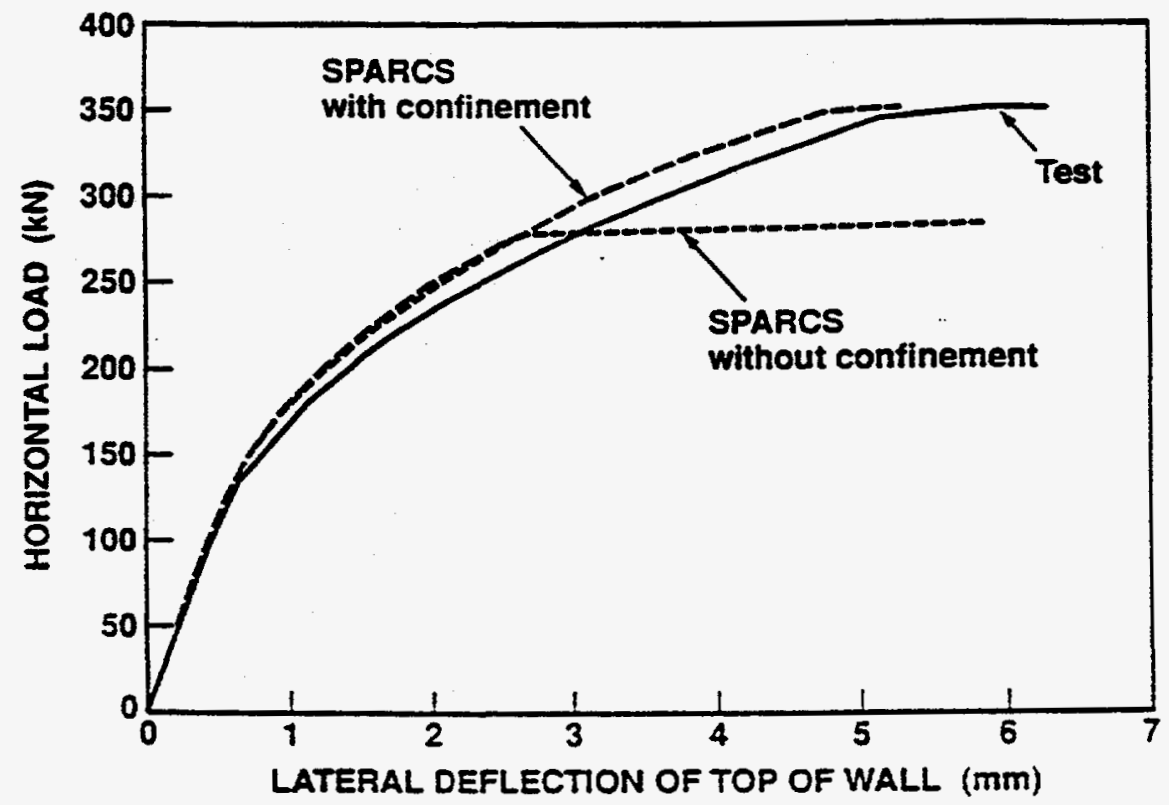

(a)

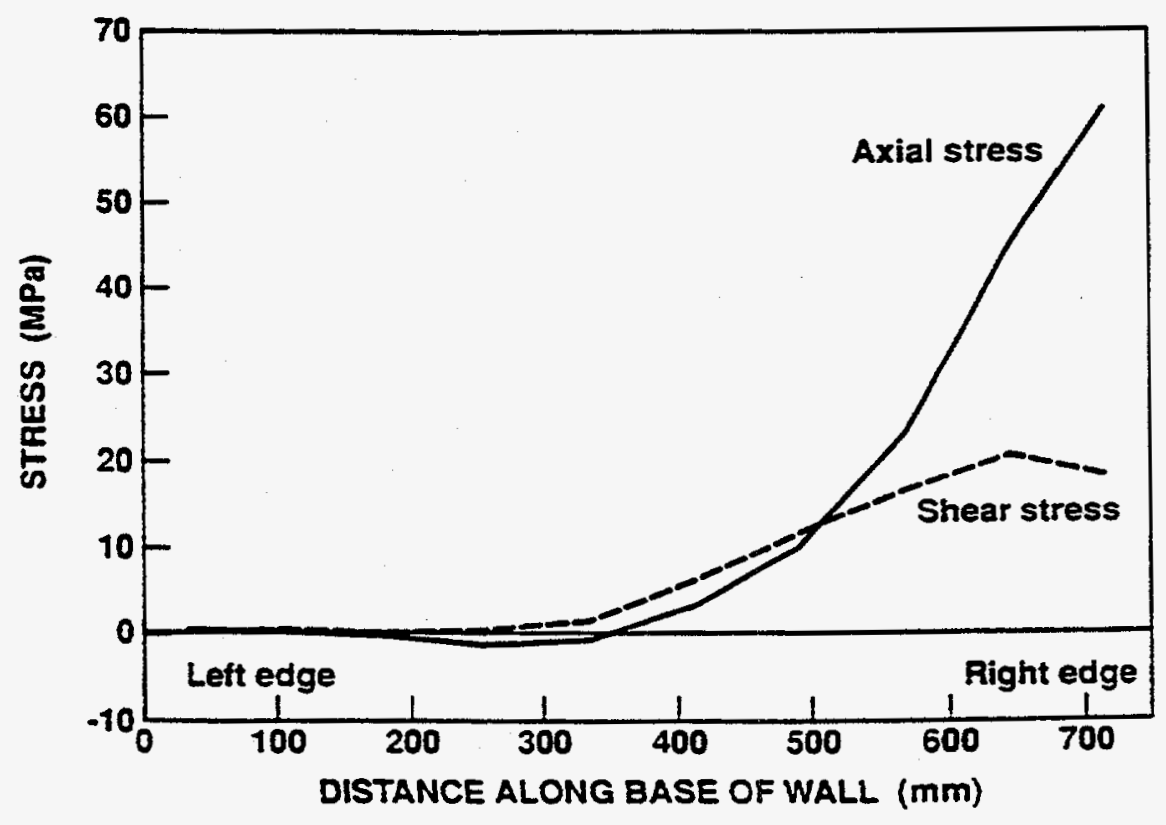

(b) 


\title{
STATIC FINITE ELEMENT ANALYSIS \\ OF SEISMIC SHEAR WALL ISP
}

\section{ADDENDUM}

\author{
F.J. Vecchio \\ Department of Civil Engineering \\ University of Toronto
}

November 11, 1995

\section{INTRODUCTION}

The results of static nonlinear finite element analyses performed for the SSWISP shear walls were previously presented and discussed in the report of September 25, 1995. Therein, it was shown that a three-dimensional analysis provided a predicted response which modelled the experimentally observed behaviour reasonably well, particularly in regards to the location and mode of failure. However, the wall strength was overpredicted by approximately $12 \%$, and the stiffness of the load-deformation response was similarly over-estimated. Possible explanations for the discrepancies included the loss of tension stiffening, bond and anchorage slip, and ratcheting effects, all arising from the fact that the walls were tested under dynamic seismic loading.

Also presented were the results of a parametric study using a two-dimensional model, suggesting that the finite element mesh used for the three-dimensional analysis was possibly too coarse. The recommendation made at that time was that the analyses be repeated with a finer descretization.

A reanalysis of the shear walls, using an improved mesh, has subsequently been undertaken. The details and results are reported herein.

\section{ELEMENT MESH SELECTION}

Recall from the previous report that the original two-dimensional model included 246 constant strain rectangular elements: the web was model with 10 (layers) $\times 15$ (columns) of elements; each flange was modelled with $10 \times 1$ elements, and the top and bottom slabs were each modelled using $2 \times 19$ elements. The same density and pattern of elements were used in modelling the web and flange components in the three-dimensional model.

Also recall from the initial study that when the element density was doubled, there was significant change in the computed response of the wall. Post-cracking deflections were increased as much as $50 \%$ in the later load stages, and the ultimate load capacity was reduced by $17 \%$. A quadrupled mesh gave essentially the same results as the doubled mesh, suggesting that a further refinement was not necessary. 
In an effort to arrive at an appropriate mesh density, without placing unnecessary demands on computation time, additional parametric studies were undertaken using twodimensional models. Complete load-deformation responses were determined using the following alternative models:

i) ISP1: web: $10 \times 15$ elements; flange: $10 \times 1$ elements;

ii) ISP10: web: $16 \times 20$ elements; flange: $16 \times 2$ elements;

iii) ISP15: web: $16 \times 20$ elements; flange: $16 \times 1$ elements;

iv) ISP16: web: $10 \times 15$ elements; flange: $10 \times 2$ elements;

v) ISP17: web: $16 \times 15$ elements; flange: $16 \times 2$ elements.

In comparing the resulting predicted load capacities, deflections, and failure modes, it was concluded that:

i) Increasing the number of layers of elements in the web and flanges, from 10 to 16 , is warranted. It allows for the bulging effect in the web to be fully realized, reducing the constraining influence of the stiff top and bottom slabs.

ii) Increasing the number of elements through the thickness of the flanges, from 1 to 2 , is also necessary. Apparently the flanges are subjected to significant flexural action; using 2 elements through the thickness allows this mechanism to be nominally included.

iii) Increasing the number of elements across the width of the web, from 15 to 20 , results in no perceptible influence.

To further optimize the model, in terms of eliminating unnecessary degrees of freedom, two additional studies were performed:

i) Model ISP18 was formulated such that the web and flanges were assumed fully fixed at their base (ie at the top of the base slab). This eliminated the need to model the base slab. The predicted ultimate load capacity, failure load, and wall bulging were unchanged. The predicted deflections, near ultimate, were reduced by about $1.2 \%$. Thus, it was concluded that this simplication is well warranted.

ii) Model ISP20 was formulated such that the top slab was modelled with $19 \times 1$ elements, reduced from $19 \times 2$. Using only one layer of elements for the top slab resulted in no change to the predicted load capacity or failure mode. Near ultimate load, the lateral deflections were increased by about $1.4 \%$, and the wall bulging at mid-height was increased by $3.0 \%$. Less elements through the thickness of the top slab perhaps afforded less opportunity for redistribution of forces. Nevertheless, the losses in accuracy were minor in consideration of the exorbitant number of degrees of freedom consumed in modelling the top and bottom slabs in the 3-D model.

In consideration of the results discussed above, the finite element mesh for the revised three-dimensional analysis was selected as shown in Figure 1. A total of 4950 degrees and 1026 elements were used: the web was modelled by $16 \times 15 \times 1$ elements; each flange was 
represented by $16 \times 2 \times 9$ elements; and the top slab was modelled by $1 \times 21 \times 10$ elements. The wall was assumed fully fixed at the interface to the base slab. All other modelling details, material properties, constitutive models and analysis parameters were as previously used.

\section{RESULTS OF THREE-DIMENSIONAL ANALYSIS}

Under a monotonic lateral load applied at the mid-depth of the top slab, the shear wall was predicted to experience a brittle flexural/shear failure near the base. The predicted maximum strength of the wall was $1740 \mathrm{kN}$ (177.5 tonf). The mode of failure, illustrated in Figure 2, involved a crushing/shear failure of the concrete in a large region of the web, centred along a plane approximately $300 \mathrm{~mm}$ above the base. The failure was accompanied by extensive yielding of the vertical reinforcement in the left flange. Relative to the results of the previous analysis, this represents a decrease in the predicted ultimate load by about $5 \%$ (far less than the $17 \%$ observed in the 2-D studies). The failure mode was essentially unchanged.

Figure 3 documents the predicted locations of first cracking, first yielding and first crushing. The predicted crack patterns are represented in Figure 4. Shown in Figure 5 is the horizontal displacement of the top slab (at its mid-depth). Figures 6 and 7 provide the vertical relative displacements of the left and right flanges, respectively. The strain in the vertical reinforcement in the left flange, at the base (element 1), is given in Figure 8; and the strain in the horizontal reinforcement in the web (element 287) is given in Figure 9. The shear strain and principal compressive stress in the web, near the compression toe (ie element 84), are given in Figures 10 and 11, respectively. Finally, the bulging of the wall, at mid-height, is represented in Figure 12.

The first cracks exhibited by the structure were shear cracks in the web, in the bottom left corner region, occurring at a load of $392 \mathrm{kN}$. By $490 \mathrm{kN}$ load, the web was essentially fully cracked. The first flexural cracks appeared on the outside surface of the left flange, at the base and near the web, at a load of $687 \mathrm{kN}$. By a load of $981 \mathrm{kN}$, the cracking at the base of the left flange extended across the full width of the flange, and penetrated the full thickness. With increasing load, the zone of cracking in the left flange progressed gradually upward such that by ultimate load, the flange sustained horizontal flexural cracking along its full height. The right flange sustained vertical flexural cracks, visible on the outside surface, along the web joint. These first appeared near the base, at a load of $981 \mathrm{kN}$, and gradually propagated upward. The right flange also experienced horizontal flexural cracking on the outside surface just below the top slab. Relative to the previous analysis, significant changes include the earlier onset of both the shear crack in the web and flexural cracks in the left flange, and the emergence of flexural cracks in the right flange.

The vertical rebar in the left flange was again the first reinforcement to yield occurring at a load of $1226 \mathrm{kN}$. As before, the location of first yieiding was towards the outer tip of the flange, just above the base. Yielding across the full width of the flange was achieved at a load of $1472 \mathrm{kN}$, with the zone of yielding gradually progressing upward to about mid-height by ultimate load. Yielding of the web wall vertical rebar occurred at a load of $1570 \mathrm{kN}$, in the bottom left comer region. As load increased, the yield zone progressed inward along the base. Note that relative to the previous analysis, first yielding of the vertical reinforcement 
occurred at about $14 \%$ lower load, and yielding of the web rebar occurred at about $6 \%$ lower load. Once again, the web wall horizontal rebar was not highly stressed and did not yield.

As before, the concrete in the web zone was highly stressed and dictated ultimate capacity and failure mode. First crushing occurred at a load of $1472 \mathrm{kN}$ in the compression toe region (elements 84 and 135). By a load of $1620 \mathrm{kN}$, this region was in post-peak response, and crushing was widespread over the lower right quadrant of the web. At ultimate load, a crushing shear failure occurred along a plane centred approximately $300 \mathrm{~mm}$ above the base. Relative to the previous analysis, the first crushing occurred at approximately $8 \%$ less lateral load and the failure zone was more widespread.

\section{DISCUSSION}

The finer mesh used in the reanalysis allowed for two important factors to be more realistically modelled. First, the greater number of element layers used for representing the web reduced any artificial constraint arising from the very stiff top and bottom slabs. Secondly, using two elements through the thickness of the flanges allowed the analysis to nominally account for flexural action in the flange walls and thus greater flexibility. Each of these two factors acted in reducing the confinement of the web, evidenced by a more pronounced bulging of the wall at mid-height. The reduced confinement, in turn, resulted in a reduced nominal strength for the concrete and a greater influence from compression softening effects. Hence, the ultimate load attainable was reduced. The lower degree of confinement also gave rise to a less stiff load-deformation response, and first cracking, first yielding and first crushing occurring at about 10 to $20 \%$ lower loads.

The finer mesh size had much less influence in the three-dimensional analysis than in did in the two-dimensional analysis; the failure load was reduced by only $5 \%$ in the 3-D study whereas it was reduced by $17 \%$ in the $2-D$ study. The reasons for this relate back to the three-dimensional effects discussed in the previous report. In the $2-D$ analysis, with the total lateral stiffness of the flanges concentrated in the plane of the web, the web confinement effects were over-estimated. Hence, the reduced confinement introduced by the finer mesh had a proportionally greater influence.

Previous remarks concerning the effects of bond and anchorage slip, loss of tension stiffening, and ratcheting action are still valid in rationalizing the differences the experimental and analytical responses.

\section{PARAMETRIC STUDIES}

The parametric studies previously performed using the two-dimensional finite element models provided some enlightenment regarding the influence of various parameters. It was also clear from the results, however, that the two-dimensional models failed to accurately account for some three-dimensional effects. Hence, some additional studies were performed using the three-dimensional finite element model described above. 
An analysis was performed in which tension stiffening effects were not considered. As previously discussed, it is customary to discount tension stiffening when attempting to nominally consider cyclic load effects in a static load analysis. Shown in Figure 13 is a comparison of the predicted load-deformation responses of the wall. Ignoring post-cracking tensile stresses in the concrete results in a significantly more flexible deflection response, and a $4.5 \%$ reduction in the estimated failure load. The results more closely resemble the experimental behaviour. Failure mode was unaffected.

It was previously suggested that the finer mesh resulted in a lowering of the predicted strength of the wall because it better allowed for wall expansion. The ensuing larger tensile strains gave rise to a greater influence from compression softening effects. To test this hypothesis, an analysis series was performed in which the compression softening formulations were suppressed. Shown in Figure 14 is the predicted response that resulted. Relative to the standard analysis, the wall response is considerably stiffer in the later load stages, and the ultimate load is increased by about $7 \%$. Thus, contrary to the findings from the 2-D parametric study previously discussed, compression softening effects did play a significant role in influencing the predicted behaviour of the wall.

Finally, to ascertain the importance of confinement effects, another analysis was run in which strength and stiffness enhancement due to confinement were suppressed (that is, the strength of the concrete was limited to the cylinder strength). Shown in Figure 15 is the loaddeformation response predicted accordingly. The stiffness of the wall is essentially unaffected, and the ultimate load capacity is reduced by only about $4 \%$. Also, there is little change in the mode of failure, or in the predicted crack patterns. Thus, confinement effects are of minor importance in this structure.

\section{CONCLUSIONS}

The reanalysis produced a predicted failure load still approximately $7 \%$ higher than the experimentally observed value. Also, the predicted load-deflection response remained somewhat stiffer than the observed behaviour. The discrepancies, however, were substantially reduced compared to the results of the initial analysis previously reported which used a coarser mesh. The remaining disparity between predicted and observed behaviour can be put down to the effects of reversed cyclic load; it must be remembered that the analyses presume static monotonically increasing lateral load. In particular, discounting post-cracking tensile stresses in the concrete, as one is apt to do for cyclic load conditions, produces results closer to the experimentally observed behaviour. The predicted mode of failure remained in good agreement to the experimental resuits.

Other conclusions previously derived from the initial three-dimensional analysis, and from the two-dimensional parametric studies, remain valid and relevant. 


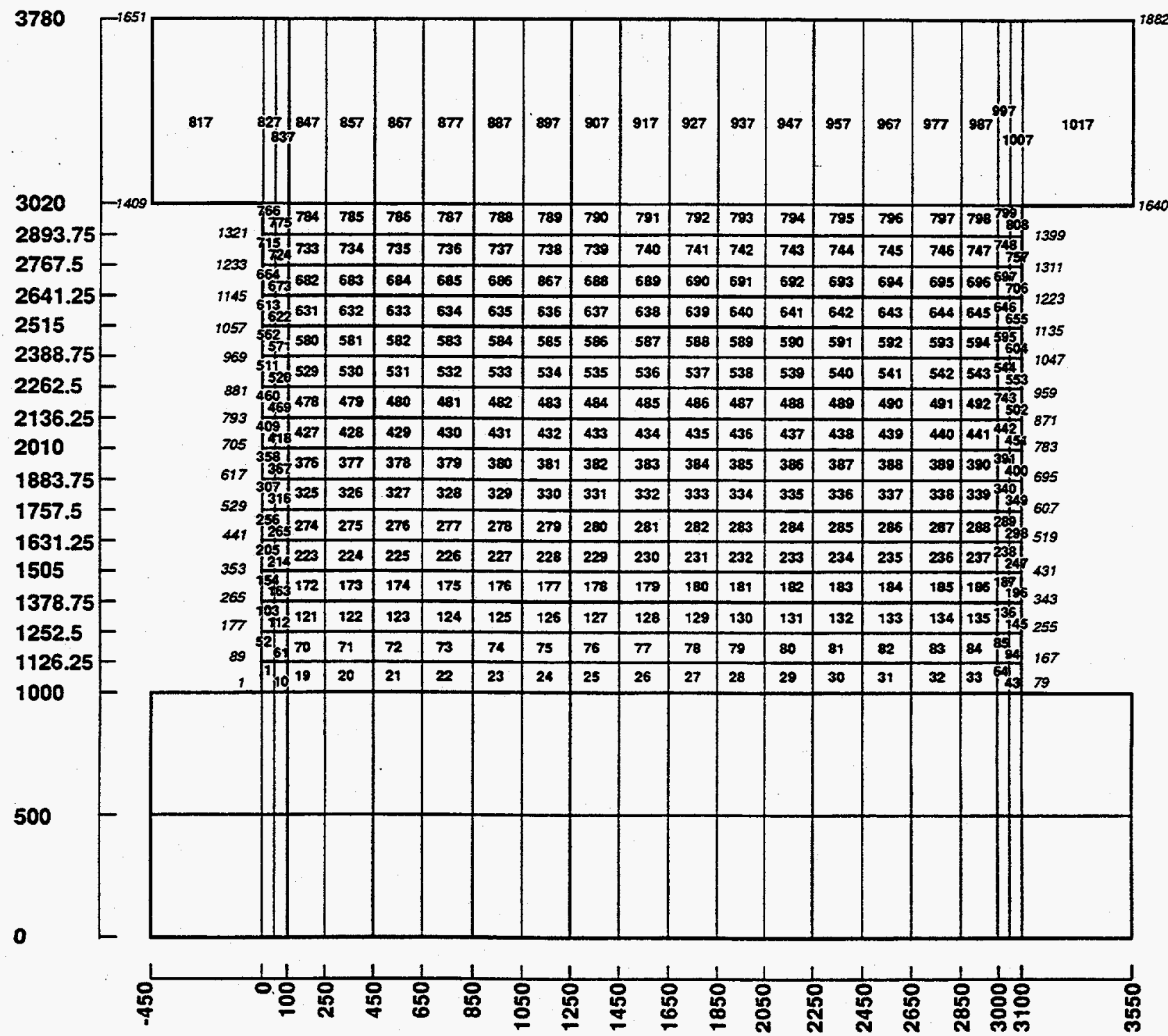

Figure 1

Element Scheme for SPARCS Model (finer mesh) 


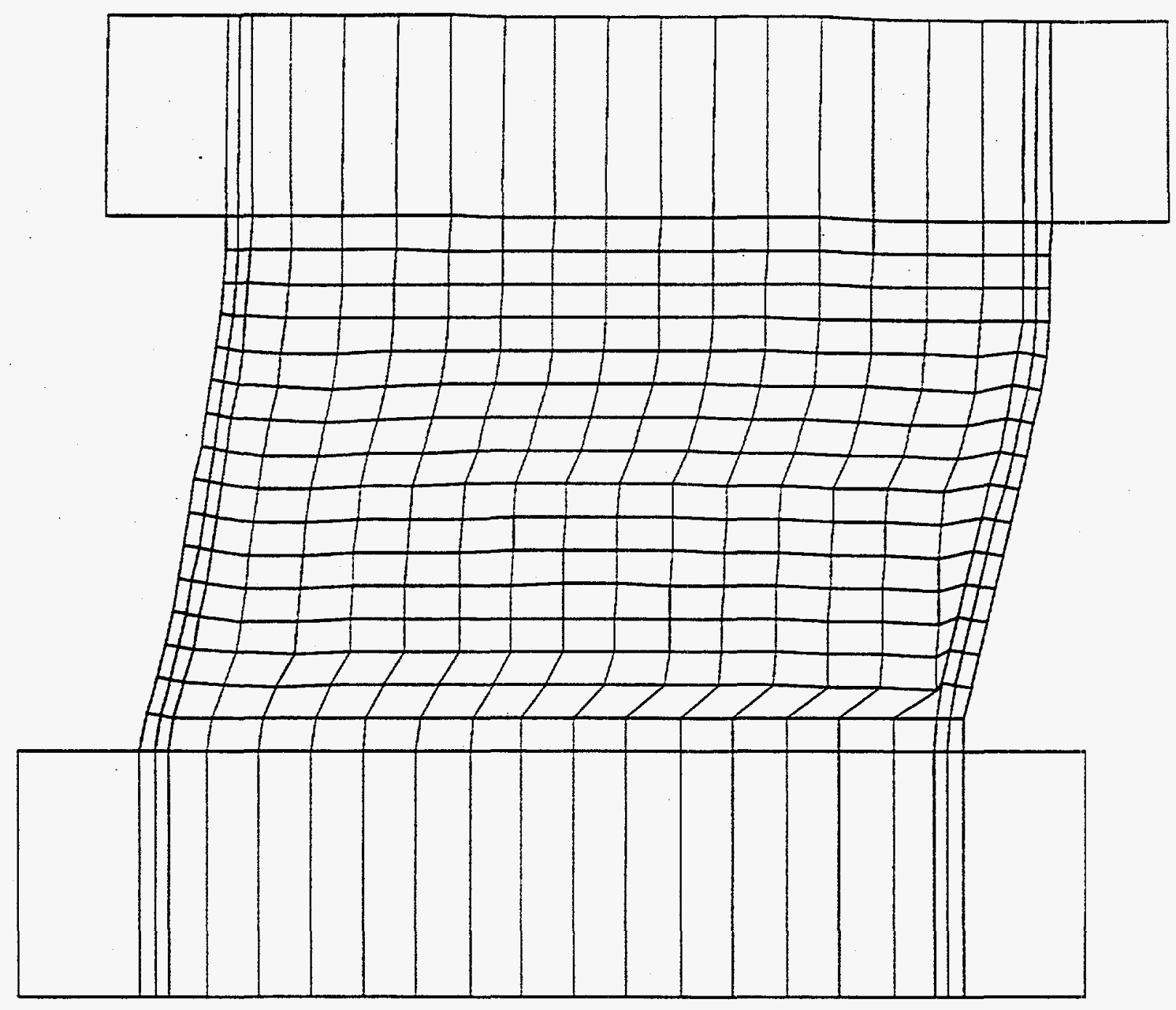

Figure 2

Predicted Failure Mode From 3-D Analysis (ISP3) 


\begin{tabular}{|c|c|c|}
\hline \multirow[t]{2}{*}{ Phenomena } & \multicolumn{2}{|c|}{ Top Slab } \\
\hline & $\begin{array}{l}\text { Horizontal Displacement } \\
(\mathrm{mm})\end{array}$ & $\begin{array}{l}\text { Horizontal Force } \\
\text { (N) }\end{array}$ \\
\hline $\begin{array}{l}\text { (a) Flange wall } \\
\text { Initiation point of bending crack }\end{array}$ & 0.855 & $685 \times 10^{3}$ \\
\hline $\begin{array}{l}\text { (b) Web wall } \\
\text { Initiation point of shear crack }\end{array}$ & 0.427 & $390 \times 10^{3}$ \\
\hline $\begin{array}{l}\text { (c) Flange wall vertical rebar } \\
\text { Initiation point of yielding }\end{array}$ & 3.357 & $1225 \times 10^{3}$ \\
\hline $\begin{array}{l}\text { (d) Web wall vertical rebar } \\
\text { Initiation point of yielding }\end{array}$ & 7.107 & $1570 \times 10^{3}$ \\
\hline $\begin{array}{l}\text { (e) Web wall horizontal rebar } \\
\text { Initiation point of yielding }\end{array}$ & $n / a$ & n/a \\
\hline (f) Crush point & 5.829 & $1470 \times 10^{3}$ \\
\hline Maximum load & 10.859 & $1740 \times 10^{3}$ \\
\hline
\end{tabular}

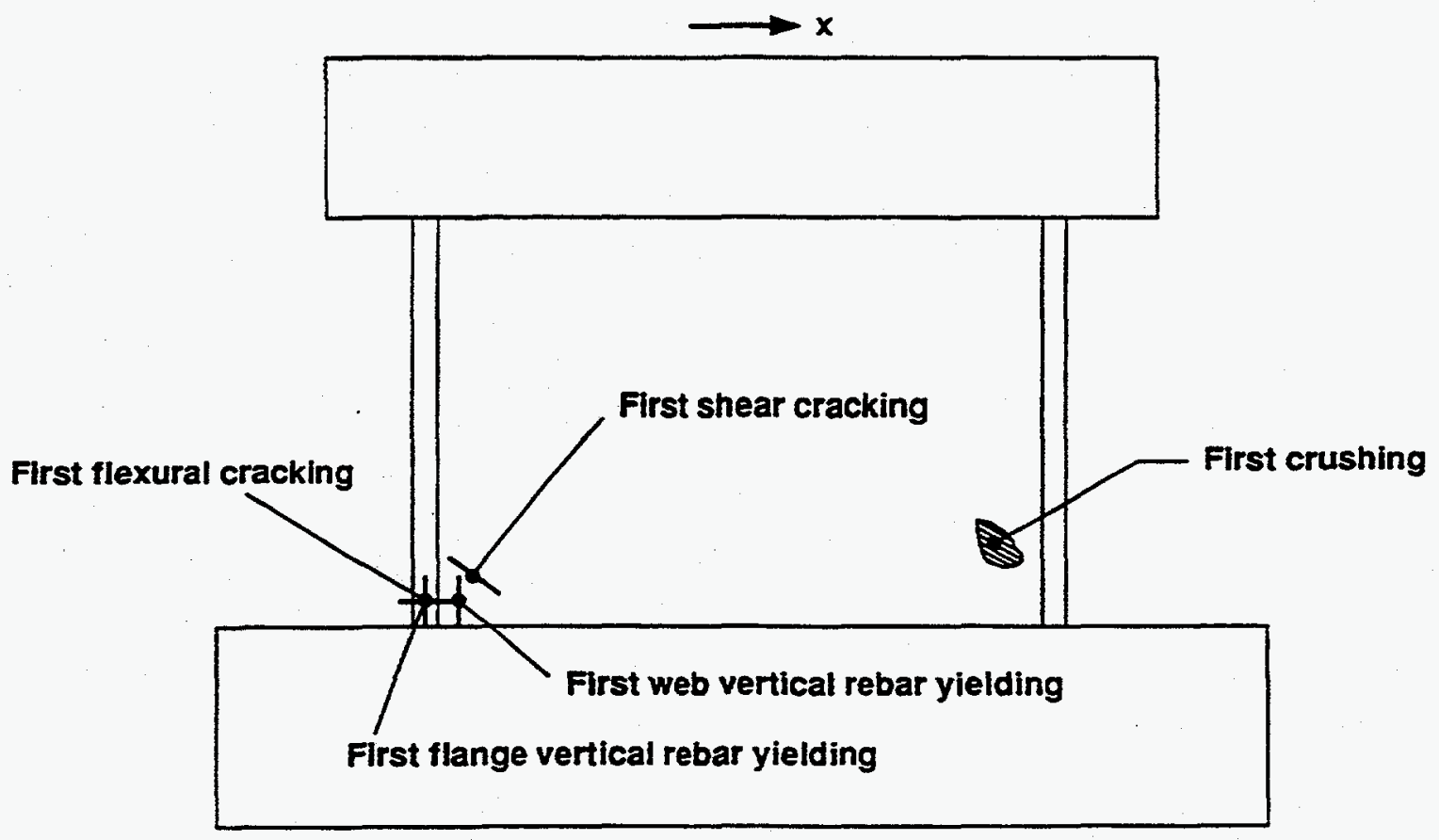

Figure 3

Summary of Calculated Results 


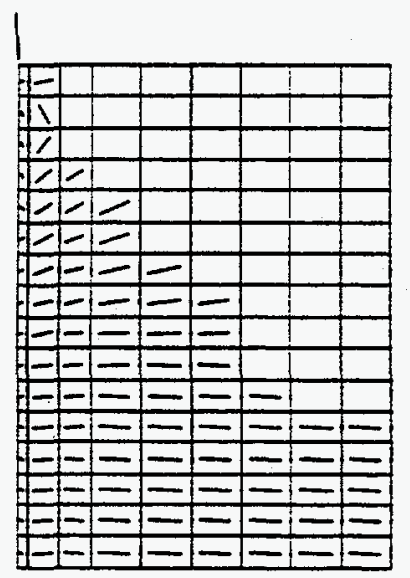

$\mathcal{Q}$

C face (flange wall)

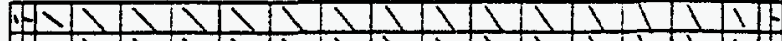

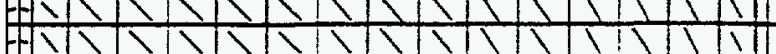

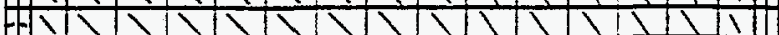

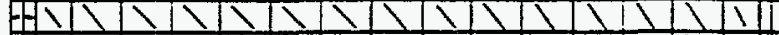

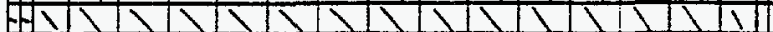

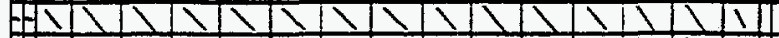

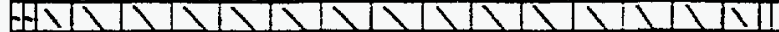

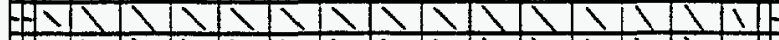

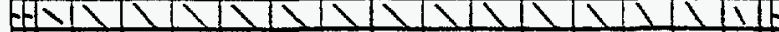

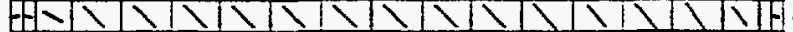

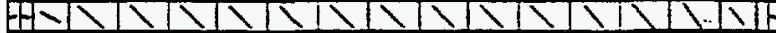

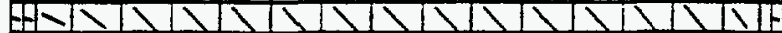

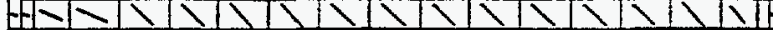

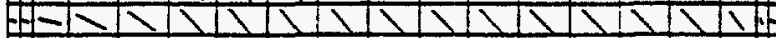

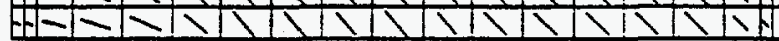

A face (web wall)

(a) At 2/3 of Maximum Load (1175 kN)

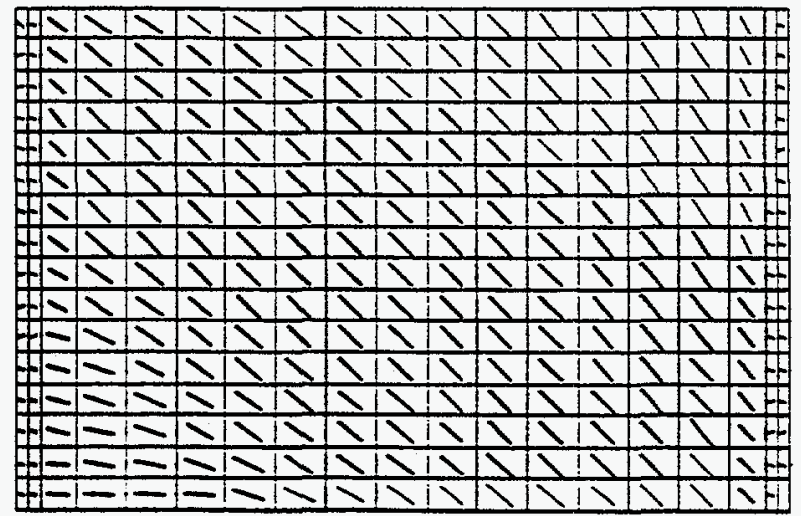

$\xi$

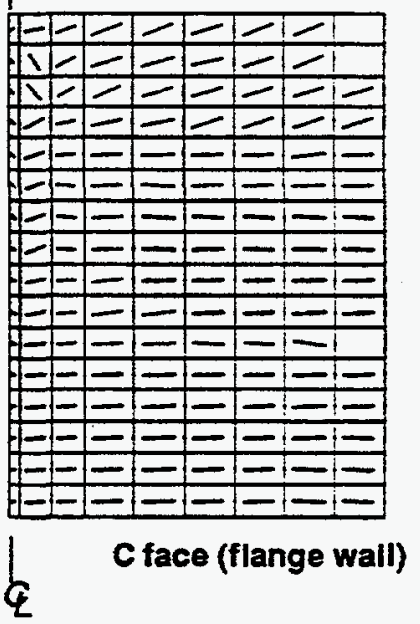
A face (web wall)

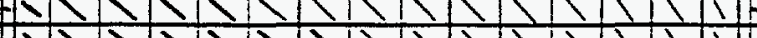

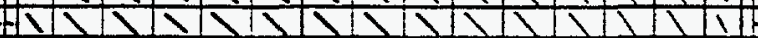
4101011010111001101110

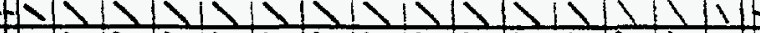

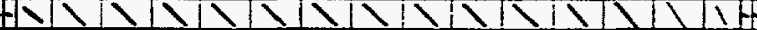
- 101010101010101000011

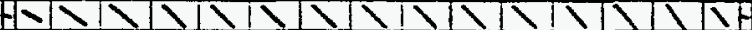

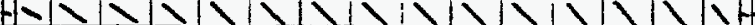
- > $>10010$

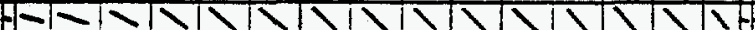
- $-1>>10101010101010$ 210101010

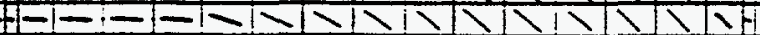

(b) At Maximum Load (1740 kN)
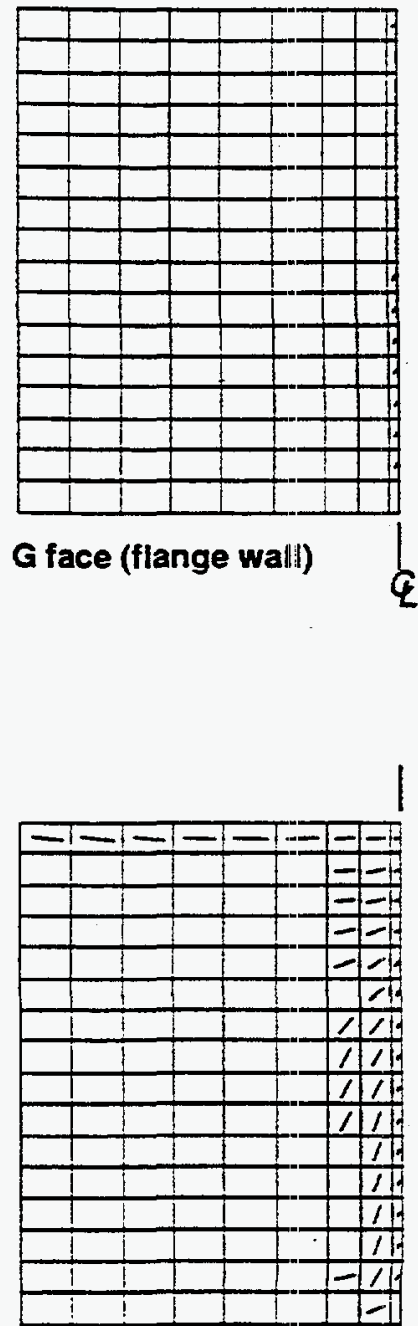

G face (flange wall)

Figure 4

Predicted Crack Patterns 


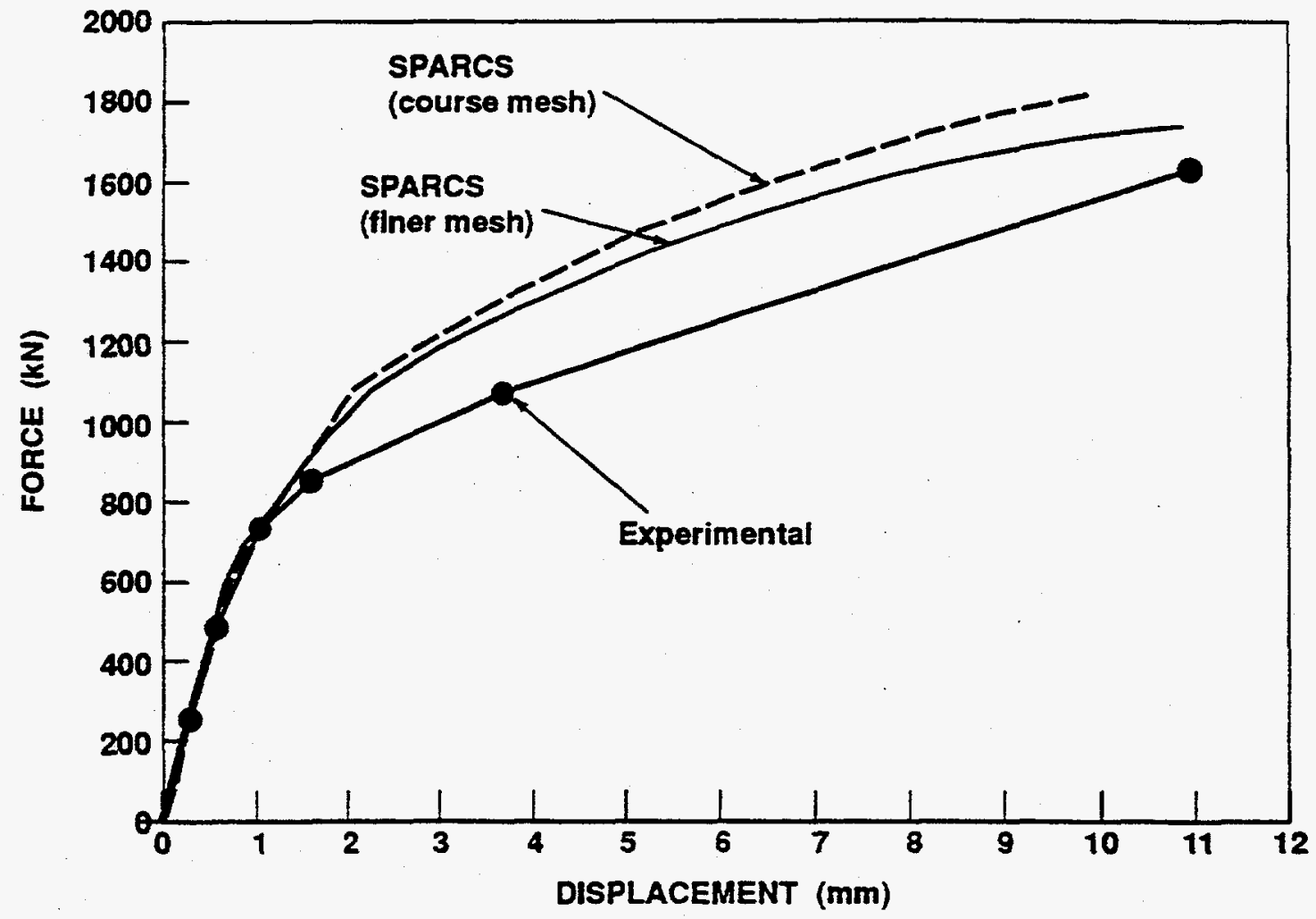

Figure 5

Horizontal Displacement of Top Slab

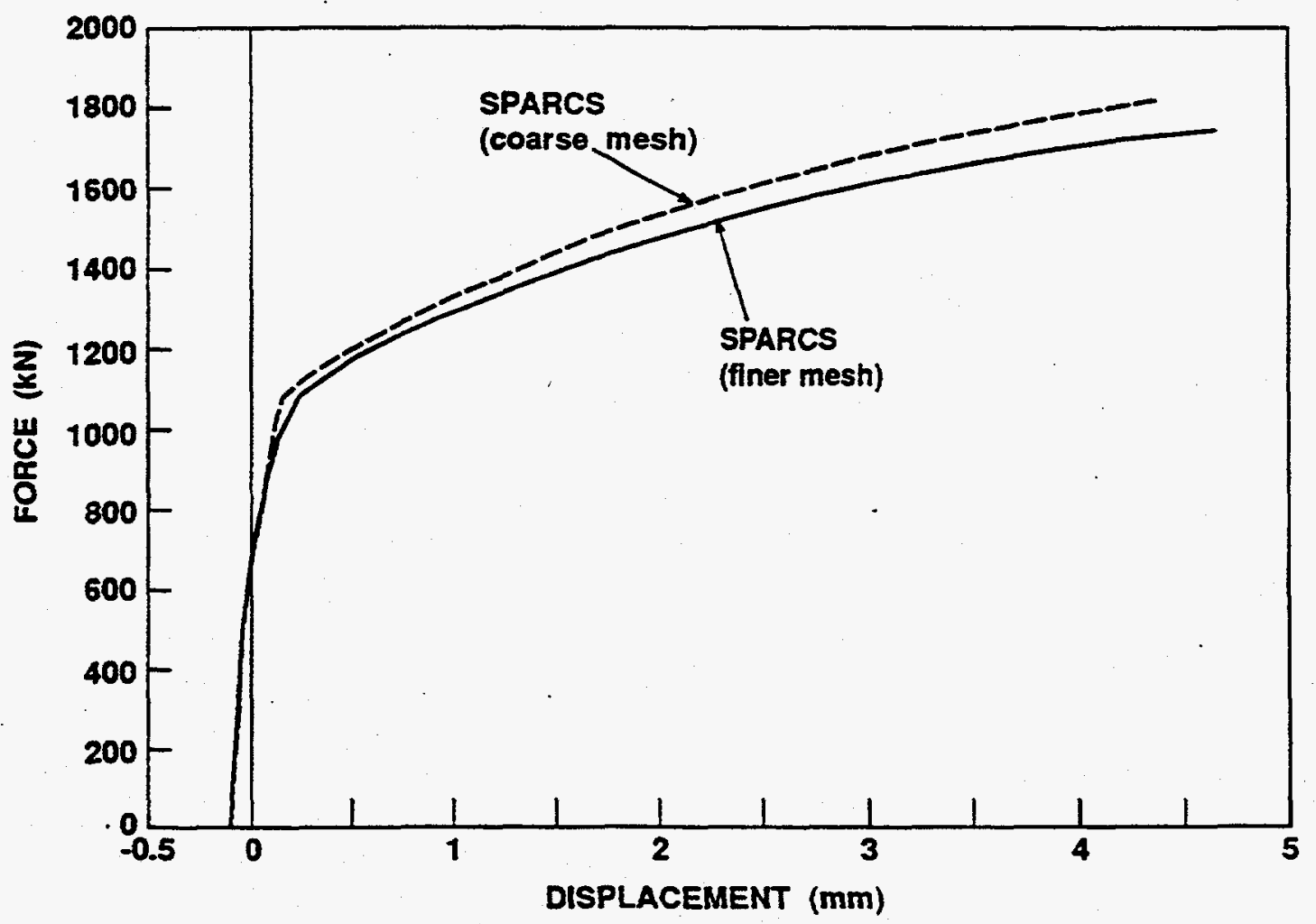

Figure 6

Vertical Relative Displacement - Left Flange 


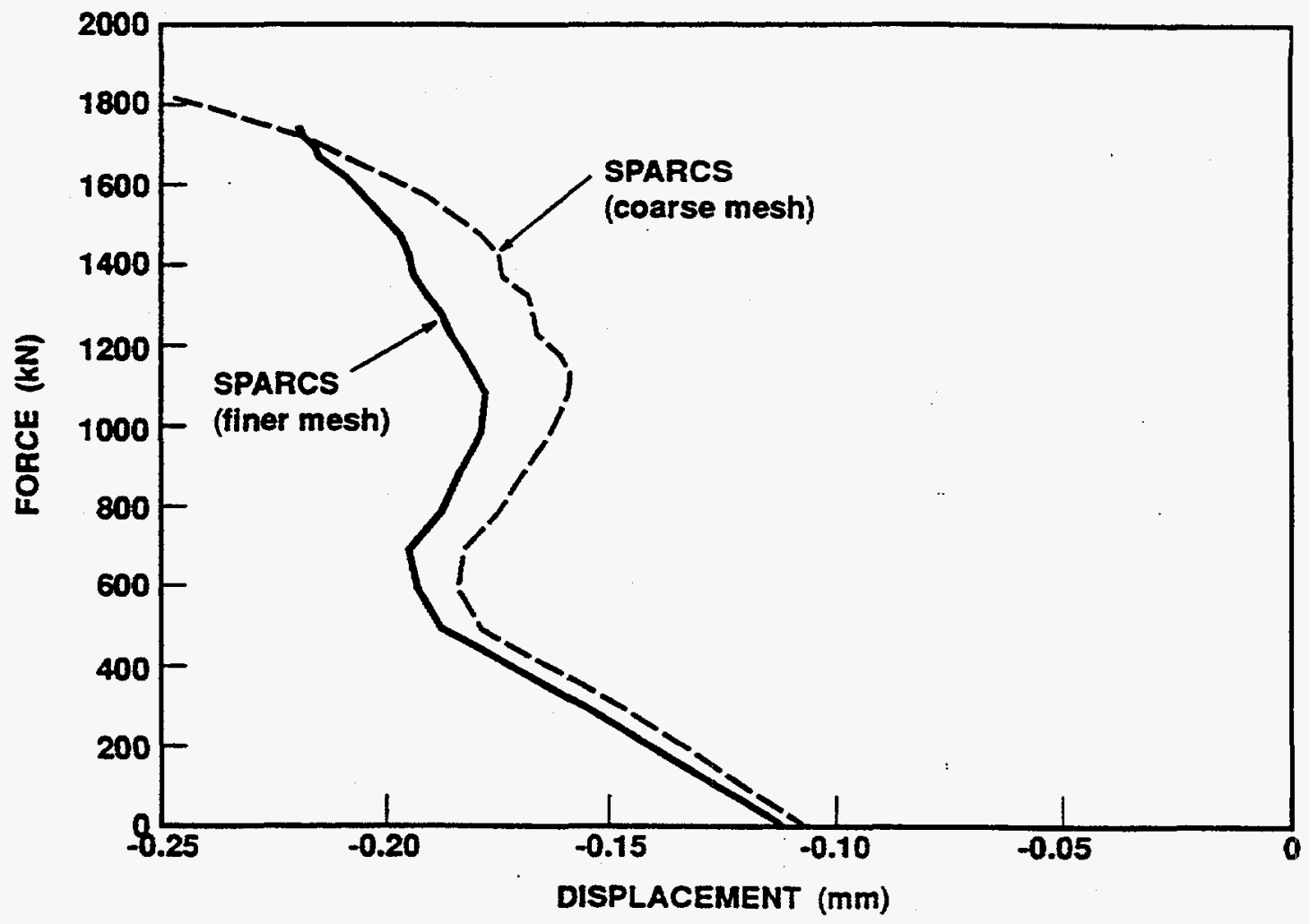

Figure 7

Vertical Relative Displacement - Right Flange

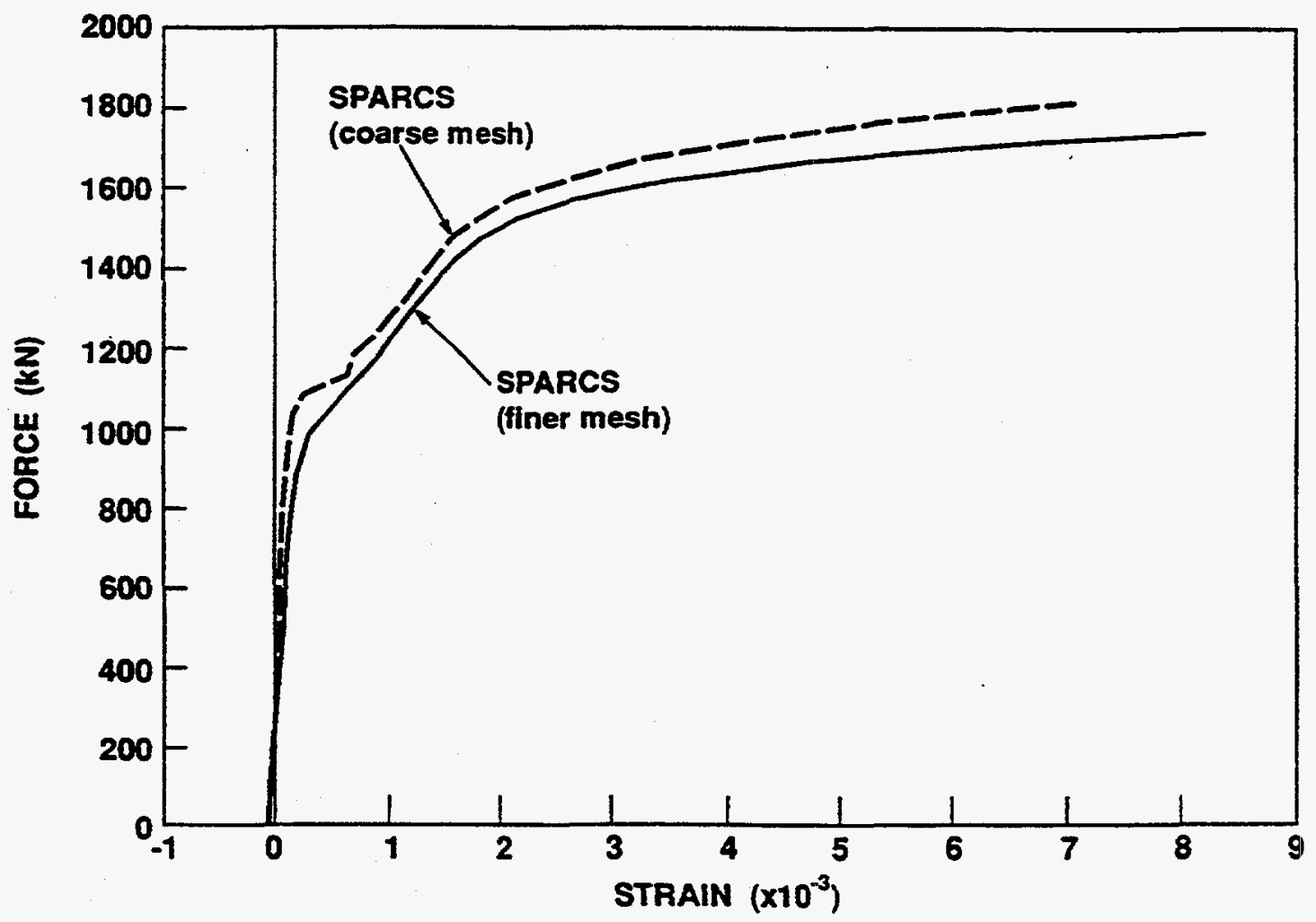

Figure 8

Vertical Rebar Strain - Base of Left Flange 


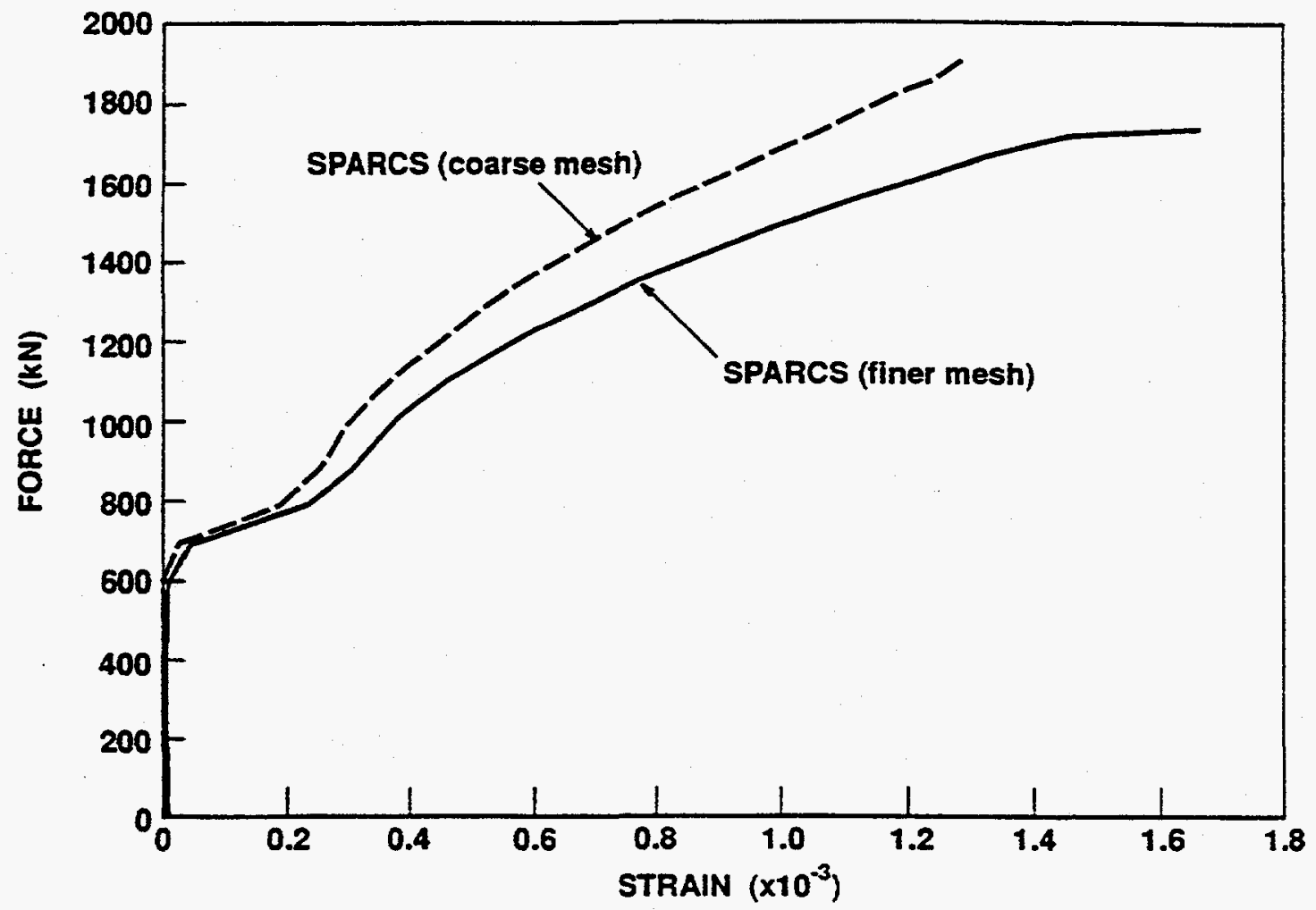

Figure 9

Strain in Horizontal Rebar in Web

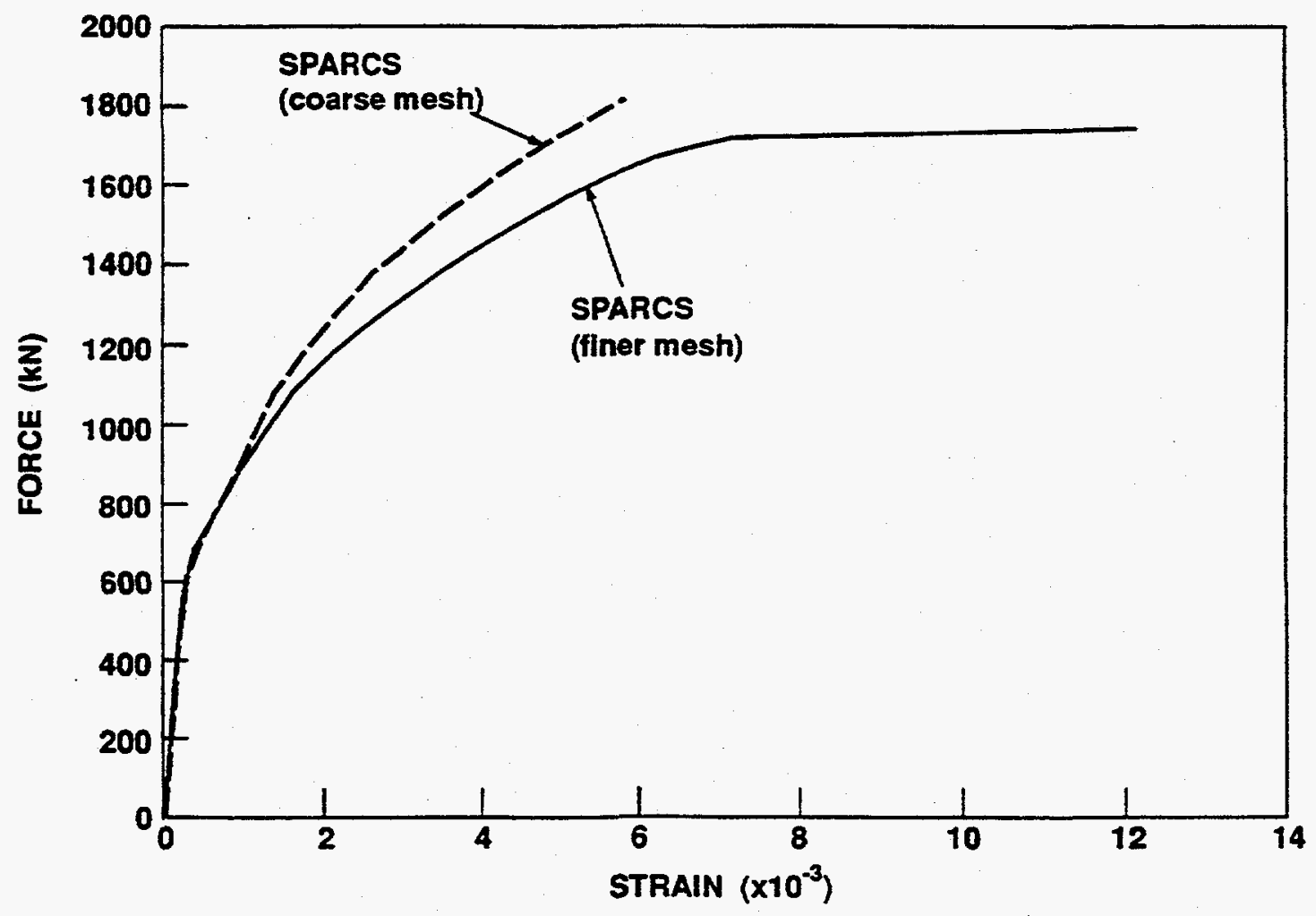

Figure 10

Shear Strain in Web 


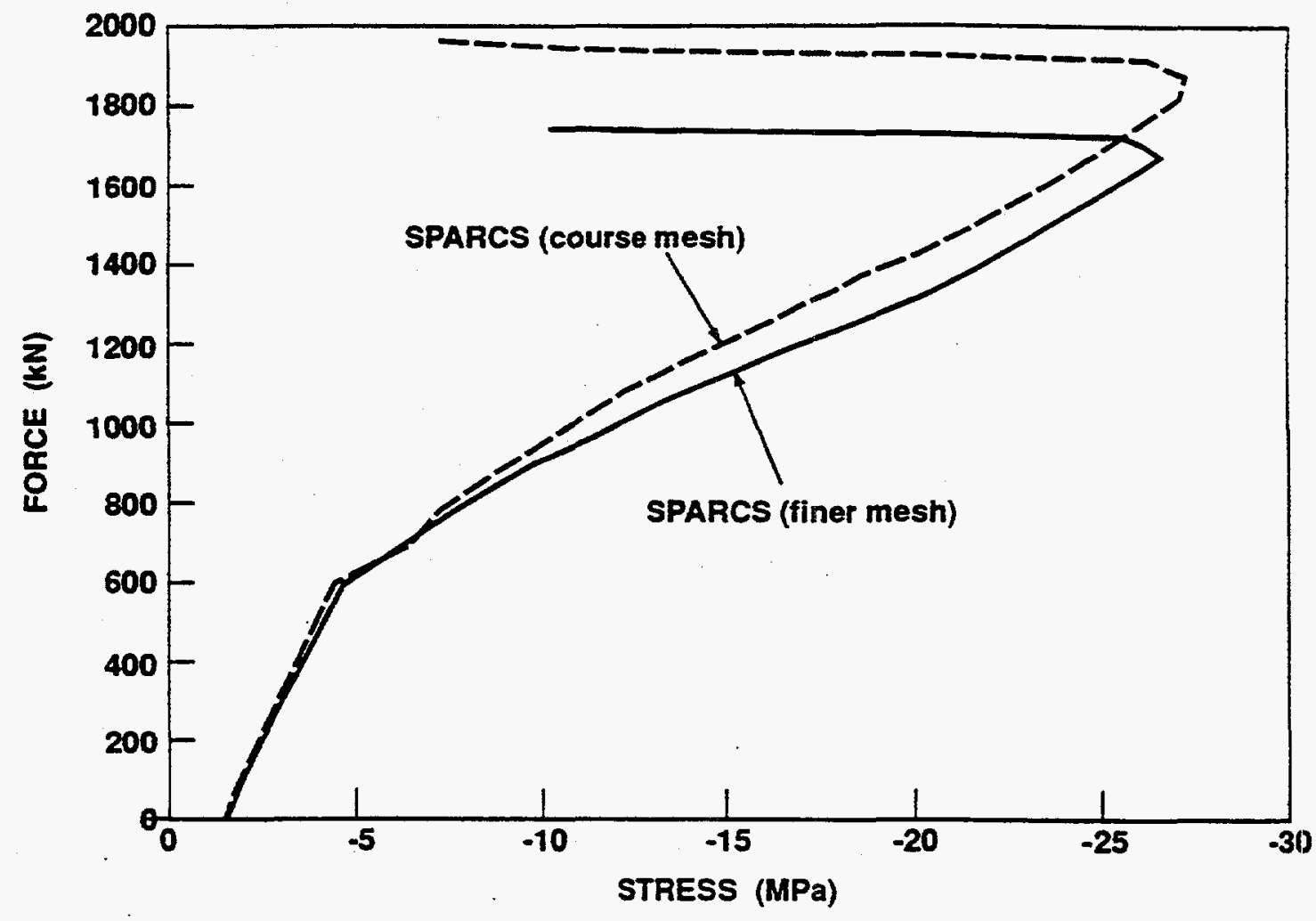

Flgure 11

Concrete Compressive Stress at Toe of Web

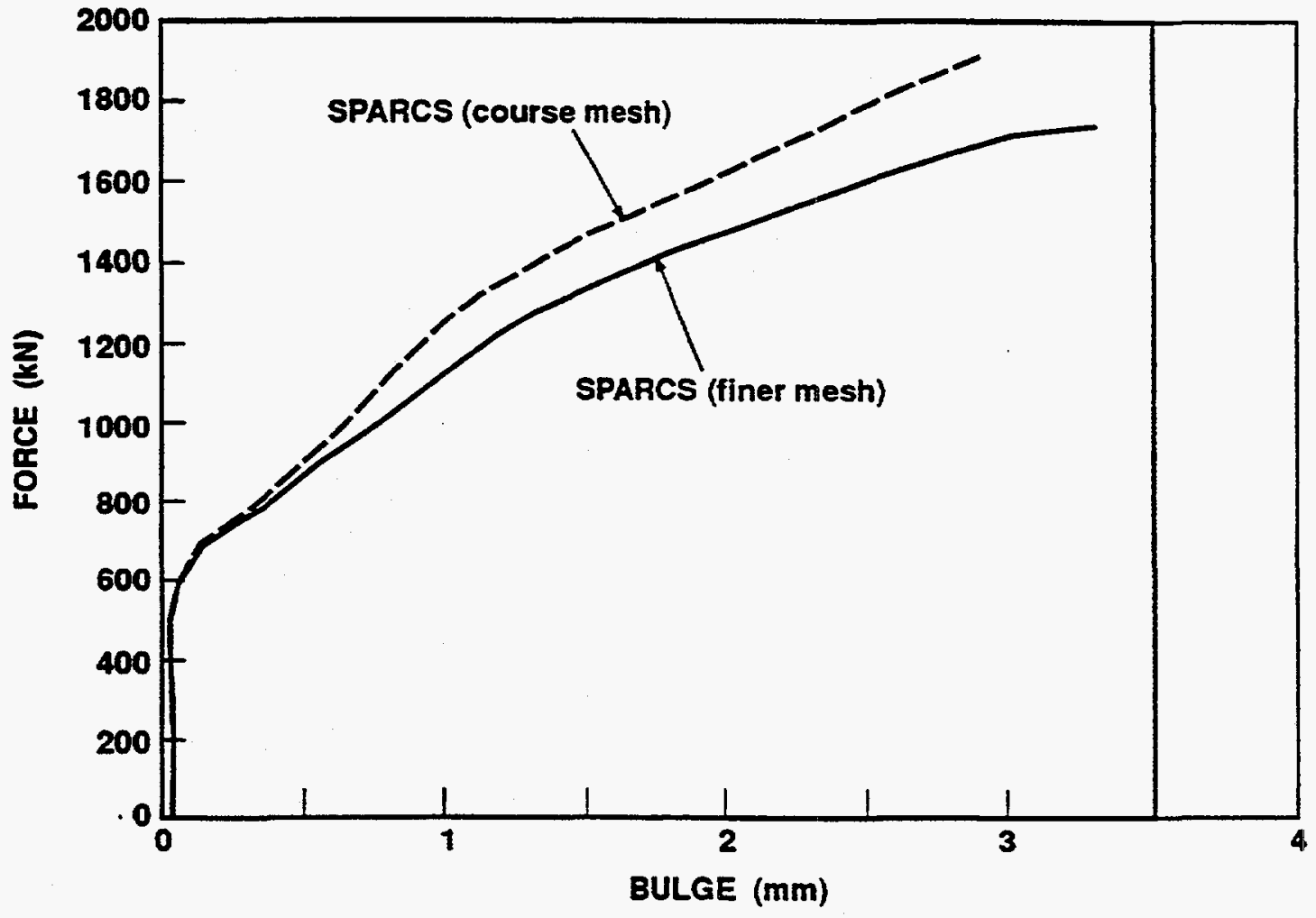

Figure 12

Bulging of Web at Mid-Height 


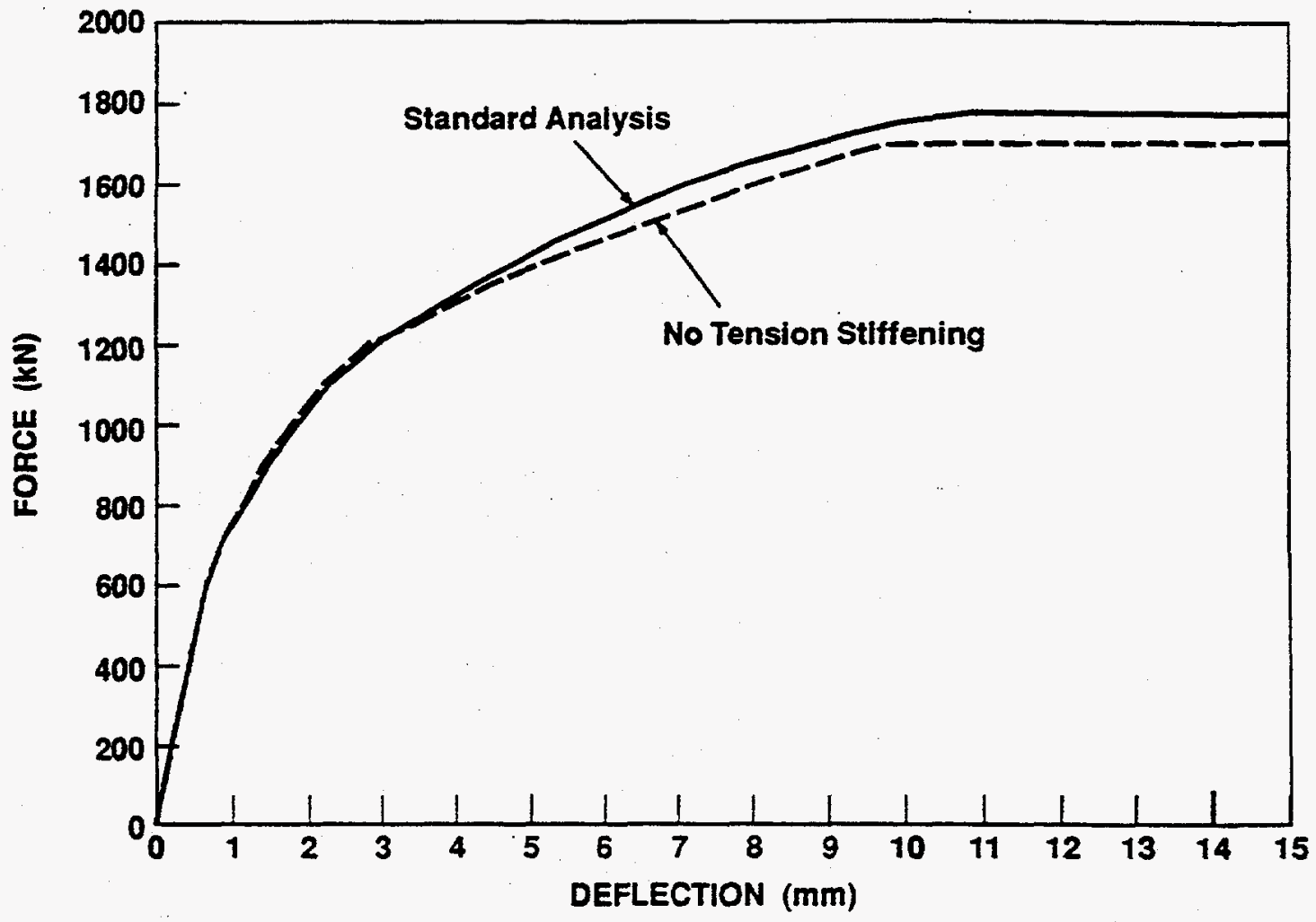

Figure 13

Influence of Tension Stiffening

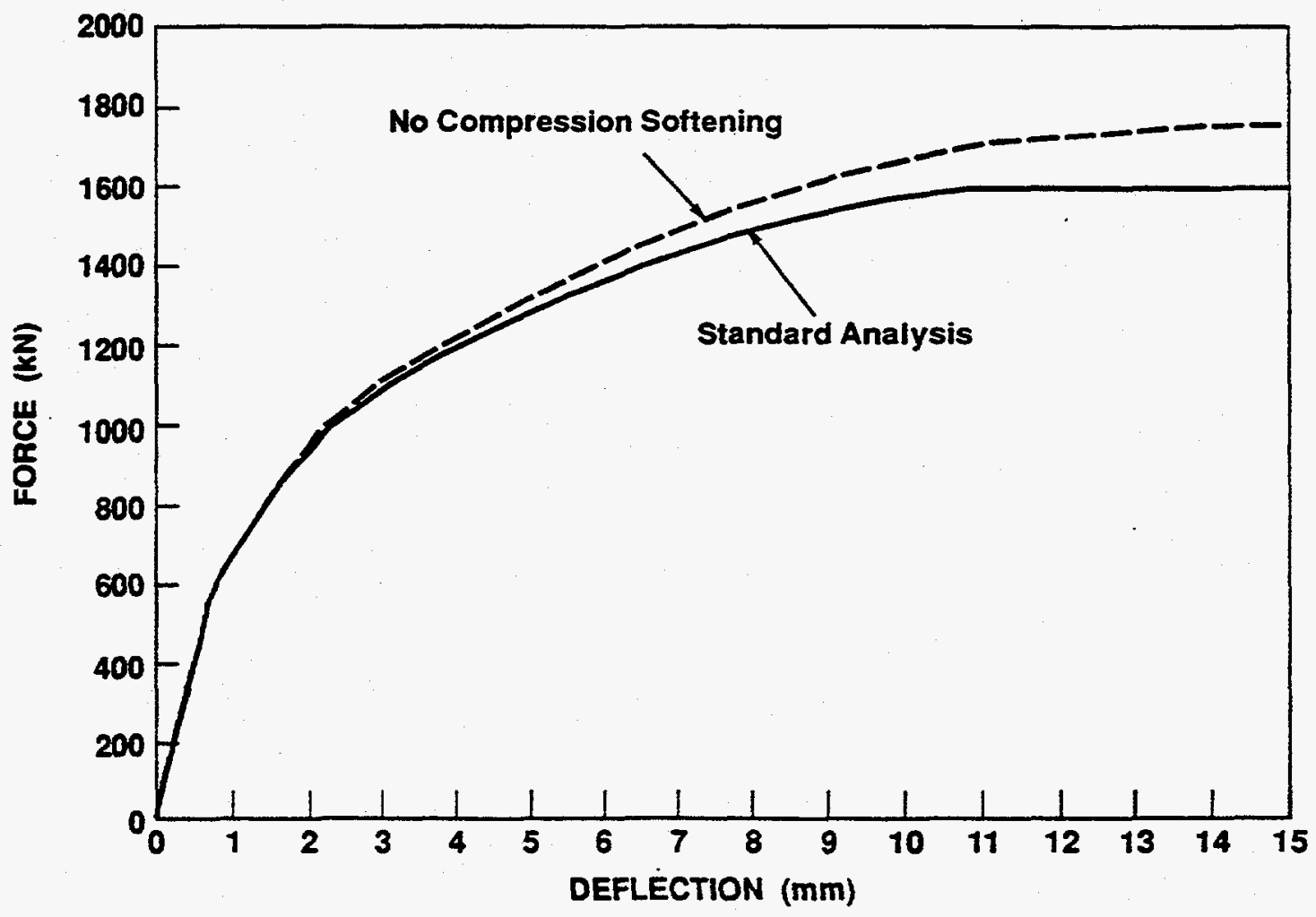

Figure 14

Influence of Compression Softening 


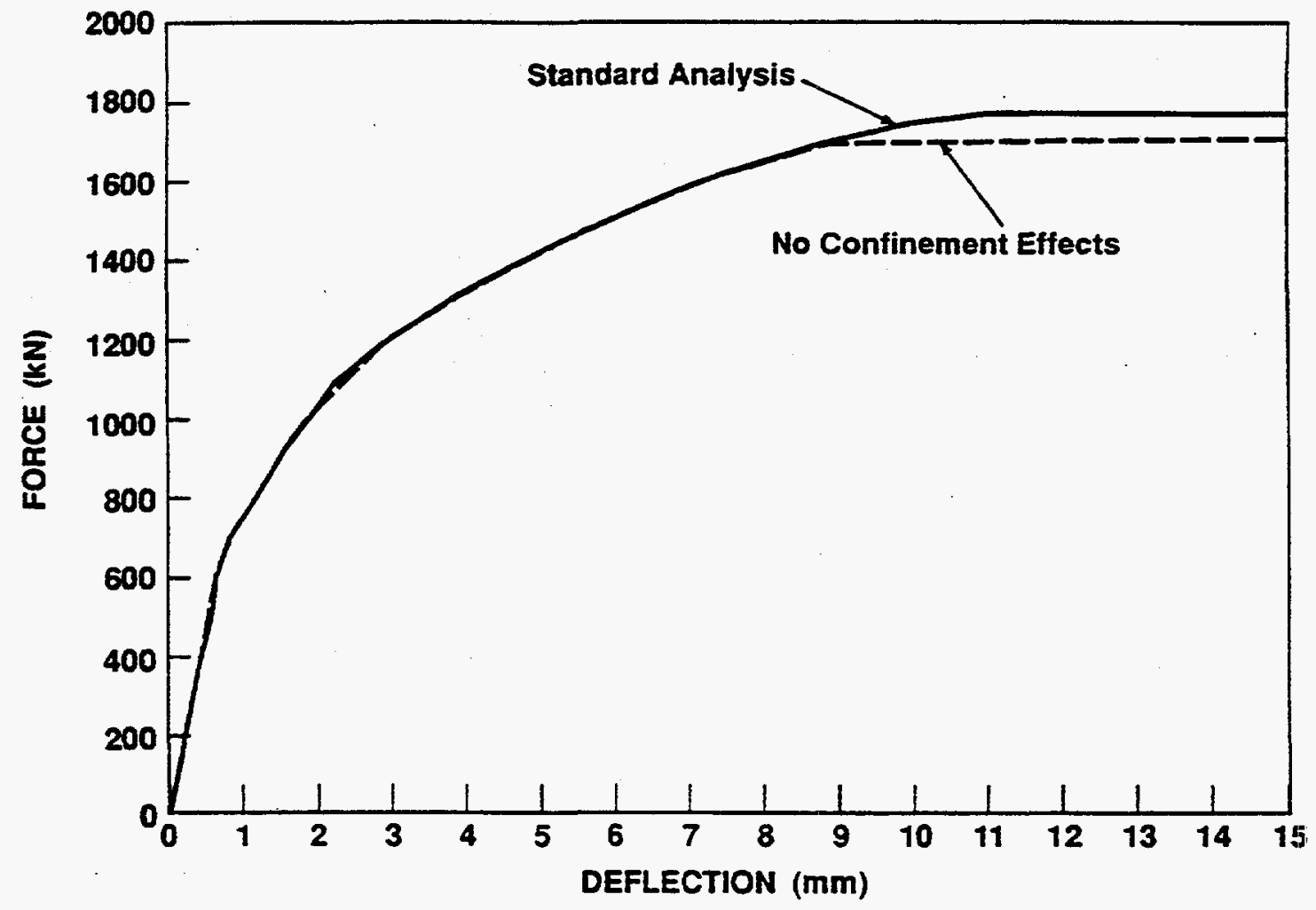

Figure 15

Influence of Confinement Effects 


\title{
APPENDIX B \\ MONOTONIC LOAD TO COLLAPSE ANALYSIS \\ OF NUPEC SHEAR WALL ISP SPECIMEN
}

\author{
By \\ F.C. Filippou and T. Balan \\ Department of Civil Engineering \\ University of California, Berkeley \\ Performed for \\ Brookhaven National Laboratory \\ Under the Sponsorship of the \\ U.S. Nuclear Regulatory Commission
}

November 1995

B-1 


\title{
MONOTONIC LOAD TO COLLAPSE ANALYSIS OF NUPEC SHEAR WALL ISP SPECIMEN
}

\author{
by
}

Filip C. Filippou and Toader Balan

Department of Civil Engineering

University of California, Berkeley 


\section{Introduction}

This. report addresses the monotonic load to collapse analysis of a reinforced concrete shear wall specimen that was subjected to dynamic response studies with a large-scale, highperformance shaking table at NUPEC's Tadotsu Engineering Laboratory in 1991. The analyses are conducted with the general purpose finite element computer program MIRAGE. The report describes the model of the specimen and the analytical results.

Appendices address the concrete and reinforcing steel constitutive relations that form the basis of the model. The concrete constitutive model used in the analyses is a hypoelastic orthotropic model based on a stress-equivalent uniaxial strain relation that is generalized to take into account triaxial stress conditions. This is discussed in detail in Appendix A. Several aspects of concrete behavior are represented by the model including triaxial nonlinear stress-strain behavior, tensile cracking, compression crushing and strain-softening. In this study the model is simplified to biaxial plane stress conditions by setting the out-of-plane normal stress equal to zero. The reinforcing steel is modeled with an embedded reinforcing layer model. This model is discussed in Appendix B along with two alternative models, the embedded reinforcing bar and the oriented medium model. The former becomes important for the inclusion of the bond-slip effect of individual reinforcing bars in possible future studies. The latter is necessary in three dimensional finite element models.

The analytical results show good agreement of the analytical predictions with the experimental results. Appendix D provides a summary of the results of the study and also supplies the specific information requested in the Specification Report of the Seismic Shear Wall ISP (Document NU-SSWISP-D008 with revised pages from NU-SSWISP-D009).

Further studies into the cyclic and dynamic behavior of the specimen are presently under consideration. 


\section{Basic Characteristics of Shear Wall Specimen}

The -geometry of the test specimen is shown in Figs. 1(a) and (b). The specimen has an Ishape cross section with a web of $75 \mathrm{~mm}$ thickness, $3000 \mathrm{~mm}$ length measured from top flange center to bottom flange center and $2000 \mathrm{~mm}$ clear height. The resulting shear span ratio is 0.8 . The flange walls are $100 \mathrm{~mm}$ thick and 2980 long.

The arrangement of reinforcement in the web and flange walls is shown in Figs. 2 (a) and (b). Deformed bars D6@70 with nominal diameter of $6.35 \mathrm{~mm}$ at a spacing of $70 \mathrm{~mm}$ are arranged in two layers of the web wall to form the vertical and horizontal reinforcement (Fig. 2b).

Two reinforcing layers were also present in the flange walls with D6@175 bars for the vertical and D6@70 for the horizontal reinforcement. As an exception, D6@70 bars were used for the vertical reinforcement at the intersections of the web and flange walls (Fig. 2a).

The material properties of D6 reinforcing bars, as used in the web and flange walls, comply with Japanese Industrial Standard (JIS) SD345:

$$
\begin{array}{cll}
\text { Modulus of elasticity: } & E_{s}=18.9 \cdot 10^{3} & \mathrm{kgf} / \mathrm{mm}^{2} \\
\text { Yield Strength: } & \sigma_{y}=38.70 & \mathrm{kgf} / \mathrm{mm}^{2} \\
\text { Nominal cross sectional area: } & A_{s}=32.0 & \mathrm{~mm}^{2}
\end{array}
$$

Normal concrete with coarse aggregate having a nominal maximum grain size of $10 \mathrm{~mm}$ was used for the web and flange walls. The concrete material parameters are:

$$
\begin{array}{cll}
\text { Modulus of elasticity: } & E_{0}=23.2 \cdot 10^{2} & \mathrm{kgf} / \mathrm{mm}^{2} \\
\text { Poisson' s ratio: } & v_{0}=0.167 & \\
\text { Uniaxial Compression Strength: } & R_{c}=2.880 & \mathrm{kgf} / \mathrm{mm}^{2} \\
\text { Uniaxial Tensile Strength: } & R_{c}=0.244 & \mathrm{kgf} / \mathrm{mm}^{2}
\end{array}
$$

The weight of the top slab of the specimen and the own weight of the shear wall amounted to 122.0 tonf. The consequent vertical compressive stress in the walls is $\sigma_{z}=0.150 \mathrm{kgf} / \mathrm{mm}^{2}$. 


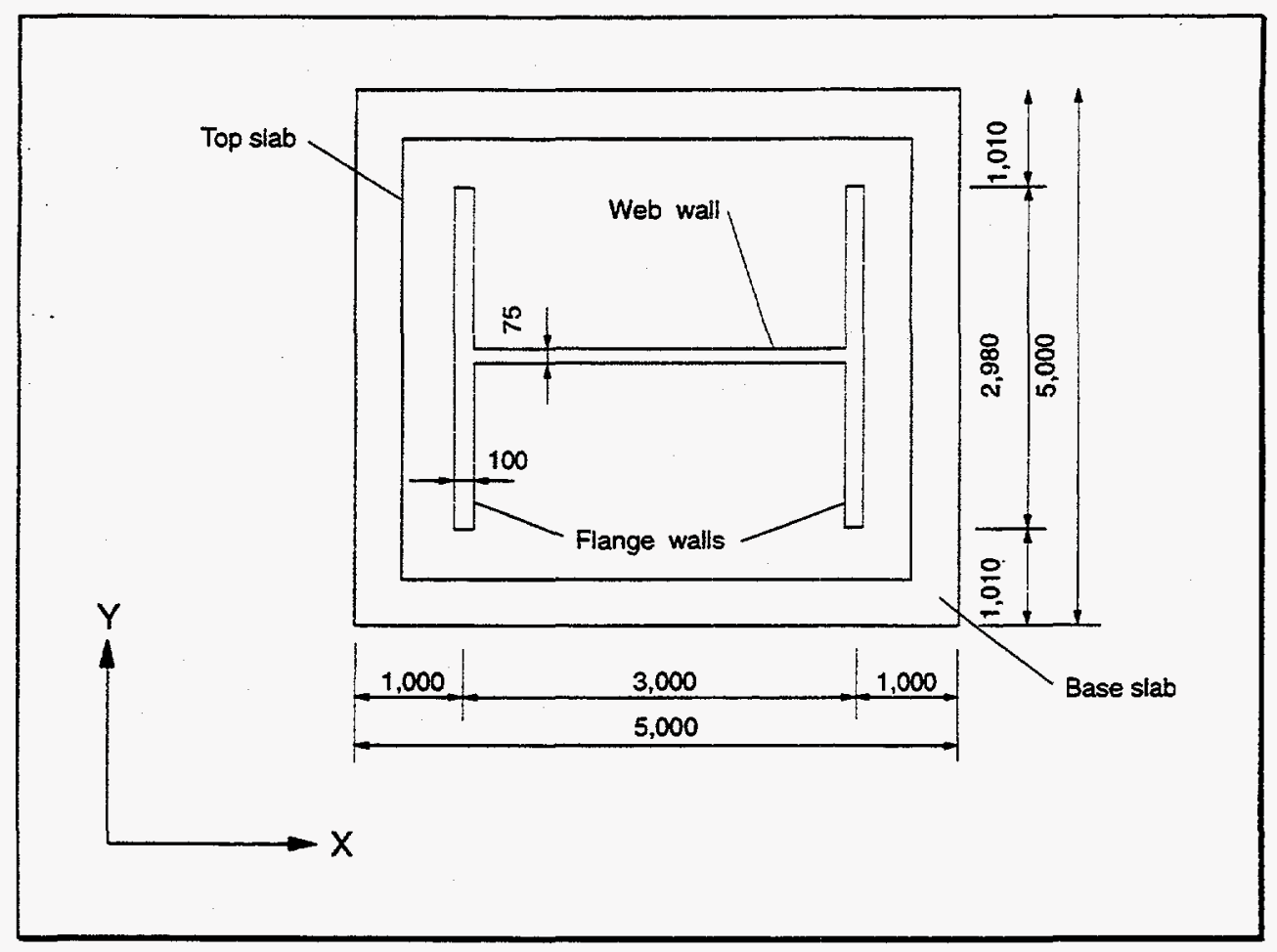

a) Plan

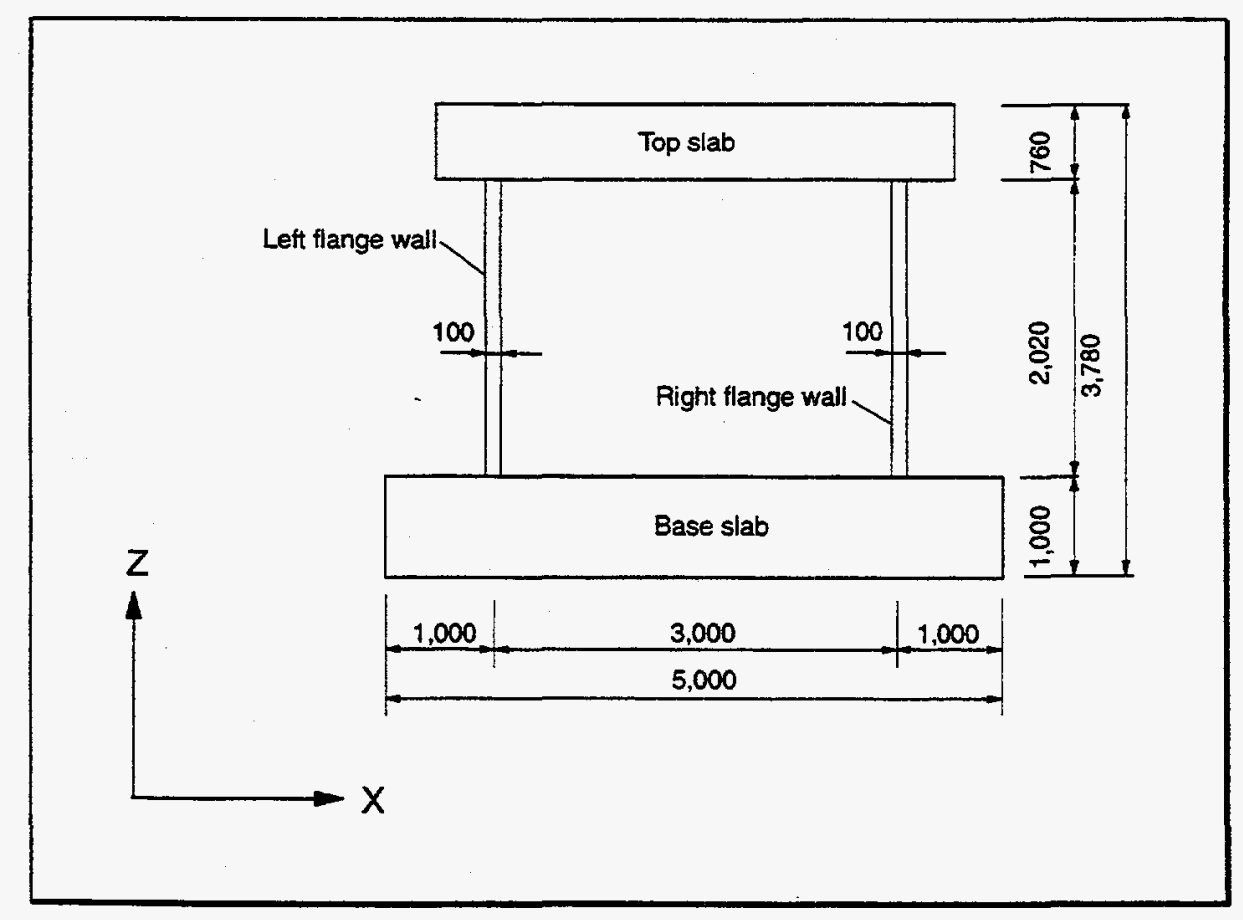

b) Web side elevation

Figure 1 Reinforced Concrete Shear Wall Specimen U-1 


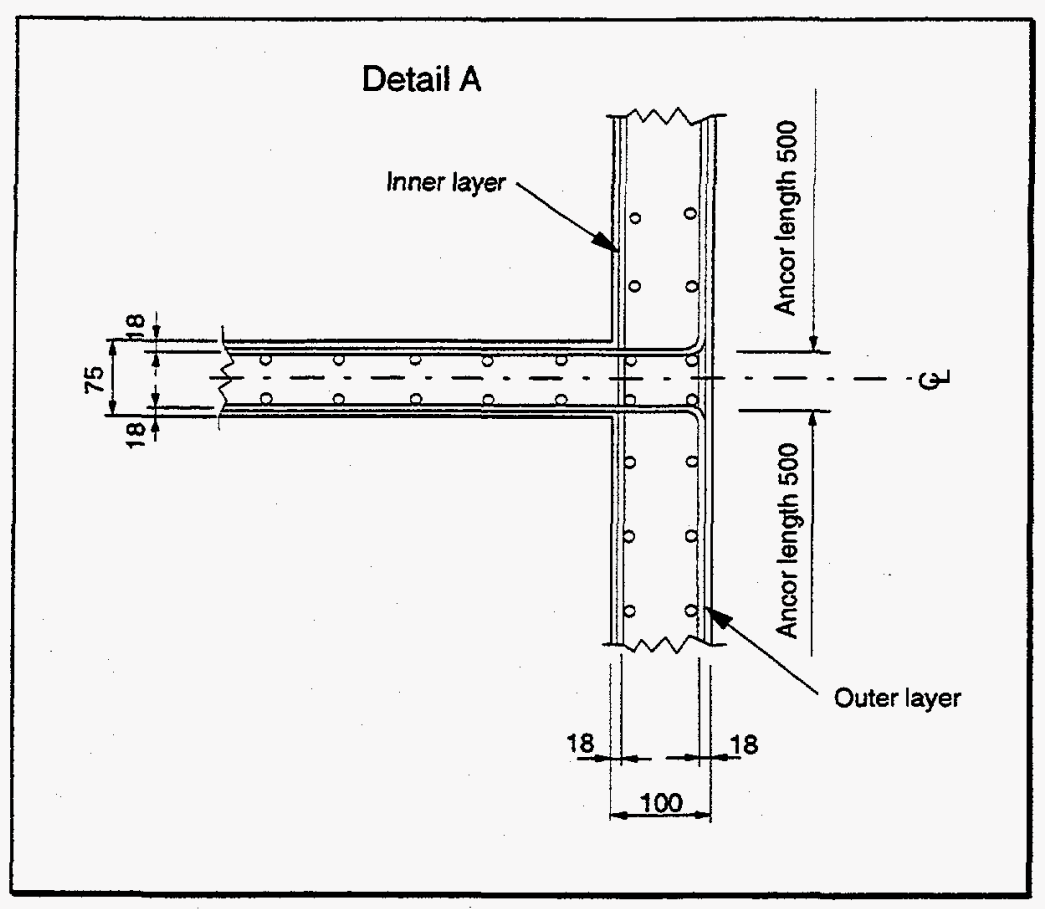

a) Detail of vertical reinforcement

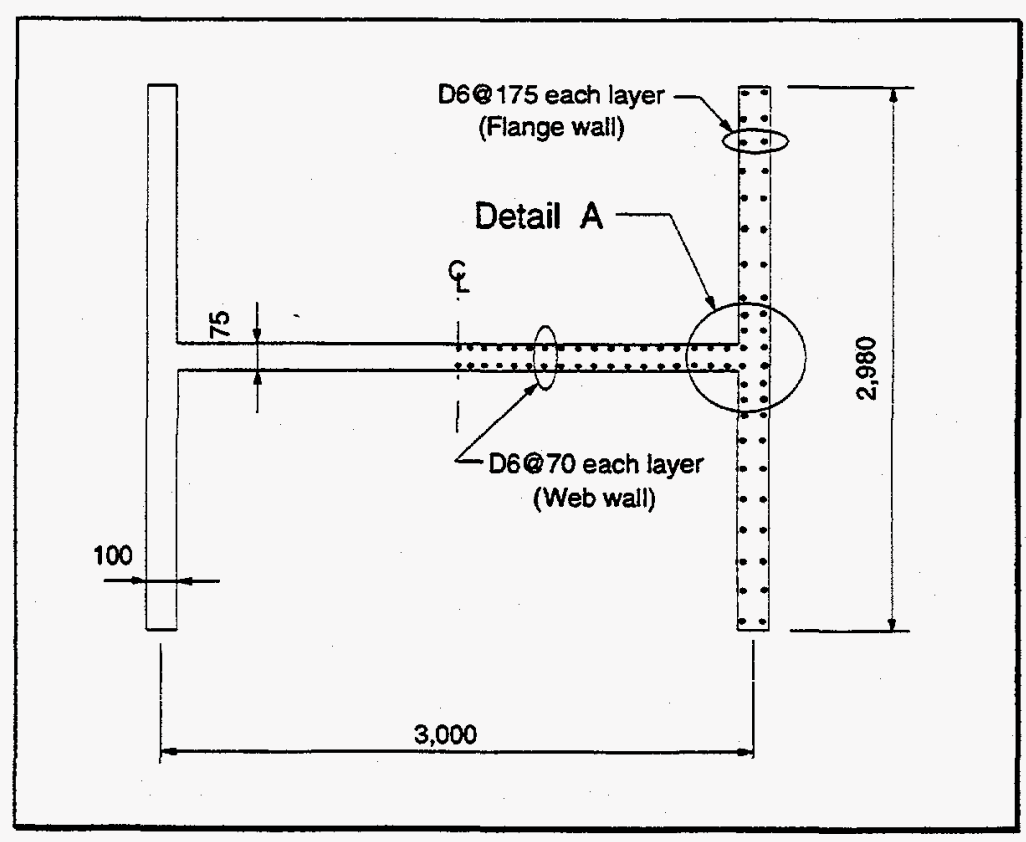

b) Plan of walls reinforcement

Figure 2 Reinforcing Arrangement 


\section{Finite Element Model of the Structure}

The model of shear wall specimen U-1 was developed with the general purpose finite element analysis program MIRAGE. Making use of symmetry in the geometry and loading arrangement of the specimen the model represents one half of the actual specimen, as shown in Fig. 3.

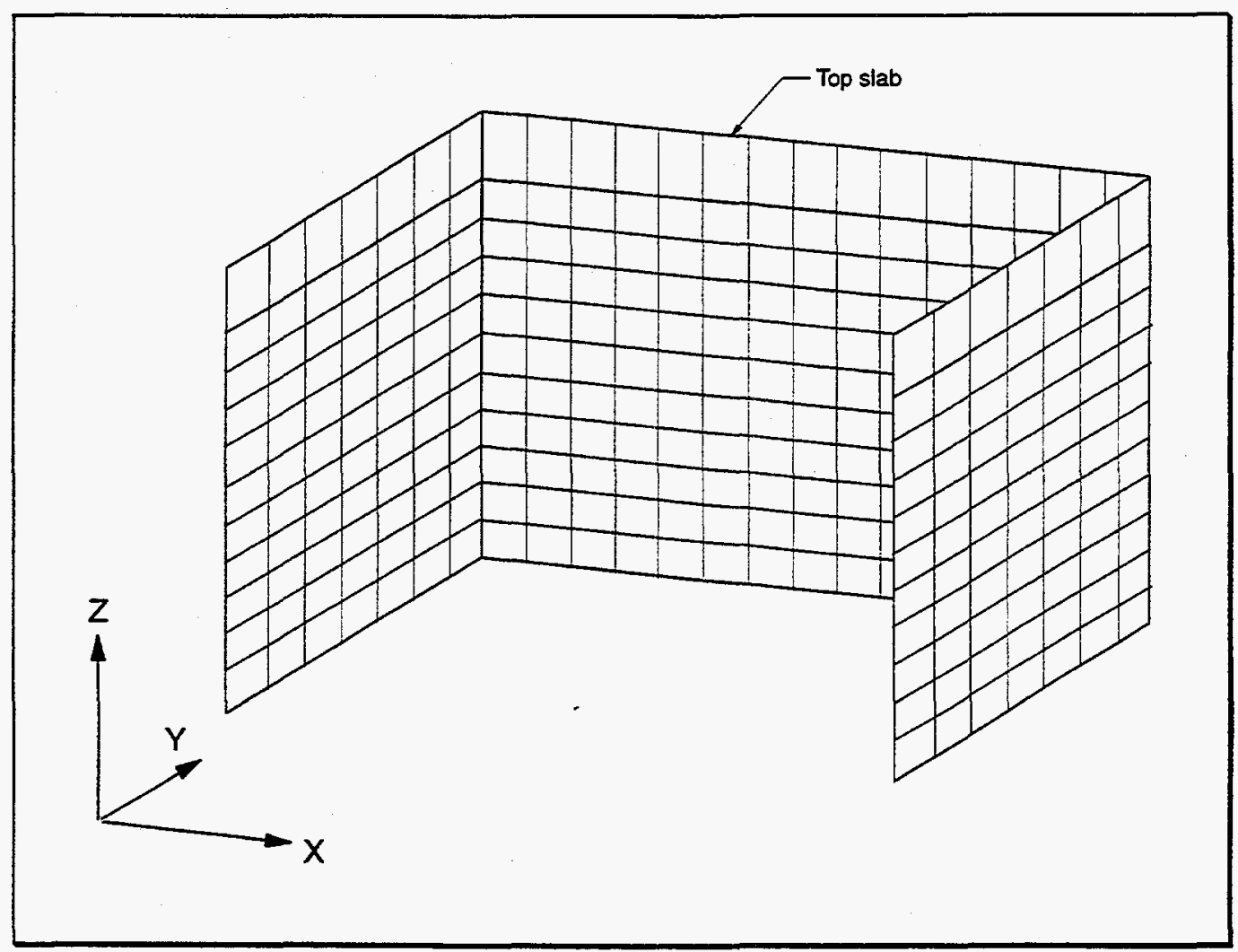

Figure 3 Three-Dimensional Thin-Walled Finite Element Model

On account of the cross section configuration of the specimen, with a ratio of wall height to thickness from 30 to 40 , the specimen is idealized as a three dimensional thin-walled structure with finite elements in condition of plane stress. An alternative three dimensional model with solid brick elements was also investigated, but is not pursued further in this report. Under the thin-wall assumption a quadrilateral plane stress finite element was used in the finite element model of the shear wall specimen.

The model of one-half of the shear wall specimen has 360 nodes and consists of 319 quadrilateral plane stress elements. The boundary conditions of the model were imposed by fixing the displacements along the global $\mathrm{X}, \mathrm{Y}$, and $\mathrm{Z}$-axes for all nodes at the base slab. On account of the lack of out-of-plane stiffness of the membrane element all nodal out-of plane displacements were also restrained in the analysis.

The material behavior of reinforced concrete was described by a two-dimensional orthotropic material model. Appendix A describes the general three-dimensional version of the 
model in program MIRAGE. The two-dimensional model of this study was obtained by setting the out-of-plane normal stress equal to zero in the model of Appendix A. The reinforcing steel is modeled with an embedded reinforcing layer model, as described in Appendix B.

The following material parameters were used for concrete:

$$
\begin{array}{cll}
\text { Modulus of elasticity: } & E_{0}=23.2 \cdot 10^{2} & \mathrm{kgf} / \mathrm{mm}^{2} \\
\text { Poisson' s ratio: } & \mathrm{v}_{0}=0.167 & \\
\text { Uniaxial Compression Strength: } & R_{\mathrm{c}}=2.880 & \mathrm{kgf} / \mathrm{mm}^{2} \\
\text { Uniaxial Tensile Strength: } & R_{\mathrm{r}}=0.244 & \mathrm{kgf} / \mathrm{mm}^{2}
\end{array}
$$

The reinforcement was smeared into an equivalent reinforcing steel layer in the mid-wall surface with uniaxial properties in each reinforcing direction (Fig. 4):

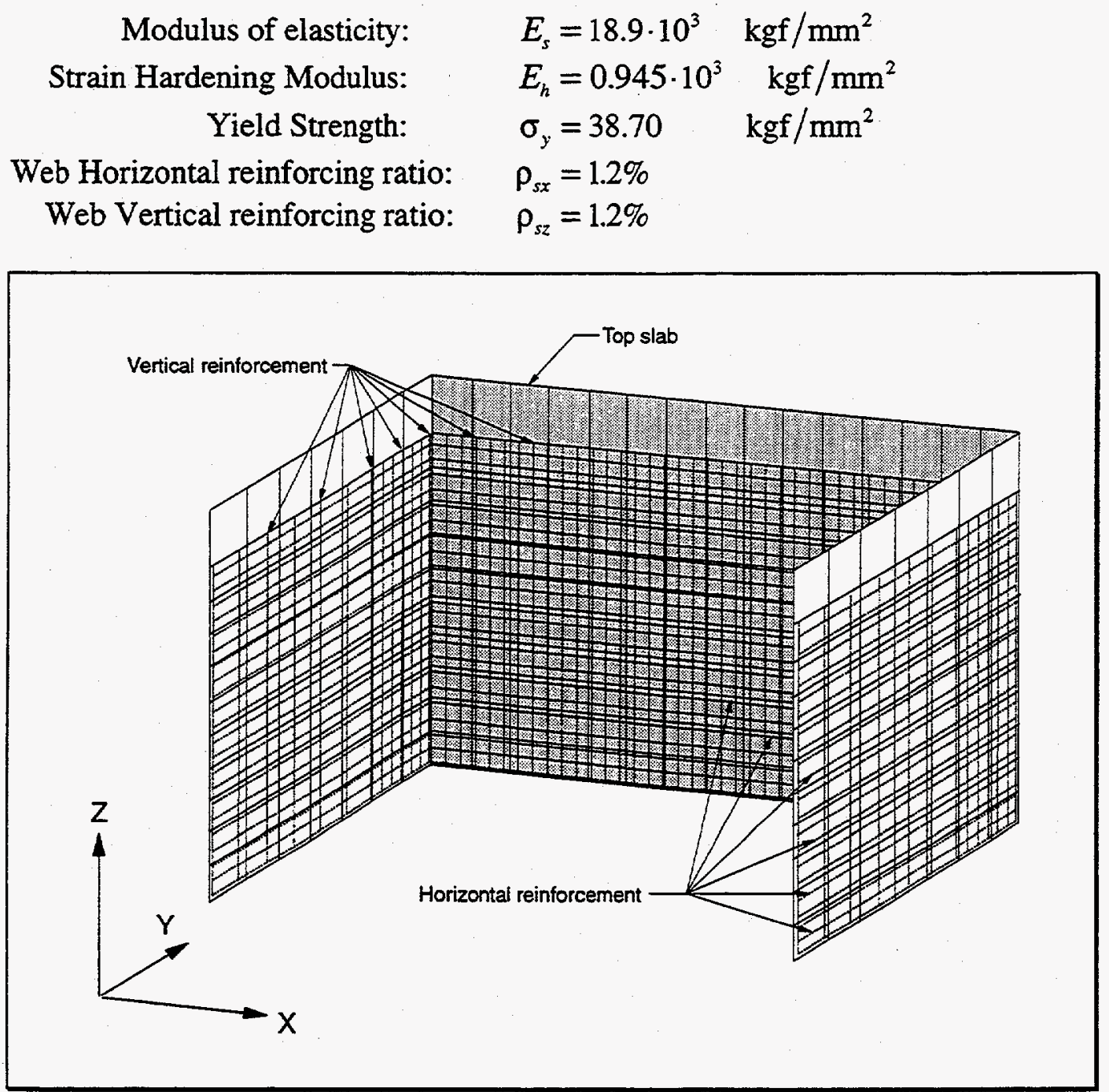

Figure 4 Reinforcement Layout in Finite Element Model 
The vertical reinforcing steel ratio $\rho_{s z}$ was $0.48 \%$ in the flange walls and $0.9 \%$ over a distance of $500 \mathrm{~mm}$ in the anchor zones between flange and web walls (Fig. 2a).

Figs. 3 and 4 show that the effect of the top slab on the structural behavior of the shear wall specimen was taken into account by the arrangement of a top row of quadrilateral membrane elements which remained linear elastic during the entire analysis. These elements account for the position of the applied load and the load distribution into the lateral load elements of the shearwall specimen. The following material properties are used for these special membrane elements:

$\begin{aligned} \text { Modulus of elasticity: } & E_{0}=23.2 \cdot 10^{2} \mathrm{kgf} / \mathrm{mm}^{2} \\ \text { Poisson's ratio: } & v_{0}=0.2\end{aligned}$

\section{Specification of Loading History}

The specification of lateral and vertical loads in several load steps is shown in Fig. 5. The vertical load of $P_{1}=122.0$ tonf simulates the weight of the top slab and other attached devices and was applied first. It was distributed to the nodes at the top of the model. The lateral load was gradually applied in subsequent steps guided by the maximum acceleration level of the five shaking table dynamic tests (Runs 1 through 5 in Fig. 5) up to a maximum value of $P_{2}=158.0$ tonf which was slightly less than the maximum acceleration level during Run 5:

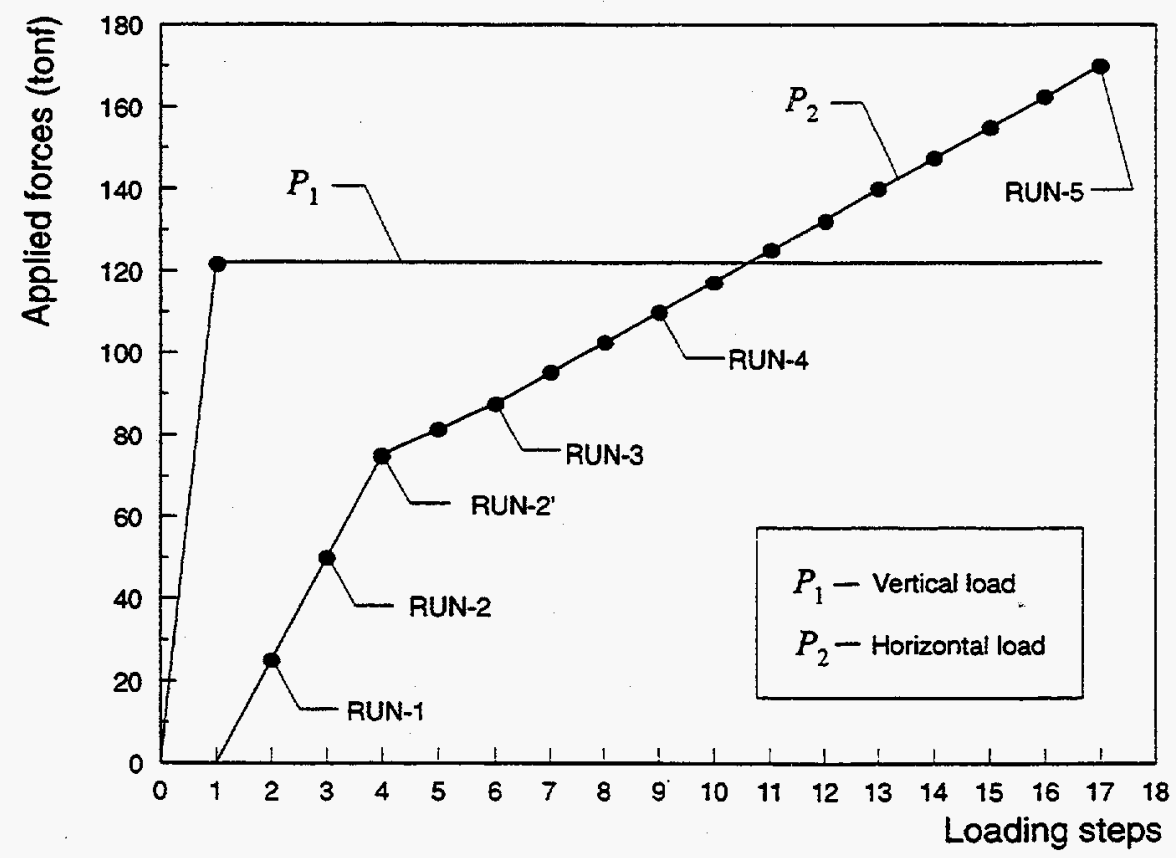

Figure 5 Loading History

- At load step 02 a horizontal load of 25.0 tonf (maximum acceleration during RUN-1)

- At load step 03 a horizontal load of 50.0 tonf (maximum acceleration during RUN-2)

- At load step 04 a horizontal load of 75.0 tonf (maximum acceleration during RUN-2') 
- At load step 06 a horizontal load of 87.5 tonf (maximum acceleration during RUN-3)

- At load step 09 a horizontal load of 110.0 tonf (maximum acceleration during RUN-4)

- At load step 16 a horizontal load of 162.0 tonf (less than the maximum acceleration during RUN-5). This load was not attained by the model.

The iterative solution process in each load step had an upper bound of 25 iterations.

\section{Comparison of Analytical with Experimental Results}

Under the load-controlled loading procedure the use of the classical Newton-Raphson iteration process for the solution of the nonlinear equilibrium equations at each load step only allowed the tracing of the nonlinear response of the shear wall specimen up to the ultimate lateral strength of the model, which was reached at a horizontal force of approximately $P_{2}=158.0$ tonf . Fig. 6 compares the analytical with the experimental load-displacement response of the shear wall specimen U-1. The analysis terminated at the end of the last branch by the singularity of the structure stiffness matrix. The use of displacement control procedures will afford the possibility of investigating the post-peak response of the specimen. In any case, the model underestimates the lateral strength that the specimen attained in Run 5. This might be due to the difference between dynamic and static behavior that was observed in other shaking table studies.

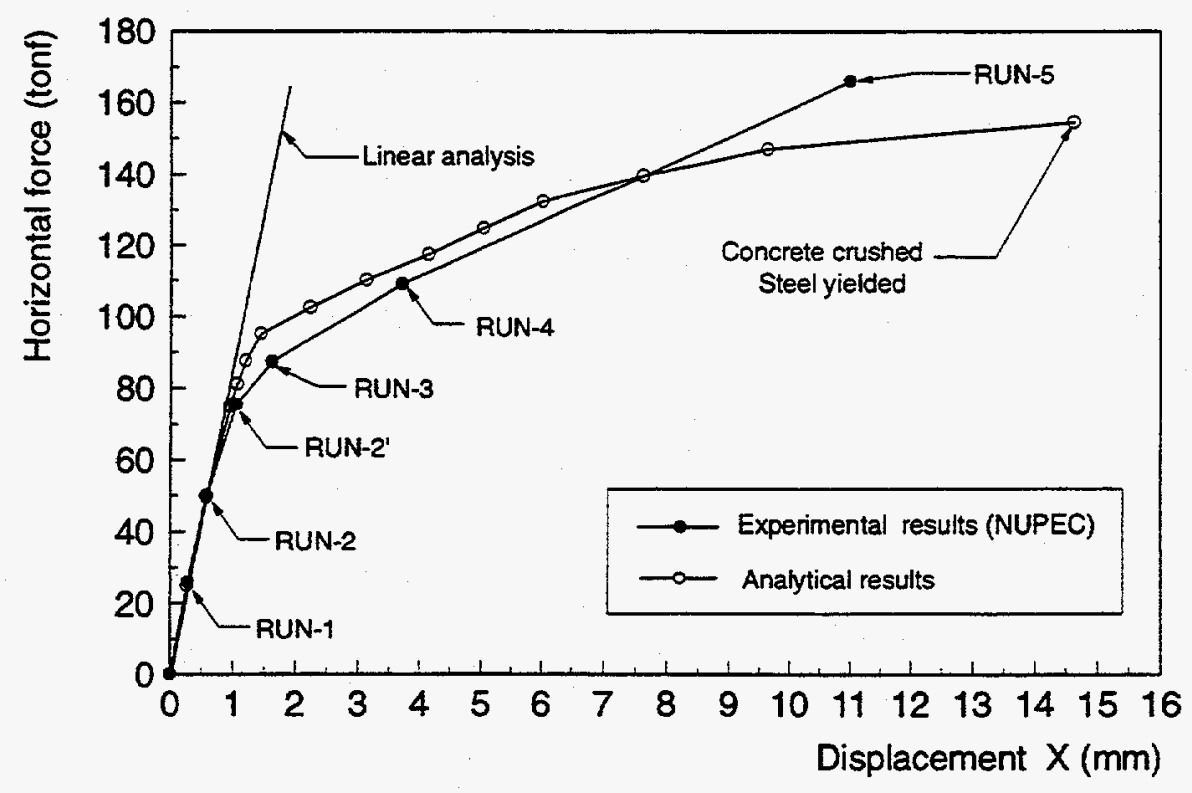

Figure 6 Load Displacement Response of the Top Slab Center

The load-displacement response up to ultimate consisted of 16 load steps. During the 16th load step crushing of several concrete elements and yielding of reinforcing steel at critical locations lead to the singularity of the structure stiffness matrix with consequent lack of 
convergence in the Newton-Raphson iteration of the equilibrium equations. It is interesting that few iterations were required from the first to the 4th load step, but this number gradually increased from the 5th to the 15 th load step as nonlinear behavior set in. The number of iterations for each load- step are given in Table 1 in Appendix D. During the 16th load step, 11 iterations were performed before the structure stiffness matrix became indefinite.

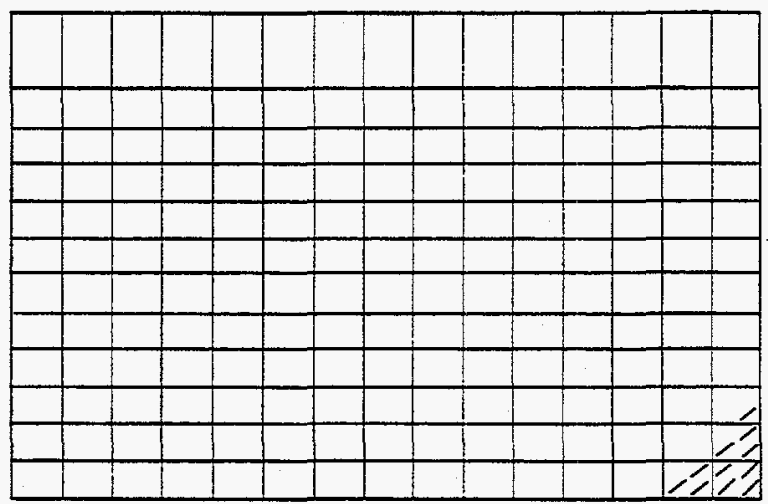

a) Horizontal force $P_{2}=50.0$ tonf

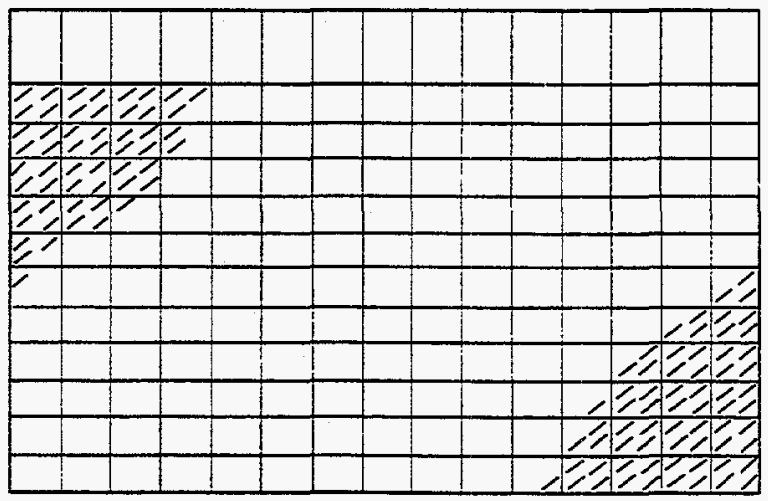

b) Horizontal force $P_{2}=75.0$ tonf

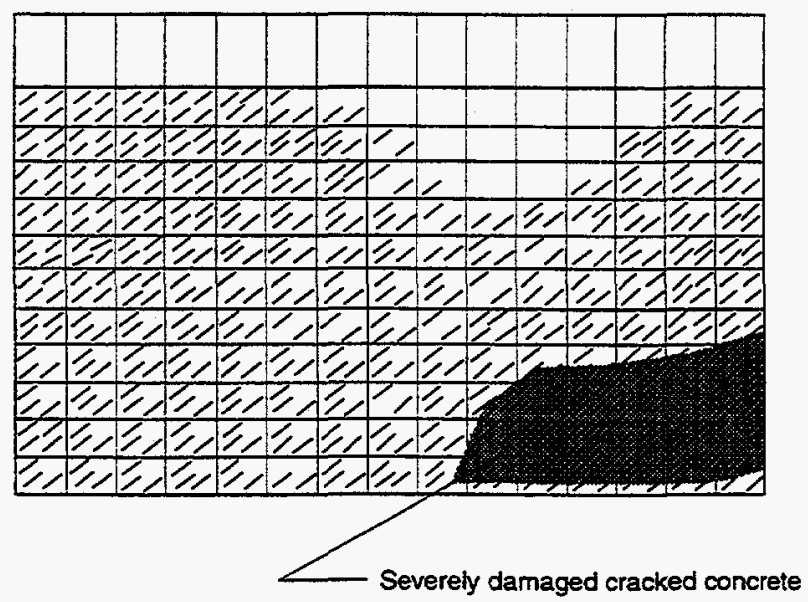

c) Horizontal force $P_{2}=158.0$ tonf

Figure 7 Analytical Crack Patterns in Web Wall 
Figs. 7 and 8 show the analytical crack distributions in the web and flange walls, respectively, for different loading stages.

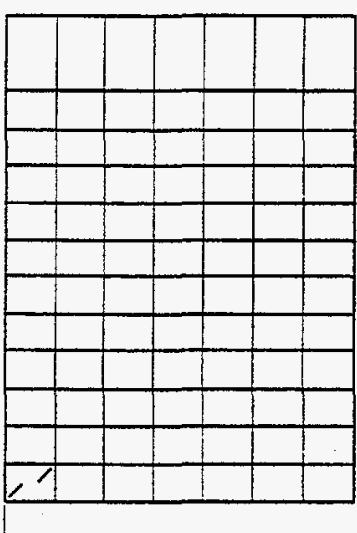

$\dot{q} \quad$ Left flange

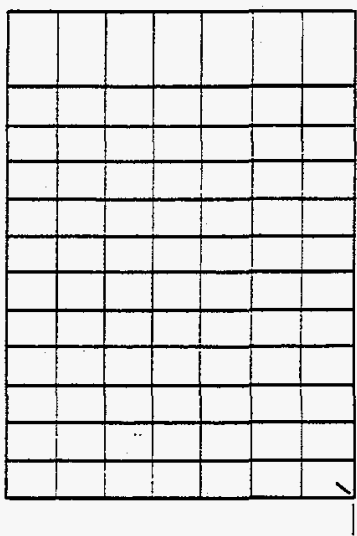

Right flange

a) Horizontal force $P_{2}=110.0$ tonf

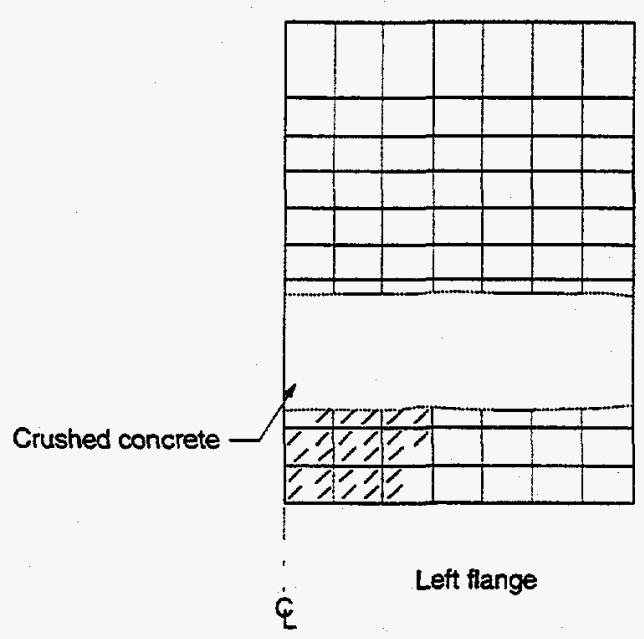

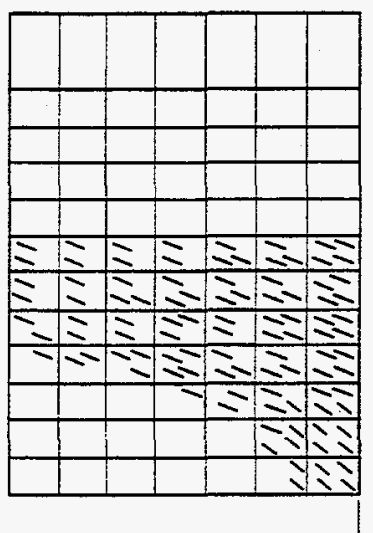

Right flange

b) Horizontal force $P_{2}=158.0$ tonf

Figure 8 Analytical Crack Patterns in Flange Walls 


\section{APPENDIX A. CONCRETE MATERIAL MODEL}

The three-dimensional cyclic concrete stress-strain relation is based on a hypoelastic orthotropic model with a stress-equivalent uniaxial strain relation that is generalized to account for triaxial stress conditions. The proposed model includes the effects of triaxial nonlinear stressstrain behavior, tensile cracking, compression crushing and strain-softening of concrete under general cyclic loading conditions. The biaxial plane stress model in this study was derived from the general model by setting the out-of-plane normal stress equal to zero.

Since plain concrete cannot sustain large relative displacements, the material model formulation assumes small deformations. Even under small deformations, rigid body rotations may be significant in some cases $[3,6,15]$. These large rotation effects are included in the finite element implementation by a total Lagrangian formulation [4].

The following description of the material model is based on engineering stresses $\sigma_{i j}$ and strains $\varepsilon_{i j}$ for the sake of convenience. For the inclusion of large rotation effects the total Lagrangian stress and strain variables should replace the corresponding engineering variables in the finite element implementation. The numerical implementation of the material model allows for cyclic loading conditions and the opening and closing of tensile cracks.

\section{Stress Strain Relations}

The following section describes the stress-strain relations of the model prior to tensile cracking and compression crushing. These general multiaxial relations are based on the equivalent uniaxial strain concept $[2,10]$.

\section{Triaxial Stress-Strain Relations}

It is well established that concrete could be treated as an initially isotropic material that exhibits deformational anisotropy during loading $[9,11-13,15,20]$. Consequently, in structures where the stress state at every point is defined by three principal stresses, concrete can be characterized during loading as a nonlinear orthotropic medium with the directions of orthotropy coincident with the principal stress directions $[3,9,11,16]$. In this approach the increntental constitutive relations of concrete in reference to the orthotropic axes $(1,2,3)$ are:

$$
\left\{\begin{array}{l}
d \sigma_{1} \\
d \sigma_{2} \\
d \sigma_{3} \\
d \tau_{12} \\
d \tau_{31} \\
d \tau_{31}
\end{array}\right\}=\frac{1}{\Omega}\left[\begin{array}{cccccc}
E_{1}\left(1-v_{23} v_{32}\right) & E_{1}\left(v_{21}+v_{23} v_{31}\right) & E_{1}\left(v_{31}+v_{21} v_{32}\right) & 0 & 0 & 0 \\
E_{2}\left(v_{12}+v_{13} v_{32}\right) & E_{2}\left(1-v_{13} v_{31}\right) & E_{2}\left(v_{32}+v_{12} v_{31}\right) & 0 & 0 & 0 \\
E_{3}\left(v_{13}+v_{12} v_{23}\right) & E_{3}\left(v_{23}+v_{13} v_{21}\right) & E_{3}\left(1-v_{12} v_{21}\right) & 0 & 0 & 0 \\
0 & 0 & 0 & G_{12} \Omega & 0 & 0 \\
0 & 0 & 0 & 0 & G_{23} \Omega & 0 \\
0 & 0 & 0 & 0 & 0 & G_{31} \Omega
\end{array}\right]\left\{\begin{array}{l}
d \varepsilon_{1} \\
d \varepsilon_{2} \\
d \varepsilon_{3} \\
d \gamma_{12} \\
d \gamma_{23} \\
d \gamma_{31}
\end{array}\right\}
$$

where 
$d \sigma_{i}(i=1,2,3)=$ normal stress increments in $i$-direction;

$d \tau_{i j}(i, j=1,2,3)=$ shear stress increments in plane $i-j$;

$v_{i j}(i, j=1,2,3)=$ transverse strain ratio for strain in $j$-direction caused by stress in $i$-direction;

$\Omega=1-v_{21} v_{12}-v_{31} v_{13}-v_{32} v_{23}-v_{12} v_{23} v_{31}-v_{21} v_{32} v_{13} ;$

$E_{i}(i=1,2,3)=$ total secant modulus of elasticity in $i$-direction of orthotropy;

$G_{i j}(i, j=1,2,3)=$ total secant shear modulus in plane $i-j$;

$d \varepsilon_{i}(i=1,2,3)=$ normal strain increments in $i$-direction;

$d \gamma_{i j}(i, j=1,2,3)=$ shear strain increments in plane $i-j$.

Eq. (1) can be written in matrix form as:

$$
\{d \sigma\}=[D]_{L}\{d \varepsilon\}
$$

where

$\{d \sigma\}=$ vector of stress increments;

$\{d \varepsilon\}=$ vector of strain increments;

$[D]_{L}=$ incremental concrete material matrix.

The transformation of material matrix $[D]_{L}$ to a non-orthotropic set of axes $(1,2,3)$ and the invariance condition for the shear moduli under this transformation yields the following relation

$$
G_{i j}=\frac{E_{i} E_{j}}{E_{i}\left(1+v_{i j}\right)+E_{j}\left(1+v_{j i}\right)}
$$

which defines the incremental concrete shear modulus in Eq. (1).

Since the material matrix $[D]_{L}$ in Eq. (2) is defined with reference to the directions of orthotropy, it must be transformed to the global coordinate system of the structure before assembly of the element stiffness matrix. This requires the following transformation:

$$
[D]_{G}=[T]^{T}[D]_{L}[T]
$$

where matrix $[T]$ takes the following form according to Lekhnitskii (1963): 


$$
[T]=\left[\begin{array}{cccccc}
m_{11}^{2} & m_{12}^{2} & m_{13}^{2} & 2 m_{11} m_{12} & 2 m_{12} m_{13} & 2 m_{11} m_{13} \\
m_{21}^{2} & m_{22}^{2} & m_{23}^{2} & 2 m_{21} m_{22} & 2 m_{22} m_{23} & 2 m_{21} m_{23} \\
-m_{31}^{2} & m_{32}^{2} & m_{33}^{2} & 2 m_{31} m_{32} & 2 m_{32} m_{33} & 2 m_{31} m_{33} \\
m_{11} m_{21} & m_{12} m_{22} & m_{13} m_{23} & m_{11} m_{22}+m_{12} m_{21} & m_{12} m_{23}+m_{13} m_{22} & m_{11} m_{23}+m_{13} m_{21} \\
m_{21} m_{31} & m_{22} m_{32} & m_{23} m_{33} & m_{21} m_{32}+m_{22} m_{31} & m_{22} m_{33}+m_{23} m_{32} & m_{21} m_{33}+m_{23} m_{31} \\
m_{11} m_{31} & m_{12} m_{32} & m_{13} m_{33} & m_{31} m_{12}+m_{32} m_{11} & m_{32} m_{13}+m_{33} m_{12} & m_{31} m_{13}+m_{33} m_{11}
\end{array}\right]
$$

$m_{i j}$ denotes the direction cosines of the principal stress $\sigma_{i}(i=1,2,3)$-axes relative to the global coordinate system $X_{j}(j=1,2,3)$ - of the structure.

\section{Equivalent Uniaxial Strains}

For the incremental constitutive relation in Eq. (1) it is necessary to describe now the determination of the nine incremental moduli. To this end the concept of equivalent uniaxial strain, as proposed in [10], is used in the material model. The method is briefly summarized as follows:

Let Eq. (1) be written as:

$$
\left\{\begin{array}{l}
d \sigma_{1} \\
d \sigma_{2} \\
d \sigma_{3} \\
d \tau_{12} \\
d \tau_{23} \\
d \tau_{31}
\end{array}\right\}=\left[\begin{array}{cccccc}
E_{1} & 0 & 0 & 0 & 0 & 0 \\
0 & E_{2} & 0 & 0 & 0 & 0 \\
0 & 0 & E_{3} & 0 & 0 & 0 \\
0 & 0 & 0 & G_{12} & 0 & 0 \\
0 & 0 & 0 & 0 & G_{23} & 0 \\
0 & 0 & 0 & 0 & 0 & G_{31}
\end{array}\right]\left\{\begin{array}{l}
d \varepsilon_{u 1} \\
d \varepsilon_{u 2} \\
d \varepsilon_{u 3} \\
d \gamma_{12} \\
d \gamma_{23} \\
d \gamma_{31}
\end{array}\right\}
$$

where

$$
\left.\begin{array}{l}
d \varepsilon_{u 1}=\frac{1}{\Omega}\left[\left(1-v_{23} v_{32}\right) d \varepsilon_{1}+\left(v_{21}+v_{23} v_{31}\right) d \varepsilon_{2}+\left(v_{31}+v_{21} v_{32}\right) d \varepsilon_{3}\right] \\
d \varepsilon_{u 2}=\frac{1}{\Omega}\left[\left(v_{12}+v_{13} v_{32}\right) d \varepsilon_{1}+\left(1-v_{13} v_{31}\right) d \varepsilon_{2}+\left(v_{32}+v_{12} v_{31}\right) d \varepsilon_{3}\right] \\
d \varepsilon_{u 3}=\frac{1}{\Omega}\left[\left(v_{13}+v_{12} v_{23}\right) d \varepsilon_{1}+\left(v_{23}+v_{13} v_{21}\right) d \varepsilon_{2}+\left(1-v_{12} v_{21}\right) d \varepsilon_{3}\right]
\end{array}\right\}
$$

The variables $d \varepsilon_{u i}(i=1,2,3)$ in Eq. (7) represent the equivalent uniaxial strain increments, expressed in terms of the actual incremental strains $d \varepsilon_{i}(i=1,2,3)$.

The equivalent uniaxial strain increments can be determined from Eq. (6):

$$
d \varepsilon_{u i}=\frac{d \sigma_{i}}{E_{i}}(i=1,2,3)
$$

Eq. (8) yields the total equivalent uniaxial strains by integration over the load path, i.e. 


$$
\varepsilon_{u i}=\int \frac{d \sigma_{u i}}{E_{i}}(i=1,2,3)
$$

Eq. (8) shows that the equivalent uniaxial strain increment represents the strain increment in the $i$-direction that the material would exhibit under a uniaxial stress increment with the other stresses kept equal to zero. In reality, $d \varepsilon_{u i}$ depends on the three-dimensional stress state, so that $\varepsilon_{u i}$ and $d \varepsilon_{u i}{ }^{\prime}$ are not collinear with the corresponding stress along the load path. Both are, therefore, fictitious quantities, except for the uniaxial case, and are only significant as a measure for the variation of material parameters.

Eqs. (7) are defined with respect to the material axes of orthotropy. Since these coincide with the current directions of principal stress, it follows that $d \varepsilon_{u i}$ must be defined with respect to the current axes of orthotropy. The last statement implies equivalence between the equivalent strain parameters of the elasto-plastic theory and the equivalent uniaxial strains in Eq. (7).

\section{Monotonic Uniaxial Stress- Equivalent Strain Relation}

The approach in the proposed model follows the method in [10] and assumes that the actual stresses are functions of the current equivalent uniaxial strains, whose components are, in turn, determined from the actual strain increments.

In the description of the uniaxial stress-equivalent strain relation the uniaxial compressive stress-strain relationship in [22] is generalized, in a manner similar to that proposed in [2], to describe both tensile and compressive response under monotonic loading. Rewriting this relation in terms of equivalent uniaxial strains according to [12] results in

$$
\sigma_{i}=R_{c i} \frac{K_{i}\left(\frac{\varepsilon_{u i}}{\varepsilon_{c i}}\right)}{1+A_{i}\left(\frac{\varepsilon_{u i}}{\varepsilon_{c i}}\right)+B_{i}\left(\frac{\varepsilon_{u i}}{\varepsilon_{c i}}\right)^{2}+C_{i}\left(\frac{\varepsilon_{u i}}{\varepsilon_{c i}}\right)^{3}} \quad(i=1,2,3)
$$

where the following notation is used:

$$
\begin{gathered}
K_{i}=E_{0} \frac{\varepsilon_{c i}}{R_{c i}} ; \quad K_{\varepsilon i}=\frac{\varepsilon_{f i}}{\varepsilon_{c i}} ; \quad K_{\sigma i}=\frac{R_{c i}}{R_{f i}} \\
A_{i}=C_{i}+K_{i}-2 ; \quad B_{i}=1-2 \cdot C_{i} ; \quad C_{i}=K_{i} \frac{\left(K_{\sigma i}-1\right)}{\left(K_{\varepsilon i}-1\right)^{2}}-\frac{1}{K_{\varepsilon i}}
\end{gathered}
$$




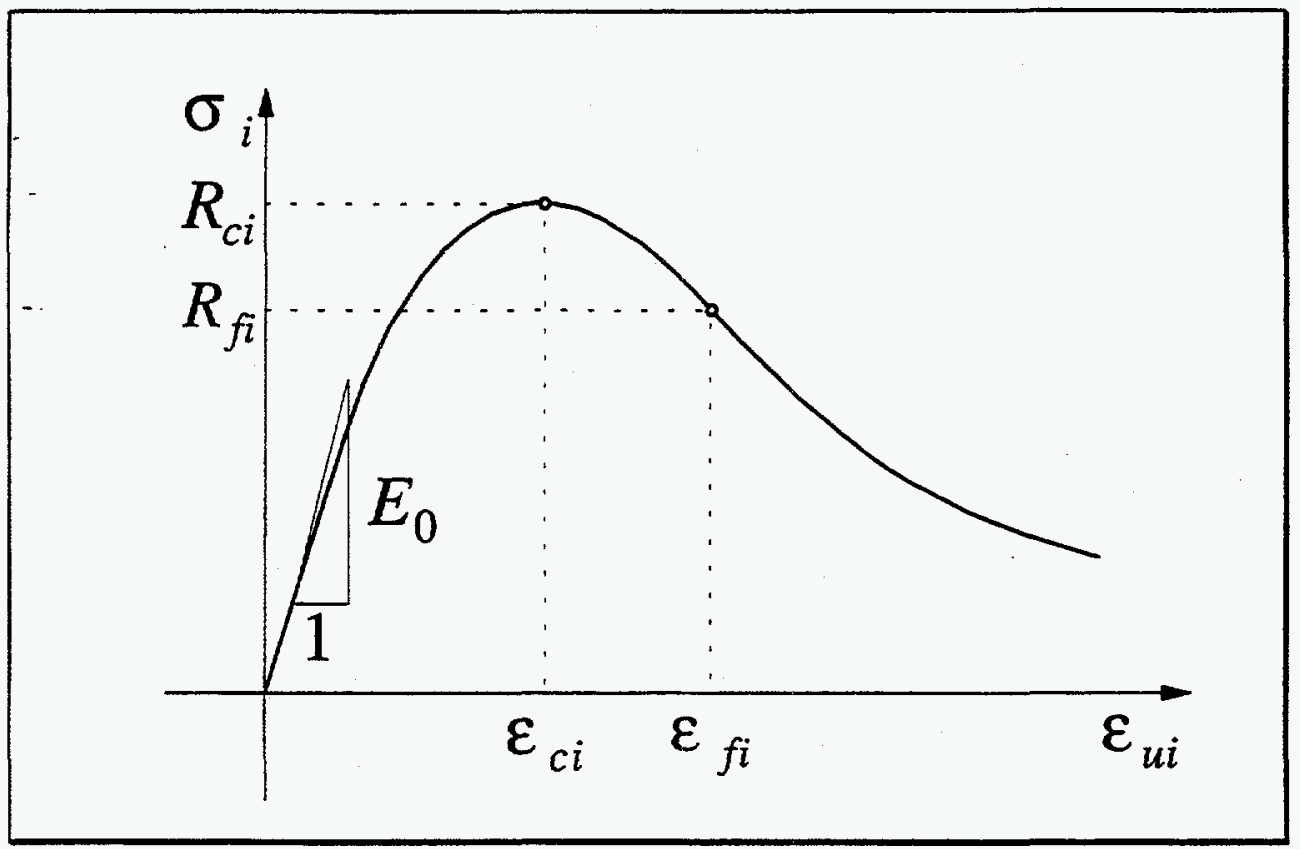

Fig. A.1 Monotonic Uniaxial Stress-Equivalent Strain Curve

Figure A.1 depicts the monotonic curve described by Eq. (9a) as well as the variables that appear in the definitions of Eq. (9b). More specifically:

$E_{0}=$ initial modulus of elasticity;

$\varepsilon_{u i}=$ equivalent uniaxial strain in $i$-direction;

$R_{c i}=$ concrete strength in $i$-direction associated with current principal stress ratio;

$\varepsilon_{c i}=$ corresponding equivalent uniaxial strain;

$R_{f i}, \varepsilon_{f i}=$ control point coordinates on descending branch of stress-equivalent strain curve.

The division of Eq. (9a) by $\varepsilon_{u i}$ yields the required incremental secant modulus of concrete for Eq. (1):

$$
E_{i}=\frac{E_{0}}{1+A_{i}\left(\frac{\varepsilon_{u i}}{\varepsilon_{c i}}\right)+B_{i}\left(\frac{\varepsilon_{u i}}{\varepsilon_{c i}}\right)^{2}+C_{i}\left(\frac{\varepsilon_{u i}}{\varepsilon_{c i}}\right)^{3}} \quad(i=1,2,3)
$$

\section{Transverse Strain Ratios}

Before implementing the proposed concrete stress-strain relation it is also necessary to define the transverse strain ratios $v_{i j}$ in Eq. (1). The following expression in [1] defines these ratios: 


$$
v_{i j}=\sqrt{v_{u i} v_{u j} \frac{E_{i}}{E_{j}}} \quad(i, j=1,2,3)
$$

where $v_{u i}$ is the uniaxial transverse strain ratio that is axially symmetric about the $i$-axis and is defined as a cubic function of the corresponding equivalent uniaxial strain

$$
v_{u i}=v_{0}\left[1+A_{i}\left(\frac{\varepsilon_{u i}}{\varepsilon_{c i}}\right)+B_{i}\left(\frac{\varepsilon_{u i}}{\varepsilon_{c i}}\right)^{2}+C_{i}\left(\frac{\varepsilon_{u i}}{\varepsilon_{c i}}\right)^{3}\right](i=1,2,3)
$$

where

$$
v_{0}=\text { initial Poisson's ratio; }
$$

$A_{i}, B_{i}, C_{i}=$ parameters determined by Eq. $(9 \mathrm{c})$, where the parameter $K_{i}=\frac{1}{2 v_{0}}$

It follows that Eq. (11) satisfies the following conditions of symmetry:

$$
E_{1} v_{21}=E_{2} v_{12} ; E_{2} v_{32}=E_{3} v_{23} ; \quad E_{3} v_{13}=E_{1} v_{31}
$$

Variable $\Omega$ in Eq. (1) should be always positive. Thus, the limiting value of $v_{u i}$ in Eq. (12) is 0.5 . This value corresponds to a limit of zero incremental volume change. As noted in $[9,13,17]$ the point at which this limit is reached corresponds to the onset of unstable micro crack propagation which gives rise to the experimentally observed dilatancy phenomenon in the vicinity of the concrete material strength.

\section{Loading, Unloading and Reloading Criteria}

The state of material loading or unloading affects the evaluation of the stress-strain relations in Eq. (1). The loading or unloading conditions are defined by a loading function [1]

$$
f=\sqrt{\frac{\varepsilon_{u 1}^{2}+\varepsilon_{u 2}^{2}+\varepsilon_{u 3}^{2}}{\varepsilon_{c 1}^{2}+\varepsilon_{c 2}^{2}+\varepsilon_{c 3}^{2}}}
$$

where $\varepsilon_{u i}, \varepsilon_{c i}(i=1,2,3)$ are the parameters in Eq. (9b).

The condition of material loading is

$$
f>f_{\max }
$$

and the condition of unloading is

$$
f \leq f_{\max }
$$

where $f_{\max }$ is the maximum value of the loading function in Eq. (13) during the previous loading history. 
Under loading conditions the principal stresses are used in the determination of the secant modulus $E_{i}$ for the corresponding equivalent uniaxial strain $\varepsilon_{u i}$ of each principal direction according to Eq. (10).

Under conditions of unloading and reloading to the stress state from which unloading occurred, the material is assumed to be isotropic. In this case the initial Young's modulus $E_{0}$ and Poisson's ratio $v_{0}$ are used in the incremental stress-strain relations of Eq. (1).

\section{Ultimate Surfaces}

The determination of incremental moduli in Eq. (10) depends on the specification of the parameters in Eq. (9b). Since these parameters vary with the principal stress ratio, this can be done most conveniently by the specification of a surface in principal stress space that defines three $R_{c i}$ values for each stress ratio, and a corresponding surface in equivalent uniaxial strain space for the definition of three $\varepsilon_{c i}$ values that correspond to these $R_{c i}$ values (Fig. A.1).

\section{Ultimate Strength Surface}

The surface in stress space that defines the ultimate strength values $R_{c i}$ for any principal stress ratio is usually called the "failure surface". Because this name is somewhat misleading in the context of a material with strain softening behavior, this surface is called in the following the "ultimate strength surface".

The ultimate strength surface of concrete is described in this study by a modification of the five-parameter model in [7]. The modified surface is described by the following equation, as shown in Fig. A.2:

$$
\frac{R_{t}}{R_{c}} \tau^{2}+\frac{\left(R_{c}^{2}-R_{t}^{2}\right)}{2 R_{c}}\left[\frac{R_{t}}{R_{c}}\left(\tau+3 \sigma_{0}\right)+2\left(1-\frac{R_{t}}{R_{c}}\right) \sigma_{1}\right]=R_{c} R_{\tau}
$$

where

$$
\sigma_{0}=\frac{1}{3}\left(\sigma_{1}+\sigma_{2}+\sigma_{3}\right) ; \quad \tau=\sqrt{\left(\sigma_{1}^{2}+\sigma_{2}^{2}+\sigma_{3}^{2}-\sigma_{1} \sigma_{2}-\sigma_{2} \sigma_{3}-\sigma_{3} \sigma_{1}\right)}
$$

and $R_{c}, R_{t}=$ uniaxial compression and tensile material strength, respectively.

Eq. (14) defines the ultimate strength surface in the principal stress plane $\sigma_{1}, \sigma_{2}, \sigma_{3}$ and describes a smooth convex surface (see Fig. A.2) with curvature approaching zero when the brittleness $\frac{R_{t}}{R_{c}} \rightarrow 1$ and/or $\sigma_{0} \rightarrow \infty$. 


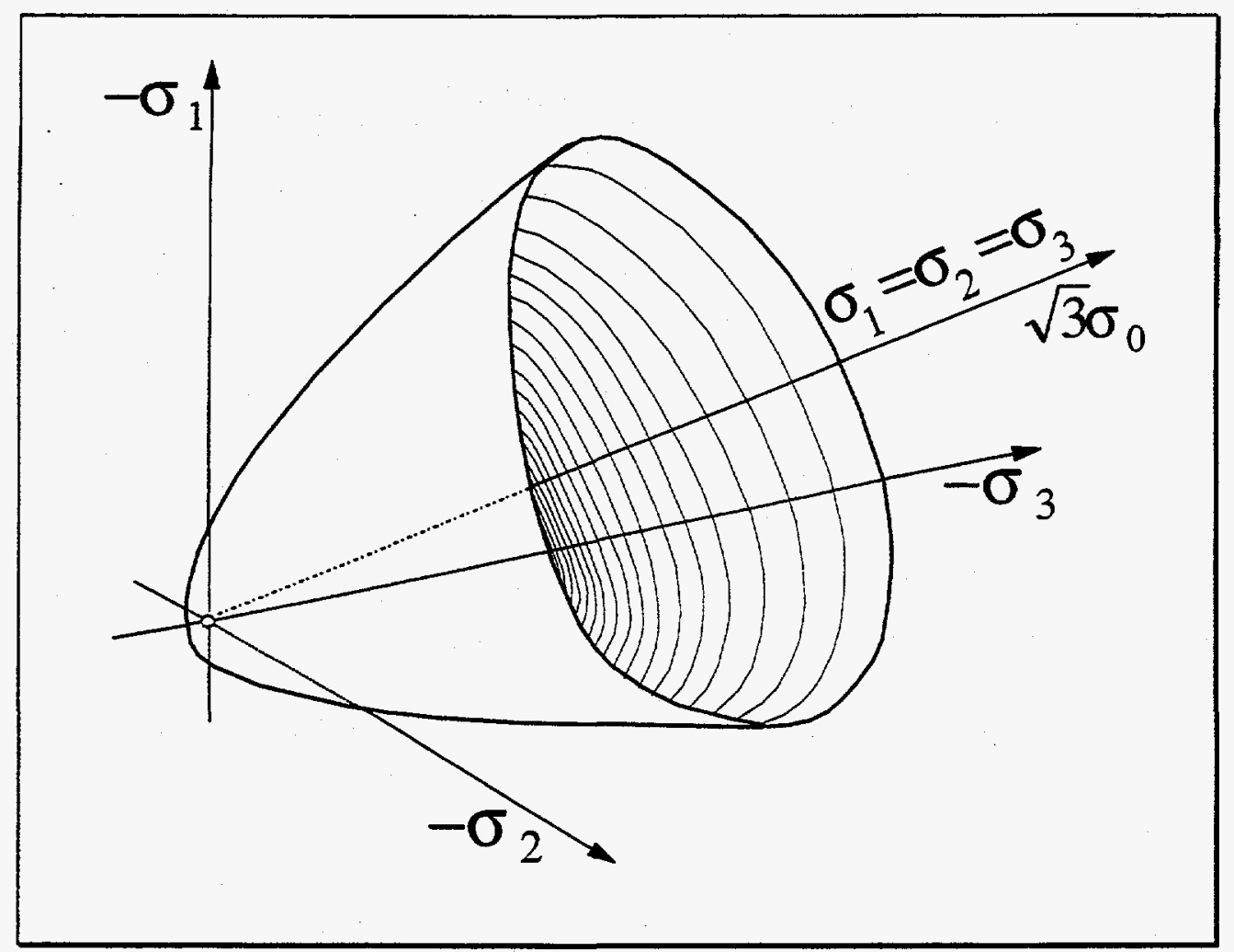

Fig. A.2 Concrete Failure Surface

\section{Ultimate Strain Surface}

For the evaluation of the ultimate equivalent uniaxial strains $\varepsilon_{c i}$ a surface is defined in equivalent uniaxial strain space according to the proposal in [11]. In this study the ultimate equivalent uniaxial strain surface has the same form as the ultimate stress surface. For the surface definition the equivalent uniaxial strains $\varepsilon_{u 1}, \varepsilon_{u 2}$ and $\varepsilon_{u 3}$ replace the principal stresses $\sigma_{1}, \sigma_{2}$ and $\sigma_{3}$, respectively, in Eq. (14) and the ultimate uniaxial compression and tensile strains $\varepsilon_{c}, \varepsilon_{t}$ replace the uniaxial compression and tensile strength $R_{c}$ and $R_{t}$, respectively.

Finally, the concrete stress-strain relation in Eq. $(9 \mathrm{~b})$ requires the values of $\sigma_{f i}$ and $\varepsilon_{f i}$ that define the coordinates of a point on the descending branch of the monotonic stressequivalent uniaxial strain curve in Fig. A.1. The definition of this point is not possible on a rigorous experimental basis, as already noted in $[13,15,17]$, since the descending branch of the stress-strain curve is highly dependent on the properties of the test apparatus and, thus, generally unavailable from a statically determinate test setup.

The following parameter values are used in this study:

$\sigma_{f i}=0.85 \cdot R_{c i}$ and $\varepsilon_{f i}=1.41 \cdot \varepsilon_{c i}$ under compression loading, and

$\sigma_{f i}=0.25 \cdot R_{c i}$ and $\varepsilon_{f i}=4.0 \cdot \varepsilon_{c i}$ under tension loading. 


\section{Current Material Strength}

The determination of the three current concrete strength values $R_{c 1}, R_{c 2}$ and $R_{c 3}$ is based on the ultimate strength surface in Eq. (14) as follows: if point $M_{c}\left(\sigma_{1}, \sigma_{2}, \sigma_{3}\right)$ on the loading trajectory in principal stress space represents the current stress state of the material in Fig. A.3, then a line that extends from the origin through the current stress point penetrates the ultimate strength surface at point $M_{r}\left(R_{c 1}, R_{c 2}, R_{c 3}\right)$, where $R_{c i}$ is the required current concrete strength of concrete in the $i$-direction.

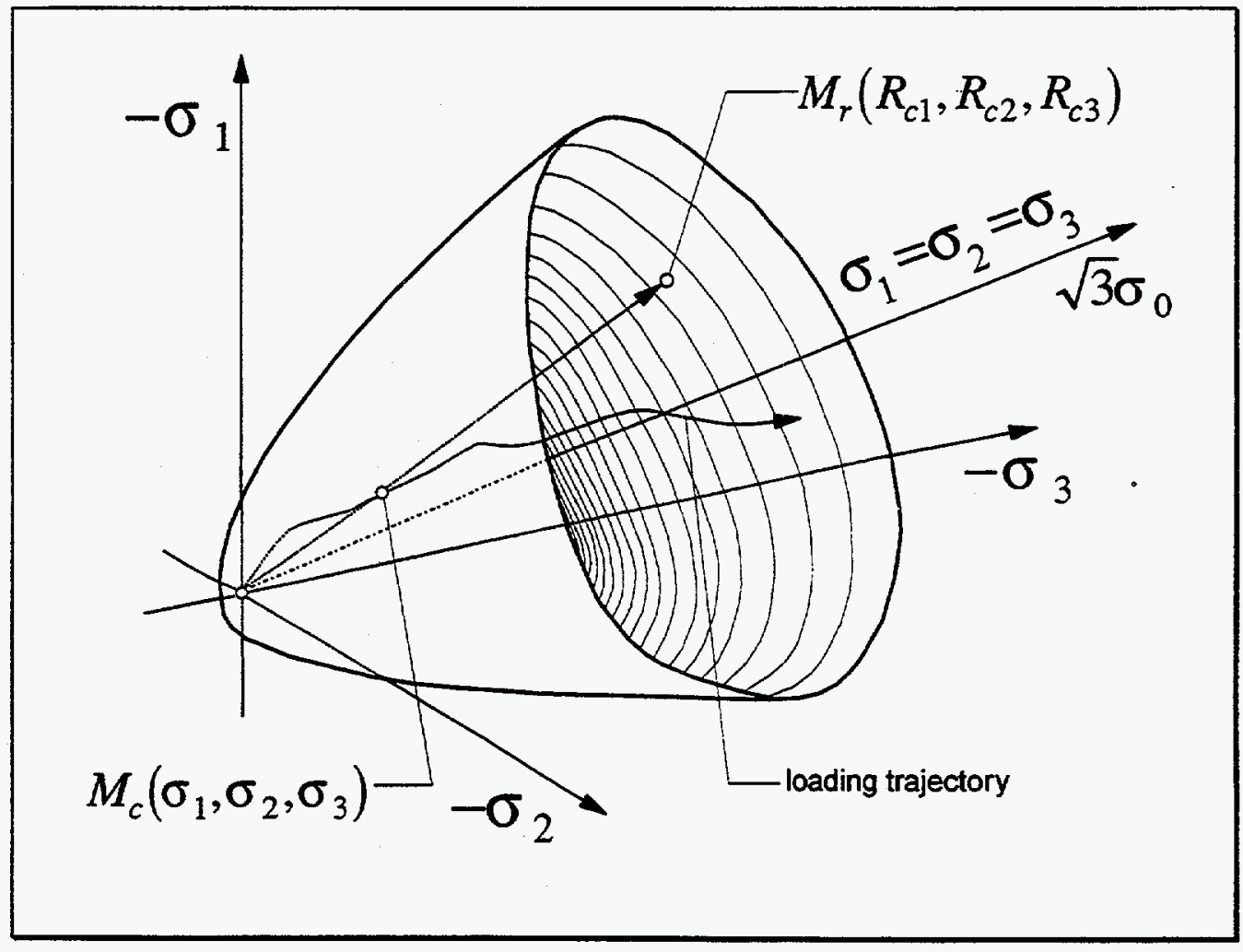

Fig. A.3 Definition of Current Material Strength

In a similar manner, the ultimate surface in the equivalent uniaxial strain space is used for the determination of the current ultimate equivalent uniaxial strains $\varepsilon_{c 1}, \varepsilon_{c 2}$ and $\varepsilon_{c 3}$.

\section{Uniaxial Stress-Strain Relation under Load Reversal}

For the representation of concrete behavior under cyclic load reversals the monotonic envelope curve in Eq. (9a) is modified to the following form in terms of the equivalent uniaxial strain: 


$$
\sigma_{i}=R_{c i} \frac{K_{i}\left(\frac{\varepsilon_{u i}}{\varepsilon_{c i}-\varepsilon_{o i}}\right)}{1+A_{i}\left(\frac{\varepsilon_{u i}}{\varepsilon_{c i}-\varepsilon_{o i}}\right)+B_{i}\left(\frac{\varepsilon_{u i}}{\varepsilon_{c i}-\varepsilon_{o i}}\right)^{2}+C_{i}\left(\frac{\varepsilon_{u i}}{\varepsilon_{c i}-\varepsilon_{o i}}\right)^{3}} \quad(i=1,2,3)
$$

where

$\varepsilon_{o i}=$ strain that corresponds to complete material unloading in the $i$-direction of orthotropy;

$R_{c i}, A_{i}, B_{i}, C_{i}=$ parameters in Eqs. (9b) and (9c).

The following rules govern the cyclic behavior of the equivalent uniaxial stress-strain relation in Eq. (15), as depicted in Fig. A.4:

- Unloading from a point $A\left(\sigma_{i}, \varepsilon_{u j}\right)$ on the envelope curve takes place along a straight line connecting the point at which unloading starts to a point $B\left(0, \varepsilon_{o i}\right)$ on the strain axis in Fig. A.4. $\varepsilon_{o i}$ is defined by the relation:

$$
\varepsilon_{o i}=\left\{\begin{array}{cc}
\varepsilon_{u i}-\frac{\sigma_{i}}{E_{0}} & \text { for } \varepsilon_{u i} \leq \varepsilon_{c i} \\
\frac{E_{0}\left(\varepsilon_{u i}-\varepsilon_{c i}\right)-R_{c i}}{E_{0}} & \text { for } \left.\quad \varepsilon_{u i}\right) \varepsilon_{c i}
\end{array} \quad(i=1,2,3)\right.
$$

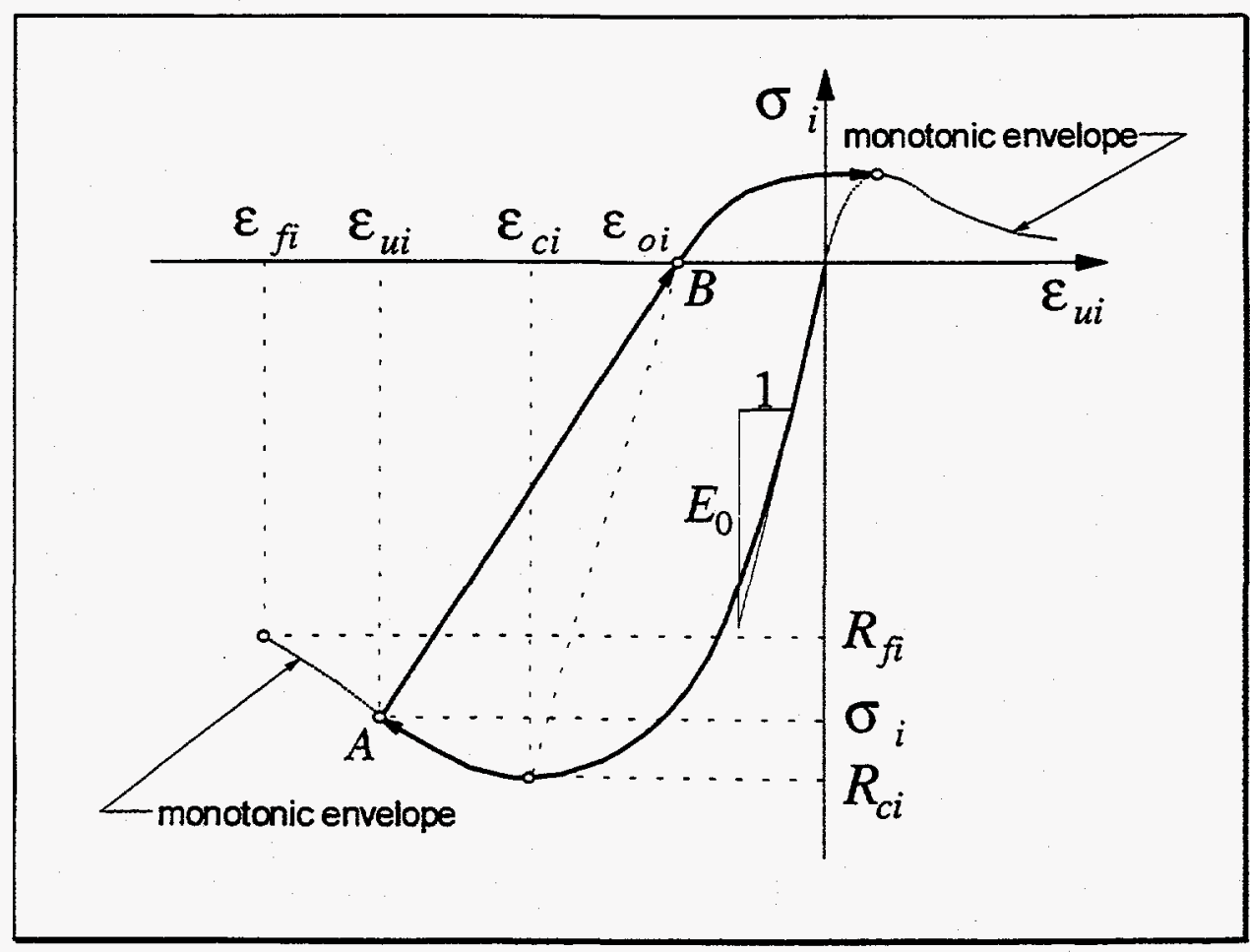

Fig. A.4 Uniaxial Stress-Strain Curve under Load Reversal 
The parameters in the definition of $\varepsilon_{o i}$ in Eq. (16) are specified in Fig. A.4.

- The material reloads to the stress state from which unloading took place as long as the loading function in Eq. (13) satisfies the condition

$$
f \leq f_{\max }
$$

- Reloading after partial unloading takes place along the unloading line and rejoins the original (re)loading envelope once it reaches the point from which unloading initiated.

- The material reloads in the opposite direction once the strain passes through point $B\left(0, \varepsilon_{o i}\right)$ in Eq. (16). At point $B\left(0, \varepsilon_{o i}\right)$ the value of $f_{\max }$ is set to zero, thus, initiating a new path of the stress-strain history.

The proposed rules of cyclic material behavior do not account for cyclic damage of concrete. The importance of this effect on the cyclic behavior of reinforced concrete structures merits a special study but is beyond the scope of the present report.

\section{Post Peak Behavior}

An important aspect of the failure behavior of concrete is the formation and propagation of tensile cracks. To model the cracking behavior of concrete under tensile stress a smeared crack approach is adopted according to the original work in $[13,15,21]$.

Another important aspect of the failure behavior of concrete is compression crushing.

The ultimate strain surface is used in the identification of the type of material failure, as discussed in the following.

\section{Post Peak Behavior after Cracking}

According to the smeared crack approach cracks form at the integration points of the finite element in a plane perpendicular to the corresponding principal tensile stress direction when the equivalent uniaxial strain in that direction pierces the ultimate strain surface. The effect of this material failure is that the material stiffness is reduced in the failure plane in the direction normal and parallel to the crack, the transverse strain ratios become equal to zero in the crack plane and the stress normal to the crack is equal to zero. This process is described in the following for the formation of a single crack plane in more detail.

After the material has cracked, it starts to strain-soften in the principal stress direction until the corresponding equivalent uniaxial strain $\varepsilon_{u 1}$ reaches the value of $\varepsilon_{f 1}$ in Fig. A.5. When this happens, stress $\sigma_{1}$ reduces to zero and the material has no more stiffness in the direction of this stress. With the assumption that Poisson's ratio in the crack plane reduces to zero after cracking, the stress-strain relation for cracked concrete in Eq. (1) takes the following form: 


$$
\left\{\begin{array}{l}
d \sigma_{1} \\
d \sigma_{2} \\
d \sigma_{3} \\
d \tau_{12} \\
d \tau_{23} \\
d \tau_{31}
\end{array}\right\}=\frac{1}{\Omega}\left[\begin{array}{cccccc}
E_{1}\left(1-v_{23} v_{32}\right) & 0 & 0 & 0 & 0 & 0 \\
0 & E_{2} & E_{2} v_{32} & 0 & 0 & 0 \\
0 & E_{3} v_{23} & E_{3} & 0 & 0 & 0 \\
0 & 0 & 0 & G_{12} \Omega & 0 & 0 \\
0 & 0 & 0 & 0 & G_{23} \Omega & 0 \\
0 & 0 & 0 & 0 & 0 & G_{31} \Omega
\end{array}\right]\left\{\begin{array}{l}
d \varepsilon_{1} \\
d \varepsilon_{2} \\
d \varepsilon_{3} \\
d \gamma_{12} \\
d \gamma_{23} \\
d \gamma_{31}
\end{array}\right\}
$$

where

$$
\begin{gathered}
\Omega=1-v_{23} v_{32} ; \quad G_{12}=\frac{E_{1} E_{2}}{\left(E_{1}+E_{2}\right)} ; \\
G_{23}=\frac{E_{2} E_{3}}{E_{2}\left(1+v_{23}\right)+E_{3}\left(1+v_{32}\right)} ; \quad G_{31}=\frac{E_{3} E_{1}}{\left(E_{3}+E_{1}\right)}
\end{gathered}
$$

$E_{1}$ is the total secant modulus of the corresponding stress-strain point on the descending branch of the stress-equivalent uniaxial strain relation in Fig. A.5. The parameters $R_{c 1}, \varepsilon_{c 1}$ and $R_{f 1}, \varepsilon_{f 1}$ of the stress-strain relation correspond to the multiaxial conditions at the instant of crack formation.

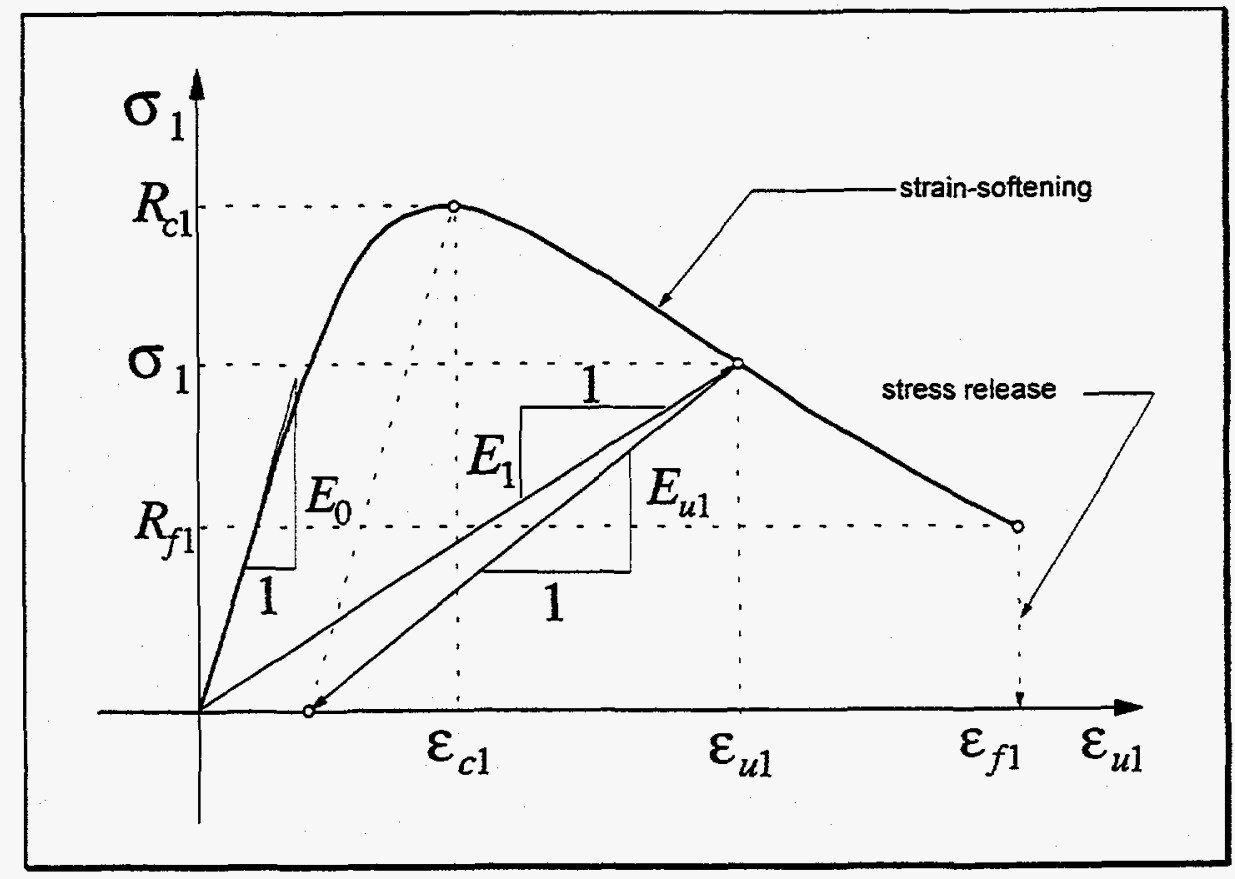

Fig. A.5 Strain Softening Behavior under Compression Loading

Once a crack plane has formed, it is checked in each subsequent solution step whether the failure is still active. The failure is considered to be passive provided the normal strain across the crack plane becomes less than the equivalent uniaxial strain at which the failure occurred initially and is active otherwise. Therefore, a tensile crack plane may repeatedly be active and passive. 
Once a crack forms, the element checks whether the crack is active during subsequent. solution steps. The crack is passive if the normal strain across the crack plane is less than the equivalent uniaxial strain at which the crack formed initially. Otherwise, the crack is considered active. A tensile crack may, thus, be activated and deactivated many times.

If a tensile crack plane has developed, the previous material constitutive relations are established for the direction perpendicular to the crack plane. Thus, the stress conditions parallel and normal to the crack plane are used for establishing the material matrix instead of the principal stress directions used for the undamaged material. Once a crack has formed, subsequent cracks can only form in planes perpendicular to the first crack plane. Thus, the direction of the second or third crack plane is fixed at any integration point once one or two cracks have formed.

The material may also fail in compression crushing after tensile cracking in one or: two directions has taken place. This condition is identified by the position of the equivalent uniaxial strain(s) along the crack plane(s) relative to the ultimate strain surface.

If unloading of the cracked material occurs in the strain-softening region, characterized by the condition $f \leq f_{\max }$, the material matrix is defined by Eq. (17) if $E_{1}$ is replaced by the unloading modulus $E_{u 1}$ given by the relation

$$
E_{u i}=E_{0} \frac{\sigma_{i}}{E_{0}\left(\varepsilon_{u i}-\varepsilon_{c i}\right)+R_{c i}}
$$

where all parameters are specified in Fig. A.5.

\section{Post Peak Behavior after Compression Crushing}

Under multiaxial stress conditions compression crushing of the material is identified with the help of the ultimate strain surface. The material model experiences crushing when the current equivalent uniaxial strain pierces the ultimate strain surface.

After the material reaches the state of compression crushing, it is assumed that it strainsoftens in all directions until the minimum equivalent uniaxial strain $\varepsilon_{c 3}$ reaches $\varepsilon_{f 3}$. When the current value of $\varepsilon_{c 3}$ reaches the value of $\varepsilon_{f 3}$, all stresses are completely released and the material has zero stiffness for subsequent loading. With the assumption that Poisson's ratio $v_{i j}$ is equal to zero after compression crushing, the constitutive relation in Eq. (1) takes the following form for the crushing material:

$$
\left\{\begin{array}{l}
d \sigma_{1} \\
d \sigma_{2} \\
d \sigma_{3} \\
d \tau_{12} \\
d \tau_{23} \\
d \tau_{31}
\end{array}\right\}=\left[\begin{array}{cccccc}
E_{1} & 0 & 0 & 0 & 0 & 0 \\
0 & E_{2} & 0 & 0 & 0 & 0 \\
0 & 0 & E_{3} & 0 & 0 & 0 \\
0 & 0 & 0 & G_{12} & 0 & 0 \\
0 & 0 & 0 & 0 & G_{23} & 0 \\
0 & 0 & 0 & 0 & 0 & G_{31}
\end{array}\right]\left\{\begin{array}{l}
d \varepsilon_{1} \\
d \varepsilon_{2} \\
d \varepsilon_{3} \\
d \gamma_{12} \\
d \gamma_{23} \\
d \gamma_{31}
\end{array}\right\}
$$


where

$$
G_{12}=\frac{E_{1} E_{2}}{\left(E_{1}+E_{2}\right)} ; \quad G_{23}=\frac{E_{2} E_{3}}{\left(E_{2}+E_{3}\right)} ; \quad G_{31}=\frac{E_{3} E_{1}}{\left(E_{3}+E_{1}\right)}
$$

$E_{i}$ is the total secant modulus of the corresponding stress-strain point on the descending branch of the stress-equivalent uniaxial strain relation. The parameters $R_{c i}, \varepsilon_{c i}$ and $R_{f i}, \varepsilon_{f i}$ of the stressstrain relation correspond to the multiaxial conditions at the instant of crushing.

If unloading of the crushing material in the strain-softening region occurs, as represented by the condition $f \leq f_{\max }$, the material matrix is defined by Eq. (19), where $E_{i}$ is replaced by the unloading modulus in Eq. (18). 


\section{APPENDIX B. REINFORCING STEEL MODELS}

Three different approaches are possible for the representation of reinforcing steel in threedimensional reinforced concrete structures:

- the embedded reinforcing bar model;

-the equivalent reinforcing layer model;

the oriented reinforcing medium model.

The most widely used model represents the reinforcement with discrete one-dimensional truss elements, which are assumed to be pin connected and possess three degrees of freedom at each node. A significant advantage of the discrete representation, in addition to its simplicity, is that it can also include the slip of reinforcing steel with respect to the surrounding concrete. This effect might be pursued in possible future studies, which are now under consideration. Thus, a short description of the model is provided below, even though this model was not used in the present study.

The model used in this study is the equivalent reinforcing layer model. In the analysis of three-dimensional structures it is computationally convenient to use a distributed steel model, in which the reinforcement is represented by an oriented reinforcing continuum medium. Since a three-dimensional model might be of interest in possible future studies, the oriented reinforcing continuum model is also described in this Appendix.

\section{Embedded Reinforcing Bar Model}

In embedded bar model the reinforcing steel is represented by one-dimensional truss element which is embedded in the concrete element, as shown in Fig. B.1a. The nodes of the truss element do not need to coincide with the concrete element nodes. The end displacements of the steel element are assumed to be compatible with the boundary displacements of the concrete element, that perfect bond is implied. The discrete embedded steel element can be extended, as proposed by Filippou et al. [12], to include the effect of bond-slip between reinforcing bar and surrounding concrete.

For the one-dimensional truss element with constant strain the stiffness matrix in local coordinate system is given by

$$
\left\{\begin{array}{l}
N_{1} \\
N_{2}
\end{array}\right\}=\frac{E_{s} A_{s}}{L}\left[\begin{array}{cc}
1 & -1 \\
-1 & 1
\end{array}\right]\left\{\begin{array}{l}
q_{1} \\
q_{2}
\end{array}\right\}
$$

or

$$
\{N\}=\left[{ }^{t} K_{L 0}\right]_{s}\{q\},
$$




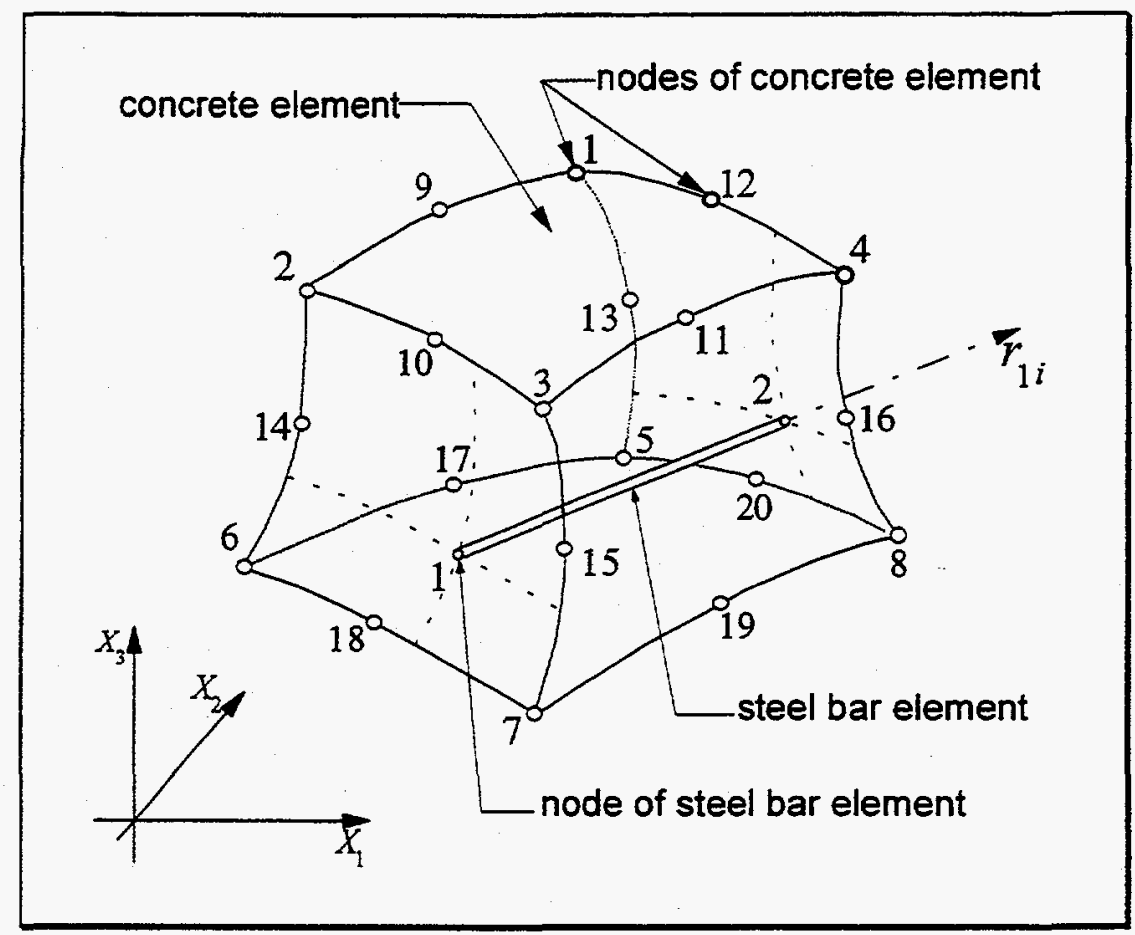

(a) Embedded Reinforcing Steel Bar

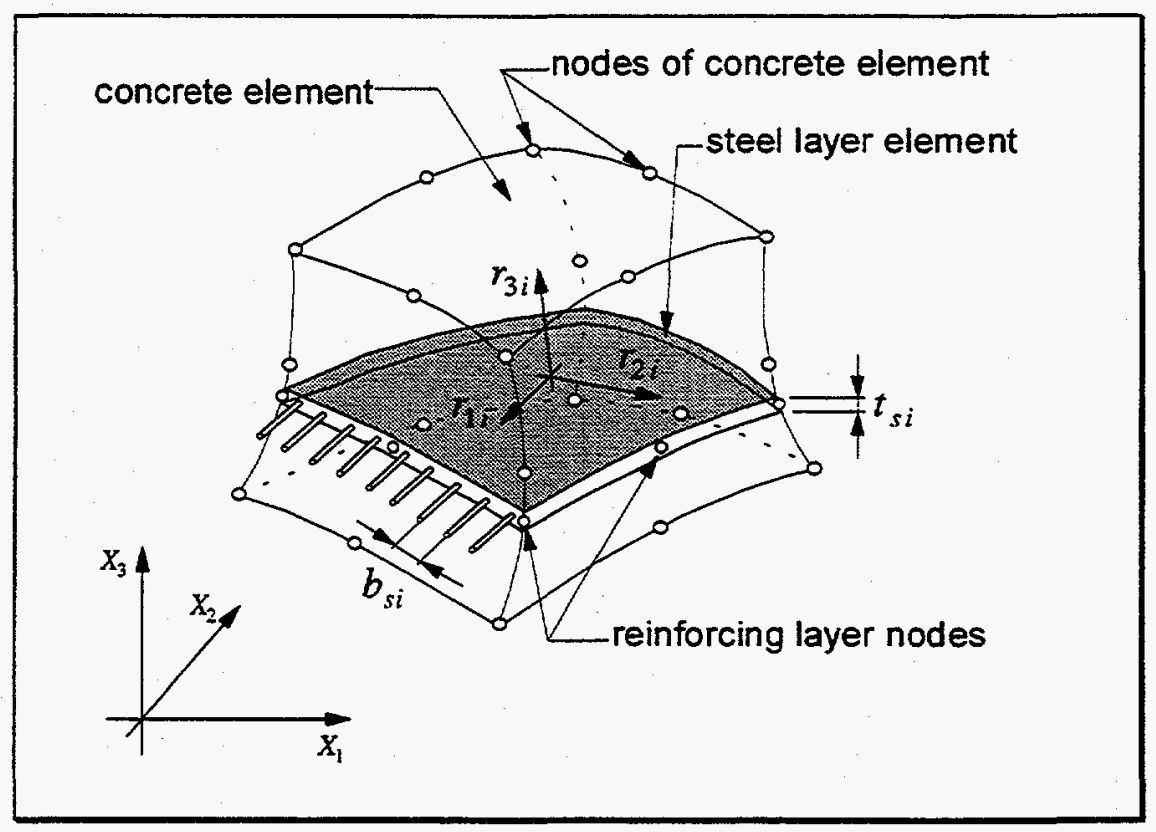

(b) Embedded Equivalent Reinforcing Steel Layer

Fig. B.1 Embedded Reinforcing Steel Models

where

$E_{s}=$ modulus of elasticity of steel;

$A_{s}=$ cross-sectional area of steel bar;

$L=$ length of steel bar; 
$N_{i}, q_{i}=$ axial end forces and displacements $(i=1,2)$.

Eq. (21) can be expressed relative to the global coordinate system by applying a rotation with angles $\alpha, \beta$ and $\gamma$, which are the angles between the axis of the reinforcing bar and the global $X_{i}(i=1,2,3)$-axes of the structure. It can be expressed by the following relation:

$$
\left\{\begin{array}{l}
N_{1 X_{1}} \\
N_{1 X_{2}} \\
N_{1 X_{3}} \\
N_{2 X_{1}} \\
N_{2 X_{2}} \\
N_{2 X_{3}}
\end{array}\right\}=[T]^{T}\left[K_{L 0}\right]_{s}[T]\left\{\begin{array}{l}
q_{1 X_{1}} \\
q_{1 X_{2}} \\
q_{1 X_{3}} \\
q_{2 X_{1}} \\
q_{2 X_{2}} \\
q_{2 X_{3}}
\end{array}\right\},
$$

where

$$
[T]=\left[\begin{array}{cccccc}
\cos \alpha & \cos \beta & \cos \gamma & 0 & 0 & 0 \\
0 & 0 & 0 & \cos \alpha & \cos \beta & \cos \gamma
\end{array}\right]
$$

Since the nodes of the reinforcing bar element do not generally coincide with the nodes of the concrete element (Fig. B.1a) Eq. (22) has to undergo another transformation before it can be assembled together with the concrete element stiffness matrix. This can be expressed by the following relation:

$$
\left[K_{G L}\right]_{s}=\left[T_{1}\right]^{T}[T]^{T}\left[K_{L O}\right]_{s}[T]\left[T_{1}\right],
$$

where the transformation matrix $\left[T_{1}\right]$ has the following form:

$$
\left[T_{1}\right]=\left[\begin{array}{lllllll}
A_{1,1} & A_{2,1} & A_{3,1} & \cdot & . & A_{20,1} \\
A_{1,2} & A_{2,2} & A_{3,2} & . & . & \cdot & A_{20,2}
\end{array}\right]
$$

and submatrix $A_{i, j}$ takes the form

$$
A_{i, j}=\left[\begin{array}{ccc}
N_{i}\left(\xi_{j}, \eta_{j}, \zeta_{j}\right) & 0 & 0 \\
0 & N_{i}\left(\xi_{j}, \eta_{j}, \zeta_{j}\right) & 0 \\
0 & 0 & N_{i}\left(\xi_{j}, \eta_{j}, \zeta_{j}\right)
\end{array}\right]
$$

In Eq. (26) $N_{i}$ are the shape functions for $i$-node of the isoparametric concrete element, and $\xi_{j}, \eta_{j}, \zeta_{j}$ are the normalized coordinates of $j$-node of the reinforcing bar element.

\section{Embedded Equivalent Reinforcing Layer Model}

In this model the reinforcing bars inside of concrete element are replaced by an equivalent steel layer with distributed uniaxial material properties in each reinforcing direction. The 
equivalent steel layer element has thickness $t_{s i}$ (Fig. B.1b) that is determined by the following relation:

$$
t_{s i}=\frac{A_{s i}}{b_{s i}}
$$

where

$A_{s i}=$ cross-sectional area of reinforcing bar in $i$-direction;

$b_{s i}=$ spacing of reinforcing bars in $i$-direction.

Since the equivalent steel layer element has uniaxial properties in direction of $r_{1 i}$-axis of the reinforcing bars, the incremental constitutive relation takes the simple form:

$$
\left\{\begin{array}{l}
d \sigma_{1}^{i} \\
d \sigma_{2}^{i} \\
d \tau_{12}^{i}
\end{array}\right\}=\left[\begin{array}{ccc}
E_{1}^{s i} & 0 & 0 \\
0 & 0 & 0 \\
0 & 0 & G_{12}^{s i}
\end{array}\right]\left\{\begin{array}{l}
d \varepsilon_{1}^{i} \\
d \varepsilon_{2}^{i} \\
d \gamma_{12}^{i}
\end{array}\right\}
$$

where

$$
E_{1}^{s i}=\rho_{s i} E_{s i} ; \quad G_{12}^{s i}=\frac{\rho_{s i} E_{s i}}{2\left(1+v_{s i}\right)} ; \quad v_{s i}=\rho_{s i} v_{s}
$$

${ }^{\prime} E_{s i}=$ incremental secant modulus of reinforcement in $i$-direction;

$\rho_{s i}=$ reinforcing ratio in $i$-direction;

$v_{s}=$ Poisson's ratio of reinforcement.

Eq. (27) can be written in matrix form as

$$
\left\{d \sigma^{i}\right\}=\left[D_{L O}\right]_{s i}\left\{d \varepsilon^{i}\right\}
$$

in which

$\left\{d \sigma^{i}\right\}=$ vector of incremental stresses in reinforcing layer with bars in $i$-direction;

$\left\{d \varepsilon^{i}\right\}=$ vector of incremental strains in reinforcing layer with bars in $i$-direction;

$\left[D_{L O}\right]_{s i}=$ incremental material matrix of reinforcing layer.

Local material matrix $\left[D_{L o}\right]_{s i}$ from Eq. (28) is used now to derive the stiffness matrix of the equivalent reinforcing steel layer by standard procedures:

$$
\left[K_{L O}\right]_{s i}=\int_{V}[B]^{T}\left[D_{L O}\right]_{s i}[B] d V
$$

where $[B]$ is a matrix operator relating the strain to the nodal displacements (assuming plane stress behavior) of the steel layer element. 
The stiffness matrix $\left[K_{L O}\right]_{s i}$ in Eq. (29), which is defined in the local coordinates system $r_{1 i}, r_{2 i}, r_{3 i}$ need to be transformed to the global system $X_{1}, X_{2}, X_{3}$ before it can be used to form the stiffness matrix of the reinforced concrete element. By the rules of orthogonal transformation the stiffness matrix of reinforcing layer in the global coordinates becomes

$$
\left[K_{G L}\right]_{s i}=[T]^{T}\left[K_{L O}\right]_{s i}[T]
$$

in which

$$
[T]=\left[\begin{array}{cccccc}
L & 0 & 0 & . & . & \cdot \\
0 & L & 0 & & \\
0 & 0 & L & & \\
\cdot & & & . & \\
\cdot & & & & \cdot \\
. & & & & & .
\end{array}\right]
$$

is a diagonal matrix built up of $L$ submatrices in a number equal to that of the nodes in the reinforcing layer element, and $L$ is a $3 \times 3$ matrix of direction cosines of angles formed between the local and global axes.

Since the nodes of the reinforcing layer element do not generally coincide with the nodes of the concrete element (Fig. B.1b) stiffness matrix in Eq. (30) has to undergo another transformation before it can be assembled together with the concrete element stiffness matrix. This can be formally expressed by relation

$$
\left[K_{G L}\right]_{s i}=\left[T_{1}\right]^{T}[T]^{T}\left[K_{L O}\right]_{s i}[T]\left[T_{1}\right]
$$

where the transformation matrix $\left[T_{1}\right]$ has the following form:

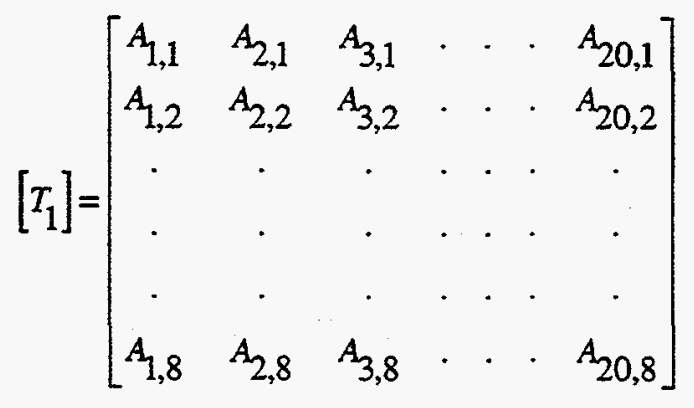

and submatrix $A_{i, j}$ has exactly the same form as Eq. (26).

\section{Oriented Reinforcing Medium Model}

In the oriented medium model the reinforcing steel is assumed to be distributed over the concrete element at a certain orientation defined by three axes $r_{1 i}, r_{2 i}$ and $r_{3 i}$ (Fig. B.2). The oriented steel medium element has the dimensions of concrete element. With reference to the 
reinforcing axes the incremental constitutive relationship for oriented steel medium can be expressed as:

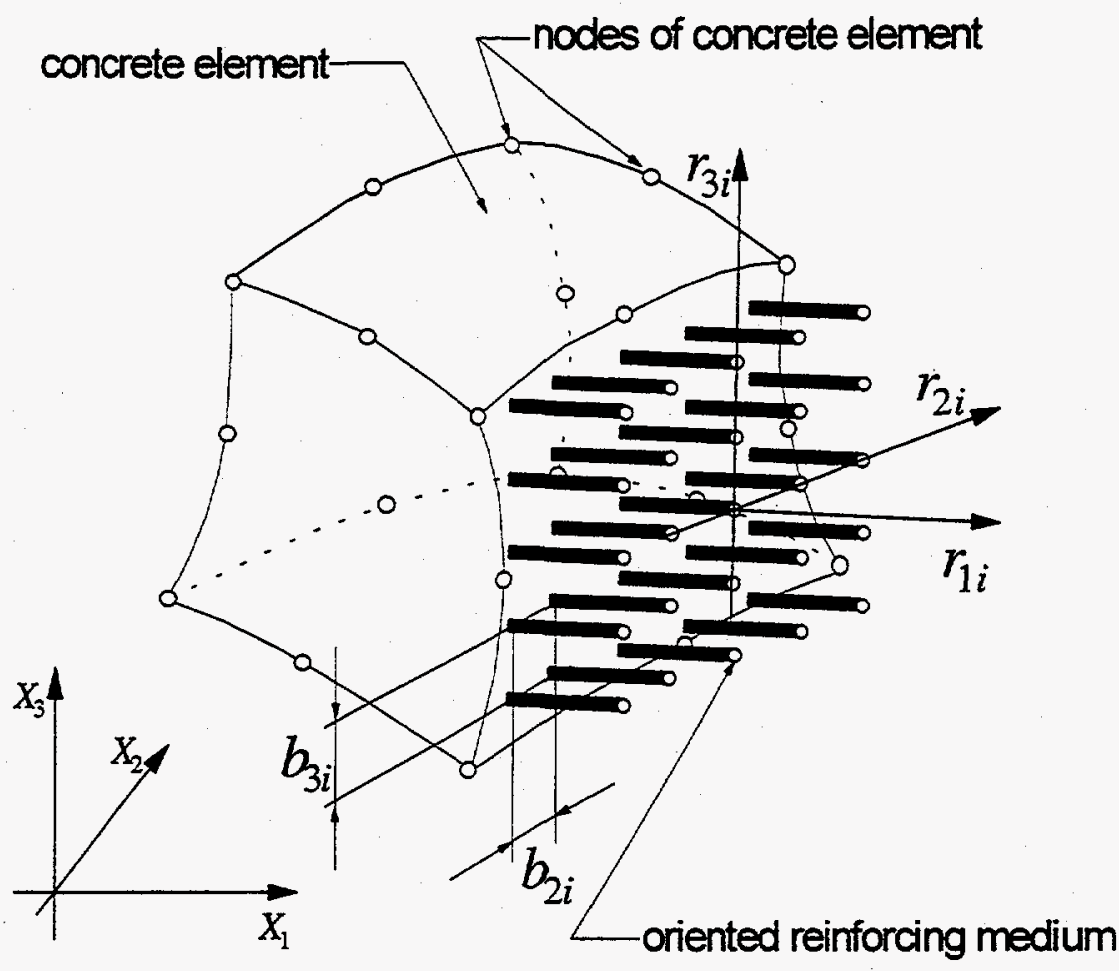

Fig. B.2 Oriented Reinforcing Steel Medium Model

$$
\left\{\begin{array}{l}
d \sigma_{1}^{i} \\
d \sigma_{2}^{i} \\
d \sigma_{3}^{i} \\
d \tau_{12}^{i} \\
d \tau_{23}^{i} \\
d \tau_{31}^{i}
\end{array}\right\}=\left[\begin{array}{cccccc}
E_{1}^{s i} & 0 & 0 & 0 & 0 & 0 \\
0 & 0 & 0 & 0 & 0 & 0 \\
0 & 0 & 0 & 0 & 0 & 0 \\
0 & 0 & 0 & G_{12}^{s i} & 0 & 0 \\
0 & 0 & 0 & 0 & 0 & 0 \\
0 & 0 & 0 & 0 & 0 & G_{31}^{s i}
\end{array}\right]\left\{\begin{array}{c}
d \varepsilon_{1}^{i} \\
d \varepsilon_{2}^{i} \\
d \varepsilon_{3}^{i} \\
d \gamma_{12}^{i} \\
d \gamma_{23}^{i} \\
d \gamma_{31}^{i}
\end{array}\right\}
$$

where

$$
E_{1}^{s i}=\mu_{s i} E_{s i} ; \quad G_{12}^{s i}=G_{31}^{s i}=\frac{\rho_{s i} E_{s i}}{2\left(1+v_{s i}\right)} ; \quad v_{s i}=\rho_{s i} v_{s}
$$

${ }^{t} E_{s i}=$ incremental secant modulus of reinforcement in $r_{1 i}$-axis of reinforcing;

$\rho_{s i}=$ ratio of reinforcing in the direction of $r_{1 i}$-axis;

$v_{s}=$ Poisson's ratio of reinforcing medium.

The reinforcing ratio is determined from the following relation: 


$$
\rho_{s i}=\frac{A_{s i}}{b_{2 i} b_{3 i}}
$$

where

$A_{s i}=$ cross-sectional area of one reinforcing bar in $r_{1 i}$-axis;

$b_{2 i}, b_{3 i}=$ spacing of reinforcing bars in the direction of $r_{2 i}$ and $r_{3 i}$-axes (Fig. B.2).

The constitutive relation (34) can be written in a matrix form:

$$
\left\{d \sigma^{i}\right\}=\left[D_{L o}\right]_{s i}\left\{d \varepsilon^{i}\right\}
$$

in which

$\left\{d \sigma^{i}\right\}=$ vector of incremental stresses in reinforcement medium with bars oriented along the $i$-direction of reinforcing;

$\left\{d \varepsilon^{i}\right\}=$ vector of incremental strains in reinforcement medium with bars oriented along the $i$-direction of reinforcing;

$\left[D_{L O}\right]_{s i}=$ incremental material matrix of reinforcing medium.

Since the material matrix $\left[D_{L o}\right]_{s i}$ in Eq. (35) is defined with reference to the reinforcing direction, it must be transformed to the global coordinate system before the element stiffness matrices can be assembled. This is accomplished exactly as in Eqs. (4) and (5) for concrete, where $m_{i j}$ now represents the direction cosines of reinforcing steel axes with reference to the $X_{i}(i=1,2,3)$-axes of the global coordinate system.

\section{Stress-Strain Relation for Reinforcing Steel}

The stress-strain behavior of reinforcing steel is described by the nonlinear relation which includes isotropic strain hardening. The relation is computationally efficient and agrees very well with experimental results from cyclic tests on reinforcing steel bars.

The relation can be presented in following form:

$$
{ }^{k} \sigma_{s i}=\frac{{ }^{k} \sigma_{y i}}{2}\left(1-b_{i}\right)\left[1+\frac{\left(1+b_{i}\right)}{\left(1-b_{i}\right)} \frac{\varepsilon_{s i}{ }^{k} \varepsilon_{y i}}{}-\sqrt{\left(\frac{\varepsilon_{s i}}{{ }^{k} \varepsilon_{y i}}-1\right)^{2}+{ }^{k} \delta_{i}}\right] \quad(k=0,1, \ldots, n)
$$

where

$$
b_{i}=\frac{E_{h i}}{E_{o i}}
$$

in which 


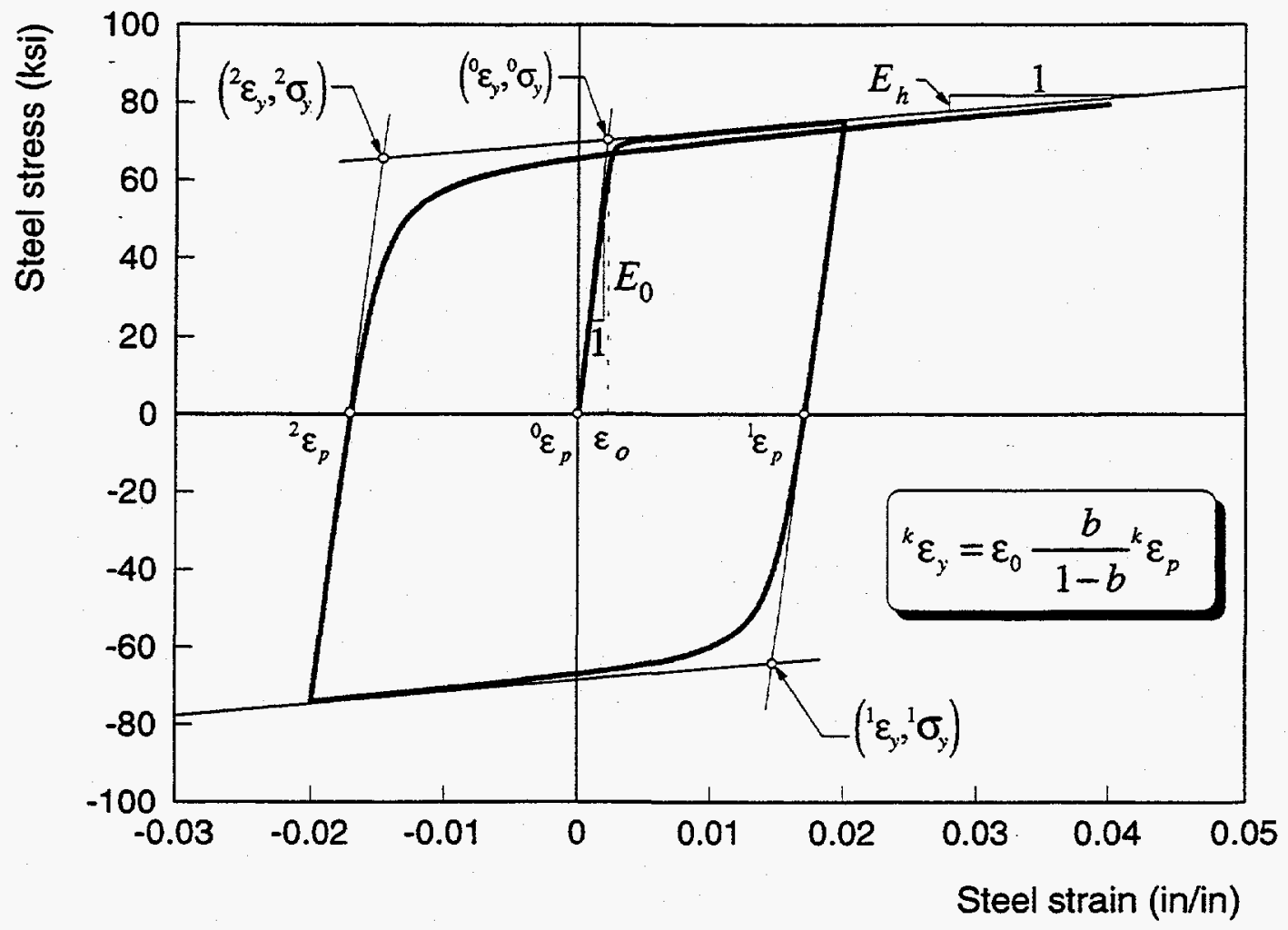

Fig. B.3 Hysteretic Stress-Strain Relation for Reinforcing Steel

$E_{o i}=$ initial elasticity modulus of steel bar, oriented along the $i$-direction of reinforcing; $E_{h i}=$ hardening modulus of steel bar

and

$$
{ }^{k} \sigma_{y i}=E_{o i}{ }^{k} \varepsilon_{y i} ; \quad{ }^{k} \varepsilon_{y i}=\varepsilon_{o i}-\frac{b_{i}}{1-b_{i}}{ }^{k} \varepsilon_{p i} \quad(k=0,1, \ldots, n)
$$

where

$$
\varepsilon_{o i}=\frac{\sigma_{o i}}{E_{o i}}
$$

Eq. (37) readily yields

$$
E_{s i}=\frac{E_{o i}}{2}\left(1-b_{i}\right)\left[\frac{{ }^{k} \varepsilon_{y i}}{\varepsilon_{i}}+\frac{1+b_{i}}{1-b_{i}}-\sqrt{\left(1-\frac{{ }^{k} \varepsilon_{y i}}{\varepsilon_{i}}\right)^{2}+{ }^{k} \delta_{i}}\right] \quad(k=0,1, \ldots, n)
$$

which defines the incremental secant modulus of reinforcement in the $i$-direction of reinforcing. 
Eq. (37) represents a hyperbolic curve transition from a straight line asymptote with slope $E_{o i}$ to another asymptote with slope $E_{h i}$ (Fig. B.3). Stress ${ }^{k} \sigma_{y i}$ and strain ${ }^{k} \varepsilon_{y i}$ are the coordinates of the point where these two asymptotes of the branch under consideration meet; ${ }^{k} \varepsilon_{p i}$ is the strain at the point where complete unloading after the $k$-th reversal took place (Fig. B.3), and ${ }^{k} \xi$ is a parameter that influences the shape of the transition curve and allows a good representation of the Bauschinger effect. As Fig. B.3 shows, ${ }^{k} \varepsilon_{p i}$ and ${ }^{k} \varepsilon_{y i}$ are updated after each strain reversal.

Parameter ${ }^{k} \delta$ is considered dependent on the ratio of the strain at the current asymptote intersection point and strain $\varepsilon_{o i}$. The expression for ${ }^{k} \delta$ takes the following form:

$$
{ }^{k} \delta_{i}=\frac{\delta_{o}}{1-b_{i}}\left(1+\frac{{ }^{k} \varepsilon_{y i}}{\varepsilon_{o i}}\right)^{2} \quad(k=0,1, \ldots, n)
$$

where parameter $\delta_{o}$ is the value of variable ${ }^{k} \delta$ during first loading, ${ }^{k} \varepsilon_{y i}$ and $\varepsilon_{o i}$ are specified in Eq. (38) (see Fig. B.3).

$$
\delta_{o}=0.01 ; \quad \varepsilon_{o i}=\frac{\sigma_{o i}}{E_{o i}}
$$

Eq. (40) shows that parameter ${ }^{k} \delta$ is updated following a strain reversal. Definition of the variable ${ }^{k} \delta$ remains valid in case that reloading occurs after partial unloading.

Some clarification is needed in connection with the set of rules for unloading and reloading which complement Eqs. (38), allowing for a generalized loading history. The analytical model has a memory extending over all previous branches of the stress-strain history, it would follow the previous reloading branch, as soon as the new reloading curve reached it. It should be noted that reloading after partial unloading rejoin the original loading curve after reaching the point from which unloading started, and continues on the envelope curve. 


\section{APPENDIX C-REFERENCES}

[1] Balan, T. A., A constitutive relationship for structurally nonuniform materials in a complex stressed state, Strength of Materials, No. 10, 1351-1357, 1986.

[2] Bashur, F. K. \& Darwin, D., Nonlinear biaxial law for concrete, J. Struct. Div., ASCE, 104(-1), 157-170, 1978.

[3] Bathe, K.-J. \& Ramaswamy, S., On three-dimensional nonlinear analysis of concrete structures, Nucl. Engrg. Design, 52(3), 385-409, 1979.

[4] Bathe, K.-J., Finite element procedures in engineering analysis, Prentice-Hall, Englewood Cliffs, New Jersey, 1982.

[5] Bazant, Z. P. \& Bhat, P. D., Endochronic theory of inelasticity and failure of concrete, $J$. Engrg. Mech. Div., ASCE, 102(4), 701-722, 1976.

[6] Berg, O. Y., Physical basis of concrete and reinforced concrete strength theory, Gosstehizdat, Moscow, 1961 (in Russian).

[7] Bich, P. M., Variant of a strength theory for concrete, Beton i Zhelezobeton, No. 6, 2838, 1980 (in Russian).

[8] Cedolin, L., Crutzen, Y. R. J. \& Poli, S. D., Triaxial stress-strain relationship for concrete, J. Engrg Mech. Div., ASCE, 103(3), 423-439, 1977.

[9] Chen, W. F., Plasticity in reinforced concrete, McGraw-Hill, New York, N.Y., 1982.

[10] Darwin, D. \& Pecknold, D. A., Nonlinear biaxial stress-strain law for concrete, J. Engrg. Mech. Div., ASCE, 103(2), 229-241, 1977.

[11] Elwi, A. A. \& Murray, D. W., A 3D hypoelastic concrete constitutive relationship, $J$. Engrg. Mech. Div., ASCE, 105(4), 623-641, 1979.

[12] Filippou, F. C., Popov, E. P.\& Bertero, V. V., Effects of bond deterioration on hysteretic behavior of reinforced concrete joints, EERC Report 83-19, Earthquake Engineering Research Center, Berkeley, CA. 1983.

[13] Geniev, G. A., Kissiuk, V. N. \& Tiupin, G. A., Theory of plasticity of concrete and reinforced concrete, Stroiizdat, Moscow,1974 (in Russian).

[14] Gorodetskii, A. S., et al., MIRAGE a package of finite element programs for static and dynamic analysis of structures, Edited by Research Institute of Automated Systems in Construction (NIIASS), Kiev, Ukraine, 1994.

[15] Karpenko, N. I., Theory of deformation of reinforced concrete with cracks, Stroiizdat, Moscow, 1976 (in Russian).

[16] Karpenko, N. I., On the development of the theory for analysis of massive reinforced concrete structures accounting for cracking, Stroitelnaia Mekhanika $i$ Raschyot Sooruzhenii, No. 2, 28-35, 1980 (in Russian).

[17] Kotsovos, M. D. \& Newman, J. B., Behavior of concrete under multiaxial stresses, $A C I$ J., 74(9), 443-446, 1977. 
[18] Kupfer, H. B., Hilsdorf, H. K. \& Rusch, H., Behavior of concrete under biaxial stresses, ACI J., 66(8), 656-666, 1969.

[19] Lekhnitskii, S. G., Theory of elasticity of an anisotropic elastic body, Edited by Julius J. Brandstatter, Holden Day, Inc., San Francisco, CA, 1963.

[20] Pisarenko, G. S. \& Lebedev, A. A., Deformation and strength of materials in a complex stressed state, Naukova Dumka, Kiev ,1976 (in Russian).

[21] Rashid, Y. R., Analysis of prestressed concrete pressure vessels, Nucl. Engrg. Diesign, 7(4), 334-344, 1968.

[22] Saenz, I. P., Discussion of "Equation for the stress-strain curve of concrete." by P. Desayi and S. Krishan, ACI J., 61(9), 1229-1235, 1964. 


\section{APPENDIX D. SHORT SUMMARY ACCORDING TO NU-SSWISP-D008}

This Appendix provides a short summary of the results of the study and, in particular, supplies the information requested in the Specification Report of the Seismic Shear Wall ISP (Document NU-SSWISP-D008 with revised pages from NU-SSWISP-D009). Repeated figures are numbered according to their numbers in the main body of this report, so as to facilitate cross-reference.

\section{General Information}

1. Professor Filip C. Filippou and Dr. Toader Balan

2. Department of Civil Engineering, University of California, Berkeley

3. Davis Hall, Berkeley, CA $94720-1710$

4. $\mathrm{Ph}: 510-642-4020$

5. Fax: $510-643-8928$

6. E-mail: filippou@ce.berkeley.edu

\section{Computer Information}

1. Gateway 2000 , Pentium $60 \mathrm{Mhz}$

2. $16 \mathrm{MB}$ of RAM memory

\section{Code information}

1. Nonlinear Static FEM Model

2. MIRAGE original

3. New concrete and steel constitutive model

4. $\# \mathrm{DOF}=542$

5. Newton-Raphson Iteration

\section{Static Analysis Condition}

1. Load control

2. 43 minutes and 59 seconds

3. 16 load steps of lateral load. First three steps with increment of 25 tonf, all subsequent steps with increments in Table 1. Max. Step Size 7.5 tonf, Min. Step Size 6.25 tonf.

4. Singularity of stiffness matrix 


\section{Model Description}

The model of shear wall specimen U-1 was developed with the general purpose finite element analysis program MIRAGE. Making use of symmetry in the geometry and loading arrangement of the specimen the model represents one half of the actual specimen, as shown in Fig. 3.

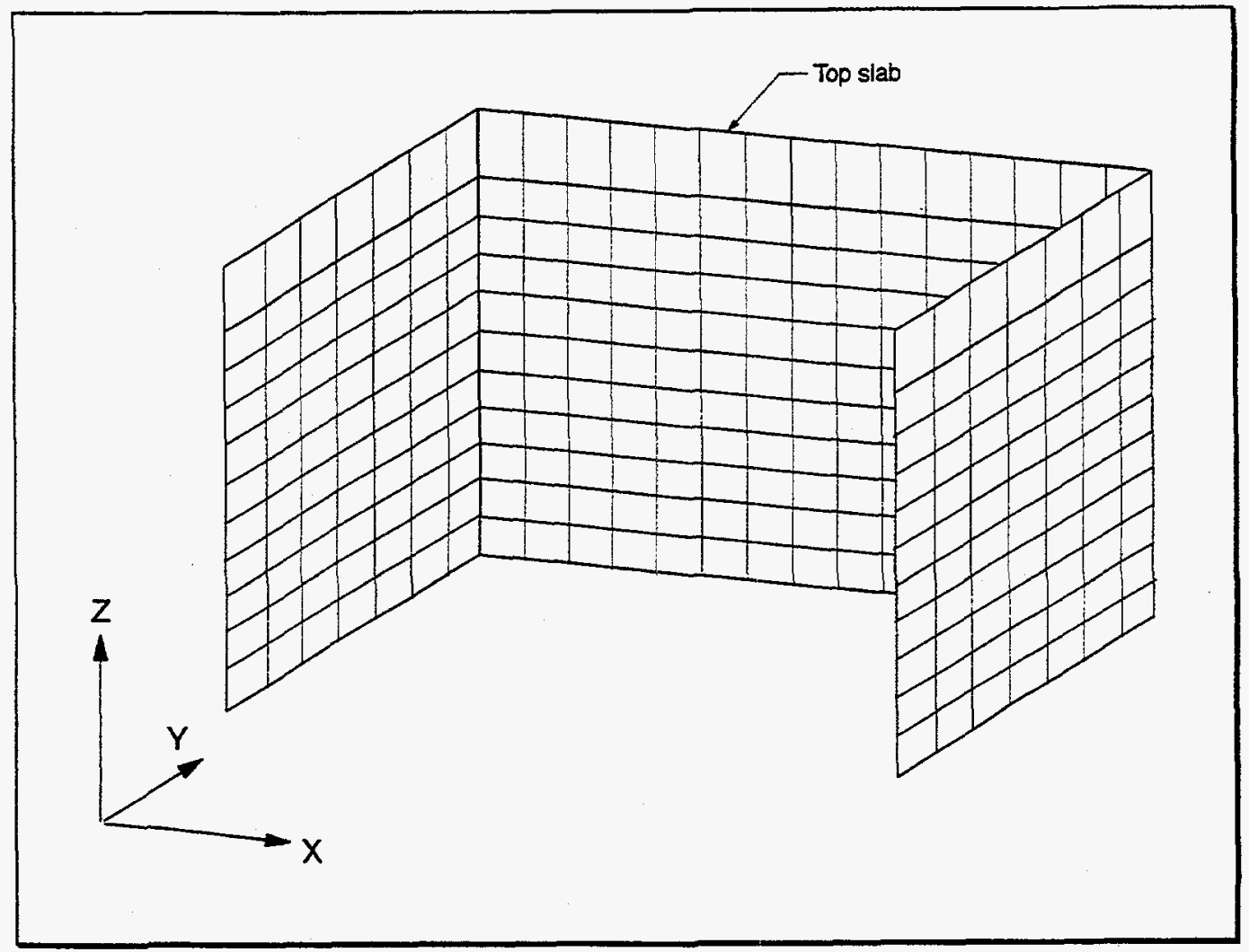

Figure 3 Three-Dimensional Thin-Walled Finite Element Model

On account of the cross section configuration of the specimen, with a ratio of wall height to thickness from 30 to 40 , the specimen is idealized as a three dimensional thin-walled structure with finite elements in condition of plane stress. An alternative three dimensional model with solid brick elements was also investigated, but is not pursued further in this report. Under the thin-wall assumption a quadrilateral plane stress finite element was used in the finite element model of the shear wall specimen.

The model of one-half of the shear wall specimen has 360 nodes and consists of 319 quadrilateral plane stress elements. The boundary conditions of the model were imposed by fixing the displacements along the global $\mathrm{X}, \mathrm{Y}$, and $\mathrm{Z}$-axes for all nodes at the base slab. On account of the lack of out-of-plane stiffness of the membrane element all nodal out-of plane displacements were also restrained in the analysis. 
Constitutive Law of Concrete

see Appendix A

Constitutive Law of Reinforcing Steel

see Appendix B

No shear stiffness due to dowel action of reinforcement

Bond-Slip between Concrete and Reinforcing Steel

No bond-slip effect in the model

No Size effect included (tension softening)

No Tension stiffening 


\section{TABLES}

\begin{tabular}{|c|c|c|c|c|c|}
\hline $\begin{array}{c}\text { Load } \\
\text { Step }\end{array}$ & $\begin{array}{c}\text { Horizontal } \\
\text { Force (tonf) }\end{array}$ & $\begin{array}{c}\text { Horizontal Displ. } \\
(\mathrm{mm})\end{array}$ & $\begin{array}{c}\text { Vertical Displ. } \\
\text { Left (mm) }\end{array}$ & $\begin{array}{c}\text { Vertical Displ. } \\
\text { Right }(\mathrm{mm})\end{array}$ & $\begin{array}{c}\text { No. of } \\
\text { iterations }\end{array}$ \\
\hline 1 & 0 & 0 & -0.1157 & -0.1157 & 3 \\
\hline 2 & 25 & 0.2734 & -0.1455 & -0.0876 & 2 \\
\hline 3 & 50 & 0.5798 & -0.1768 & -0.0602 & 3 \\
\hline 4 & 75 & 0.9581 & -0.2094 & -0.0318 & 8 \\
\hline 5 & 81.25 & 1.0657 & -0.2179 & -0.0242 & 8 \\
\hline 6 & 87.5 & 1.2045 & -0.2264 & -0.0163 & 10 \\
\hline 7 & 95 & 1.4497 & -0.2372 & -0.0014 & 10 \\
\hline 8 & 102.5 & 2.2351 & -0.2453 & 0.0306 & 10 \\
\hline 9 & 110 & 3.1362 & -0.2504 & 0.0403 & 10 \\
\hline 10 & 117.43 & 4.1467 & -0.2642 & 0.0510 & 16 \\
\hline 11 & 124.86 & 5.0482 & -0.2781 & 0.0618 & 16 \\
\hline 12 & 132.29 & 6.0328 & -0.2908 & 0.0721 & 16 \\
\hline 13 & 139.72 & 7.6219 & -0.3041 & 0.0829 & 18 \\
\hline 14 & 147.16 & 9.6428 & -0.3174 & 0.0839 & 16 \\
\hline 15 & 154.58 & 14.6341 & -0.3307 & 0.0850 & 16 \\
\hline
\end{tabular}

Table 1 Load Displacement Response and No. of Iterations

\begin{tabular}{|c|c|c|c|}
\hline $\begin{array}{c}\text { Load } \\
\text { Step }\end{array}$ & $\begin{array}{c}\text { Horizontal } \\
\text { Force (tonf) }\end{array}$ & $\begin{array}{c}\text { Horizontal Displ. } \\
\text { (mm) }\end{array}$ & Phenomenon \\
\hline 1 & 0 & 0 & \\
\hline 2 & 25 & 0.2734 & \\
\hline 3 & 50 & 0.5798 & Initiation of Shear Crack in Web Wall (b) \\
\hline 4 & 75 & 0.9581 & \\
\hline 5 & 81.25 & 1.0657 & \\
\hline 6 & 87.5 & 1.2045 & \\
\hline 7 & 95 & 1.4497 & \\
\hline 8 & 102.5 & 2.2351 & \\
\hline 9 & 110 & 3.1362 & $\begin{array}{c}\text { Initiation of Bending Crack in Flange Wall (a) } \\
\text { Initial Yielding of Vertical and Horizontal Rebar } \\
\text { in Web Wall (d), (e) Fig. 10 }\end{array}$ \\
\hline & & & \\
\hline 10 & 117.43 & 4.1467 & \\
\hline 11 & 124.86 & 5.0482 & \\
\hline 12 & 132.29 & 6.0328 & \\
\hline 13 & 139.72 & 7.6219 & \\
\hline 14 & 147.16 & 9.6428 & \\
\hline 15 & 154.58 & 14.6341 & \\
\hline 16 & 158 & & \\
\hline & & & \\
\hline
\end{tabular}

Table 2 Observations during Load-Displacement Response 


\section{FIGURES}

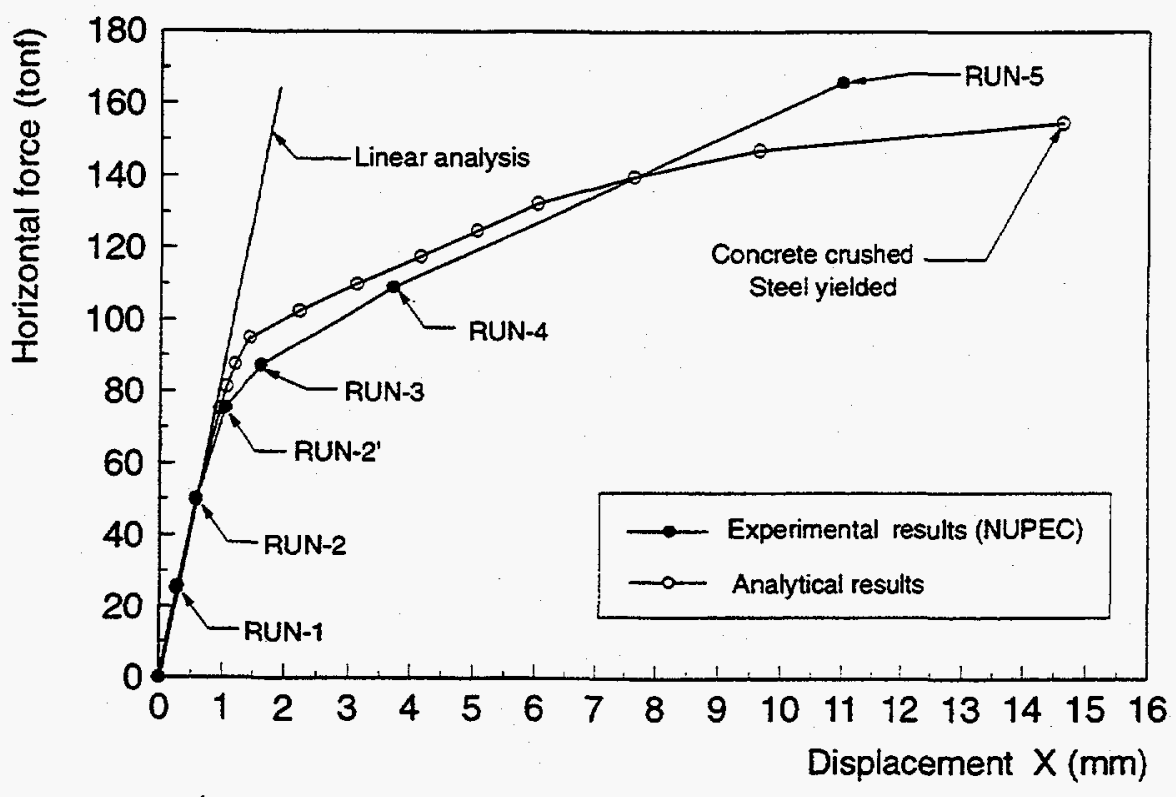

Figure 6 Load Displacement Response of the Top Slab Center 


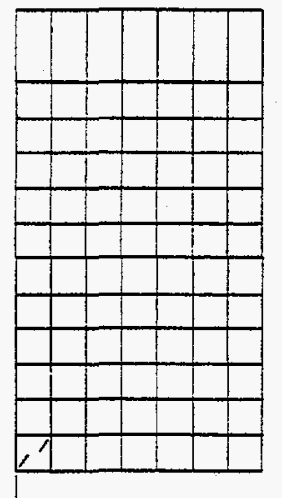

$\varepsilon$

Let flange

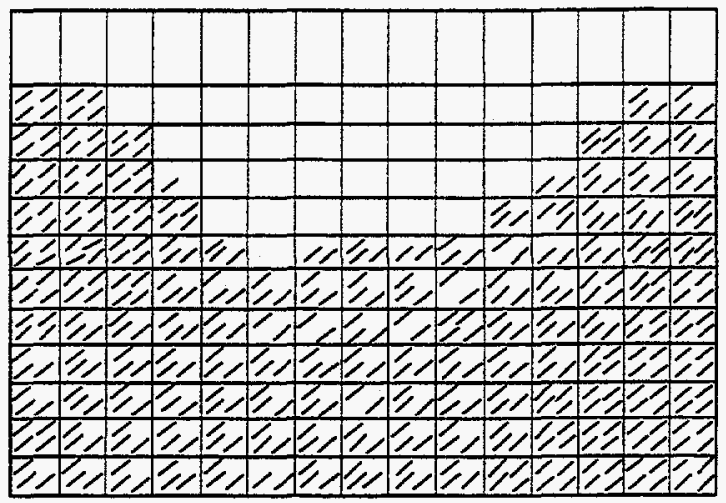

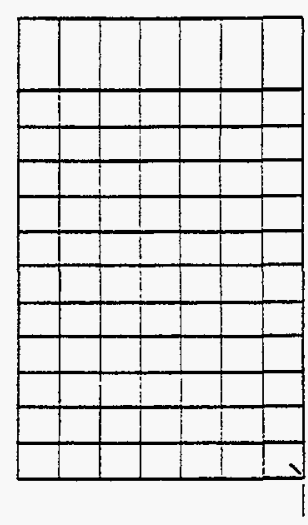

Pight flange

(a) Horizontal force $P_{2}=110.0$ tonf
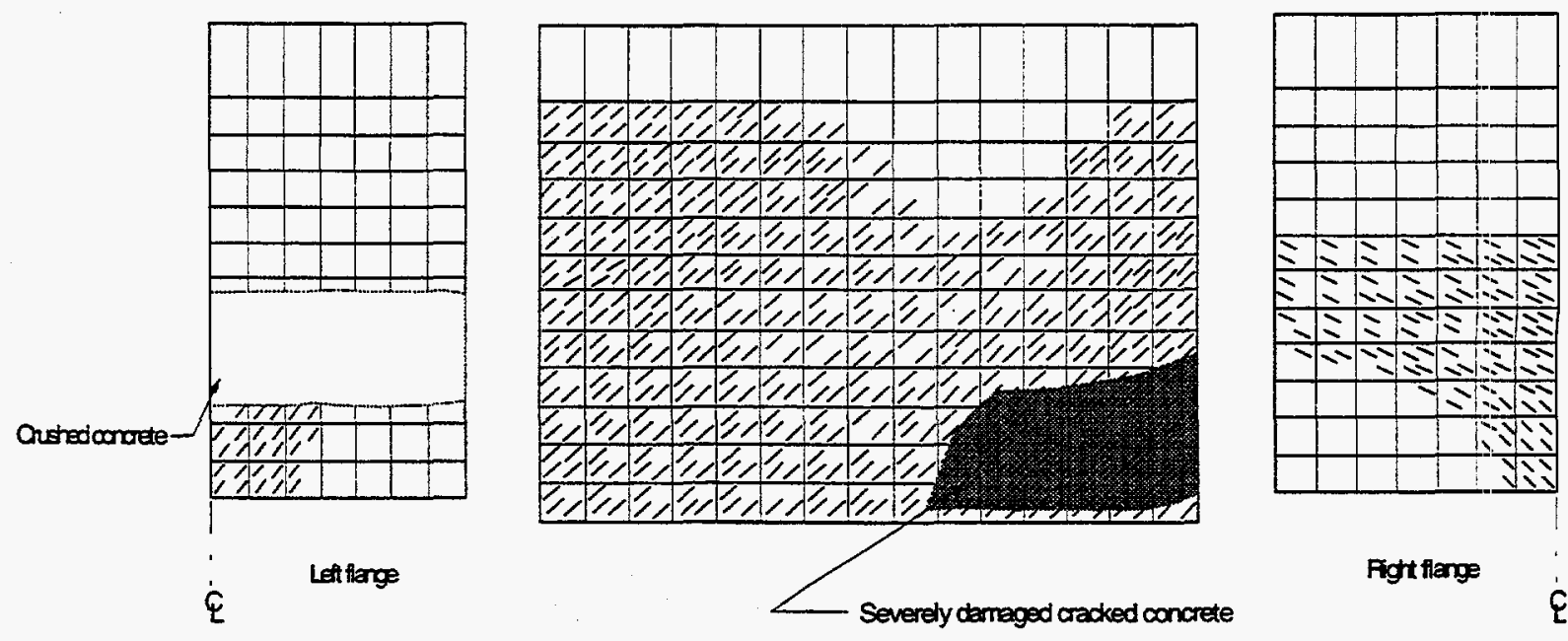

(b) Horizontal force $P_{2}=158.0$ tonf

Figures 7-8 Predicted Crack Patterns in the Web and Flange Walls 


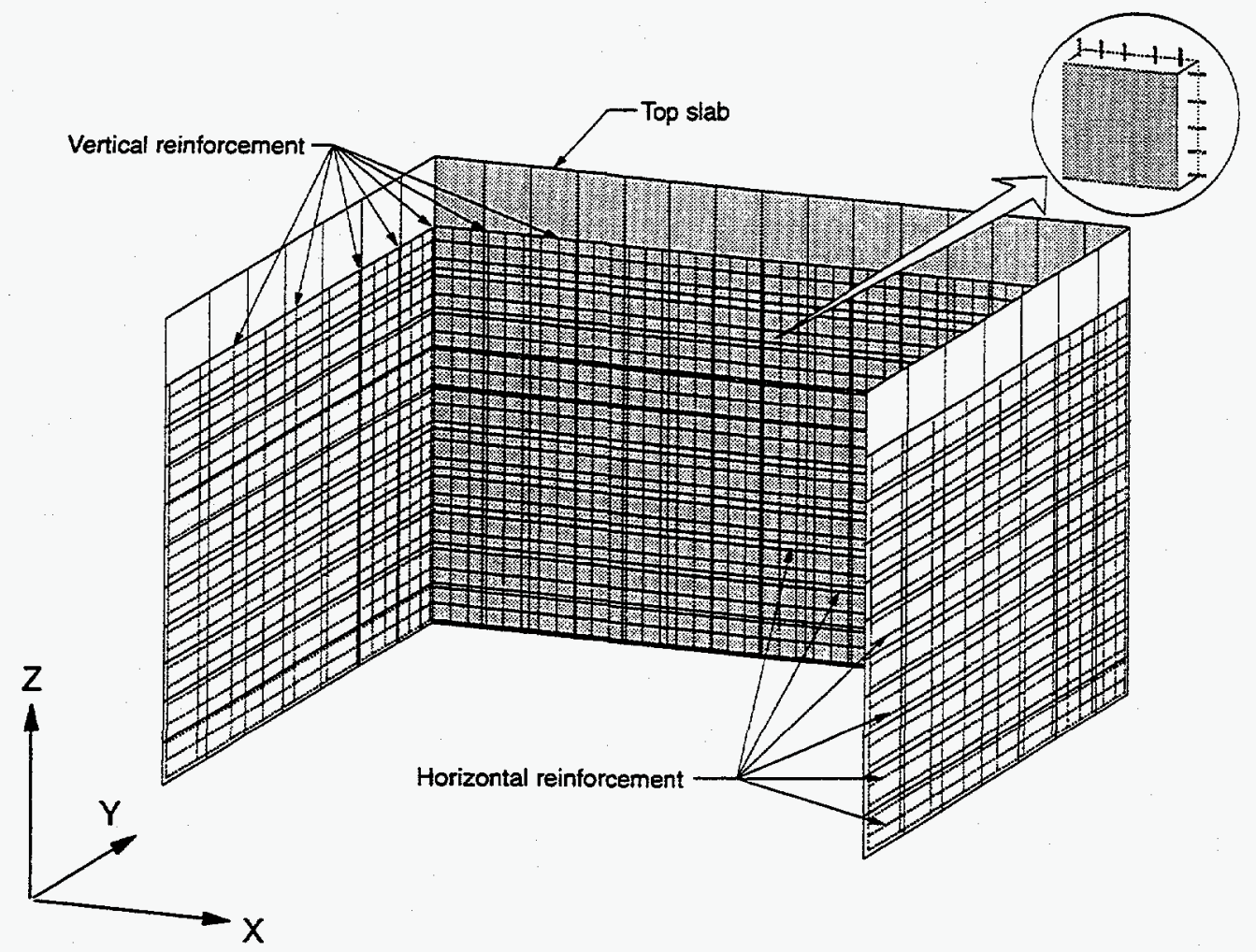

Figure 9 Reinforcement Arrangement in Finite Element Model

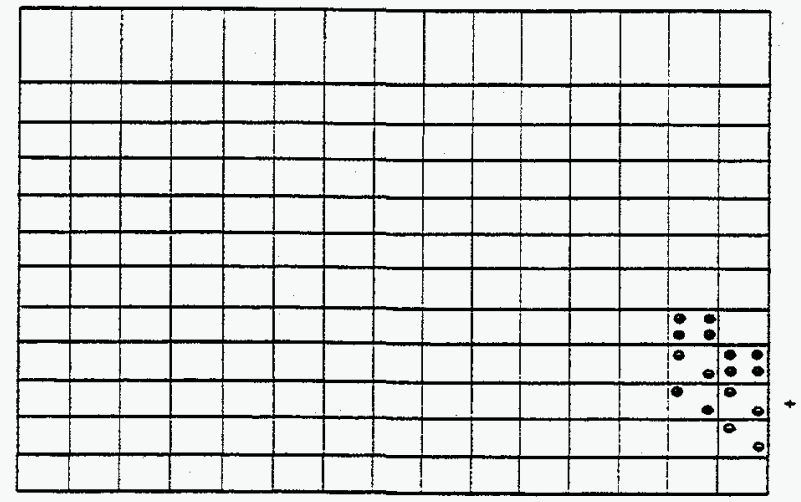

- Yielding of tensioned rebar

- Yielding of compressed rebar

Figure 10 Yielding of Web Wall Reinforcement at Horizontal Force $P_{2}=110.0$ tonf 


\section{APPENDIX C \\ COLLECTION OF \\ RECORDED TIME HISTORIES \\ FOR RUN-1}

C -1 


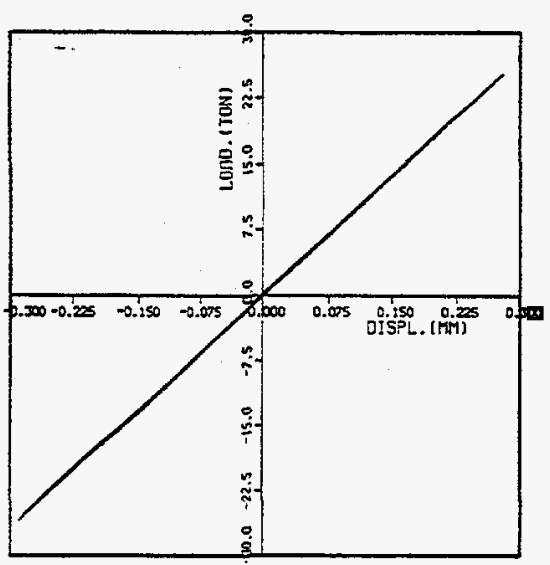

Figure C.1 Horizontal

force-deformation

relationship of top slab

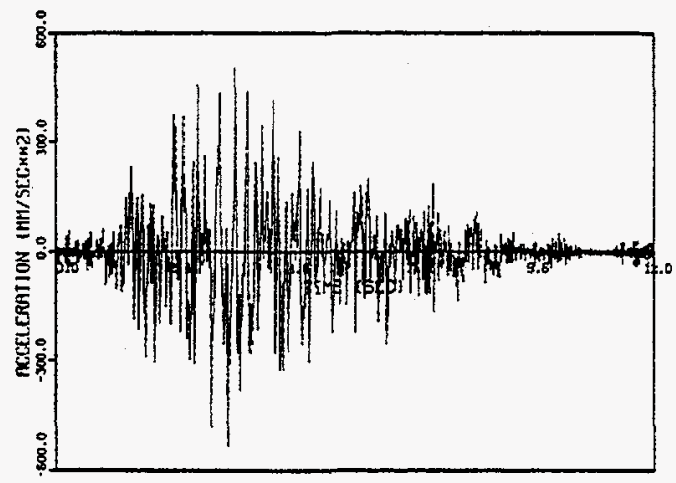

Figure C.3 AXB

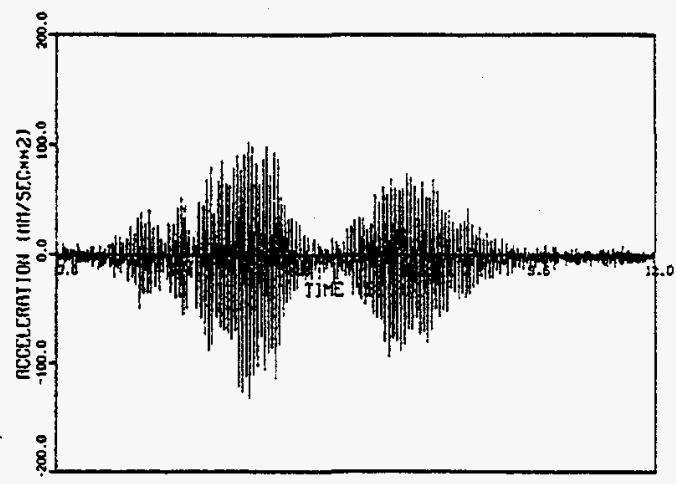

Figure C.5 AZBL

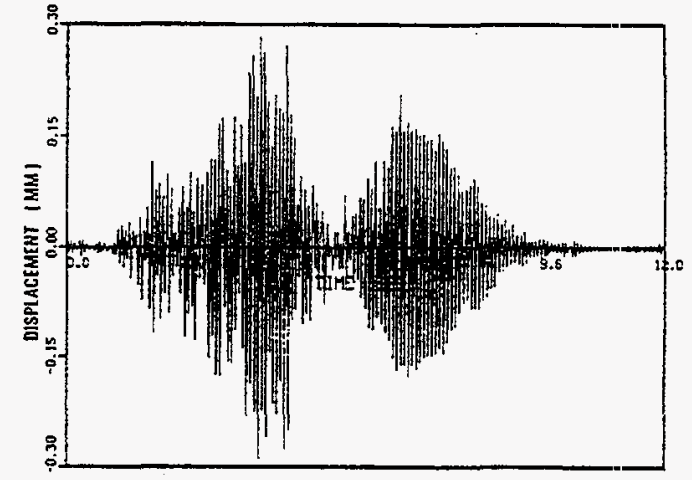

Figure C.2 DXT

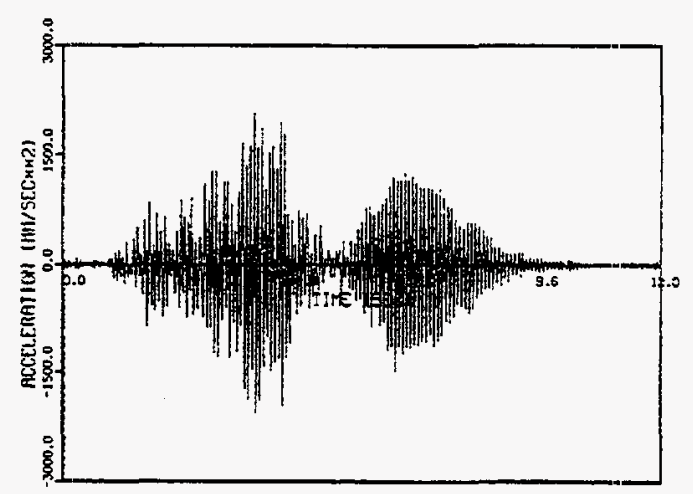

Figure C.4 AXT

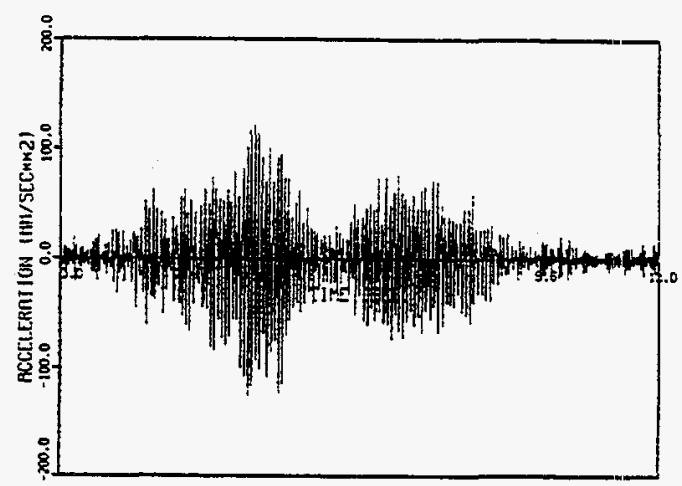

Figure C.6 AZBR 


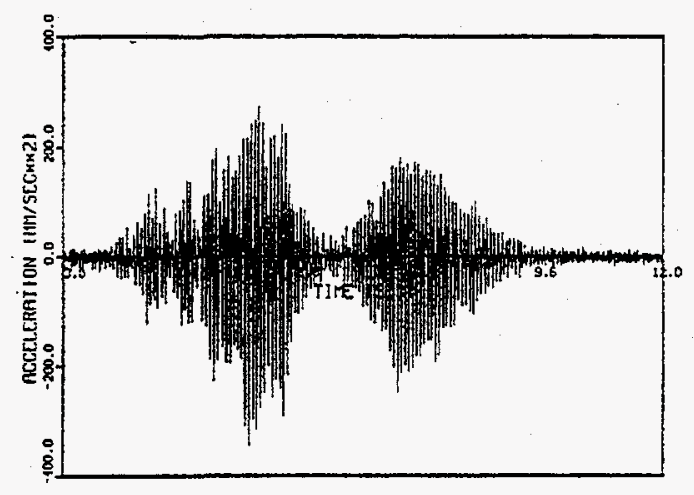

Figure C.7 AZTL

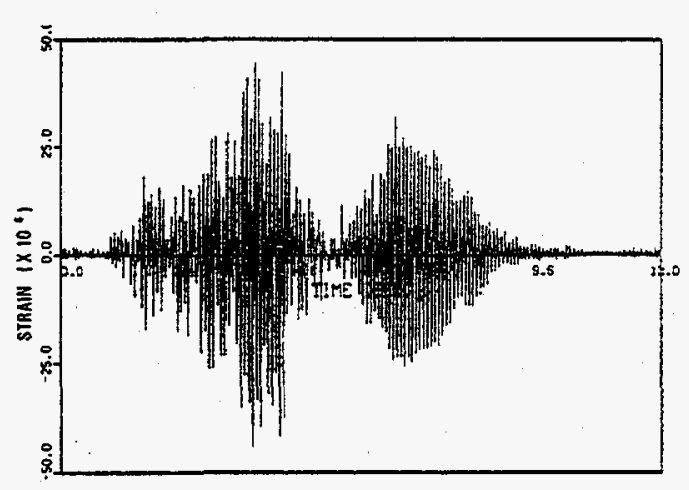

Figure C.9 FV2CL

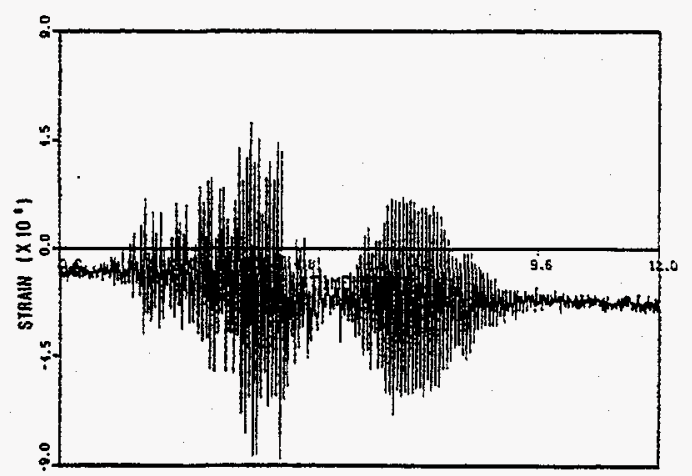

Figure C.11 WH3B

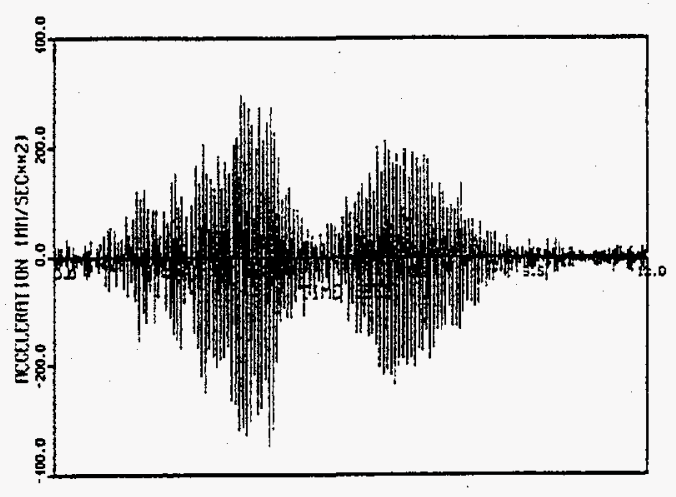

Figure C.8 AZTR

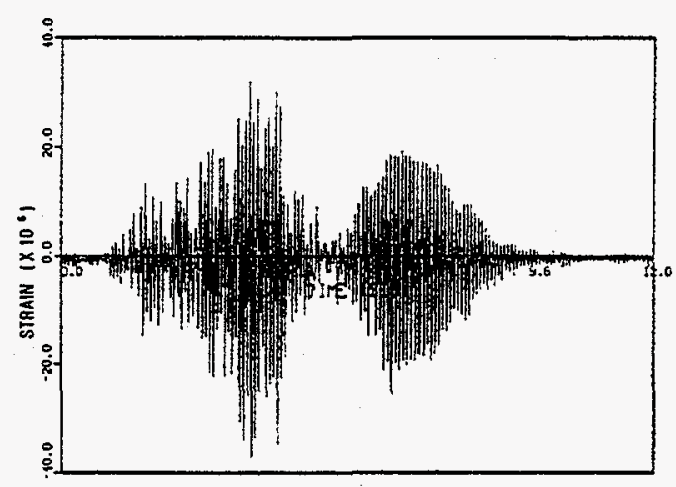

Figure C.10 FV2CR

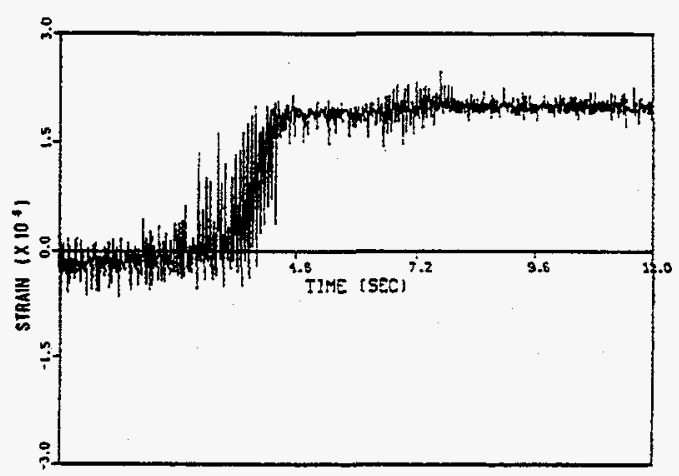

Figure C.12 WH3C 


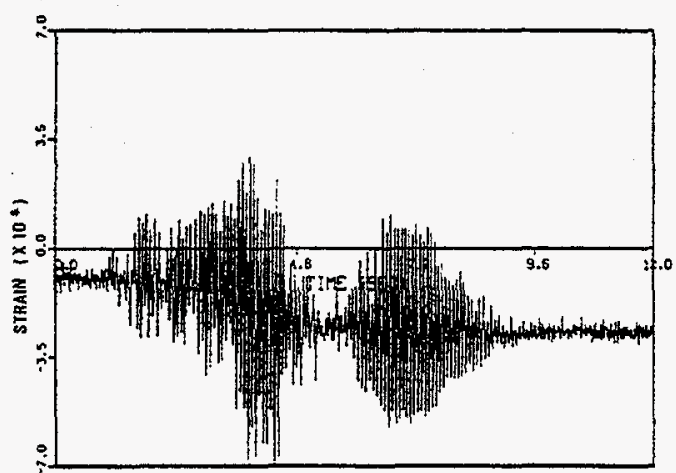

Figure C.13 WH3D

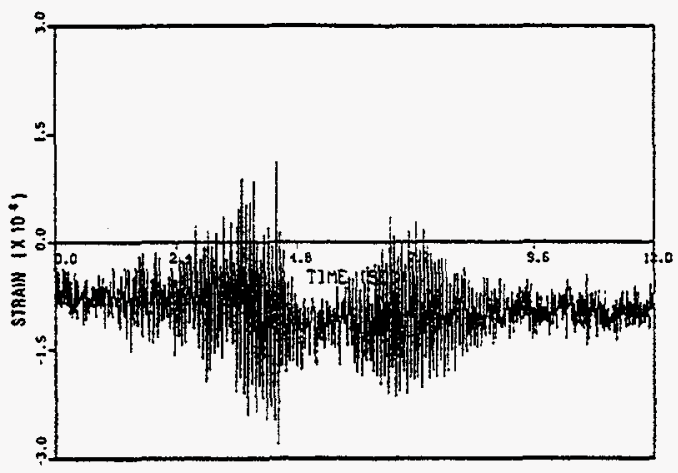

Figure C.15 WH4C

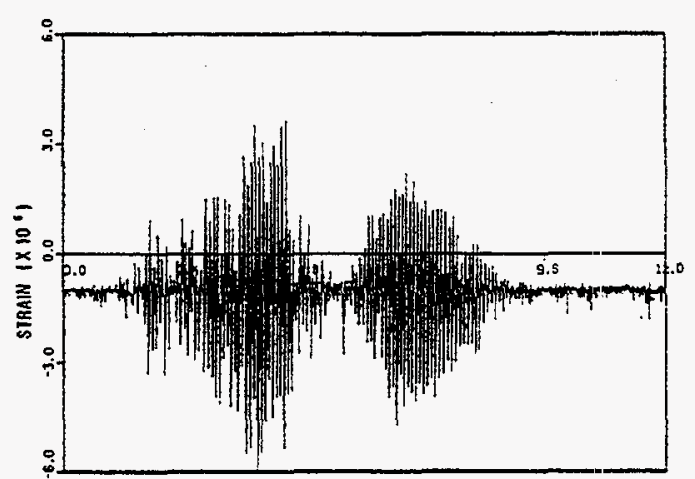

Figure C.14 WH4B

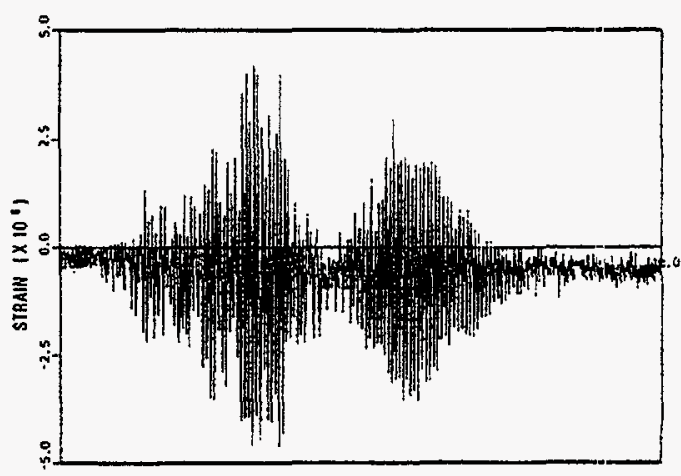

Figure C.16 WH4D 


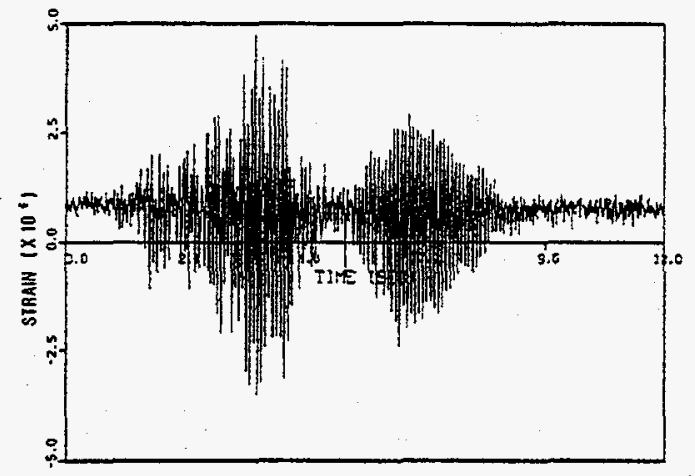

Figure C.17 WHSB

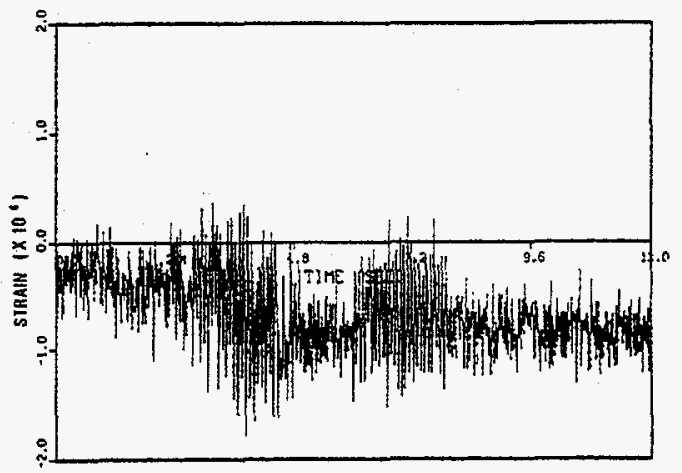

Figure C.18 WH5C

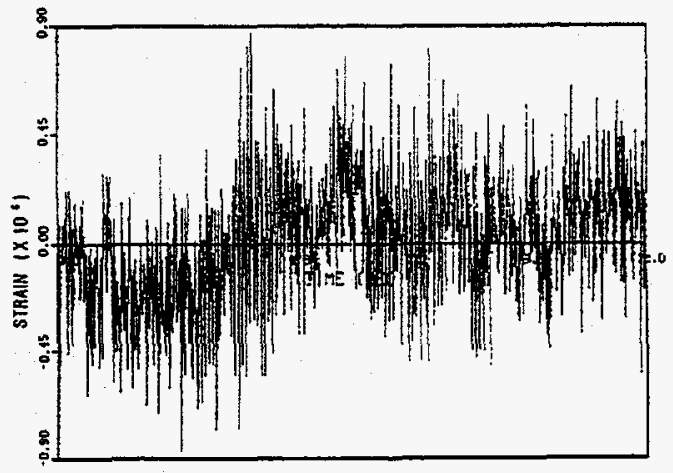

Figure C.19 WH5D 


\section{APPENDIX D \\ COLLECTION OF \\ RECORDED TIME HISTORIES \\ FOR RUN-4}




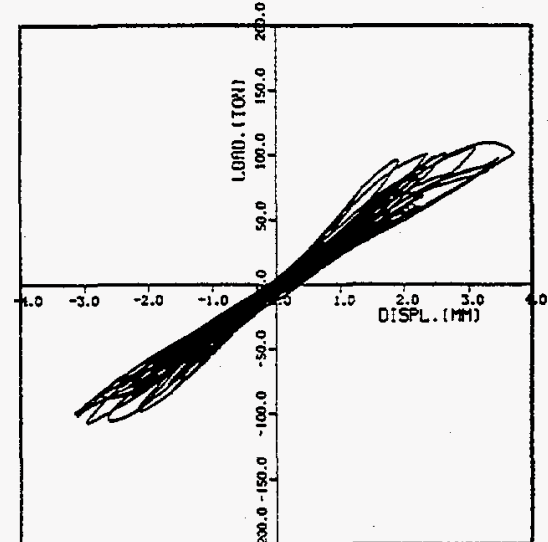

Figure D.1 Horizontal

force-deformation

relationship of top slab

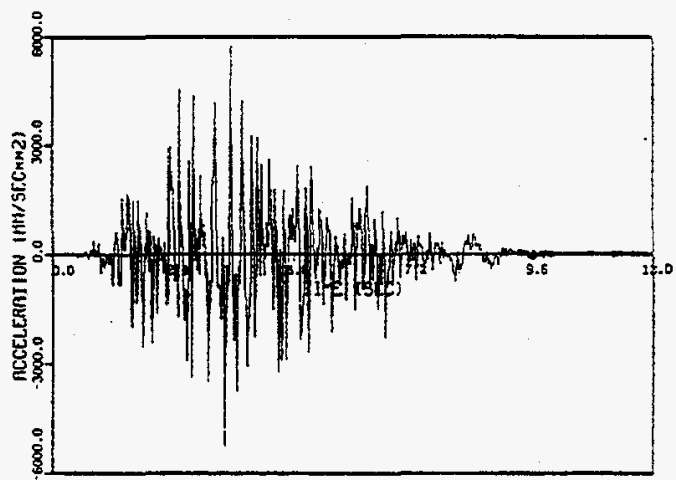

Figure D.3 AXB

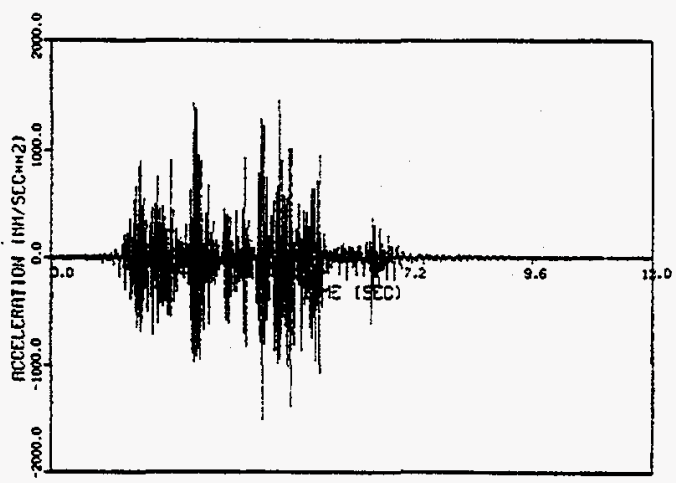

Figure D.5 AZBL

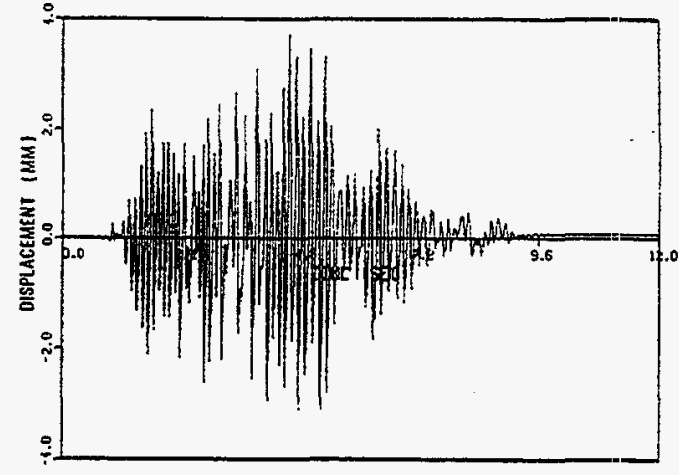

Figure D.2 DXT

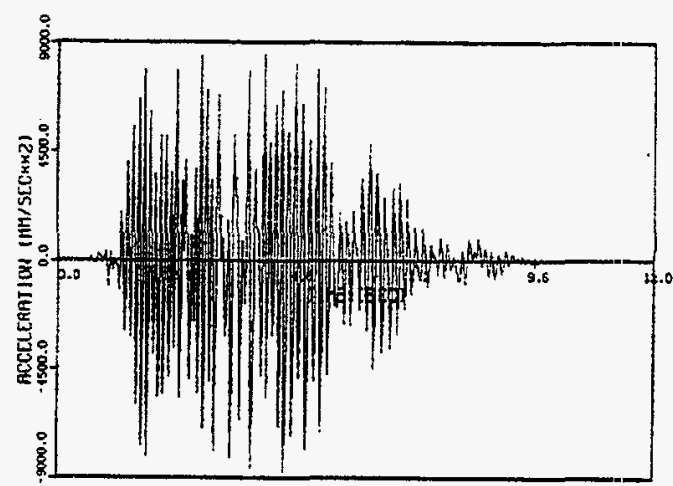

Figure D.4 AXT

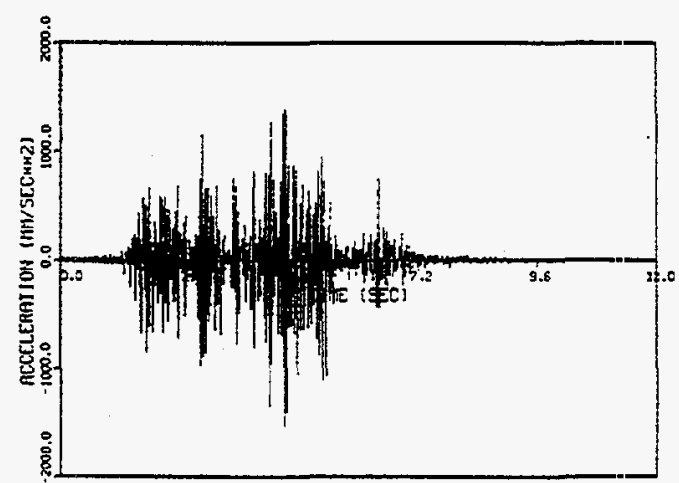

Figure D.6 AZBR 


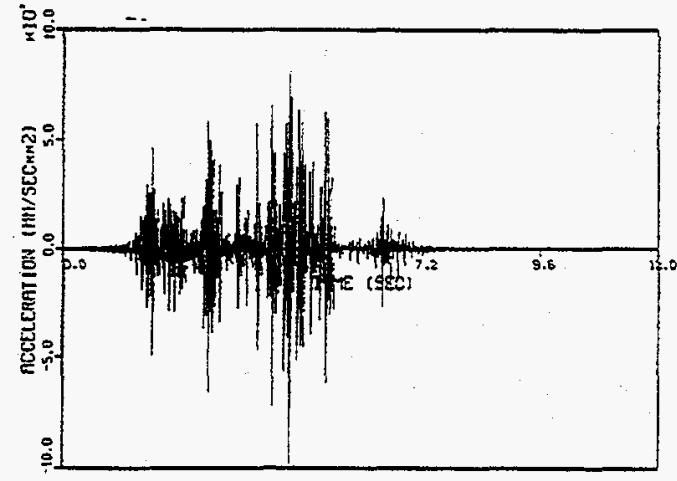

Figure D.7 AZTL

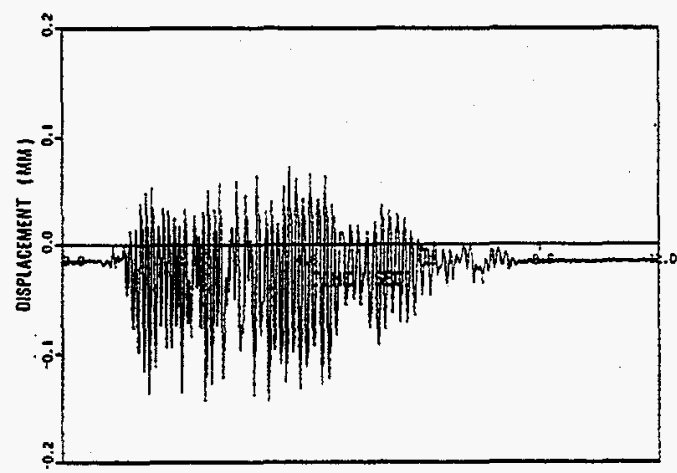

Figure D.9 DV11

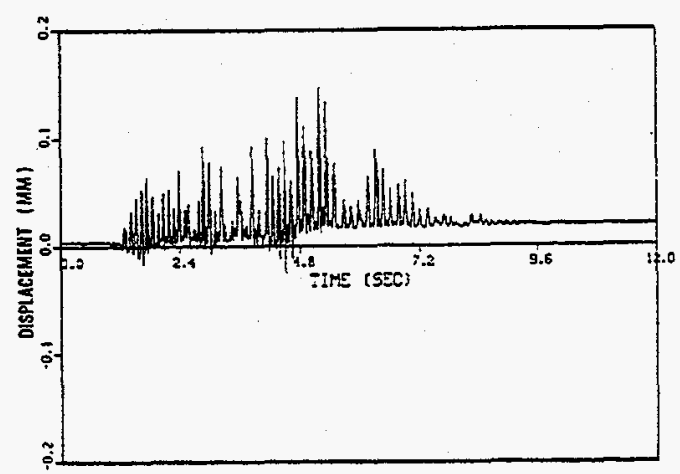

Figure D.11 DV13

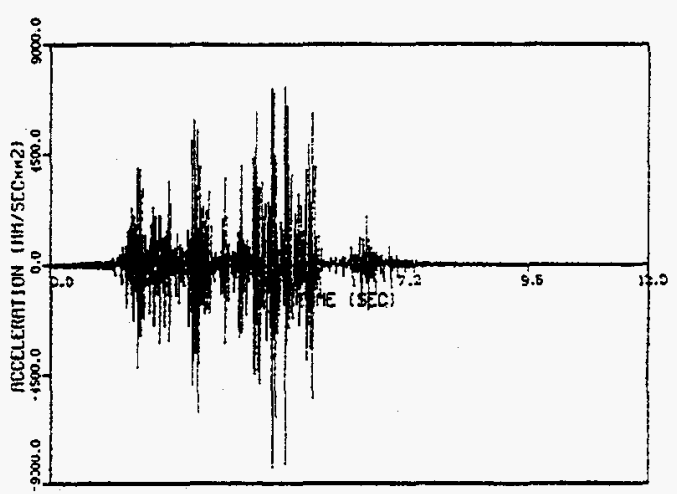

Figure D.8 AZTR

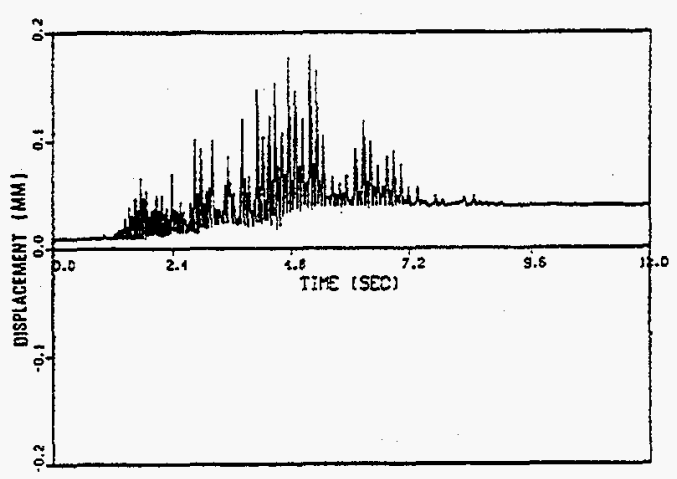

Figure D.10 DV12

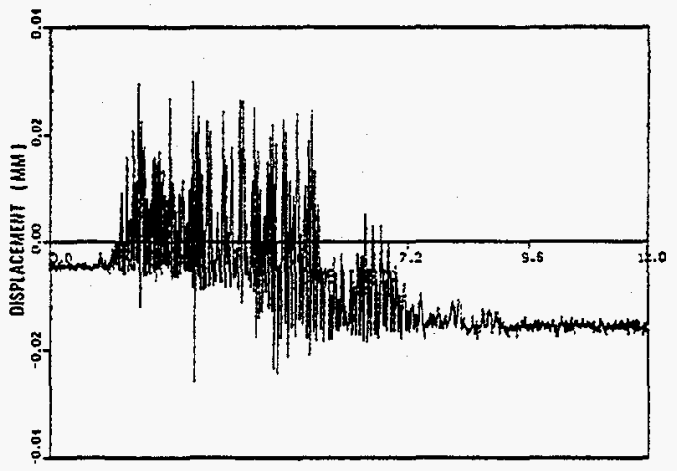

Figure D.12 DV14 


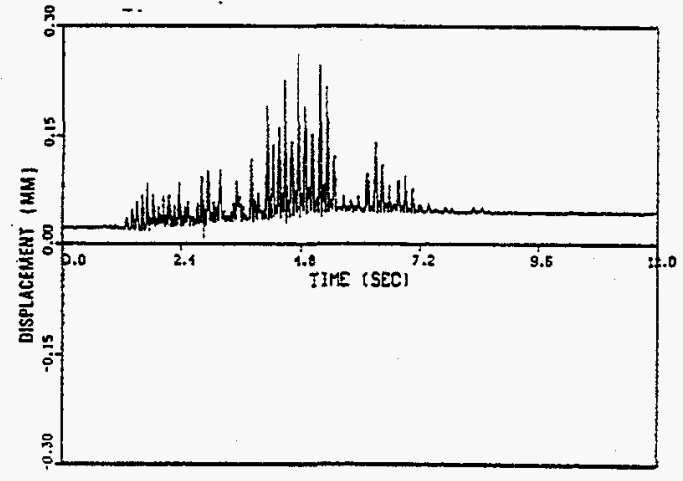

Figure D.13 DV15

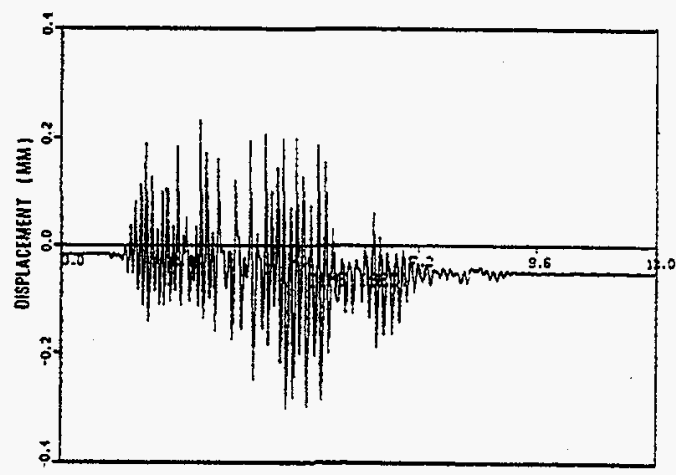

Figure D.15 DV18

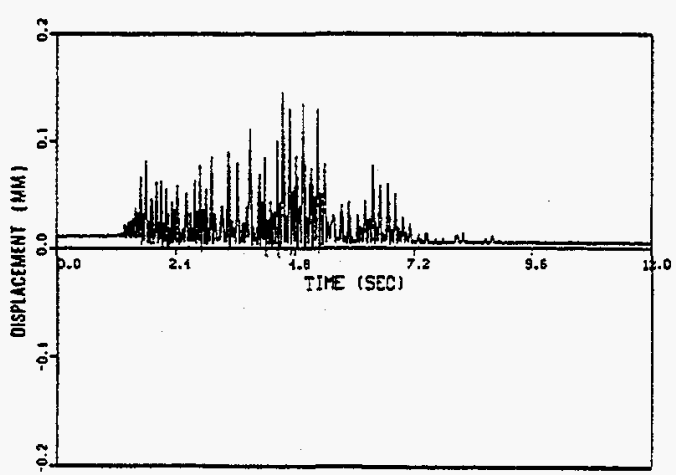

Figure D.17 DV22

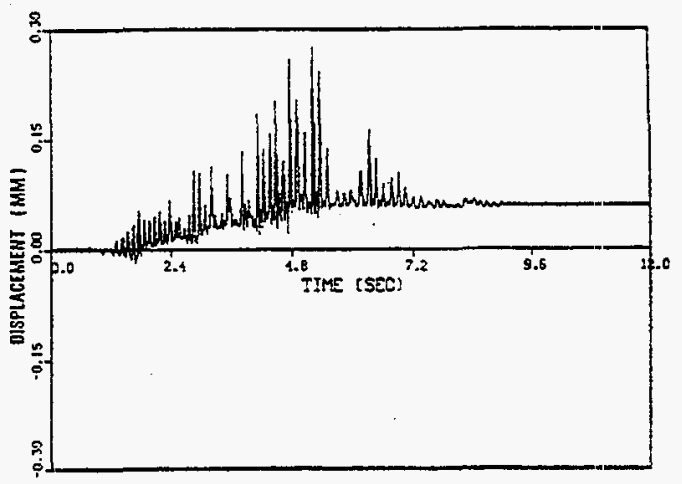

Figure D.14 DV16

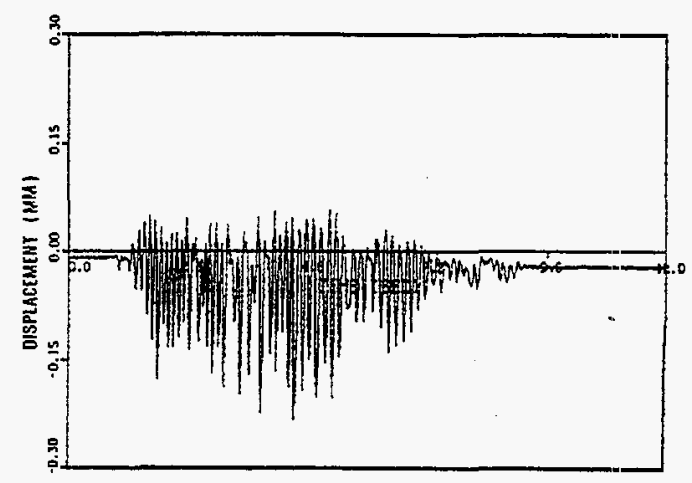

Figure D.16 DV21

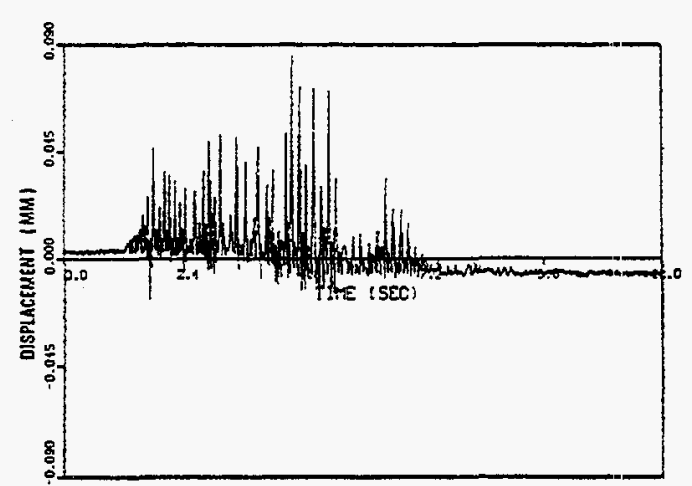

Figure D.18 DV23 


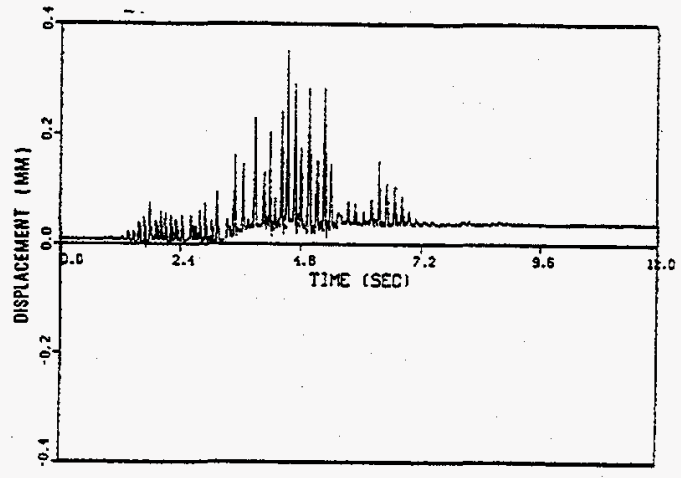

Figure D.19 DV24

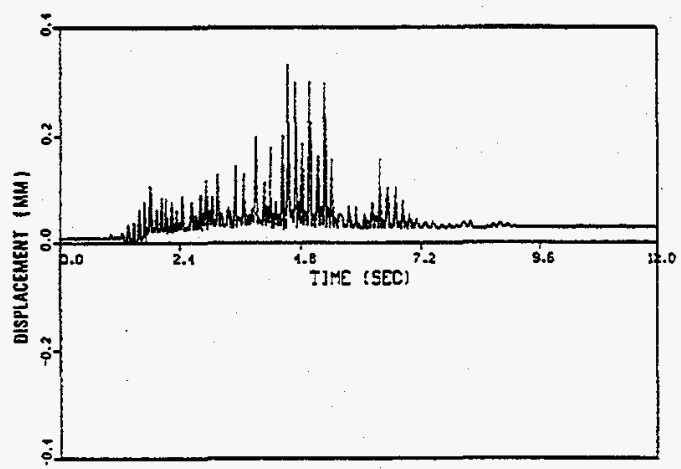

Figure D.21 DV26

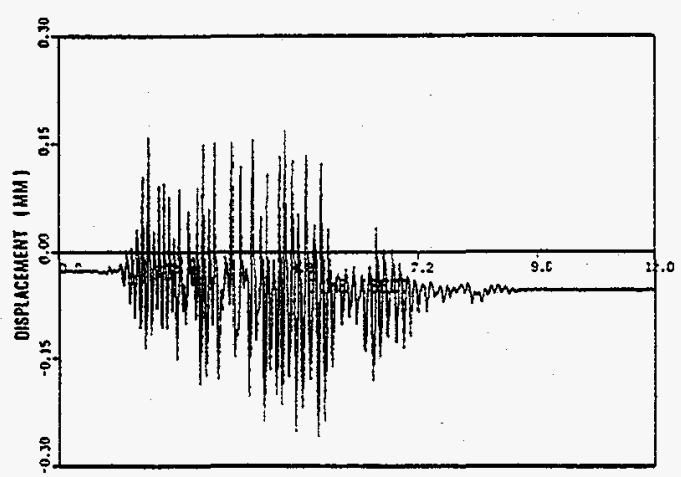

Figure D.23 DV28

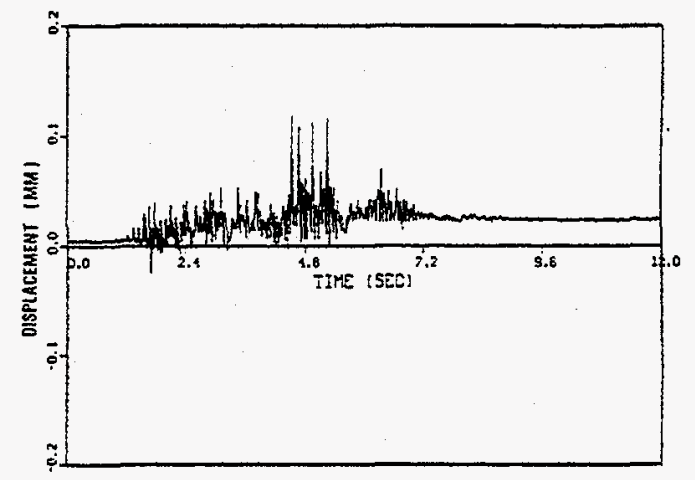

Figure D.20 DV25

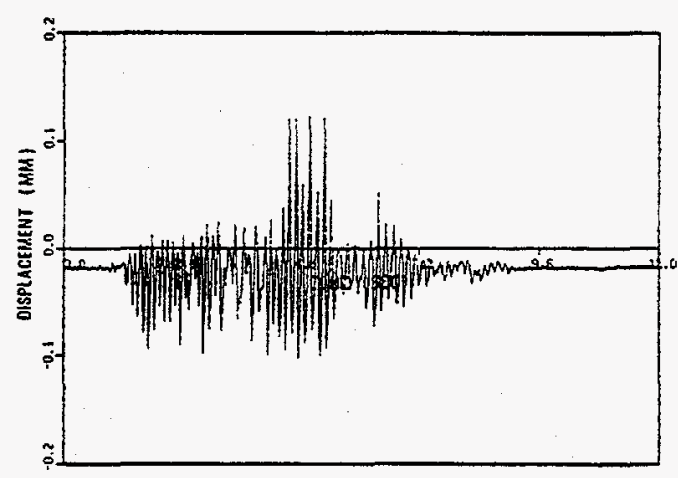

Figure D.22 DV27

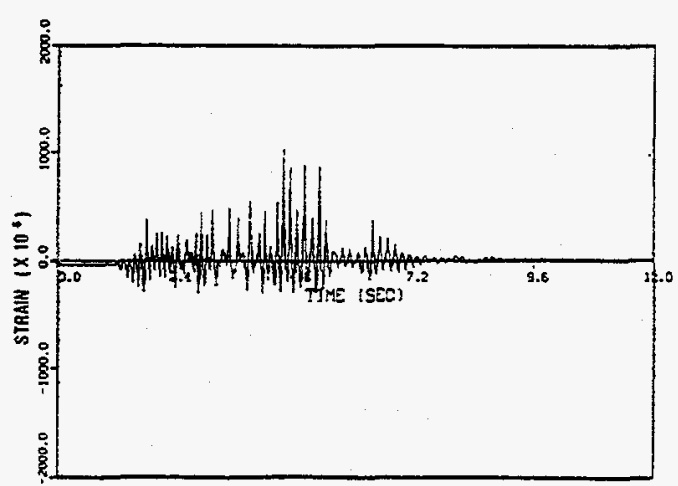

Figure D.24 FV2CL 


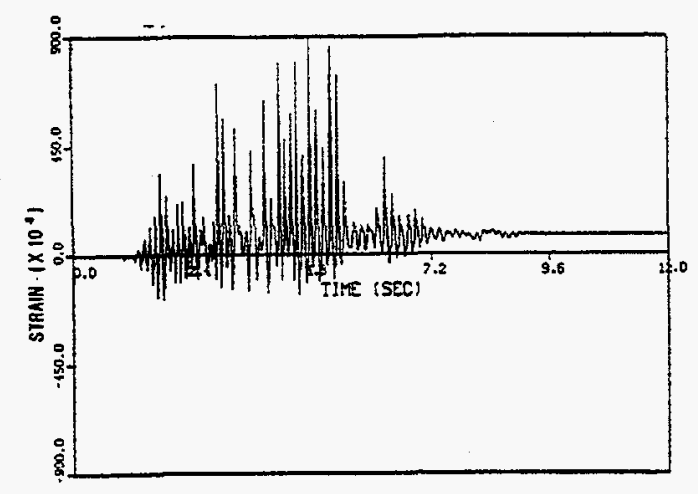

Figure D.25 FV2CR

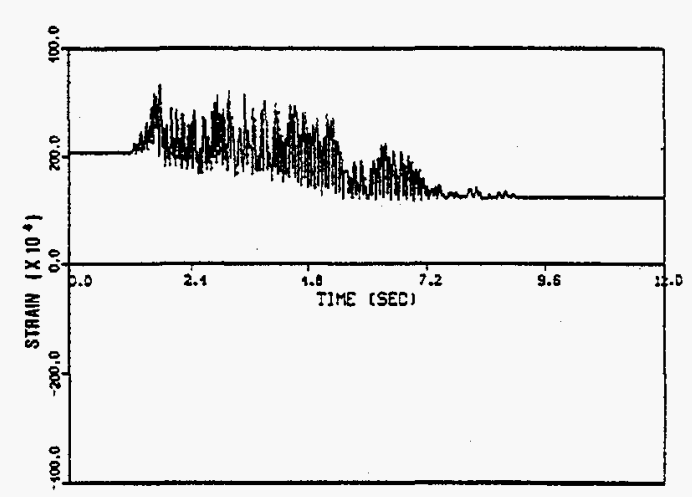

Figure D.27 WH3C

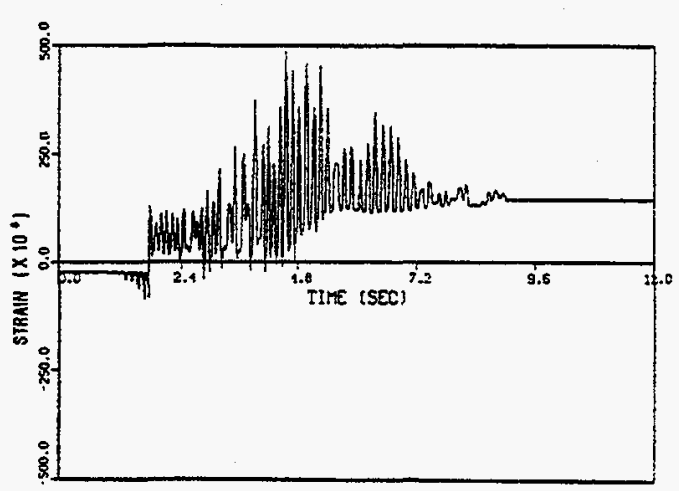

Figure D.29 WH4B

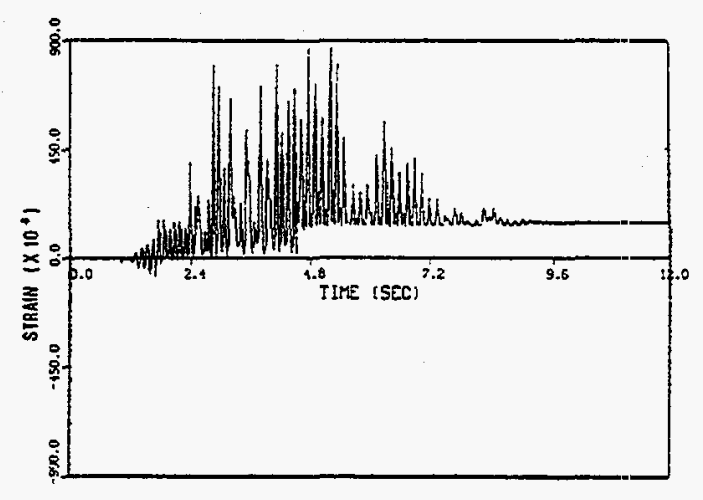

Figure D.26 WH3B

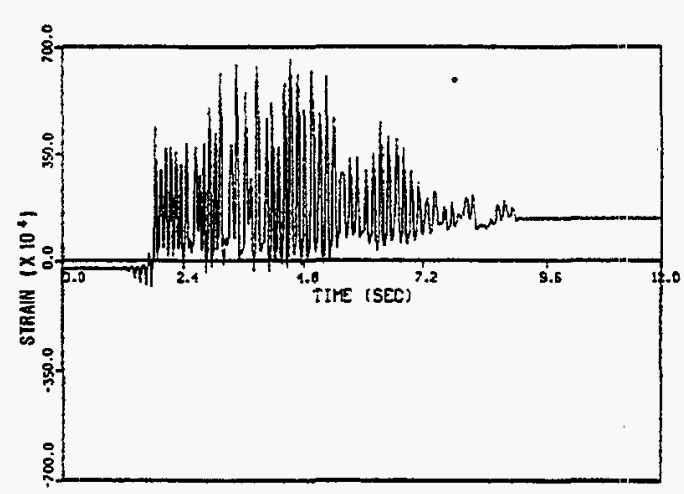

Figure D.28 WH3D

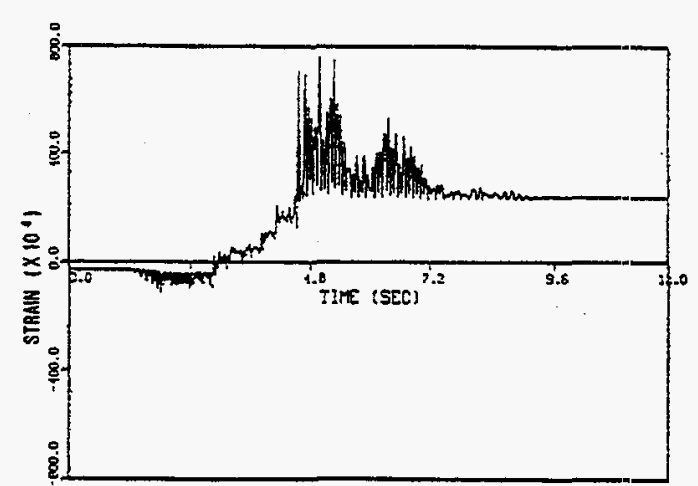

Figure D.30 WH4C 


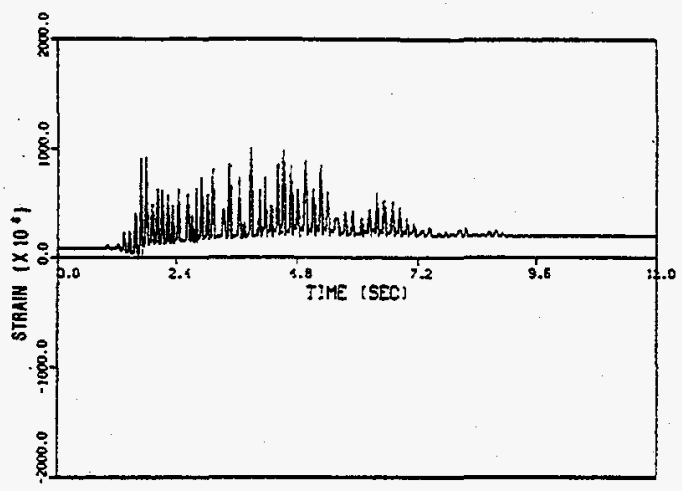

Figure D.31 WH4D

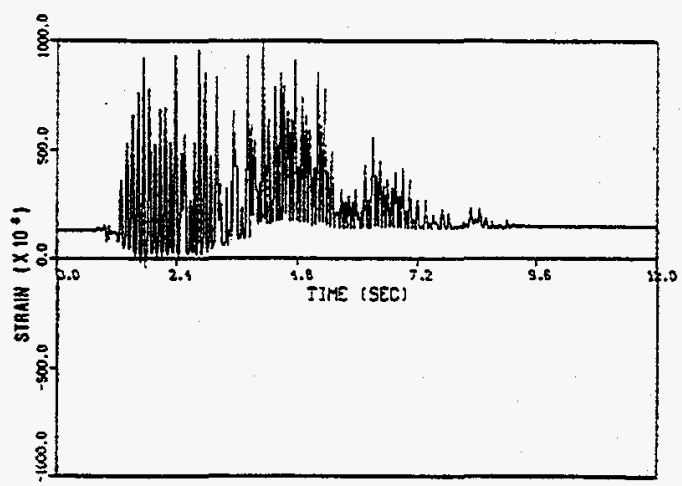

Figure D.33 WH5C

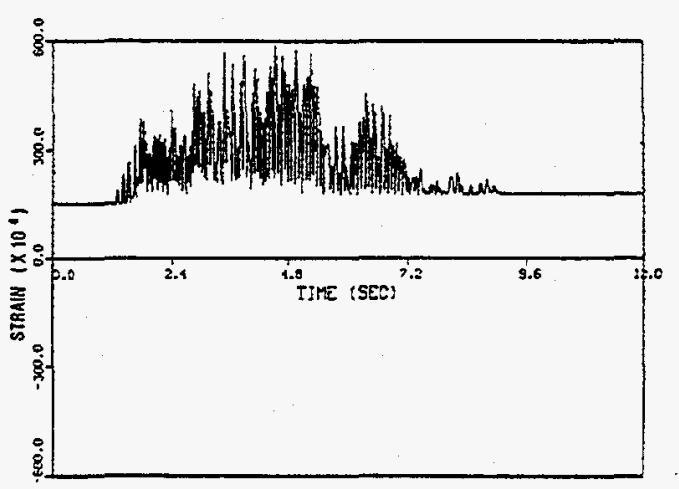

Figure D.32 WH5B

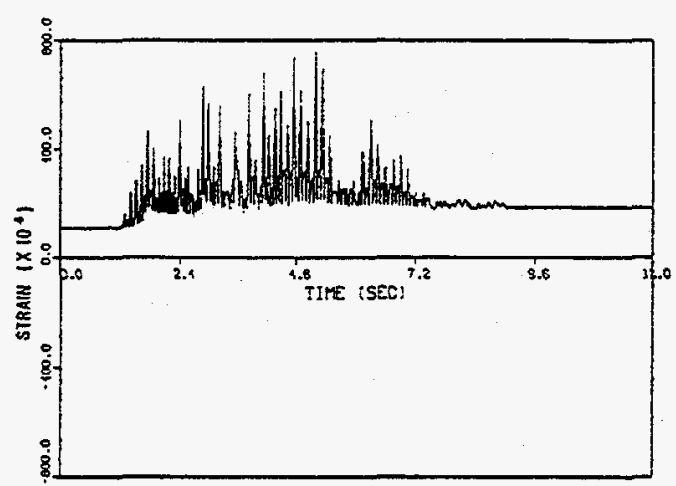

Figure D.34 WH5D 


\section{APPENDIX E}

\section{COLLECTION OF}

\section{RECORDED TIME HISTORIES}

FOR RUN-5 


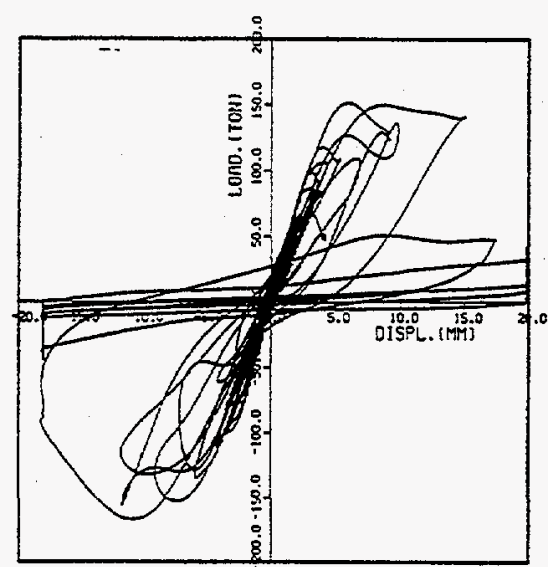

Figure E.1 Horizontal

force-deformation relationship of top slab

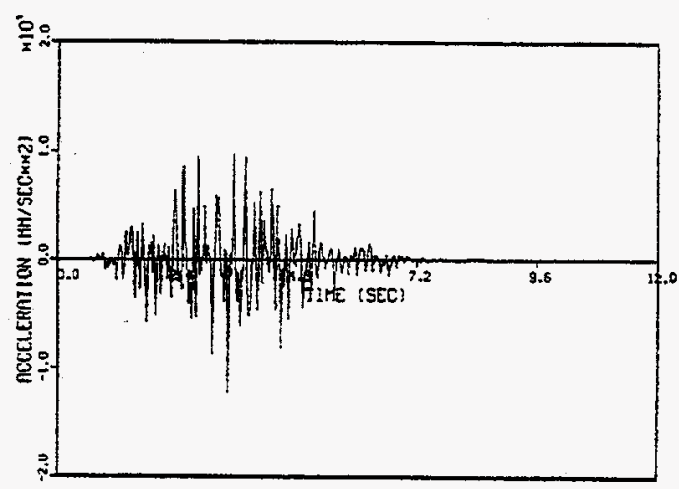

Figure E.3 AXB

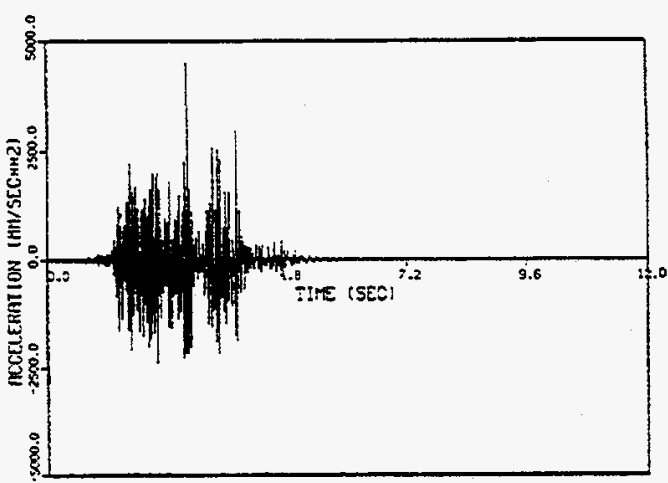

Figure E.5 AZBL

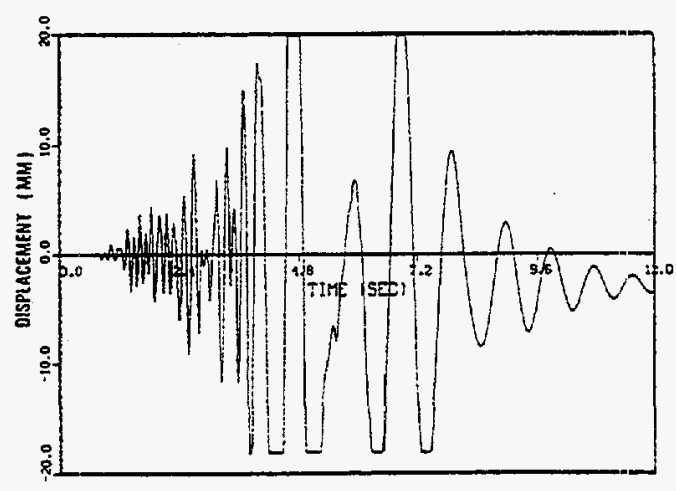

Figure E.2 DXT

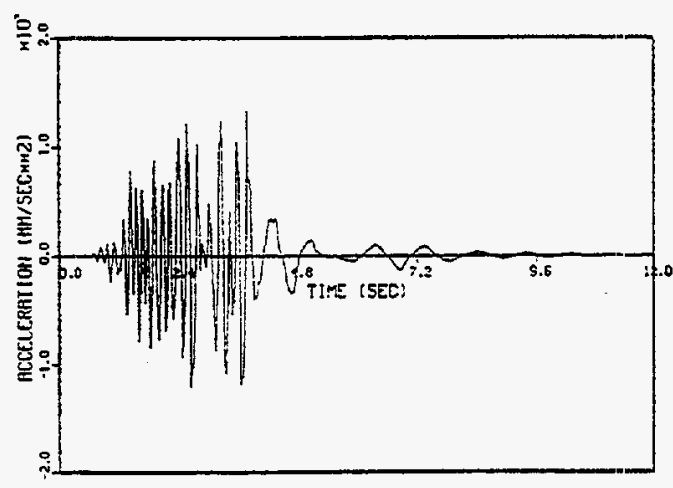

Figure E.4 AXT

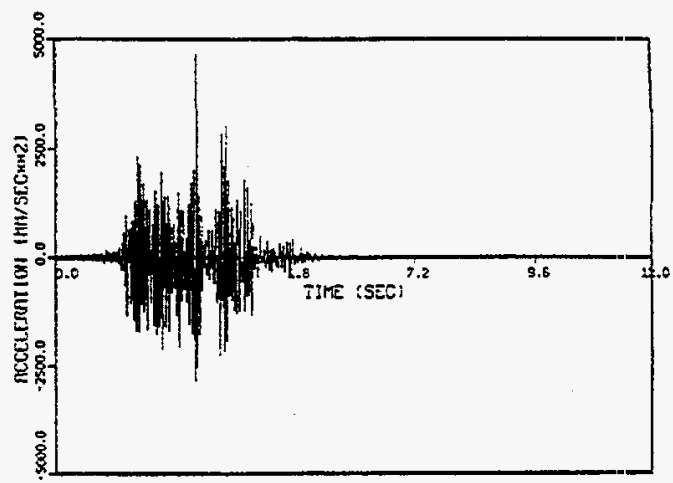

Figure E.6 AZBR 


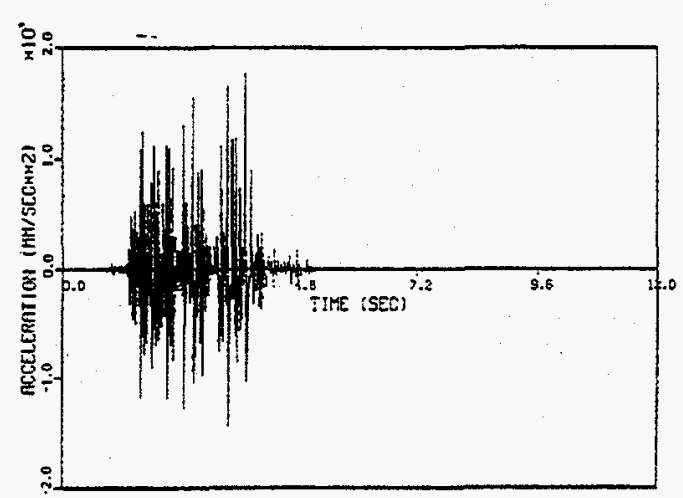

Figure E.7 AZTL

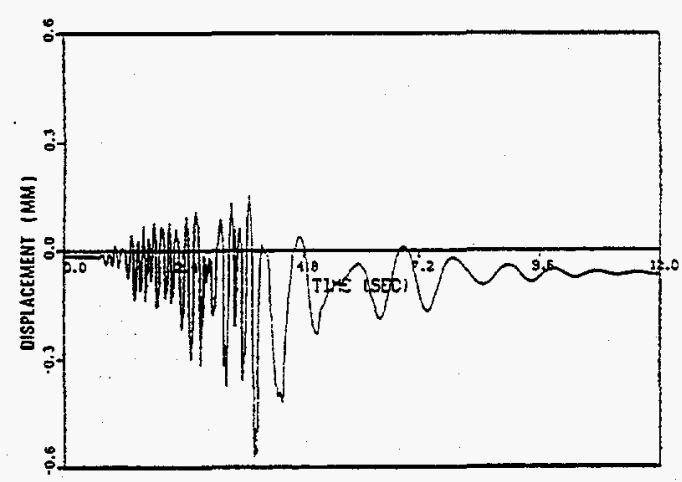

Figure E.9 DV11

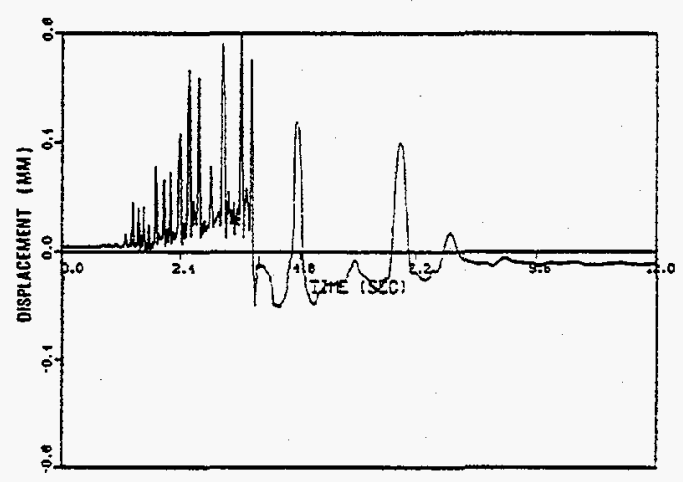

Figure E.11 DV13

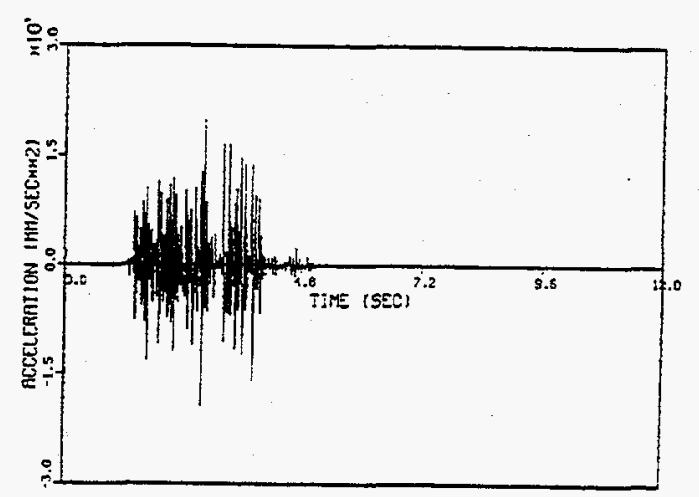

Figure E.8 AZTR

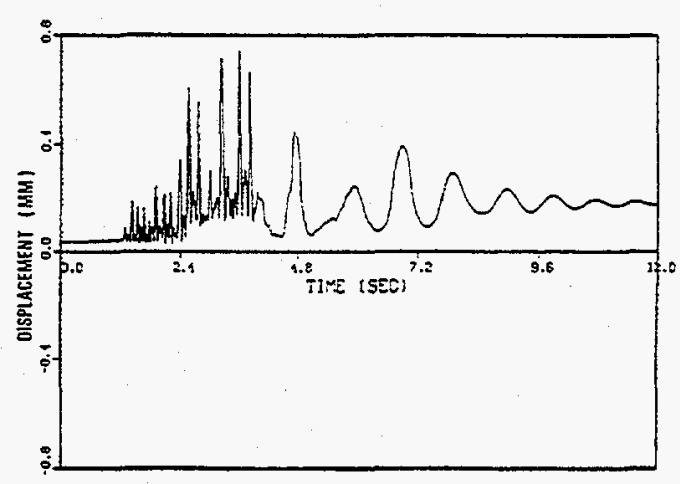

Figure E.10 DV12

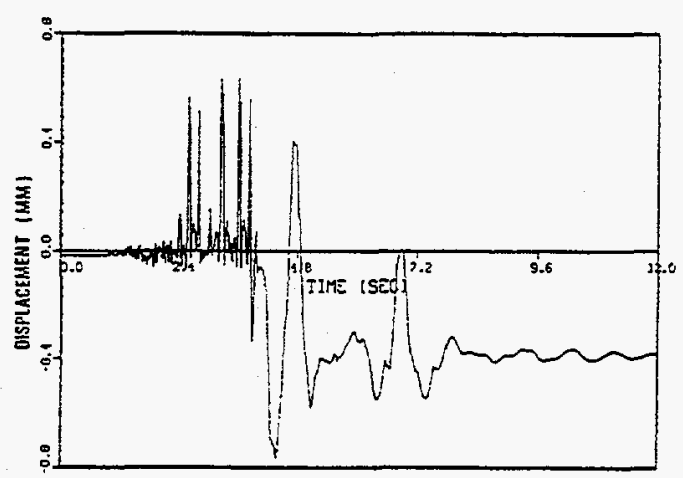

Figure E.12 DV14 


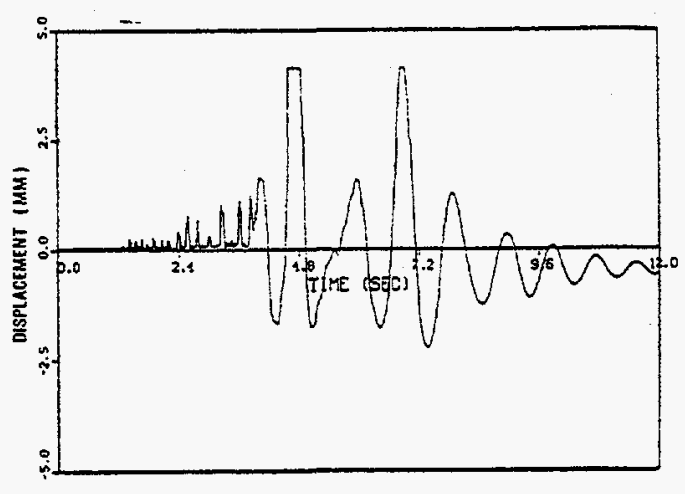

Figure E.13 DV15

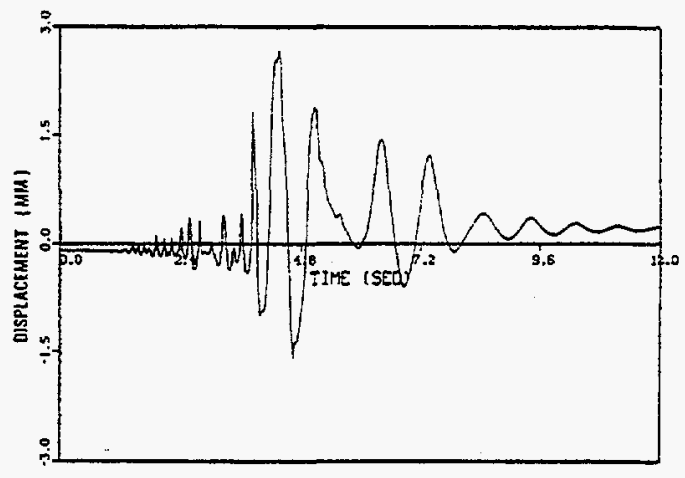

Figure E.15 DV17

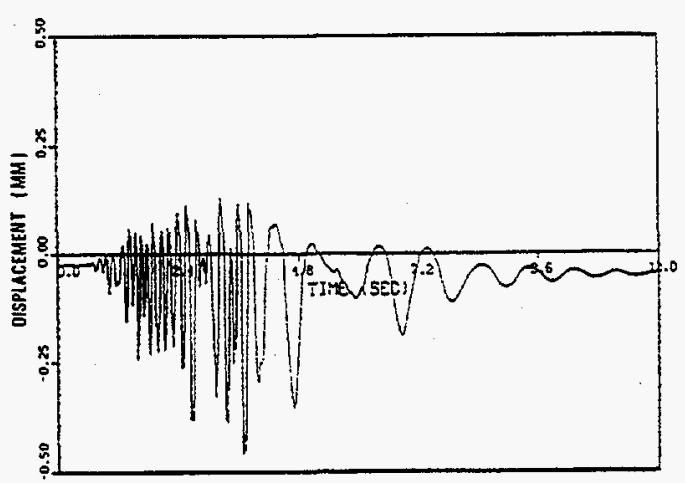

Figure E.17 DV21

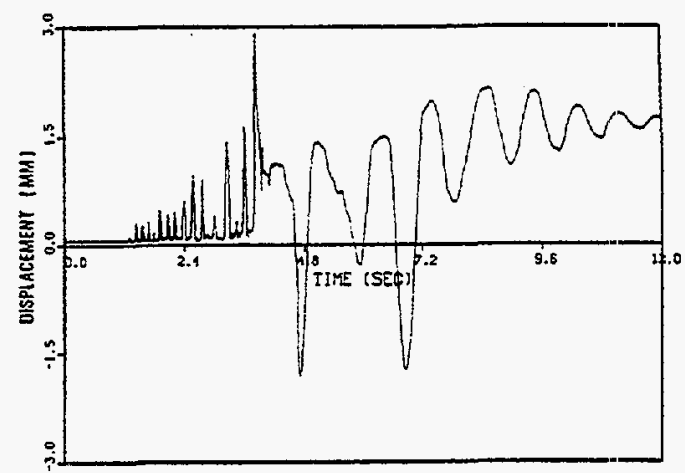

Figure E.14 DV16

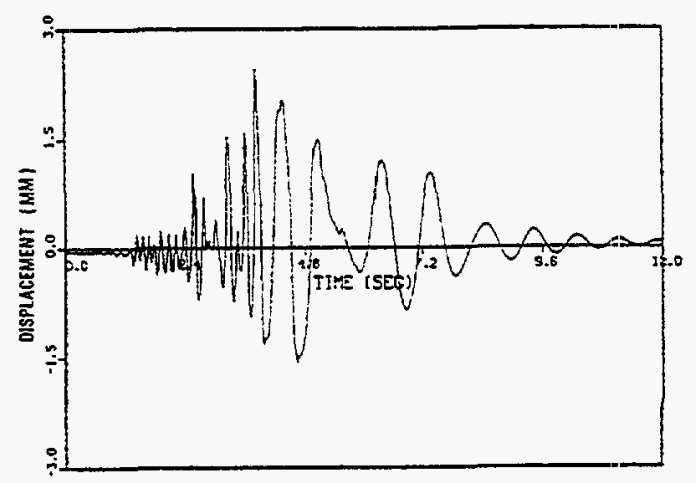

Figure E.16 DV18

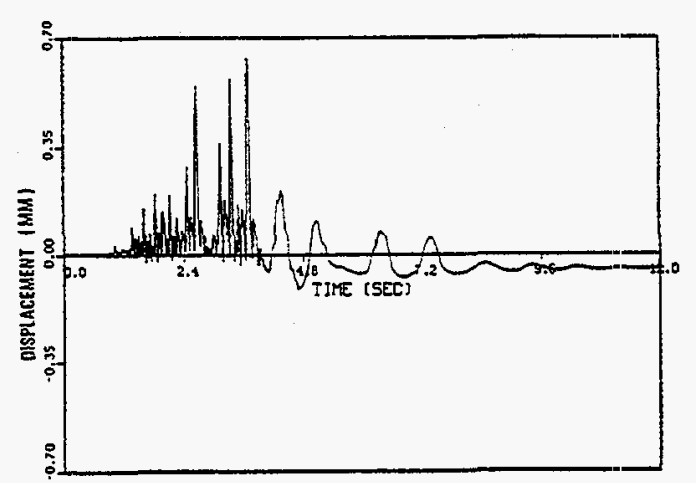

Figure E.18 DV22 


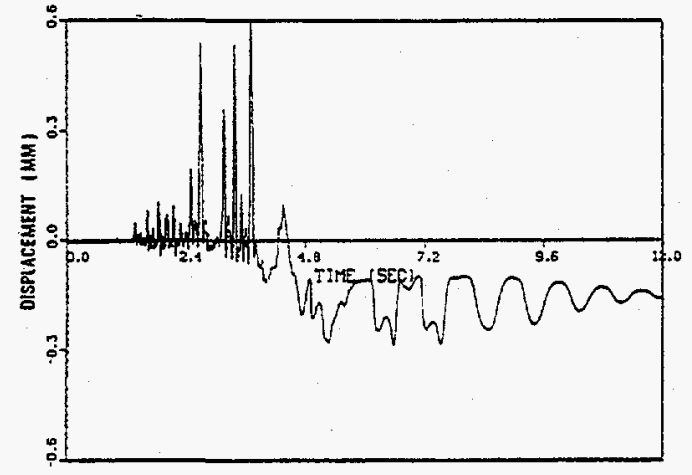

Figure E.19 DV23

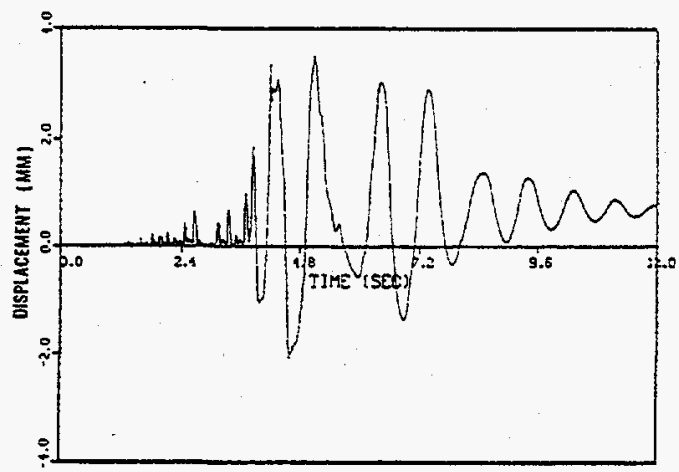

Figure E.21 DV25

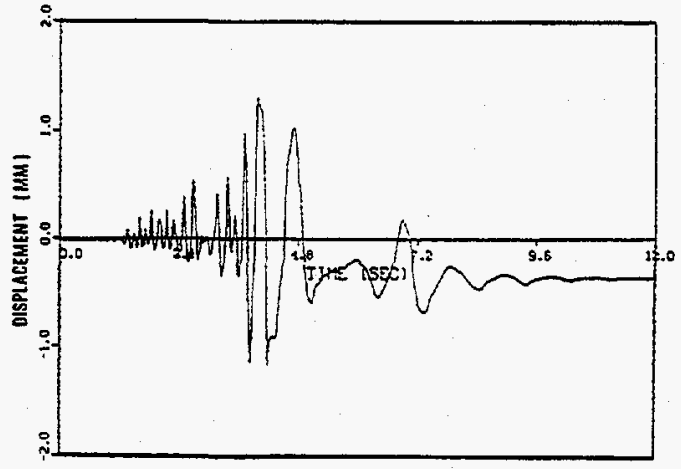

Figure E.23 DV27

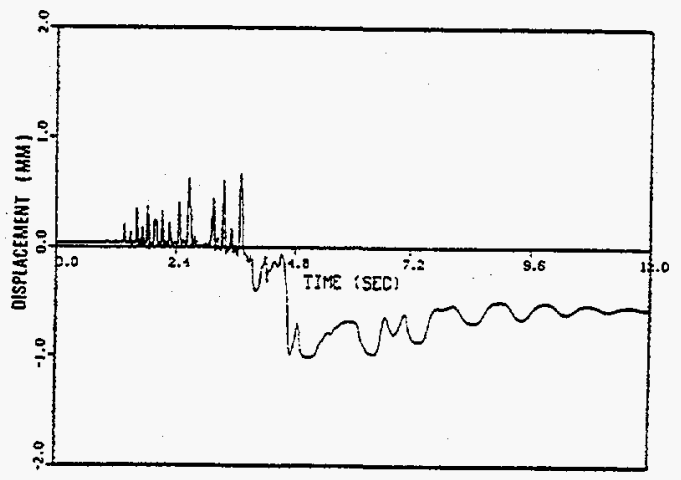

Figure E.20 DV24

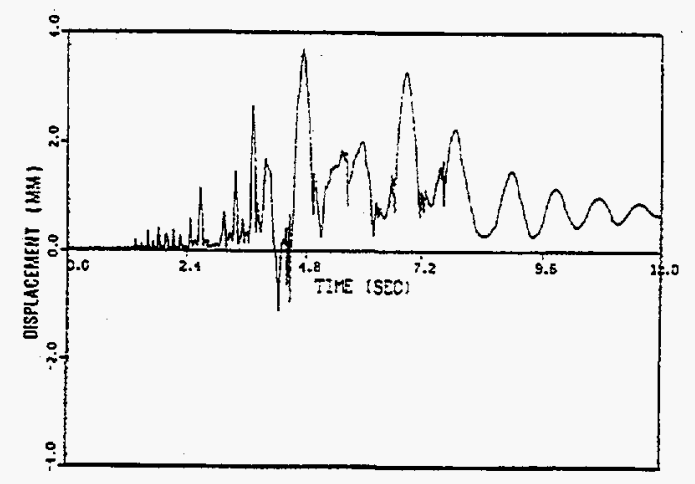

Figure E.22 DV26

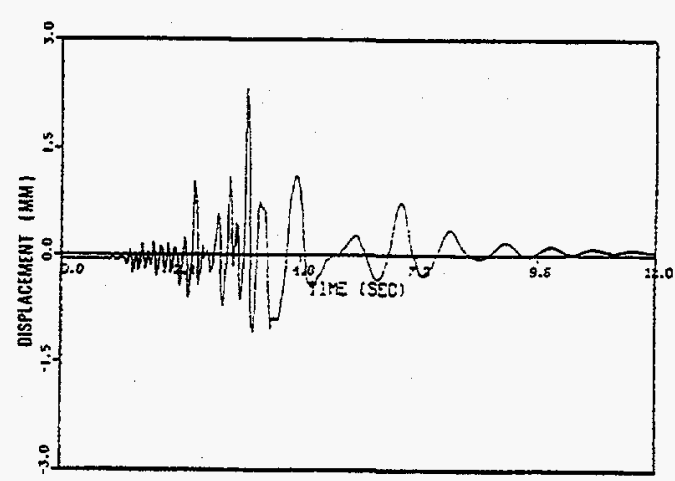

Figure E.24 FV28 


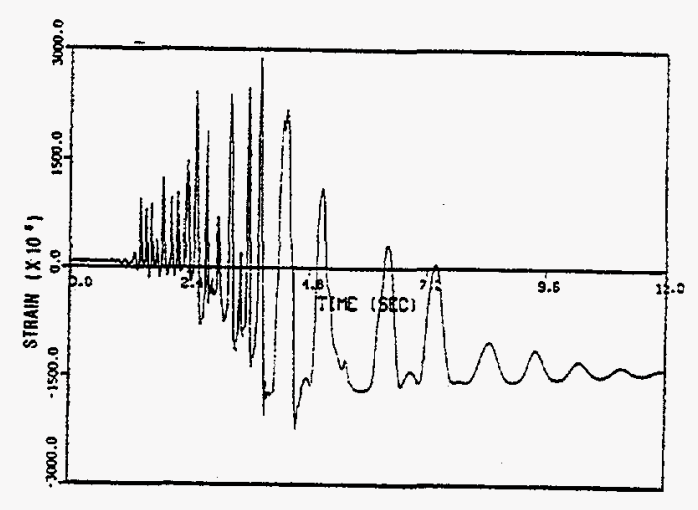

Figure E.25 FV2CR

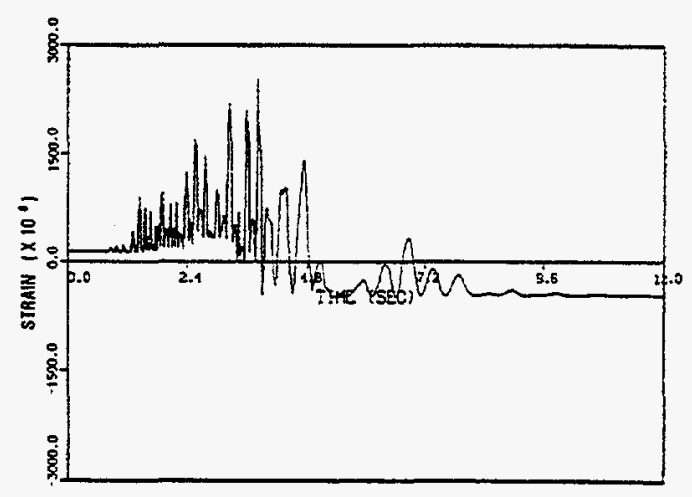

Figure E.27 WH3B

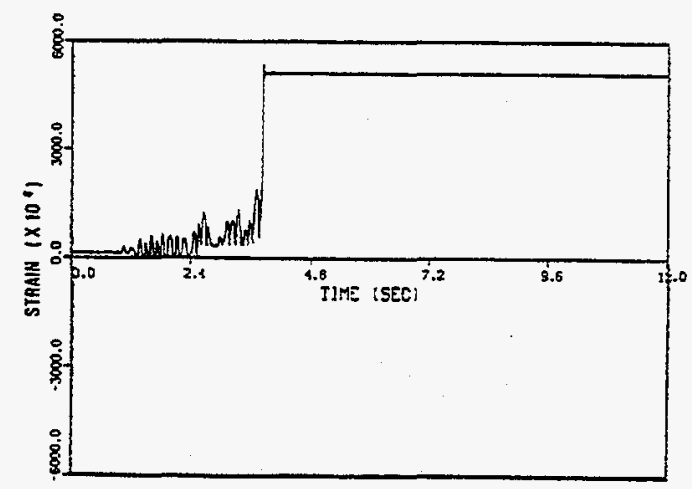

Figure E.29 WH3D

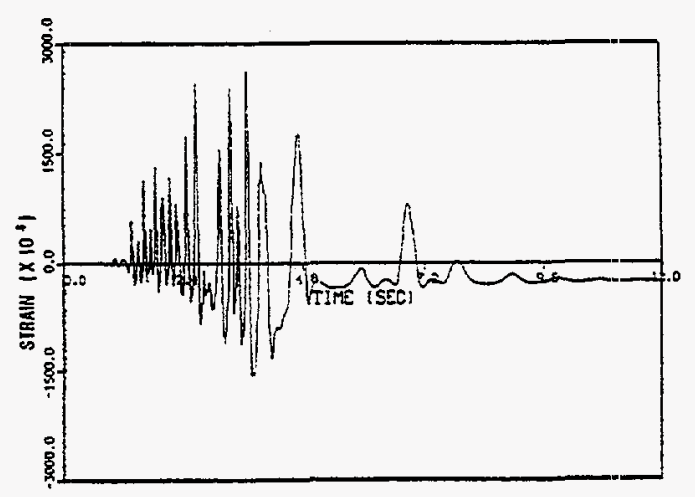

Figure E.26 FV2CL

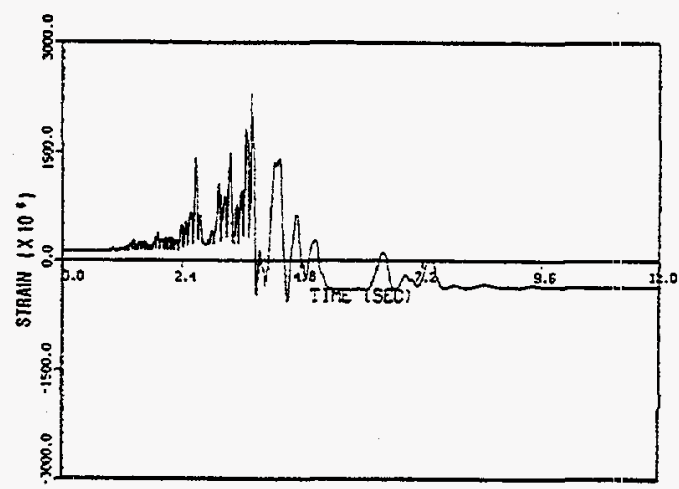

Figure E.28 WH3C

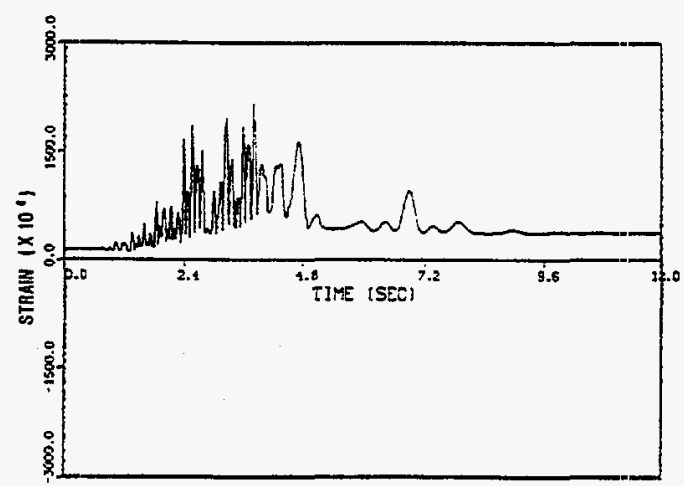

Figure E.30 WH4B 


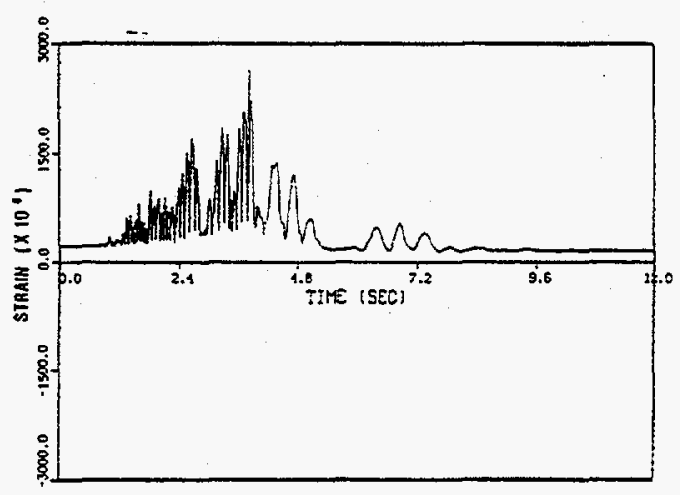

Figure E.31 WH4C

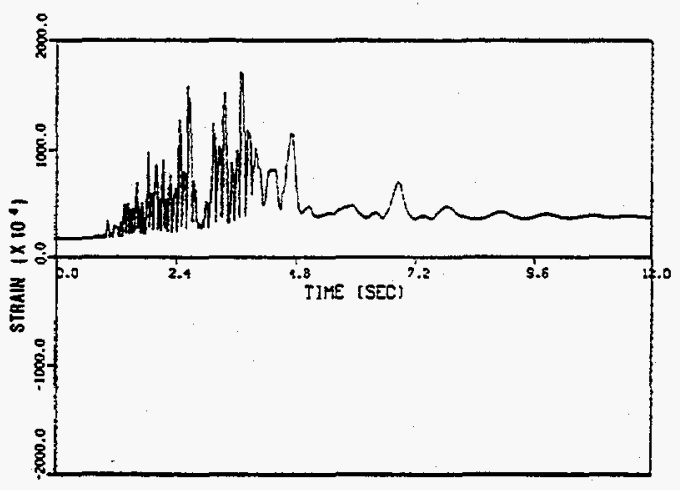

Figure E.33 WH5B

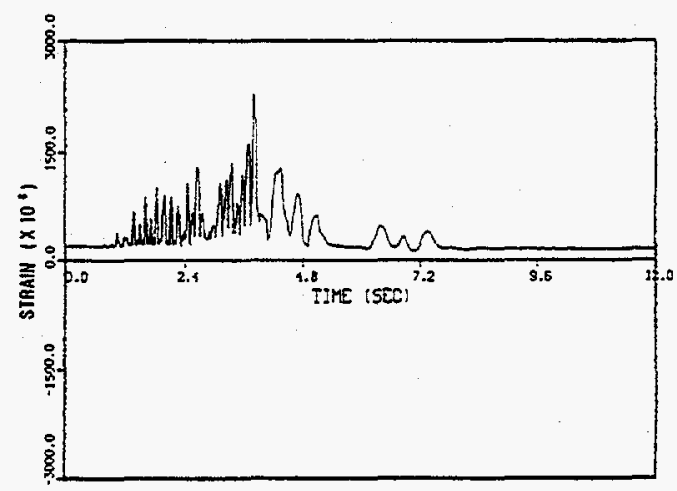

Figure E.32 WH4D

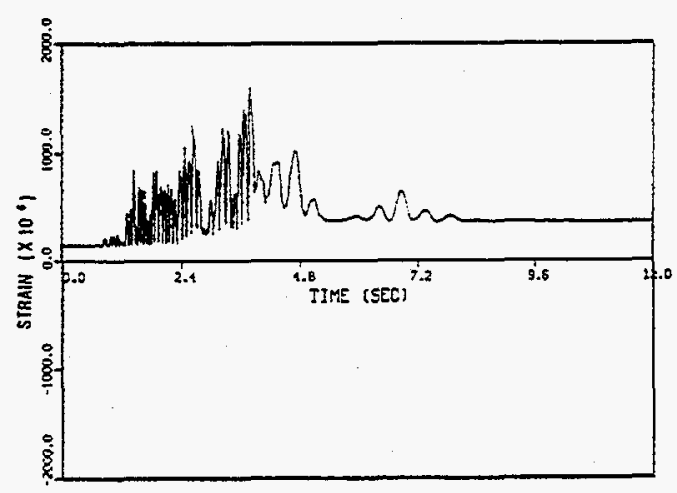

Figure E.34 WH5C

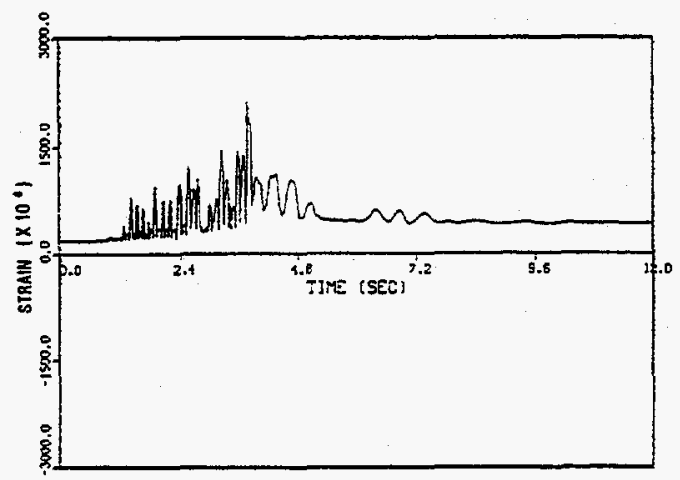

Figure E.29 WH5D 


\begin{tabular}{|c|c|}
\hline $\begin{array}{l}\text { BIBLIOGRAPHCLEAR REGULATORY COMMISSION } \\
\text { (See instructions on the reverse) }\end{array}$ & $\begin{array}{l}\text { 1. REPORT NUMBER } \\
\text { (Assignod by NRC, Add Vol., Supp., Rev., } \\
\text { and Addendum Numbers, if any.) } \\
\text { NUREG/CR-6554 } \\
\text { BNL-NUREG-52530 }\end{array}$ \\
\hline 2. TMLE AND SUBTILE & \\
\hline & 3. DATE REPORT PUBLISHED \\
\hline Finite Element Analyses for Seismic Shear Wall International Standard Problem & \begin{tabular}{c|r} 
MONTH & YEAR \\
ApriT & 1998 \\
\end{tabular} \\
\hline & $\begin{array}{l}\text { 4. FIN OR GRANT NUMBER } \\
\text { W6249. }\end{array}$ \\
\hline 5. AUTHOR(S) & $\begin{array}{l}\text { 6. TYPE OF REPORT } \\
\text { Technical }\end{array}$ \\
\hline Y. J.Park, C. H. Hofmayer & $\begin{array}{l}\text { 7. PERIOD COVERED (melusive Dates) } \\
06 / 30 / 94-09 / 30 / 97\end{array}$ \\
\hline $\begin{array}{l}\text { 8. PERFORMING ORGANZATION - NAME AND ADDRESS (fF NRC, provide Division, Offiee or Region, U.S. Nuctear Regulatory Commission, anc } \\
\text { name and mailing odtross.) } \\
\text { Department of Advanced Technology } \\
\text { Brookhaven National Laboratory } \\
\text { Upton, NY } 11973-5000\end{array}$ & mailing addresss, if contractor, provide \\
\hline 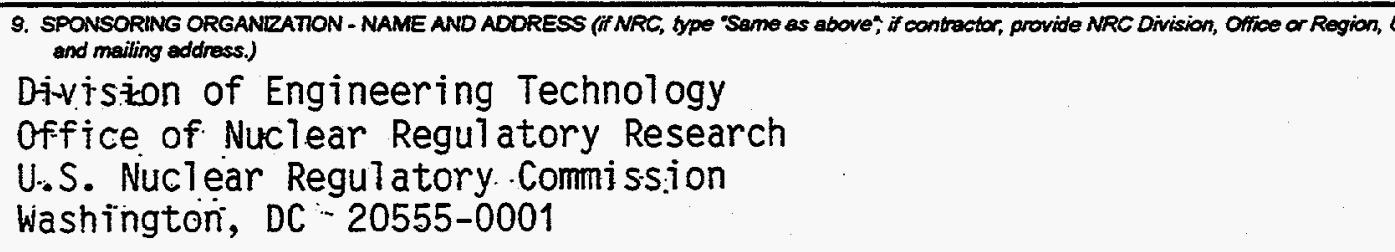 & 1.S. Nucteor Regulatory Commission, \\
\hline $\begin{array}{l}\text { 10. SUPPLEMENTARY NOTES } \\
\text { N. C. Chokshi, NRC Project Manager }\end{array}$ & \\
\hline 11. ABSTRACT (200 words or less) & \\
\hline $\begin{array}{l}\text { Two identical reinforced concrete (RC) shear walls, which consist of web, flanges and mas } \\
\text { were tested up to ultimate failure under earthquake motions at the Nuclear Power Enginee } \\
\text { Tadotsu Engineering Laboratory, Japan. NUPEC provided the dynamic test results to the } \\
\text { Economic Cooperation and Development), Nuclear Energy Agency (NEA) for use as an Int } \\
\text { (ISP). The shear walls were intended to be part of a typical reactor building. One of the ma } \\
\text { Shear Wall ISP (SSWISP) was to evaluate various seismic analysis methods for concrete } \\
\text { seismic margin assessment. It also offered a unique opportunity to assess the state-of-the- } \\
\text { analysis of reinforced concrete shear wall structures under severe earthquake loadings. As } \\
\text { workshops, Brookhaven National Laboratory (BNL) performed finite element analyses unde } \\
\text { Nuclear Regulatory Commission (USNRC). Three types of analysis were performed, i.e., } \\
\text { cyclic static and dynamic analyses. Additional monotonic static analyses were performed b } \\
\text { of the University of Toronto (UT) and F. Filippou of the University of California at Berkeley (l) }\end{array}$ & $\begin{array}{l}\text { ive top and bottom slabs, } \\
\text { ing Corporation's (NUPEC) } \\
\text { ECD (Organization for } \\
\text { ernational Standard Problem } \\
\text { jor objectives of the Seismic } \\
\text { ructures used for design and } \\
\text { art in nonlinear dynamic } \\
\text { a participant of the SSWISP } \\
\text { the sponsorship of the U.S. } \\
\text { nonotonic static (push-over), } \\
\text { two consultants, F. Vecchio } \\
\text { JCB). }\end{array}$ \\
\hline $\begin{array}{l}\text { The analysis results by BNL and the consultants were presented during the second worksh } \\
\text { 1996. A total of } 55 \text { analyses were presented during the workshop by } 30 \text { participants from } 1 \\
\text { major findings on the presented analysis methods, as well as engineering insights regarding } \\
\text { of the FEM codes are described in detail in this report. }\end{array}$ & $\begin{array}{l}\text { op in Yokohama, Japan in } \\
1 \text { different countries. The } \\
\text { the applicability and reliability }\end{array}$ \\
\hline 12 KEY WORDSIDESCRRPTORS (List words or phrases that will assist researchers in loceding the report.) & 13. AVALABILITY STATEMENT \\
\hline Reinforced Concrete, Shear Walls, Seismic Analysis, Shaking Table Tests, Finite Element & Unlimited \\
\hline Model, Dynamic Analysis, Constitutive Model, Nonlinear Analysis & $\begin{array}{l}\text { 14. SECURTY CLASSIFICATION } \\
\text { (This Page) } \\
\text { Unclassified } \\
\text { (This Report) } \\
\text { Unclassified }\end{array}$ \\
\hline & 15. NUMBER OF PAGES \\
\hline & 16. PRICE \\
\hline
\end{tabular}

